\title{
WestVirginiaUniversity
}

THE RESEARCH REPOSITORY @ WVU

Graduate Theses, Dissertations, and Problem Reports

2013

\section{Natural Kenaf Fiber Reinforced Composites as Engineered Structural Materials}

David B. Dittenber

West Virginia University

Follow this and additional works at: https://researchrepository.wvu.edu/etd

\section{Recommended Citation}

Dittenber, David B., "Natural Kenaf Fiber Reinforced Composites as Engineered Structural Materials" (2013). Graduate Theses, Dissertations, and Problem Reports. 280.

https://researchrepository.wvu.edu/etd/280

This Dissertation is protected by copyright and/or related rights. It has been brought to you by the The Research Repository @ WVU with permission from the rights-holder(s). You are free to use this Dissertation in any way that is permitted by the copyright and related rights legislation that applies to your use. For other uses you must obtain permission from the rights-holder(s) directly, unless additional rights are indicated by a Creative Commons license in the record and/ or on the work itself. This Dissertation has been accepted for inclusion in WVU Graduate Theses, Dissertations, and Problem Reports collection by an authorized administrator of The Research Repository @ WVU.

For more information, please contact researchrepository@mail.wvu.edu. 


\title{
Natural Kenaf Fiber Reinforced Composites as Engineered Structural Materials
}

\author{
David B. Dittenber
}

\author{
Dissertation submitted to the \\ College of Engineering and Mineral Resources at \\ West Virginia University in \\ partial fulfillment of the requirements \\ for the degree of
}
Doctor of Philosophy
in
Civil Engineering

\author{
Approved by \\ Hota V.S. GangaRao, PhD, PE, Chair \\ Udaya B. Halabe, PhD, PE \\ Lian-Shin Lin, PhD, PE \\ Ruifeng Liang, $\mathrm{PhD}$ \\ Ever J. Barbero, PhD \\ Stephen R. Ayers, PhD
}

\section{Department of Civil and Environmental Engineering}

\author{
Morgantown, West Virginia \\ 2013
}

Keywords: innovative materials; sustainability; weathering; structural insulated panels 


\title{
ABSTRACT \\ Natural Kenaf Fiber Reinforced Composites as Engineered Structural Materials
}

\author{
David B. Dittenber \\ Constructed Facilities Center, West Virginia University
}

Compared to most synthetic fibers, natural fibers are low-cost, easy to handle, have good strength- and stiffness-to-weight ratios, and require only around $20-40 \%$ of the production energy. Natural composites also offer the long-term potential to create large-volume, biodegradable structural components using only renewable resources. Two of the main challenges facing natural fiber reinforced polymer (NFRP) composites' adoption as infrastructure materials are their higher moisture absorption and lower mechanical properties when compared to synthetic composites. The objective of this work was to examine these issues and to provide a comprehensive evaluation of NFRP's ability to act as a structural material. Issues explored in this evaluation include: chemical treatment of fibers to reduce moisture absorption and improve bonding, mechanical characterization of laminates, weathering and durability, manufacture of large-scale components, theoretical modeling, and sustainability assessment.

As a chemical treatment, aligned kenaf fibers were treated with sodium hydroxide (alkalization) in different concentrations and durations and then manufactured into kenaf fiber / vinyl ester composite plates. This aligned fiber composite configuration was found to possess flexural strength and stiffness over 2 times higher than for a pultruded natural fabric composite. Single fiber tensile properties and composite flexural properties, both in dry and saturated environments, were assessed. Alkalization was shown to increase single fiber tensile strength by $\sim 35-70 \%$ and composite flexural strength by $\sim 12-15 \%$ over untreated fibers, while stiffness was improved by as much as $50 \%$ in both single fiber tensile tests and composite flexural tests. A treatment of $5 \% \mathrm{NaOH}$ for around 16 hours was found to produce the most promising mechanical results, although with little improvement to moisture performance.

Based on ASTM standard testing, a comparison of flexural, tensile, compressive, and shear mechanical properties was also made between an untreated kenaf fiber reinforced composite, a chemically treated kenaf fiber reinforced composite, a glass fiber reinforced composite, and oriented strand board (OSB). The mechanical properties were evaluated for dry samples, samples immersed in water for 50 hours, and samples immersed in water until saturation ( 2700 hours). On average, at 40\% fiber volume fraction, a kenaf composite with bidirectional reinforcement had a flexural strength and stiffness of $28 \mathrm{ksi}$ and 1.09 Msi, respectively, and a shear strength and stiffness of $6 \mathrm{ksi}$ and $0.35 \mathrm{Msi}$, respectively. At the same volume fraction, a kenaf composite with unidirectional reinforcement had a tensile strength and stiffness of $25 \mathrm{ksi}$ and $3.3 \mathrm{Msi}$, respectively, and a compressive strength and stiffness of $17 \mathrm{ksi}$ and 3.35 Msi, respectively. When the mechanical properties of the kenaf composite were divided by the material density to get the specific properties, they were found to be comparable to the glass fiber composite's specific properties.

Since NFRPs are more vulnerable to environmental effects than synthetic fiber composites, a series of weathering and environmental tests were conducted on the kenaf fiber composites. The environmental conditions studied include real-time outdoor weathering, elevated temperatures, immersion in different $\mathrm{pH}$ solutions, and UV exposure. In all of these 
tests, degradation was found to be more pronounced in the NFRPs than in the glass FRPs; however, in nearly every case the degradation was less than $50 \%$ of the flexural strength or stiffness.

Using a method of overlapping and meshing discontinuous fiber ends, large mats of fiber bundles were manufactured into composite facesheets for structural insulated panels (SIPs). The polyisocyanurate foam cores proved to be poorly matched to the strength and stiffness of the NFRP facesheets, leading to premature core shear or delamination failures in both flexure and compressive testing. Despite the poor performance due to the core material, theoretical calculations show that kenaf SIPs offer the potential to be 2-4 times stronger and more than 2.5 times stiffer than the OSB SIPs that are already common in the construction industry, if significant improvements can be made to the core and the core-facesheet interface.

The NFRPs were found to match well with the theoretical stiffness prediction methods of classical lamination theory, finite element method, and Castigliano's method in unidirectional tension and compression, but are less accurate for the more bond-dependent flexural and shear properties. With the acknowledged NFRP matrix bonding issues, the over-prediction of these theoretical models indicates that the flexural stiffness of the kenaf composite may be increased by up to $40 \%$ if a better bond between the fiber and matrix can be obtained. Natural FRP strength prediction using maximum stress and strain criteria was again found to be relatively accurate in unidirectional tension and compression and significantly less so in flexure and shear.

The sustainability of NFRPs was examined from two perspectives: environmental and socioeconomic. The environmental sustainability was assessed by a simple comparison of five International Residential Code-based wall designs: OSB SIPs, glass fiber composite SIPs, kenaf fiber composite SIPs, stick-framing, and insulating concrete forms. The environmental impact metrics of embodied energy and contributed $\mathrm{CO}_{2}$ were examined for each of the designs. The analysis results indicated that the stick-framing and OSB SIP wall designs were the cheapest and most eco-friendly, while the composite SIPs were the least eco-friendly. While the kenaf fibers themselves possess excellent sustainability characteristics, costing less while possessing a lesser environmental impact than the glass fibers, the vinyl ester resin used in the composites is environmentally hazardous and inflated the cost and embodied energy of the composite SIPs. Consistent throughout all the designs was a correlation between the respective costs of the raw materials and the respective environmental impacts.

The socioeconomic study looked at the sustainability of natural fiber reinforced composite materials as housing materials in developing countries. A literature study on the country of Bangladesh, where the fibers in this study were grown, showed that the jute and kenaf market would benefit from the introduction of a value-added product like natural fiber composites. The high rate of homeless and inadequately housed in Bangladesh, as well as in the US and throughout the rest of the world, could be somewhat alleviated if a new, affordable, and durable material were introduced. While this study found that natural fiber composites possess sufficient mechanical properties to be adopted as primary structural members, the two major remaining hurdles needing to be overcome before natural fiber composites can be adopted as housing materials are the cost and sustainability of the resin system and the moisture resistance/durability of the fibers. 


\section{ACKNOWLEDGEMENTS}

Oh, the depth of the riches both of the wisdom and knowledge of God! How unsearchable are His judgments and unfathomable His ways! For who has known the mind of the Lord, or who became His counselor? Or who has first given to Him that it might be paid back to him again? For from Him and through Him and to Him are all things. To Him be the glory forever. Amen.

Romans 11:33-36

I would like to thank my advisor, Dr. Hota GangaRao, for encouraging me to apply for a research fellowship, for helping me develop this research plan, for giving constant feedback on the results and drafts I passed along to him, for serving as the chair of my review committee, and especially for guiding me as I have grown as a researcher and an academic over the past several years.

I would also like to thank the other members of my review committee for spending the time to evaluate my work and provide constructive feedback: Dr. Udaya Halabe, Dr. Lian-Shin Lin, Dr. Ruifeng Liang, Dr. Ever Barbero, and Dr. Stephen Ayers.

Many people at West Virginia University provided assistance with this research work over the past five years. Among faculty and staff, my thanks go out to Mark Skidmore for technical assistance, to Jerry Nestor for manufacturing assistance, to Lynne Jacobs for administrative assistance, and to Dr. Xueyan Song, Dr. Sushant Agarwal, Dr. John Zondlo, and Gabriela Perhinschi for sharing their equipment. Among my fellow students, I would especially like to thank Praveen Majjigapu, who helped with much of the composite manufacturing work and environmental testing, and acknowledge the contributions of many others: Fatimah Saleem, Brian Walker, M. Asif Qureshi, Ken Donald, Denny Dispennette, and Daniel Estep.

I am also grateful to several members of industry who were also supportive throughout this research, providing advice, materials, or both: Hugh McKee of Bast Fibers, LLC, Bhyrav Mutnuri and Bridgett Diehl of Bedford Reinforced Plastics, Bob Pfeifer of Fiber-Tech Industries, Inc., Charlie Fisher of Ashland, Larry Dickinson of 3F, LLC, Dustin Troutman of Creative Pultrusions, Inc., Daniel Heberer of Huntsman International, LLC, and Jim Steinmetz of Gelest, Inc.

Funding for this work was provided by a National Science Foundation Graduate Research Fellowship, with a supplementary summer ASEE Engineering Innovation Fellowship served at Bedford Reinforced Plastics. I would like to thank NSF and ASEE for providing me with this opportunity to focus exclusively on my research and both the WVU graduate school and BRP for administering the fellowship programs.

Finally, I am very grateful for my family and friends who have supported me during my time at West Virginia University, especially my wonderful wife Rachel, who has never known me to not be working on this dissertation. Needless to say, we are both excited to see what life has in store for us after it is finished! 


\section{TABLE OF CONTENTS}

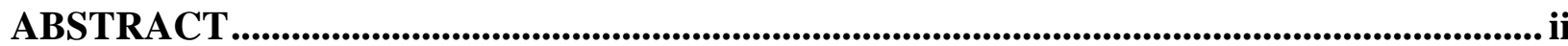

ACKNOWLEDGEMENTS ……........................................................................................... iv

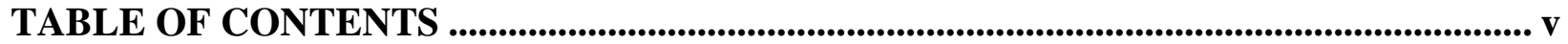

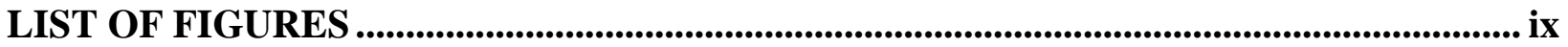

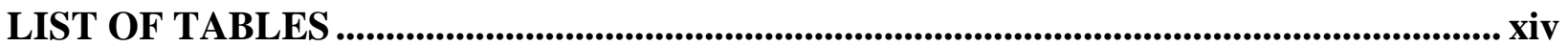

NOMENCLATURE AND UNITS................................................................................. xvii

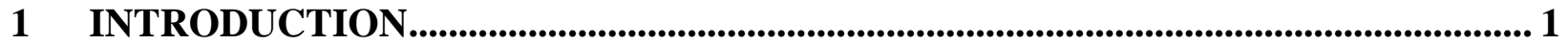

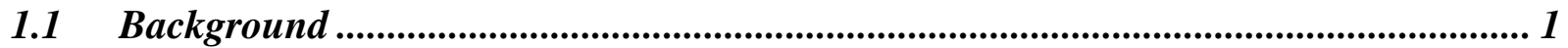

1.2 Objectives............................................................................................................................ 1

1.3 Organization ....................................................................................................................... 2

2 LITERATURE REVIEW .................................................................................... 4

2.1 Abstract................................................................................................................................ 4

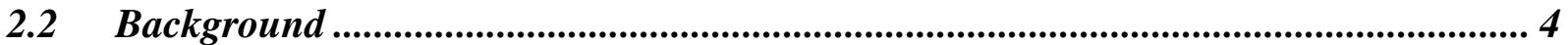

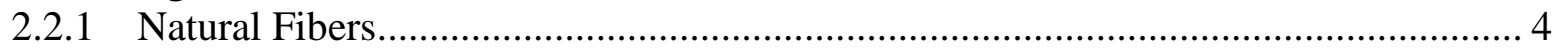

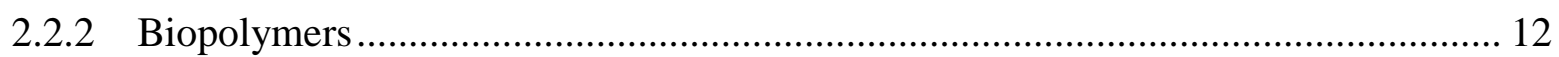

2.2.3 Natural Fiber Reinforced Composites - Mechanical Properties.................................. 13

2.2.4 Composites as Construction Materials.................................................................... 16

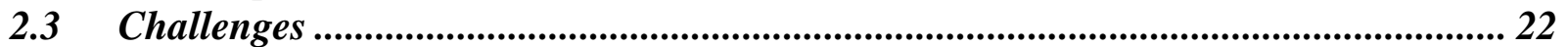

2.3.1 Moisture Absorption / Hydrophilic Nature............................................................... 22

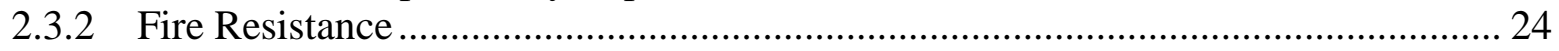

2.3.3 Mechanical Properties and Durability ................................................................... 25

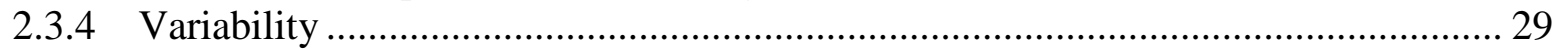

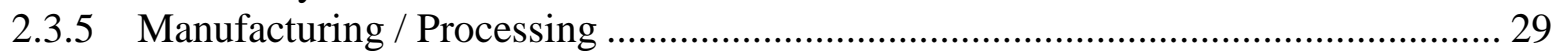

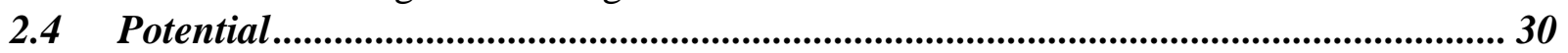

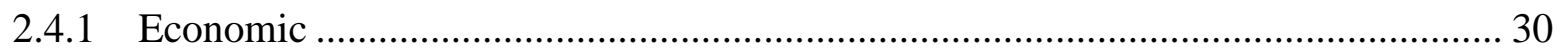

2.4.2 Environmental............................................................................................... 31

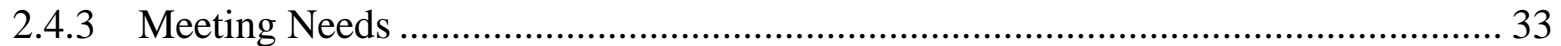

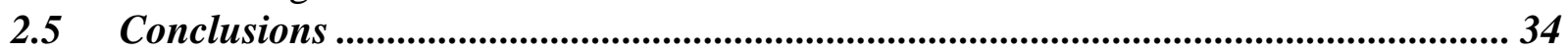

3 PRELIMINARY TESTING RESULTS …......................................................................... 35

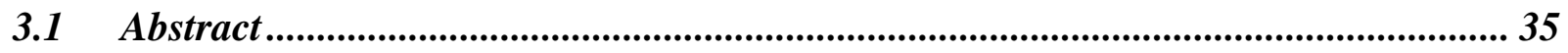

3.2 Introduction and Scope...................................................................................................... 35

3.3 Materials and Manufacturing Processes ..................................................................... 35

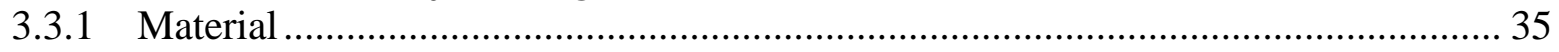

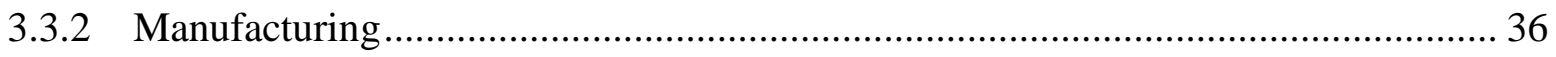

3.3.3 Sample Preparation .................................................................................................. 38

3.4 Experimental Methodology.............................................................................................. 39

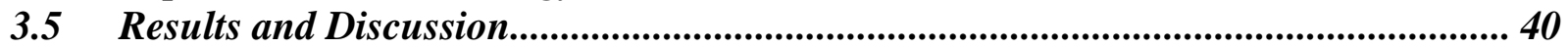

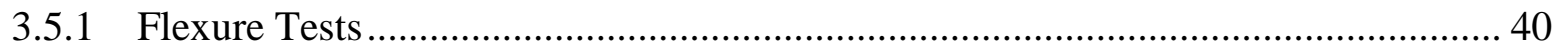

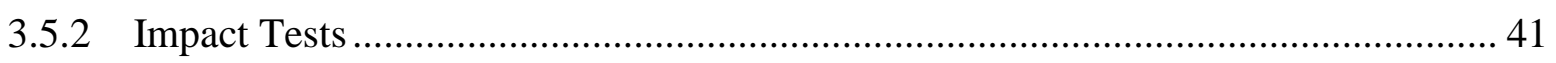

3.5.3 Single Fiber Tension Tests................................................................................ 42

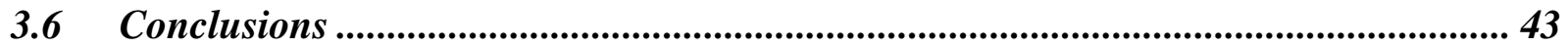

4 FIBER SURFACE TREATMENT CHARACTERIZATION......................................... 44

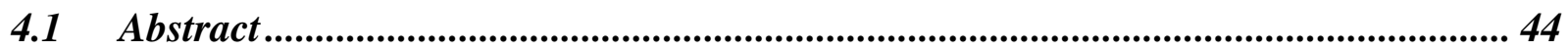

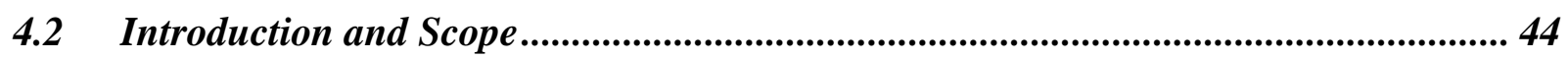


4.3 Materials and Manufacturing Processes ...................................................................... 45

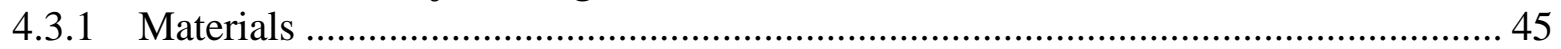

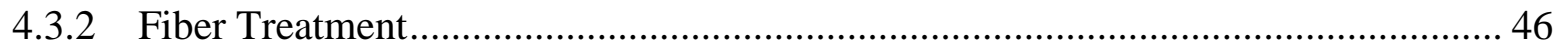

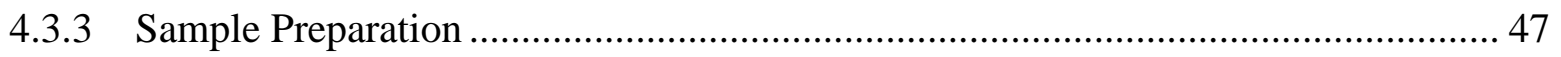

4.4 Experimental Methodology................................................................................................ 50

4.4.1 Single Fiber Tensile Testing …………………................................................... 51

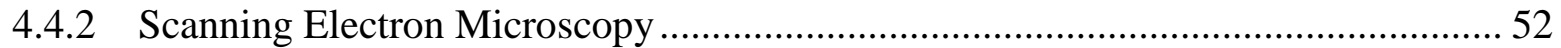

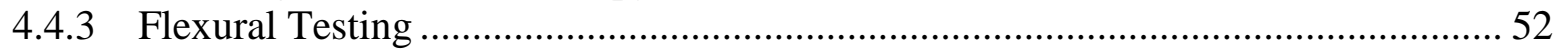

4.4.4 Moisture Absorption Testing ..................................................................................... 53

$4.5 \quad$ Results and Discussion................................................................................................. 53

4.5.1 Single Fiber Tension Testing ............................................................................ 53

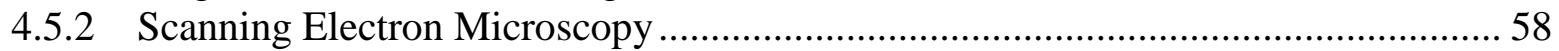

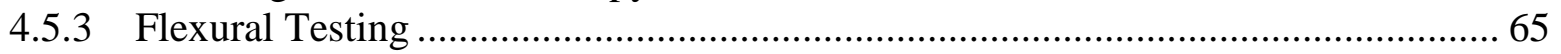

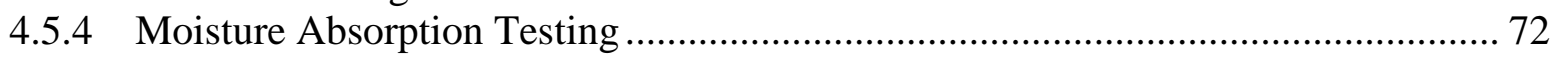

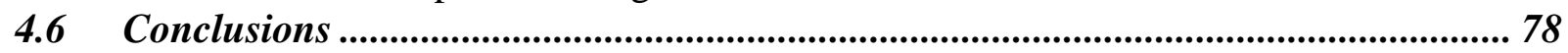

5 MECHANICAL CHARACTERIZATION ..................................................................... 79

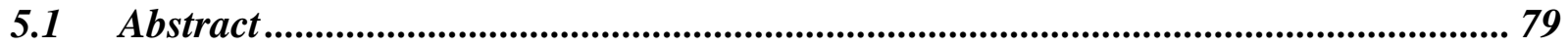

5.2 Introduction and Scope....................................................................................................... 79

5.3 Materials and Manufacturing Processes .......................................................................... 80

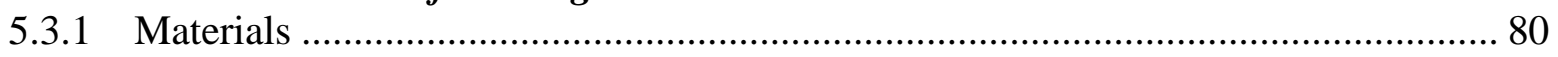

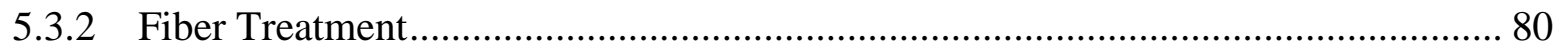

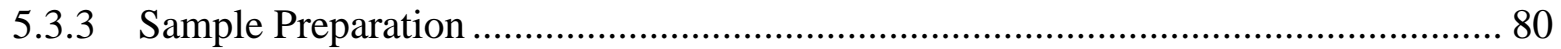

5.4 Experimental Methodology.................................................................................................... 83

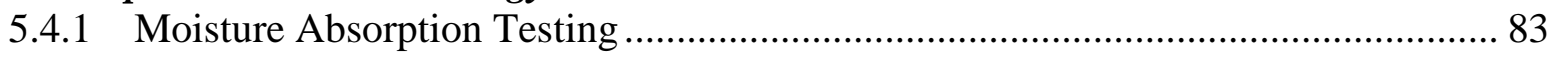

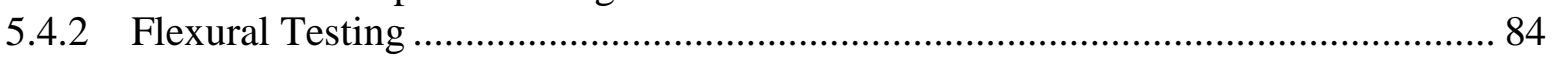

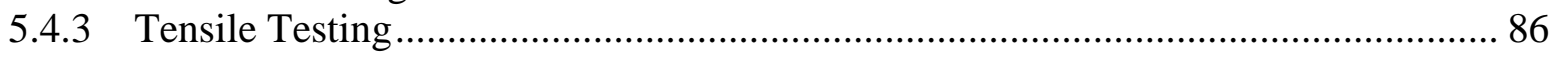

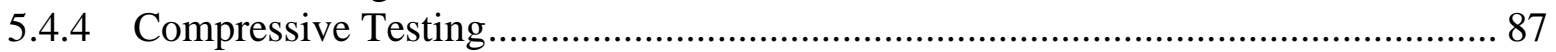

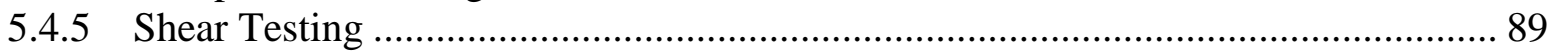

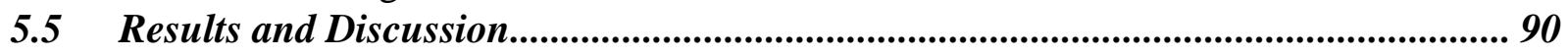

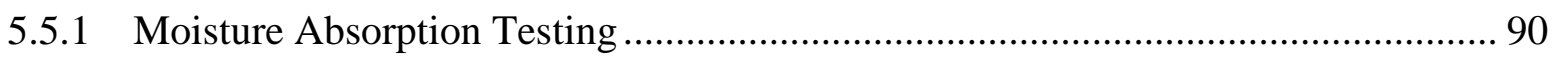

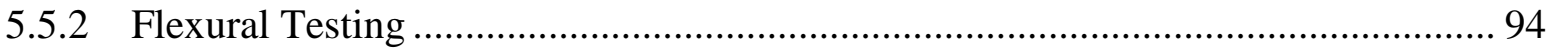

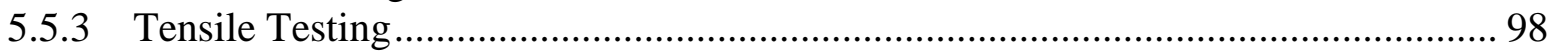

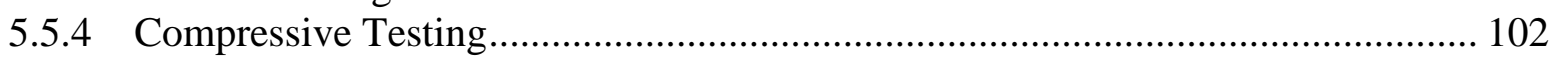

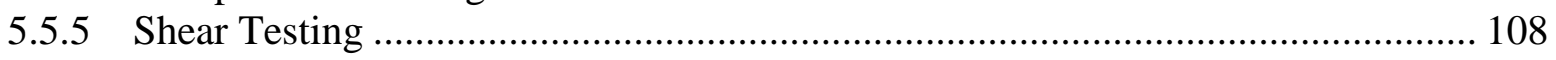

5.5.6 Specific Properties ........................................................................................... 111

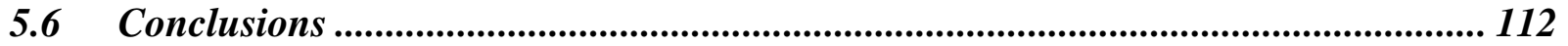

6 WEATHERING AND ENVIRONMENTAL TESTING ................................................ 114

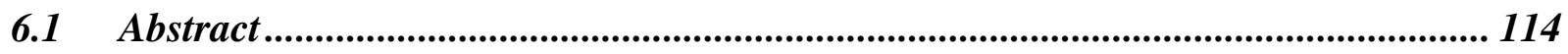

6.2 Introduction and Scope....................................................................................................... 114

6.3 Materials and Manufacturing Processes ...................................................................... 115

6.3.1 Materials ............................................................................................... 115

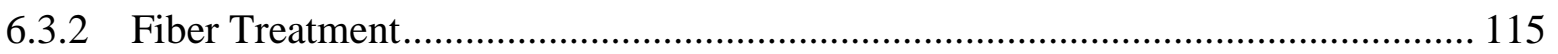

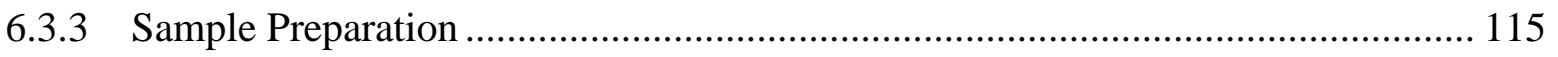

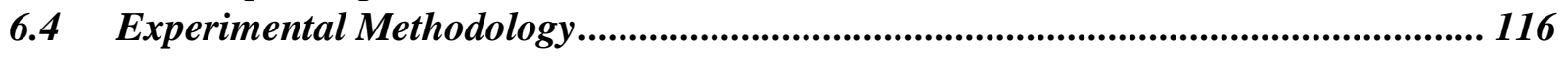

6.4.1 Real-time Weathering Tests................................................................................... 116

6.4.2 Accelerated Aging Tests ........................................................................................ 119 


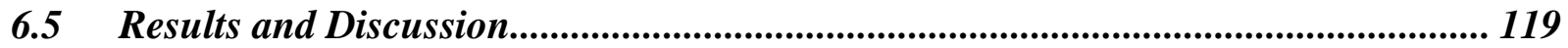

6.5.1 Real-time Weathering Tests.......................................................................... 119

6.5.2 Accelerated Aging Tests ....................................................................................... 125

6.6 Design Considerations ............................................................................................... 134

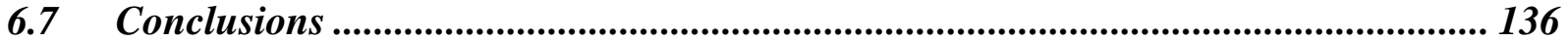

7 STRUCTURAL COMPONENT TESTING ..................................................................... 138

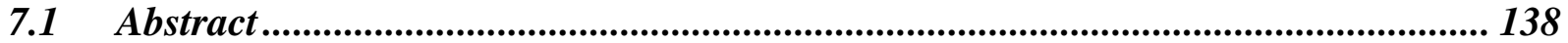

$7.2 \quad$ Introduction and Scope.................................................................................. 138

$7.3 \quad$ Materials and Manufacturing Processes .................................................................... 138

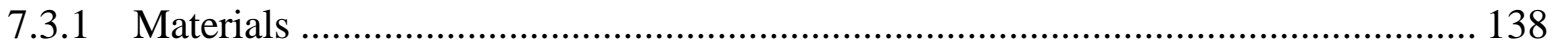

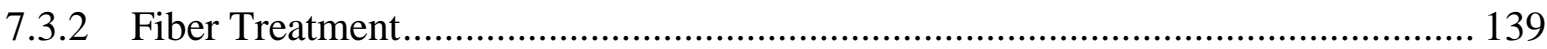

7.3.3 Sample Preparation .......................................................................................... 139

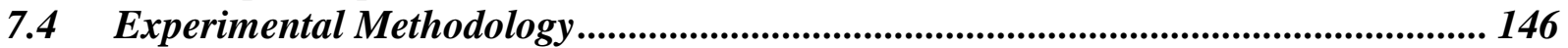

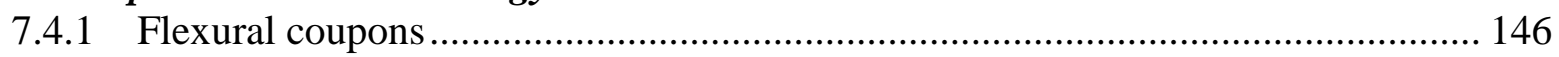

7.4.2 Flexure test setup .......................................................................................... 147

7.4.3 Compressive test setup.................................................................................. 148

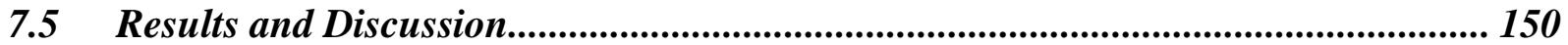

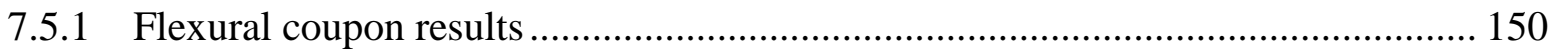

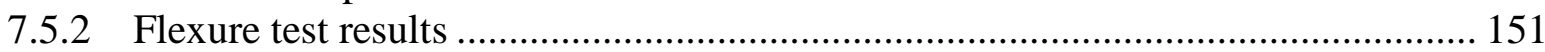

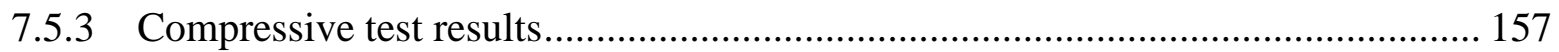

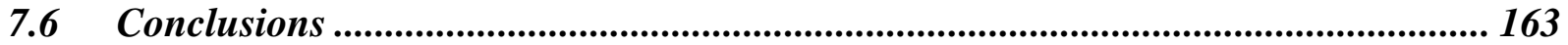

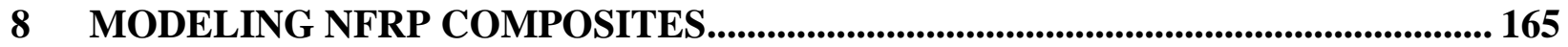

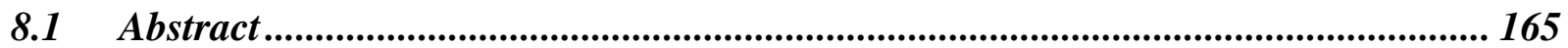

8.2 Introduction and Scope................................................................................................. 165

8.2.1 Mathematical Modeling of Composites.................................................................. 165

8.2.2 Properties and Assumptions................................................................................ 166

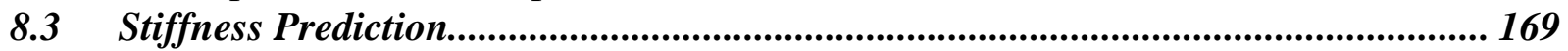

8.3.1 Lamina Engineering Constants ................................................................................ 169

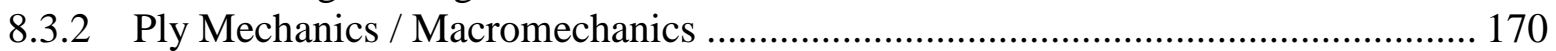

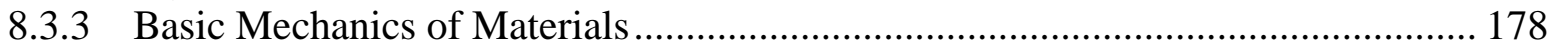

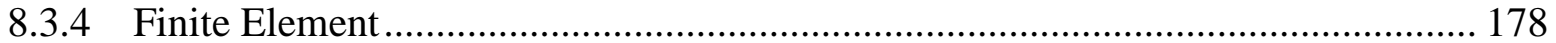

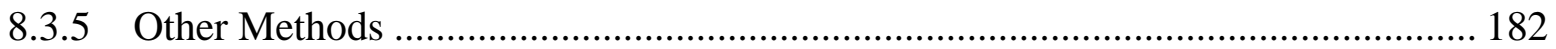

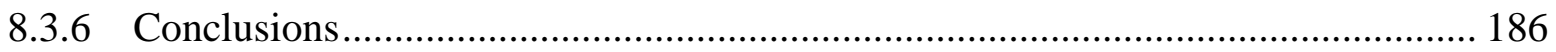

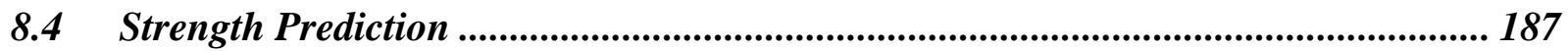

8.4.1 Lamina Strength and Strain Properties .................................................................... 187

8.4.2 Laminate Strain and Stress............................................................................... 190

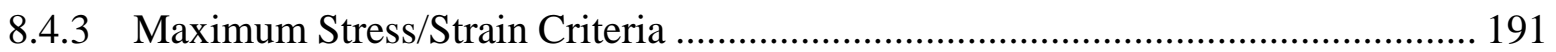

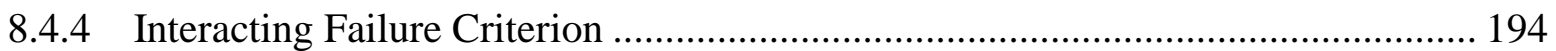

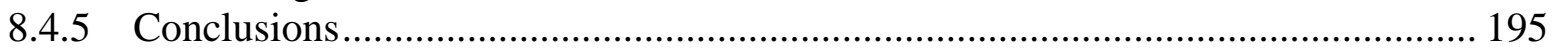

8.5 Moisture Effects ............................................................................................................ 195

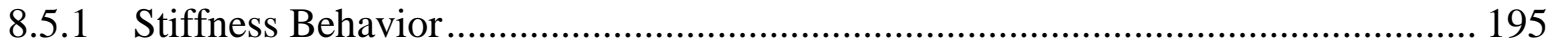

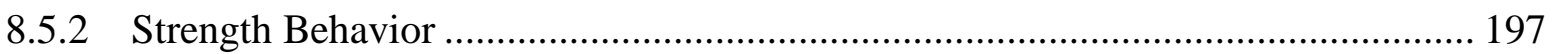

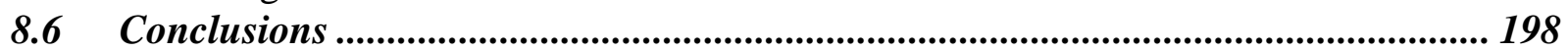

9 SUSTAINABILITY OF NFRP COMPOSITES .............................................................. 200

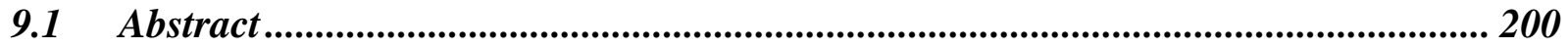

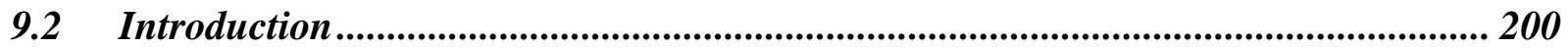


$9.3 \quad$ Environmental Impacts.................................................................................................... 201

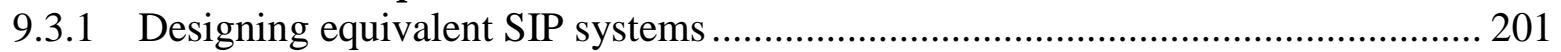

9.3.2 Material Usage for each Wall Design .................................................................. 204

9.3.3 Environmental Impact for each Wall Design............................................................ 207

9.4 Socioeconomic impacts .................................................................................................... 218

9.4.1 Bangladesh Case Study …….......................................................................... 218

9.5 Conclusions ..................................................................................................................... 224

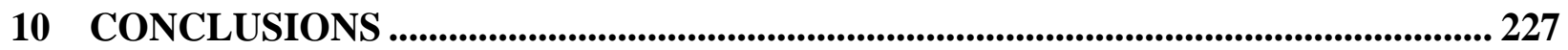

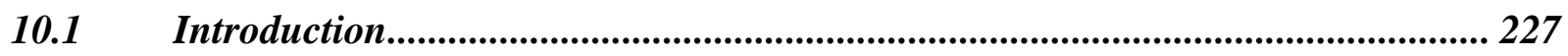

$10.2 \quad$ Main Chapter Conclusions.............................................................................................. 227

10.2.1 Literature Review Conclusions ......................................................................... 227

10.2.2 Preliminary Testing Conclusions ........................................................................ 228

10.2.3 Fiber Surface Treatment Characterization Conclusions ...................................... 228

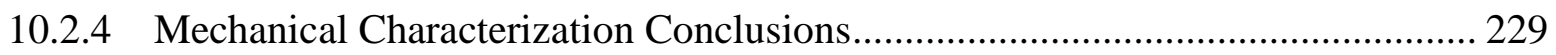

10.2.5 Weathering and Environmental Testing Conclusions............................................ 230

10.2.6 Structural Component Testing Conclusions …………............................................. 231

10.2.7 Modeling NFRP Composites Conclusions ……….............................................. 231

10.2.8 Sustainability of NFRP Composites Conclusions ................................................ 232

$10.3 \quad$ Specific Contributions.............................................................................................. 233

$10.4 \quad$ Recommendations .......................................................................................................... 234

APPENDIX A - MECHANICAL TESTING RESULTS.................................................. 236

APPENDIX B - MONTHLY WEATHERING SAMPLE IMAGES....................................... 242

APPENDIX C - EIO-LCA DETAILED RESULTS................................................................... 248

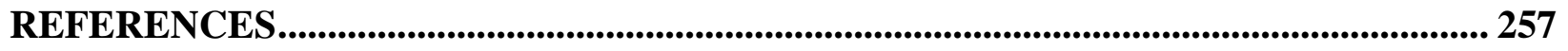




\section{LIST OF FIGURES}

Figure 1-1 - Chapter flow and key topics.................................................................................. 3

Figure 2-1 - General structure of natural fibers (John and Anandjiwala 2008) ……..................... 5

Figure 2-2 - Relationship between tensile properties and fiber location within kenaf plant (Ochi 2002)...

Figure 2-3 - Comparison of potential specific modulus value ranges between natural fibers and

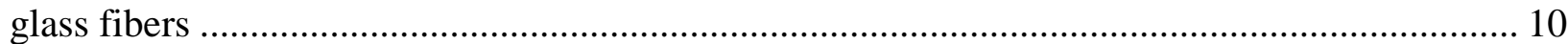

Figure 2-4 - Cost per weight comparison between glass and natural fibers ................................. 10

Figure 2-5 - Cost per unit length (capable of resisting $100 \mathrm{kN}$ load) comparison between glass and natural fibers

Figure 2-6 - Relationship between tensile mechanical properties and kenaf fiber volume fraction

(Ochi 2008) 14

Figure 2-7 - Relationship between flexural mechanical properties and kenaf fiber volume

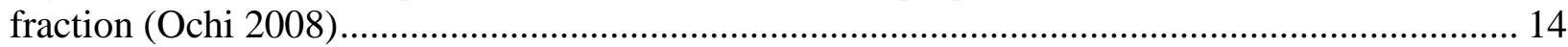

Figure 2-8 - Construction with SIPs (Johnston and Gibson 2008) ………………………….... 19

Figure 2-9 - Results of BASF Life-Cycle Analysis of traditional SIPs (SIPA 2009) .................... 20

Figure 3-1 - Compression molded flax/phenolic plate ( 10 in x 15 in)........................................ 37

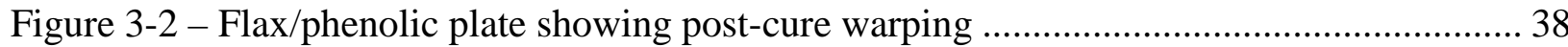

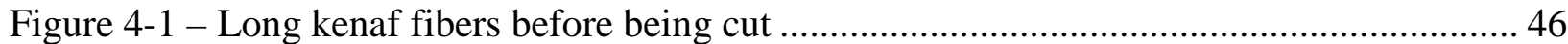

Figure 4-2 - Fibers cut to size, before surface treatment............................................................. 46

Figure 4-3 - Dried, treated kenaf fibers ................................................................................ 48

Figure 4-4 - Combed fibers and tension clamps for stitching ..................................................... 48

Figure 4-5 - Stitched mats laid out in quadrants in mold ............................................................. 49

Figure 4-6 - Completed kenaf-reinforced composite plate ......................................................... 50

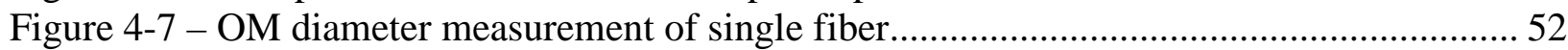

Figure 4-8 - Flexural testing of coupons ............................................................................. 52

Figure 4-9 - Average single fiber ultimate tensile strength (with SD bars) …………………....... 54

Figure 4-10 - Average single fiber tensile stiffness (with SD bars) ........................................... 54

Figure 4-11 - Weibull distribution of tensile strength of kenaf fibers $(\alpha=125.9, \beta=1.957) \ldots \ldots . . .57$

Figure 4-12 - Weibull distribution of tensile strain in kenaf fibers $(\alpha=0.0117, \beta=2.408)$........... 57

Figure 4-13 - Weibull distribution of tensile stiffness in kenaf fibers $(\alpha=17.1, \beta=2.431) \ldots \ldots \ldots . . .58$

Figure 4-14 - Untreated fibers in D510A matrix, different fiber shapes and sizes ...................... 58

Figure 4-15 - Untreated fibers in D510A matrix, filaments and lumens, poor bonding ............... 59

Figure 4-16 - Untreated fibers in D510A matrix, longitudinal structure ..................................... 59

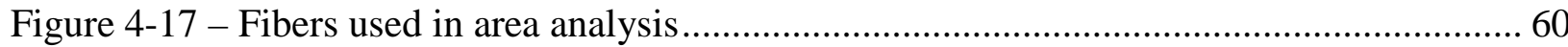

Figure 4-18 - 5\%, 40 min treated fibers in D510A matrix, fiber distribution...................................... 62

Figure 4-19 - 5\%, 40 min treated fibers in D510A matrix, poor bonding ……………….............. 62

Figure 4-20 - 5\%, 40 min treated fibers in D510A matrix, longitudinal, poor bonding ............... 63

Figure 4-21 - 5\%, 16 hrs treated fibers in D510A matrix, cracks and voids................................... 64

Figure 4-22 - 5\%, 16 hrs treated fibers in D510A matrix, reduced gap size and penetration....... 64

Figure 4-23 - 5\%, 16 hrs treated fibers in D510A matrix, improved bonding....................................... 65

Figure 4-24 - 5\%, 16 hrs treated fibers in D510A matrix, longitudinal, poor bonding ................. 65

Figure 4-25 - Longitudinal flexural strength of manufactured composites ..................................... 66

Figure 4-26 - Longitudinal flexural stiffness of manufactured composites .................................... 67

Figure 4-27 - Transverse flexural strength of manufactured composites....................................... 67 
Figure 4-28 - Transverse flexural stiffness of manufactured composites ..................................... 68

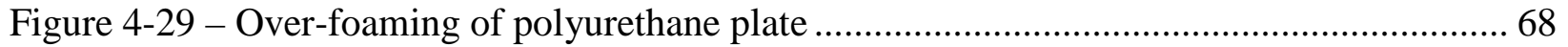

Figure 4-30 - Polyurethane plate removed from mold............................................................ 69

Figure 4-31 - Longitudinal flexural strength normalized for $40 \% \mathrm{FVF}\left[0_{4} /\right.$ fabric $/ 90_{4} /$ fabric $\leftarrow$

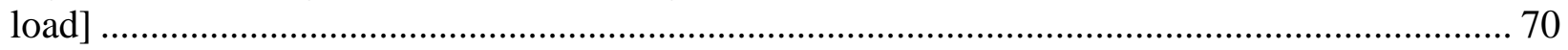

Figure 4-32 - Longitudinal flexural stiffness normalized for 40\% FVF $\left[0_{4} /\right.$ fabric $/ 90_{4} /$ fabric $\leftarrow$

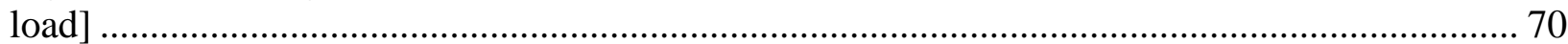

Figure 4-33 - Transverse flexural strength normalized for $40 \%$ FVF $\left[90_{4} /\right.$ fabric $/ 0_{4} /$ fabric $\leftarrow$

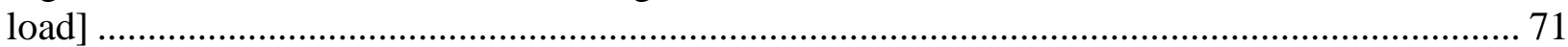

Figure 4-34 - Transverse flexural stiffness normalized for 40\% FVF $\left[90_{4} /\right.$ fabric $/ 0_{4} /$ fabric $\leftarrow$

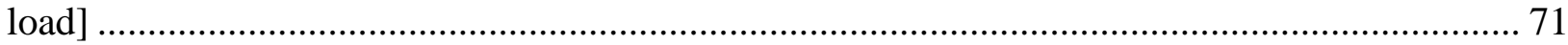

Figure 4-35 - Moisture absorption rate of untreated fiber / Derakane 510A composite ............... 72

Figure 4-36 - Relative moisture absorption rate over first 50 hours ............................................. 73

Figure 4-37 - Moisture absorption equilibrium, in \% total weight of composite.......................... 73

Figure 4-38 - Relative moisture absorption rate over first 50 hours .......................................... 74

Figure 4-39 - Moisture absorption equilibrium, in \% weight of fiber reinforcement ................... 74

Figure 4-40 - Flexural strength/stiffness retention for saturated Derakane 510A samples........... 77

Figure 5-1 - Layups for natural fiber reinforced composite mechanical test samples .................. 81

Figure 5-2 - Prepared coupons for mechanical testing (from left, GFRP, OSB, untreated NFRP,

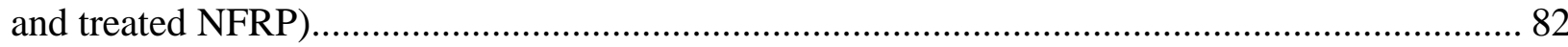

Figure 5-3 -Treated natural fiber reinforced composite coupons for mechanical testing (from top,

left to right: tensile, shear, D695 compression, D3410 compression, and flexure) ....................... 82

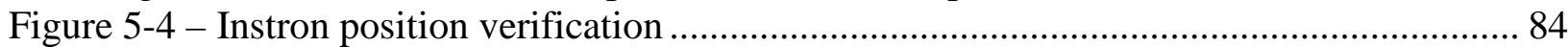

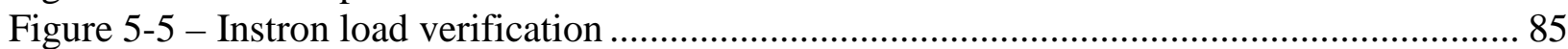

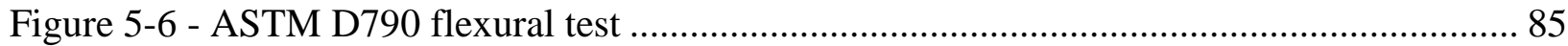

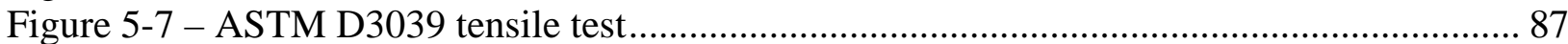

Figure 5-8 - ASTM D695 compressive test with support jig....................................................... 88

Figure 5-9 - ASTM D3410 compressive test (C-clamp only to keep sample from falling over

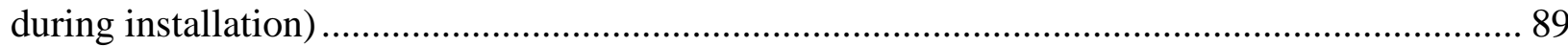

Figure 5-10 - Iosipescu / V-notch shear test (ASTM D5379)..................................................... 90

Figure 5-11 - Plot of moisture absorption rates for untreated and treated kenaf fiber reinforced

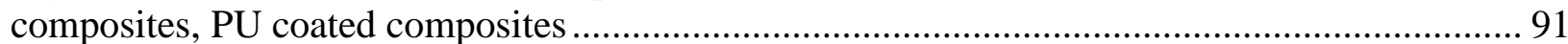

Figure 5-12 - Logarithmic plot of moisture absorption rates from Figure 5-11 ........................... 92

Figure 5-13 - Load-deflection plots for dry, flexural, untreated kenaf samples ........................... 94

Figure 5-14 - ASTM D790 flexural strength comparison (with max/min bars) ............................ 95

Figure 5-15 - ASTM D790 tangent modulus comparison (with max/min bars) ........................... 95

Figure 5-16 - Load-deflection plots for saturated, flexural, untreated kenaf samples ................... 96

Figure 5-17 - Kenaf composite tensile face rupture failure mode in flexure ................................ 97

Figure 5-18 - Kenaf composite compressive face local buckling failure mode in flexure .......... 97

Figure 5-19 - Stress-strain plots for dry, tensile, untreated kenaf samples ....................................... 98

Figure 5-20 - ASTM D3039 tensile strength comparison (with max/min bars) ............................ 99

Figure 5-21 - ASTM D3039 tensile chord modulus comparison (with max/min bars) ................ 99

Figure 5-22 - ASTM D3039 ultimate tensile strain comparison (with max/min bars) ................ 100

Figure 5-23 - Kenaf composite tensile failure with splitting along fabric interface ................... 101

Figure 5-24 - Typical kenaf composite tensile failure due to rupture ......................................... 101 
Figure 5-25 - Stress-strain plots for dry, ASTM D695-based compressive, untreated kenaf

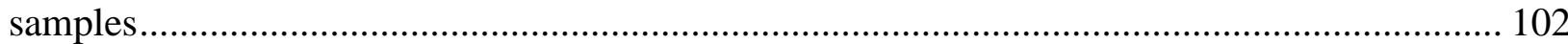

Figure 5-26 - ASTM D695 compressive strength comparison (with max/min bars)................ 103

Figure 5-27 - ASTM D695 modulus of elasticity comparison (with max/min bars)................ 103

Figure 5-28 - ASTM D695 ultimate compressive strain comparison (with max/min bars) ...... 104

Figure 5-29 - ASTM D695 compressive test resulting in shear failure at loading end.............. 105

Figure 5-30 - ASTM D3410 ultimate compressive stress comparison (with max/min bars) .... 106

Figure 5-31 - ASTM D3410 compressive chord modulus comparison (with max/min bars).... 106

Figure 5-32 - ASTM D3410 ultimate compressive strain comparison (with max/min bars) .... 107

Figure 5-33 - Average compressive strength results ...................................................... 107

Figure 5-34 - ASTM D3410-based compressive test sample exhibiting shear failure mode .... 108

Figure 5-35 - Stress-strain plots for dry, shear, untreated kenaf samples................................ 108

Figure 5-36 - ASTM D5379 ultimate in-plane shear strength comparison (with max/min bars)

Figure 5-37 - ASTM D5379 in-plane shear chord modulus comparison (with max/min bars). 109

Figure 5-38 - ASTM D5379 ultimate in-plane shear strain comparison (with max/min bars).. 110

Figure 5-39 - Typical kenaf reinforced composite failure between notches, as seen on fabric

surface.

Figure $6-1$ - Real-time weathering test fixture ........................................................... 117

Figure 6-2 - Omega temperature datalogger (image from www.omega.com)........................ 117

Figure 6-3 - Real-time weathering test fixture installed on roof-top location .......................... 118

Figure 6-4 - Real-time weathering \% weight change, by month ........................................ 121

Figure 6-5 - Real-time weathering \% apparent density change, by month .............................. 122

Figure 6-6 - Real-time weathering flexural strength degradation, by month ........................... 123

Figure 6-7 - Real-time weathering flexural stiffness degradation, by month ......................... 123

Figure 6-8 - Weathering-induced end splitting or "brooming" (9 months) .............................. 125

Figure 6-9 - Weathering-induced cracks along longitudinal fiber layers (9 months) ............... 125

Figure 6-10 - Weathering-induced black areas with higher swelling in transverse fiber layers (11

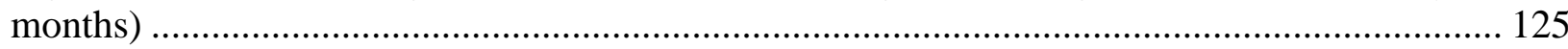

Figure 6-11 - Untreated kenaf strength vs. temperature (with max/min bars)........................ 126

Figure 6-12 - Treated kenaf strength vs. temperature (with max/min bars) ........................... 127

Figure 6-13 - Glass FRP strength vs. temperature (with max/min bars)................................. 127

Figure 6-14 - Untreated kenaf stiffness vs. temperature (with max/min bars) ........................ 127

Figure 6-15 - Treated kenaf stiffness vs. temperature (with max/min bars) ........................... 128

Figure 6-16 - Glass FRP stiffness vs. temperature (with max/min bars) ................................ 128

Figure 6-17 - Glass FRP strength vs. immersion treatment (with max/min bars; \% weight

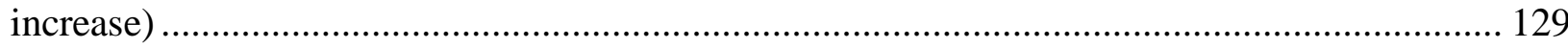

Figure 6-18 - Glass FRP stiffness vs. immersion treatment (with max/min bars; \% weight

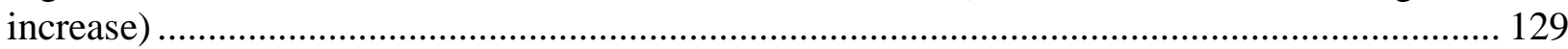

Figure 6-19 - Kenaf FRP strength vs. immersion treatment (with max/min bars; \% weight

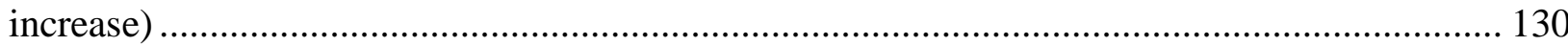

Figure 6-20 - Kenaf FRP stiffness vs. immersion treatment (with max/min bars; \% weight

increase) .................................................................................................................. 130

Figure 6-21 - UV-induced color change for (a) untreated kenaf FRP, (b) treated kenaf FRP, (c)

treated/coated kenaf FRP, (d) glass FRP, and (e) OSB ....................................................... 132

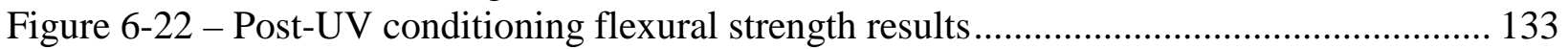


Figure 6-23 - Post-UV conditioning flexural stiffness results ........................................... 133

Figure 7-1 - Different fiber 'joint' types tested .................................................................. 140

Figure 7-2 - Tensile strength comparison between different fiber 'joint' designs.................... 141

Figure 7-3 - Tensile modulus comparison between different fiber 'joint' designs ................... 141

Figure 7-4 - Ultimate Tensile strain comparison between different fiber 'joint' designs.......... 142

Figure $7-5-12$ in x 48 in stitched mats with 12 in long fibers ............................................. 143

Figure 7-6 - 60” x 26” 90-degree and 0-degree layers made up of 5 and 2 separate mats, respectively

Figure 7-7 - Mat placement diagrams for manufacturing of two adjacent 60 ” x 13 ” sandwich

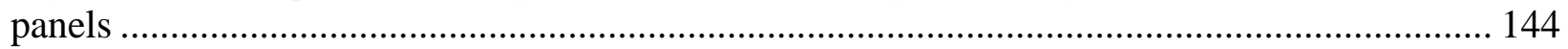

Figure 7-8 - Completed structural insulated panels from Fiber-Tech .................................... 145

Figure 7-9 - As-received delamination at composite/foam interface (worst corner) ................ 146

Figure 7-10 - Flexural coupons from Fiber-Tech Panel cut-off material................................. 147

Figure 7-11 - Skin panel thickness comparison between OSB and NFRP SIPs ...................... 148

Figure 7-12 - Flexural test setup of NFRP SIP ............................................................... 148

Figure 7-13 - Compressive test setup for both NFRP and OSB SIPs (before removal of midspan

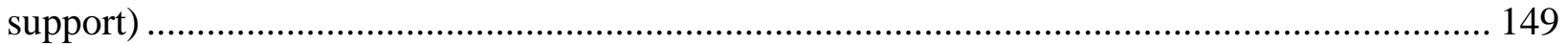

Figure 7-14 - Dry fibers and voids in Fiber-Tech Industries panels (worst cases) ................... 150

Figure 7-15 - Load vs. deflection for flexure of NFRP SIP over 27 in span (not to failure)..... 151

Figure 7-16 - Strain measurements from flexure of NFRP SIP over 27 in span (not to failure) 152

Figure 7-17 - Load vs. deflection for flexure of NFRP SIP over 45 in span (to failure) ........... 153

Figure 7-18 - Strain measurements from flexure of NFRP SIP over 45 in span (to failure) ..... 153

Figure 7-19 - Load vs. deflection for flexure of OSB SIP over 45 in span (to failure) .............. 154

Figure 7-20 - Shear and delamination flexural failures in foam core of NFRP SIP ................. 155

Figure 7-21 - Shear and delamination flexural failures in foam core of OSB SIP .................. 155

Figure 7-22 - Load vs. strain-determined deflection of NFRP SIP in braced compressive test (not

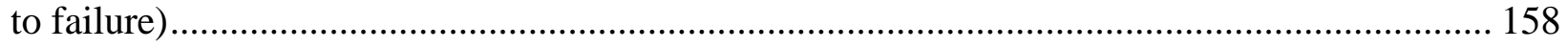

Figure 7-23 - Strain measurements from braced compressive test of NFRP SIP (not to failure)

Figure 7-24 - Stress vs. strain for each facesheet during braced compressive test of NFRP SIP (not to failure) 159

Figure 7-25 - Load vs. strain-determined deflection of NFRP SIP in un-braced compressive test

(to failure) 160

Figure 7-26 - Strain measurements from un-braced compressive test of NFRP SIP (to failure)160 Figure 7-27 - Stress vs. strain for each facesheet during un-braced compressive test of NFRP SIP

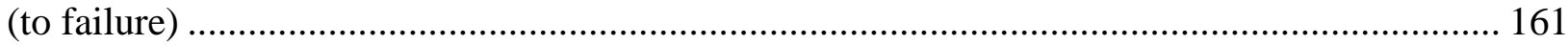

Figure 7-28 - Complete panel delamination compressive failure of NFRP SIP ...................... 162

Figure 7-29 - End-buckling compressive failure of OSB SIP ............................................. 162

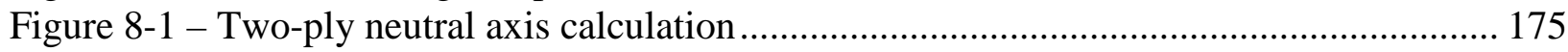

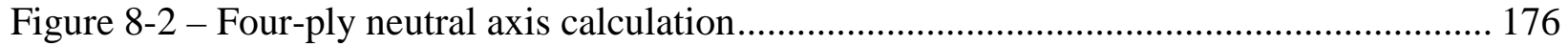

Figure 8-3 - Characteristic FE compression test results, treated kenaf composite, X-displacement 180

Figure 8-4 - Characteristic FE tension test results, treated kenaf composite, X-displacement .. 180 Figure 8-5 - Characteristic FE flexure test results, treated kenaf composite, Z-displacement ... 181 Figure 8-6 - Characteristic FE shear test results, treated kenaf composite, Y-displacement...... 181 Figure 8-7 - Laminate coordinate system and thicknesses................................................... 183 
Figure 9-1 - Partial process flow diagram for NFRP SIP ................................................. 209

Figure 9-2 - Flooded housing in Bangladesh (Ahmed 2005) ................................................ 220

Figure 9-3 - Kutcha-style house in Bangladesh (Ahmed 2005)........................................... 221

Figure 9-4 - Semi-pucca-style house in Bangladesh (Ahmed 2005) ...................................... 221

Figure 9-5 - Pucca-style house in Bangladesh (Ahmed 2005).............................................. 222

Figure B-1 - Samples collected at the end of April 2012; 1 month exposure ........................... 242

Figure B-2 - Samples collected at the end of May 2012; 2 months exposure........................... 242

Figure B-3 - Samples collected at the end of June 2012; 3 months exposure.......................... 243

Figure B-4 - Samples collected at the end of July 2012; 4 months exposure .......................... 243

Figure B-5 - Samples collected at the end of August 2012; 5 months exposure ...................... 244

Figure B-6 - Samples collected at the end of September 2012; 6 months exposure................. 244

Figure B-7 - Samples collected at the end of October 2012; 7 months exposure ...................... 245

Figure B-8 - Samples collected at the end of November 2012; 8 months exposure .................. 245

Figure B-9 - Samples collected at the end of December 2012; 9 months exposure .................. 246

Figure B-10 - Reverse side of samples collected at the end of December 2012; 9 months

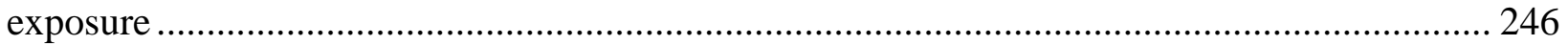

Figure B-11 - Samples collected at the end of January 2013; 10 months exposure ................. 247

Figure B-12 - Samples collected at the end of February 2012; 11 months exposure ............... 247 


\section{LIST OF TABLES}

Table 2-1 - Factors affecting fiber quality at each stage of processing............................................ 6

Table 2-2 - Compiled Properties of Natural Fibers............................................................................. 9

Table 2-3 - Mechanical properties of long, bast (and sisal) fiber reinforced composites from

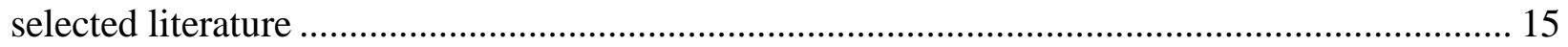

Table 2-4 - Sources from composite mechanical properties (Table 2-3) ..................................... 16

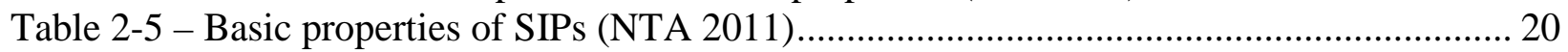

Table 3-1 - Materials for Plate Manufacturing................................................................................ 36

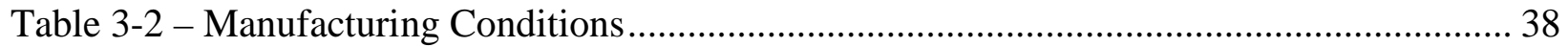

Table 3-3 - Preliminary Flexure and Impact Testing Results ....................................................... 40

Table 3-4 - Single fiber tension test results and published values................................................ 42

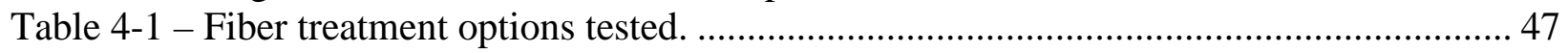

Table 4-2 - Composite manufacturing details ............................................................... 50

Table 4-3 - Single fiber tension tests on Owens Corning E-glass and Toray carbon fibers ......... 56

Table 4-4 - Moisture deformation comparison between resin systems.......................................... 76

Table 4-5 - Moisture deformation comparison between fiber treatments in Derakane 510A resin

Table 4-6 - Flexural strength/stiffness retention for saturated samples, by resin system ............. 77

Table 5-1 - Mechanical testing samples, \% wt. moisture absorption at 50 hours and at saturation

Table 5-2 - Swelling and curvature in mechanical testing samples due to moisture absorption . 94

Table 5-3 - Comparison of dry, normalized FVF mechanical properties .................................. 111

Table 5-4 - Comparison of specific properties (strength or stiffness divided by composite

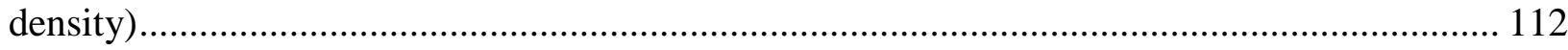

Table 6-1 - Weathering conditions during experiment compared to Morgantown historical averages....................................................................................................................... 120

Table 6-2 - Precipitation and average temperature from same day as well as the four days preceding sample removal; accumulated freeze-thaw cycles for each set of samples ................ 121 Table 6-3 - Moisture effect factors, $\mathrm{C}_{\mathrm{M}}$, from NDS 2012, Table 4A for dimensional lumber and from experimental testing of saturated kenaf composites.......................................................... 134 Table 6-4 - Temperature factors, $C_{t}$, from NDS 2012, Table 2.33 for dimensional lumber and from experimental testing of kenaf composites for up to 12 days of exposure ............................ 135 Table 6-5 - Design factors, $\mathrm{C}_{\mathrm{UV}}$, based on ASTM D4329, Cycle A UV exposure for over 1000

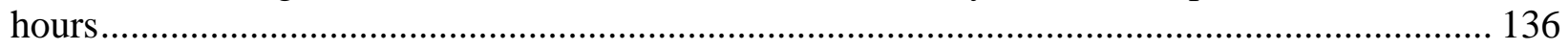

Table 6-6 - Design Factors, $\mathrm{C}_{\mathrm{OE}}$, based on outdoor exposure in WV for 1 year ........................ 136 Table 7-1 - Manufacturer-given foam core strength and stiffness properties (along with average

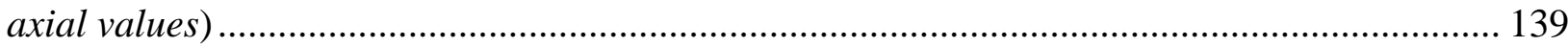
Table 7-2 - Flexural coupon testing results and comparison to compression molded samples (at

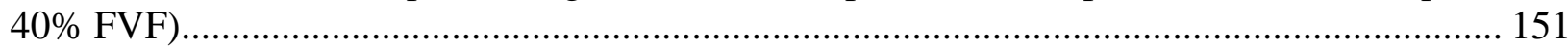

Table 8-1 - Derakane 510A-40 mechanical properties used for modeling ................................ 166

Table 8-2 - Fiber and fabric constituent mechanical properties used for modeling.................... 167

Table 8-3 - Average coupon dimensions from mechanical testing used for modeling............... 168 Table 8-4 - Laminate properties from mechanical testing used for modeling (normalized at $40 \%$

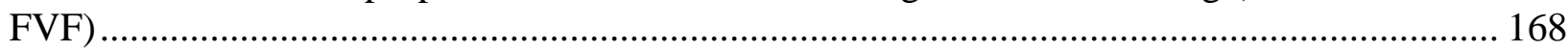

Table 8-5 - Longitudinal modulus rule of mixtures results for 40\% FVF (in Msi) .................... 169 
Table 8-6 - Lamina engineering constants for 40\% FVF used for modeling.......................... 170

Table 8-7 - Reduced lamina stiffness matrix coefficients (Msi) ........................................... 171

Table 8-8 - ABD matrices for flexural coupon layups and dimensions, $A$ : (ksi-in), $B$ : (ksi-in $\left.{ }^{2}\right), D$ :

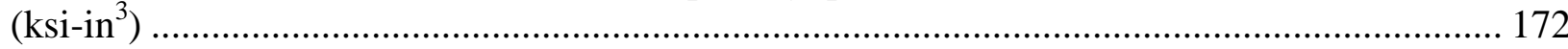

Table 8-9 $-\alpha \beta \delta$ matrices for flexural coupon layups and dimensions, $\alpha$ : $(\mathrm{ksi}-\mathrm{in})^{-1}, \mathrm{~b}:\left(\mathrm{ksi}_{-1 n^{2}}\right)^{-1}$, $\delta:\left(\mathrm{ksi}^{-i \mathrm{n}^{3}}\right)^{-1}$

Table 8-10 - Application of equivalent laminate moduli equations (in Msi).......................... 174

Table 8-11 - Adjusted neutral axis depths based on unbalanced laminates .............................. 176

Table 8-12 - ABD matrices from Table 8-8 adjusted for neutral axis ..................................... 177

Table 8-13 $-\alpha \beta \delta$ matrices from Table 8-9 adjusted for neutral axis........................................ 177

Table 8-14 - Neutral axis adjusted equivalent bending laminate moduli (in Msi)................... 178

Table 8-15 - Summed stiffness method laminate bending moduli (in Msi)............................ 178

Table 8-16 - Finite element laminate moduli results (in Msi).............................................. 182

Table 8-17 - Average extensional modulus method laminate bending moduli (in Msi)........... 182

Table 8-18 - Flexural rigidities of given materials and laminate thicknesses ......................... 184

Table 8-19 - Midpoint deflection of given materials and dimensions .................................... 185

Table 8-20 - Castigliano's method laminate bending moduli (in Msi) ................................... 186

Table 8-21 - Comparison of stiffness model errors................................................................ 187

Table 8-22 - Longitudinal lamina tensile strengths............................................................. 188

Table 8-23 - Assumptions for determining lamina properties with CADEC software ............. 189

Table 8-24 - Lamina strength properties for each lamina reinforcing material ........................ 189

Table 8-25 - Lamina failure strain properties for each lamina reinforcing material.................. 190

Table 8-26 - Laminate load conditions leading to failure .................................................... 190

Table 8-27 - Applicable stress and strain ratios for each material, test type, and failure mode at

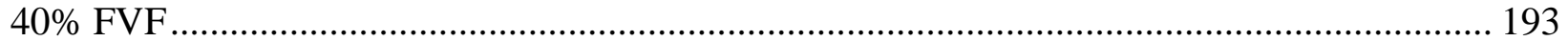

Table 8-28 - Applicable stress ratios from interacting failure criterion.................................. 194

Table 8-29 - Reduced stiffness of untreated kenaf reinforced composite at saturation ............ 197

Table 8-30 - Reduced stiffness of treated kenaf reinforced composite at saturation ................. 197

Table 8-31 - Coefficients of moisture expansion for kenaf laminae..................................... 198

Table 9-1 - OSB SIP Properties and sources in 2012 IRC .................................................. 202

Table 9-2 - Insulating properties for construction materials included in the following designs 202

Table 9-3 - GFRP and KFRP SIP design properties, meeting or exceeding 2012 IRC OSB SIP

specifications............................................................................................................... 204

Table 9-4 - Weight (in lbs) of raw material for each SIP wall design for 30 x $50 \mathrm{ft}$ building... 205

Table 9-5 - Weight (in lbs) of raw material for stick-framing design for 30 x $50 \mathrm{ft}$ building ... 205

Table 9-6 - Weight (in lbs) of raw material for insulating concrete forms design for 30 x $50 \mathrm{ft}$

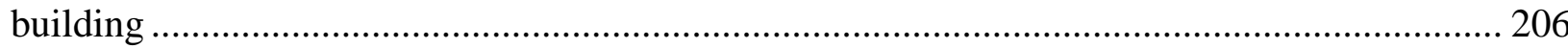

Table 9-7 - EIO-LCA Sectors and detailed sectors for each raw material............................... 211

Table 9-8 - Embodied Energy, $\mathrm{CO}_{2}$ contribution, and cost by weight for each material .......... 212

Table 9-9 - Sources corresponding to data in Table 9-8 .......................................................... 213

Table 9-10 - Embodied Energy, $\mathrm{CO}_{2}$ contribution, and cost comparisons for the five wall

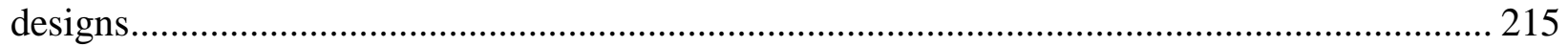

Table 9-11 - Environmental impact divided by total cost of construction materials ................ 217

Table 10-1 - Comparison between fiber treatment methods (improvement over untreated in

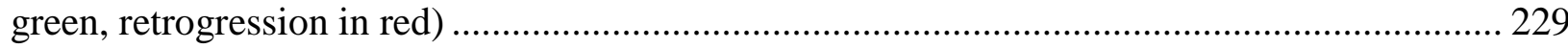


Table A-1 - Dry, non-normalized mechanical testing results ............................................... 236

Table A-2 - 50 hours immersion, non-normalized mechanical testing results......................... 237

Table A-3 - Saturated, non-normalized mechanical testing results ..................................... 238

Table A-4 - Dry, VF-normalized mechanical testing results .............................................. 239

Table A-5 - 50 hours immersion, VF-normalized mechanical testing results ......................... 240

Table A-6 - Saturated, VF-normalized mechanical testing results ...................................... 241

Table C-1 - Key to column headings in EIO-LCA detailed results ....................................... 248

Table C-2 - OSB SIP Design: Economic activity for raw materials ...................................... 249

Table C-3 - OSB SIP Design: Embodied energy by source for raw materials ........................ 249

Table C-4 - OSB SIP Design: Greenhouse gases in equivalent lbs of $\mathrm{CO}_{2}$ for raw materials .. 250

Table C-5 - GFRP SIP Design: Economic activity for raw materials.................................... 250

Table C-6 - GFRP SIP Design: Embodied energy by source for raw materials ...................... 251

Table C-7 - GFRP SIP Design: Greenhouse gases in equivalent lbs of $\mathrm{CO}_{2}$ for raw materials 251

Table C-8 - KFRP SIP Design: Economic activity for raw materials.................................... 252

Table C-9 - KFRP SIP Design: Embodied energy by source for raw materials ....................... 252

Table C-10 - KFRP SIP Design: Greenhouse gases in equivalent lbs of $\mathrm{CO}_{2}$ for raw materials

253

Table C-11 - Stick-Framing Design: Economic activity for raw materials ............................ 253

Table C-12 - Stick-Framing Design: Embodied energy by source for raw materials................ 254

Table C-13 - Stick-Framing Design: Greenhouse gases in equivalent lbs of $\mathrm{CO}_{2}$ for raw

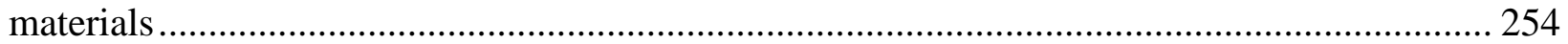

Table C-14 - ICF Design: Economic activity for raw materials ........................................... 255

Table C-15 - ICF Design: Embodied energy by source for raw materials............................... 255

Table C-16 - ICF Design: Greenhouse gases in equivalent lbs of $\mathrm{CO}_{2}$ for raw materials......... 256 


\section{NOMENCLATURE AND UNITS}

In the following text, a number of terms and acronyms frequently used may be ambiguous or may not be familiar to all readers. For easy reference, the below definitions apply throughout the entirety of the text:

- $\alpha \beta \delta$ Matrix: Plate compliance matrix for a composite material

- ABD Matrix: Plate stiffness matrix for a composite material

- Alkalization: Chemical treatment in which fibers are soaked in an alkali solution

- ASTM: American Society for Testing and Materials (formerly, now ASTM international)

- Bast fibers: Fibers obtained from the stem or trunk of plants

- CAM: Cylindrical assemblage model

- CLT: Classical laminate plate theory

- $\mathrm{CO}_{2}$ : Carbon dioxide

- EIO-LCA: Economic Input-Output Life Cycle Assessment

- ELM: Equivalent laminate moduli

- EPS: Expanded polystyrene

- Fabric weight in oz: Measured per square yard

- FE: Finite element

- FRP: Fiber reinforced polymer

- FVF: Fiber volume fraction

- GDP: Gross domestic product

- GFRP: Glass fiber reinforced polymer

- ICF: Insulating concrete form

- IRC: International Residential Code

- IROM: Inverse rule of mixtures

- KFRP: Kenaf fiber reinforced polymer

- Layup: Lamina orientations for a laminate, listed in order

- LCA: Life cycle assessment (or life cycle analysis)

- LCI: Life cycle inventory analysis

- LCIA: Life cycle impact assessment

- LEED: Leadership in Energy and Environmental Design

- LVDT: Linear variable differential transformer

- MEKP: Methyl ethyl ketone peroxide

- NA: Neutral axis

- NaOH: Sodium hydroxide

- NDS: National Design Specification (for wood construction)

- NFRP: Natural fiber reinforced polymer

- OM: Optical microscopy

- OSB: Oriented strand board

- PALF: Pineapple leaf fiber

- PE: Polyethylene

- PIR: Polyisocyanurate

- PLA: Polylactic acid

- PMM: Periodic microstructure model 
- PP: Polypropylene

- PU or PUR: Polyurethane

- R-value: relative insulating value, with unit of $\mathrm{Hr}-\mathrm{ft}^{2}-{ }^{\circ} \mathrm{F} / \mathrm{BTU}$

- RH: Relative humidity

- ROM: Rule of mixtures

- RT: Room temperature

- RTM: Resin transfer molding

- SD: Standard deviation

- SEM: Scanning electron microscopy

- SIP: Structural insulated panel

- Specific properties: Strength or stiffness divided by the density of the material

- SPP: Stress partitioning parameter technique

- UV: Ultraviolet light

- VE: Vinyl ester

- VF: Volume fraction

- WVU-CFC: West Virginia University Constructed Facilities Center

All units in the following work have been converted to US customary units with the following logical exceptions:

- Micro-scale measurements - e.g. $\mu \mathrm{m}, \mathrm{nm}$

- Temperatures or other measurements used to directly describe equipment - e.g. $10 \mathrm{kN}$ load cell

- Values taken from literature and combined in tabular format (Table 2-2, Table 2-3)

- Direct quotes, plots, or images from other sources

In situations where rounded values converted inelegantly from a source (e.g. 0.787 in from $20 \mathrm{~mm}$ ), the original value has been included in parenthesis following the US value. Most of the numerical values reported from literature were originally in SI units. The following conversions have been applied, when necessary:

- $1 \mathrm{psi}=6894.757 \mathrm{~Pa}$

- 1 in $=25.4 \mathrm{~mm}$

- $1 \mathrm{lbm}=0.4535924 \mathrm{~kg}$

- $1 \mathrm{lbf}=4.448222 \mathrm{~N}$

- $1 \mathrm{lb} / \mathrm{in}^{3}=27.7 \mathrm{~g} / \mathrm{cm}^{3}$

- $1{ }^{\circ} \mathrm{F}=9 / 5^{\circ} \mathrm{C}+32$

- $1 \mathrm{ft}^{3}=0.0283168 \mathrm{~m}^{3}$

- $1 \mathrm{BTU}=1.05506 \mathrm{~kJ}$

- 1 opsy $\left(\mathrm{oz} / \mathrm{yd}^{2}\right)=33.935 \mathrm{gsm}\left(\mathrm{g} / \mathrm{m}^{2}\right)$ 


\section{INTRODUCTION}

\subsection{Background}

Concrete, steel, and timber are the most important construction materials of physical infrastructure. However, construction costs, largely due to the costs of these materials, have been increasing in recent years significantly above the level of general inflation, and research has been similarly increasing for advanced materials that can offer improvements (Liang and Hota 2009). In recent years, synthetic, petroleum-based fiber reinforced polymer (FRP) composites have been attractive alternatives to conventional construction materials due to a number of factors, including: Higher strength and stiffness with reference to specific gravity; higher fatigue strength and impact energy absorption capacity; better resistance to corrosion, fire, acids, and natural hazardous environments; longer service life and lower life-cycle costs; non-conductivity and non-toxicity (Liang and Hota 2009).

The expanded use of natural FRP composites composed of natural fibers and/or biopolymers would provide long term benefits to infrastructure. Natural FRPs have the potential to eventually be lighter-weight and lower-cost than many synthetic composites (Brouwer 2000). Natural fibers are easier to handle and have good thermal and acoustic insulation properties (Brouwer 2000). However, products need to be designed in order to take advantage of natural fibers' unique properties, rather than just substituting the fibers for other materials in similar configurations, if the true potential of natural fiber reinforced composites is going to be reached (Rowell 1997). In addition to their weight and cost potentials, the main impetus in pursuing the use of natural FRPs instead of synthetic FRPs is the ecological benefit: natural composites offer the potential to create large-volume, biodegradable structural components using only renewable resources. Using materials like natural composites that reduce construction waste and increase energy efficiency would provide a solution to immediate infrastructure needs while promoting the concept of sustainability (Humphreys 2009).

One of the main concerns with natural FRP composites is their reputation for not possessing sufficient mechanical properties to compete with glass FRPs as primary structural materials, despite the fact that their specific fiber properties are often near or better than those of glass fibers. Of secondary concern is their behavior in different environments, as natural fibers do not possess the inherent environmental resistance of glass fibers. This research addresses these issues as it presents a comprehensive evaluation of the real potential of natural fiber reinforced composites as structural materials.

\subsection{Objectives}

The objectives of this study are to:

1. Demonstrate natural fiber reinforced composites' potential to be used as primary structural components in infrastructure applications.

2. Review existing research on natural FRPs, evaluating their challenges and potential. 
3. Explore the improvement to moisture resistance and flexural properties of kenaf FRPs through the use of chemical fiber surface treatments and composite coatings.

4. Evaluate mechanical properties of kenaf FRPs in flexure, tension, compression, and shear, in both dry and wet environments, and compare with glass FRPs.

5. Assess the weathering and durability of kenaf FRPs, both in real-time and accelerated aging environments.

6. Design a method to scale up fabrication for full-size components, construct a kenaf FRP SIP, and compare to OSB SIPs.

7. Demonstrate that the theoretical laminate property prediction potential of natural FRPs is comparable to that of glass FRPs.

8. Evaluate the sustainability aspects of kenaf FRP SIPs, in the contexts of environmental impacts and socioeconomic, human impacts.

\subsection{Organization}

As a part of this research, nearly 1400 experimental results were generated under a wide variety of tests and conditions, as well as two full chapters of theoretical analyses based on the gathered data. In order to most efficiently present these findings, this dissertation is divided into ten chapters based on the main topic explored within each chapter. A visual outline of the flow and dependencies of the chapters, as well as their major subtopics, is shown in Figure 1-1. 


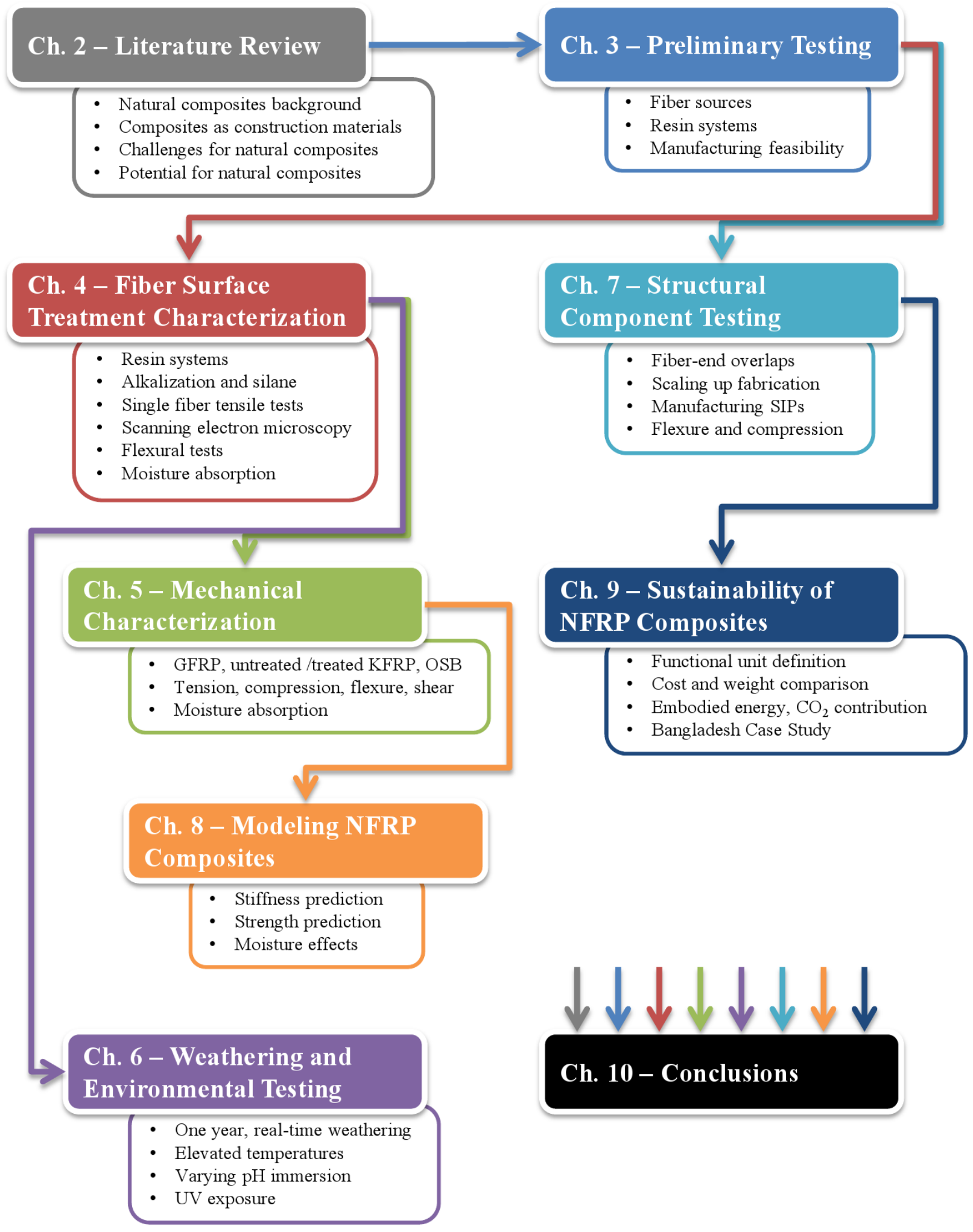

Figure 1-1 - Chapter flow and key topics 


\section{LITERATURE REVIEW}

\subsection{Abstract}

Compared to most synthetic fibers, natural fibers are low-cost, are easier to handle, have good specific mechanical properties, and require only around $20-40 \%$ of the production energy. Using natural materials and modern construction techniques reduces construction waste and increases energy efficiency while promoting the concept of sustainability. Several drawbacks of natural composites which would be even more pronounced in their use in infrastructure include their higher moisture absorption, inferior fire resistance, lower mechanical properties and durability, variation in quality and price, and difficulty using established manufacturing practices when compared to synthetic composites. Many researchers have been working to address these issues, with particular attention paid to the surface treatment of fibers and improving the fiber / matrix interface. Because of their positive economic and environmental outlook, as well as their ability to uniquely meet human needs worldwide, natural composites are showing a good potential for use in infrastructure applications.

\subsection{Background}

There is a large variety of materials being researched for use in natural composites, with varying physical and chemical properties. Not every 'natural' product is as environmentally friendly as their classification would indicate. Biodegradability does not necessarily directly coincide with products made from natural materials - some synthetic materials are biodegradable, and some natural ones are not (Mohanty et al. 2001). An ideal natural composite would be composed only of short-cycle renewable plant resources and would be fully biodegradable under controllable conditions. The challenge in the development of biodegradable biopolymers is maintaining stability during storage and usage, but then allowing for degradation after disposal (Mohanty et al. 2002). If several advances are made with natural composites, there are a number of potential applications within the infrastructure industry where they would be able to compete with glass fiber reinforced composites and wood products.

\subsubsection{Natural Fibers}

A single natural filament generally has a diameter on the order of about $10 \mu \mathrm{m}$ and is itself a type of natural composite material (see Figure 2-1). Each fiber contains a primary cell wall and three secondary cell walls. The cell walls are made up of a lignin-hemicellulose matrix and microfibrils, which are oriented in different directions in each cell wall. The microfibrils each have a diameter of the order of about $10 \mathrm{~nm}$, and are made up of around 30-100 cellulose molecules (John and Anandjiwala 2008); these microfibrils have been found to possess better mechanical properties for manufacturing composites alone than combined in an individual fiber (Ibrahim et al. 2010a). The microfibrillar angle, or the average angle at which the microfibrils are oriented off of the axis of the filament, is thought to be responsible for a number of mechanical properties of the fiber, as smaller angles generally lead to higher strength and stiffness and larger angles to better ductility (John and Anandjiwala 2008). Between species, and even between fibers of the same species, the fiber cell walls differ in their composition and their microfibrillar angle. Filaments are bonded into a bundle by lignin and then attached to the stem by pectin. The lignin and the pectin are both weaker polymers than the cellulose, so they must be removed if the 
fibers are going to be effective as composite reinforcements. Most of the pectin is removed when the bundles are separated from the rest of the stem by retting (soaking) and scutching (beating).

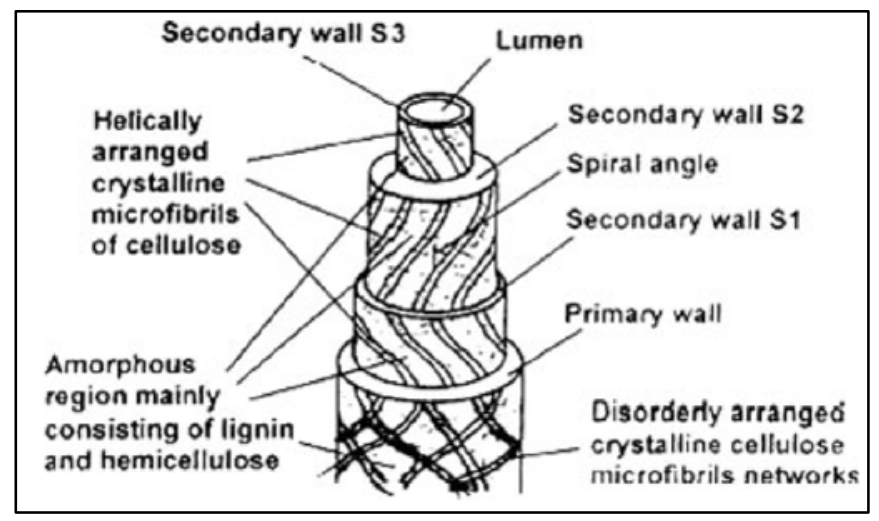

Figure 2-1 - General structure of natural fibers (John and Anandjiwala 2008)

The three main chemical components of a natural fiber are cellulose, hemicellulose, and lignin. The hemicellulose forms cross-linking molecules with the cellulose, forming the main structural component of the cell. The lignin provides additional strength and coupling on the hemicellulose-cellulose network and in some cases acts as a protective barrier. In many applications, fibers are used as bundles still bonded together by pectin; while they require less processing, these bundles have lower strength capacity than the individual fibers (Stamboulis et al. 2000). The length of the reinforcing fibers can also have a significant effect on the strength and stiffness of the composite, particularly in situations where there is poor interfacial bonding (Saha 2011).

\subsubsection{Harvesting and Processing}

Natural fibers can be harvested from the stem, leaves, or seeds of various plants. Fibers collected from the stem are called 'bast' fibers. Examples of bast fibers are flax, hemp, and jute. Leaf fibers are generally coarser than bast fibers (Brouwer 2000). Sisal (agave) and abaca are common leaf fibers, and are particularly useful for stiff binder twines and seawater applications, respectively. Fibers obtained from seeds tend to be coarser and more brittle than bast fibers (Bismarck et al. 2005). Cotton and coir (coconut) are examples of common seed fibers.

There are several different stages of production, and within each stage there are several factors that can influence fiber quality, as shown in Table 2-1. At the plant growth stage, fiber quality is affected by the plant species, the crop production, the location, and the climate. The temperatures at which the plants are grown, as well as the location of the fibers within the plant have both been shown to clearly influence fiber properties, as seen in Figure 2-2. The harvesting stage timing is important because as fibers ripen, the secondary cell wall occupies more of the plant stalk and increases mechanical strength. However, simultaneously the fibers get coarser and adhere more firmly to each other and to the surrounding plant structure, which makes fiber extraction more difficult. The fiber extraction stage involves some process of retting or decortication by which the fibers and the non-useful biomass are separated. Practical yield of flax fibers throughout processing is $27-30 \%$ by mass for fibers between $0.79-7.87$ in (20-200 
$\mathrm{mm}$ ), and 4-9\% by mass for fibers less than 0.79 in $(20 \mathrm{~mm})$ (Munder et al. 2005). Retting methods can be divided into four categories: Biological (such as the traditional 'dew retting'), Mechanical, Physical, and Chemical.

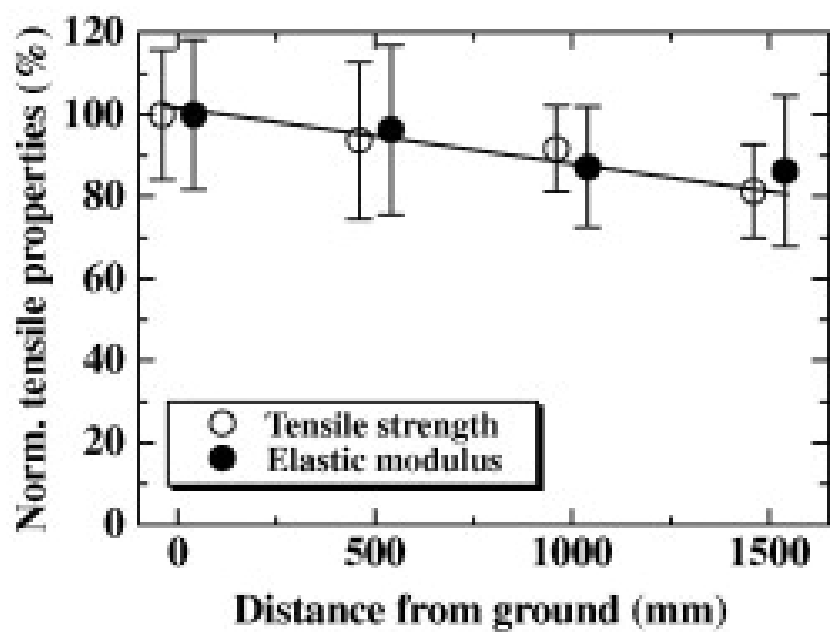

Figure 2-2 - Relationship between tensile properties and fiber location within kenaf plant (Ochi 2002)

Table 2-1 - Factors affecting fiber quality at each stage of processing

\begin{tabular}{|c|c|}
\hline Stage & Factors Affecting Fiber Quality \\
\hline Plant Growth & $\begin{array}{ll}\text { - } & \text { Species of plant } \\
\text { - } & \text { Crop cultivation } \\
\text { - } & \text { Crop location } \\
\text { - } & \text { Fiber location in plant } \\
\text { - } & \text { Local climate }\end{array}$ \\
\hline Harvesting Stage & $\begin{array}{l}\text { - Fiber ripeness, which affects: } \\
\text { o cell wall thickness } \\
\text { o coarseness of fibers } \\
\text { o adherence between fibers \& surrounding structure }\end{array}$ \\
\hline Fiber Extraction Stage & $\begin{array}{l}\text { - } \quad \text { Decortication process } \\
\text { - Type of retting method }\end{array}$ \\
\hline Supply Stage & $\begin{array}{ll}\text { - } & \text { Transportation conditions } \\
\text { - } & \text { Storage conditions } \\
\text { - } & \text { Age of fiber }\end{array}$ \\
\hline
\end{tabular}

Dew retting involves leaving the mown fibers lay in the fields until microorganisms (fungi, mainly) have degraded the cortex, allowing the fibers to be separated. During this process, the plant must be turned over at least once; after retting is complete, the fibers are dried. Over-retting can cause the fiber cellulose to begin degrading, while under-retting can make it 
difficult to separate the fibers. The duration of dew retting is from 3-6 weeks, and is highly dependent on weather conditions. Dew retting is only possible in humid zones - it will not work in arid regions (Brouwer 2000). Every six to seven years, the unpredictability of dew retting results in a lost fiber crop (Bismarck et al. 2005). A variation of dew retting is stand retting, in which the plants are terminated by overheating their bases, and then allowed to dry 1-2 days. This process is more reliable and allows more control than dew retting, but adds cost due to the need for special equipment (Bismarck et al. 2005).

Cold water retting, another traditional type of biological retting, involves immersing bundles of fiber in water tanks or natural bodies of water to allow bacteria to break down the pectin. While this process is effective and only takes 7-14 days, it causes a bad odor and environmental pollution due to the fermentation wastewater; as a result it has been banned in Germany for many years (Bismarck et al. 2005). Warm water retting, an accelerated form of cold water retting, is also banned throughout the rest of Europe due to the environmental impact.

Mechanical retting involves the mechanical separation of fibers that have been slightly field retted or technically dried. Fibers produced by this method (called green fibers) are coarser, but less sensitive to property variation, and may have reduced properties due to physical damage.

Plants subjected to ultrasound retting, a form of physical retting, are placed in a hot water bath that contains alkali and surfactants and then exposed to high intensity ( $1 \mathrm{~kW}, 40 \mathrm{kHz}$ ) ultrasound to separate the fibers (Bismarck et al. 2005). While the retting solution is a byproduct, it is not necessarily wasted and can be used to produce fuel or other applications and fibers are separated well enough to be used for technical or non-textile applications. The steam explosion method utilizes steam under pressure and elevated temperatures to separate the fibers, resulting in extremely fine fibers. The Duralin process involves steam heating in an autoclave, then drying and heating of the fibers. The fibers obtained by this process are bundles, rather than individual fibers.

Enzyme retting, a form of chemical retting, uses pectin-degrading enzymes to separate the fibers and can produce high quality fibers but is, so far, cost prohibitive for most applications. Similarly, chemical and surfactant retting involves immersing the plants in heated tanks of water solutions containing sulfuric acid, chlorinated lime, sodium or potassium hydroxide, or soda ash (sodium carbonate) to dissolve the pectin (Bismarck et al. 2005). Again, these processes produce high quality fibers, but add cost to the final product.

Fibers retted by different methods generally have different coloring, with water and enzyme retting producing light fibers and dew retting producing dark, non-uniform fibers (Dodd and Akin 2005). However, it has been shown that the resulting ultimate stress of the fiber is generally independent of the retting process (Hu et al. 2010). With infrastructure applications in mind, the process of dew (or stand) retting is likely to produce reasonable quality fibers at the lowest cost.

\subsubsection{Comparison to Glass Fibers}

The physical and mechanical properties for natural fibers in Table 2-2 were assembled from several sources (Mohanty et al. 2001; John and Anandjiwala 2008; Bismarck et al. 2005; Beckwith 2003; Barbero 2011; Fowler et al. 2006; Malkapuram et al. 2009; Mohanty et al. 2000; Oksman 2001; Rowell et al. 1997; Satyanarayana et al. 2009; Symington et al. 2009; Tan et al. 2012). Due to the high degree of variability inherent with natural fibers and their testing, many of 
the values listed in Table 2-2 have large ranges. The specific modulus was approximated using the average of the extreme values of stiffness and the average of the extreme values of density found in the literature. Figure 2-3 illustrates the comparison between the specific moduli of glass and natural fibers, a favorable comparison for several types of natural fibers, and additionally the wide range each type of fiber may have for the specific modulus obtained from published stiffness and density values.

A cost per weight comparison between natural and glass fibers is shown in Figure 2-4 (using values from the same published sources as Table 2-2 along with an approximation of the cost of bamboo fibers from (Ray et al. 2005)). However, since natural fibers have much lower densities and strengths than glass fibers, a better way to look at the cost comparison is shown in Figure 2-5. In this figure, the range of values for cost per weight is multiplied by the range of values for the fiber density and an assumed $22.48 \mathrm{kip}(100 \mathrm{kN})$ load and divided by the range of values for tensile strength. The resulting range of values indicates the potential cost per length of fiber material capable of resisting the $22.48 \mathrm{kip}(100 \mathrm{kN})$ load. This figure better illustrates how only some natural fibers are primed to compete with glass fibers, particularly jute, sisal, kenaf, bamboo, and lower-cost flax or hemp. 
Table 2-2 - Compiled Properties of Natural Fibers

\begin{tabular}{|c|c|c|c|c|c|c|c|c|c|c|c|c|c|c|c|}
\hline 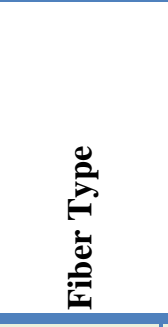 & 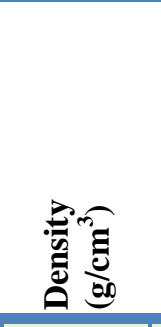 & 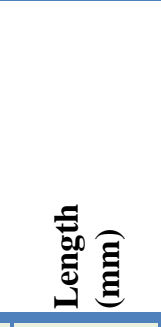 & 壱 & 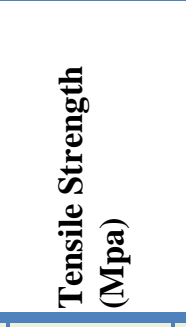 & 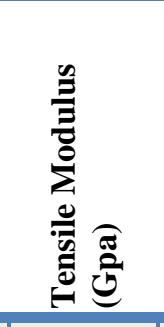 & 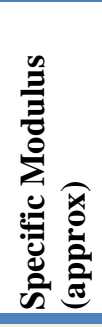 & 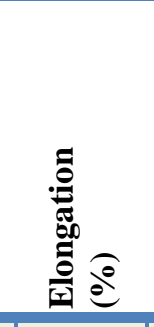 & 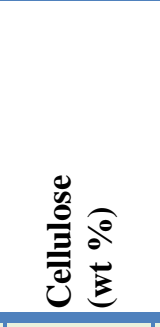 & 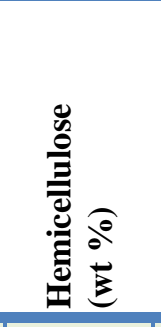 & 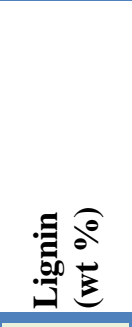 & 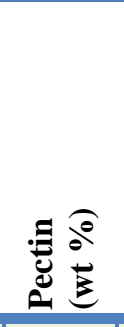 & 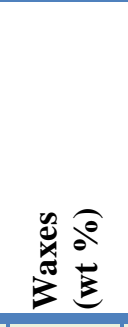 & 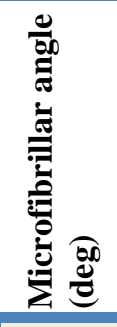 & 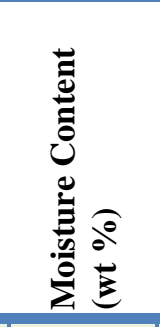 & 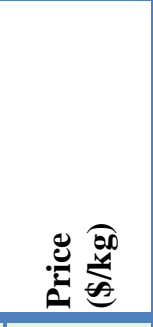 \\
\hline E-Glass & 2.5-2.59 & - & $<17$ & $2000-3500$ & $70-76$ & 29 & $1.8-4.8$ & - & - & - & - & - & - & - & $1.60-3.25$ \\
\hline Abaca & 15 & - & - & $400-980$ & $6.2-20$ & 9 & $1.0-10$ & $56-63$ & $20-25$ & $7-13$ & 1 & 3 & - & $5-10$ & $1.54-2.53$ \\
\hline Alfa & 0.89 & - & - & 35 & 22 & 25 & 5.8 & 45.4 & 38.5 & 14.9 & - & 2 & - & - & - \\
\hline Bagasse & 1.25 & $10-300$ & $10-34$ & $222-290$ & $17-27.1$ & 18 & 1.1 & $32-55.2$ & 16.8 & $19-25.3$ & - & - & - & - & - \\
\hline Bamboo & $0.6-1.1$ & $1.5-4$ & $25-40$ & $140-800$ & $11-32$ & 25 & $2.5-3.7$ & 26-65 & 30 & $5-31$ & - & - & - & - & - \\
\hline Banana & 1.35 & $300-900$ & $12-30$ & 500 & 12 & 9 & $1.5-9$ & $63-67.6$ & $10-19$ & 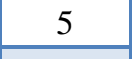 & - & - & - & $8.7-12$ & - \\
\hline Coir & $1.15-1.46$ & $20-150$ & $10-460$ & $95-230$ & $2.8-6$ & 4 & $15-51.4$ & $32-43.8$ & $0.15-20$ & $40-45$ & $3-4$ & - & $30-49$ & 8.0 & $0.22-0.44$ \\
\hline Cotton & $1.5-1.6$ & $10-60$ & $10-45$ & 287-800 & $5.5-12.6$ & 6 & $3-10$ & $82.7-90$ & 5.7 & $<2$ & $0-1$ & 0.6 & - & 7.85-8.5 & $1.54-2.20$ \\
\hline Curaua & 1.4 & 35 & $7-10$ & $87-1150$ & $11.8-96$ & 39 & 1.3-4.9 & 70.7-73.6 & 9.9 & $7.5-11.1$ & - & - & - & - & - \\
\hline Flax & $1.4-1.5$ & 5-900 & $12-600$ & $343-2000$ & 27.6-103 & 45 & $1.2-3.3$ & $62-72$ & 18.6-20.6 & $2-5$ & 2.3 & $1.5-1.7$ & $5-10$ & $8-12$ & $0.33-1.54$ \\
\hline Hemp & 1.4-1.5 & $5-55$ & $25-500$ & $270-900$ & 23.5-90 & 40 & $1-3.5$ & $68-74.4$ & $15-22.4$ & $3.7-10$ & 0.9 & 0.8 & $2-6.2$ & $6.2-12$ & $0.33-1.65$ \\
\hline Henequen & 1.2 & - & - & $430-570$ & $10.1-16.3$ & 11 & $3.7-5.9$ & $60-77.6$ & $4-28$ & 8-13.1 & - & 0.5 & - & - & - \\
\hline Isora & $1.2-1.3$ & - & - & $500-600$ & - & - & $5-6$ & 74 & - & 23 & - & 1.09 & - & - & - \\
\hline Jute & $1.3-1.49$ & $1.5-120$ & $20-200$ & $320-800$ & $8-78$ & 30 & $1-1.8$ & $59-71.5$ & 13.6-20.4 & $11.8-13$ & $0.2-0.4$ & 0.5 & 8.0 & $12.5-13.7$ & $0.30-0.33$ \\
\hline Kenaf & 1.4 & - & - & 223-930 & $14.5-53$ & 24 & $1.5-2.7$ & $31-72$ & 20.3-21.5 & $8-19$ & $3-5$ & - & - & - & $0.33-0.66$ \\
\hline Nettle & - & - & - & 650 & 38 & - & 1.7 & 86 & 10 & - & - & 4 & - & $11-17$ & - \\
\hline Oil Palm & $0.7-1.55$ & - & $150-500$ & $80-248$ & $0.5-3.2$ & 2 & $17-25$ & $60-65$ & - & $11-29$ & - & - & $42-46$ & - & - \\
\hline Piassava & 1.4 & - & - & $134-143$ & $1.07-4.59$ & 2 & $7.8-21.9$ & 28.6 & 25.8 & (5) & - & - & - & - & - \\
\hline PALF & 0.8-1.6 & $900-1500$ & $20-80$ & $180-1627$ & $1.44-82.5$ & 35 & $1.6-14.5$ & $70-83$ & - & 5-12.7 & - & - & 14.0 & 11.8 & - \\
\hline Ramie & $1.0-1.55$ & $900-1200$ & $20-80$ & $400-1000$ & 24.5-128 & 60 & $1.2-4.0$ & 68.6-85 & 13-16.7 & $0.5-0.7$ & 1.9 & 0.3 & 7.5 & 7.5-17 & $1.54-2.42$ \\
\hline Sisal & $1.33-1.5$ & 900 & $8-200$ & 363-989 & $9.0-38$ & 17 & $2.0-7.0$ & $60-78$ & $10.0-14.2$ & $8.0-14$ & 10.0 & 2.0 & $10-22$ & $10-22$ & $0.36-0.66$ \\
\hline
\end{tabular}




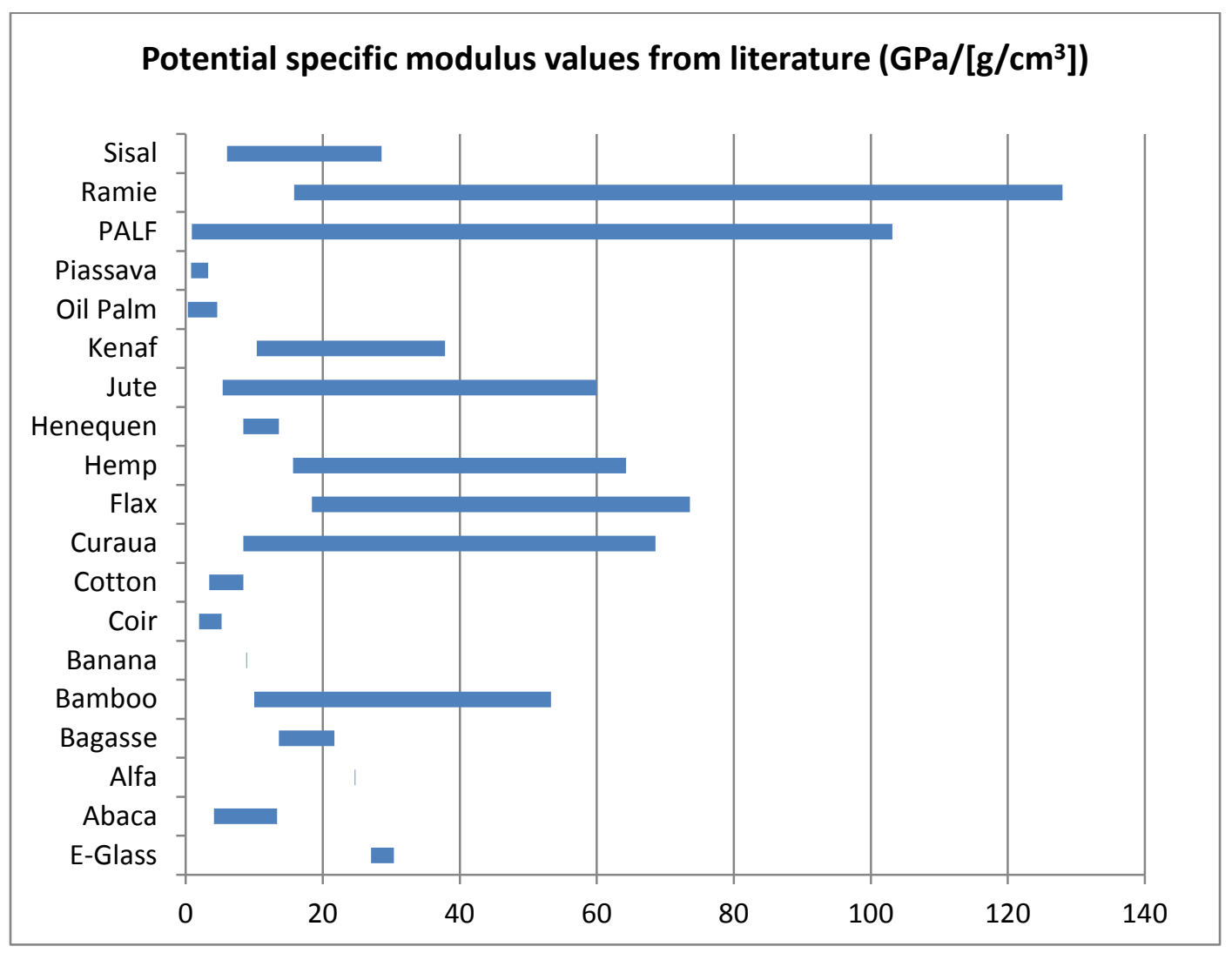

Figure 2-3 - Comparison of potential specific modulus value ranges between natural fibers and glass fibers

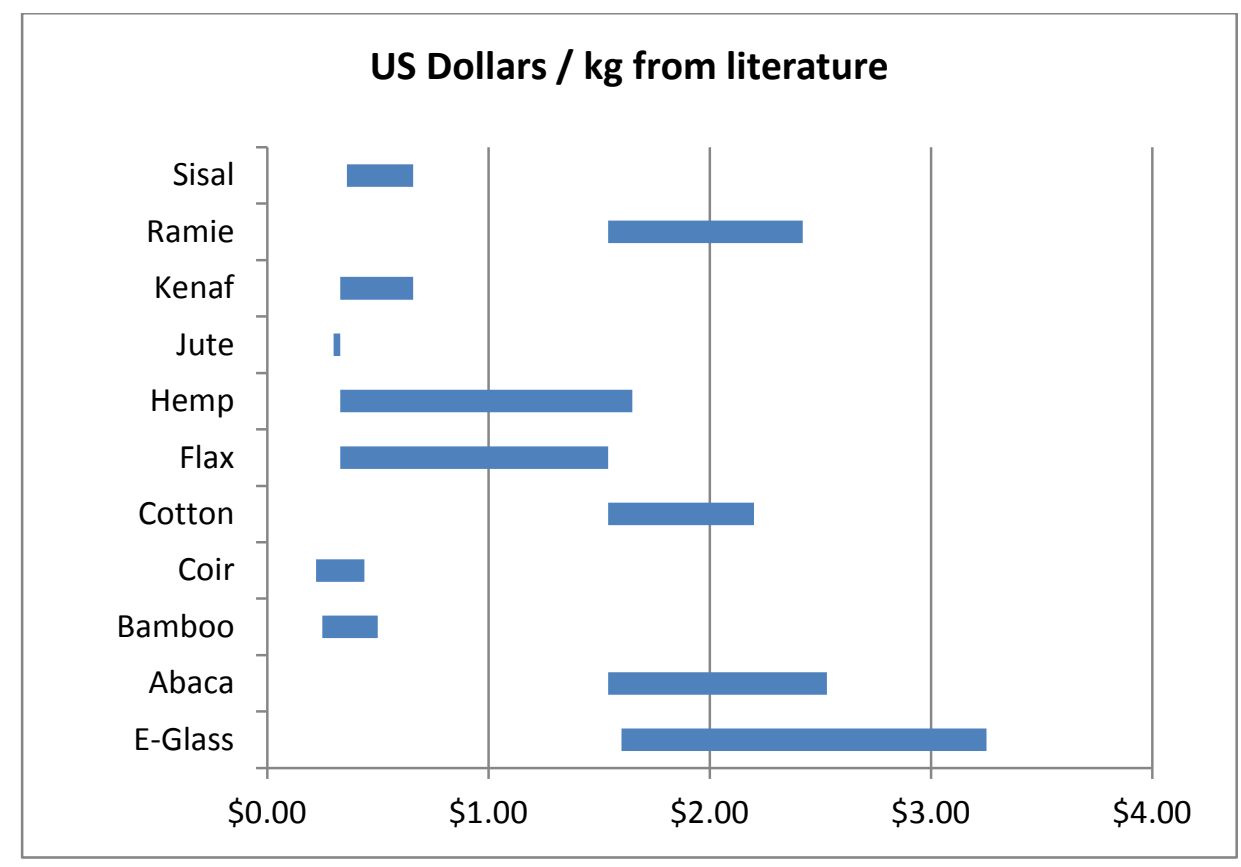

Figure 2-4 - Cost per weight comparison between glass and natural fibers 


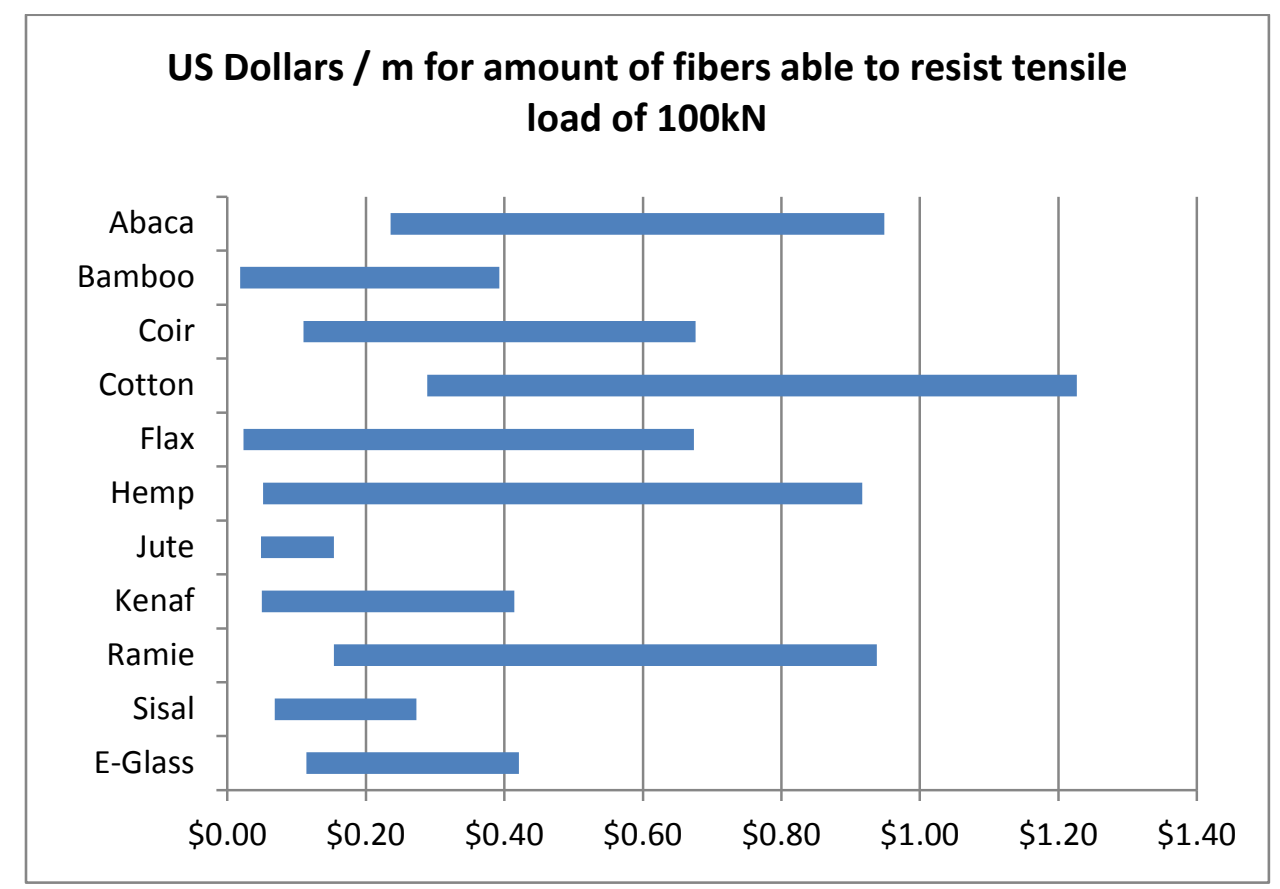

Figure 2-5 - Cost per unit length (capable of resisting $100 \mathrm{kN}$ load) comparison between glass and natural fibers

The common advantages of natural fibers, when considered alongside glass fibers are: Good specific mechanical properties due to low density, renewable resource with low energy consumption, low cost and low investment, easier handling and processing, recyclability, and good thermal and acoustic insulation (Brouwer 2000). Kim et al. (2012) found that at high strain rates (impact loading), natural fiber reinforced composites had improved energy absorption compared to glass fiber reinforced composites. On the other hand, the disadvantages include: Lower strength, variability in quality, higher moisture absorption, limited processing temperatures, lower durability, and more inferior fire resistance. Bast fibers, in general, seem to have the best properties for structural applications; of those, flax offers the best potential combination of low cost, light weight, and high strength and stiffness. Jute is more common, but is not as strong or stiff as flax (Beckwith 2003). Hemp fibers are highly water resistant. Kenaf does not need much care before or during growth and is fairly disease resistant. It is also considered a weed and as a result is one of the least expensive natural fibers (Williams et al. 2011). Ramie fibers have excellent fiber properties and high potential for use as reinforcing fibers in polymer composites. Henequen, a close relation to sisal, is tough and resilient, but only has marginal mechanical properties. Pineapple fibers are very hygroscopic. Straw has low cellulose content and poor mechanical properties, but can be used to manufacture decent fiberboards or lightweight core materials (Mo et al. 2005).

Overall, natural fibers have lower densities and can generally be found for cheaper (per unit weight) than glass fibers, although their strength is usually significantly less. Because of their good specific modulus values, natural fibers can be preferable to glass fibers in applications where stiffness and weight are primary concerns (Beckwith 2003). 


\subsubsection{Biopolymers}

The combined effect of the depletion of petroleum resources and increasing environmental regulations is generating increasing interest in the development of new materials that are environmentally compatible and independent of fossil fuels. The US Department of Agriculture and the US Department of Energy have set goals of having at least $10 \%$ of all basic chemical building blocks be created from renewable, plant-based sources in 2020, increasing to 50\% by 2050 (Mohanty et al. 2005b). One of the major factors holding back the development of biopolymers is their cost. Biopolymers have historically cost anywhere from about twice to ten times as much as comparable petroleum-based resins, with starch-based resins and polylactic acid (PLA) generally being the most affordable among them. Another difficulty in producing bio-based plastics that are similar to petrol-based plastics lies in the fact that crude oil is only made up of hydrogen and carbon atoms (hydrocarbons), while plant based sugars and oils are made up of hydrogen, carbon, and oxygen atoms (Evans 2011). Therefore plant-based plastics can generally only be similar to, not identical to, petrol-based plastics. Innovation is needed for the development of new ways to produce identical plastics from plant sources, with some companies already having success producing PE and PP from ethanol (Evans 2011).

There are many polymer products claiming to be 'natural' or 'bio-based,' but in some cases these descriptions can be misleading: bio-based and biodegradable are not synonymous. The term 'natural fiber reinforced composite' generally refers to natural fibers in any sort of polymeric matrix (thermoplastic or thermoset; natural or synthetic). 'Completely biodegradable composites' (or, in most cases, 'biocomposites') refer to natural fibers in biodegradable matrices. Combinations of natural and synthetic fibers, which are often used to achieve higher mechanical properties, are referred to as 'hybrid composites' (Mohanty et al. 2001).

Until biopolymers are more extensively available and affordable, it is often easier to incorporate natural fibers into a synthetic matrix in order to create semi-disposable composites (usually only through incineration) which can, in some cases, produce favorable life cycle analysis results, and to develop the natural fiber technology in anticipation of eventual structuralquality biocomposites. As an alternative to fully biodegradable composites and incineration disposal, Grozdanov et al. (2010) recycled biocomposites by milling them to particle size and using them as eco-friendly reinforcements in polymer mortars.

Polypropylene (PP) has frequently been reinforced with natural fibers (Brouwer 2000; Stamboulis et al. 2000; Malkapuram et al. 2009; Rowell et al. 1997; Hoang et al. 2010; Czigany 2004; Deliglio 2010; Wambua et al. 2003; Pervaiz and Sain 2003; Joshi et al. 2004; Chang et al. 2009b; Oksman et al. 2003) and possesses the greatest potential of any synthetic thermoplastic matrix, despite its shortcomings of low stiffness, temperature dependency, and creep behavior, due to its recyclability. Most often these composites are made by melt-mixing the PP and natural fibers and injection/compression molding, although this often damages the fibers. The use of PP is attractive due to its recyclability, although there is degradation of properties after each recycling (Hoang et al. 2010). Most applications for natural fiber and synthetic thermoplastic systems seem to be focused in the automotive industry (Mohanty et al. 2001).

Just as there is a wide variety of natural fibers available as raw materials, there are also many options for biopolymers. Starch-based thermoplastics are easy to work with, but do not perform well in the presence of humidity (Mohanty et al. 2001). The additive polycaprolactone can improve water resistance, and is a good compatibilizer for starch; with its contribution, 
bleached jute has been shown to work well with starch polymers (Mohanty et al. 2001). Bakare et al. (2010) produced a sisal-reinforced, rubber seed oil-based polyurethane that possessed good mechanical and moisture resistance properties. Natural polymeric materials can also be made from tung oil, corn oil, peanut oil, sunflower oil, safflower oil, walnut oil, linseed oil, or fish oils ( $\mathrm{Li}$ and Larock 2005). By far the most common biopolymers, and those that seem to offer the greatest potential for competing with synthetic polymers in terms of cost and performance, are polylactic acid and soy-based resins.

Natural thermoplastic resins, such as polylactic acid (PLA), already have some market exposure. PLA is made from renewable agricultural raw materials (such as dextrose), fermented to form lactic acid and then polymerized. It has a broad range of applications due to its ability to be stress crystallized, thermally crystallized, impact modified, filled, copolymerized, and processed in a variety of polymer processing equipment (Henton et al. 2005). PLA is fully biodegradable by hydrolysis to lactic acid, and eventually to water and carbon monoxide. In long-term use, since PLA is produced from field waste products, PLA can potentially become a $\mathrm{CO}_{2}$ sink and contribute to the reduction of greenhouse gases. PLA production has historically been limited due to its high cost, although more recently the cost has fallen and demand has risen such that supply constraints are the more recent restriction (MarketsandMarkets 2011). However, with the opening of several major, new production facilities in the past few years, global output is expected to increase (Malveda et al. 2009). Even with the intrinsic reduction in cost that comes with increased production, PLA biopolymers are expected to remain more expensive than comparable synthetic polymers, at least for the near future (Malveda et al. 2009). PLA has been used, along with natural fiber, by a number of researchers to produce fully biodegradable composites (Ochi 2008; Oksman et al. 2003; Nishino et al. 2003; Hu and Lim 2007; Åkesson et al. 2006; Riedel and Nickel 1999; Plackett and Sodergard 2005).

Soy-based resins are currently the focus of a large portion of biopolymer research. Soy protein concentrates (SPC) and soy protein isolates (SPI) are two common variations of soy products made by purification of defatted soy flour. Soy protein polymers have relatively low strength and high moisture absorption, so they are commonly blended with other natural or biodegradable polymers to create soy-based bioplastics (Mohanty et al. 2001; Mohanty et al. 2005a; Netravali et al. 2007; Haq et al. 2009; Dweib et al. 2005; Netravali 2003). As long as the prices and mechanical properties of biopolymers remain uncompetitive, this hybridization is the most realistic option. However, some recent efforts have produced improved mechanical and moisture resistant properties in soy protein resins (Kumar and Zhang 2008; Kumar and Zhang 2009) and composites (Williams and Wool 2000). Epoxidized soybean oil (ESO) is also commonly used as a resin, but it produces a low crosslink density and limited thermal/mechanical properties unless blended with other materals (Sundaraman et al. 2008).

\subsubsection{Natural Fiber Reinforced Composites - Mechanical Properties}

Many researchers have developed composites using natural fibers as the primary reinforcement material. The highest structural (mechanical) properties are usually found in composites manufactured using bast fibers (jute, flax, kenaf, hemp) or some leaf fibers (sisal, PALF) due to those fibers' relatively high strength and stiffness values. While a large portion of natural fiber reinforced composite research focuses on using short or milled fibers in thermoplastic matrices, those composites generally have a difficult time being mechanically competitive with long (or continuous) fiber and woven fabric reinforced composites. A 
compilation of published long, bast (or sisal) fiber reinforced composite material properties is shown in Table 2-3 (sources in Table 2-4).

A trend that can be observed in Table 2-3 is that the fiber loading (either weight fraction or volume fraction) of the composite significantly impacts the resulting composite's mechanical properties, although this is far from the only factor. The relationship between volume fraction and tensile/flexural mechanical properties was thoroughly studied by Ochi (2008), with the plots generated as a result of that study shown in Figure 2-6 and Figure 2-7. Up to about 50\% FVF, the increase in properties appears to be linearly related to the fiber volume fraction, tapering off for fiber loadings beyond that. Saheb and Jog (1999) found that a FVF of around $60 \%$ seemed to optimize mechanical properties.

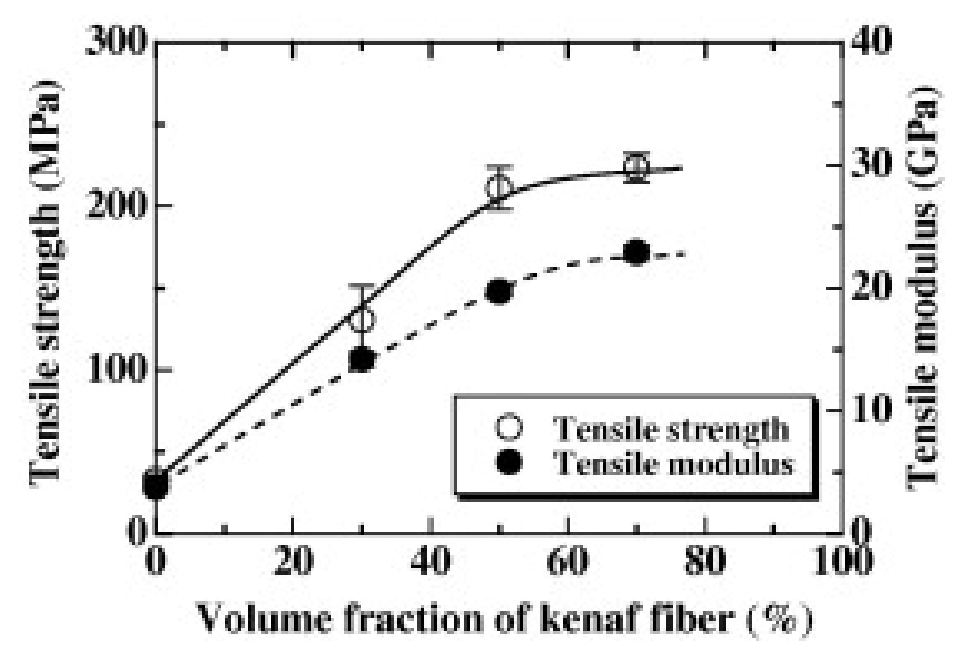

Figure 2-6 - Relationship between tensile mechanical properties and kenaf fiber volume fraction (Ochi 2008)

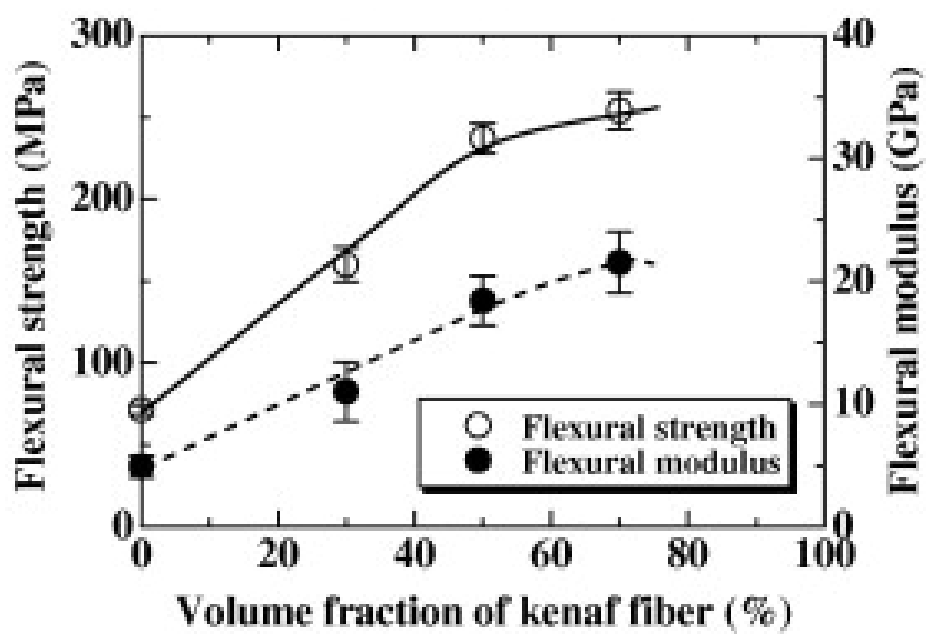

Figure 2-7 - Relationship between flexural mechanical properties and kenaf fiber volume fraction (Ochi 2008) 
Table 2-3 - Mechanical properties of long, bast (and sisal) fiber reinforced composites from selected literature

\begin{tabular}{|c|c|c|c|c|c|c|c|c|c|c|c|c|}
\hline Fiber Species & Matrix Type & Reinforcement Type & Fiber Content & 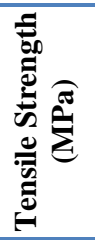 & 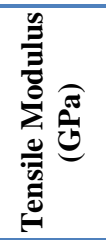 & 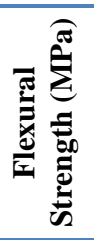 & 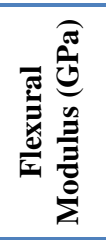 & 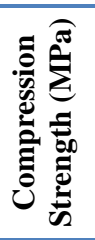 & 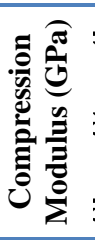 & 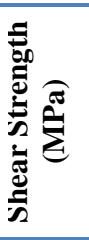 & 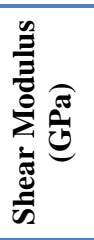 & Source \\
\hline Flax & AESO & Loose long fibers & $34-40 \% \mathrm{WF}$ & 30 & 4.70 & 64 & 4.20 & & & & & [1] \\
\hline Hemp & AESO & Non-woven, oriented mat & $20 \% \mathrm{WF}$ & 35 & 4.40 & 51 & 2.70 & & & & & [1] \\
\hline Sisal & Rubber seed oil-based PU & Loose long fibers & $25-30 \% \mathrm{WF}$ & 119 & 2.16 & 103 & 3.25 & & & & & [2] \\
\hline Hemp/Wool (6:1) & Polyester & Pultruded strands & $35 \% \mathrm{WF}$ & 122 & 16.84 & 180 & 11.50 & 52 & 4.40 & & & [3] \\
\hline Hemp/Wool (6:1) & Polyurethane & Pultruded strands & $35 \% \mathrm{WF}$ & 123 & 18.91 & 146 & 12.20 & 51 & 4.90 & & & [3] \\
\hline Hemp/Wool (6:1) & Vinyl ester & Pultruded strands & $35 \% \mathrm{WF}$ & 113 & 15.27 & 145 & 13.00 & 49 & 4.50 & & & [3] \\
\hline Jute & Isothalic polyester & Bi-directional woven fabric & $44 \% \mathrm{WF}$ & 80 & 9.58 & 122 & 7.64 & 83 & 1.84 & 21 & 1.74 & [4] \\
\hline Kenaf & PLLA & "Like paper" & $70 \% \mathrm{VF}$ & 60 & 6.00 & & & & & & & [5] \\
\hline Hemp & Cellulose Acetate & Bi-directional woven fabric & $37-43 \% \mathrm{VF}$ & 54 & 5.40 & 95 & 6.56 & & & 12 & 1.09 & {$[6]$} \\
\hline Hemp & PHB & Bi-directional woven fabric & $37-45 \% \mathrm{VF}$ & 56 & 5.50 & 65 & 5.05 & & & 10 & 0.88 & [6] \\
\hline Flax & Vinyl ester & Bi-directional woven fabric & & & & 155 & 5.18 & & & & & [7] \\
\hline Jute & Epoxy & Wound filaments & $33 \% \mathrm{WF}$ & 104 & 15.04 & 150 & 14.65 & 95 & & & & [8] \\
\hline Jute & Polyester & Wound filaments & $22 \% \mathrm{WF}$ & 84 & 12.21 & 125 & 8.07 & 123 & & & & [8] \\
\hline Flax & Modified SPC & Fabric & $45 \% \mathrm{WF}$ & 55 & 1.00 & & & & & & & [9] \\
\hline Flax & Modified SPC & Yarn bundles & $45 \% \mathrm{WF}$ & 126 & 2.24 & & & & & & & [9] \\
\hline Hemp & PLA & Medium (5-15 mm) fibers & $40 \% \mathrm{VF}$ & 55 & 8.50 & 113 & & & & & & [10] \\
\hline Flax & Bio-based ENVIREZ & Bi-directional woven fabric & $69 \% \mathrm{VF}$ & 85 & 10.00 & 113 & 6.96 & & & 21 & & [11] \\
\hline Kenaf & PLA & Loose long fibers & $70 \% \mathrm{VF}$ & 223 & 22.70 & 254 & 21.50 & & & & & [12] \\
\hline Kenaf & Polypropylene & Randomly distributed & $40 \% \mathrm{WF}$ & 29 & 6.80 & 28 & 2.20 & & & & & [13] \\
\hline Sisal & Polypropylene & Randomly distributed & $40 \% \mathrm{WF}$ & 33 & 5.30 & 25 & 1.70 & & & & & [13] \\
\hline Hemp & Polypropylene & Randomly distributed & $40 \% \mathrm{WF}$ & 52 & 6.80 & 54 & 5.00 & & & & & [13] \\
\hline Jute & Polypropylene & Randomly distributed & $40 \% \mathrm{WF}$ & 27 & 3.50 & 34 & 2.70 & & & & & [13] \\
\hline Jute & Polyester & 60 cm "slivers" & $60 \% \mathrm{WF}$ & 132 & 2.96 & 172 & 18.44 & & & & & [14] \\
\hline Flax & Epoxy & Loose long fibers & $40 \% \mathrm{VF}$ & 190 & 26.00 & 218 & 18.00 & & & & & {$[15]$} \\
\hline Flax & Epoxy & Wound filament rovings & $48 \% \mathrm{VF}$ & 268 & 32.00 & 282 & 23.00 & & & & & [15] \\
\hline
\end{tabular}


Table 2-4 - Sources from composite mechanical properties (Table 2-3)

\begin{tabular}{|c|c|}
\hline ID\# from Table 2-3 & Source Citation \\
\hline [1] & (Williams and Wool 2000) \\
\hline [2] & (Bakare et al. 2010) \\
\hline [3] & (Peng et al. 2012) \\
\hline [4] & (Ahmed and Vijayarangan 2007) \\
\hline [5] & (Nishino et al. 2003) \\
\hline [6] & (Christian and Billington 2009) \\
\hline [7] & (GangaRao et al. 2009) \\
\hline [8] & (Shah and Lakkad 1981) \\
\hline [9] & (Chabba and Netravali 2004) \\
\hline [10] & (Hu and Lim 2007) \\
\hline [11] & (Mutnuri et al. 2010) \\
\hline [12] & (Ochi 2008) \\
\hline [13] & (Wambua et al. 2003) \\
\hline [14] & (Dash et al. 2000) \\
\hline [15] & (Van de Weyenberg et al. 2006) \\
\hline
\end{tabular}

The best composites, in terms of high mechanical properties, listed in Table 2-3 are those cited by Ochi (2008) and Van de Weyenberg et al. (2006). While these results are very good, it should be noted that they are both concerning thin $(0.04-0.12$ in; $1-3 \mathrm{~mm})$, unidirectionally reinforced laminates with relatively high fiber loading and, in the case of (Van de Weyenberg et al. 2006), a high quality epoxy matrix. These sources mostly confirm Netravali et al.'s (2007) conclusion that most 'semi-green' or 'green' composites have maximum tensile strengths and stiffnesses in the ranges of 14.5-29 ksi (100-200 MPa) and 145-580 ksi (1-4 GPa) (although, in the case of above data, the tensile stiffness is more like 145-2900 ksi or 1-20 GPa). However, Netravali et al. (2007) showed that these are not necessarily the maximum achievable properties for biodegradable composites, as they developed an extremely high quality, "liquid crystalline cellulose" reinforced / nano-modified SPC composite with tensile strength and stiffness properties of $92.5 \mathrm{ksi}(638 \mathrm{MPa})$ and $1900 \mathrm{ksi}(13.1 \mathrm{GPa})$, respectively, and flexural strength and stiffness properties of $36.0 \mathrm{ksi}(248 \mathrm{MPa}$ ) and $4030 \mathrm{ksi}$ (27.8 GPa), respectively at around 40\% fiber volume fraction.

In their work developing natural fiber-reinforced composites for housing materials in 1974, Winfield and Winfield calculated that, in order to withstand wind loading, their material would need to have mechanical properties of around $18 \mathrm{ksi}(124 \mathrm{MPa})$ flexural strength, $9 \mathrm{ksi}$ (62 MPa) tensile strength, and $850 \mathrm{ksi}$ (5.86 GPa) flexural stiffness. While Winfield and Winfield (1974) used calcium carbonate as filler in an attempt to increase their flexural properties to these limits, it appears that advances in fiber selection, treatment, and resin system quality have all made those targets achievable with today’s natural fiber reinforced composites.

\subsubsection{Composites as Construction Materials}

Research into new materials for structural design should focus on meeting the need areas of home safety/security, affordability/constructability, sustainability/durability, and functional design (Cramer 2004). As production has increased and prices have dropped in the past few decades, synthetic composite materials have found more and more applications in infrastructure. 
Their potential for meeting the need areas of new materials is high, particularly with their capability to be used in modular designs.

\subsubsection{Current Uses}

Within the area of civil infrastructure, there are many potential applications for FRP composites that have been or are currently being explored: Bridge decks and other highway structures, pavement panels, utility poles, pipelines, turbine blades, blast resistant structures, naval platforms, marinas, chimneys/flues, railroad ties, and modular buildings and housing, among many other applications (Liang and Hota 2009; Van Erp et al. 2006; Ayers et al. 2004). In the building industry, FRP panel, modular, and open-building systems have all been explored since the 1980s (Singh and Gupta 2005a; Evernden and Mottram 2009). Composite materials can be particularly applicable for disaster relief shelters, grain or fuel storage, cisterns, septic tanks, jail facilities, cold storage facilities, military barracks, school buildings, greenhouses, and industrial factories or warehouses (Liang and Hota 2009; Omar et al. 2007).

The use of natural fibers in building materials has been explored previously: Armand G. Winfield oversaw implementation of several structural applications of jute-fiber composites in India in the late 1970s (Winfield and Winfield 1974; Winfield 1979). However, progress seems to have stalled after his advances in this particular area until recently. In the past few years, several researchers (Chang et al. 2009b; Dweib et al. 2005) and small companies have made progress with large-volume natural composites, but most of these involve some amount of synthetic polymers and many products are not intended for structural use.

\subsubsection{Modular Construction}

The estimated average construction cycle time for houses in the US is around 152 days, with actual construction operations only consuming around $25-40 \%$ of the available working time (40 hours/week) (Mullens and Hastak 2004). Disentangling the shell and infill components of houses will reduce interface complexity and conflict during construction, and will facilitate factory production of interchangeable infill components. With the current construction system, studs resist gravity loads, exterior sheathing resists weathering and lateral loads, insulation provides energy efficiency, and gypsum wallboard provides interior finish and, in some cases, fire resistance. Four separate components are needed to provide essentially four different functions - not exactly a model of efficiency.

Components that combine separate functions into one system offer the potential for significant cost reduction (Cramer 2004). Improving the connection between the sheathing and the framing in a house is one of the most effective ways of increasing the structural integrity of the house with respect to lateral loads (Cramer 2004). Advances in the housing construction industry have been slow to develop, with most common practices remaining essentially the same for decades. Major inhibitors in the advancement of housing technology have been: A low demand for revolutionary changes in performance; the dominance of housing research by specific industries vying for increased market shares, not system overhauls; liability issues; and perceived dependence on centralized infrastructures (Van Dessel and Messac 2004).

There is a growing pressure in the UK housing industry over the last few decades to re-

think and streamline the standard construction model. To improve the technology of construction, one researcher recommends that the UK industry should aim to make the following improvements (Pearce 2003): 
- Standardization of building components

- Development of lighter weight and super-strength materials

- Wider use of information technology

- Increased use of off-site manufacture

- Improved design for occupants' health and wellbeing

- Flexibility of design for changing uses over time

- More customer-centric thinking

- A holistic view of construction focusing on integrating the supply chain and thinking ahead to the management of the built environment

- Stronger investment in education and training

Many of the above improvements, along with lower costs, can be met by increasing the prevalence of off-site preassembly (Evernden and Mottram 2009). Prefabricated modular systems can provide dimensionally accurate, consistent, reliable, and cost effective products that can be assembled in any location or climate (Liang and Hota 2009). Modular, semi-permanent FRP shelters have already been used in disaster relief situations; for example, in India after the 2001 Gujarat earthquake (Baksi et al. 2010).

There are many advantages to FRP modular construction systems (Evernden and Mottram 2009; Lawson and Richards 2010):

- Ability to offer wide variety of thin-walled shapes with integrated hollow sections or cores leading to geometrically optimized systems

- Potential for mechanical interlocking connections for quick erection

- Insulation properties

- Resistance to warping/distortion

- Acoustic absorbance

- Environmental impact / sustainability

- Low density and high strength-to-mass ratio leads to smaller foundations and faster installations

- Improved quality control

- Potential for disassembly and reuse

- Economy of scale in manufacturing repeated units

- Lower whole-life cost per unit and potentially lower labor costs

Natural FRPs could possess all of the same advantages if successfully designed as modular construction materials. Additionally, the potentially lighter weight of bio-based composite construction materials would increase the possibility that they could be manufactured in a plant and then assembled on site without the need for a heavy crane (Shenton III and Wool 2004). Ultimately, however, modular systems have a long way to go in terms of quality and flexibility before they are a consumer-preferred housing option.

\subsubsection{Structural Insulated Panels}

Residential and commercial construction applications have recently benefited from the increased construction simplicity, quality control, and energy efficiency offered by Structural Insulated Panels (SIPs), which may be used as walls, floors, or roofing. SIPs typically consist of insulating cores (usually made of polyurethane, expanded polystyrene (EPS), or 
polyisocyanurate) sandwiched between oriented strand board (OSB) structural skins (see Figure 2-8).

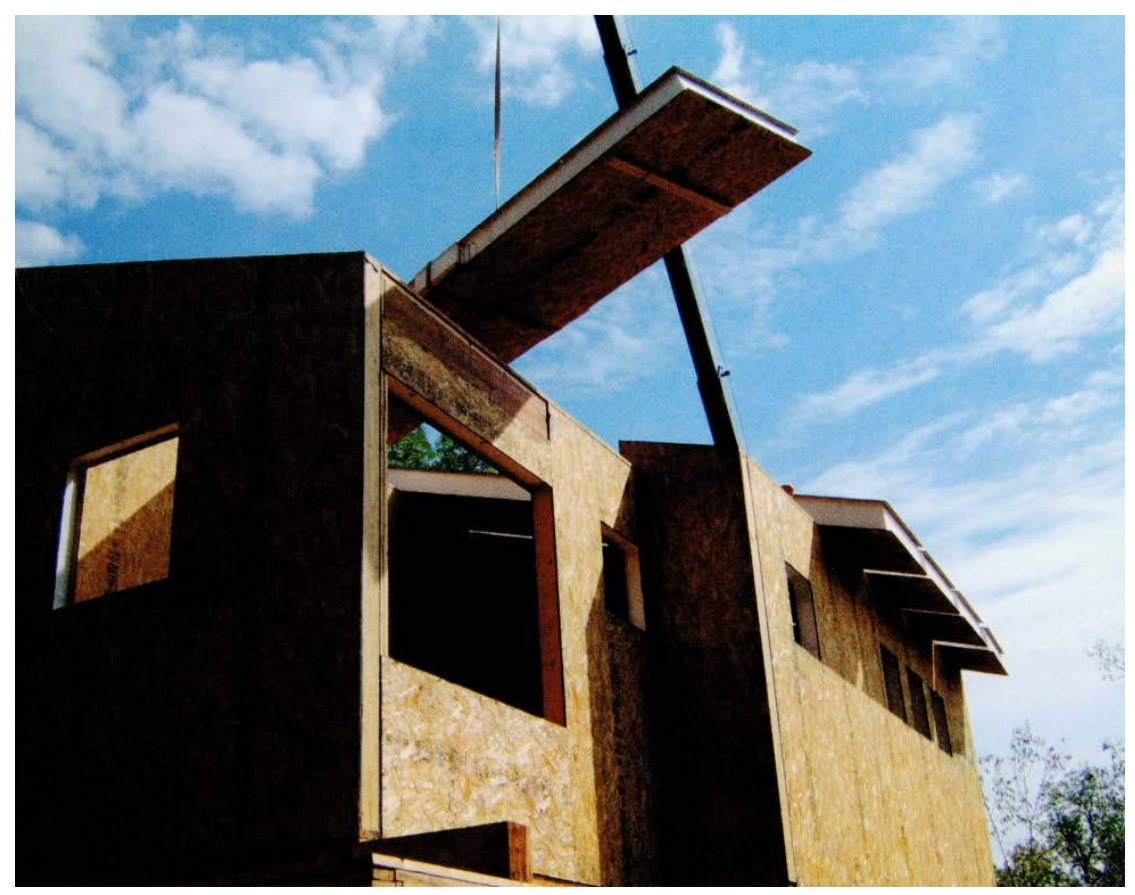

Figure 2-8 - Construction with SIPs (Johnston and Gibson 2008)

All sustainability measurement systems look at the general categories of energy efficiency and renewable energy, conservation of materials and resources, material toxicity and air quality, and waste reduction. SIPs alone may be superior to standard wood framing systems within each category (Pasma 2010), while bio-SIPs could provide even more promising sustainable solutions. SIPs can contribute up to 39 points in LEED for New Construction qualification or up to 46 points in LEED for Homes (Pasma 2010). BASF conducted a study where they evaluated the eco-efficiency of SIPs by life cycle analysis (see Figure 2-9). Their conclusions were that energy consumption was lower, necessary production, maintenance, and energy resources were reduced, less forest acreage was needed for production, emissions were less (largely due to lower energy consumption), and onsite waste was reduced (SIPA 2009). Because of their high load resistance and excellent sealing, SIPs are also of particular interest as the base elements in buildings with living roofs. While there are not specific standards for SIPs included in typical building design codes, the Structural Insulated Panel Association has published a report including minimum panel properties (see Table 2-5). 


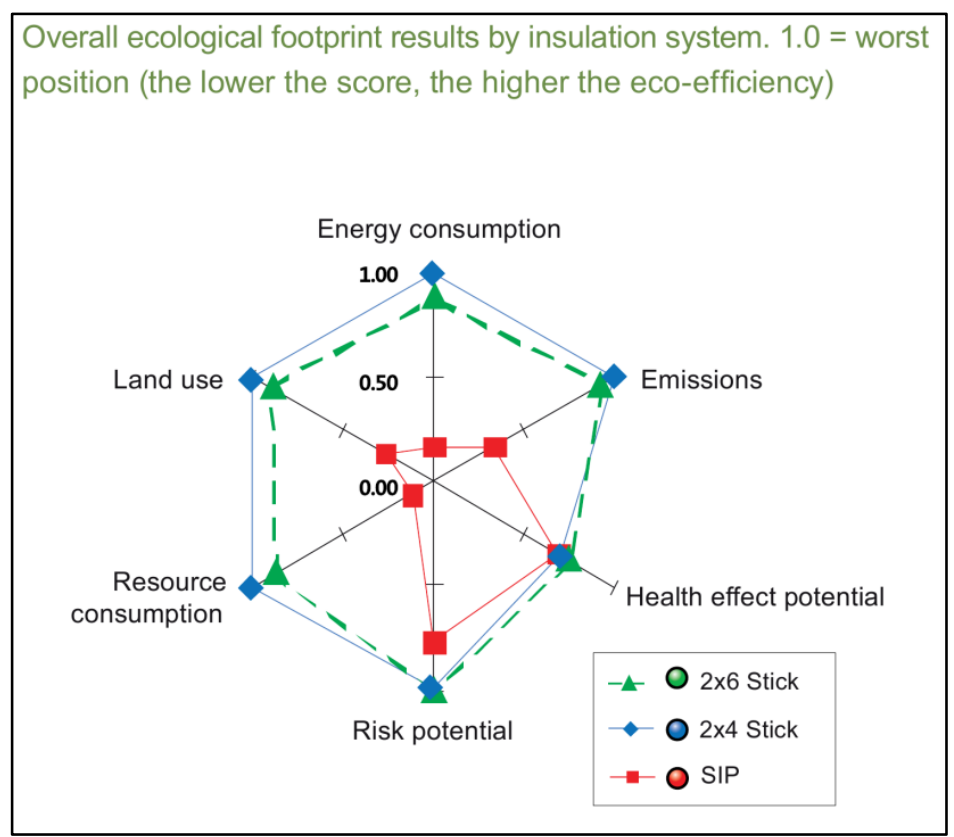

Figure 2-9 - Results of BASF Life-Cycle Analysis of traditional SIPs (SIPA 2009)

Table 2-5 - Basic properties of SIPs (NTA 2011)

\begin{tabular}{|c|c|c|}
\hline Property & Weak-Axis Bending & Strong-Axis Bending \\
\hline Allowable Tensile Stress, $\mathbf{F}_{\mathbf{t}}$ (psi) & 245 & 495 \\
\hline Allowable Compressive Stress, $F_{c}$ (psi) & 340 & 580 \\
\hline Elastic Modulus (Bending), $\mathbf{E}_{\mathrm{b}}$ (psi) & 738900 & 658800 \\
\hline Shear Modulus, G (psi) & 270 & 405 \\
\hline Allowable Core Shear Stress, $F_{v}$ (psi) & 4.5 & 5.0 \\
\hline Core Compressive Modulus, $\mathbf{E}_{\mathrm{c}}$ (psi) & 360 & 360 \\
\hline Reference Depth, $\mathbf{h}_{\mathrm{o}}$ (in.) & 4.625 & 4.625 \\
\hline Shear Depth Factor Exponent, m & 0.84 & 0.86 \\
\hline
\end{tabular}

All properties are based on a minimum panel width of 24 inches.

SIP manufacturers claim that SIPs create 'green' buildings through their energy efficiency and use of renewable resources, their creation of healthy indoor environments, and their reduction in waste during the construction process (McIntosh and Harrington 2007). In many conventionally-constructed buildings, large amounts of heat are lost or gained through air leakage or thermal bridging. Up to $40 \%$ of a home's heat loss is due to air leakage (SIPA 2009). SIPs can be used to create tighter building envelopes (about 15 times more airtight than stick framing) and better insulation (about 1.5 times higher insulation coefficient, $R$, values), which can provide environmental benefits and a significant increase in energy efficiency (Pasma 2010). However, with a better seal between indoor and outdoor air, there is some concern over the degradation of indoor air quality and the potential for SIPs to release volatile contaminants, mainly aldehydes (Little and Hodgson 2004). The advantage of reduced construction time is 
valuable, not only economically, but also for providing shelter in the face of civil emergencies. The National Association of Home Builders estimates that the construction of a 2000 sq. $\mathrm{ft}$. home produces 7000 lbs of waste, which can be significantly reduced by extensive use of SIPs (SIPA 2009).

As well as providing 'green' benefits, SIPs also provide bases for interior and exterior cladding, insulation against temperature extremes, secondary water-tightness, and structural support to resist gravity and lateral loads, performing in a manner analogous to I-beams (McIntosh and Harrington 2007). They have been shown to perform well in natural disasters, including earthquakes, hurricanes, and record snowfalls (McIntosh and Harrington 2007).

While SIPs have the inherent limitation of restricted in-wall utilities, there are a few other problems associated with SIPs, mainly in that polystyrene is difficult to recycle and OSB has a poor finish, with susceptibility to warping (McIntosh and Harrington 2007). Other potential problems with traditional SIPs include their use of formaldehyde-based adhesives, the inability of EPS to degrade post-demolition, and the smoke produced by burning EPS. Formaldehyde offgassing can be a health concern if panels are not properly cured or undergo hydrolysis. However, OSB manufacturers adamantly claim that OSB has been inaccurately accused of formaldehyde emissions associated with other fiber board products; while it does contain phenolic formaldehyde adhesives, the finished product OSB contains only trace amounts of formaldehyde that do not pose any health risk (SIPA 2009). This is likely a case of consumer misconception, but is still a minor challenge for OSB as a housing product. EPS is highly flammable, while other typical forms of insulation emit toxic fumes when exposed to flames. The use of FRPs as structural skins would provide better strength, stiffness, fatigue life, and resistance to warping, absorption and fire (with intumescent coatings). Some researchers have already used FRP in conjunction with OSB to improve the strain energy of shear walls (Davids et al. 2004).

Natural fiber / natural resin FRPs, using a natural insulator as the core, would improve mechanical properties while using economical, environmentally-safe materials and processes (McIntosh and Harrington 2007). Insulating core options include soy-based foams, corn-based foams, recycled cellulosic waste foams, and straw. Soy foams are recently developed materials with promising insulation and fire response properties. Corn foam production is a more expensive process than soy foam production, but some promising results have been found working with $\mathrm{CO}_{2}$-foamed polylactic acid (McIntosh and Harrington 2007). Recycled cellulosic waste foams tend to be a mix of bio and mineral-based components. Densely packed straw does not resist moisture, has inconsistent thermal values, and does not provide consistent structural properties.

For bio-SIPs to become common, they must meet the thermal efficiency of traditional SIPs, be manufactured from local or sustainably managed sources, be durable, result in few or no undesirable emissions, and reduce overall waste. A cost comparison is difficult to generalize, as material costs are more when houses are built only to minimum standards; however, SIPs provide a number of additional benefits that must be taken into account (Johnston and Gibson 2008). Size, weight, structural integrity, and fire resistance as well as the potential problems of pest attraction, weatherproofing, and water damage are all necessary design considerations if bioSIPs are ever going to become mainstream products. 


\subsection{Challenges}

Nearly every researcher who has worked with natural fiber composites agrees that there are significant challenges impeding their advancement into the field of primary structural components. Applications for natural fibers are often limited to interior and non-structural applications due to their lower mechanical properties and poor moisture resistance, but these properties appear to have significant room for improvement through the use of innovative surface treatments, resins, additives, and coatings. Other challenges facing natural fiber composites are fire resistance, fiber/matrix adhesion, durability, variability in quality, and manufacturing difficulties.

\subsubsection{Moisture Absorption / Hydrophilic Nature}

All natural fibers are hydrophilic in nature. This is the main drawback of natural fibers, as this causes them to have high water absorption and to be incompatible with hydrophobic polymer matrices (Mohanty et al. 2001; George et al. 2010). Glass fibers, on the other hand, are essentially moisture resistant. Historically, natural FRPs used as building materials have failed in wet conditions through surface roughening by fiber swelling or delamination (Singh and Gupta 2005a). Natural composites perform particularly poorly when immersed in water; Singh and Gupta (2005a) found that the strength of a sisal/polyester composite was 13-31\% lower when fully immersed than at 95\% RH. Ahmed and Vijayarangan (2007) found that a saturated value of $13 \%$ (by weight) moisture absorption could lead to as much as a $50 \%$ reduction in mechanical properties. However, this is not always the case, as sometimes the effect is far less pronounced or can even be positive (Bakare et al. 2010).

Biopolymers also tend to absorb more moisture than their synthetic counterparts, although a study by Alam et al. (2006) focused on one that only absorbed around 1.1\% moisture.

\subsubsection{Fiber Modification}

The most common way to reduce the moisture absorption capability of a natural fiber seems to be through the process of alkalization (also known as mercerization). Alkali treatment (usually with potassium hydroxide, $\mathrm{KOH}$, or sodium hydroxide, $\mathrm{NaOH}$ ) reduces the hydrogen bonding capacity of the cellulose, eliminating open hydroxyl groups that tend to bond with water molecules. Alkalization can also dissolve hemicellulose. The removal of hemicellulose, which is the most hydrophilic part of natural fiber structures, reduces the ability of the fibers to absorb moisture (Symington et al. 2009). However, during alkalization, the amount of amorphous cellulose (cellulose II) is increased at the expense of crystalline cellulose (cellulose I) (John and Anandjiwala 2008). This is a negative effect for moisture absorption, as crystalline cellulose does not possess hydroxyl groups readily available for bonding to water molecules like amorphous cellulose, and the overall loss of crystalline structure can lower mechanical properties (Symington et al. 2009; Pott et al. 2000a; Jin and Nava 2002).

The concentration of the alkali and the process time affects the resulting fiber properties. According to Symington et al. (2009), the best treatment to reduce moisture absorption of flax fibers is exposure to a $\mathrm{NaOH}$ solution at $\sim 2-10 \%$ concentration for $\sim 10-30$ minutes, followed by neutralization with a low-concentration acid and washing with distilled water. Most other researchers recommend a similar pattern (occasionally with a exposure time more on the order of 16-24 hours for different fiber types) of alkali treatment for either the reduction of moisture absorption or improvement of mechanical properties, with mixed results (Williams et al. 2011; 
Chang et al. 2009b; Bakare et al. 2010; Hu and Lim 2007; Mutnuri et al. 2010; Van de Weyenberg et al. 2006; Chang et al. 2009a; De et al. 2007; Ibrahim et al. 2010b; Kreže et al. 2005; Rong et al. 2001). In some cases, treatment with alkalization actually increased moisture absorption, although that could partially be due to a higher resulting fiber content (Rouison et al. 2005). If the fibers are not carefully washed after alkalization, the alkali will continue to degrade the fibers long after exposure, eventually leading to significant fiber swelling and breakdown.

Some negative aspects of the alkali treatment process include the high $\mathrm{pH}$ values, high surfactant content, polluted wastewater, and the degradation of cellulose fibers. Alternatively, Kreze et al. (2005) explored the use of pectin-dissolving enzymes. While their research showed that the alkali-treated fibers were more effective at lowering moisture absorption, the enzymetreated fibers produced less polluted wastewater.

Another process that shows promise for reducing the moisture content in natural fibers is the Duralin steam treatment process (Stamboulis et al. 2000; Bismarck et al. 2005; Pott et al. 2000a; Pott et al. 2000b). This process depolymerizes the hemicellulose and lignin into aldehyde and phenolic functionalities, which are subsequently cured into a water-resistant resin. Untreated fibers reached a maximum moisture content of over $42 \%$ in $100 \% \mathrm{RH}$ while the fibers treated by the Duralin process reached a maximum moisture content of only around 14\% (Stamboulis et al. 2000). The moisture diffusion time was also slowed for the fibers treated by the Duralin process. Some advantages of the Duralin process include the omittance of dew retting, increased fiber yield and quality, better dimensional and temperature stability, better resistance to fungal attack, and generally improved mechanical properties (Pott et al. 2000a; Pott et al. 2000b). On the other hand, there is a significant amount of added embodied energy to the fibers due to the steaming process.

\subsubsection{Matrix Modification}

In most cases, the concern about moisture absorption in natural FRPs is focused on the natural fibers, since most resins are hydrophobic and absorb very little moisture. However, soy protein resins can absorb a large amount of moisture which reduces their mechanical properties. Chabba and Netravali (2004) modified SPC resin by adding glutaraldehyde (GA) or GA plus poly(vinyl alcohol) (PVA). GA was added to increase cross-linking for better mechanical properties, while PVA was intended to improve the toughness of the resin. Both modified resins exhibited about 2\% less moisture absorption than the unmodified SPC. Kumar and Zhang (2008) mixed SPI powder with two different plasticizers and found that the resulting plastic would actually lose mass when immersed in water due to a very low moisture absorption rate and offgassing of $\mathrm{CO}_{2}$. Treating soy protein films by arylation (immersion in benzilic acid) was also shown by Kumar and Zhang (2009) to reduce moisture absorption and improve mechanical properties. In both treatment cases, the resulting soy protein plastic remained biodegradable.

Alternatively, there are some polymer coatings that can reduce moisture absorption. If the material properties of the coating are carefully selected and the coating is properly applied, diffusion of the material can be cut in half (Newill et al. 1999). In most cases, though, coatings only are effective in providing short- to medium-term protection. Doherty et al. (2007) improved moisture absorption properties by applying a natural, lignin-based coating. 


\subsubsection{Fire Resistance}

Fiber reinforced polymer composites can lose strength and stiffness at elevated temperatures. According to Sorathia et al. (1997), some tests have shown up to 50\% strength reduction at temperatures as low as $121^{\circ} \mathrm{C}$ for glass/vinyl ester composites. Due to the high thermal capacitance of glass reinforced composites, once a fire has been put out on the surface of the material, reignition can occur if there is retained internal thermal energy. The gases released could also potentially be hazardous to firefighters.

Variations in flammability of natural fibers are at least in part due to differences in chemical composition. Fibers with higher cellulose content tend to be more flammable than those with higher hemicellulose content, and char formation is generally better with higher lignin content (Chapple and Anandjiwala 2010). Silica, or ash, present in the fiber chemical composition also has been shown to be related to better fire resistance (Chapple and Anandjiwala 2010). In addition to chemical content, fiber microstructure can also be a contributing factor in determining fire resistance, with higher crystallinity and lower polymerization improving fire resistance (Chapple and Anandjiwala 2010).

Phenolic-based synthetic composites offer good fire performance at reasonable cost, when compared to other potential matrix materials. Sorathia et al. (1997) offer that "Phenolic resins have the inherent characteristics of low flammability, less smoke, low flame spread, high ignition delay, low peak heat release rate, and high oxygen index." Phenolics also tend to char during fire exposure, which can protect the core of the material and thus some of the structural integrity. If synthetic resins are to be reinforced with natural fibers, phenolics offer the best fire resistance.

There are a number of coatings or additives that can be used to improve fire resistance. Fire barriers for composites can be made from ceramics, intumescents, silicone, phenolics, ablatives, glass mats, or one of several chemical additives (Sorathia et al. 1997; Chapple and Anandjiwala 2010). The intumescent systems (coatings or additives) show particular promise as fire barrier treatments for natural fiber reinforced composite systems.

When intumescent materials are heated beyond a specific temperature, they begin to foam and expand, forming a cellular, charred surface that protects the underlying material from the heat flux or flame. The addition of intumescent zeolites, natural clays, and zinc borates to polymers has been shown to drastically enhance fire performance. Polyols are commonly used char formers in thermoplastic intumescent systems, but have problems with water solubility, compatibility, and reduced mechanical properties; polyolefin char formers, on the other hand, have improved mechanical properties and reduced water solubility (Bourbigot et al. 2004). Doherty et al. (2007) found that a natural alternative of char-forming lignin coating minimally improved fire test results. The addition of common fillers, like talc or nanoparticles, can also improve fire resistance by forming physical and heat barriers (Chapple and Anandjiwala 2010).

Currently, there are few, if any, completely biodegradable composites that can pass building flammability tests. Plackett and Sodergard (2005) concluded that no PLA composites passed the requirements for the UL 94 flammability test. Possibly the best potential for producing a flame-retardant natural fiber reinforced composite is to combine a char-forming cellulosic material and an intumescent system (Chapple and Anandjiwala 2010). 


\subsubsection{Mechanical Properties and Durability}

In a recent biocomposites project at Stanford University, researchers manufactured and tested hemp/cellulose acetate (HCA) and hemp/polyhydroxybutyrate (HPHB) biocomposites (made of bidirectional fabric, eight plies) (Christian and Billington 2009). Results showed that mechanical properties were comparable or better than lumber, but significantly less than glass or carbon fiber composites. The tensile modulus was found to be around $798 \mathrm{ksi}$ (5.5 GPa), tensile strength around $8.1 \mathrm{ksi}$ (56 MPa), and the flexural strength and stiffness were around $13.8 \mathrm{ksi}$ (95 $\mathrm{MPa})$ and $943 \mathrm{ksi}$ (6.5 GPa), respectively. Most 'semi-green' or 'green' composites have maximum tensile strengths and stiffnesses too low to be used for primary, load-bearing components (Netravali et al. 2007). One way to handle this lower performance is to use designed structural configurations that negate these disadvantages, like cellular constructions (Burgueño et al. 2004), although such designs are not suited for all applications. Hybridization is another potential solution to improve mechanical properties, but also reduces the overall attractiveness of natural FRPs. On the other hand, George et al. (2010) believed that the mechanical properties of natural fiber reinforced composites had sufficient mechanical properties for use as sustainable construction materials, but their response to environmental conditions and durability concerns was not yet adequately established.

\subsubsection{Adhesion / Wetting}

The main problem leading to reduced mechanical properties in natural FRPs is the poor compatibility/adhesion between the hydrophilic fibers and the hydrophobic matrix materials (Malkapuram et al. 2009). Many researchers have been developing treatments to modify the surface characteristics of the fibers to improve compatibility and adhesion.

The three factors affecting the bond between two materials are the mechanical interlocking, the molecular attractive forces, and the chemical bonds. According to Belgacem and Gandini (2005), chemical modifications should be limited to the superficial OH-groups (hydroxyl groups) so as not to disrupt the integrity and mechanical strength of the fibers. Ideally, hydroxyl groups in a resin would bond with the hydroxyl groups that are available in all natural fibers, creating hydrogen bonds. The bond strength between resins and fibers is significantly lowered by the presence of moisture while curing due to the fact that $\mathrm{H}_{2} \mathrm{O}$ molecules will bond with the available hydroxyl groups on the surface of the fiber, lessening the connections available for matrix bonding. When the water evaporates, this then leaves voids in the cured composite. If fibers are properly dried before a suitable matrix is introduced, then a better bond ought to result and future moisture uptake ought to be limited due to the lack of available hydroxyl bonding locations.

\subsection{Alkalization}

There are a number of different modifications that can be made in order to improve the interface between the fibers and the matrix. One of the most popular surface modifications for improving strength is alkali treatment, where the reduction of absorption capacity and the surface modification of the fibers work together to improve the mechanical properties of the composite. Most alkalization treatments intended to improve mechanical properties are conducted with a procedure similar to the treatment to reduce moisture absorption: soaking in 2-10\% concentration $\mathrm{NaOH}$ for between 10 minutes and a few hours (Symington et al. 2009; Ibrahim et al. 2010b; Kalia et al. 2009). Treatment for kenaf fibers with alkali for much longer than this may lead to 
higher individual fiber tensile properties, but worse composite properties, as the number of bonding sites would be too reduced (Williams et al. 2011). During alkalization, the cementitious materials in the fibers are removed, leading to rougher fiber topography. Even in low $\mathrm{NaOH}$ concentrations, fibers can fibrillate which increases the aspect ratio and the bondable surface area. By improving the fiber/matrix adhesion in this manner, the tensile and flexural properties of the composite are improved at the expense of a reduction in impact behavior. De et al. (2007) found that alkali treatment could improve mechanical properties of a natural fiber reinforced composite by as much as 50\%. Flexural tests conducted by Van de Weyenberg et al. (2006) showed that longitudinal strength and stiffness experienced some increases, while transverse strength and stiffness experienced sharp increases. This shows that the interface, rather than the fiber properties, was improved. This improvement is believed by Van de Weyenberg et al. (2006) to be a combination of better mechanical interlocking, due to the rougher topography and the larger number of individual fibrils, and better chemical bonding, due to the purified fiber surface being enabled to form more hydrogen bonds between hydroxyl groups on the cellulose and the resin. Hu and Lim (2007) pointed out that the alkali treatment not only improves the fiber surface and bonding, but also increases the structural material content (by the removal of nonstructural parts of the fibers), which would lead to seemingly higher composite mechanical properties at the same volume fraction of fibers. Although the overall composite strength is higher due to the improved interface, the fiber strength may be lower after alkali treatment. An alkali treatment in too great of a concentration may overly depolymerize the cells, which can lower the strength of the fiber beyond usability (Mohanty et al. 2001). While most sources agree on the theory behind the improvement of mechanical properties due to alkalization, in practice the process (for certain fibers, resins, or manufacturing processes) sometimes fails to produce significant change in the resulting composite mechanical properties or even causes a significant reduction (Rong et al. 2001; Doherty et al. 2007).

According to Chang et al. (2009b), an ultrasound treatment, when combined with an alkaline treatment, can improve pectin technology by extracting low molecular substances and depolymerizing macromolecules. Results showed conclusively that the combination of alkali treatment with ultrasound treatment is more effective in improving mechanical properties as compared to the chemical treatment alone. Rahman and Khan (2007) worked with coir fibers that were treated with ultraviolet aging, alkali treatment, and grafting with an acrylate monomer using UV radiation. The UV pretreatment resulted in an additional improvement of tensile properties, to a total increase in tensile strength of $33 \%$.

\subsection{Coupling Agents}

Other than treatment with alkaline, most chemical modifications generally involve the introduction of a third material that remains in the composite to act as a compatibilizer between the hydrophilic fibers and the hydrophobic polymers. Coupling agents can improve the bond between materials by eliminating weak boundary layers, by producing tough, deformable layers, by developing a highly cross-linked interphase region with an intermediate modulus, by improving the wettability (critical surface tension factor), by forming covalent bonds with both materials, and by altering surface acidity (Malkapuram et al. 2009).

Silanes are popular compatibilizers, and can form chemical bridges between the fibers and the matrices. Many different silane structures have been used for coupling of inorganic reinforcements and organic polymer matrices; a more limited number have been used to couple 
natural fibers and polymer matrices, most commonly trialkoxysilanes (Xie et al. 2010). By proper surface or bulking treatment with silanes, the hydroscopic natural fibers can be converted into a hydrophobic reinforcement. Surface treatments decrease the water sorption rate, while bulk treatments additionally reduce the water sorption capacity (Xie et al. 2010). Unlike alkalization, silane treatments have little effect on fiber tensile strength (Xie et al. 2010). Valadez-Gonzalez et al. (1999) found that a combination of alkalization and silane coupling agents produced higher interfacial shear strength in a natural fiber reinforced composite.

Stearic acid hydrophobizes fibers and improves dispersion and wetting, but was shown to provide no statistical improvement to fiber strength (Zafeiropoulos et al. 2007). For better fabric impregnation, low viscosity polymer dispersions are used (Malkapuram et al. 2009). Isocyanates can be chemically linked to the cellulose matrix through strong covalent bonds, and are reported to be more effective than silane treatments (Malkapuram et al. 2009). Triazine also forms covalent bonds with cellulose fibers and can reduce moisture absorption (Malkapuram et al. 2009). Acetylation is an esterification mode to introduce plasticization, and has been shown to improve dimensional stability and environmental degradation (Mohanty et al. 2001). However, in one study there was no statistical effect of acetylation on fiber strength for curing up to 2 hours, and only a detrimental effect beyond that (Zafeiropoulos et al. 2007). Maleic anhydride grafted polypropylene (MAPP) is a common compatibilizer for natural fiber / polypropylene composites, improving wettability and adhesion (Mohanty et al. 2001). Qiu et al. (2011) coated hemp fibers in unsaturated polyester resin with a solution of 1,6-diisocyanatohexane and 2hydroxyethyl acrylate dissolved in anhydrous ethyl acetate as a chemical bridge, improving tensile strength by around $30 \%$ and reducing water uptake by about $25 \%$.

\subsection{Additives}

Wood (2009) reported that researchers treated kenaf fibers with chemical retting and calcium chloride or sodium carbonate nanoparticles. The use of nanoparticles, in addition to filling micropores in the fibers, created crystalline layers that were intended to improve the interface between the fibers and resin. This modification was able to increase stiffness by about $10 \%$.

It is also common to introduce additives to the matrix material to improve adhesion and mechanical properties. Malkapuram et al. (2009) used maleated polyolefins to improve adhesion. Huang and Netravali (2009) combined SPC powder and micro bamboo fibrils (MBF) to make a fully biodegradable composite. The addition of (3-isocyanatopropyl)triethoxysilane (among other chemical modifiers) to the matrix was found to increase toughness and fracture strain, but had little effect on the strength or stiffness.

Mohanty et al. (2001) concluded that, with the proper surface modifications, those systems which used flax fibers in combination with biodegradable matrices showed promise for suitability as construction materials.

\subsubsection{Hybridization}

Hybridization, in the context of natural FRPs, generally refers to the combination of glass fibers (or, in some cases, polymer fibers (Tan et al. 2012)) and natural fibers in either a synthetic or biopolymer matrix. Hybridization was shown by to increase flexural strength and reduce water absorption properties (Ray and Rout 2005) and to significantly increase mechanical properties at a loading of only 5.7\% by weight (Burgueño et al. 2005). Drzal et al. (2004) believed that 
combining natural and glass fibers with optimized cellular structural forms would allow natural composites to compete with conventional structural materials. Haq et al. (2009) concluded about non-hybridized biopolymers that "their mechanical and thermal properties are far lower than required for structural applications.”

The main argument against fiber hybridization is that nearly all of the advantages of natural fibers (biodegradability, recyclability, easy handling and processing, and being made from renewable resources) are negated by being mixed with glass fibers. While hybridization can still be used to reduce cost and weight, and to provide some improvement to environmental effects, the hybrid systems still have reduced mechanical properties compared to glass FRPs. As with fibers, hybridization of resins can allow natural materials to be used in higher-performance applications, but much of the attraction of the natural materials is forfeited in the process.

\subsubsection{Durability}

One major concern with natural composites is their long-term behavior when exposed to different environments, such as hygrothermal aging and loading as well as prediction of lifetimes (Satyanarayana et al. 2009). The general consensus from a review of literature is that very little fatigue work has been carried out on natural fiber composites, although Hagstrand and Oksman (2001) found that a flax / melamine-formaldehyde composite performed favorably in fatigue compared to a glass-reinforced composite. Ray and Rout (2005) conclude that this will be a necessary area of research before natural fiber composites are accepted as primary structural components. More work has been done exploring the results of natural composites exposed to the durability concerns of moisture absorption and weathering than fatigue.

The growth of fungus and bacteria in natural composites due to biodegradation or moisture retention is a major concern in their development as structural materials. On samples of jute / phenolic composites exposed to humidity by Singhet al. (2000), some black spots and white patches appeared, which when viewed under a microscope were observed to be fungal hyphae. In another study by Stamboulis et al. (2000), moisture was found to cause fungus development on the surface of flax fibers after as little as three days' exposure. The Duralin treatment appeared to lessen this effect, improving the environmental durability. On the other hand, O'Dell (1997) reported that jute / UPE samples exposed to the same fungal and UV environments exhibited similar behavior to glass FRPs, indicating that whatever problems exist are shared by both products.

Weathering studies conducted by Singh and Gupta (2005b) showed that UV can cause color fading. After two years of exposure, jute / phenolic composites showed resin cracking, bulging, fibrillation, and black spots, with a tensile strength reduction of over 50\%. Mehta et al. (2006) also found that weathering produced color change, weight loss, and surface roughening. In an effort to reduce weathering, some samples were coated in polyurethane, and exhibited very little surface deterioration (Singh and Gupta 2005b). Satyanarayana et al. (1990a) found that mechanical properties deteriorate quickly with exposure to outdoor conditions for natural fiber / polyester composites. The strength was reduced by around 5-25\%, with a more pronounced reduction in wetter conditions. The same composites exposed to indoor conditions for the same duration showed no significant changes in mechanical properties. Poor weathering performance is not only a problem for natural fibers; soy protein plastic lost strength and toughness and became stiff and brittle over time, possibly due to leaching of the plasticizer additive (Mohanty et al. 2005a). The use of proper coatings (Singh and Gupta 2005a) and certain types of fiber 
modification (bleaching, alkalization, or silanes) (Dash et al. 2000; Xie et al. 2010; Joseph et al. 2006) seem to slow the effects of weathering.

\subsubsection{Variability}

Fiber variability can lead to problems in natural FRPs, particularly if they are going to be used as primary structural components. There are many factors that can lead to variation in fiber quality: crop variety, seed density, soil quality, fertilization, field location, fiber location on the plant, climate, weather conditions, and harvest timing can all impact the raw material (Bismarck et al. 2005; Sherman 1999). Additionally, variation in the cross-sectional area of the fibers, extraction processing methods, damage incurred in handling and processing, and differences in drying processes can lead to further variation in the end-use product (Mohanty et al. 2001; Symington et al. 2009). Fiber quality assurance protocols are necessary to ensure that fiber nonuniformity and dimensional variability does not lead to drastically different mechanical properties between production batches (Bismarck et al. 2005).

In addition to variability in the quality of natural fibers, there is also potentially a large degree of variability in the price and availability of fibers. For example, flax is more expensive than comparable fibers and has a price that fluctuates frequently due to climate variations (Defosse 2004). If an important growing region for a particular fiber experiences a poor growing season due to uncontrollable environmental effects, than that fiber will be less plentiful and more expensive. The best way to counteract this unreliability is to grow many types of fibers in different regions to avoid local shortfalls.

\subsubsection{Manufacturing / Processing}

Natural fibers generally cannot be processed at over $356-392^{\circ} \mathrm{F}\left(180-200^{\circ} \mathrm{C}\right)$, or over $347^{\circ} \mathrm{F}\left(175^{\circ} \mathrm{C}\right)$ for prolonged periods without a significant decrease in mechanical properties (Mohanty et al. 2001; John and Anandjiwala 2008; Ochi 2008; Fowler et al. 2006). As a result of this restriction, suitable matrix systems and processing methods need to be chosen. Most biopolymers also have low processing temperatures; for example, PLA has a $\mathrm{T}_{\mathrm{g}}$ of $\sim 136^{\circ} \mathrm{F}$ $\left(\sim 58^{\circ} \mathrm{C}\right)$ and a melting point, $\mathrm{T}_{\mathrm{m}}$, of $266-446^{\circ} \mathrm{F}\left(130-230^{\circ} \mathrm{C}\right)$, which would make it compatible with most natural fibers (Henton et al. 2005). Most synthetic composite manufacturing methods that do not require intense heat are also compatible with natural FRPs.

The simplest form of composite manufacturing, hand lay-up, works well with natural composites as it does not intrinsically involve the application of heat. However, Satyanarayana et al. (1990a) found that only 14 wt\% lignocellulosic fibers could be incorporated by the hand layup technique, while between $20-80$ wt $\%$ could be incorporated by press molding. Satyanarayana, et al. (2009) concluded that compression molding was the preferred method for production of natural fiber reinforced composites. Brouwer (2000) reported that resin transfer molding (RTM) and vacuum molding worked best for natural fibers with higher fiber volume fractions and preformed mats. O’Donnellet al. (2004) showed how vacuum-assisted RTM was able to cure large-volume natural fiber / soybean oil composites at room temperature. Pultrusion is a popular manufacturing method for synthetic fibers and, while it has only recently become a more popular area of exploration for use with natural fibers, it has high potential for use with natural fibers and/or biopolymers (Ray and Rout 2005). Lackey et al. (2004), Chandrashekhara (2004), and Peng et al. (2012) demonstrated that pultrusion of natural fiber composites was feasible, although Mutnuri et al. (2010) found that pultrusion worked much better when some glass fibers were also 
included in the lay-up with the natural fiber fabrics. Peng et al. (2012) observed that the mechanical properties of the pultruded natural fiber composite were promising even though SEM revealed that the fiber/matrix adhesion was poor. Fowleret al. (2006) and Oksmanet al. (2003) have shown that natural fibers can be successfully incorporated into thermoplastic matrices using compounding, extrusion, and injection molding; however, the potential mechanical properties of composites produced by this process are low compared to processes that use longer fibers.

As an interesting side note, Satyanarayana et al. (1990b) reported a common problem with high resin consumption in natural fiber reinforced composites. They found that the use of treated fibers could reduce resin wastage and consumption by half, although the mechanism behind this reduction is not clear.

\subsection{Potential}

Several of the major areas of interest behind the development of natural composites are the potential economic impacts, environmental impacts, and the ability of natural composite to meet social, economic, and material needs worldwide.

\subsubsection{Economic}

"The reason business should be specifying sustainable materials is not that it's just ethically the right thing to do, but for commercial longevity it's going to be the right thing to do as well," says Dr. Kristian Steele, a sustainability consultant (Graham 2011).The costs of construction materials have been increasing dramatically for several years. Building materials constitute $60-75 \%$ of the total cost of construction; this cost could be reduced by efficient use of locally grown raw materials (Satyanarayana et al. 1990a). The construction industry provides a vital role in economic sustainability, since through construction resources are transformed from one area of capital (man-made, human, social, or natural) to another, with the objective being a net gain. Built wealth accounts for around $16-22 \%$ of the total wealth of European countries (Pearce 2003). Dwellings have historically made up about a third of that amount. Considering the current house building rates in several countries, the amount of time each dwelling would need to be used before being replaced is around 133 years in the UK, 103 years in France, 78 years in the US, and 28 years in Japan (Pearce 2003). An alternative way to look at this data is that the UK completed only 3 dwellings per 1000 persons in the mid-1990s; the US completed 5.3 and Japan completed 13 per 1000. While in some ways it is good that housing stock is durable, once other considerations are factored in, such as energy efficiency, compatibility with new technology, health risks, and repair needs, these older dwellings are in all likelihood a barrier to technological advancement.

The North American demand for wood and agricultural fibers as plastic additives is projected to increase by 30\% each year in the automotive industry and as high as $60 \%$ each year in construction applications (Mohanty et al. 2001). Satyanarayana et al. (2009) and Defosse (2004) predict natural fiber industry growth rates from 10-22\% per year. The biopolymer market is predicted to increase in sales by anywhere from 16-30\% per year (Mohanty et al. 2001; Mohanty et al. 2000). Bismarck et al. (2005) and Defosse (2004) agree that current natural fiber production (particularly flax) in the US and Europe will soon be unable to meet market demands, indicating that opportunity is ripe for other regions of the world to join the market. India is already a major producer of natural fibers, but not flax. Unless government subsidies are 
provided, US and European fiber producers will not be able to compete with prices of fibers grown in developing countries.

The development of natural composites is largely motivated by greater environmental awareness, waste disposal problems, and the depletion of petrochemical resources. Several countries have passed legislation encouraging the use of recycled or bio-based products (Fowler et al. 2006). The cost of biopolymers has, in recent years, been too high to sustain the industry (Mohanty et al. 2000). Each stage of processing needed to create natural fibers or biopolymers reduces the value added of the final product, thus the simplest production method provides the greatest value (Bismarck et al. 2005). Surface modifications are necessary, yet add cost, and therefore need to be optimized in order for biocomposites to compete with glass fiber composites (Mohanty et al. 2001; Beckwith 2003). The cost ranges for each natural fiber (as seen in Figure 2-4 and Figure 2-5) are a result of crop variability and the difficulty associated with storing, transporting, and processing fibers. The real challenge lies in finding new applications for the natural FRPs which will lead to substantial price reductions. Once prices begin to fall, the potential market for natural composites will likely grow in many industries, not just in civil engineering applications.

There is some disagreement about how the future market of natural FRPs will compare to the market for synthetic FRPs. Rowell et al. (1997) and Satyanarayana et al. (1990a) do not anticipate the replacement of synthetic fibers with agricultural-based fibers, but instead think they will have their own niche in the market for non-critical applications. Conversely, Netravali et al. (2007) believe that continuing research into natural composites will eventually lead to the replacement of petroleum-based fibers and resins in many applications. If natural FRPs can be produced with strengths and stiffnesses comparable to those of synthetic FRPs, they will gain at least a significant share of the market for use in low-moisture applications where durability is less of a concern.

\subsubsection{Environmental}

Patel and Narayan (2005) define sustainable development as "development that meets the needs of present without compromising the needs of future generations to meet their own needs." This definition implies that sustainable development must include environmental, economic, and social factors. Sustainability is not as much about achieving a specific goal within a period of time, but instead is about each generation trying to provide the next generation with a reasonable chance to have an improved quality of life (QOL) (Pearce 2003). Biocomposites are promising materials in terms of sustainability due to their substitution of renewable resources for petrochemical materials, lower greenhouse gas emissions, closure of the cyclical loop from raw material growth to biodegradation, potential for lower production costs, and opportunities for growth in agricultural and chemical industries (Patel and Narayan 2005). Built structures are major factors in energy consumption and carbon dioxide emissions, making up over $46 \%$ of the total national values in the UK for each (Pearce 2003); in the US, construction and operation of buildings accounts for 39\% of total energy consumption, and 38\% of carbon dioxide emissions (SIPA 2009). While the production of cement and other building materials results in a large amount of carbon emissions, efficiently produced natural composites would provide a minimal carbon footprint due to their incorporation of $\mathrm{CO}_{2}$-absorbing natural resources.

Fowler et al. (2006) concluded that the greater environmental detriment in synthetic composites is from the petroleum-based matrix materials and that the development of high 
quality bio-matrix materials is of higher environmental concern than the development of natural reinforcements. Over less than two years, pure PLA will degrade to carbon dioxide, water, and methane, whereas petroleum-based plastics require hundreds of years ( $\mathrm{Hu}$ and $\mathrm{Lim}$ 2007). Recycled plastics are often contaminated by impurities and incompatible materials, but PLA can be reduced to lactic acid, which can then be purified and used to remake PLA with no loss in mechanical properties (Henton et al. 2005).

Construction impacts the environment in many ways: from raising social capital by providing safer and more aesthetic towns and cities to decreasing natural capital due to consumption of energy and natural resources and production of waste. Few material or energy sources are near depletion, but the environments that receive waste are of particular concern. In the UK, about $25 \%$ of construction materials end up as waste and of those about $50 \%$ are not recycled (Pearce 2003). While these numbers could be interpreted as either good or bad, there is no benchmark against which to compare them. Much of this waste is also in the form of inert (non-polluting) waste, which is less difficult to dispose. Around $25 \%$ of construction waste is packaging materials, with $10 \%$ each of timber, concrete, ceramic, plaster/cement, and insulation (Pearce 2003).

The use of large volumes of synthetic composites has already led to disposal problems. In the US alone, in 2001 over 25 million tons of plastics (including reinforced plastics) entered the municipal solid waste stream, which accounted for over $11 \%$ of the total national waste (Mohanty et al. 2005b). Construction demolition debris as a whole occupies $40 \%$ of landfill space in the US (Cramer 2004). Landfill capacity issues are a major concern - many states are within only a few years of reaching their landfill capacity.

Joshi et al. (2004) identified the four potential areas of environmental improvement offered by natural fibers as: lower environmental impact from natural fiber production compared to glass fiber production, substitution of higher volume natural fibers for some base polymer material, lower emissions while in use/transport due to weight reduction, and energy/carbon credits from end of life incineration. The $\mathrm{CO}_{2}$ contribution of fiber production depends on many growing and processing factors, such that conclusions on the eco-friendliness of natural fibers are not clear cut (Bismarck et al. 2005). Life cycle analysis (LCA) is a sustainability assessment methodology that has been developed to evaluate the environmental effect of parameters such as raw materials, energy consumption, and ultimate disposal or recycling characteristics. Several different researchers have attempted to quantify the environmental improvements natural fibers offer over synthetic fibers.

Mohanty et al. $(2001,2002)$ concluded that natural fibers' needed energy is much lower than for synthetic fibers with a pound of kenaf requiring around 6,500 BTUs (6.86 MJ) of production energy compared to 23,500 BTUs (24.8 MJ) to produce a pound of glass fiber. Mueller and Krobjilowski (2003) similarly concluded that the energy demand to produce a natural fiber nonwoven fabric only amounts to $30-40 \%$ that required to produce a glass fiber mat. Mutnuri et al. (2010) found through life cycle energy assessments that 40-60\% less energy was consumed by natural FRPs as compared to glass FRPs. The factors considered in this assessment included raw material extraction, raw material processing, raw material transportation, and manufacturing of the composite. Finally, a more comprehensive energy analysis of natural fibers compared to glass fibers by Patel and Narayan (2005) found a range of results showing anywhere from limited improvement (-14\%) to good improvement (-45\% to $-50 \%)$ for energy usage. LCA 
studies of the use stage of biomaterials showed that energy savings at this secondary stage could be even higher than at the processing stage (Patel and Narayan 2005).

While a quality life cycle assessment that proves conclusively that biocomposites are in fact 'greener' would certainly benefit the industry, no such conclusion is available, nor realistically should be made at any point in the immediate future given the numerous variables to be considered for each application. However, according to Patel and Narayan (2005) it seems that relative to conventional materials, green polymers and natural fiber composites should save at least 19,000 BTUs (20 MJ) of nonrenewable energy per kilogram of polymer, avoid at least 1 $\mathrm{kg}$ of $\mathrm{CO}_{2}$ per kilogram of polymer, and reduce most other environmental impacts by at least $20 \%$. At the very least, LCA studies strongly support further development of biomaterials.

\subsubsection{Meeting Needs}

The use of FRPs in primary structural applications would be of particular interest in countries like India, where wood is often scarce for building projects. Wood production in India has a per capita output of around $0.88 \mathrm{ft}^{3}\left(0.025 \mathrm{~m}^{3}\right)$, whereas the United States produces $66.7 \mathrm{ft}^{3}$ $\left(1.89 \mathrm{~m}^{3}\right)$ (Singh and Gupta 2005a). India already possesses a good knowledge-base in working with composites and has numerous related raw materials (Baksi et al. 2010). If natural composites could be manufactured locally, they would probably quickly become costcompetitive with other wood substitutes.

Increased demand for natural fibers can have two important socioeconomic impacts: the first in that it would generate an industrial crop source for the economic development of rural and agricultural-based regions of the world, and the second in that the use of natural FRP modular construction for housing could be a key to providing quality housing for people who currently live in extreme poverty throughout the world (Liang and Hota 2009). If natural FRPs were used to create construction modules, then countries with rural regions to grow the necessary industrial crops would be empowered to address their own poverty, employment, and housing issues with little or no outside assistance.

"How we design the built environment may hold tremendous potential for addressing many of the nations' greatest current public health concerns, including obesity, cardiovascular disease, diabetes, asthma, injury, depression, violence, and social injustice" (Von Bernuth 2004). The nature of any particular built environment affects human health, social behavior, crime, as well as civic pride. Many studies have shown that careful design of hospitals can speed patient recovery and increase staff morale, with the lower treatment times in new hospitals causing significant per-patient cost savings. Public dwellings that should be deemed unfit for habitation produce significantly lower health, higher crime, and higher fire risks, to the point where the British Medical Association has requested public action against poor housing (Pearce 2003). Studies have shown that schools with higher investment into their physical environments have better educational performance, while schools with unsatisfactory accommodations often have significantly poorer performance (Pearce 2003). Good developmental design produces better property values and encourages social responsibility, while good neighborhood design can directly decrease the occurrence of crime (Pearce 2003). As social capital declines, society must invest more and more into unproductive construction activities, such as courts, prisons, and postterrorism or post-vandalism repairs (Pearce 2003). 
Without an approach to rehabilitation and renovation that includes social systems, deployment of technology can be counterproductive and harmful (Koebel 2004). Van Rijswijk et al. (2001) explored the possibility of introducing a natural fiber-based composites industry to a developing country, and the necessary steps that would have to be taken for successful implementation. It is ideal for rural societies to manufacture products from their raw materials, rather than just selling the raw materials to a manufacturer, since there is more value added with the products and the extra jobs would create additional employment opportunities. Natural fiber composites would provide a unique industry for developing countries, due to the fact that FRP applications can begin very simple yet grow to increasingly more technical applications as the industry grows (van Rijswijk et al. 2001). When coupled with the potential to produce safe, affordable housing, the development of the natural fiber industry could potentially become a major factor in meeting human needs throughout the world.

\subsection{Conclusions}

Natural FRPs show good potential in the future for infrastructure applications. The natural fibers of flax, hemp, and ramie possess particularly impressive specific mechanical properties. The main challenges associated with moisture absorption, fire resistance, mechanical properties and durability, variability, and manufacturing / processing of natural FRPs are being addressed by many recent research efforts. Moisture absorption can be reduced through surface modification of fibers and/or by special coatings. Fire resistance can be improved by the use of intumescent coatings, which eventually may also be made from renewable resources. Mechanical properties and durability are the main areas of research into natural FRPs, and many proposed solutions have been found to improve the fiber / matrix interface. Fiber variability is itself largely uncontrollable, but the development of quality assurance protocols and diversification of fiber growing sources can address the issue before the fibers reach composite manufacturers. Natural FRPs have been successfully adapted to nearly every major manufacturing process currently used with synthetic composites, usually with few or no modifications to the processes themselves.

One of the biggest challenges of natural composites is also one of its greatest potential benefits: even though, to-date, natural composites are not as cost-effective as their synthetic equivalents, the price difference shows signs of continuing to decrease such that eventually they will not only be the more environmentally responsible choice but also more economical. While it should not be claimed that natural FRPs are currently more 'green' than traditional building materials (such as wood, steel, concrete) in all applications, life cycle analyses have shown that natural composites compare favorably to synthetic composites, using only around $20-40 \%$ as much energy. Finally, the development of a worldwide infrastructure-based natural composite industry has the potential to assist in providing poverty alleviation, higher employment, and costeffective housing in many different countries.

Future research in the field of natural composites for infrastructure applications would be

most beneficial if aimed at one of the highlighted challenge areas, particularly at continuing to improve mechanical properties, moisture resistance, and durability. 


\section{PRELIMINARY TESTING RESULTS}

\subsection{Abstract}

In order to assess the ability of the WVU-CFC to manufacture natural fiber reinforced composites, the mechanical properties of composite plates comprised of different reinforcement and resin systems were compared. Two types of flax fabric, two types of loose kenaf fibers, and E-glass were compression molded in either a phenolic or vinyl ester resin system. The main findings, through flexural testing and single fiber tension testing, were that the selected phenolic system was unsuitable as a matrix for natural fiber reinforced composites and that the kenaf fibers from Bangladesh presented the best potential as a natural reinforcing material for further research work.

\subsection{Introduction and Scope}

In order to assess the ability of the West Virginia University Constructed Facilities Center's (WVU-CFC) equipment to manufacture natural fiber reinforced composites and to obtain some initial mechanical properties for comparison to glass fiber reinforced composites, several composites plates were manufactured and mechanical testing was performed on coupons cut from those plates. Because of their relative ease of testing, static flexural tests and, to a lesser degree, Izod impact tests were chosen to determine the mechanical properties for comparison. In later stages of testing, impact characterization was abandoned and the majority of samples were tested only in flexure. At a later date, single fiber tension testing was also used to characterize fiber properties. Plates were manufactured using one of two resin systems and one of five types of fibers, in the form of fabrics or stitched loose fibers.

\subsection{Materials and Manufacturing Processes}

\subsubsection{Material}

Three different fabrics were used to manufacture the composite plates. The first material, a [0/90] plain weave, 6.6 oz (225 gsm) flax linen fabric (called 'Bedford Flax' in Table 3-1), was provided by Bedford Reinforced Plastics (BRP). This fabric was imported by BRP from Asia. The exact chemical and processing history of the fabric is unknown, beyond that the fabric manufacturer used some method of hot water scouring and bleaching. The uncertainty of this pre-processing methodology makes it difficult to assess whether the original fabric has been bonding-optimized in its surface treatment or has already been over-treated, and whether it would benefit from additional treatments.

The second material (called 'Flaxcraft Flax' in Table 3-1) was a [0/90] plain weave, 8.8 oz (300 gsm) flax linen fabric and was provided by Bast Fibers/Flaxcraft, LLC. The fibers were imported directly by this company from Bangladesh and they are high quality green (untreated) fibers. Because the fibers are green, they will theoretically have poorer bonding capability and higher moisture absorption, but they ought to respond much better to surface treatments and, post-treatment, provide better mechanical and absorption results than the Bedford Flax. 
The E-glass fabric was included in testing purely for comparative purposes. It was a [0/90] weave and had a density of around $13.3 \mathrm{oz}$ (450 gsm).

Loose kenaf fibers were obtained from two sources: directly through a contact in India and through Bast Fibers/Flaxcraft, LLC. The loose fibers were cut to length, arranged in a unidirectional pattern, and stitched to a single piece of flax fabric to make a thin 'unidirectional' mat. Two of these mats were stacked on top of each other at a right angle to make a bidirectional [0/90] composite, so as to roughly approximate the fabric composites. To test out some potential surface treatment options, one set of Bangladeshi kenaf fibers was treated by heating in linseed (flax) oil, and another by immersion in $2 \%$ sodium hydroxide for 10 minutes.

The phenolic resin used in the manufacture of the composite plates was Hexion Specialty Chemicals' Cascophen ${ }^{\circledR}$ G-1131 adhesive system. This phenol-formaldehyde thermoset resin is designed to provide strong, water-proof bonds on materials like wood and textiles and is curable at or around room temperature, although it is an adhesive resin system rather than a laminating one. The system is composed of two parts in a 5-to-1 ratio: the liquid resin (Cascophen ${ }^{\circledR}$ Adhesive G-1131-A) and the curing agent (Cascoset ${ }^{\circledR}$ Hardener G-1131-B).

A vinyl ester resin, Derakane ${ }^{\circledR} 510$ A from Ashland Chemical's Derakane ${ }^{\circledR}$ resins was also used to manufacture composite plates. The 510A resin system is intended to provide extra fire retardance, which is a particular concern with natural fiber reinforced composites.

Table 3-1 - Materials for Plate Manufacturing

\begin{tabular}{|c|c|c|c|c|c|c|}
\hline Plate & $\begin{array}{c}\text { Fabric } \\
\text { (0/90) }\end{array}$ & Surface Treatment & Matrix & Fabric layers & $\begin{array}{c}\text { Fabric } \\
\text { weight (oz) }\end{array}$ & $\begin{array}{c}\text { Calculated Fabric } \\
\text { Density (opsy) }\end{array}$ \\
\hline BF-1 & Bedford Flax & Bleached* & Phenolic & 5 & 3.88 & 6.62 \\
BF-2 & Bedford Flax & Bleached* & Phenolic & 10 & 7.83 & 6.68 \\
BF-3 & Bedford Flax & Bleached* & Phenolic & 15 & 11.43 & 6.50 \\
BF-4 & Bedford Flax & Bleached* & Phenolic & 20 & 15.20 & 6.49 \\
FF-1 & Flaxcraft Flax & None (green) & Phenolic & 10 & 13.33 & 9.00 \\
G-1 & E-glass & - & Phenolic & 10 & 14.96 & 12.77 \\
G-2 & E-glass & - & VE & 10 & 15.70 & 13.40 \\
IK-1 & Indian Kenaf & None (green) & Phenolic & - & 1.01 & - \\
IK-2 & Indian Kenaf & None (green) & VE & - & 0.73 & - \\
BK-1 & Bangladeshi Kenaf & None (green) & Phenolic & - & 1.20 & - \\
BK-2 & Bangladeshi Kenaf & None (green) & VE & - & 0.89 & - \\
BK-3 & Bangladeshi Kenaf & Linseed Oil & VE & - & 1.70 & - \\
BK-4 & Bangladeshi Kenaf & NaOH (2\%, 10 min) & VE & - & 1.94 & - \\
\hline
\end{tabular}

*Actual fabric treatment process unknown

\subsubsection{Manufacturing}

All plates were manufactured using a heated compression molding device. Rectangular steel plates were used for the upper and lower platens with a rectangular steel cut-out plate used to define the edges of the composite plate. The upper platen was sized to fit neatly inside the cut- 
out plate to provide compression. Aluminum foil was applied between platens and the resin to aid in removing the cured plate from the mold. Technick Products Tech-Lube was also sprayed on the aluminum foil to ensure easy release.

Layers of fabric were pre-cut to the mold-size before beginning manufacturing. Alternating layers of prepared resin and fabric were pressed into the mold, and a paintbrush and a roller were used to provide good resin dispersion. Once all layers of fabric were applied, the upper platen was put in place and the filled mold was installed in the compression molding fixture. Figure 3-1 shows a completed flax/phenolic plate. The loose fiber composites were manufactured on a smaller scale by compressing the resin-impregnated fiber mats between two plates.

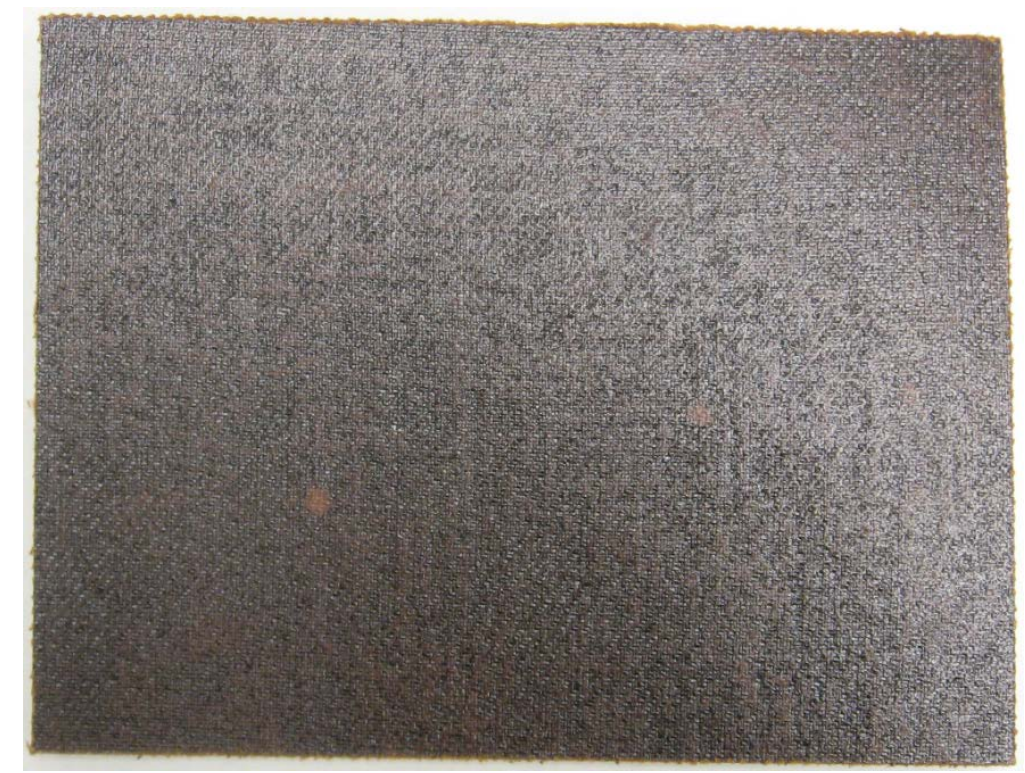

Figure 3-1 - Compression molded flax/phenolic plate ( 10 in x 15 in)

The first few tests used lower curing temperatures and pressures than necessary in order to avoid damaging the material (see Table 3-2). However, after checking manufacturing conditions with the resin manufacturer, the temperature and compression were increased. The duration of application of heat was also decreased (from 12 to 2 hours), although the load was maintained for around 24 hours for each plate in an attempt to reduce warping in later stages of curing. Before the manufacture of plate FF-1 (chronologically the last plate made in this phase), a new mold was built with a larger area (see Table 3-2) which will be used for all future compression tests. Data for the plate area and applied compression force for the kenaf reinforced composites was not recorded, although the fiber volume fraction was calculated by comparing the resulting composite weight to the initial fiber weight. 
Table 3-2 - Manufacturing Conditions

\begin{tabular}{|c|c|c|c|c|c|c|c|}
\hline Plate & $\begin{array}{c}\text { Area } \\
\left(\mathbf{i n}^{2}\right)\end{array}$ & $\begin{array}{c}\text { Thickness } \\
\text { (in) }\end{array}$ & $\begin{array}{c}\text { Lamina } \\
\text { Thickness (in) }\end{array}$ & $\mathbf{\% V f}$ & $\begin{array}{c}\text { Temp. } \\
\left({ }^{\circ} \mathbf{F}\right)\end{array}$ & $\begin{array}{c}\text { Compression } \\
\text { (psi) }\end{array}$ & $\begin{array}{c}\text { Compression time } \\
\text { (w/wo heat, hrs) }\end{array}$ \\
\hline BF-1 & 152 & 0.081 & 0.016 & 37 & 120 & 13 & $12 / 2$ \\
BF-2 & 152 & 0.178 & 0.018 & 36 & 120 & 13 & $12 / 12$ \\
BF-3 & 152 & 0.274 & 0.018 & 30 & 150 & 66 & $12 / 10$ \\
BF-4 & 152 & 0.337 & 0.017 & 32 & 150 & 66 & $12 / 10$ \\
FF-1 & 192 & 0.212 & 0.021 & 46 & 150 & 52 & $2 / 24$ \\
G-1 & 152 & 0.171 & 0.017 & 40 & 150 & 66 & $2 / 24$ \\
G-2 & 152 & 0.138 & 0.014 & 55 & 150 & 99 & $2 / 24$ \\
IK-1 & $?$ & 0.208 & - & 22 & 150 & $?$ & $2 / 24$ \\
IK-2 & $?$ & 0.138 & - & 51 & 150 & $?$ & $2 / 24$ \\
BK-1 & $?$ & 0.186 & - & 33 & 150 & $?$ & $2 / 24$ \\
BK-2 & $?$ & 0.138 & - & 38 & 150 & $?$ & $2 / 24$ \\
BK-3 & $?$ & 0.131 & - & 66 & 150 & $?$ & $2 / 24$ \\
BK-4 & $?$ & 0.124 & - & 47 & 150 & $?$ & $2 / 24$ \\
\hline
\end{tabular}

A few of the plates were removed from compression prematurely and experienced some post-cure warping due to uneven curing and cooling (see Figure 3-2). This can be partially avoided by ensuring that the fibers are dry at the time of molding, keeping pressure on the mold until full cure is achieved, and maintaining even temperatures on both the upper and lower surfaces, although for the unbalanced stitched mats it has proven extremely difficult to eliminate.

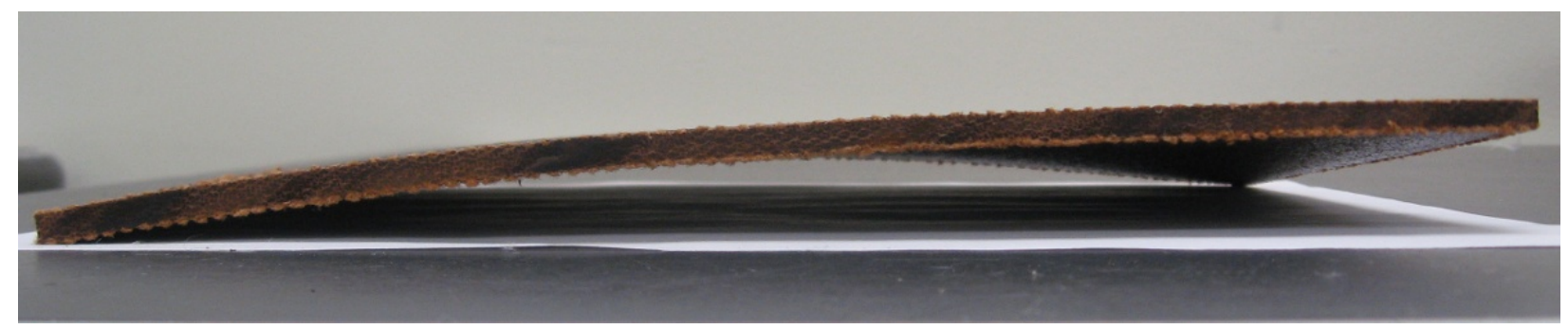

Figure 3-2 - Flax/phenolic plate showing post-cure warping

\subsubsection{Sample Preparation}

\subsubsection{Flexural sample preparation}

Samples were cut from the cured plates for flexural testing using a table saw with a carbide blade. Each sample was 0.5 inches wide and approximately 4.5 inches long, as recommended by ASTM D790. For flexural tests, no additional sample preparation is needed. 


\subsubsection{Izod impact sample preparation}

For the Izod impact testing, samples were cut to 0.5 inches wide and 2.5 inches long, as recommended by ASTM D256. A sample cutter was used to place a 0.01 inch radius notch in a flat edge at the midpoint of each sample.

\subsubsection{Single fiber tension sample preparation}

Single yarn strands were removed from the woven flax fabrics. The kenaf fiber bundles were split by hand into the smallest possible single fibers. The separated single fibers/strands were then mounted with scotch tape to lightweight cardboard frames. The gage length of each fiber was approximately 2 in, within the recommendations in ASTM D3822.

\subsection{Experimental Methodology}

\subsubsection{Flexural test method}

Flexural tests were conducted on an Instron Universal Testing Machine with a 3-point bending fixture installed. The testing procedures in ASTM D790 were used as guidelines to determine sample dimensions, test span, and testing rate. For all of the samples except for those from plate BF-2, the support span was approximately 3.7 inches; for plate BF-2, the support span was 3.0 inches. These support spans were not exactly as specified in ASTM D790, but were considered a reasonable approximation for most of the samples by student performing the tests. The testing was conducted at a crosshead speed of around $0.1 \mathrm{in} / \mathrm{min}$ for all of the samples. Three tests were conducted on coupons from each plate and the results were averaged. Strain gages were not used and the load and deflection data were collected through the Instron's built-in load cell and LVDT.

\subsubsection{Impact test method}

A cantilever beam, Izod-type pendulum impact tester was used to measure the impact energy of each composite plate. Following the procedures in ASTM D256 as a guide, the tester was calibrated before any of the samples were installed in the test apparatus. Three tests were conducted on coupons from each of the fabric-reinforced plates and the results were averaged.

\subsubsection{Single fiber tension test method}

Before tension testing, the fibers were examined under a digital optical microscope at $5 \mathrm{x}$ power, and the smallest diameter along the length was recorded. For the stress and stiffness calculations, each fiber was assumed to have a circular cross-sectional area of the recorded diameter, except for the Indian kenaf fibers. The Indian kenaf fibers seemed to be from a different kenaf family than the Bangladeshi kenaf fibers, as their cross-sections were shaped more like blades of grass (wide and flat) rather than hair (circular). For these fibers, the area was approximated as the smallest flat-side diameter multiplied by the smallest edge diameter.

The single fibers and their cardboard frames were installed in a universal testing machine outfitted with a $10 \mathrm{kN}$ load cell and wedge-style tensile clamps. Once both clamps were in place, the cardboard frame was cut away, leaving the fiber as the only source of tensile load resistance. Testing was conducted at a rate of $0.4 \mathrm{~mm} / \mathrm{min}$, although this is much slower than ASTM D3822 
requires. At least three tests were conducted on each type of fiber sample and the results were averaged.

\subsection{Results and Discussion}

\subsubsection{Flexure Tests}

A total of 39 samples (3 each kind) were tested in flexure on the Instron machine and 21 (3 each kind for fabric samples) on the Izod impact test apparatus. The kenaf fiber reinforced composites were not tested in impact after it was determined that the impact rating would not continue to be a critical part of this research. Results were averaged for the three samples cut from each of the different composite plates and are shown in Table 3-3.

Table 3-3 - Preliminary Flexure and Impact Testing Results

\begin{tabular}{|c|c|c|c|c|}
\hline Plate & $\begin{array}{l}\text { Bending Modulus } \\
\text { (ksi) }\end{array}$ & $\begin{array}{l}\text { Bending Strength } \\
\text { (ksi) }\end{array}$ & $\begin{array}{l}\text { Izod Impact Energy } \\
\text { (ft-lbf/in) }\end{array}$ & $\begin{array}{c}\text { ASTM Impact Units } \\
\left(\text { ft-lbf/in }{ }^{2}\right)\end{array}$ \\
\hline BF-1 & 471.9 & $13.02 *$ & 0.74 & 10.4 \\
\hline BF-2 & 505.5 & 11.86 & 1.44 & 8.6 \\
\hline $\mathrm{BF}-3$ & 403.3 & 10.56 & 2.44 & 9.2 \\
\hline BF-4 & 504.3 & 12.19 & 2.88 & 8.8 \\
\hline FF-1 & 264.7 & 7.50 & 1.84 & 9.1 \\
\hline G-1 & 1184.4 & 14.31 & 3.86 & 24.0 \\
\hline G-2 & 2746.9 & 74.51 & $4.00+* *$ & $31.3+* *$ \\
\hline IK-1 & 84.6 & 4.04 & - & - \\
\hline IK-2 & 991.0 & 16.41 & - & - \\
\hline BK-1 & 361.4 & 6.55 & - & - \\
\hline BK-2 & 1135.0 & 16.73 & - & - \\
\hline BK-3 & 1168.2 & 15.17 & - & - \\
\hline BK-4 & 918.3 & 16.73 & - & - \\
\hline
\end{tabular}

Bending modulus $\left(E_{B}\right)$ was calculated according to Equation (3-1), with $L=$ support span, $m=$ slope of the initial linear portion of the load-deflection curve, $b=$ width of sample, and $d$ = depth of sample:

$$
E_{B}=L^{3} m / 4 b d
$$

Bending strength $\left(\sigma_{B}\right)$ was calculated according to Equation (3-2), with the same variable definitions as shown above and $P=$ load at a given point (maximum reached during testing) on the load-deflection curve:

$$
\sigma_{B}=3 P L / 2 b d^{2}
$$


The support span for the samples cut from plate BF-1 was too large (3.7 inches instead of 1.3 inches as specified by ASTM D790) given the thinness and flexibility of the 5-ply laminate. As a result, the sample had a large deflection and slipped off the supports prematurely. The higher bending stress found for this sample should not be considered accurate.

The glass fabric composites had at least 2-4 times higher modulus values than the natural fiber composites with the same resin (phenolic). However, the glass fabric does not seem to bond well with the phenolic resin, as the strength is only slightly higher (30\%) than for the flax fabric, and its strength and stiffness do not compare well with the glass/vinyl ester composite (19\% and $43 \%$, respectively). These results indicate that the properties of the phenolic composites may be low largely due to the behavior of the selected phenolic resin rather than the variable of most interest, the fiber type.

None of the flax fabric composites performed quite as well as hoped, but considering the lack of intentional surface treatments and the somewhat arbitrary resin selection, the results are not discouraging. The Bedford flax composites (BF-1, -2, -3, -4) offered fairly consistent strength and stiffness values independent of the number of layers of fabric. The strength of the BF composites was about 1.5 times higher than that of the Flaxcraft flax composite (FF-1) and the stiffness was about twice as high. Even though the exact surface treatment for the BF is unknown, it has been through some sort of scouring and bleaching process that would remove lignin and seems to have provided some increase in bonding ability. On the other hand, the FF, while believed to be a higher quality fiber, has not undergone any surface treatment and thus is unable to bond well with conventional hydrophobic resin systems. With proper surface treatments, the FF and BF fibers would likely be fairly competitive.

The kenaf fiber reinforced phenolic composites did not appear to perform particularly well compared to the fabric reinforced phenolics. However, when placed in a vinyl ester resin, the kenaf fiber reinforced composites had fairly promising stiffness results, if not strength results. Neither of the fiber surface treatments (linseed oil or sodium hydroxide) seemed to have much impact on the mechanical properties of the resulting composite.

The failure mode of the glass/vinyl ester coupons was rupture and delamination on the surface opposite the central loading nose. The failure mode on the flax/phenolic coupons was not discernible, as they appear to have merely bent in plastic deformation. While nothing is visible on the surface, when this type of failure mode is combined with the fact that the surface treatments are not yet optimized it seems reasonable to conclude that some sort of internal slippage may be occurring due to poor bonding. From this testing, it was determined that the phenolic resin system should be discarded in future testing, and the focus should instead shift to other resin types.

In order to discern whether the flax fabric or kenaf fibers provide better reinforcement, it would have been necessary to manufacture flax/vinyl ester panels in order to compare results between vinyl ester composites instead of just the phenolic composites. However, due to the single fiber tension testing reported below, this extra testing was deemed unnecessary as the kenaf fibers were eventually selected as the focus for further testing.

\subsubsection{Impact Tests}

The value obtained by the impact tester was divided by the sample width ( 0.5 in) to obtain the impact energy in units of ft-lbf/in (see Table 3-3). In order to obtain a thickness- 
independent unit for a better comparison between the impact energy absorption of different thicknesses of laminates, ASTM D256 allows for the use of an optional, area-normalized unit of $\mathrm{ft}-\mathrm{lbf} / \mathrm{in}^{2}$. This unit is obtained by dividing the impact energy by the sample thickness below the notch (plate thickness minus the notch depth of 0.01 inches).

The impact test apparatus had too small of a pendulum weight installed to produce a measurable impact deflection for the samples cut from plate G-2. Because of this, the maximum measurement of $2.00 \mathrm{ft}-\mathrm{lbf}$ was exceeded. If future impact testing is conducted on the glass/vinyl ester composite, pendulum weight will need to be increased to avoid this problem.

The impact energy is not a particular concern for this project, but it is interesting to note, from the thickness-adjusted ASTM units, that the area-normalized impact energy absorption is about equal for all of the flax composites (BF and FF), irrespective of surface treatment. This is potentially an indicator of the better quality of the FF, and with surface treatments that material should also show an improvement in impact properties. The glass fabric composites performed significantly better ( $\sim 3$ times) than the flax fabric composites when the area-normalized impact energy was considered.

\subsubsection{Single Fiber Tension Tests}

The kenaf fibers generally possessed smaller diameters than the flax fabric strands (50$200 \mu \mathrm{m}$ as compared to $400-900 \mu \mathrm{m}$ ). Due to these smaller diameters and the resulting smaller loads, the standard deviations for the kenaf fibers were much higher than for the flax strands. The results from the single fiber tension tests, as well as a comparison to published data, are shown in Table 3-4.

Table 3-4 - Single fiber tension test results and published values

\begin{tabular}{|l|c|c|c|c|c|}
\hline \multicolumn{1}{|c|}{ Fiber } & \# of Samples & Strength (ksi) & SD & Stiffness (Msi) & SD \\
\hline BF (225gsm) & 4 & 19.4 & 4.8 & 0.754 & 0.068 \\
BF (685gsm) & 5 & 19.1 & 2.2 & 0.305 & 0.042 \\
FF (treated) & 3 & 15.7 & 2.5 & 0.247 & 0.058 \\
FF (untreated) & 3 & 28.4 & 2.6 & 0.609 & 0.128 \\
Kenaf (India) & 11 & 51.8 & 22.5 & 4.830 & 2.488 \\
Kenaf (Bangladesh) & 5 & 39.2 & 16.8 & 3.655 & 1.207 \\
\hline Published Flax (tensile) & - & $50-290$ & - & $4.00-14.94$ & - \\
Published Kenaf (tensile) & - & $32-135$ & - & $2.10-7.69$ & - \\
Published Glass (tensile) & - & $290-508$ & - & $10.15-11.02$ & - \\
\hline
\end{tabular}

The large load cell and wedge-type tensile clamps really are not suitable given the small loads (sometimes as little as $1.0 \mathrm{~N}$, or $0.01 \%$ of the load cell's capacity) and were likely responsible for some of the high standard deviation in the data. Even though the standard deviations for the kenaf fiber were quite high, it is clear that they have a greater potential to provide higher strength and stiffness than the woven flax fabrics as composite reinforcements (see Table 3-4). The kenaf tensile strength and stiffness were both within the published values, but the flax strength and stiffness were both considerably lower. These lower properties are indicative of how much of the base material's properties are lost during the processing, spinning, 
and weaving of fibers. In order to optimize natural fiber reinforced composites for mechanical properties, it seems likely that woven materials are unsuitable.

Even though the Indian kenaf provided slightly higher tensile properties, the Bangladeshi kenaf was selected as the focus for future work for three reasons:

1. Reliable supply source - Through Bast Fibers/Flaxcraft, LLC, the Bangladeshi fibers can be acquired for a reasonable cost and without having to deal with import/export complications.

2. Smaller surface area - The Indian kenaf fibers broke up into flat, blade-like individual fibers, while the Bangladeshi fibers easily split into small, circular fibers. Since the cross sectional area of the Indian kenaf is larger and more variable, it seems likely that the Bangladeshi fibers will provide a better reinforcement material.

3. Mechanical properties within standard deviations - Even though the Indian kenaf resulted in a slightly higher tensile strength and stiffness, the Bangladeshi fibers were within one standard deviation of its properties and vice versa, indicating that the difference is likely insignificant.

\subsection{Conclusions}

Preliminary results have shown that WVU-CFC has the potential to manufacture natural fabric composites by compression molding; however, mechanical testing has shown that these composites likely need to be modified by the use of surface treatments or substitution of different constituent materials in order to achieve the desirable mechanical properties.

The untreated flax fabric had poorer flexural properties than the bleached fabric despite a high quality rating from the supplier, suggesting composite mechanical properties should be improvable with a better bond between the fabric and resin. At this stage, flax fabric composites are only able to meet about $40 \%$ of the stiffness and $67 \%$ of the strength of glass fabric composites, although even the glass fabric does not seem to perform well in the Cascophen ${ }^{\circledR}$ phenolic resin system. Vinyl ester seemed to provide significantly better mechanical properties for both the glass and kenaf reinforced composites.

After comparing the results of single fiber tension testing, the Bangladeshi kenaf fiber was selected as the focal point of continuing research due to its availability, surface area consistency, and good tensile properties.

The following testing will focus on trying alkalization as a surface treatment and testing other resin systems to improve mechanical properties, as well as assessing the moisture absorption characteristics of the different composites. 


\section{FIBER SURFACE TREATMENT CHARACTERIZATION}

\subsection{Abstract}

Before a composite part is manufactured, the surface treatment method applied to the natural fibers is responsible for reducing the moisture absorption capacity and enhancing the chemical and mechanical bonding potential between the fibers and the matrix. Two common surface treatments used with bast (stem) fibers are alkalization, in which the chemical makeup and physical surface of the fibers are modified by soaking in a strong alkali solution and treatment with silane coupling agents. Stitched kenaf fibers were treated with sodium hydroxide and/or a silane for different concentrations and durations, and then used to reinforce vinyl ester or polyurethane in compression-molded composite plates. The mechanical properties of the treated fibers were compared by tensile tests of individual fibers and of the resulting composites by three-point flexural tests. Scanning electron microscopy was used to evaluate composite quality and fiber / matrix bonding. Moisture absorption was monitored for each variation of composite until saturation. Alkalization was shown to increase single fiber tensile strength by $\sim 35-70 \%$ and composite flexural strength by as much as $12-15 \%$ over untreated fibers. Stiffness was improved by as much as $50 \%$ in both single fiber tensile tests and composite flexural tests. A treatment of $5 \% \mathrm{NaOH}$ for around 16 hours was found to produce the most promising mechanical results, although with little or no improvement to moisture performance.

\subsection{Introduction and Scope}

Natural fiber reinforced composites have the potential to eventually be lighter-weight and lower-cost than many synthetic composites, as well as being easier to handle (Brouwer 2000; Beckwith 2003; Dittenber and GangaRao 2011). They potentially also offer improved sustainability and eco-friendly characteristics (Joshi et al. 2004; Dittenber and GangaRao 2011). Natural composites are already used in many automotive applications as interior paneling, but are not generally used in applications as primary structural elements due to their lower mechanical properties and reduced environmental performance (Brouwer 2000; Netravali et al. 2007; Chabba and Netravali 2004). However, with the use of appropriate resin systems and fiber surface treatments, as well as other additives and coatings, natural fiber composites should be able to achieve mechanical and environmental performance adequate enough to compete with glass fiber composites in structural applications.

Natural fibers that are collected from the stems of plants are called 'bast' fibers, and are generally the class of fibers best suited for structural reinforcement. Examples of bast fibers are flax, hemp, jute, and kenaf. Kenaf, a relative of jute grown most commonly in India and Southeast Asia, produces fibers with good tensile properties which are representative of high quality bast fibers. Kenaf has published strength and stiffness values in the ranges of 32-135 ksi and 2.10-7.69 Msi, respectively (John and Anandjiwala 2008; Bismarck et al. 2005; Symington et al. 2009).

One of the most important factors in developing a successful natural composite is the surface treatment method applied to the fibers before manufacturing. Natural fibers are inherently hydrophilic, causing them to absorb a relatively large amount of moisture and creating incompatibility with most hydrophobic polymer matrices (Mohanty et al. 2001; Malkapuram et 
al. 2009). Treatment of natural fibers with an alkali solution, such as sodium hydroxide, reduces the hydrogen bonding capacity of the cellulose in the natural fibers and dissolves hemicellulose, which is the most hydrophilic part of the fiber structure (Symington et al. 2009). The resulting physically and chemically modified fiber surface can better bond with synthetic polymer resins, giving improved composite mechanical properties.

In addition to alkalization, treatment with coupling agents, such as silanes, has also been shown to improve the bond between natural and synthetic materials by the formation of a chemical bridge. While silane treatments do not necessarily increase the mechanical properties of natural fiber composites, they can reduce the moisture absorption capacity (Xie et al. 2010). Through a combination of alkalization and silane treatments, the mechanical properties and moisture absorption characteristics of a natural fiber composite can be improved, leading to a product more suitable for structural applications.

Single fiber tension tests, scanning electron microscopy, flexural tests, and moisture absorption tests were used to determine the best possible alkalization surface treatment (along with one set of silane samples) for kenaf fibers and the best reinforcing matrix (of two vinyl esters, a phenolic, and polyurethane).

\subsection{Materials and Manufacturing Processes}

\subsubsection{Materials}

Kenaf fibers were imported from Bangladesh by Bast Fibers LLC, both in a long fiber ( 8-10 ft), unprocessed form and in a loosely woven, $6.28 \mathrm{oz}$ (213 gsm) fabric. The loose fibers had been dew retted, stripped of any additional plant core materials, and washed, but were otherwise untreated (green). The fabric likewise had not undergone any prior chemical treatments.

Two types of vinyl ester were used with the kenaf reinforcement. Derakane 510A is one of Ashland's epoxy vinyl ester resins designed particularly for fire retardance and chemical resistance. Derakane 8084 is a toughened version of the epoxy vinyl ester resin and was selected with the expectation that its improved toughness might provide better mechanical properties when reinforced with natural fibers, which in many cases have higher strains to failure than glass fibers. A Huntsman polyurethane system was also reinforced with the kenaf fibers and tested. The system was a combination of RIMline SK97014 polyol and Suprasec 9701 isocyanate. A phenolic system, Hexion Specialty Chemical's Cascophen G-1131 was only tested with the untreated fibers (as discussed in the previous chapter). This adhesive proved to bond poorly with the fibers and provided minimal strength and stiffness as a manufactured composite. 


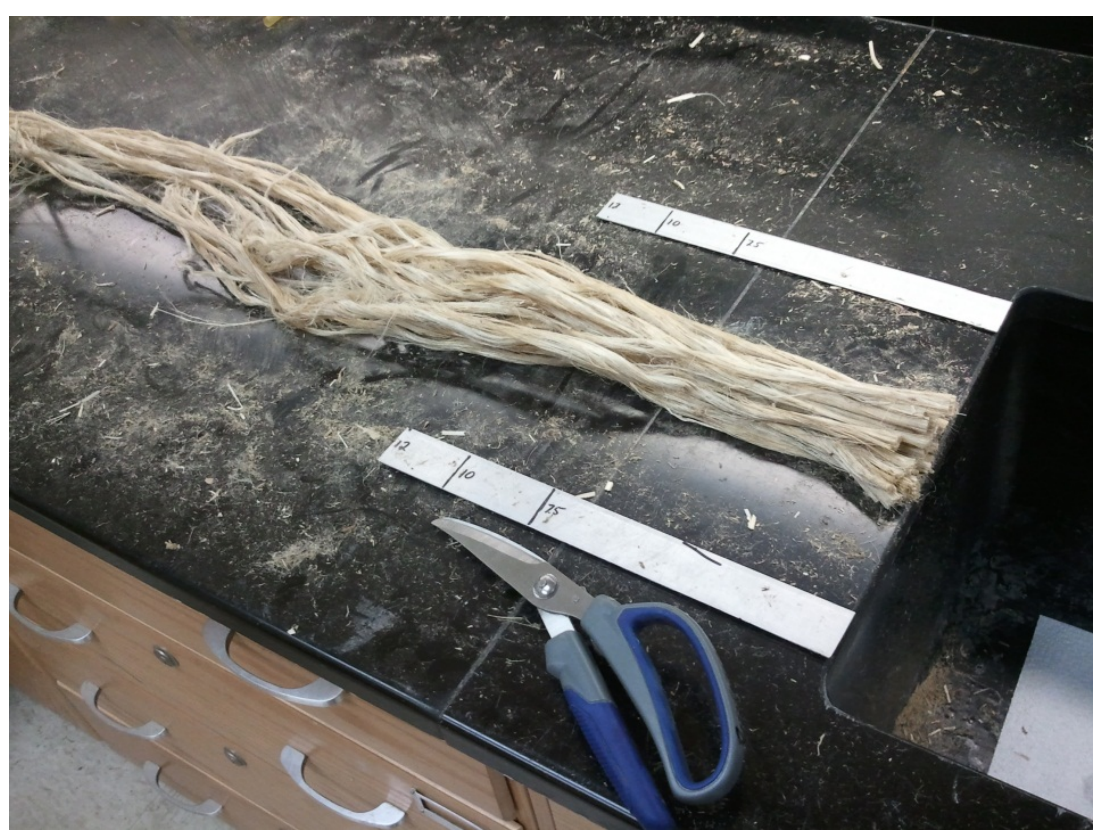

Figure 4-1 - Long kenaf fibers before being cut

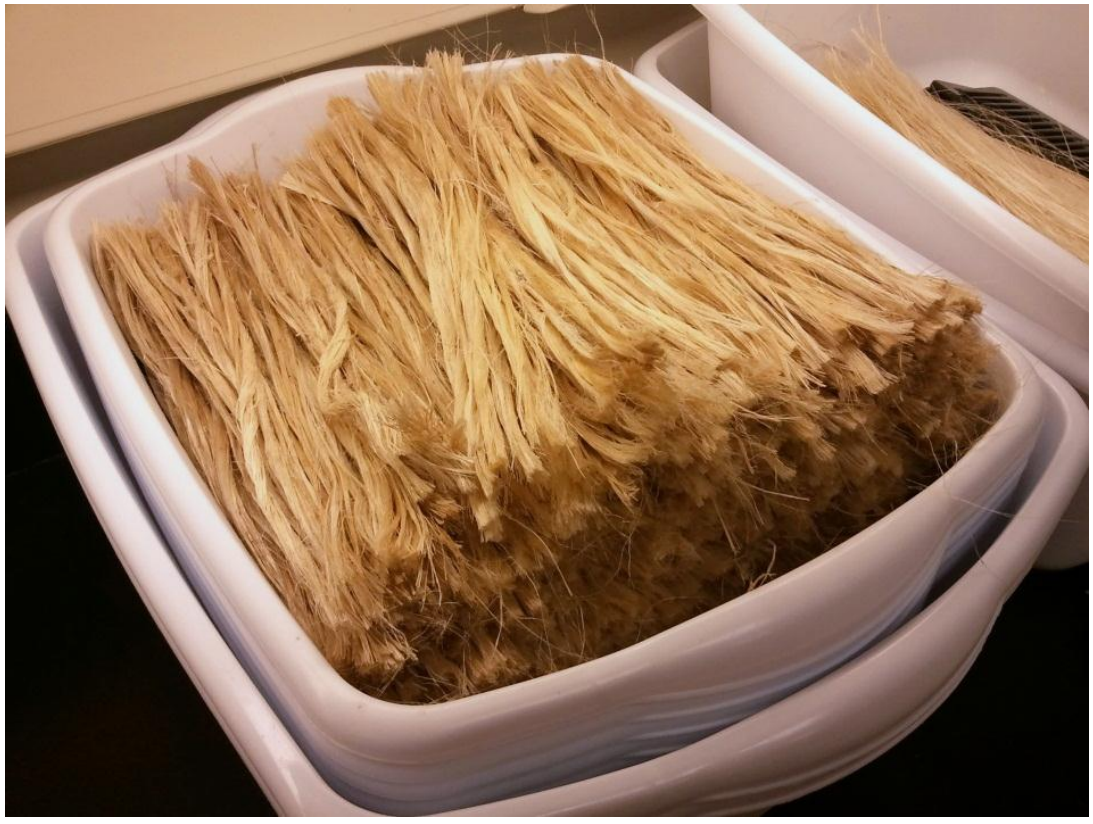

Figure 4-2 - Fibers cut to size, before surface treatment

\subsubsection{Fiber Treatment}

The long kenaf fibers (Figure 4-1) were cut to size (Figure 4-2) and any non-fibrous debris was removed. From a review of literature (Symington et al. 2009; Chang et al. 2009b; Ibrahim et al. 2010b), it appeared that, on average, a treatment of 5\% concentration by weight sodium hydroxide $(\mathrm{NaOH})$ at room temperature for around 30 minutes produced good mechanical and moisture resistance results, although other sources advised much longer treatments in order to optimize fiber properties (Williams et al. 2011). The $\mathrm{pH}$ of the sodium 
hydroxide solutions was around 12.8 for $2 \%$ concentration and 13.0 for $5 \%$ or $10 \%$ concentration, indicating that concentrations higher than around 5\% would not produce higher $\mathrm{pH}$ values, as confirmed by $\mathrm{pH}$ calculations. Therefore, $5 \%$ concentration was selected as the main sodium hydroxide concentration for the study. As a control, some fibers were left in their green, untreated state. Nine different levels of room temperature sodium hydroxide treatment and one treatment at elevated temperature were applied to the fibers, as shown in Table 4-1. After removal from the sodium hydroxide and squeezing to remove excess liquid, the fibers were immersed in 5\% acetic acid (distilled white vinegar) for 3-5 minutes to neutralize whatever $\mathrm{NaOH}$ remained on the fibers. The fibers were then removed from the acetic acid, squeezed to remove excess liquid and then rinsed and soaked in distilled water for 3-5 minutes. After removal from the distilled water, the fibers were allowed to air dry on wire cooling racks at room temperature for 24 hours before being dried in an oven at $160^{\circ} \mathrm{F}$ for $\sim 8$ hours. The fibers were turned and shuffled several times while in the oven to ensure even drying.

Table 4-1 - Fiber treatment options tested.

\begin{tabular}{|ccc|}
\hline Alkali concentration & Soaking Time & Additional treatment \\
\hline Green, untreated & n/a & w/ \& w/o silane \\
$2 \% \mathrm{NaOH}$ & $10 \mathrm{~min}$ & w/ \& w/o silane \\
$5 \% \mathrm{NaOH}$ & $20 \mathrm{~min}$ & $\mathrm{w} /$ \& w/o silane \\
$5 \% \mathrm{NaOH}$ & $40 \mathrm{~min}$ & w/ \& w/o silane \\
$5 \% \mathrm{NaOH}$ & $60 \mathrm{~min}$ & no silane \\
$5 \% \mathrm{NaOH}$ & $90 \mathrm{~min}$ & no silane \\
$10 \% \mathrm{NaOH}$ & $2 \mathrm{hrs}$ & no silane \\
$5 \% \mathrm{NaOH}$ & $8 \mathrm{hrs}$ & no silane \\
$5 \% \mathrm{NaOH}$ & $16 \mathrm{hrs}$ & no silane \\
$5 \% \mathrm{NaOH}$ & $24 \mathrm{hrs}$ & no silane \\
$5 \% \mathrm{NaOH}$ & $16 \mathrm{hrs}, 130^{\circ} \mathrm{F}$ & no silane \\
\hline
\end{tabular}

As an additional treatment, some of the fibers were also soaked in a bath of aminosilane solution ( $2 \%$ by mass of Gelest Inc.'s Aminopropyltriethoxysilane) for 3 minutes, followed by 30 minutes of curing at $230^{\circ} \mathrm{F}$, a product and procedure recommended by the manufacturer. The fibers were then allowed to air dry overnight on wire racks before being oven dried once more for 2 hours at $160^{\circ} \mathrm{F}$.

\subsubsection{Sample Preparation}

\subsubsection{Single fiber tension samples}

Fiber bundles were split by hand into elementary fibers then mounted with scotch tape in lightweight cardboard frames. The gage length of each fiber was approximately 2 in. 


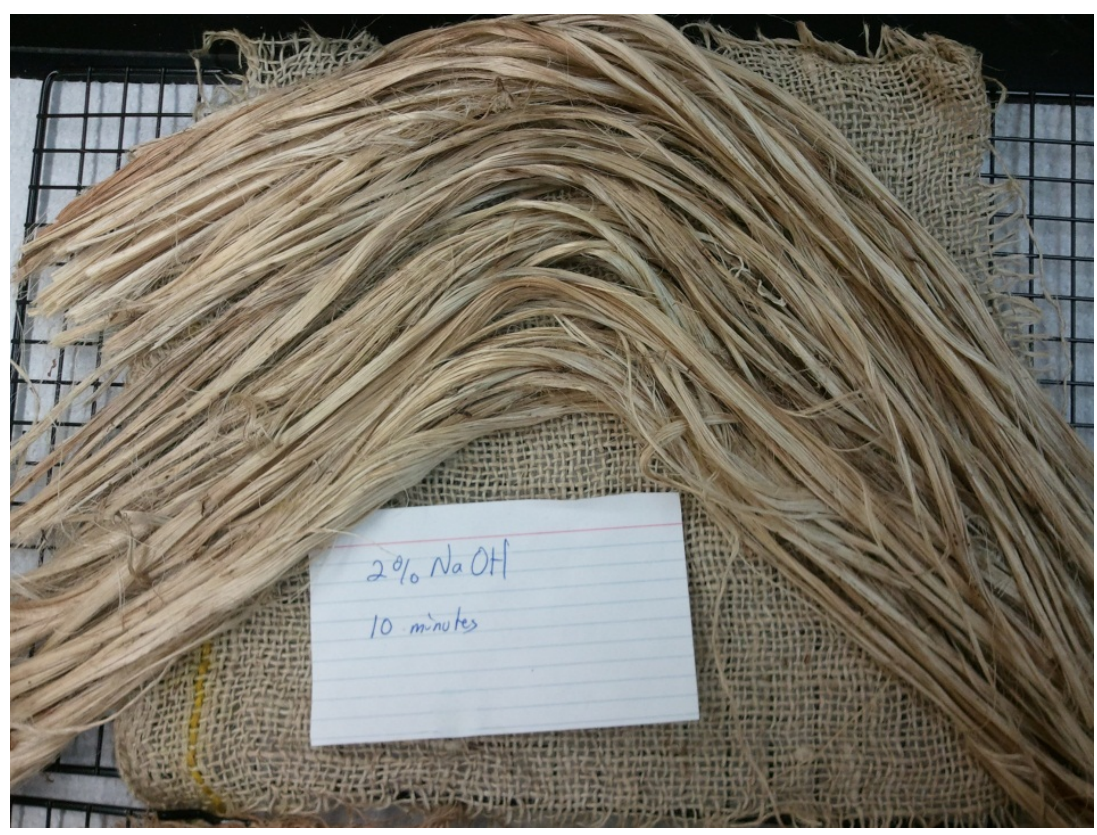

Figure 4-3 - Dried, treated kenaf fibers

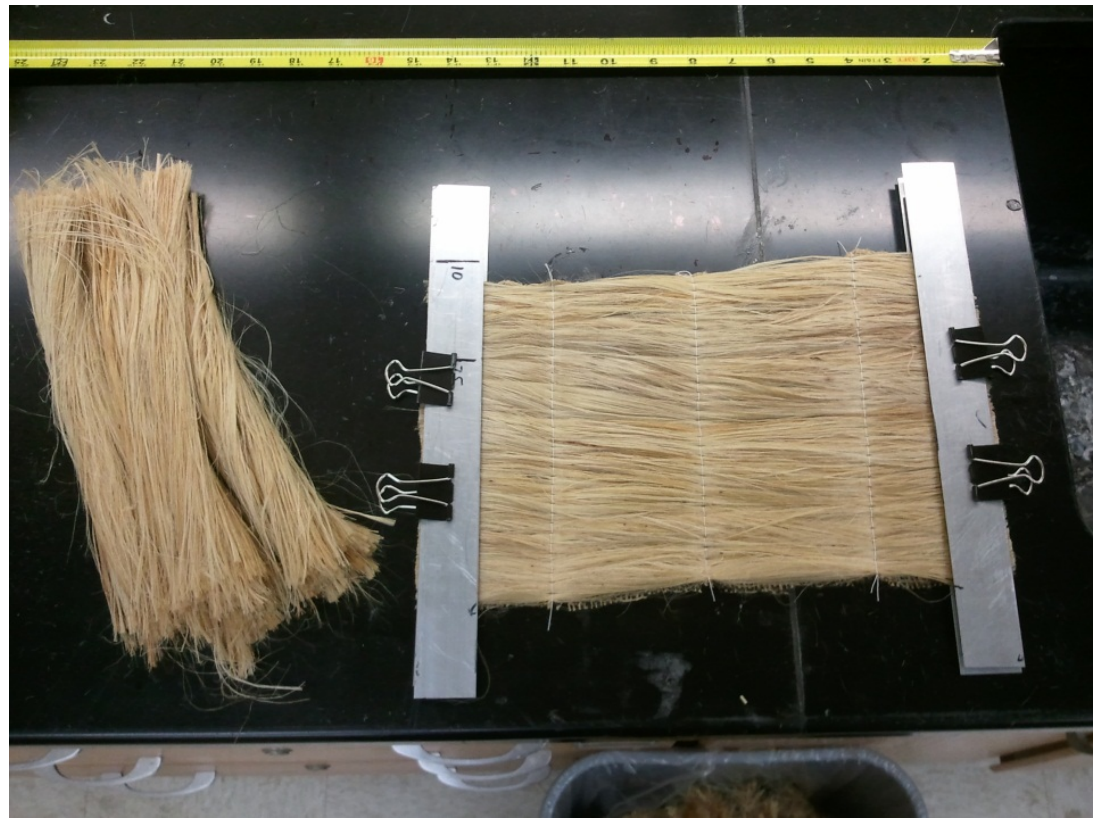

Figure 4-4 - Combed fibers and tension clamps for stitching

\subsubsection{Mat preparation}

The long kenaf fibers were stitched into unidirectional 'mats,' using the 6.28 opsy (213 gsm) kenaf fabric as a backing material. After the treatment and drying process, the fibers (Figure 4-3), were combed to align the main reinforcing fibers and to remove any tangled and misaligned fibrils. The fibers were laid out by hand on the kenaf fabric so that each fiber was continuous across the width of the fabric and gently tensioned with clamps on either end (Figure 4-4). A commercial sewing machine was used to lay 2-3 lines of transverse stitching with 
polyester thread along each $5 \times 7.5$ in mat (Figure 4-5). For each resin type and treatment option a single longitudinal and a single transverse mat were stitched with roughly the same fiber content by mass, resulting in a pair of mats with a combined ratio of approximately 3:3:1 by mass of longitudinal fibers, transverse fibers, and fabric. Each composite plate therefore had an unsymmetrical layup of [fabric/90/fabric/0]. This layup was selected for flexural testing, rather than a unidirectional, symmetric layup, in order to get a better approximation of how a 2-D, bidirectional panel (a potential end-use product) might be expected to perform. Before manufacturing the fiber reinforced composite, all of the fiber mats were dried in an oven at $160^{\circ} \mathrm{F}$ for 1 hour and sealed in plastic bags.

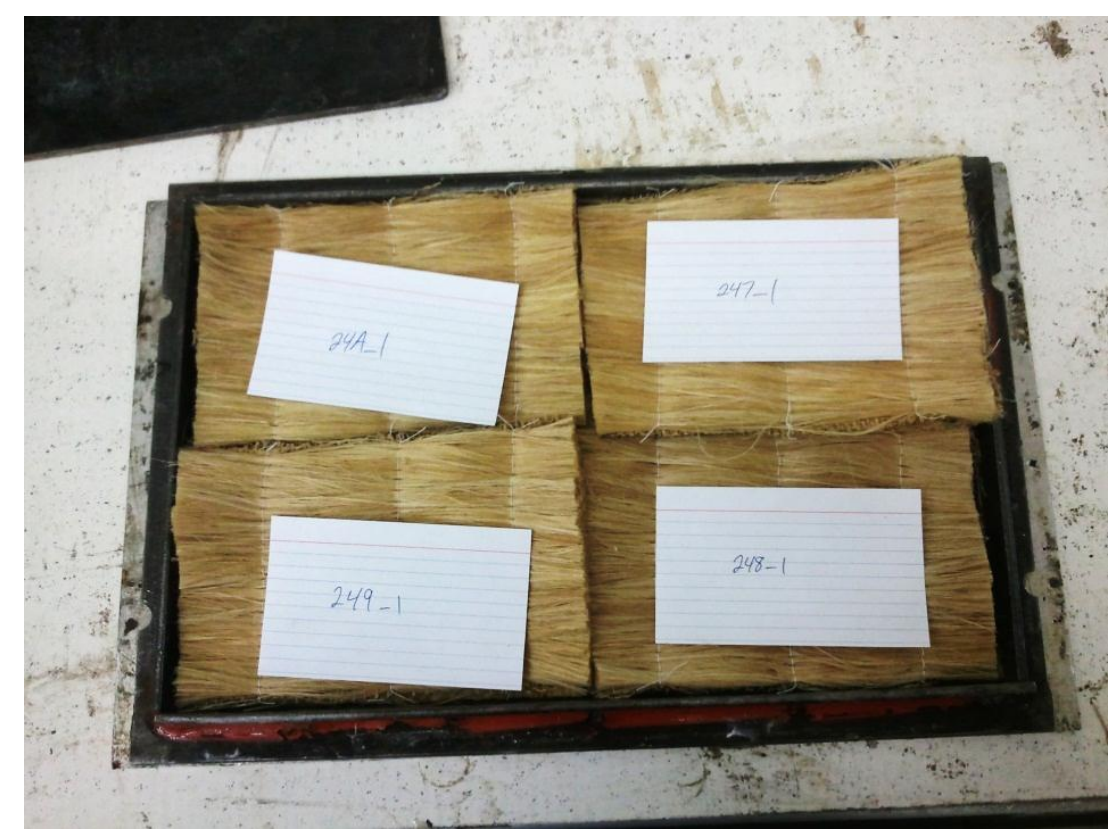

Figure 4-5 - Stitched mats laid out in quadrants in mold

\subsubsection{Composite manufacturing}

The kenaf reinforced composite plates were all manufactured by heated compression molding. Technick Products Techlube 25 was used as the mold release agent and all of the steel mold parts were covered with aluminum foil to ease the removal of the plate from the mold. For each resin type, a $10 \times 15$ in plate was manufactured with each quadrant consisting of a separate pair of mats (Figure 4-6) treated by one of the processes listed in Table 4-1. The top plate was free floating within the inside edge of the side bars in order to provide the best possible compression, albeit at the expense of consistent plate thickness. The resin was applied to each of the fiber mats by hand and spread evenly with a paintbrush. The manufacturing details for each plate are shown in Table 4-2, and were determined from the manufacturer's technical data sheets and previous experience. 


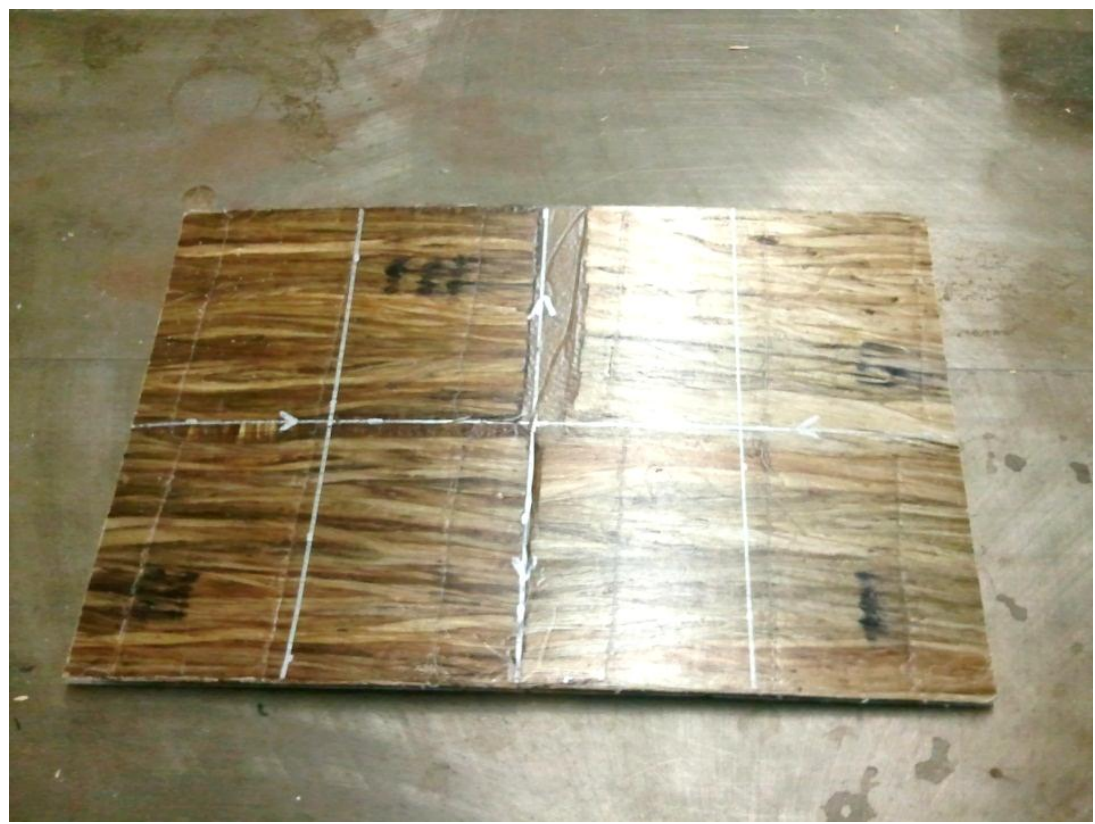

Figure 4-6 - Completed kenaf-reinforced composite plate

Table 4-2 - Composite manufacturing details

\begin{tabular}{|c|c|c|c|c|}
\hline Resin system & Mix & $\begin{array}{c}\text { Temp } \\
\left({ }^{\circ} \mathrm{F}\right)\end{array}$ & $\begin{array}{l}\text { Pressure } \\
\text { (psi) }\end{array}$ & $\begin{array}{c}\text { Cure Time } \\
\text { (hrs) }\end{array}$ \\
\hline $\begin{array}{l}\text { Derakane 510A } \\
\text { Vinyl Ester }\end{array}$ & $\begin{array}{l}\text { Resin } \\
+0.75 \% \text { MEKP } \\
+0.25 \% \text { Co Naph. }\end{array}$ & 150 & 40 & $\begin{array}{l}2 @ \text { temp \& press } \\
+24 @ \text { RT \& press }\end{array}$ \\
\hline $\begin{array}{l}\text { Derakane } 8084 \\
\text { Vinyl Ester }\end{array}$ & $\begin{array}{l}\text { Resin } \\
+1.00 \% \text { MEKP } \\
+0.20 \% \text { Co Naph. }\end{array}$ & 150 & 40 & $\begin{array}{l}2 \text { @ temp \& press } \\
+24 @ \mathrm{RT} \& \text { press }\end{array}$ \\
\hline $\begin{array}{l}\text { RIMline } 97014 \\
\text { Polyurethane }\end{array}$ & $\begin{array}{l}1.5: 1 \text { (by wt) } \\
\text { isocyanate:polyol }\end{array}$ & 285 & 40 & $\begin{array}{l}0.7 @ \text { ramp to temp ( } 8 \\
\text { press) } \\
+0.3 @ \text { temp \& press } \\
+24 @ \text { RT \& press }\end{array}$ \\
\hline $\begin{array}{l}\text { Cascophen G- } \\
1131 \text { Phenolic }\end{array}$ & $\begin{array}{l}\text { 5:1 (by wt) } \\
\text { G-1131-A:B }\end{array}$ & 150 & 40 & $\begin{array}{l}2 \text { @ temp \& press } \\
+24 @ \text { RT \& press }\end{array}$ \\
\hline
\end{tabular}

\subsection{Experimental Methodology}

The evaluation criteria applied to the natural fiber treatments consisted of four parts: the tensile performance of the single fibers, a qualitative analysis of the manufactured composites by imaging through scanning electron microscopy, the flexural performance of the manufactured composites, and the moisture absorption resistance performance of the composite. Flexural performance was selected as the metric for the composite mechanical properties due to the 
relative ease of sample preparation and testing and the ability to evaluate stiffness properties without the need of a strain measurement device.

\subsubsection{Single Fiber Tensile Testing}

Single fiber tensile tests are the most common way to measure fiber tensile properties. According to ASTM D3822, optical microscopy (OM) is used to measure the diameter of a single fiber, with the cross section assumed to be approximately round. For fabricated, synthetic fibers, this assumption is reliable and provides good tensile properties. Natural fibers, on the other hand, are more irregularly shaped and often are made up of groupings of elementary fibers (or filaments). Even the elementary fibers themselves do not typically have round cross sections, but rather polygonal shapes with 5-7 sides (Hu et al. 2010). Additionally, natural fibers can have a central hollow structure (lumen) which cannot be accounted for with the OM measurement technique.

The diameter size was found to be as much as $50 \%$ different along the length of a single fiber. To overcome diameter variations, possible solutions are to average 5 diameters or to measure only the smallest diameter. Neither of these methods can adequately take into consideration defects that may lead to premature fiber failure, however.

$\mathrm{Hu}$ et al. (2010) recommend a procedure in an attempt to provide a better evaluation of single fiber tensile properties. They propose selecting a fiber without visible defects, failing the fiber, encapsulating the failed end in resin, polishing it down to a flat surface, and using SEM to measure the exact cross sectional area. This method showed improved standard deviations with an average strength value around roughly $70 \%$ the average obtained by the OM diameter method, although in some cases the resulting strength was higher. While the results do have an improved standard deviation, these SEM standard deviation values are based on 6 samples instead of the 20 samples for OM for each method. The testing speed used by Hu et al. (2010) was also 24 times higher than recommended in ASTM D3822, which may skew results.

When these concerns were taken into account along with the significant increase in preparation / analysis time necessary to encapsulate the failed fibers in resin and measure their diameter through SEM, it was determined that the OM method would be sufficient for the determination of single fiber tensile properties in this research.

Before tension testing, the fibers were examined under a digital optical microscope at $5 \mathrm{x}$ power, and the smallest diameter along the length was recorded (Figure 4-7). From these measurements, the average minimum diameter used for the cross-sectional area calculations was around $43 \mu \mathrm{m}$, as opposed to a reported value for average fiber diameters of $150 \mu \mathrm{m}$ (Lavadiya and Fronk 2011). For the stress and stiffness calculations, each fiber was assumed to have a circular cross-sectional area of the recorded diameter. The single fibers and their cardboard frames were installed in a universal testing machine outfitted with a $10 \mathrm{~N}$ load cell and light, pneumatic tensile clamps (an improvement over the methodology in the previous chapter). Once both clamps were in place, the cardboard frame was cut away, leaving the fiber as the only source of tensile load resistance. Testing was conducted at a rate of $4 \mathrm{~mm} / \mathrm{min}$, approximately the testing speed ASTM D3822 recommends. Three to five tests were conducted on each type of fiber sample and the results were averaged. 


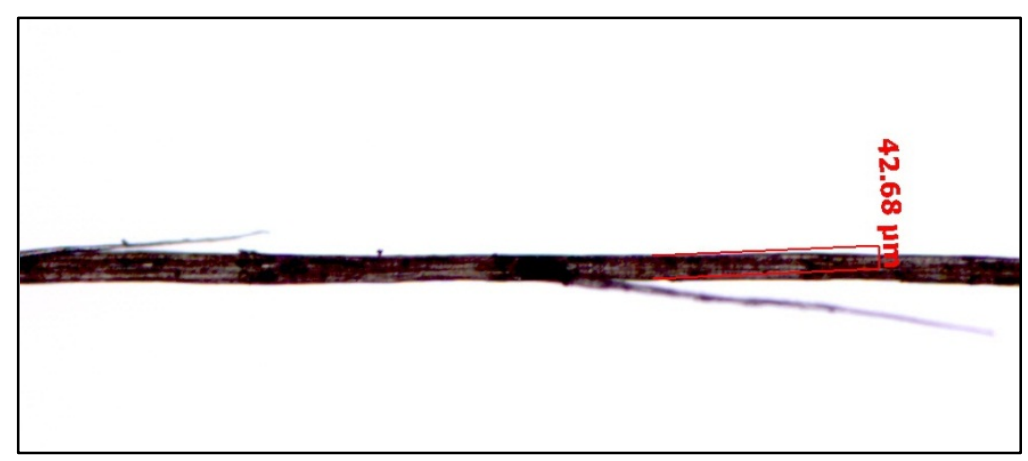

Figure 4-7 - OM diameter measurement of single fiber

\subsubsection{Scanning Electron Microscopy}

Cross-sectional slices of each of three manufactured composite samples were examined by scanning electron microscopy (SEM). The imaged samples consisted of the Derakane 510A resin reinforced by untreated fibers, fibers treated with $5 \% \mathrm{NaOH}$ for 40 minutes, and fibers treated with $5 \% \mathrm{NaOH}$ for 16 hours. The samples were embedded in a polymer disk and wetpolished for ten minutes each with a progression of 180 grit, 240 grit, and 600 grit grinding papers, as well as a polishing cloth. A $5 \mathrm{~nm}$ gold-palladium spatter coating was applied to the prepared surface prior to imaging. Samples were imaged at a variety of resolutions and locations using a combination of lower and upper detectors.

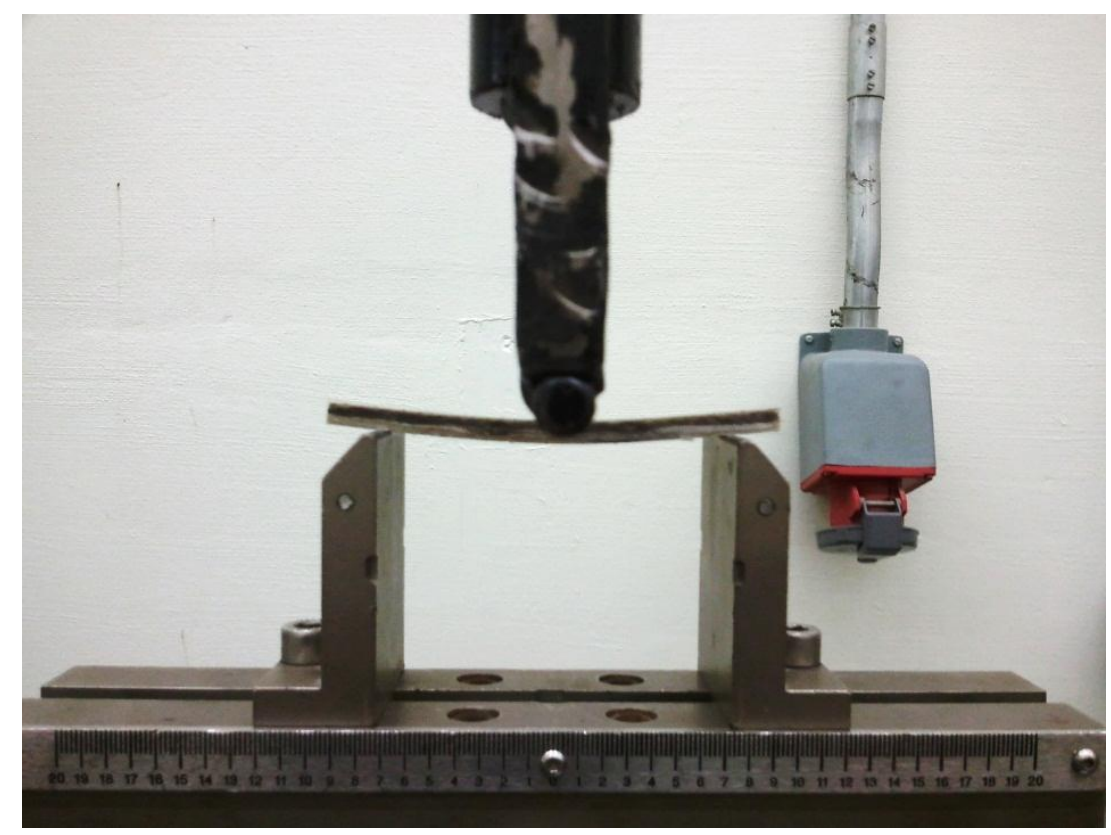

Figure 4-8 - Flexural testing of coupons

\subsubsection{Flexural Testing}

Flexural testing was carried out on a universal testing machine following the guidelines in ASTM D790 (see Figure 4-8). Each coupon was cut from the larger plate, had a thickness of $0.15-0.23$ in and a width of 0.5 in, and was tested over a support span of 3 in at a testing rate of 
$0.08 \mathrm{in} / \mathrm{min}$. A cross-calibration of the testing equipment (90 kN capacity Instron $8500 \mathrm{UTS}$ ) with a more accurate load transducer $(9 \mathrm{kN})$ confirmed that the recorded loads were within $2 \%$ error of actual, even at the relatively low loads at which the flexural tests were conducted. Similarly, displacement was confirmed to be comfortably within $2 \%$ error.

Tests were conducted on coupons cut in both the longitudinal $\left[0_{4} /\right.$ fabric $/ 90_{4} /$ fabric $\leftarrow$ load] and transverse $\left[90_{4} /\right.$ fabric $/ 0_{4} /$ fabric $\leftarrow$ load] directions. All of the coupons were tested with the midpoint load in contact with the fabric surface, in order to provide the greatest flexural strength and stiffness for the longitudinal samples. Five tests were conducted on each type of longitudinal sample and three tests were conducted on each type of transverse sample, with the results averaged, respectively.

\subsubsection{Moisture Absorption Testing}

Each sample exposed to moisture absorption testing had roughly the same dimensions as the flexural testing coupons. Each sample was weighed to the nearest $0.0001 \mathrm{~g}$ before being immersed in room temperature distilled water. Periodically, each sample was removed, patted dry with a paper towel, and reweighed over the course of several weeks.

\subsection{Results and Discussion}

\subsubsection{Single Fiber Tension Testing}

Single fiber tension testing produces very high standard deviations $(>10-50 \%$ of the average) due to the small loads, small cross-sections, and inherent variability of natural materials. Due to the inherent irregularities in natural fibers, the tensile results shown in Figure 4-9 and Figure 4-10 are the averages once values outside of a single standard deviation for each fiber treatment type are removed (along with the new SD bars). However, even with this scattered data, it was observed that the average untreated fiber strength (91.5 ksi) was within the range of values reported in literature (32-135 ksi), while the average stiffness (10.15 Msi) was considerably higher than the reported range (2.10-7.69 Msi), indicating that the fibers were between average and excellent quality (John and Anandjiwala 2008; Bismarck et al. 2005; Symington et al. 2009). 


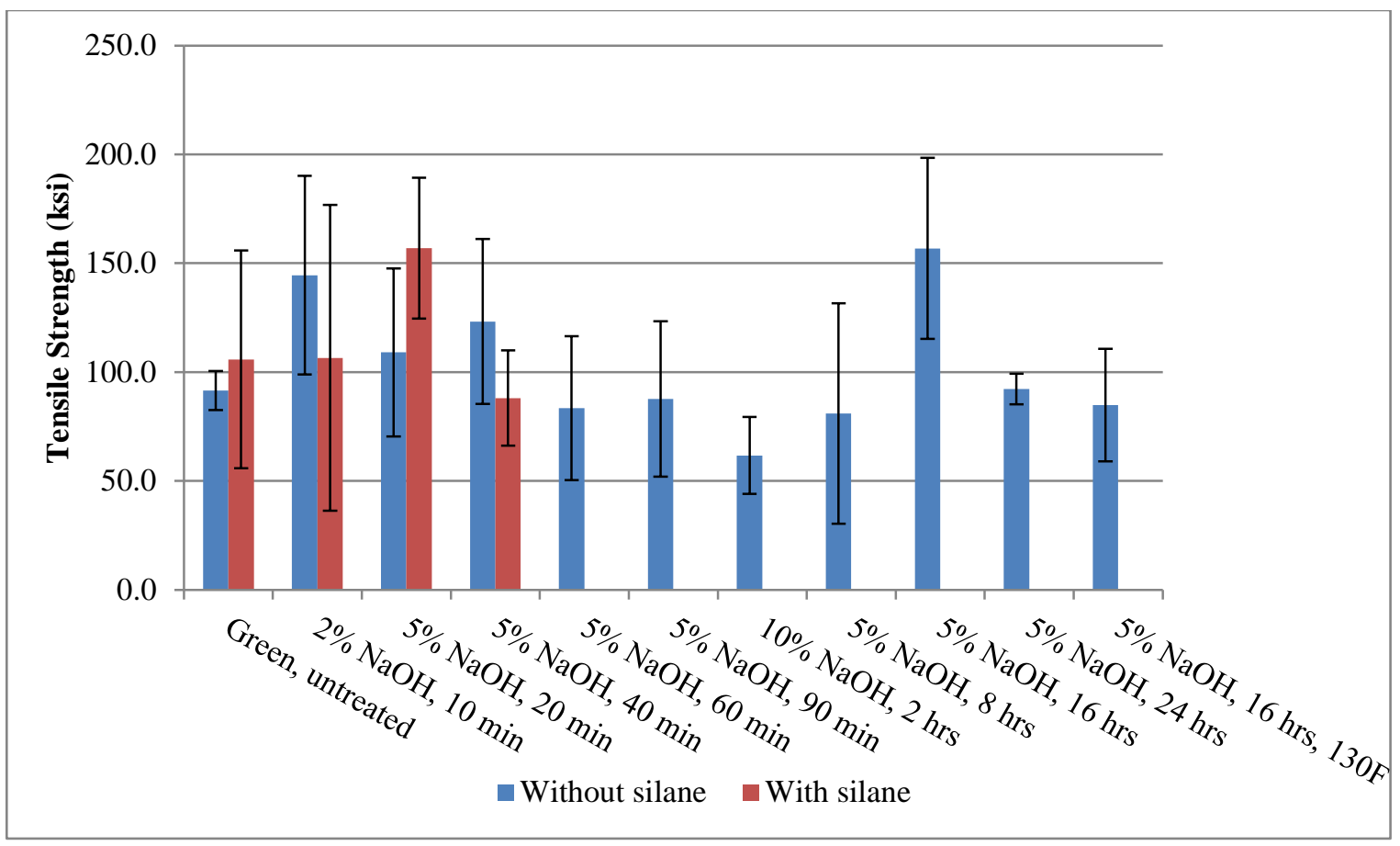

Figure 4-9 - Average single fiber ultimate tensile strength (with SD bars)

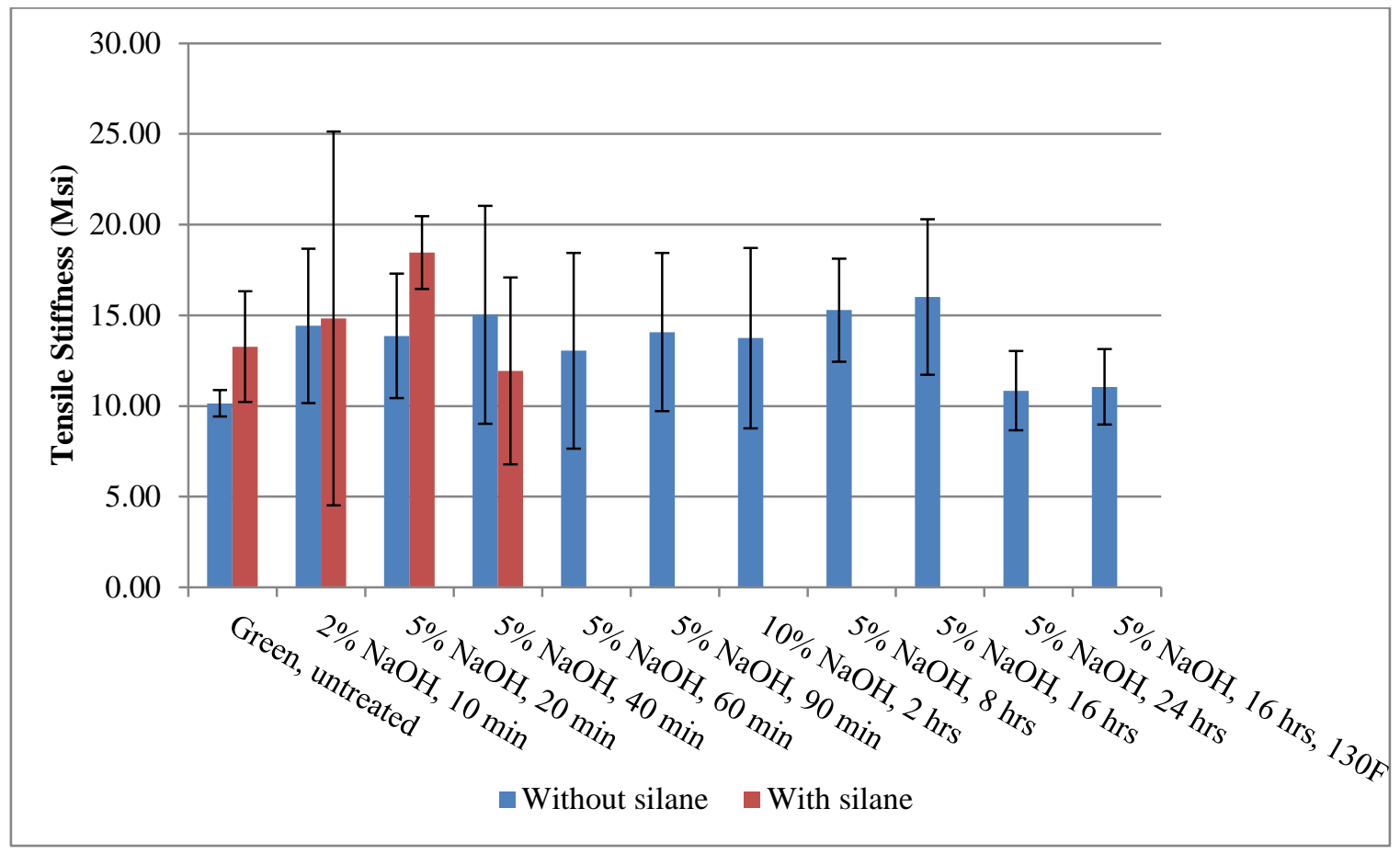

Figure 4-10 - Average single fiber tensile stiffness (with SD bars) 
The maximum consistent strength obtained by using the explored surface treatments was around 110-140 ksi from the fibers with light to medium strength alkali treatment (2-5\% for 10$40 \mathrm{~min}$ ). Higher strength (concentration or duration) alkali treatments only reduced the strength of the fiber, by as much as $50-60 \%$, with the exception of the fibers treated with $5 \% \mathrm{NaOH}$ for 16 hours which had the highest overall strength. Although it is possible to conclude from this data that this surface treatment produces the greatest improvement, these very high values are more likely a product of a small sample size (7 fiber samples) drawn from a particularly highquality fiber bundle, considering the reduction in strength at both 8 hours and 24 hours of treatment (conversely, the fibers treated for either 8 or 24 hours may have both been of poorer quality).

The failure strain of the fibers follows a similar trend to the one shown in Figure 4-9, with a max failure strain of around $2 \%$ for the fibers treated with $2 \% \mathrm{NaOH}$ for 10 minutes, and then generally decreasing with higher strength alkalization to around $0.7 \%$ (1.3\% for 5\%, 16 hours, however), suggesting an increasing brittleness of the fibers with harsher alkali treatments.

Almost all of the treated fibers displayed an increase in stiffness of around $40-50 \%$ over the untreated fibers, to an average stiffness of around $14.5 \mathrm{Msi}$, although the harsher alkali treatments (5\%, $24 \mathrm{hrs}$; 5\%, $26 \mathrm{hrs}, 130^{\circ} \mathrm{F}$ ) resulted in considerably lower stiffnesses. The best improvements to strength and stiffness by alkalization are $71 \%$ and 58\%, respectively, for 16 hours of $5 \%$ alkali treatment. These values compare well with published improvements of $61 \%$ and $42 \%$ for $16-24$ hours of $7 \%$ alkali treatment (Williams et al. 2011), although the authors point out that this improvement does not necessarily correspond to improved composite properties, most likely due to bonding issues between the fibers and resin.

The silane-treated fibers did not seem to consistently display either improved or reduced tensile properties (potentially due to the high standard deviations), and therefore the effect of the silane treatment on fiber tensile properties is assumed, on average, to be small ( $<10 \%$ increase) or negligible.

Because of the good strength and unexpectedly high stiffness properties observed in the kenaf fibers, additional fiber tensile tests were run on glass and carbon fibers with known properties in an attempt to confirm the test methodology. The results of these additional tests are shown in Table 4-3. Assuming these trends correspond to trends with the kenaf fibers (and that the publisher-provided values are realistic), it appears from the experimental values that the method may have a tendency to underestimate the strength while providing a fairly reasonable value for the stiffness. However, the brittleness of glass fibers can reduce fiber strength by as much as 50\% due to damage incurred during handling and processing (Barbero 2011). If this assumption is extended to carbon fibers as well, the first set of experimental results in Table 4-3 appears to be reasonable. If a further assumption is applied that man-made glass and carbon fibers are less likely to be unexpectedly strong/stiff and more likely to possess lower properties only due to damage, then the second set of experimental values in Table 4-3, which is the average of only the highest $50 \%$ of experimental results, ought to be closer to optimal values.

While the average strength of the E-glass fibers appears to be much closer to the actual value (only $\sim 20 \%$ low as opposed to $\sim 40 \%$ low), the carbon fibers still are not producing anywhere near the expected strength results (45\% low). Even when the maximum observed values are examined (the third set of experimental data in Table 4-3), the strength of the carbon fibers is still nowhere near the expected strength (37\% low), although the stiffness is very high. 
These results provide additional evidence that single fiber tension tests produce highly scattered results, even on man-made fibers, but as a whole the single fiber tensile testing method appears to provide good stiffness results and low-to-good strength results.

As further evidence that some of the single fiber tension testing results should not be considered to be terribly precise, all of the 101 natural fiber samples tested were plotted together in a single histogram and fitted with Weibull distributions. Barbero (Barbero 2011) recommends the application of a Weibull distribution for an accurate representation of fiber strengths; in this case, the strain and stiffness were also fit with Weibull distributions reasonably well. The resulting plots are shown in Figure 4-11 (ultimate tensile strength of kenaf fibers), Figure 4-12 (ultimate strain), and Figure 4-13 (stiffness).

The main points to take away from the application of the Weibull distribution to the natural fiber data are: 1) as with synthetic fibers, a Weibull distribution appears to be able to fairly accurately fit the strength (as well as strain and stiffness) of natural fibers, and 2) if there were really significant differences in the fiber properties as a result of surface treatment, it seems unlikely that they would so neatly fit a Weibull distribution when considered as an entire group. Therefore, it is likely that at least some of the variation in natural fiber tensile properties between different surface treatments is due to inherent variation in the fibers themselves.

Table 4-3 - Single fiber tension tests on Owens Corning E-glass and Toray carbon fibers

\begin{tabular}{|c|c|c|c|}
\hline Published Values & \# & $\sigma_{\text {ult }}(\mathbf{k s i})$ & E (Msi) \\
\hline E-Glass & - & 290 & 10.4 \\
\hline T700S Carbon & - & 711 & 33.4 \\
\hline \multicolumn{4}{|c|}{ Experimental Values with 1 SD Contraction } \\
\hline E-Glass & 6 & 167 & 10.2 \\
\hline T700S Carbon & 4 & 335 & 27.5 \\
\hline \multicolumn{4}{|c|}{ Average of High $50 \%$ Experimental Values } \\
\hline E-Glass & 6 & 229 & 11.0 \\
\hline T700S Carbon & 3 & 393 & 35.7 \\
\hline \multicolumn{4}{|c|}{ Maximum Experimental Values } \\
\hline E-Glass & 1 & 317 & 11.6 \\
\hline T700S Carbon & 1 & 450 & 50.5 \\
\hline
\end{tabular}




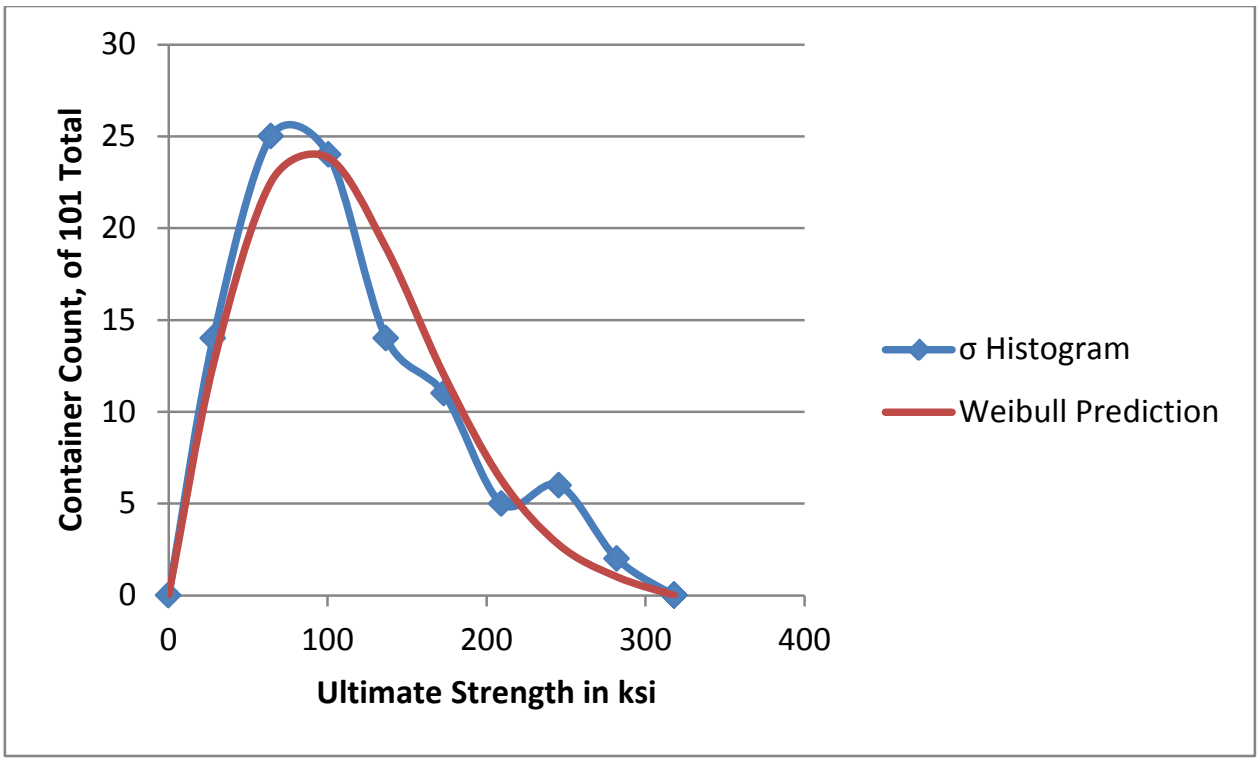

Figure 4-11 - Weibull distribution of tensile strength of kenaf fibers $(\alpha=125.9, \beta=1.957)$

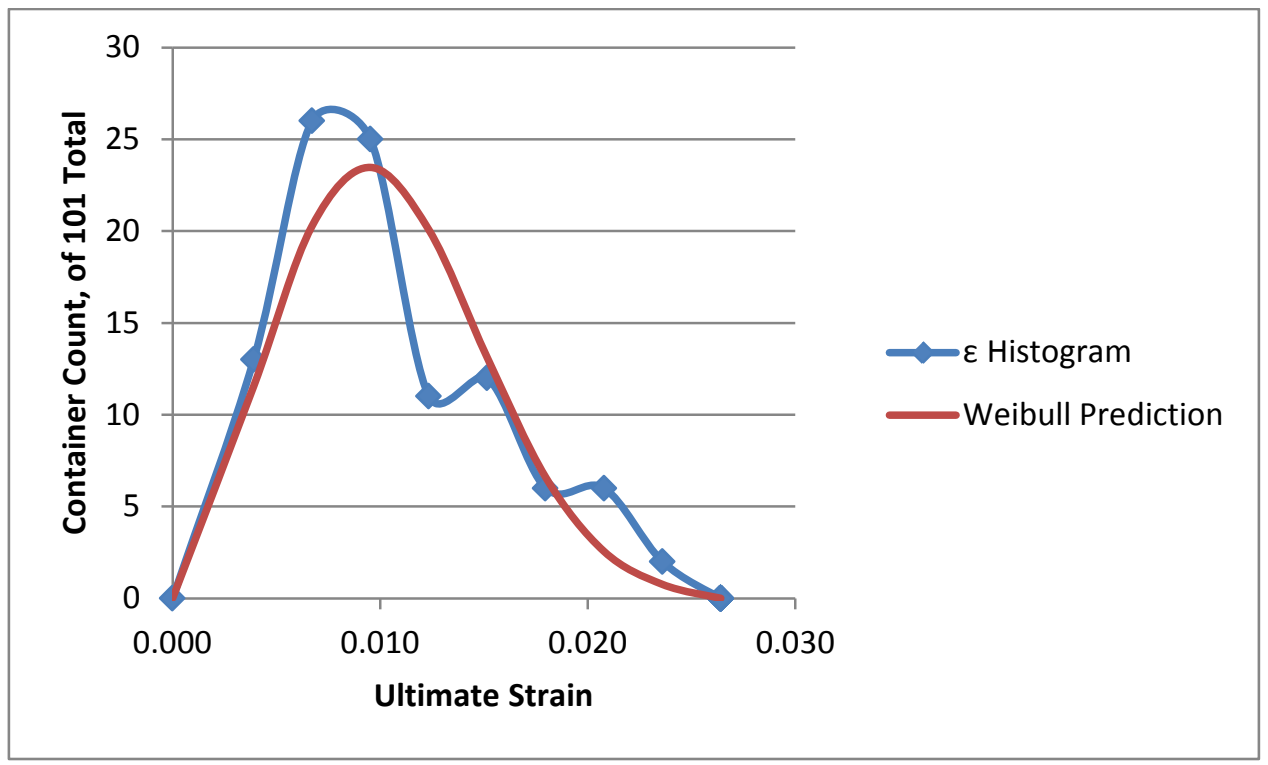

Figure 4-12 - Weibull distribution of tensile strain in kenaf fibers $(\alpha=0.0117, \beta=2.408)$ 


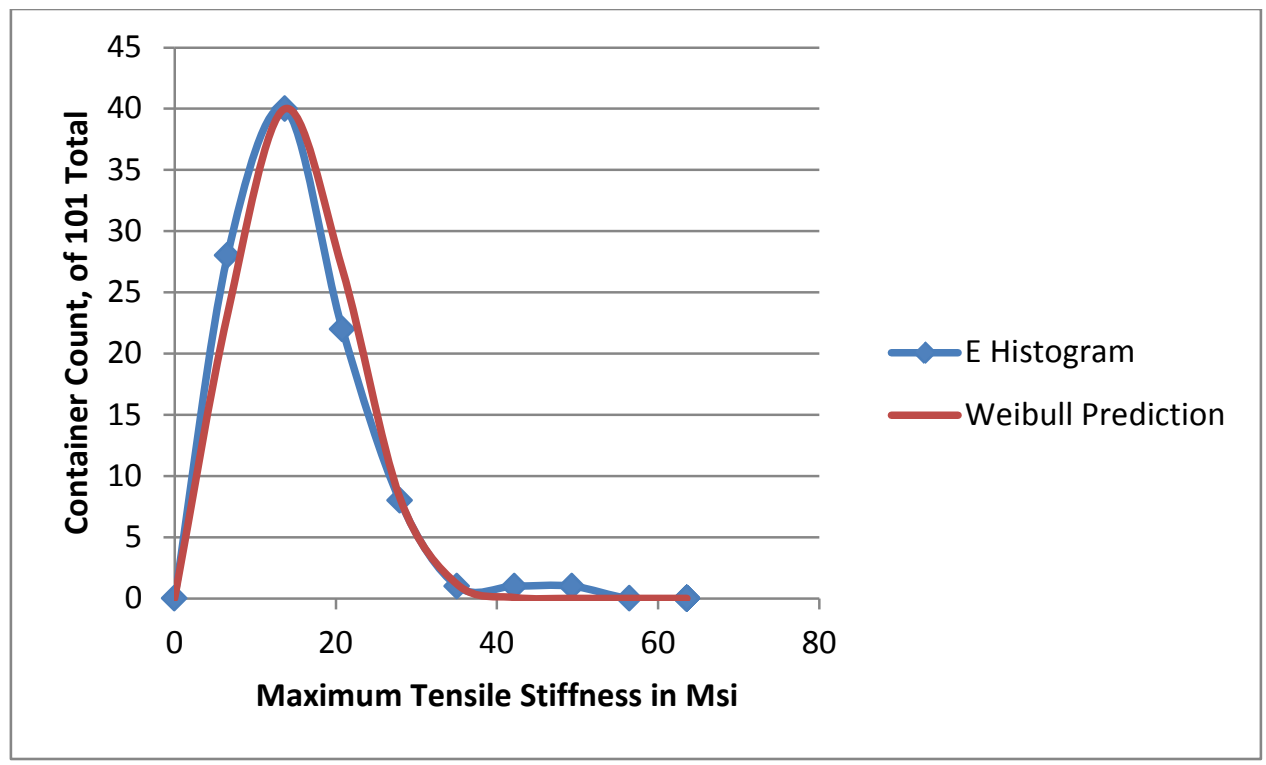

Figure 4-13 - Weibull distribution of tensile stiffness in kenaf fibers $(\alpha=17.1, \beta=2.431)$

\subsubsection{Scanning Electron Microscopy}

SEM images taken from the Derakane 510A / untreated fiber composite are shown in Figure 4-14, Figure 4-15, and Figure 4-16. SEM images from the Derakane 510A / 5\% NaOH, 40 minute-treated fiber composite are shown in Figure 4-18, Figure 4-19, and Figure 4-20. SEM images from the Derakane 510A / 5\% NaOH, 16 hour treated fiber composite are shown in Figure 4-21, Figure 4-22, Figure 4-23, and Figure 4-24.

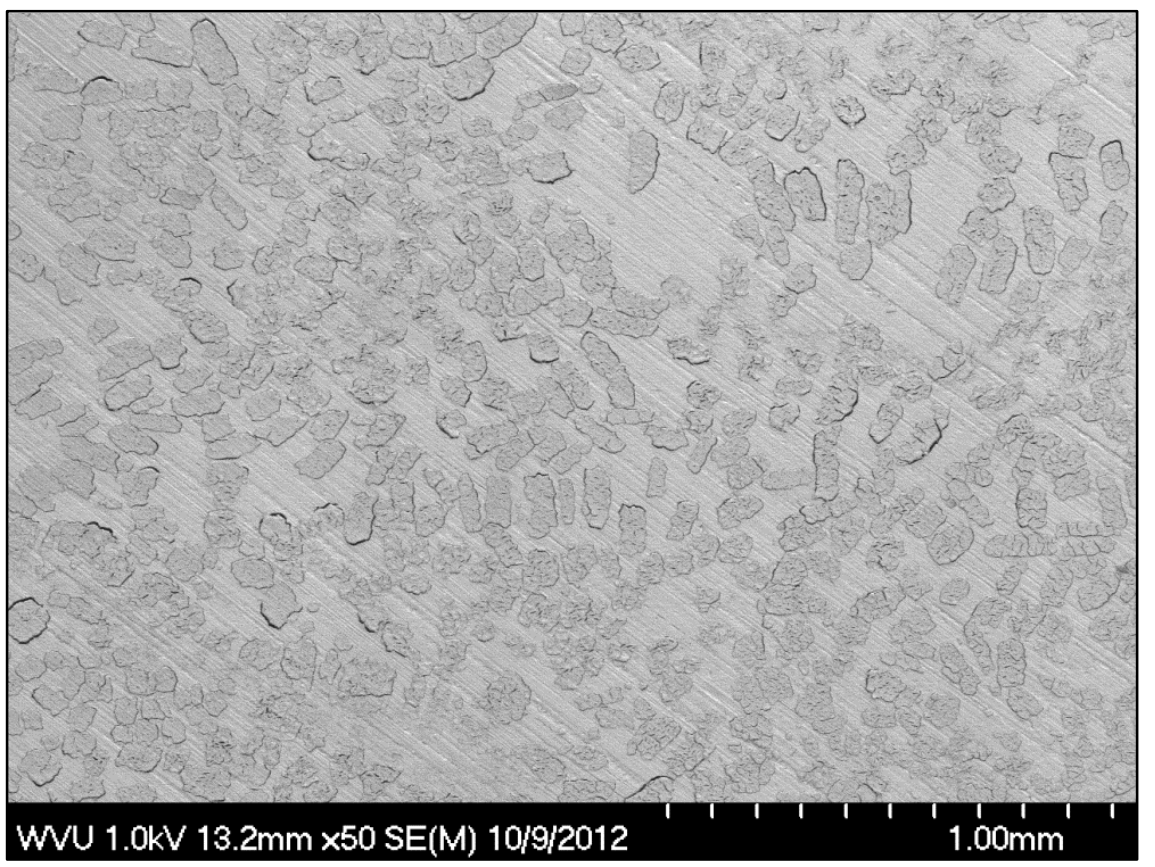

Figure 4-14 - Untreated fibers in D510A matrix, different fiber shapes and sizes 


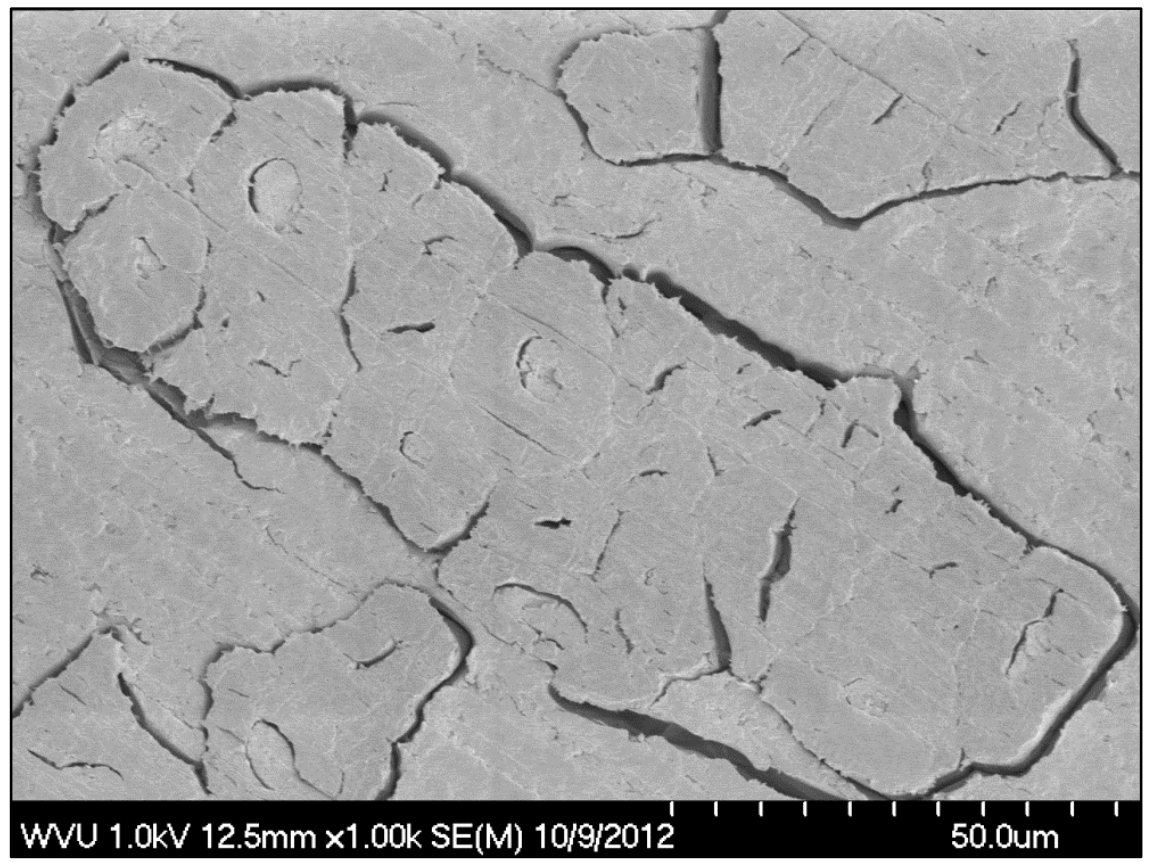

Figure 4-15 - Untreated fibers in D510A matrix, filaments and lumens, poor bonding

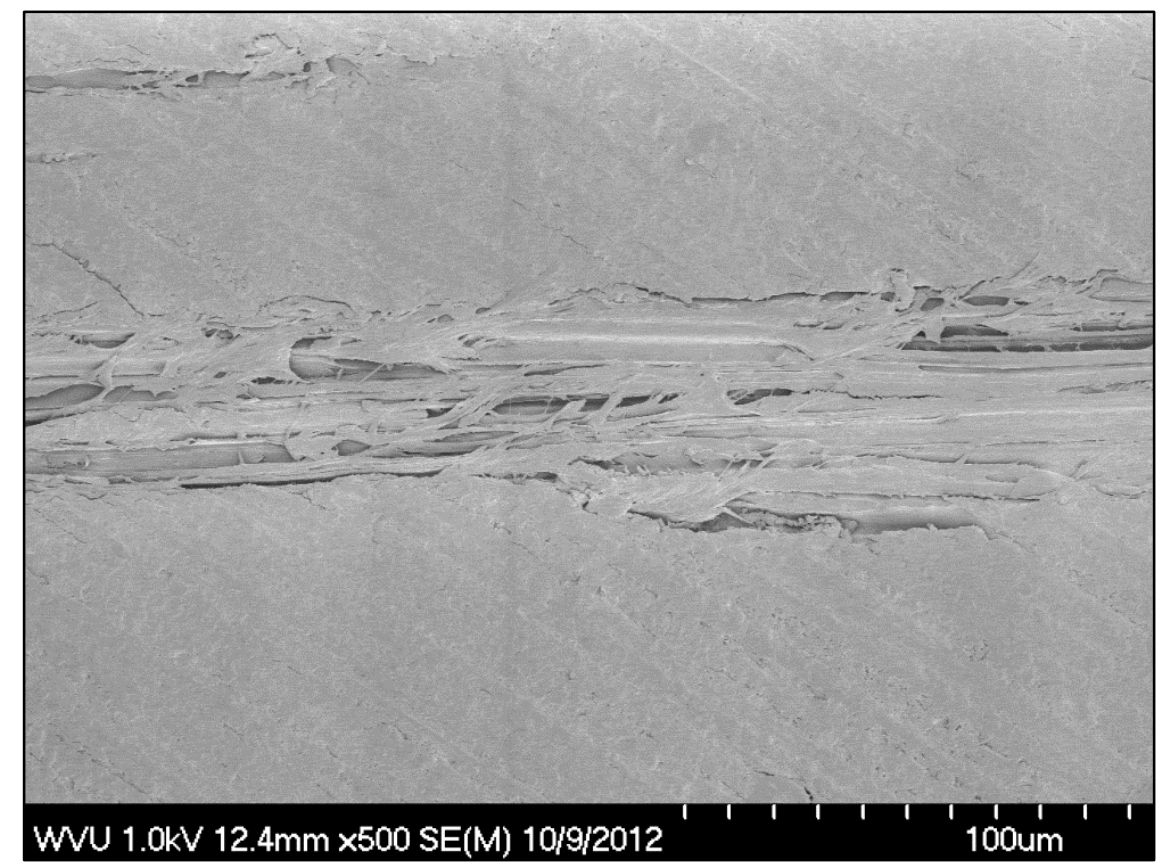

Figure 4-16 - Untreated fibers in D510A matrix, longitudinal structure

One of the first things to take notice of in Figure 4-14 is the variation in shape and size of the fibers. Some fibers are rounder and quite small, but others are much more oblong and as much as 3-5 times larger than the smallest fibers. As pointed out by $\mathrm{Hu}$ et al.(Hu et al. 2010), 
fibers are made up of groups of filaments and thus can possess a variety of shapes, and even the filaments themselves are more polygonal than round. This can be seen very clearly in Figure 4-15, where a single fiber nearly fills the screen, stretching from the top left to the bottom right. That fiber consists of a number of polygonal shapes (filaments). Each filament has a hollow center that appears as either a crack or a circle (called the lumen) and sometimes there are also additional small cracks or gaps between the filaments. If the smallest diameter of the fiber shown in Figure 4-15, or of one of the many oblong-shaped fibers in Figure 4-14, was measured by the OM method and the assumption was made that the fiber was round, the area of the fiber would be significantly underestimated, resulting in higher estimated tensile strengths and stiffnesses than the actual fiber value. Based on the findings of $\mathrm{Hu}$ et al. (Hu et al. 2010), where they stated that their improved method of area measurement resulted in a reduction to $70 \%$ of the mechanical properties of the fiber, and an estimation based on the fiber shapes in Figure 4-14, it seems reasonable to assume that the typical area of a fiber is around 1.5 times the area calculated from the minimum diameter OM method. This would result in a reduction to about $2 / 3$ of the tensile strengths and stiffnesses shown in Figure 4-9 and Figure 4-10.

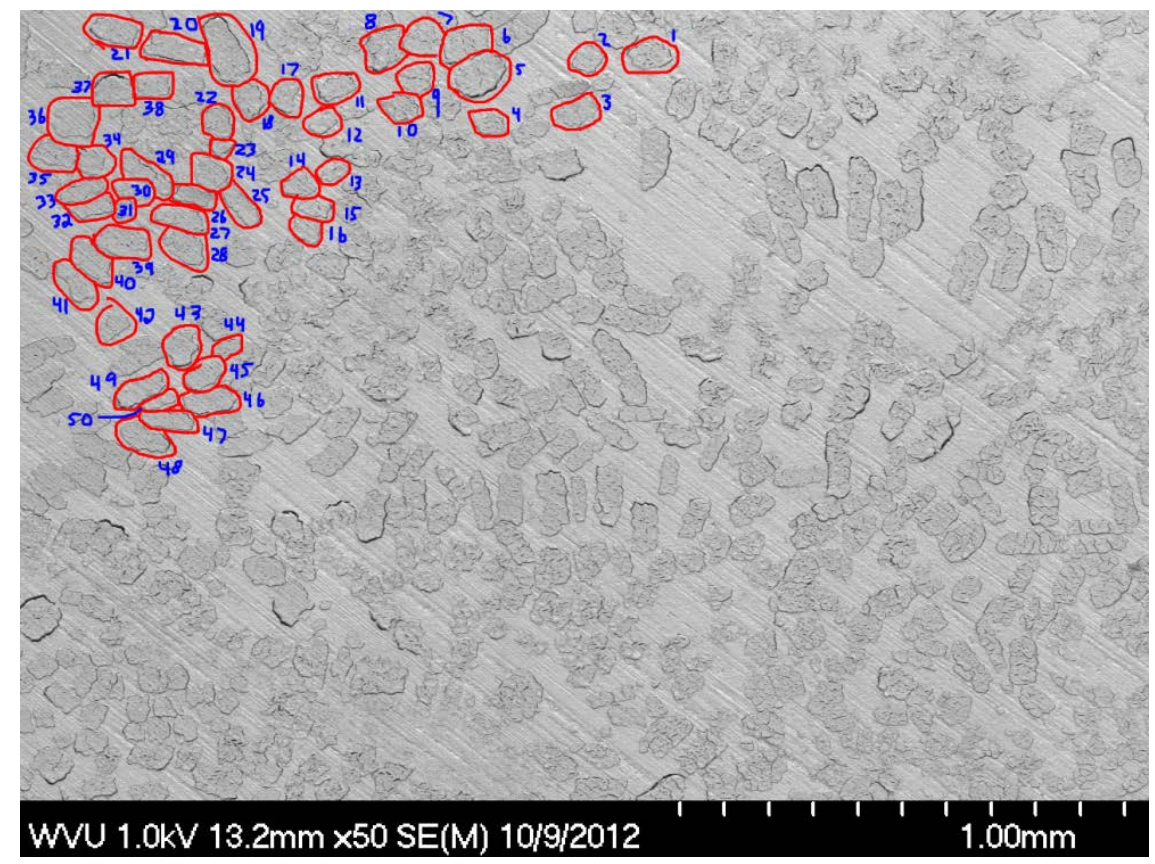

Figure 4-17 - Fibers used in area analysis

In order to confirm the assumption that the actual area of the fibers should be approximately $150 \%$ that obtained by measuring the minimum diameter and assuming a circular cross-section, an analysis of one of the better quality SEM images was conducted. First, fifty fibers were selected (as shown in Figure 4-17, with analyzed fibers circled in red and numbered in blue) with the only selection criteria being a clearly defined outline. Next, for each of these fibers, an imaging tool was used to measure the length in pixels of the minimum diameter, and from this the "assumed circular" area in pixels could be calculated. A second analysis of each fiber constituted filling the fiber area with a virtual paintbrush, after which another imaging tool was used to count the total number of filled pixels. Finally, the average area by the pixel 
counting method was divided by the minimum diameter method, resulting in an average value of $151.8 \%$. The assumption that the fiber area is approximately $\mathbf{1 5 0} \%$ that calculated based on the minimum diameter measurement method will be used in the application of theoretical models in later chapters.

Another important characteristic from Figure 4-15 is the presence of the dark line or 'gap' around the majority of the perimeter of the fiber. This empty space, small as it may be, implies that: 1) there is poor chemical bonding between the fiber and the resin, 2) moisture on the surface of the fiber at the time of manufacture prevented the resin from bonding with the fiber, 3) due to the heating of the curing process and during the subsequent several months between manufacture and imaging the fiber shrunk as it gave up moisture, or 4) some combination of the above three possibilities acted on the composite. While it is difficult to conclusively determine what primarily caused this gap (and it was likely a combined effect), the fact remains that if the fiber and matrix were more chemically compatible then the gap should be reduced or eliminated regardless of the other factors.

Figure 4-16 is an image taken of a fiber in a 90-degree layer of the composite, essentially splitting a fiber along its axis. In this image one can again detect at least a partial gap between the fiber surface and resin. Also of interest is the structure of the filaments: from this and other images, it appears that many of them are not strictly aligned along the axis of the fiber, but instead may form a sort of helical or twisted pattern.

The amount of detail in the images of the treated fiber composites was less than in the images of the untreated fibers (as in Figure 4-19 and Figure 4-23, for example), although this was likely due to poorer preparation of those samples for SEM rather than a significant change of the fibers' structures. As such it is much more difficult to make out the separate filament structures that make up a single fiber. There are still some interesting observations to be made from these images, however. 


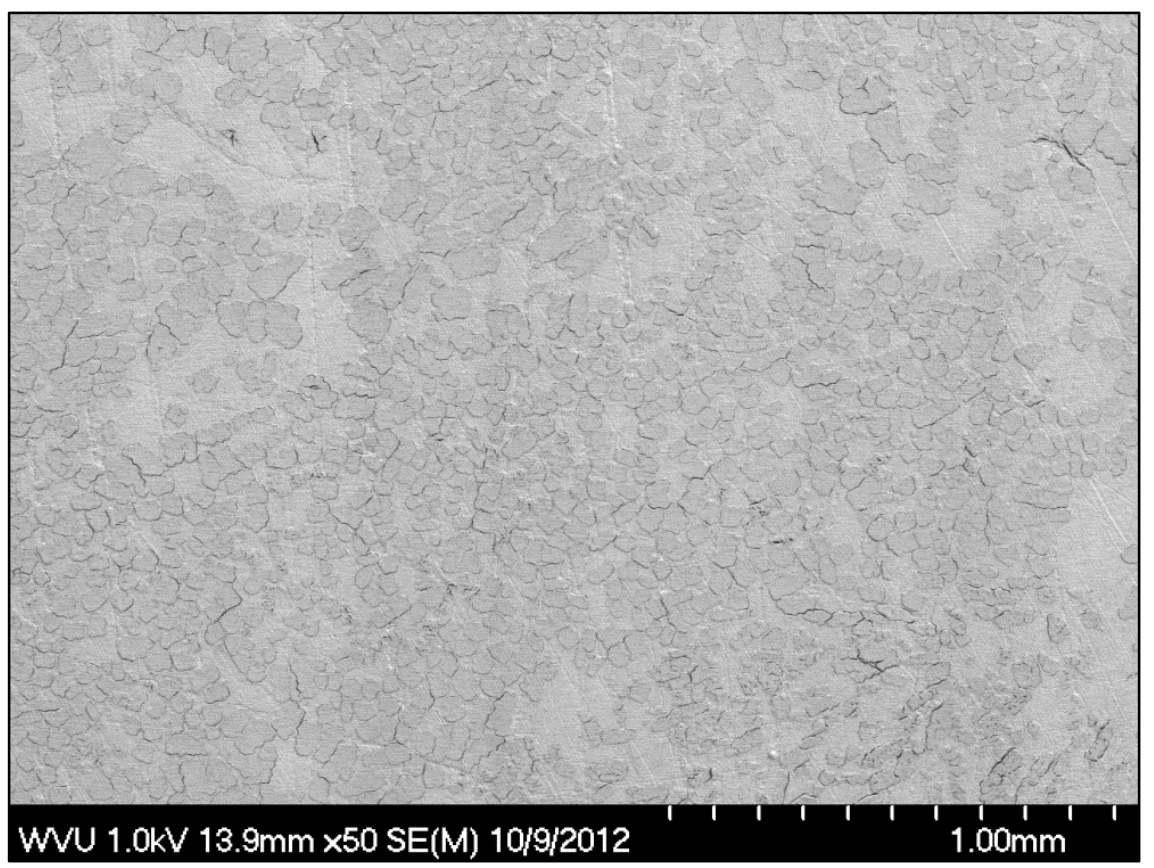

Figure 4-18 - 5\%, 40 min treated fibers in D510A matrix, fiber distribution

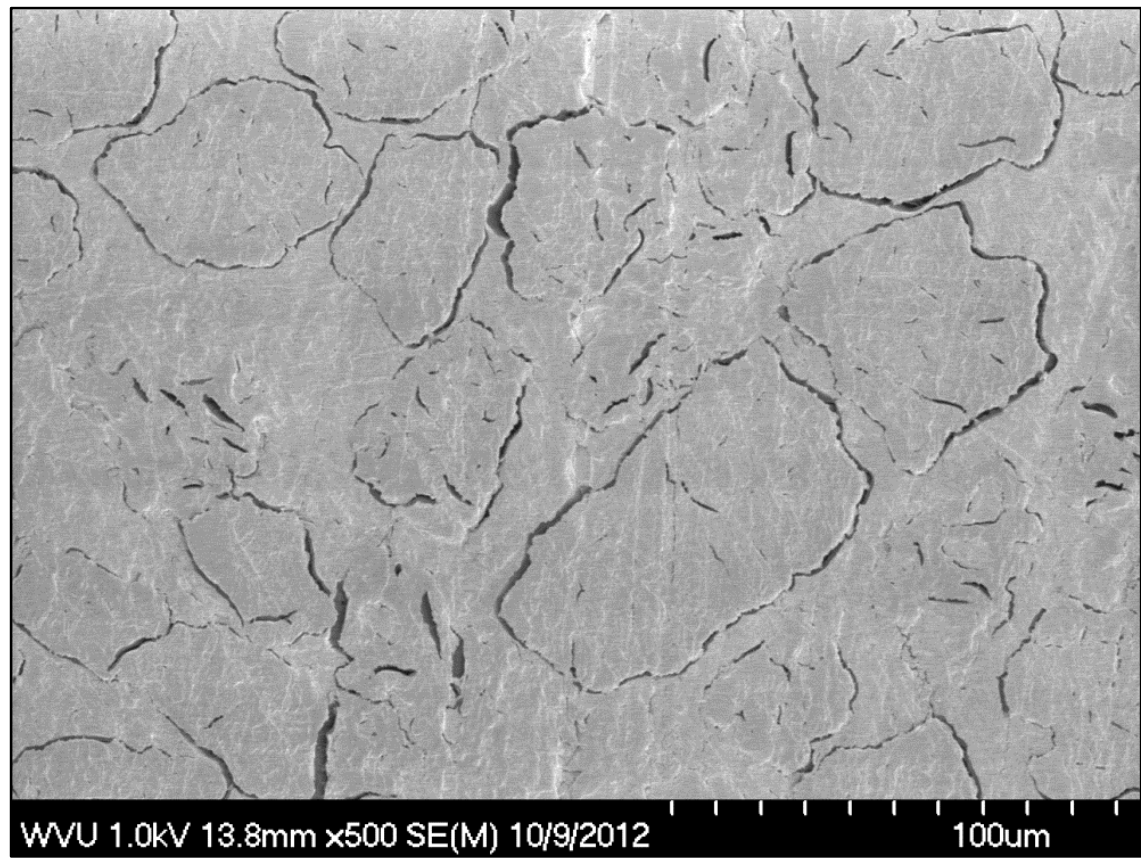

Figure 4-19 - 5\%, 40 min treated fibers in D510A matrix, poor bonding 


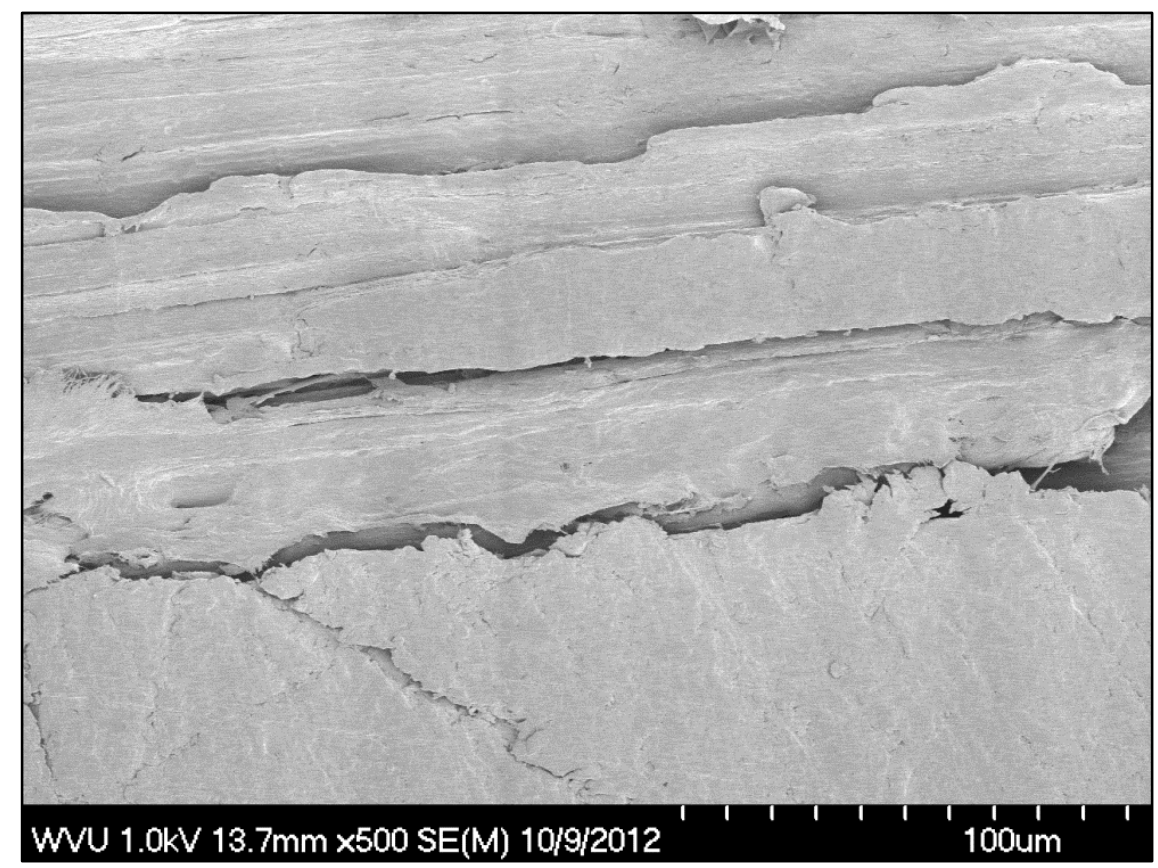

Figure 4-20 - 5\%, 40 min treated fibers in D510A matrix, longitudinal, poor bonding

Figure 4-18 offers a wider angle of a 0-direction surface of the composite, and from this it can be seen that a good fiber distribution is obtained by using stitched mats in a compression molding fixture. There are few and only small resin rich areas, minimal voids, and no areas where the resin was unable to reach fibers. However, that does not necessarily mean that the treatment process ( $5 \% \mathrm{NaOH}$ for 40 minutes) produced a significant chemical change in the fibers - from Figure 4-19 (view of 0-direction fiber) and Figure 4-20 (view of 90-direction fiber) it appears that the gaps between the fibers and the matrix are approximately the same as those seen around the untreated fibers.

While Figure 4-21 also shows good fiber distribution for the composite reinforced with $5 \% \mathrm{NaOH}, 16$ hour treated fibers, there are also a few voids and larger cracks. While the quality is again poorer for these images than for those of the untreated fibers, it would appear from Figure 4-22 and Figure 4-23 that the gaps between the fibers and the matrix are less frequent and considerably smaller. This implies that the $5 \% \mathrm{NaOH}, 16$ hour treatment may have an overall positive effect on the chemical bonding between the fibers and the matrix. The presence of this positive effect is difficult to confirm and the extent is difficult to quantify, however, as some fibers (such as the one shown in Figure 4-24) still have significant gaps around them. 


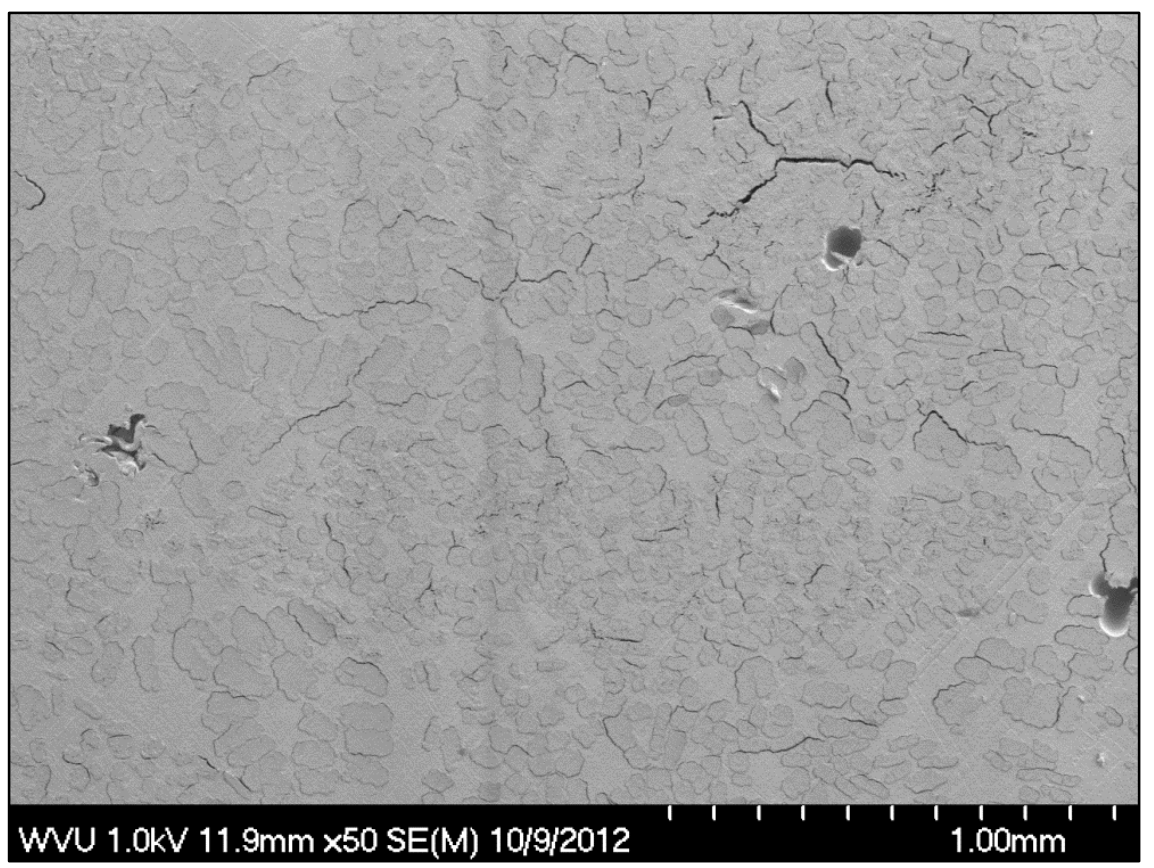

Figure 4-21 - 5\%, 16 hrs treated fibers in D510A matrix, cracks and voids

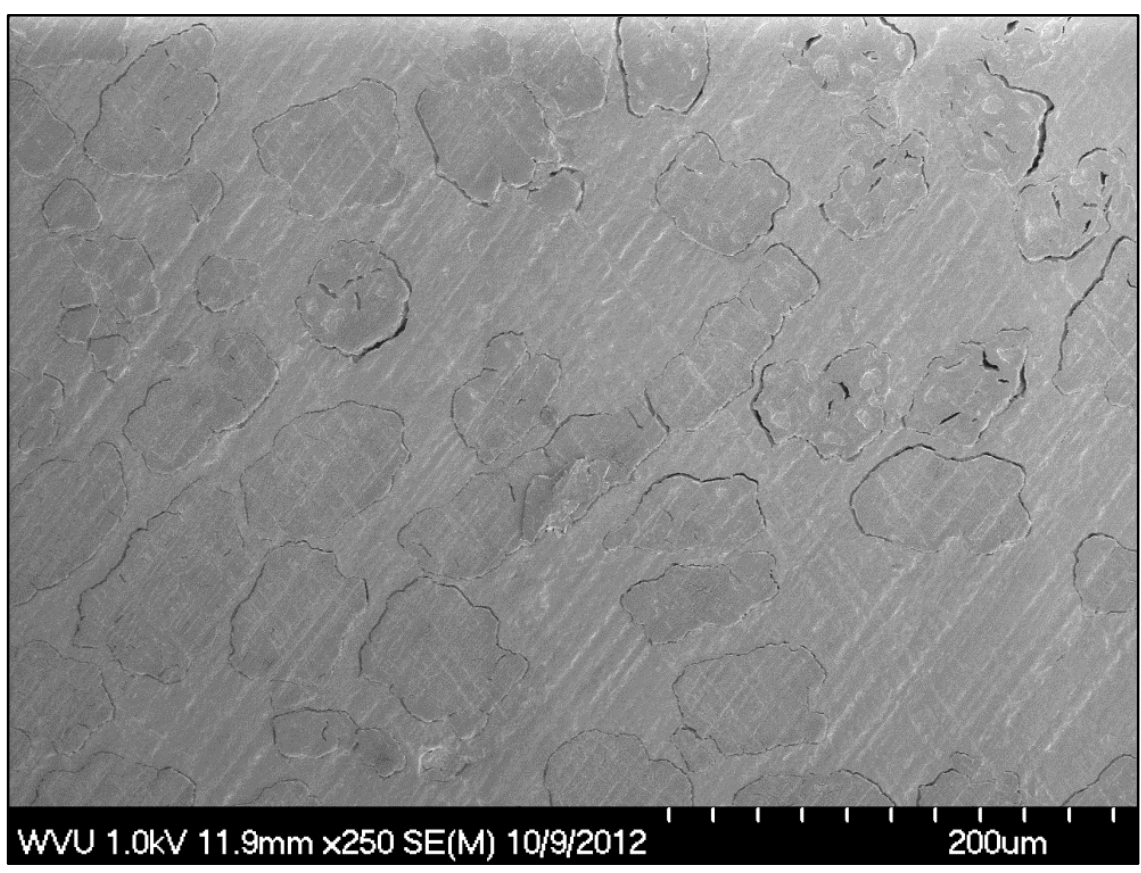

Figure 4-22 - 5\%, 16 hrs treated fibers in D510A matrix, reduced gap size and penetration 


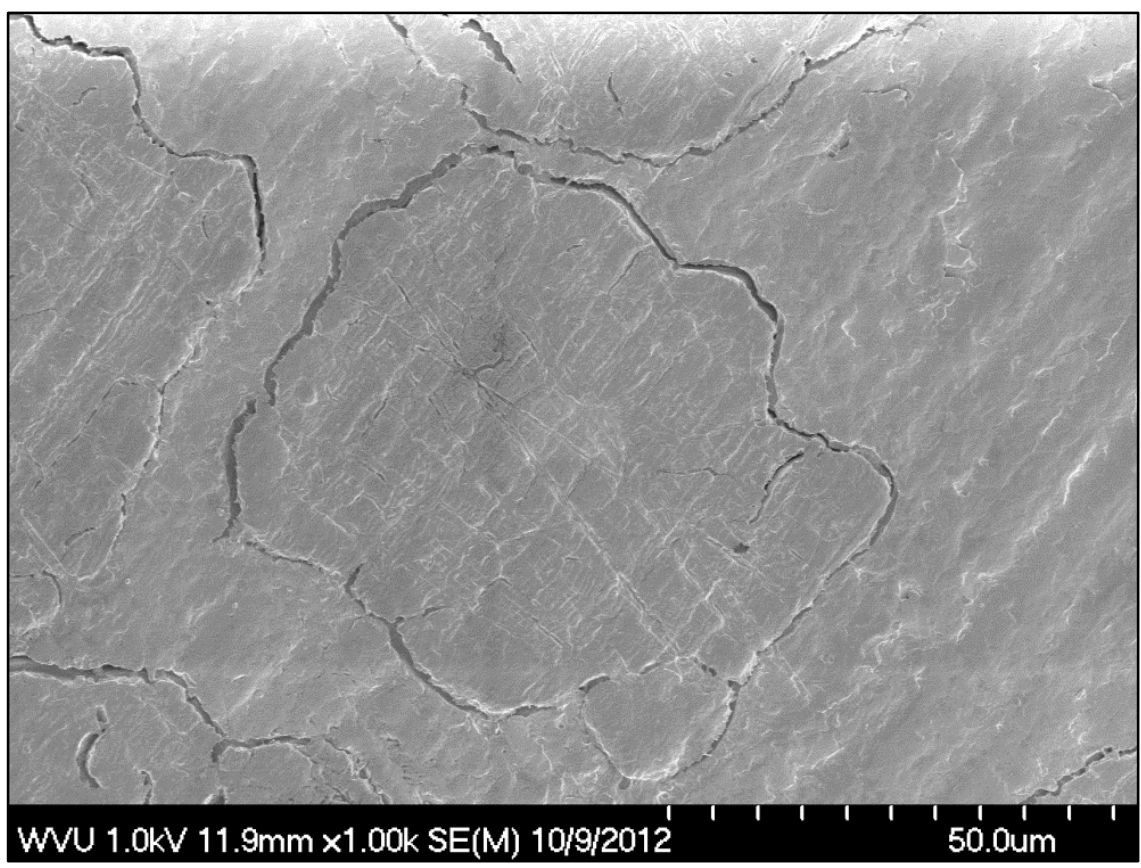

Figure 4-23 - 5\%, 16 hrs treated fibers in D510A matrix, improved bonding

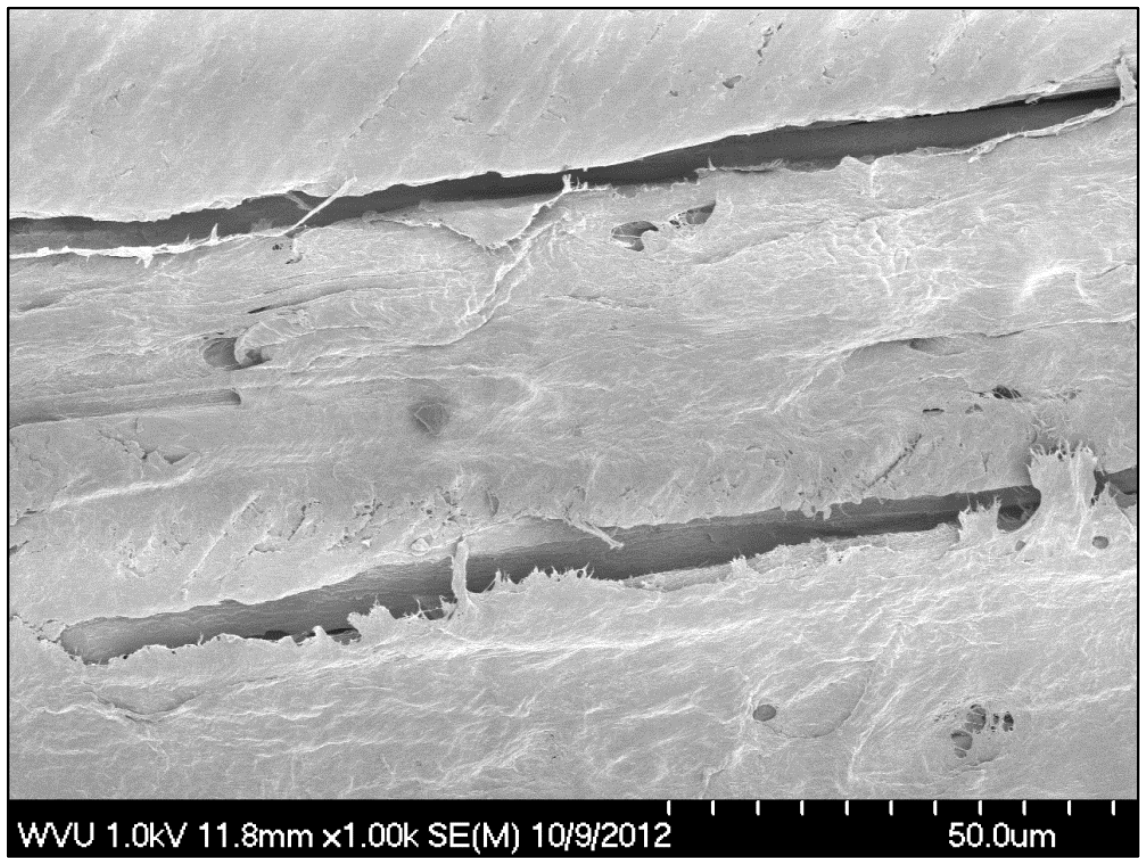

Figure 4-24 - 5\%, 16 hrs treated fibers in D510A matrix, longitudinal, poor bonding

\subsubsection{Flexural Testing}

The flexural stress at failure and the tangent modulus were calculated according to ASTM D790. Figure 4-25 contains the longitudinal strength results and Figure 4-26 contains the longitudinal stiffness results. The results of the transverse tests (Figure 4-27 and Figure 4-28) 
were far more scattered, and were generally around 30\% the strength and 65\% the stiffness of the longitudinal tests.

Both of the vinyl ester systems produced similar strength and stiffness results. The polyurethane did not produce comparable mechanical properties, probably due to mishandling during preparation and the presence of moisture in the fibers. Even though the fibers were dried, plant-based products still contain some moisture, and that moisture may have caused to polyurethane to foam and create microvoids. Additionally, the handling of the polyurethane during the preparation and application to the fibers may have led to the introduction of air bubbles and foaming (Figure 4-29). Even though the completed plate had a good finish (Figure 4-30), the fact that at least some foaming took place within the composite was also evident due to the low density of the plate $\left(0.031 \mathrm{lb} / \mathrm{in}^{3}\right.$ instead of the expected ROM density of $\left.0.045 \mathrm{lb} / \mathrm{in}^{3}\right)$, indicating entrapped air. The phenolic system had even lower performance than the polyurethane, and was only tested with the untreated fibers. Of the resin systems explored, the Derakane 510A and the Derakane 8084 seem to provide the best strength, with the Derakane 8084 providing the best stiffness.

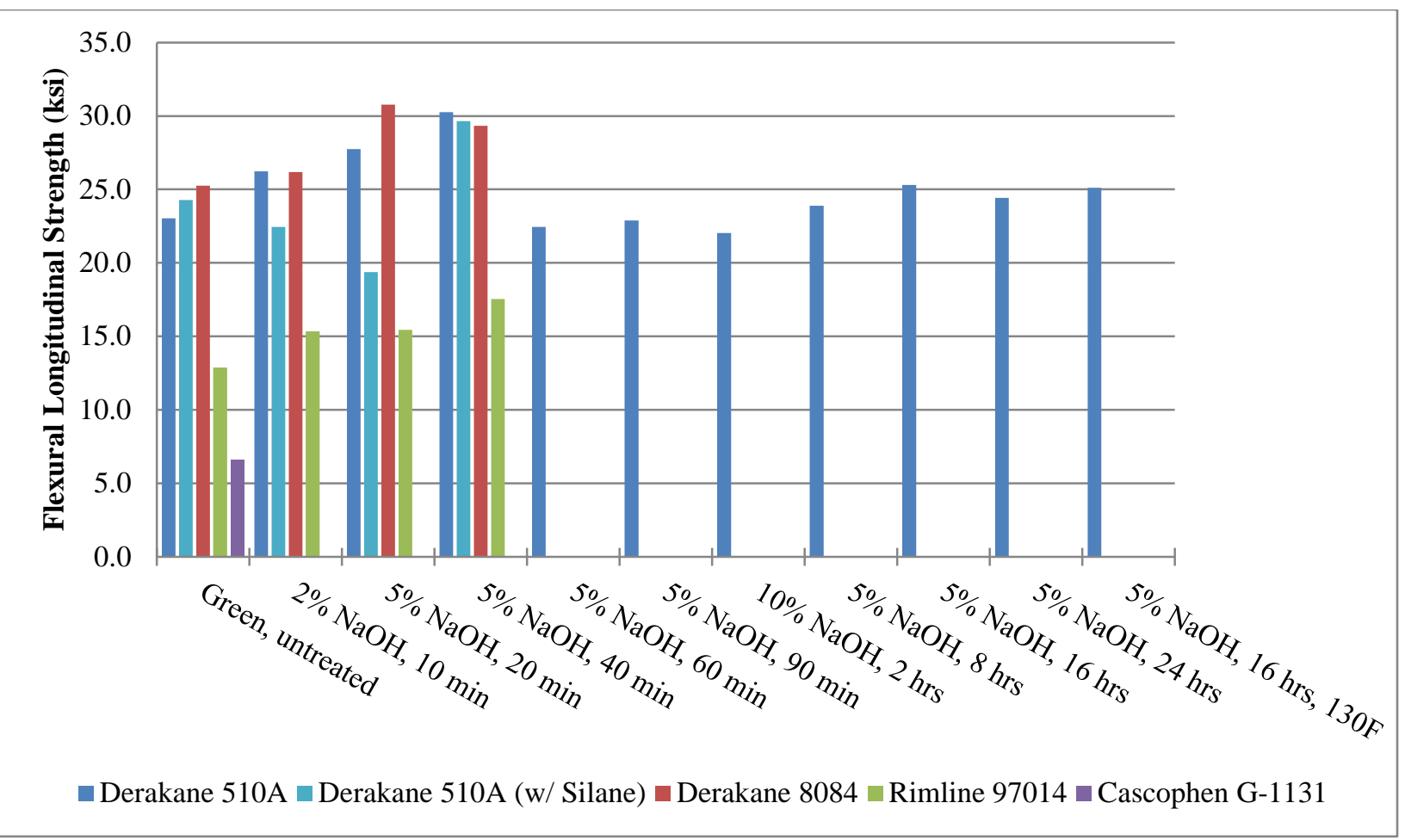

Figure 4-25 - Longitudinal flexural strength of manufactured composites 


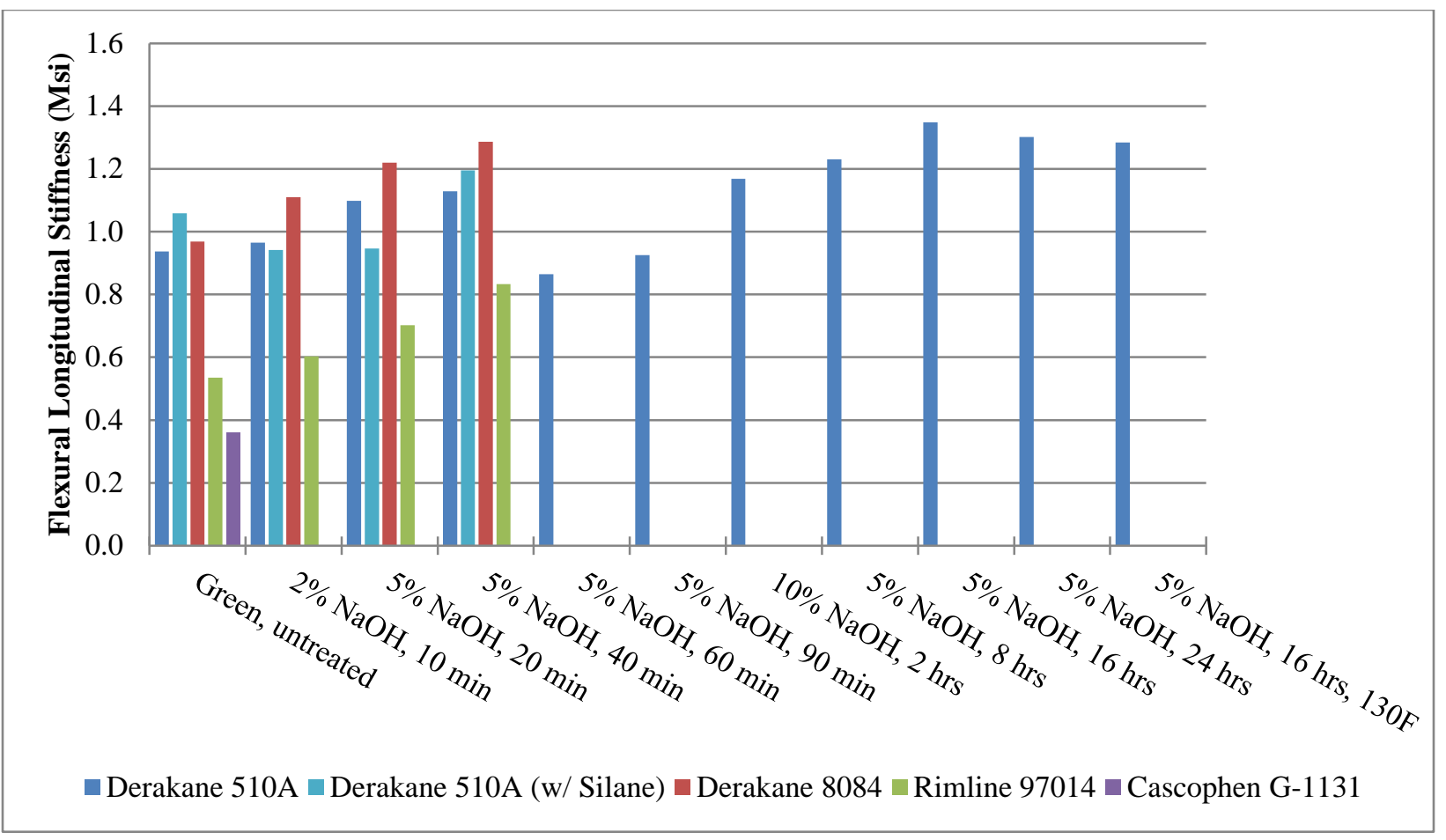

Figure 4-26 - Longitudinal flexural stiffness of manufactured composites

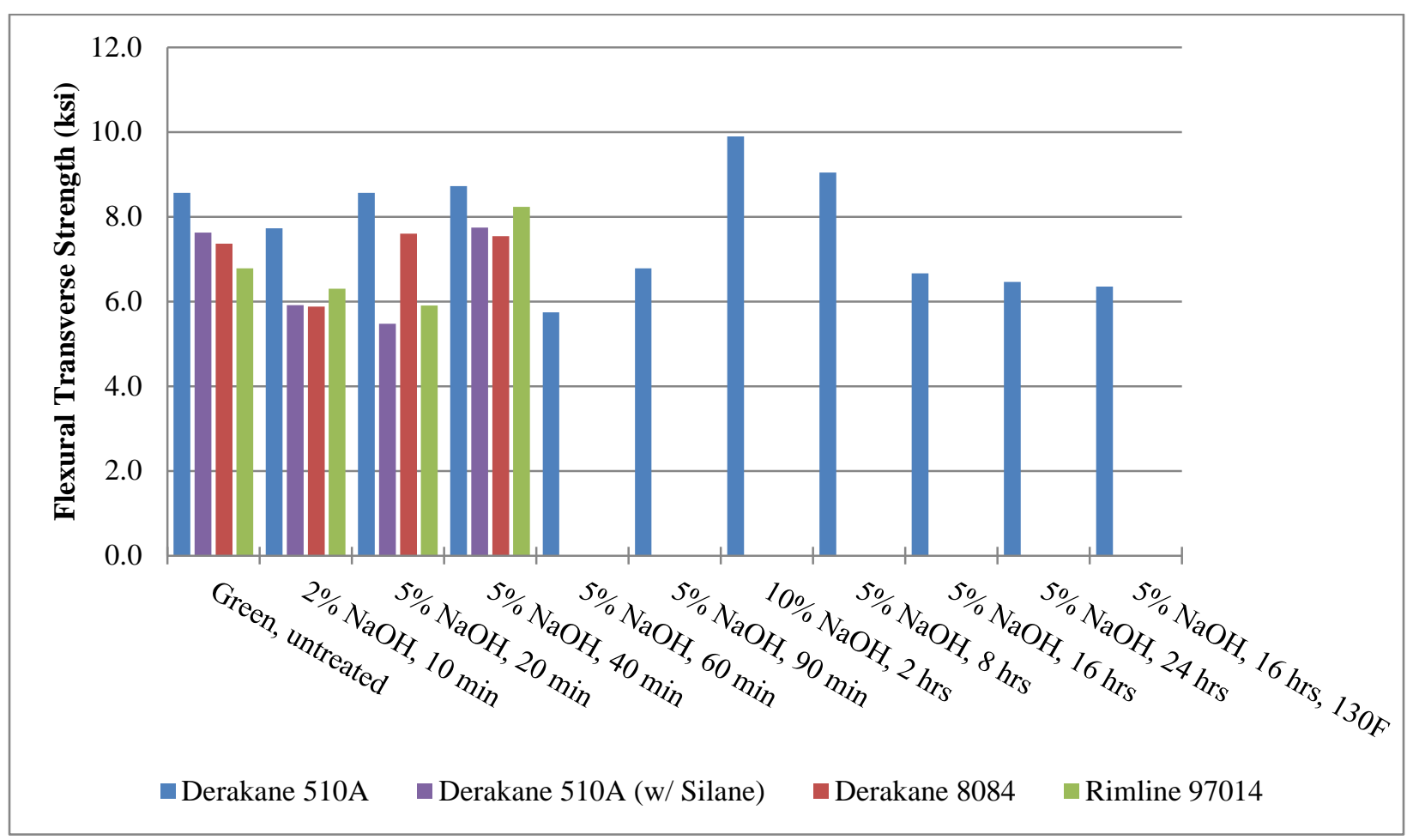

Figure 4-27 - Transverse flexural strength of manufactured composites 


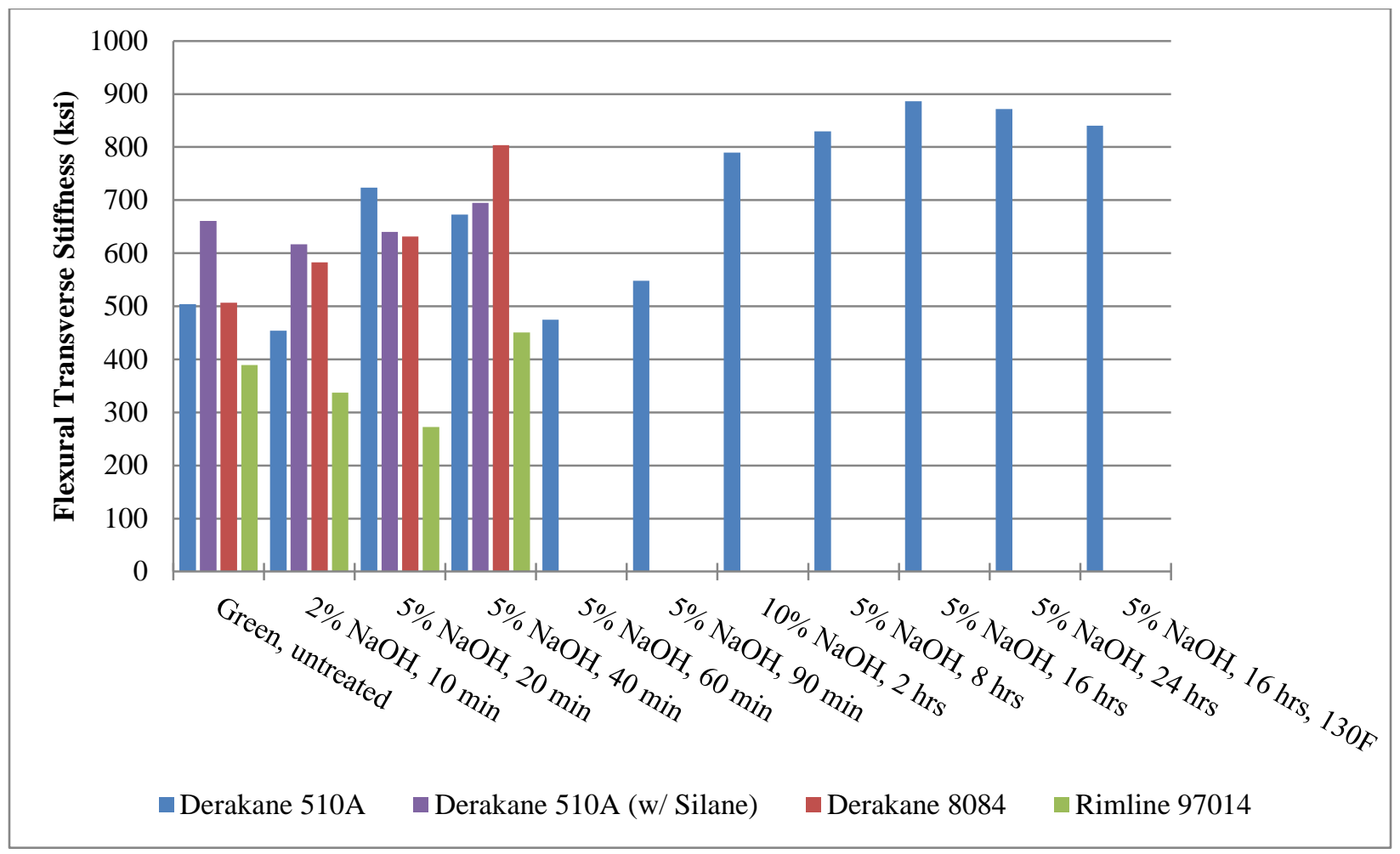

Figure 4-28 - Transverse flexural stiffness of manufactured composites

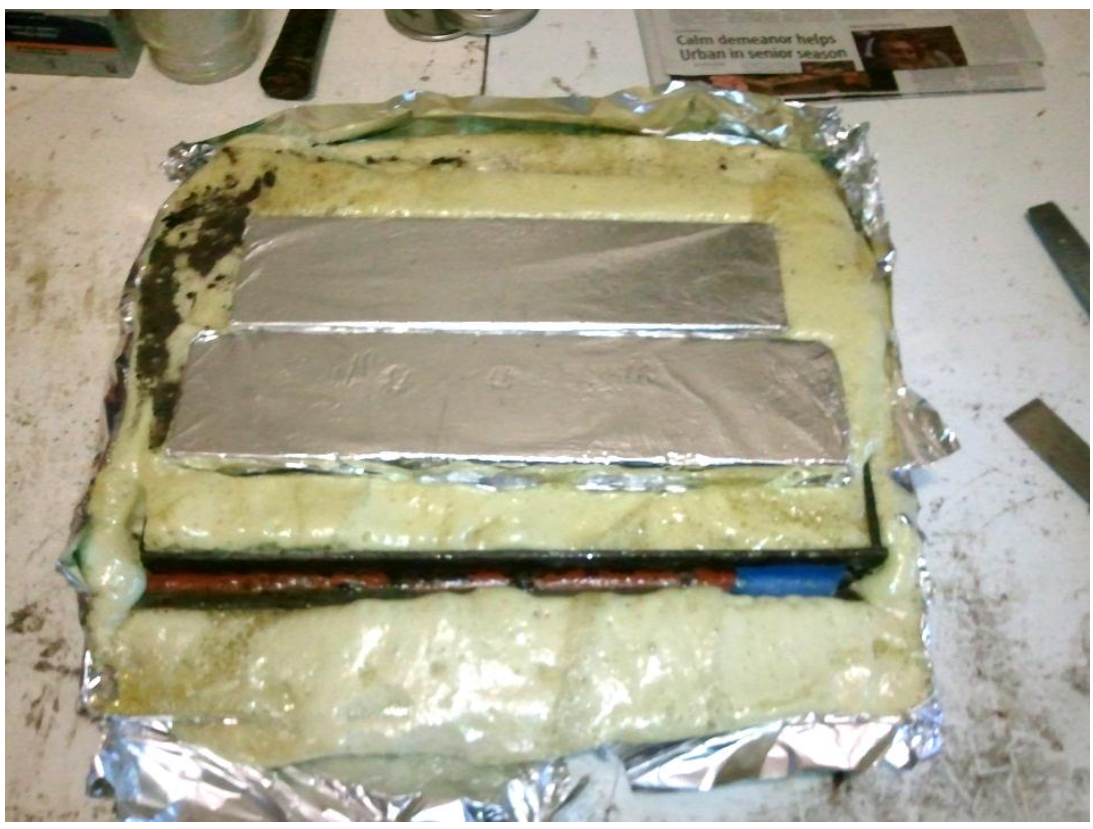

Figure 4-29 - Over-foaming of polyurethane plate 


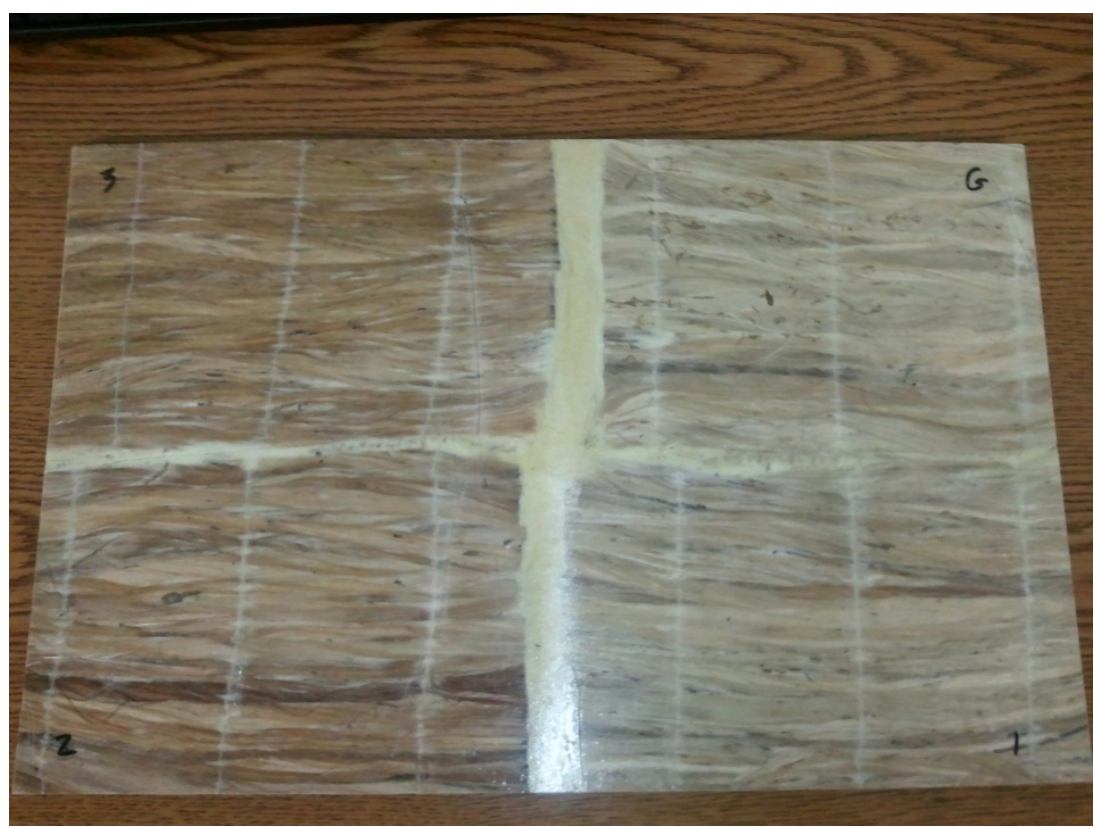

Figure 4-30 - Polyurethane plate removed from mold

The longitudinal strength and stiffness results show a clear increase in the mechanical properties of the composite with light alkalization for each resin system. However, when Figure 4-25 is compared to Figure 4-9, it can be seen that the highest fiber tensile strength does not necessarily correspond to the best composite flexural strength. This suggests that the fibers which underwent a medium alkali treatment were able to better bond with the resin, in some cases more than making up for their reduced tensile strength. The best flexural properties were observed in the composites manufactured with vinyl ester resin with a medium strength alkali treatment (5\% for 20-40 min). Coupons from these composites exhibited flexural strengths of around $29 \mathrm{ksi}$ and flexural stiffnesses of around $1100 \mathrm{ksi}$. The stiffest flexural coupons were those that underwent the high alkali treatment (5\% for 8-24 hours), with a stiffness of around $1300 \mathrm{ksi}$, although these coupons only had a middling strength of around $25 \mathrm{ksi}$. The presence of silane did not seem to consistently affect either the flexural strength or stiffness of the manufactured composites, and can therefore be assumed, on average, to have only a small ( $<10 \%$ decrease) or negligible impact.

However, if the strength and stiffness values are divided by the volume fraction of fibers (FVF) (Longitudinal: Figure 4-31, Figure 4-32 respectively; Transverse: Figure 4-33, Figure 4-34 respectively) to account for the variation (22-53\%) in the FVF between each composite plate and then multiplied by an average volume fraction of $40 \%$, the silane-treated composites exhibit comparable strength and around 18\% higher stiffness. When examined from this perspective, the ideal alkali treatment also seems to shift more toward the medium alkali treatments for strength $(5 \%, 60-90 \mathrm{~min})$ and even more toward the high alkali treatments for stiffness (5\%, 16-24 hours). The transverse properties were again fairly scattered, but the transverse flexural stiffness also seems to favor the high alkali treatments. 


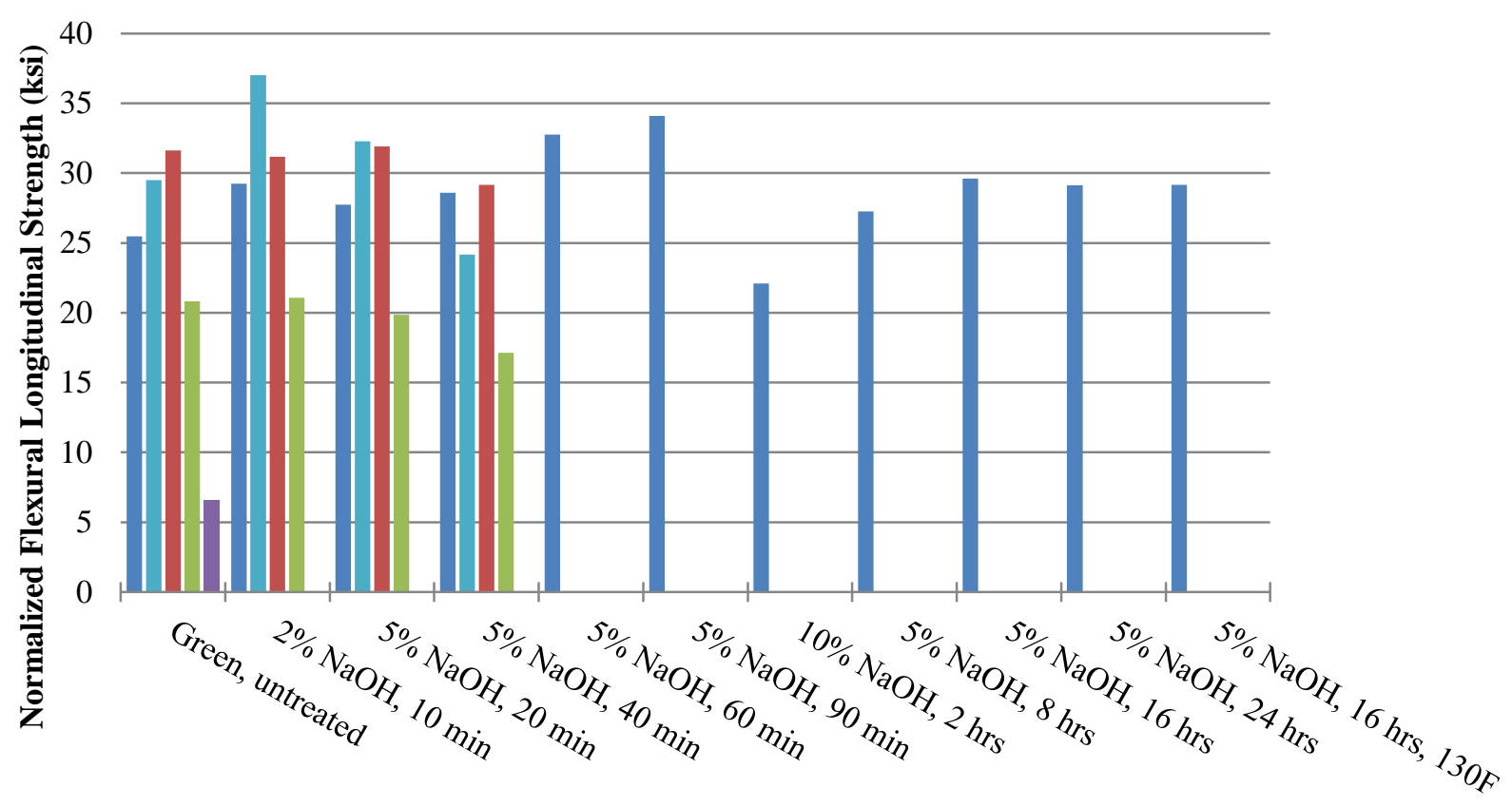

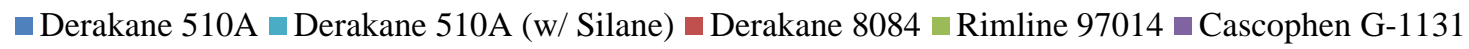

Figure 4-31 - Longitudinal flexural strength normalized for $40 \%$ FVF $\left[0_{4} /\right.$ fabric $_{9} \mathbf{9 0}_{4} /$ fabric $\leftarrow$ load]

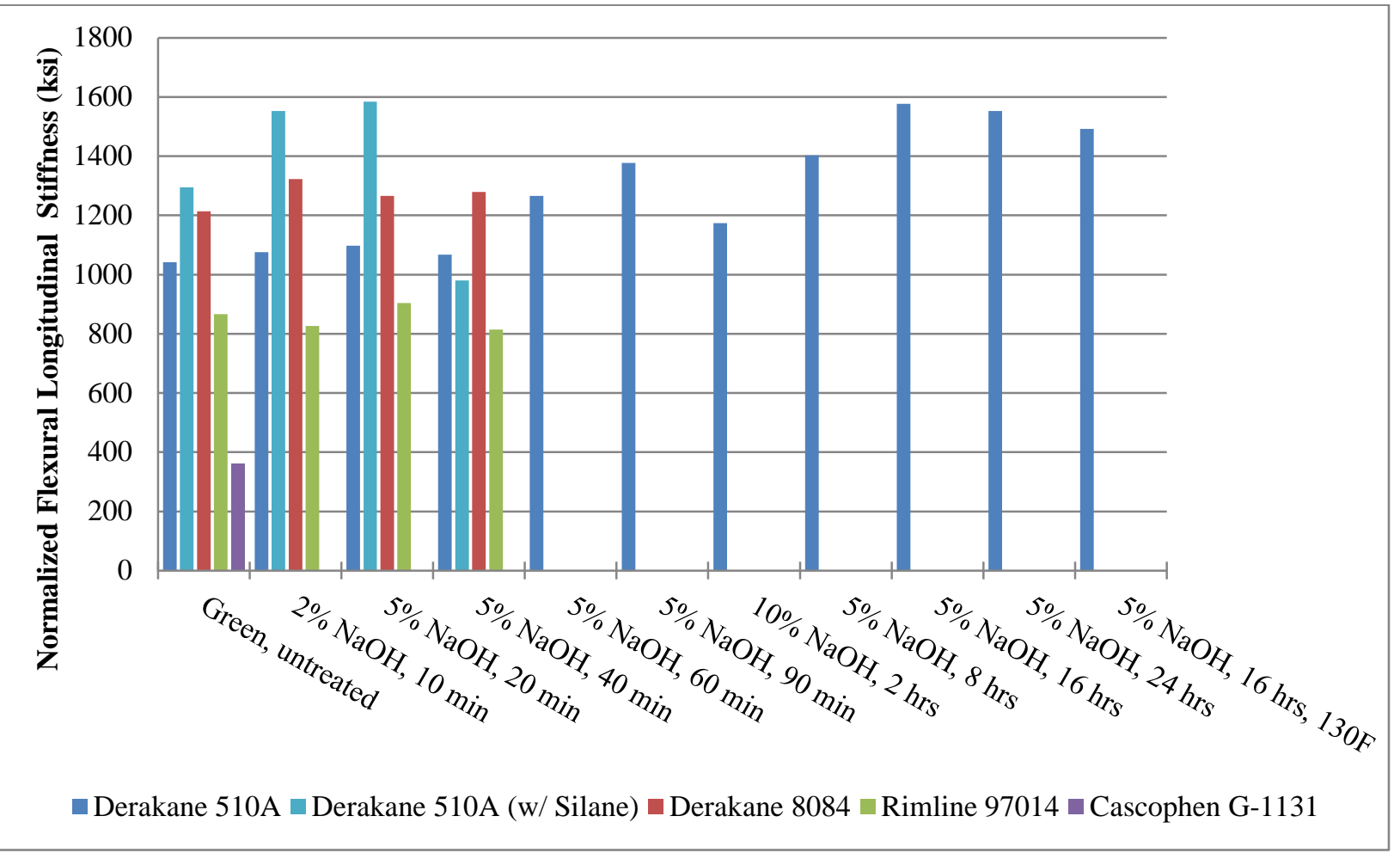

Figure 4-32 - Longitudinal flexural stiffness normalized for $40 \%$ FVF $\left[0_{4} /\right.$ fabric/90 $/$ /fabric $\leftarrow$ load $]$ 


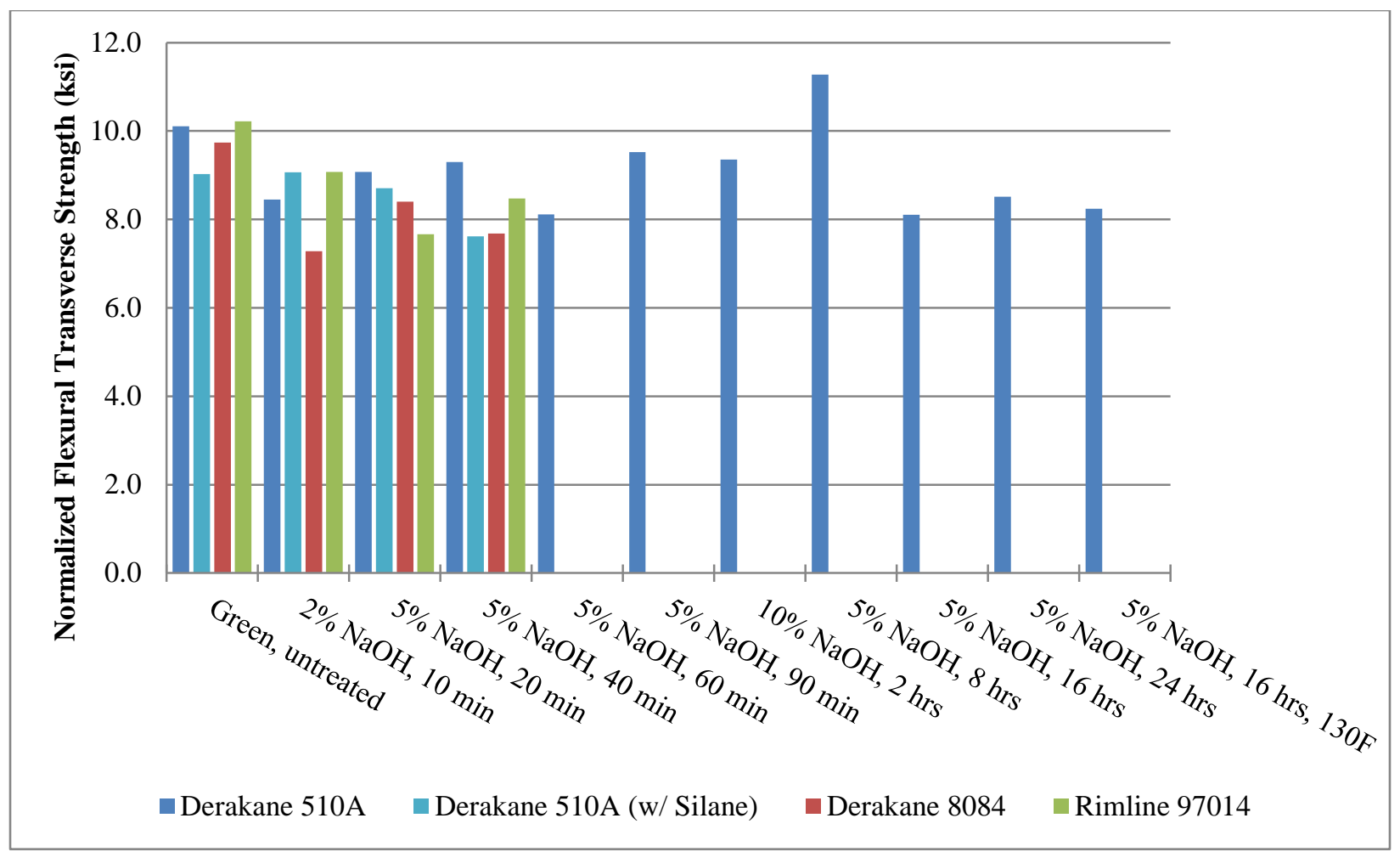

Figure 4-33 - Transverse flexural strength normalized for $40 \%$ FVF $\left[90_{4} / f_{\text {fabric }} / 0_{4} /\right.$ fabric $\left.\leftarrow l o a d\right]$

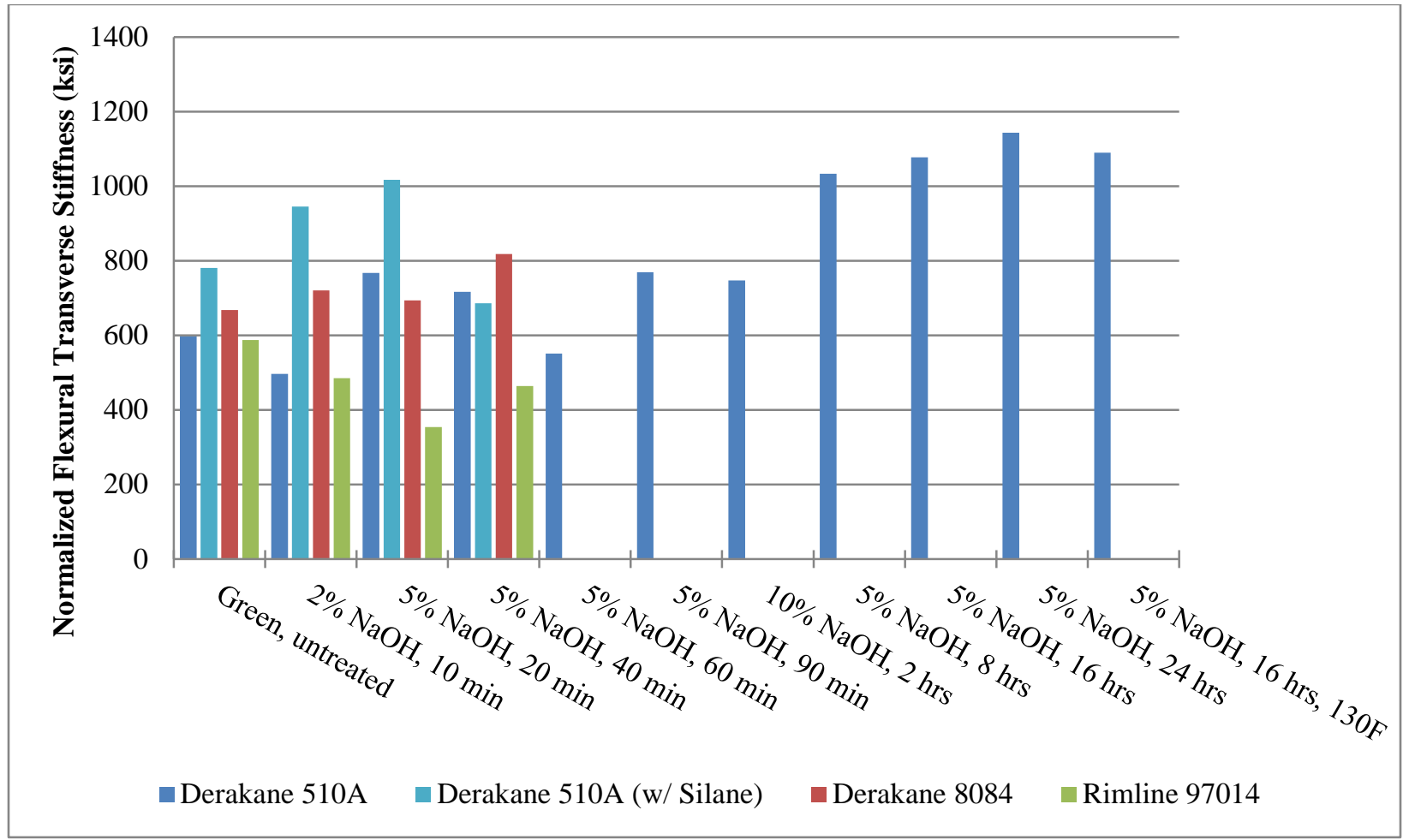

Figure 4-34 - Transverse flexural stiffness normalized for $40 \%$ FVF $\left[90_{4} /\right.$ fabric $/ 0_{4} /$ fabric $\leftarrow$ load $]$ 


\subsubsection{Moisture Absorption Testing}

\subsubsection{Moisture uptake}

On average, all of the moisture absorption coupons absorbed around 50\% of their total moisture uptake after 50 hours and around 95\% of their total uptake after 500 hours, with equilibrium reached around 1000 hours. The first set of samples (different resin types) was immersed for around 3100 hours, the second set (lighter alkali treatments) for 1873 hours, and the last set (harsher alkali treatments) for around 600 hours due to a haste to progress on to the next phase of research. The assumption was that the exposure time did not significantly matter for these durations, but only whether the samples were saturated; this may have been incorrect and contributed to the lower saturation point and better saturated mechanical properties observed from the third set of samples.

Figure 4-35 shows the moisture uptake rate over the first 100 hours of immersion for the composite manufactured with untreated fibers and Derakane 510A, considered the control in the relative absorption rate comparison (over the first 50 hours of exposure) shown in Figure 4-36. The absorption equilibrium for each of the different resin types is shown in Figure 4-37. From these plots, it can be inferred that the moisture absorption rate and equilibrium point are closely, if not directly, related. The vinyl ester composites were vastly superior to the polyurethane composite in terms of moisture absorption, likely due to the presence of microvoids in the polyurethane coupons. Peng et al. (2012) found that natural fiber reinforced polyurethane had a high moisture absorption rate of around 18\% over the first 24 hours and around 36\% over 144 hours, which closely matches the results of this test. However, the vinyl ester composite in this test only had about half the moisture absorption reported from the same source. The Derakane 510A also consistently absorbed less moisture and more slowly than the Derakane 8084. Neither the rate nor the equilibrium point seemed to be significantly affected by alkalization, with the best composite (Derakane 510A resin) absorbing around $10 \%$ of its own weight regardless of the fiber treatment process.

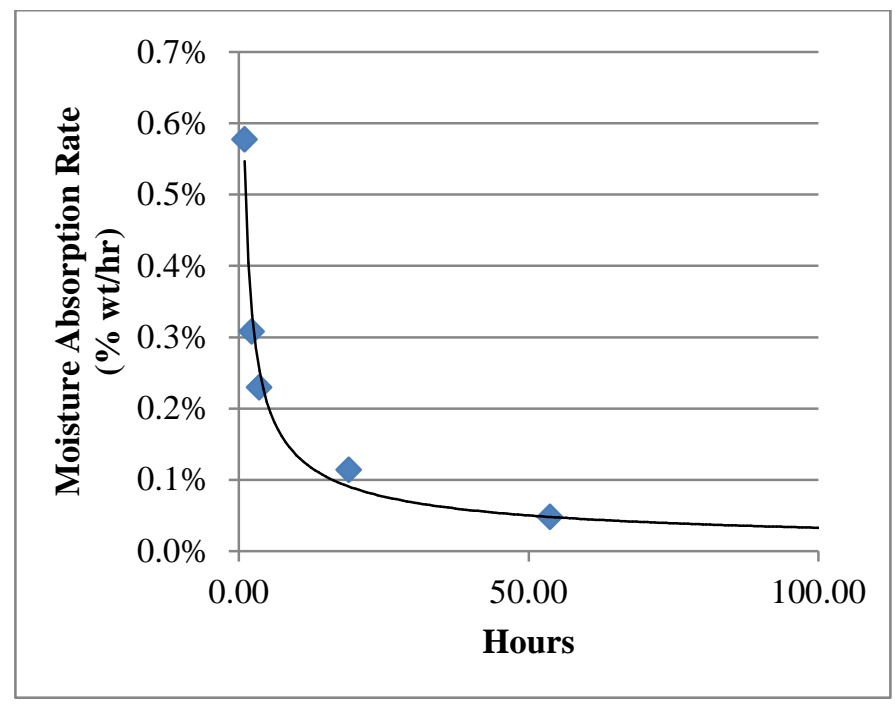

Figure 4-35 - Moisture absorption rate of untreated fiber / Derakane 510A composite 


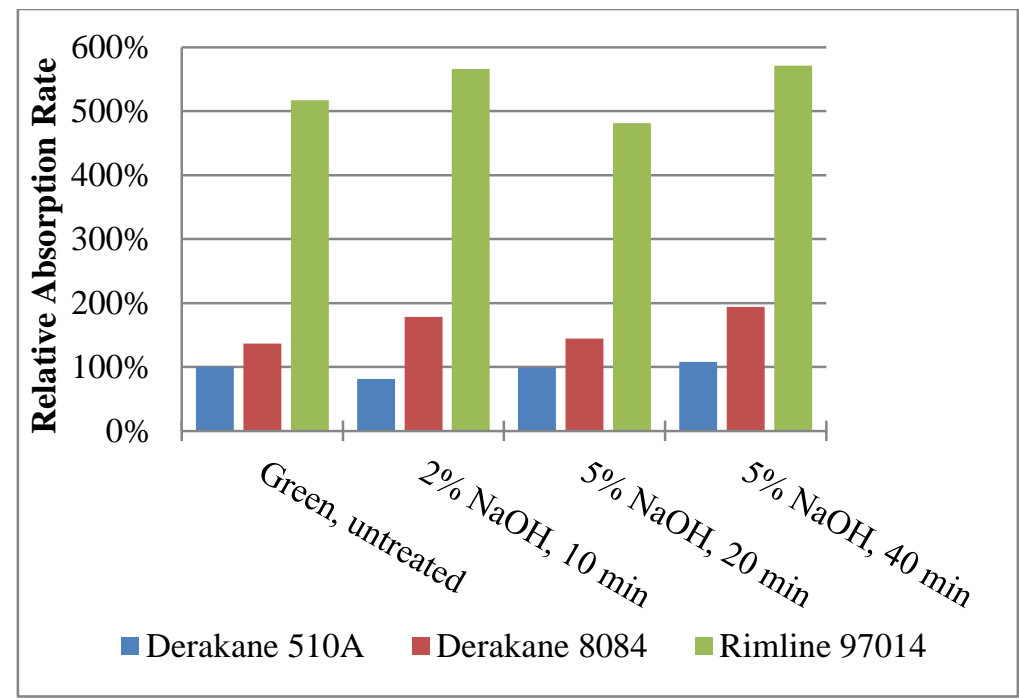

Figure 4-36 - Relative moisture absorption rate over first 50 hours

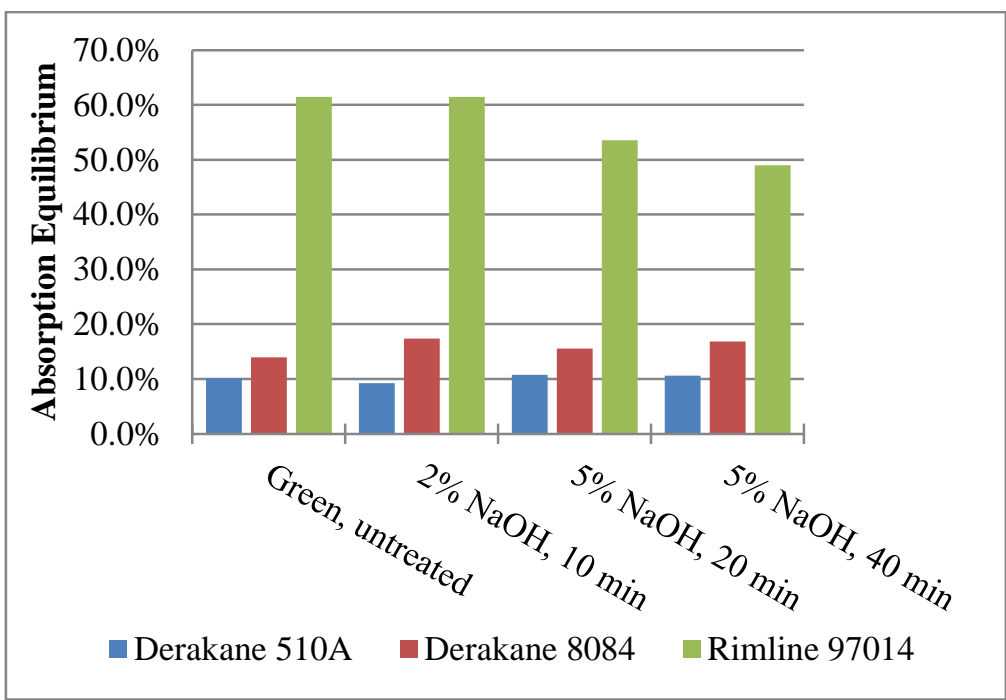

Figure 4-37 - Moisture absorption equilibrium, in \% total weight of composite 


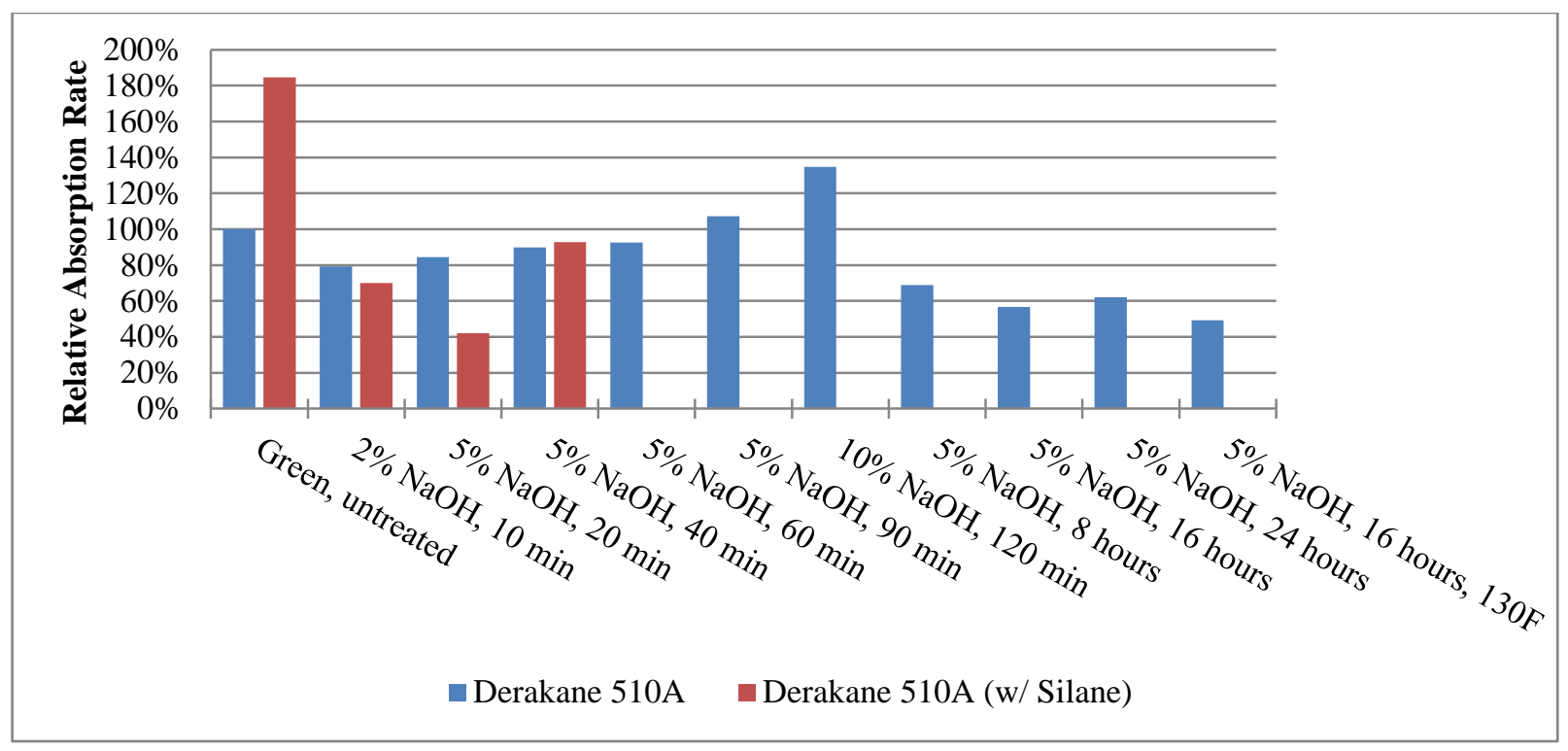

Figure 4-38 - Relative moisture absorption rate over first 50 hours

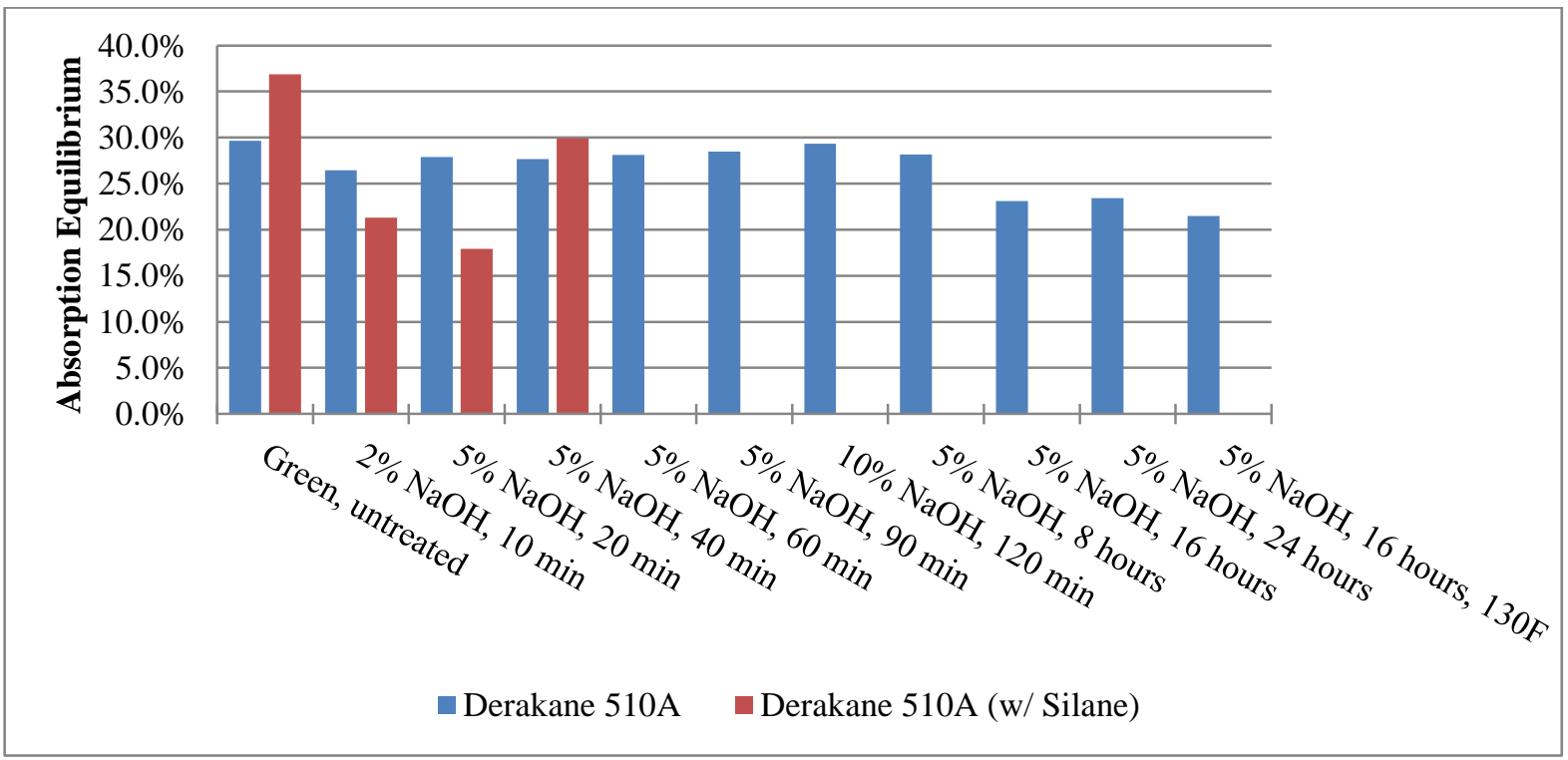

Figure 4-39 - Moisture absorption equilibrium, in \% weight of fiber reinforcement

A closer examination of the Derakane 510A composites included a look at the higher strength alkali treatments and silane treatments. Figure 4-38 again shows the absorption rates relative to the control rate in Figure 4-35.

Figure 4-39 is a plot of the absorption equilibrium points for each type of surface treatment with respect to the fiber weight rather than the total weight, to correct for some variation in FVF. From Figure 4-38 it appears that a light alkali treatment can improve the moisture absorption properties of the composite, particularly when combined with treatment with silanes. This moisture absorption improvement vanishes with the medium alkali treatments and 
then seems to reappear with the harsher alkali treatments. The harsher alkali treated fibers, while nearly saturated, were not exposed to moisture for the same length of time, although that has no effect on the moisture absorption rate. In light of Figure 4-39, though, all of the moisture absorption behavior may have more to do with the differing fiber loadings and less to do with the alkali treatments. The bond quality between the fibers and resin may also contribute to moisture absorption and rate, as a gap at the interface could cause a wicking action that would accelerate absorption.

The presence of silane seems to worsen the moisture absorption properties of the untreated fibers, but when combined with an alkali treatment, can decrease the moisture absorption rate and equilibrium point by as much as $40-50 \%$. The optimum equilibrium point for the composite treated with silanes is still around $5-6 \%$ of the total weight, however, which is still higher than ideal for a structural composite.

\subsubsection{Moisture-induced swell and curvature}

After the samples had reached saturation (or a point estimated to be within around 3\% of saturation), their width, thickness, and transverse deformation were measured in order to assess the swell and curvature of the materials. In every case both the thickness and the width were increased and, due to the unbalanced layup, nearly every sample had unbalanced swelling leading to a midspan deflection towards the fabric face (opposite the longitudinal fibers). The radius of curvature was the method selected to describe this deformation and can be calculated using Equation (4-1) and Equation (4-2), where $L$ is the length of the sample and $\delta$ is the transverse midspan deflection. Equation (4-1) was derived from a calculation for finding the radius of a circle passing through three points, and simplified such that the three points represent the endpoints and the midspan of each sample. While the actual deformed shape of the samples may be better approximated by a parabolic function, the 3-point circle method was an easy way to obtain the curvature from only a few measurements, and is accurate enough for the subsequent analysis.

$$
\begin{gathered}
\text { Radius }=\sqrt{(L / 2)^{2}+\left(\frac{\delta}{2}-\frac{(L / 2)^{2}}{2 \delta}\right)^{2}} \\
\text { Curvature }=\frac{1}{\text { Radius }}
\end{gathered}
$$

The average moisture deformation results for each of the three resin systems, after normalization for $40 \%$ FVF, are shown in Table 4-4. Note that, using the above definition of curvature, a higher value indicates a smaller radius, and thus more deflection. From the results it can be seen that the swelling in both directions was in the order of Polyurethane $>$ Derakane 8084 > Derakane 510A, as would be expected from their relative moisture absorption equilibriums. The additional silane treatment may have resulted in a small reduction in moisture deformation, although this decrease is not very significant and may have been purely the result of the shorter immersion time. The polyurethane composite had the least curvature, although this is 
consistent with the belief that the resin system itself (or rather microvoids within the resin system) was to blame for the high moisture absorption, rather than the fibers. Due to the unbalanced layup, moisture absorption by the fibers would more significantly contribute to curvature, while moisture absorption by the resin system would result in more uniform swelling.

Table 4-4 - Moisture deformation comparison between resin systems

\begin{tabular}{|lrrrr|}
\hline Resin System & Curvature (in ${ }^{-1}$ ) & Width Swell & Thick Swell & Hours \\
\hline Derakane 510A & 0.031 & $3.9 \%$ & $10.7 \%$ & 3100 \\
Derakane 510A w/ Silane & 0.038 & $3.2 \%$ & $7.8 \%$ & 1873 \\
Derakane 8084 & 0.049 & $5.8 \%$ & $15.3 \%$ & 3100 \\
Polyurethane & 0.021 & $6.7 \%$ & $25.8 \%$ & 3100 \\
\hline
\end{tabular}

As previously stated, the different exposure times (see Table 4-5) for the three sets of samples may have led to moisture absorption data skewed in favor of the second two sets of samples (1873 and 600 hours of immersion), despite the assumption that all of the samples were approximately saturated. Table 4-5 has been normalized for 40\% FVF. With the understanding that the shorter immersion times may have inadvertently led to lower moisture deformations, there appears to be a general trend that harsher alkali treatments lead to greater moisture deformations. In general, it appears that an assumption of around 5\% width swell and 10-15\% thickness swell at saturation would be appropriate for these composites. Note that the width swell does not closely match the thickness swell due to the alignment of around $50 \%$ of the fiber content in the width direction from the [0/90] layup, in addition to the two bidirectional fabric layers. These fibers restrict the swelling in the width direction (resulting in a somewhat trapezoidal cross-section), while the swelling in the thickness direction is essentially unconstrained.

Table 4-5 - Moisture deformation comparison between fiber treatments in Derakane 510A resin

\begin{tabular}{|c|c|c|c|c|}
\hline Fiber Treatment & Curvature (in $\left.{ }^{-1}\right)$ & Width Swell & Thick Swell & Hours \\
\hline Green, untreated & 0.020 & $2.7 \%$ & $7.9 \%$ & $3100 / 1873$ \\
\hline $2 \% \mathrm{NaOH}, 10 \mathrm{~min}$ & 0.018 & $3.6 \%$ & $6.8 \%$ & 3100 \\
\hline $5 \% \mathrm{NaOH}, 20 \mathrm{~min}$ & 0.039 & $4.7 \%$ & $12.1 \%$ & 3100 \\
\hline $5 \% \mathrm{NaOH}, 40 \mathrm{~min}$ & 0.057 & $4.6 \%$ & $14.7 \%$ & 3100 \\
\hline $5 \% \mathrm{NaOH}, 60 \mathrm{~min}$ & 0.047 & $3.8 \%$ & $9.7 \%$ & 1873 \\
\hline $5 \% \mathrm{NaOH}, 90 \mathrm{~min}$ & 0.047 & $4.3 \%$ & $7.2 \%$ & 1873 \\
\hline $10 \% \mathrm{NaOH}, 120 \mathrm{~min}$ & 0.075 & $3.9 \%$ & $20.7 \%$ & 1873 \\
\hline 5\% NaOH, 8 hrs & 0.043 & $3.0 \%$ & $10.3 \%$ & 600 \\
\hline 5\% NaOH, 16 hrs & 0.051 & $1.4 \%$ & $9.7 \%$ & 600 \\
\hline 5\% NaOH, 24 hrs & 0.030 & $2.7 \%$ & $9.5 \%$ & 600 \\
\hline 5\% NaOH, 16 hrs, 130F & 0.039 & $2.9 \%$ & $6.9 \%$ & 600 \\
\hline
\end{tabular}




\subsubsection{Saturated composite flexural properties}

After the saturated samples were weighed and measured, they were tested in 3-point bending using the same parameters as the dry samples. The strength and stiffness were evaluated with respect to the original properties, however (discussed further in the next chapter). The resulting average strengths and stiffnesses were divided by the dry properties and are shown (along with their standard deviations) in Table 4-6 for the first set (3100 hours) of moisture absorption data. As expected from an inverse relationship with their relative moisture absorption equilibrium points, generally Derakane 510A > Derakane $8084>$ Polyurethane in terms of strength and stiffness retention (although, in terms of stiffness retention, the two vinyl esters possess essentially the same values). Treatment with silanes did not have any significant effect on the Derakane 510A composite's flexural property retention. At saturation, the Derakane 510A composites were still able to provide around $74 \%$ of the strength and $68 \%$ of the stiffness of the dry composites.

Table 4-6 - Flexural strength/stiffness retention for saturated samples, by resin system

\begin{tabular}{|lcccc|}
\hline Resin System & Avg \% Strength Retention & SD & Avg \% Stiffness Retention & SD \\
\hline Derakane 510A & $74 \%$ & $11 \%$ & $68 \%$ & $10 \%$ \\
Derakane 8084 & $64 \%$ & $8 \%$ & $70 \%$ & $15 \%$ \\
Polyurethane & $50 \%$ & $7 \%$ & $41 \%$ & $3 \%$ \\
\hline
\end{tabular}

The comparisons between flexural strength/stiffness retention values for the different fiber treatment methods (in Derakane 510A resin) are shown in Figure 4-40. From this plot, it appears that the lighter alkali treated composites are better able to retain their stiffness at saturation while the harsher alkali treated composites are better able to retain their strength at saturation (although this may be, again, due to the shorter immersion period for the harsher alkali treated composites).

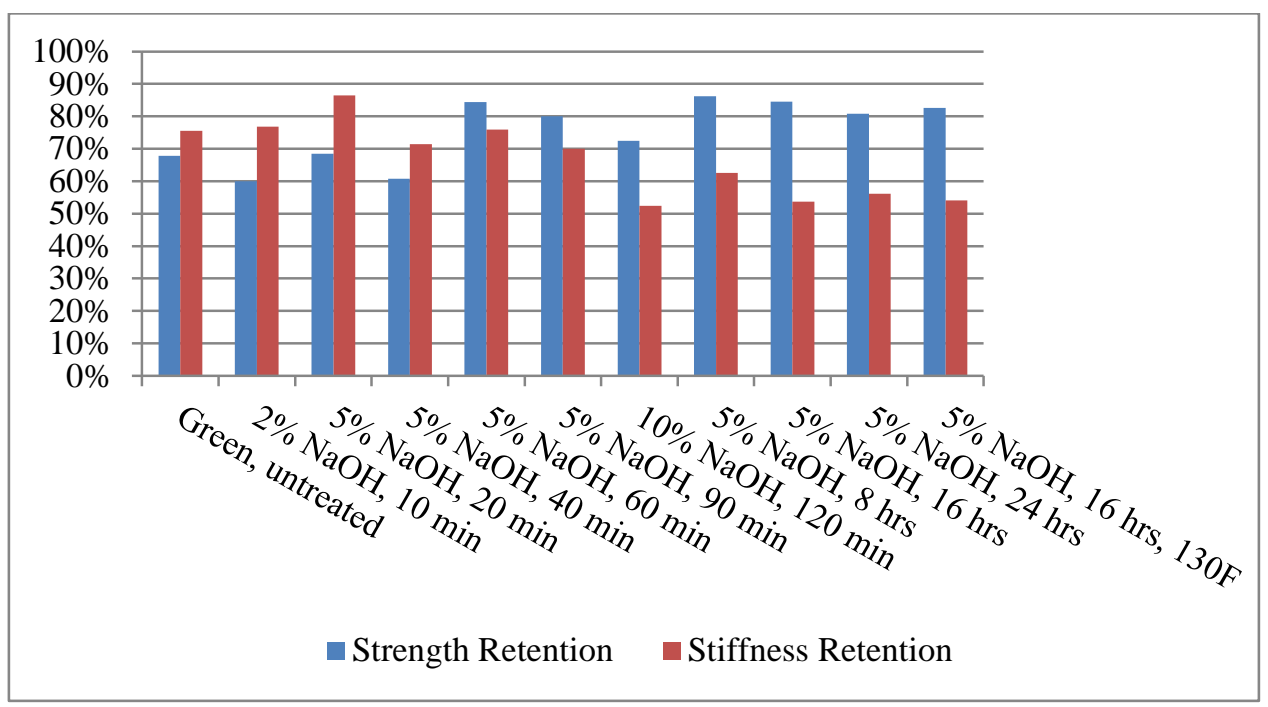

Figure 4-40 - Flexural strength/stiffness retention for saturated Derakane 510A samples 


\subsection{Conclusions}

Of the resin systems tested, both of the vinyl esters outperformed the polyurethane and phenolic resins with regard to flexural properties and moisture absorption. The Derakane 8084 exhibited slightly higher flexural stiffness, but when its higher moisture absorption characteristics are taken into consideration, the Derakane $510 \mathrm{~A}$ is a better choice for a kenaf fiber reinforced composite.

The kenaf fibers obtained from Bangladesh seemed to be of between average and excellent quality when their tensile properties were compared to published results. Light alkalization with $5 \% \mathrm{NaOH}$ for $20-40$ minutes (or for $2 \% \mathrm{NaOH}$ for 10 minutes) resulted in a single fiber tensile strength of around $80 \mathrm{ksi}$ and stiffness of around 10 Msi (after 150\% area adjustment), increases of around 30-50\% over untreated fibers. Meanwhile, alkalization with 5\% $\mathrm{NaOH}$ for 16 hours resulted in a strength of around $100 \mathrm{ksi}$ and 10.5 Msi (after 150\% area adjustment), increases of around 50-70\% over untreated fibers.

The strength of the individual fiber does not directly correspond to the strength of the resulting composite, indicating that alkalization not only affects the fiber but also the interface between the fiber and the matrix. Light alkalization with from 2-5\% $\mathrm{NaOH}$ for 10-40 minutes resulted in a composite flexural strength of around $29 \mathrm{ksi}$ and stiffness of around $1.1 \mathrm{Msi}$, increases of around 5-10\% over composites manufactured with untreated fibers. Alkalization with $5 \% \mathrm{NaOH}$ for 16 hours resulted in a composite flexural strength of around $30 \mathrm{ksi}$ and stiffness of around $1.5 \mathrm{Msi}$, increases of around $12 \%$ and 50\%, respectively. An additional increase in stiffness (up to around 18\%) may be achievable by the use of silanes in stiffnesscritical situations where the added cost can be justified.

The treated fibers seemed to absorb less moisture, although this could at least in part be due to prematurely assuming the fibers had reached their saturation point. With that in mind, the mechanical behavior of the treated fiber composites at saturation is of even more concern - with little or no improvement over the untreated fiber composites, the mechanical behavior of the treated fiber composites would likely become even worse with continued exposure to moisture.

It appears that there are potentially three different surface treatment results - green, light alkali treatment (2-5\% $\mathrm{NaOH}, 10-40 \mathrm{~min}$ ), and strong alkali treatment (5\% $\mathrm{NaOH}$, 8+ hours). The light alkali treatments may only have cleaned the surface of the fibers, removing waxes and oils, without actually changing the physical or chemical structure of the fibers. The fibers treated with $5 \% \mathrm{NaOH}$ for 16 hours do appear to offer a significant advantage over untreated fibers in both single fiber tension tests and dry-condition composite flexural stiffness. SEM results corroborate this conclusion, with a reduced gap visible between the fiber perimeters and the resin matrix. However, the addition of the 16 hour treatment (along with the other treatments of 8 hours or longer) to the testing matrix occurred at a later stage of this research effort; most of the previously completed research work that is covered in the following chapters was planned and executed under the assumption that a treatment of $5 \% \mathrm{NaOH}$ for 40 minutes was the best possible treatment process, and that it was only a marginal improvement over untreated fibers at that. In future work, fiber treatment methods closer to that of the $5 \% \mathrm{NaOH}$ for 16 hours should be further explored and used for the manufacturing of test samples and components. 


\section{MECHANICAL CHARACTERIZATION}

\subsection{Abstract}

A comparison of flexural, tensile, compressive, and shear properties was made between an untreated kenaf fiber reinforced composite, a chemically treated kenaf fiber reinforced composite, a glass fiber reinforced composite, and OSB. The mechanical properties were evaluated for dry samples, samples immersed in water for 50 hours, and samples immersed in water until saturation (2700 hours). In most tests, the OSB mechanical properties were nearly an order of magnitude below the other materials' properties. The only consistent significant difference between the untreated and treated kenaf composites was that the treated kenaf composites tended to experience greater strength and stiffness reductions in the presence of moisture. Both of the kenaf reinforced composites had a tendency to lose stiffness and increase ductility in the presence of moisture. On average, at 40\% FVF, the bidirectional kenaf reinforced composites had a flexural strength and stiffness of $28 \mathrm{ksi}$ and $1.09 \mathrm{Msi}$, respectively, and a shear strength and stiffness of $6 \mathrm{ksi}$ and $0.35 \mathrm{Msi}$, respectively. On average, at $40 \% \mathrm{FVF}$, the unidirectional kenaf reinforced composites had a tensile strength and stiffness of $25 \mathrm{ksi}$ and 3.3 Msi, respectively, and a compressive strength and stiffness of $17 \mathrm{ksi}$ and $3.35 \mathrm{Msi}$, respectively. When these properties are divided by the material density, they are shown to be very comparable to the glass fiber reinforced composite’s specific properties.

\subsection{Introduction and Scope}

Before natural fiber reinforced composites can be used as primary structural members, their mechanical performance must be carefully studied. Since one of the main concerns with using natural fibers in a synthetic resin is the chemical bond between the fibers and the matrix, understanding the mechanical performance in a variety of loading conditions is particularly important. With this potentially poor bonding, the flexural, compressive, tensile, and shear behavior may not follow trends similar to those of glass fiber reinforced composites.

A comparison was made between the mechanical properties of an untreated natural fiber reinforced composite, a composite reinforced with treated fibers (5\% $\mathrm{NaOH}$ for 40 minutes; see previous chapter's conclusions), and a glass fiber reinforced composite. For each composite type, the resin matrix was kept the same and an attempt was made to keep the fiber architecture and FVF constant. OSB is commonly used as the structural skin material on structural insulated panels, one potential application for natural fiber reinforced composites. As a result, OSB coupons were also tested with the intent to highlight the potential increase in mechanical properties and resistance to weathering if natural fiber composites were substituted for OSB.

In addition to an evaluation of the mechanical properties of the composites immediately following manufacture and sample preparation, samples were also tested after immersion in water both after 50 hours and after reaching saturation. One of the main concerns with natural fiber reinforced composites (as compared to glass reinforced composites) is their tendency to absorb moisture and their resulting degradation in mechanical properties. In these tests OSB and 
glass were again tested in addition to the two different natural fiber reinforced composites in order to provide some basis for comparison of results.

\subsection{Materials and Manufacturing Processes}

\subsubsection{Materials}

The loose kenaf fibers discussed in the previous chapter were stitched to a light bidirectional kenaf fabric (at a weight ratio of around 5-to-1 fibers to fabric) in order to create essentially unidirectional kenaf fiber mats.

A roll of unidirectional E-glass fibers loosely stitched (not intended as reinforcement) in the transverse direction was used for the manufacture of the glass reinforced composite samples.

The resin system used for all of the composites in this chapter was Ashland's Derakane 510A vinyl ester, as discussed in the previous chapters. A MEKP catalyst and a Cobalt Naphthenate promoter were added to the resin at concentrations of $0.75 \%$ weight and $0.25 \%$ weight, respectively.

The OSB used was a standard $1 / 4$ inch utility panel purchased from a local hardware store.

As a secondary test to demonstrate the potential use of coatings on natural fiber reinforced composites, three additional flexural samples reinforced with the treated kenaf were painted with a urethane protective coating before undergoing the absorption-to-saturation test. The urethane coating used was Minwax Helmsman Spar Urethane, applied according to the manufacturer's instructions.

\subsubsection{Fiber Treatment}

As discussed at length in the previous chapter, the untreated kenaf fibers were simply cut and combed before stitching. The treated kenaf fibers were immersed in $5 \% \mathrm{NaOH}$ for 40 minutes, rinsed in 5\% acetic acid (white vinegar), rinsed in distilled water, and then oven dried at $160^{\circ} \mathrm{F}$ for 6-8 hours. After drying, the fibers were combed before stitching.

No surface treatments were applied to the glass fibers or OSB panels.

\subsubsection{Sample Preparation}

\subsubsection{Plate manufacturing}

Before manufacturing the fiber reinforced composite, all of the fiber mats were dried in an oven at $160^{\circ} \mathrm{F}$ for 1 hour and sealed in plastic bags. Different layups of kenaf mats were used for the tension/compression samples and the flexure/shear samples, as shown in Figure 5-1. The plates manufactured with glass fibers followed the same basic structure as the kenaf mats, only without the fabric layers. The flexure and shear coupon layup [0/90] was selected because it will more closely approximate the layup used for the full-size panels. The tension and compression coupon layup [0/0] was selected in order to more accurately measure the tensile and compressive properties of a single unidirectional lamina for modeling purposes.

The 10 inch by 15 inch kenaf and glass reinforced composite plates were all manufactured by heated compression molding. Technick Products Techlube 25 was used as the mold release agent and all of the steel mold parts were covered with aluminum foil to ease the 
removal of the plate from the mold. The top plate was free floating within the inside edge of the side bars in order to provide the best possible compression, albeit at the expense of consistent plate thickness. The resin was applied to each of the fiber mats by hand and spread evenly with a paintbrush. Once installed in the compression molding device, the composite mat was compressed to around 40 psi and raised to $150^{\circ} \mathrm{F}$ for 2 hours. After 2 hours, the heating elements were switched off and pressure was maintained for 24 additional hours.

Due to the structure of the glass fibers (tightly packed bundles of unidirectional fibers loosely stitched from one to another) and the decision to use a mold which applied constant pressure rather than a constant thickness, more of the resin was squeezed out of the mold during the manufacture of the glass fiber reinforced composites. This led to glass fiber volume fractions more on the order of $50-55 \%$ (and thinner plates) instead of the $35-40 \%$ achieved with kenaf fibers using the exact same fiber volumes and molding parameters. Because of this inconsistency, nearly all of the mechanical results in this chapter are divided by their respective fiber volume fraction, as determined by comparing the weight of the included fiber/fabric with the weight of the manufactured composite, and then multiplied by $40 \%$ (an average VF) in order to normalize the results for a better comparison of properties. A detailed listing of the pre- and post-normalization results is included in Appendix A.
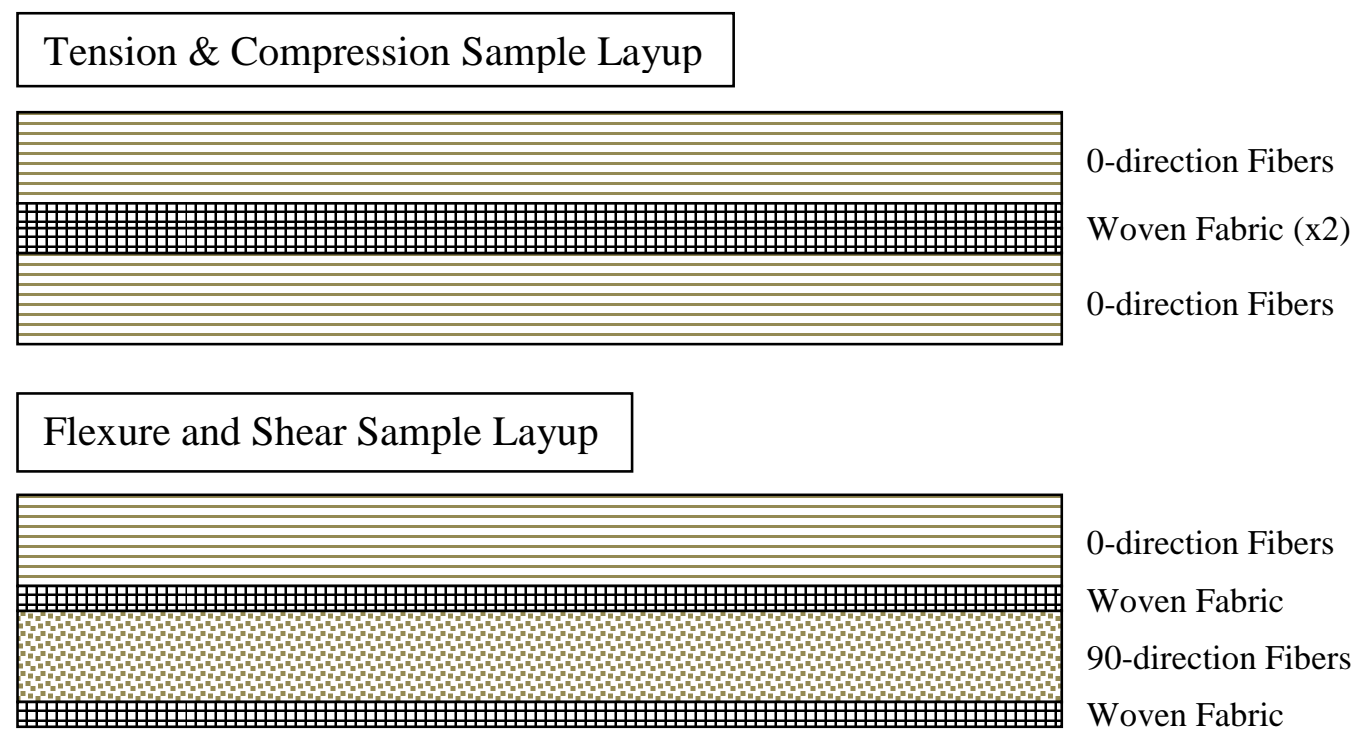

Figure 5-1 - Layups for natural fiber reinforced composite mechanical test samples

\subsubsection{Coupon preparation}

Each of the test methods studied (tension, flexure, compression, shear) required coupon samples of different dimensions (see Figure 5-2 and Figure 5-3). For nearly every test material (glass, OSB, untreated kenaf, treated kenaf) at each test condition (dry, 50 hours, saturated) and test type (flexure, tension, shear, $2 \mathrm{X}$ compression), three samples were tested and the results were averaged. All of the coupons were cut from larger plates using a table saw. A carbide blade was used to cut the OSB and natural fiber reinforced samples and a diamond-tipped blade was 
used to cut the glass reinforced samples. Unless otherwise noted, all of the samples were cut, stored, and tested at room temperature $\left(65-80^{\circ} \mathrm{F}\right)$ and typical indoor humidity $(50-60 \% \mathrm{RH})$.

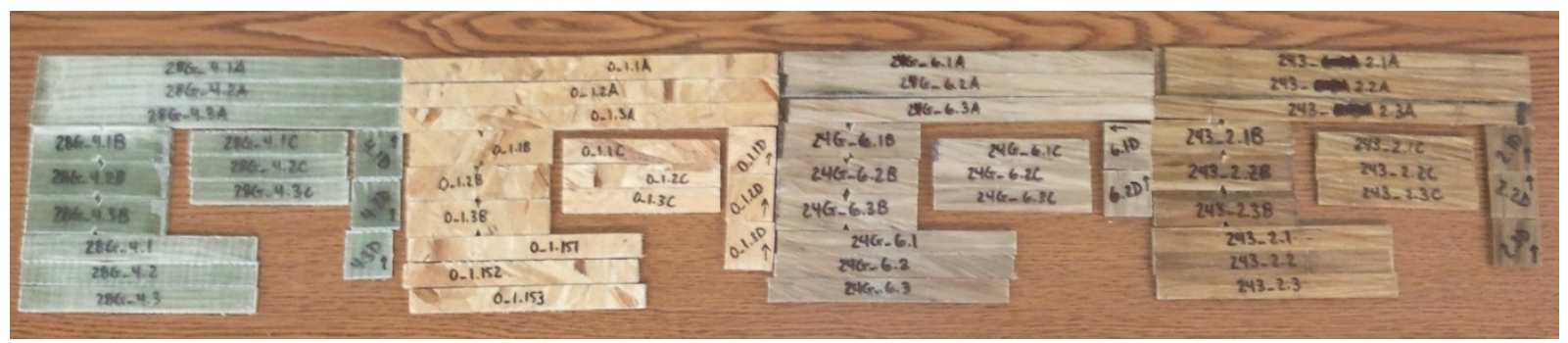

Figure 5-2 - Prepared coupons for mechanical testing (from left, GFRP, OSB, untreated NFRP, and treated NFRP)

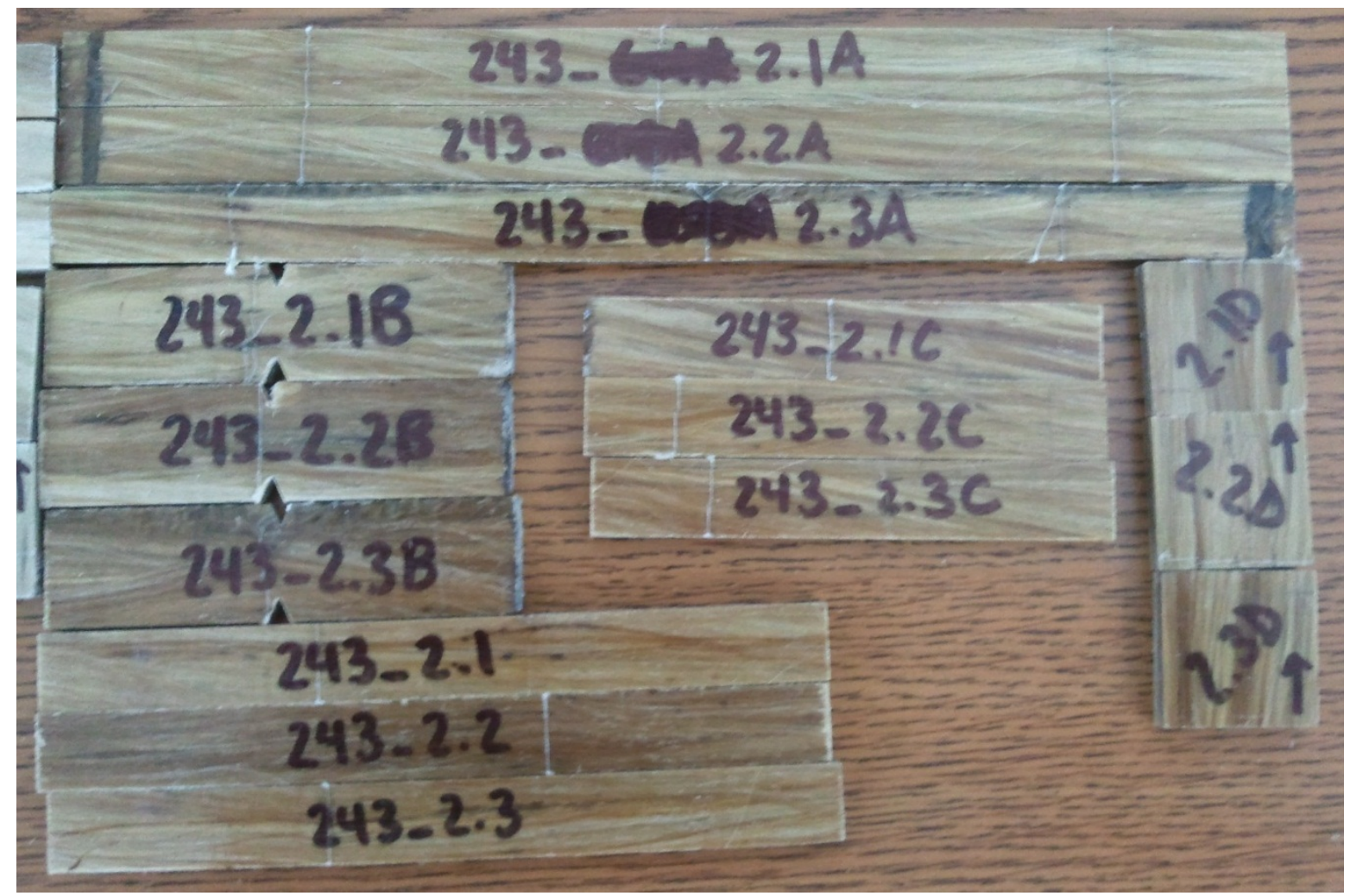

Figure 5-3 -Treated natural fiber reinforced composite coupons for mechanical testing (from top, left to right: tensile, shear, D695 compression, D3410 compression, and flexure)

The coupons for tensile testing (those whose ID numbers end with an 'A' in Figure 5-3) were cut according to the specimen geometry requirements in ASTM D 3039 - Standard Test Method for Tensile Properties of Polymer Matrix Composite Materials. In general, the dimensions used were 8 inches long by 0.5 inches wide by the thickness (between 0.12-0.27 inches). For all of the glass reinforced samples and some of the natural fiber reinforced samples, 2 inch steel tabs were affixed by Ashland's Pliogrip structural adhesive; once it was discovered 
that the natural fiber reinforced samples did not need end tabs in order to get satisfactory failure modes, their use was discarded. Vishay Micro-Measurements' general purpose strain gages (120 ohm, $1 / 4$ inch gage length, linear pattern) were applied in the longitudinal direction at the midspan for all of the samples except for the OSB post-moisture absorption test samples.

The coupons for flexural testing (those whose ID numbers do not end with a letter in Figure 5-3) were cut according to ASTM D 790 - Standard Test Methods for Flexural Properties of Unreinforced and Reinforced Plastics and Electrical Insulating Materials. In general, the dimensions used were 5 inches long by 0.5 inches wide by the thickness (between 0.11-0.30 inches). No strain gages are required for flexural modulus calculations.

The coupons for shear testing (those whose ID numbers end with a 'B' in Figure 5-3) were cut according to the specimen geometry requirements in ASTM D 5379 - Standard Test Method for Shear Properties of Composite Materials by the V-notched Beam Method. In general, the dimensions used were 3.0 inches long by 0.75 inches wide by the thickness $(0.11-0.28$ inches). The notches were cut with a $90^{\circ}$ mill cutting bit so that the notched width was around 0.45 inches. Vishay Micro-Measurements’ general purpose strain gages (350 ohm, 0.187 inch gage length, shear/torque pattern) were applied in the longitudinal direction at the midspan of the 0-direction face (perpendicular to shear loading) for all of the samples except for the OSB postmoisture absorption test samples, which had no gages. The current composite manufacturing method currently produces samples suitable only for the determination of in-plane shear properties using this test method, not interlaminar shear properties.

The coupons for the compressive testing were cut according to the same parameters used by Blandford (2010). This includes a modification of the geometry requirements of ASTM D 695 - Standard Test Method for Compressive Properties of Rigid Plastics as proposed in SACMA SRM 1R-94, wherein rectangular samples are substituted for dog-bone samples. The resulting sample geometry used was 3.3125 inches long by 0.5 inches wide by the thickness (0.11-0.23 inches). A second set of compressive tests was also run using the geometry specifications selected by Blandford (2010), loosely based on the requirements of ASTM D 3410 - Standard Test Method for Compressive Properties of Polymer Matrix Composite Materials with Unsupported Gage Section by Shear Loading. The modified sample geometry used was 1.0 inch long by 1.0 inch wide by the thickness (0.12-0.26 inches). Inaccessibility to compressive clamps dictated this modification, as the additional length of the samples for the use of tabs was ignored. The first compressive test method was recommended by Blandford (2010) as a better method of obtaining modulus of elasticity results, while the second test method was shown to provide good ultimate failure stress values. Vishay Micro-Measurements' general purpose strain gages (120 ohm, $1 / 4$ inch gage length, linear pattern) were applied in the longitudinal direction at the midspan of the gage area for all of the samples except for the OSB post-moisture absorption test samples.

\subsection{Experimental Methodology}

\subsubsection{Moisture Absorption Testing}

Each sample was weighed to the nearest $0.0001 \mathrm{~g}$ before being immersed in room temperature water. Periodically over the course of testing (four times over 50 hours; fourteen times over 2700 hours), each sample was removed from immersion, patted dry with a paper 
towel, and reweighed. Saturation was determined to be the point at which the moisture absorption rate for all of the samples was less than $0.005 \%$ by weight per day. After reaching saturation, the samples were not only reweighed, but also remeasured to evaluate the swelling and curvature effects of moisture absorption.

The OSB samples that were exposed to moisture absorption held too much moisture to allow for the application of strain gages, and therefore their stiffness properties were only evaluated in the dry state.

\subsubsection{Flexural Testing}

Due to the relatively low loading used for flexural testing ( $150 \mathrm{lbs}$ ), several calibration confirmation tests were run on the $90 \mathrm{kN}$ (20 kip) Instron comparing the load results to an external $9 \mathrm{kN}$ (2 kip) load cell and the position results to caliper measurements. The position results are plotted in Figure 5-4 and the load results are plotted in Figure 5-5. For the load and position ranges used in all of the test methods, the load and position measurements should each be correct to within $2 \%$ error.

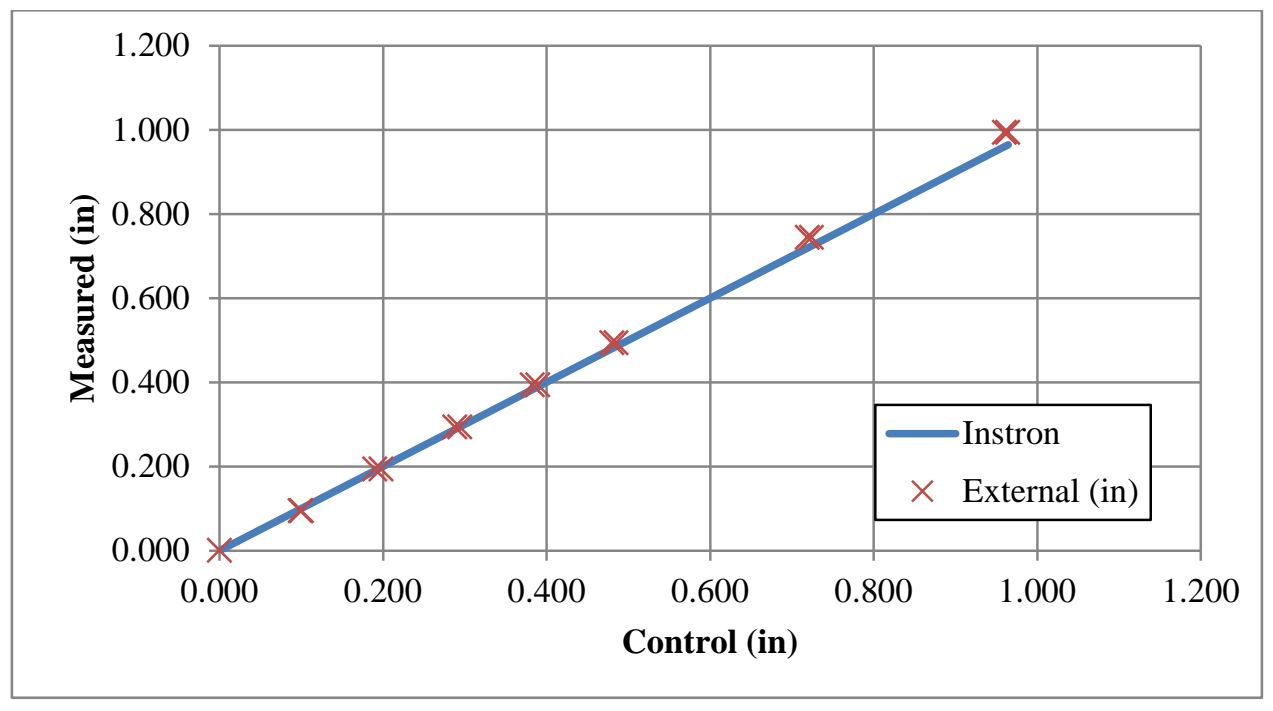

Figure 5-4 - Instron position verification 


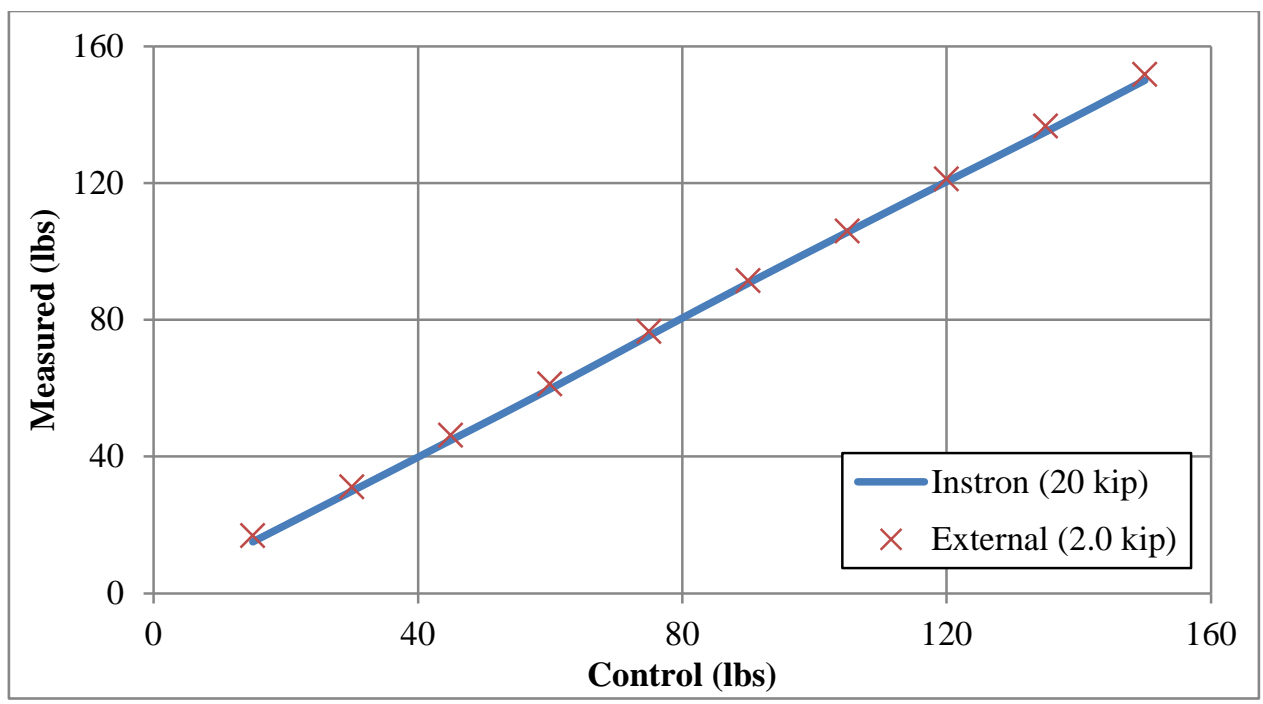

Figure 5-5 - Instron load verification

Flexural testing was conducted based on the guidelines of ASTM D790. The three point bending fixture had a rounded loading nose and supports with an adjustable support span (see Figure 5-6). Tests were conducted at various spans depending on the thickness of the material to be tested, roughly following the guidelines in the standard of a support span of 16 (tolerance \pm 1 ) times the depth of the beam. For most of the tests, this meant that the support span for the glass reinforced samples was 2.25 inches, 3.30 inches for the treated kenaf reinforced samples, and 4.0 inches (limited by coupon length) for the untreated kenaf reinforced and OSB samples. An approximated crosshead rate $(R)$ of 0.08 inches/min was obtained based off of the standard's equation (5-1), where $Z$ is $0.01, L$ is the support span, and $d$ is the depth of the beam.

$$
R=Z L^{2} / 6 d
$$

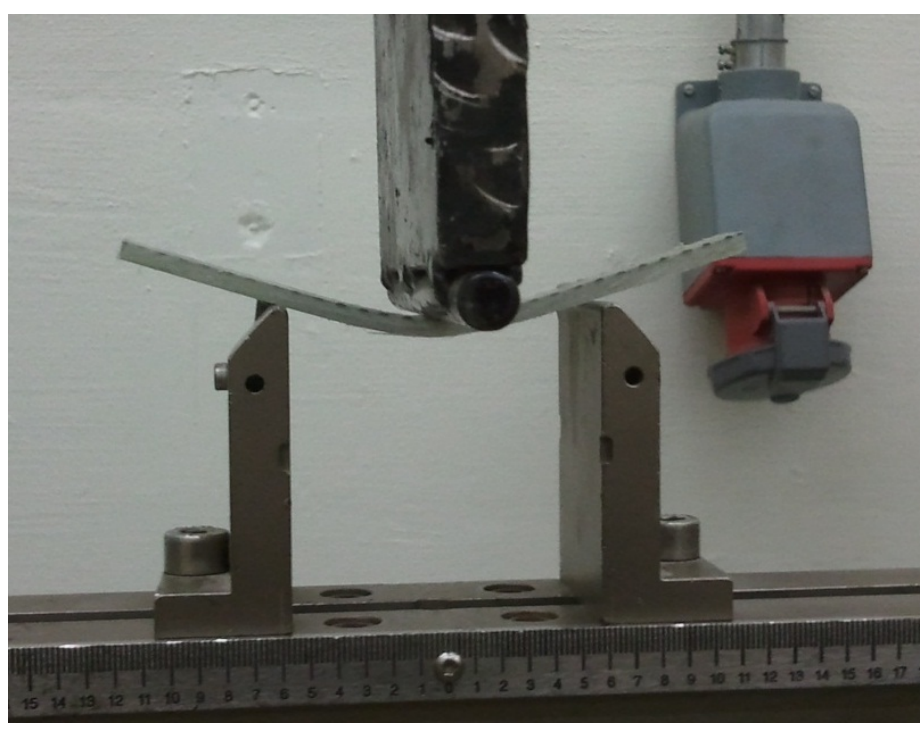

Figure 5-6 - ASTM D790 flexural test 
Specimens were tested until the occurrence of an abrupt loss in load resistance, after which the load failed to continue to increase. The flexural stress $\left(\sigma_{f}\right)$ was calculated according to ASTM D790's equation (5-2), where $P$ is the maximum load before failure, $L$ is the support span, $b$ is the width of the beam, and $d$ is the depth of the beam. The tangent modulus of elasticity $\left(E_{B}\right)$ was calculated according to the standard's equation (5-3), where $m$ is the slope of the tangent to the steepest initial straight line portion of the load-deflection curve.

$$
\begin{gathered}
\sigma_{f}=3 P L / 2 b d^{2} \\
E_{B}=L^{3} m / 4 b d^{3}
\end{gathered}
$$

The slope of the tangent to the steepest initial straight line portion of the load deflection curve is a poorly constrained value, particularly when dealing with materials that fail at relatively low loads and that exhibit non-linear behavior fairly early in the testing process, such as with natural fiber composites. The variation between arbitrary visual methods and algorithm-based computer methods was observed to result in errors as high as $15 \%$ when obtaining these slopes. For consistency, a computer method was used for the analysis of all flexural samples where $m$ was obtained as the maximum slope of linear regressions across any set number of data points (usually around 100, depending on the total number of data points in the test) above a lower threshold (usually around $4 \mathrm{lbs}$, to avoid the initial data scatter).

\subsubsection{Tensile Testing}

Tensile testing was conducted based on the guidelines of ASTM D3039. Wedge-style grips were used to hold each end of a sample (see Figure 5-7). The overall sample length was shorter than the standard recommended length (8 inches instead of 10 inches), but within the minimum length requirements (gripping $+2 \mathrm{x}$ width + gage length). Samples were tested at a crosshead rate of $0.05 \mathrm{in} / \mathrm{min}$. Specimens were tested until the occurrence of an abrupt loss in load resistance, after which the load failed to continue to increase. 


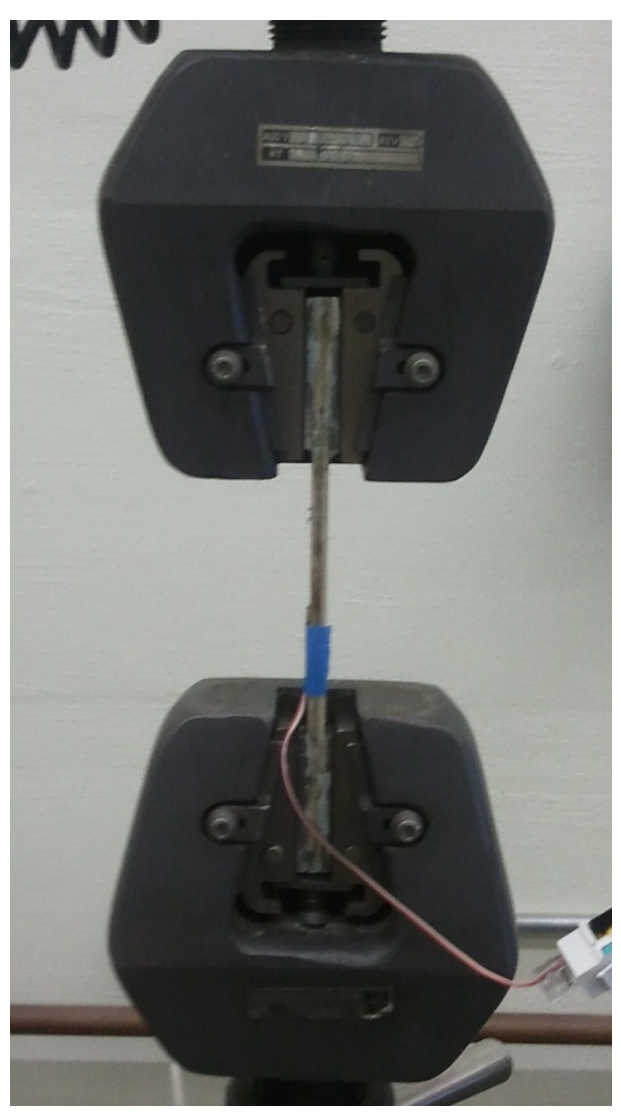

Figure 5-7 - ASTM D3039 tensile test

The ultimate tensile strength $\left(F^{t u}\right)$ was calculated according to the standard's equation (5-4), where $P^{\max }$ is the maximum force before failure and $A$ is the average cross-sectional area. When possible, the tensile chord modulus of elasticity $\left(E^{\text {chord }}\right)$ was calculated according to the standard's equation (5-5), where $\Delta \sigma$ is the difference between the applied tensile stresses at 1000 and $3000 \mu \varepsilon$, and $\Delta \varepsilon$ is 0.002 . In situations where one of the stresses at the desired strains was not available, the nearest value to the desired strain was selected and $\Delta \varepsilon$ increased or reduced accordingly.

$$
\begin{gathered}
F^{t u}=P^{\max } / A \\
E^{\text {chord }}=\Delta \sigma / \Delta \varepsilon
\end{gathered}
$$

\subsubsection{Compressive Testing}

Two separate types of compressive testing were used to evaluate the compressive properties of the materials: one based on the guidelines of ASTM D695 and one based on the guidelines of ASTM D3410.

For the test based on ASTM D695, samples were affixed in a support jig to prevent weak-axis buckling and then placed between two compressive plates (see Figure 5-8). Within the support jig, the span of the intended failure location is 1.5 inches, although typically some samples still fail due to brooming at the top, unsupported end. Samples were tested at a 
crosshead rate of $0.05 \mathrm{in} / \mathrm{min}$. Specimens were tested until the occurrence of an abrupt loss in load resistance, after which the load failed to continue to increase. The compressive strength was calculated as the maximum compressive load divided by the original cross-sectional area. The modulus of elasticity was calculated as the slope of the line tangent to the initial linear portion of the load-strain curve divided by the cross-sectional area.

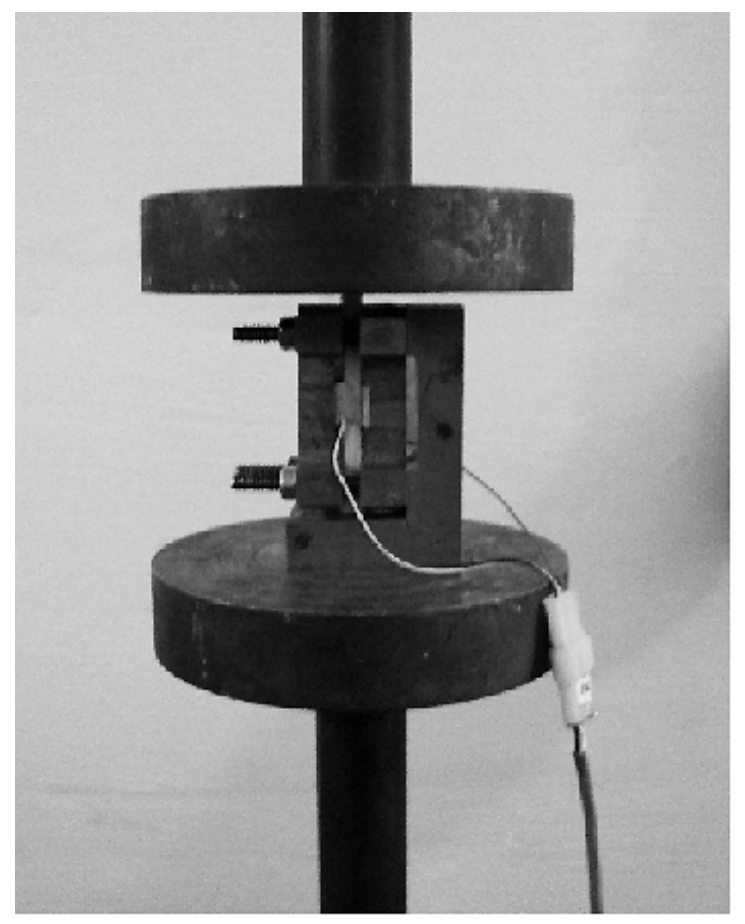

Figure 5-8 - ASTM D695 compressive test with support jig

For the test based on ASTM D3410, samples were stood freely on one end between two compression plates or held loosely upright by a small C-clamp, if needed (see Figure 5-9). Samples were tested at a crosshead rate of $0.05 \mathrm{in} / \mathrm{min}$. Specimens were tested until the occurrence of an abrupt loss in load resistance, after which the load failed to continue to increase. The ultimate compressive strength $\left(F^{c u}\right)$ was calculated according to the standard's equation (5-6), where $P^{\max }$ is the maximum force before failure and $A$ is the cross-sectional area. When possible, the compressive chord modulus of elasticity $\left(E^{\text {chord }}\right)$ was calculated according to the standard's equation (5-7), where $\Delta \sigma$ is the difference between the applied compressive stresses at 1000 and $3000 \mu \varepsilon$, and $\Delta \varepsilon$ is 0.002 . In situations where one of the stresses at the desired strains was not available, the nearest value to the desired strain was selected and $\Delta \varepsilon$ increased or reduced accordingly.

$$
\begin{gathered}
F^{c u}=P^{\max } / A \\
E^{\text {chord }}=\Delta \sigma / \Delta \varepsilon
\end{gathered}
$$




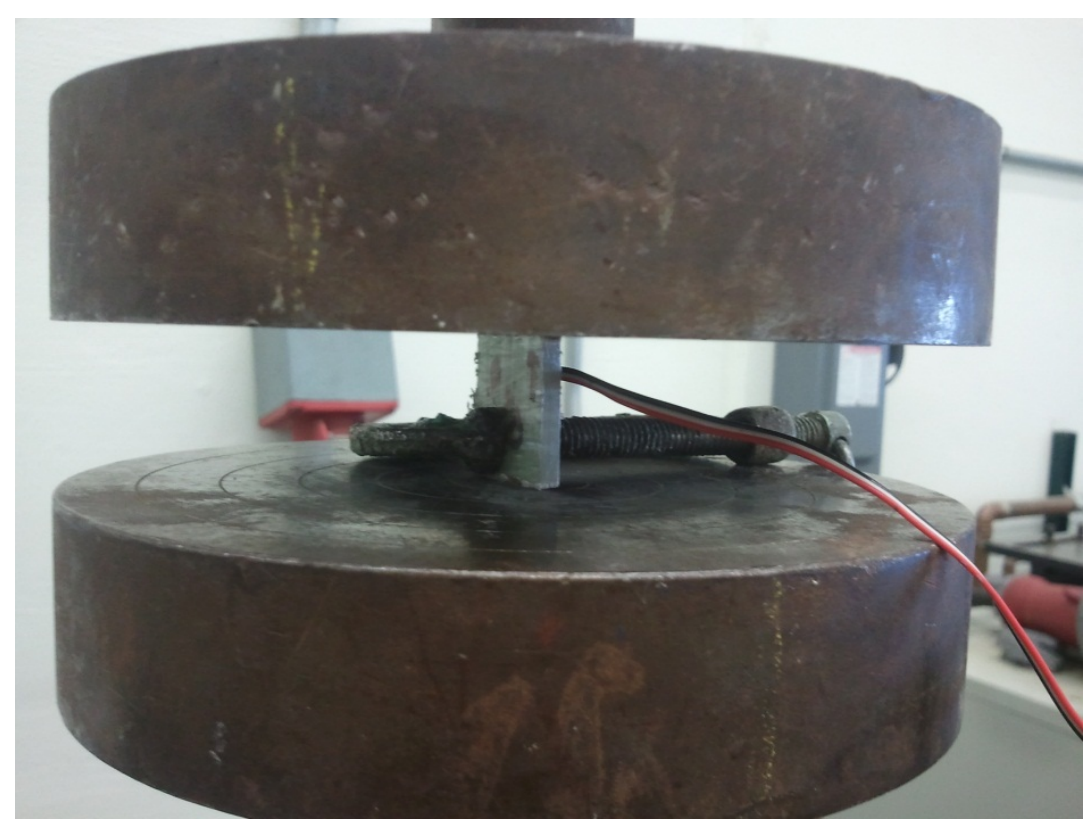

Figure 5-9 - ASTM D3410 compressive test (C-clamp only to keep sample from falling over during installation)

\subsubsection{Shear Testing}

Shear testing was conducted based on the guidelines of ASTM D5379. An Iosipescu (or V-notched beam) test fixture was used to apply in-plane shear loading to the [0/90] samples (see Figure 5-10). After samples were installed in the fixture, the adjustment wedge screws were only tightened by hand to avoid excessive preloading. Samples were tested at a crosshead rate of 0.05 $\mathrm{in} / \mathrm{min}$. Specimens were tested until the occurrence of an abrupt loss in load resistance, after which the load failed to continue to increase.

The strain gages used for shear testing collected the sum of the strains in both the $+45^{\circ}$ and $-45^{\circ}$ directions, directly recording the shear strain $(\gamma)$. The ultimate shear strain $\left(\gamma^{a}\right)$ was calculated according to the standard's equation (5-8). The ultimate shear strength $\left(F^{u}\right)$ was calculated according to the standard's equation (5-9), where $P^{u}$ is the lower of the ultimate load or the load at $5 \%$ shear strain, and $A$ is the cross-sectional area. The shear chord modulus of elasticity ( $G^{\text {chord }}$ ) was calculated according to the standard's equation (5-10), where $\Delta \tau$ is the difference in shear stress between a data point at or near $2000 \mu \varepsilon$ and a data point at or near 6000 $\mu \varepsilon$, and $\Delta \varepsilon$ is the difference between the two strain points (generally very near to 0.004 ).

$$
\begin{gathered}
\gamma^{a}=\min \left\{\begin{array}{l}
5 \%(\text { or } 50,000 \mu \varepsilon) \\
\gamma \text { at ultimate load }
\end{array}\right. \\
F^{u}=P^{u} / A \\
G^{\text {chord }}=\Delta \tau / \Delta \gamma
\end{gathered}
$$




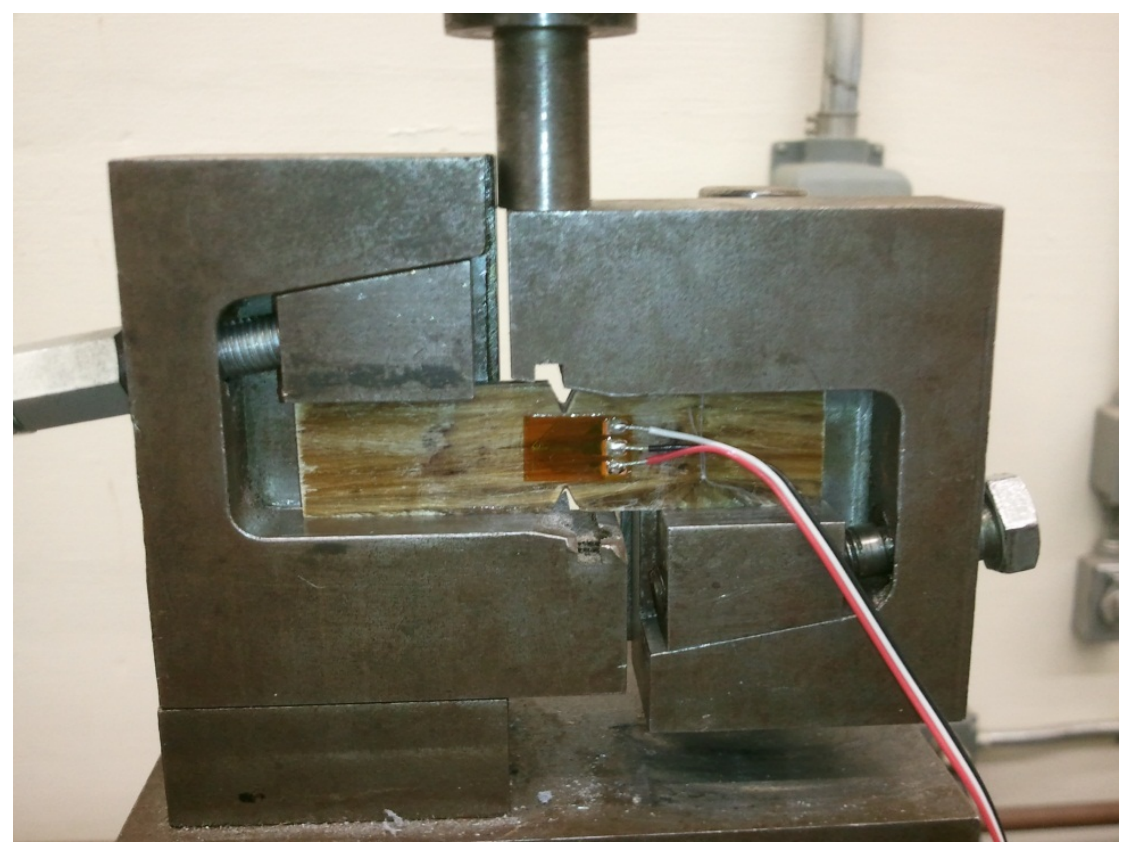

Figure 5-10 - Iosipescu / V-notch shear test (ASTM D5379)

\subsection{Results and Discussion}

\subsubsection{Moisture Absorption Testing}

\subsubsection{Moisture uptake}

The moisture absorption rate was monitored by individually weighing the surface-dried samples four times for the samples immersed for 50 hours and fourteen times for the samples immersed for 2700 hours (until saturation). The average percent weight of moisture absorption for each sample at 50 and 2700 hours is shown in Table 5-1.

As expected, the OSB absorbed far more water weight than any of the fiber-reinforced composites, and at a much faster rate. The glass reinforced composites had a negligible weight gain which, since the fibers themselves absorb practically no moisture, is likely due to a combination of resin moisture absorption and filling of microvoids. None of the natural fiber reinforced composites exhibited particularly good moisture absorption resistance at saturation, as they all absorbed in excess of $10 \%$ of their own weight in water. From the 50 hour data, as well as the linear (Figure 5-11) and logarithmic (Figure 5-12) plots of weight gain due to moisture absorption, it would appear that the untreated kenaf has a reduced moisture absorption rate when compared to the treated kenaf composite samples. However, this may have been due to the slightly lower volume fraction and corresponding better resin encapsulation of the fibers in the untreated fibers. While a correction was made to normalize for volume fraction, that correction does not necessarily include the encapsulation effects. Regardless of the moisture absorption rates and the reasons for their slower uptake, the untreated kenaf samples still ended up absorbing around 2.5\% more moisture by weight, a $21 \%$ increase over the saturated moisture content of the treated kenaf samples. From these results it can be concluded that the treatment 
process has little to no effect on the moisture absorption rate, but can reduce the amount of moisture absorbed at saturation by a significant amount. This reduction was of greater significance than the reduction observed in previous tests.

Table 5-1 - Mechanical testing samples, \% wt. moisture absorption at 50 hours and at saturation

\begin{tabular}{|lrr|}
\hline \multicolumn{1}{|c}{ Sample Type } & $\mathbf{5 0}$ hours & $\mathbf{2 7 0 0}$ hours \\
\hline Untreated Kenaf & $4.44 \%$ & $13.93 \%$ \\
Treated Kenaf & $6.53 \%$ & $11.56 \%$ \\
Treated/Coated Kenaf & $1.91 \%$ & $11.79 \%$ \\
Glass & $0.05 \%$ & $0.11 \%$ \\
OSB & $79.80 \%$ & $130.85 \%$ \\
\hline
\end{tabular}

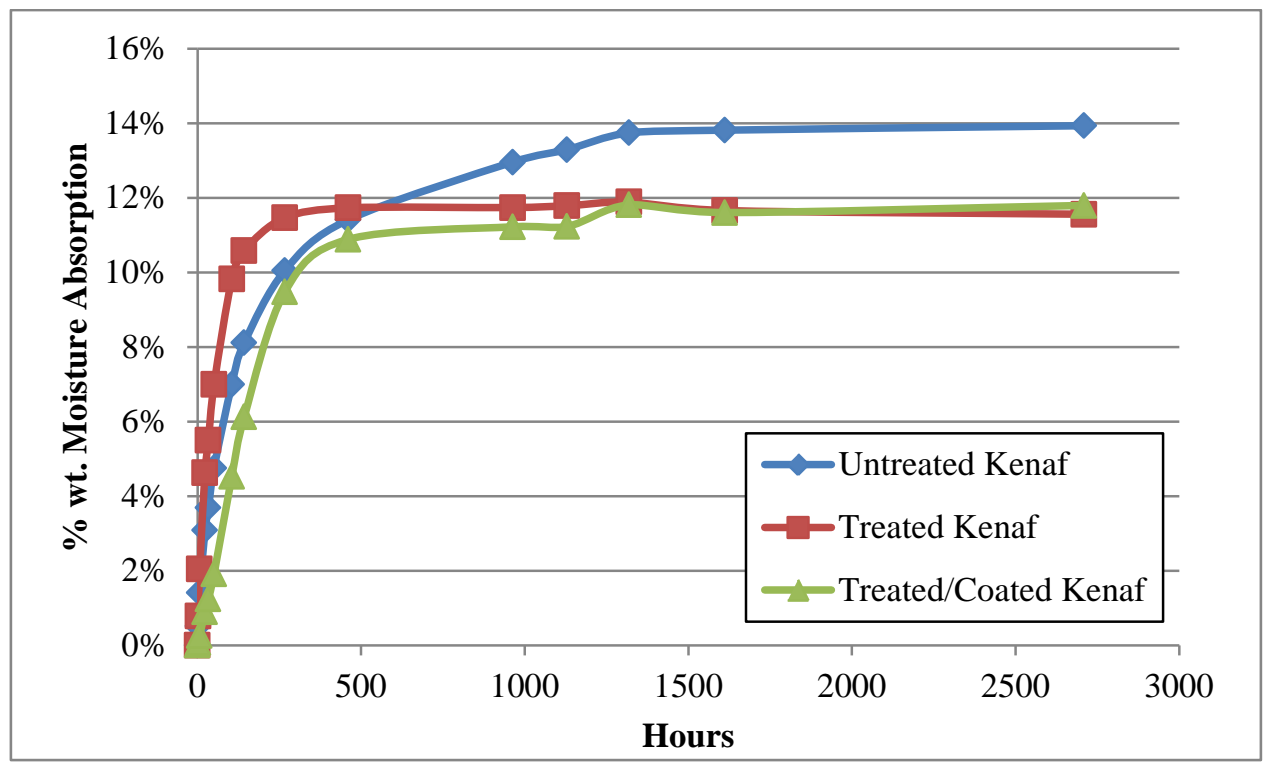

Figure 5-11 - Plot of moisture absorption rates for untreated and treated kenaf fiber reinforced composites, PU coated composites 


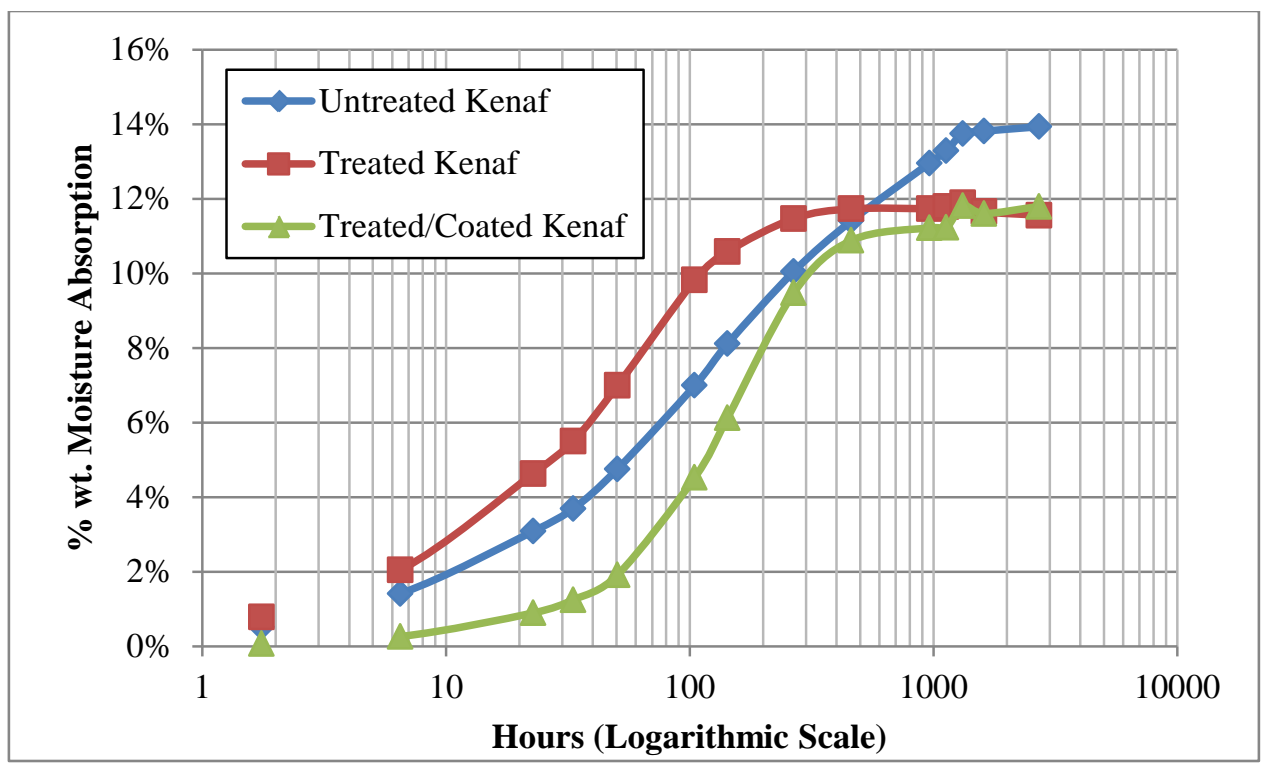

Figure 5-12 - Logarithmic plot of moisture absorption rates from Figure 5-11

Perhaps the most enlightening result of examining the moisture absorption rates and saturation points was the performance of the kenaf sample coated with the urethane weatherresistant coating. Particularly obvious on the logarithmic plot of moisture absorption (Figure $5-12)$ is the reduction of the moisture absorption rate over the early time of exposure (0-100 hours). During this period, the coated samples absorbed about the same amount of moisture as the uncoated samples immersed for $1 / 10^{\text {th }}$ of the time. However, once the samples had been immersed for in excess of 1000 hours, the effect of the coating seems to have become negligible as the moisture eventually found its way past the urethane and resin barriers and saturated the fibers. This failure of the coating could also be visually observed through the appearance of large bubbles beneath the coating surface as the immersion tests continued. Experimentation with other coatings could likely produce an even better reduction of the moisture absorption rate, but would also likely fail to reduce the long-term saturation point. From these observations, it can be concluded that the presence of a coating on natural fiber reinforced composites can provide an immense improvement in moisture resistance in humid or saturated environments as long as the exposure is not prolonged beyond 50-100 hours.

\subsubsection{Moisture-induced swell and curvature}

Swelling and curvature measurements were only taken for the saturated samples after their final weight measurement, so there was no data collected on the rates. However, since these dimensional instabilities are caused by moisture absorption, it is reasonable to assume that they would fairly closely correspond with the moisture absorption rates. The average percent swelling in the thickness, width, and length dimensions and the average curvature are shown for each material type in Table 5-2. Note: While measurements were recorded after moisture absorption to evaluate swelling, the original, dry sample measurements were used in the strength and stiffness calculations for the saturated samples.

As was seen with the moisture uptake results, the OSB had by far the greatest thickness and length swelling, and no significant curvature changes due to its randomly oriented, 
essentially balanced reinforcement. The glass fiber reinforced composite samples' swelling results also mirrored their moisture uptake results, as there was very little swelling of any dimension. A very slight average curvature was observed, although in the opposite direction one would expect due to fiber swelling (center of curvature on the side of the 90-degree face). This very small negative curvature value is reasonable considering that any dimensional change would have to be due to moisture effects on the resin, since the fibers absorb no moisture.

As found with previous tests, the natural fiber reinforced composites proved to possess fairly significant dimensional instability in the presence of moisture. Also similar to previous tests, the treated kenaf samples exhibited greater swelling and curvature than the untreated kenaf samples. This disparity is not solely due to the percent of moisture absorbed, as the untreated kenaf actually contained more moisture at saturation, and must therefore be attributed either to the slightly higher thickness and corresponding improved fiber encapsulation of the untreated kenaf samples or to the chemical/physical modifications caused by the surface treatment process. Most likely, the treated kenaf had a better bond with the resin system, resulting in fewer gaps at the fiber matrix interfaces. The untreated kenaf, with its poorer bonding, would have some room for the fibers to swell within the matrix, filling the voids introduced by chemical incompatibility, before any global dimensional changes could be observed. This hypothesis is supported by the fact that the average treated sample density was $4.7 \%$ higher than the average untreated sample before immersion; after saturation, the untreated sample density was actually $1.4 \%$ higher than the treated sample density, indicating that some previously present voids had been filled by the swollen, saturated fibers. (The SEM images collected in the previous chapter provide additional evidence of reduced gaps at the fiber-matrix interface for composites made with treated fibers.)

Global swelling of the natural fiber reinforced composites in each of the width and length directions was reduced by the presence of longitudinal fibers in that direction; width swelling in a unidirectional composite would likely be much closer to the percentage of thickness swelling (10-15\% as opposed to 3-4\%). The swelling of this unbalanced, bidirectional reinforcement led the kenaf reinforced samples to have a somewhat trapezoidal profile (instead of rectangular) when the cross-section was viewed from the 0 - or 90-degree axes, and also led directly to the fairly high observed curvatures. In a balanced laminate there is no reason to expect curvature of anywhere near this scale to occur, even with the natural fiber reinforced composites' high moisture absorption properties. The overall volume changes were $+13.77 \%$ for the untreated kenaf samples, $+20.60 \%$ for the treated kenaf samples, $+21.87 \%$ for the treated and coated kenaf samples, $+0.50 \%$ for the glass samples, and $+60.82 \%$ for the OSB. The high degree of swelling in the natural fiber reinforced composites prohibits their usage in most high-humidity or fullyimmersed applications, at least until significant improvements to the material are made via surface treatments and coatings. 
Table 5-2 - Swelling and curvature in mechanical testing samples due to moisture absorption

\begin{tabular}{|lcccc|}
\hline \multicolumn{1}{c}{ Sample Type } & Thickness Swell & Width Swell & Length Swell & Curvature $\left(\right.$ in $\left.^{-1}\right)$ \\
\hline Untreated Kenaf & $9.7 \%$ & $3.4 \%$ & $0.3 \%$ & 0.033 \\
Treated Kenaf & $15.6 \%$ & $3.7 \%$ & $0.6 \%$ & 0.051 \\
Treated/Coated Kenaf & $16.7 \%$ & $3.5 \%$ & $0.9 \%$ & 0.039 \\
Glass & $0.0 \%$ & $0.2 \%$ & $0.3 \%$ & -0.003 \\
OSB & $57.5 \%$ & $0.8 \%$ & $1.3 \%$ & 0.000 \\
\hline
\end{tabular}

\subsubsection{Flexural Testing}

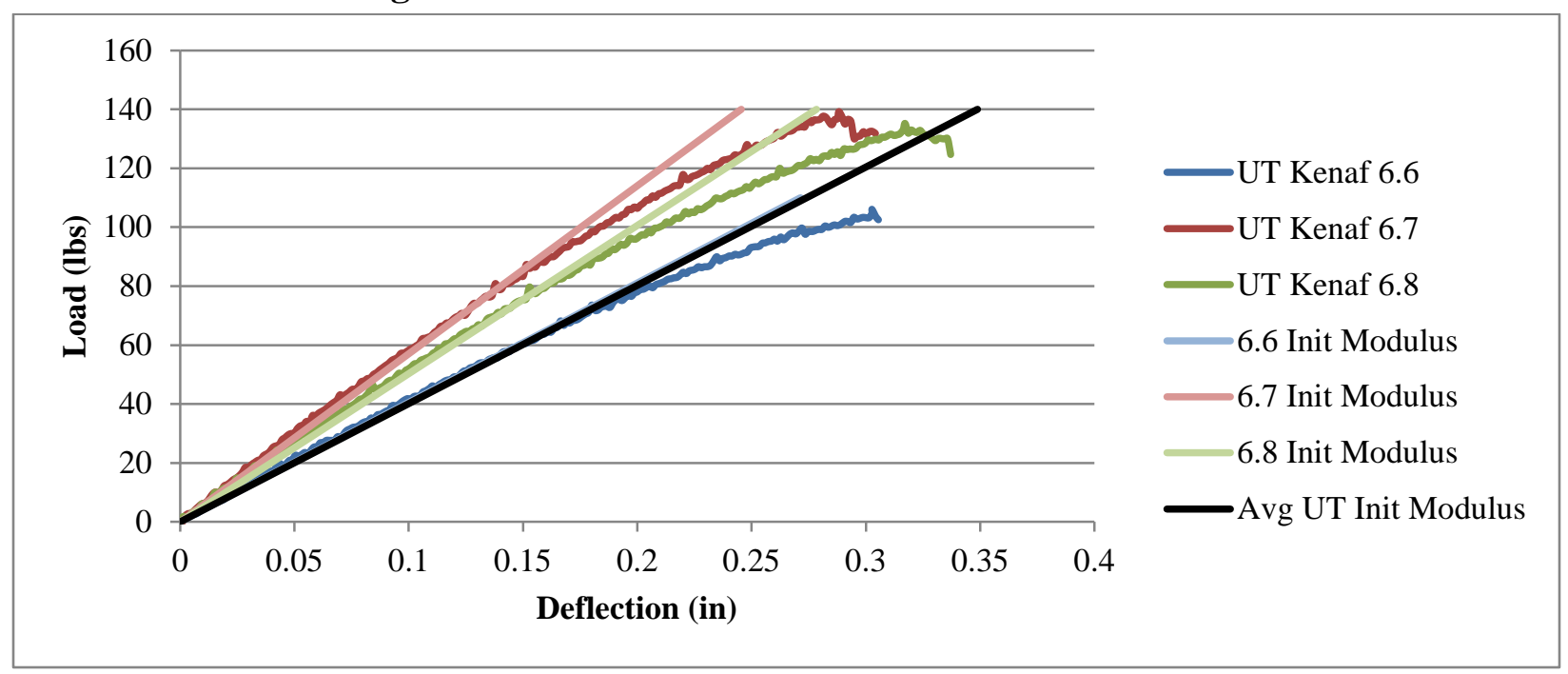

Figure 5-13 - Load-deflection plots for dry, flexural, untreated kenaf samples

As representative plots of the flexural load-deflection behavior of kenaf fiber composites, plots of the three dry samples are shown in Figure 5-13, along with the calculated initial slopes and the average initial slope of all dry, flexural, untreated kenaf samples. All of the flexural strength and stiffness testing results (after normalization to 40\% FVF) are plotted in Figure 5-14 and Figure 5-15, respectively. On average, the bidirectional kenaf reinforced composite had a flexural strength and stiffness of around $28 \mathrm{ksi}$ and $1.09 \mathrm{Msi}$, respectively, at $40 \% \mathrm{FVF}$.

One common trend between these plots and many of the other mechanical testing results is an unexpectedly high degree of variation in the glass reinforced composite laminates. This was likely due to poor manufacturing quality, as the glass fibers had no stitching through the thickness and as a result had a tendency to shift around during layup and compression, creating areas of higher- and lower-than-expected fiber volume fraction. With regard to their flexural behavior, however, the difference in strength between the saturated samples and the other two samples is large enough to appear significant. This reduction is therefore likely a result of some degradation of the resin system, since the fibers should lose no strength in the presence of water and a reduction of matrix strength would be particularly noticeable in flexural testing. This does not, however, explain the observed increase in flexural stiffness at saturation. 
In all of the tests run, flexural and otherwise, it quickly became evident that OSB is not a suitable comparison material, as it generally only possesses a fraction of the properties of either the natural or glass reinforced composites and it degrades very quickly when exposed to moisture. On the other hand, it was made clear that the natural fiber reinforced composites will potentially outperform OSB in any construction application in every consideration except for price. A better comparison for future tests would potentially be high quality engineered or solid wood components.

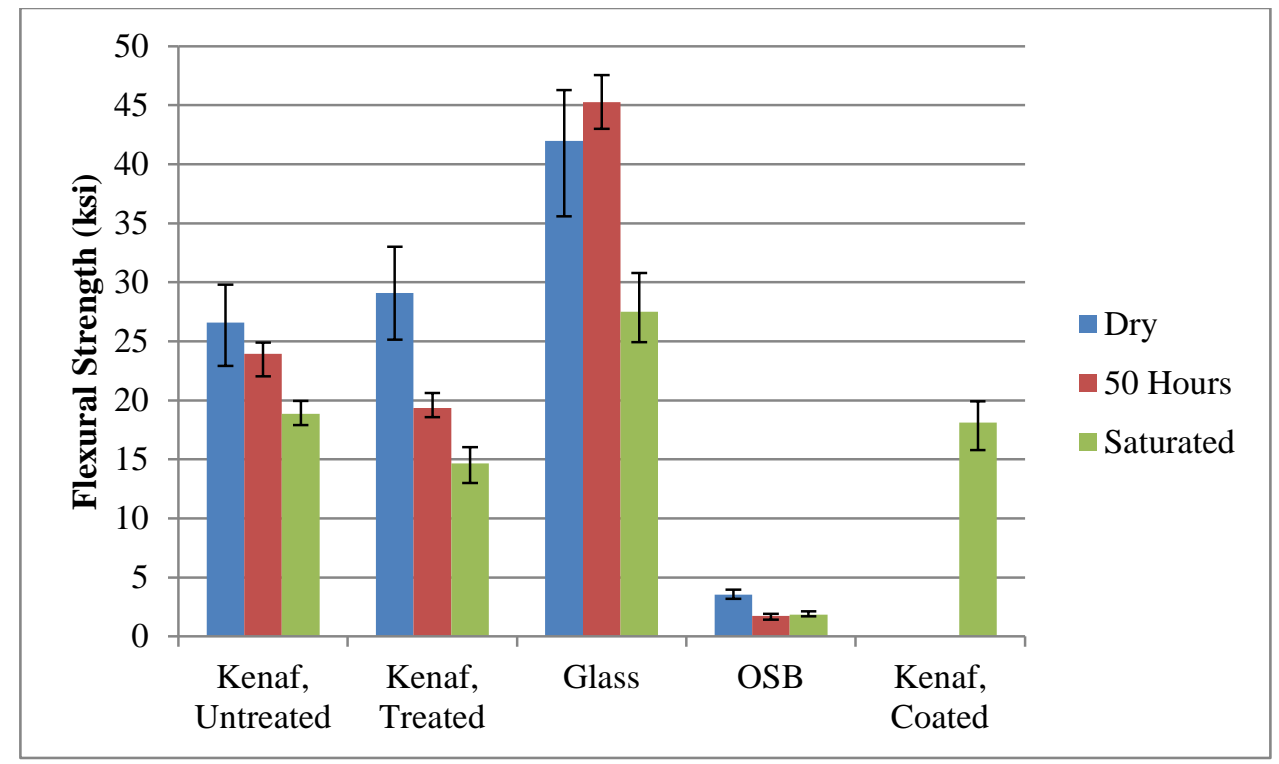

Figure 5-14 - ASTM D790 flexural strength comparison (with max/min bars)

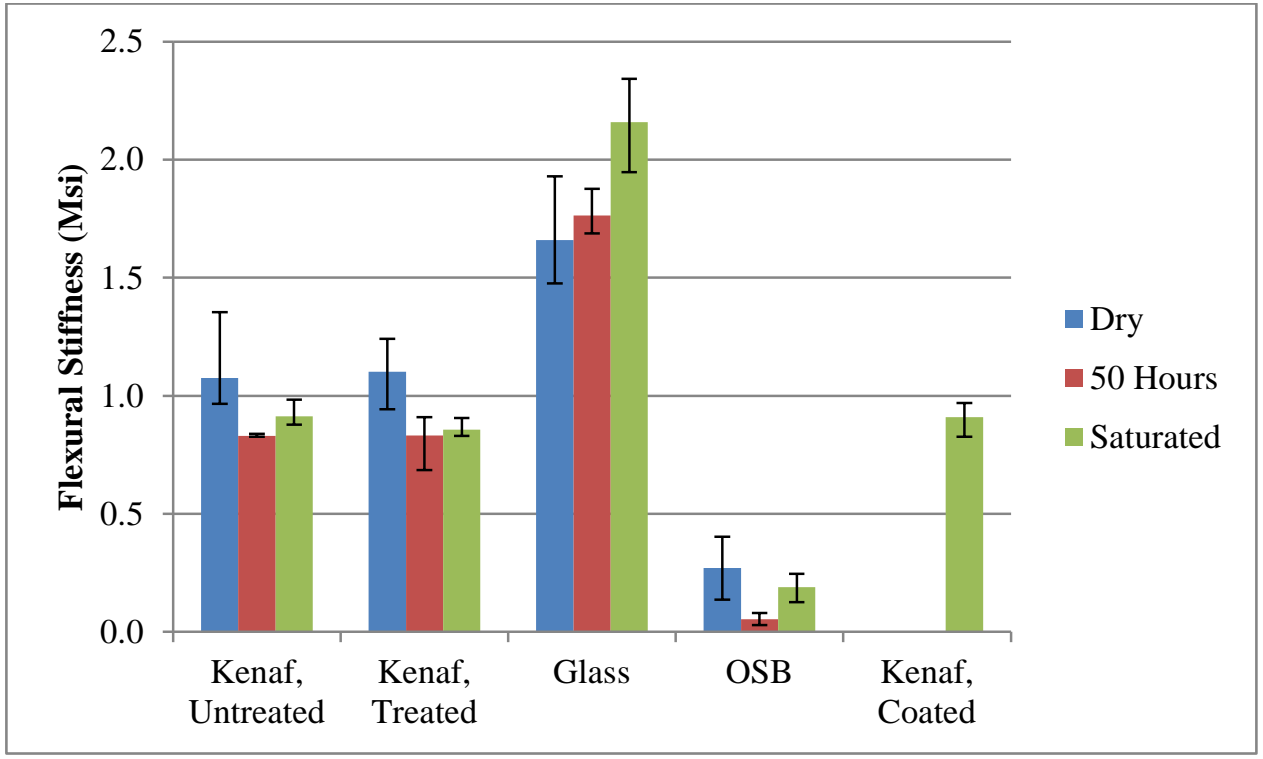

Figure 5-15 - ASTM D790 tangent modulus comparison (with max/min bars) 
The treated and untreated kenaf samples generally exhibited fairly similar flexural performance, particularly with regard to stiffness. As characteristic plots of the saturated moisture behavior, the flexural load-deflection response of three of the saturated, untreated kenaf composites is shown in Figure 5-16, along with the calculated initial slopes and the same average initial slope of all dry, flexural, untreated kenaf samples from Figure 5-13. Both the treated and untreated samples lost stiffness when immersed for 50 hours, but experienced no further reduction at saturation. A potential mechanism causing this reduction in stiffness due to increased moisture absorption is likely the microstructure of the individual natural fiber, which is composed of microfibrils helically wound through the several layers of cell walls. This microfibrillar angle has been proposed by several researchers to significantly affect the fiber stiffness (John and Anandjiwala 2008, Bismarck et al. 2005). As the individual fibers swell from moisture absorption, the microfibrillar angle with respect to the longitudinal axis would increase, reducing the stiffness yet increasing the strain to failure. The tensile and compressive test results will provide more support for this hypothesis as the stiffness and strain results can be directly compared.

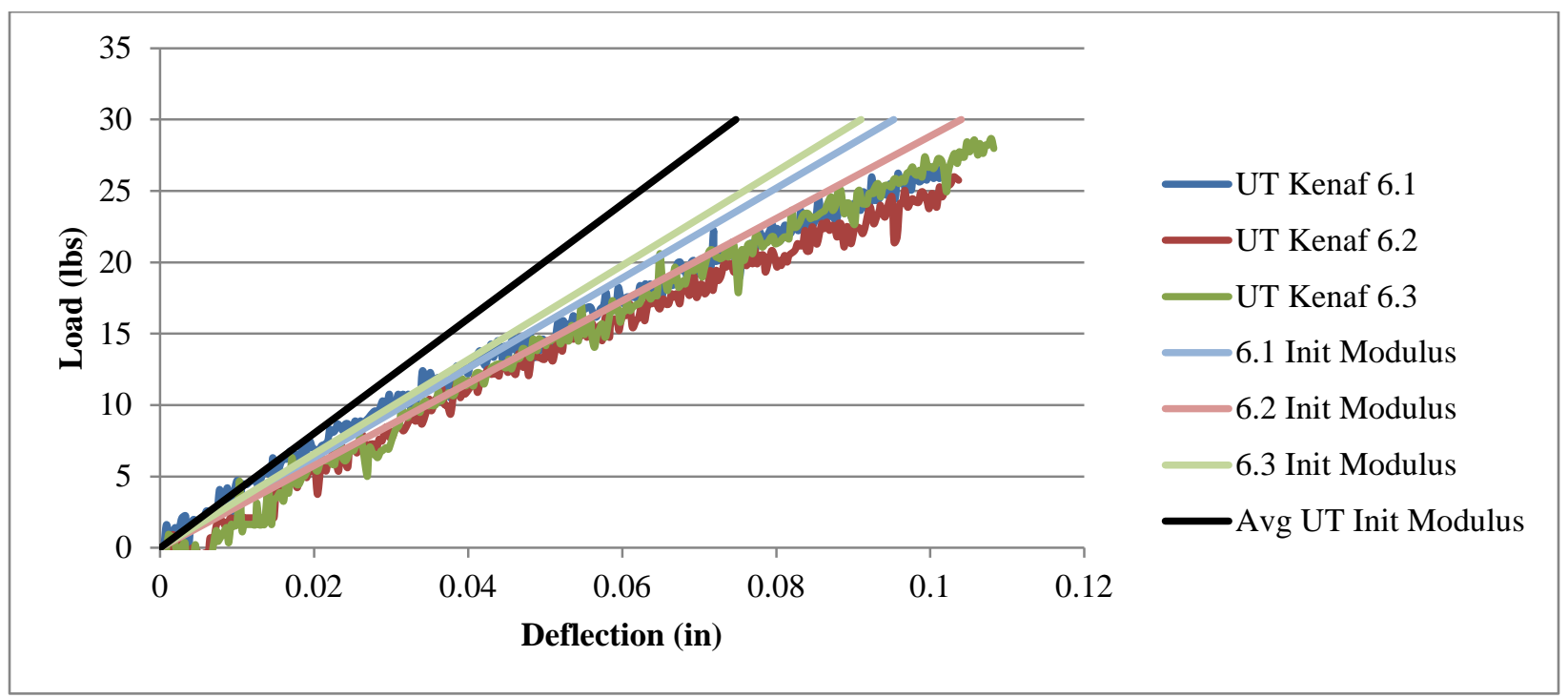

Figure 5-16 - Load-deflection plots for saturated, flexural, untreated kenaf samples

The perceived increase in stiffness at saturation could, in part, be due to the increased thickness (and therefore bending moment of inertia) that results from the moisture-induced swelling. As stated previously, dry sample measurements were used for calculations instead of the swollen, saturated sample measurements. In practice, a component would be designed and selected based off of dry dimensions, and by using the dry sample measurements to calculate properties, a designer could predict the percent reduction in a moist environment without having to account for the swollen dimensions. To obtain a reasonable approximation of the saturated material properties with respect to the saturated dimensions, the changes in the cross-sectional area and moment of inertia recorded in Table 5-2 can be used to adjust material properties accordingly. 
In terms of strength, the treated kenaf composite had a slightly higher dry strength, but appeared to degrade to a greater extent than the untreated kenaf composite in the presence of moisture. This would appear to be further support for the hypothesis that the treated kenaf has a better bond, and thus a reduced gap at the fiber-matrix interface. Due to the better bonding the treated kenaf samples exhibit better dry behavior, but due to the reduced gap size the fibers cause more matrix damage as they absorb moisture and expand. The coating only slowed the moisture absorption rate, and since those samples reached roughly the same saturation point as the uncoated samples, the coated samples do not have significantly different saturated properties from the uncoated samples.

If saturated, it appears that the kenaf reinforced composites would typically only possess around $60 \%$ of their original flexural strength and around $80 \%$ of their original flexural stiffness.

The failure mode of the kenaf composites in flexure was typically tensile face rupture (see Figure 5-17) or a combination of tensile face rupture and compressive face local buckling (see Figure 5-18). The tensile failure would sometimes also be visible in the 90-direction layer as a small crack above the $0 / 90$ interface. With higher moisture absorption, the compressive failure mode seemed to become much more prevalent.

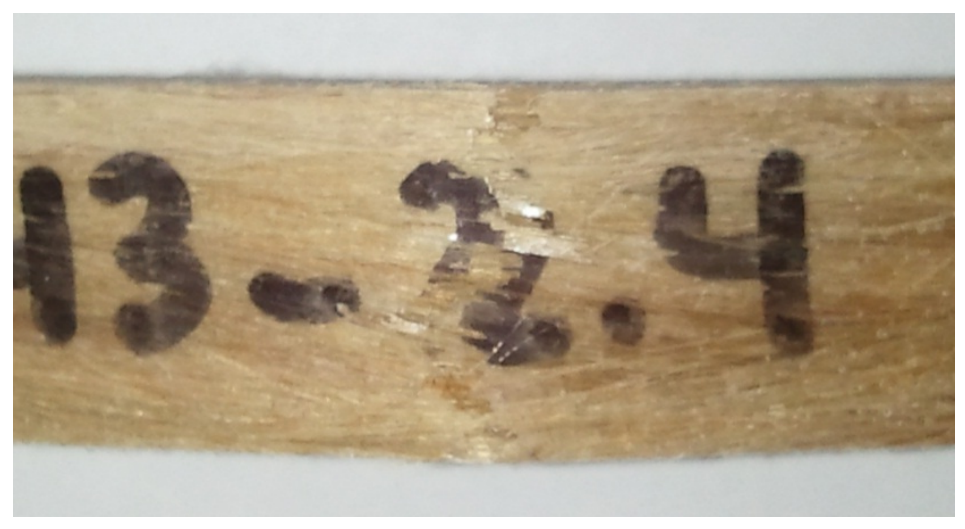

Figure 5-17 - Kenaf composite tensile face rupture failure mode in flexure

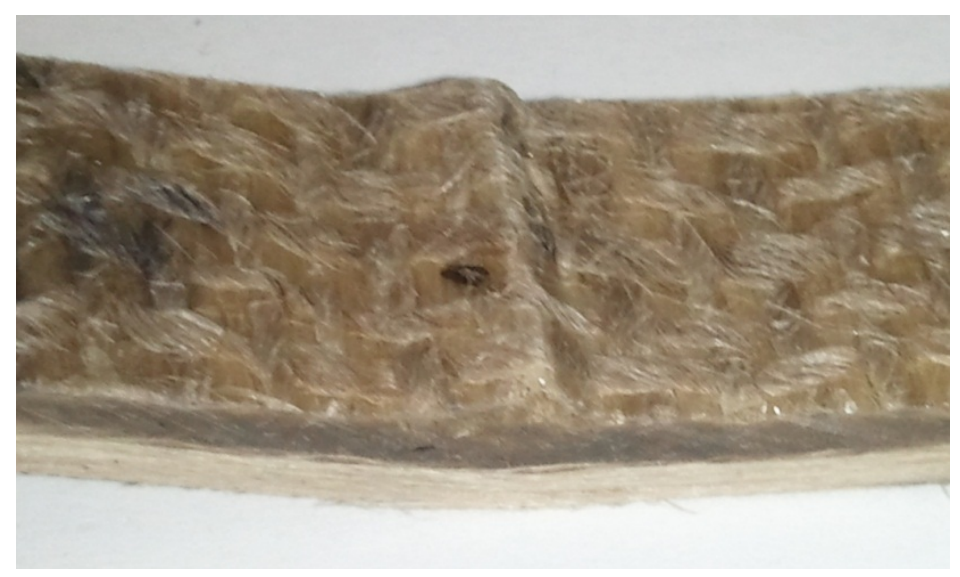

Figure 5-18 - Kenaf composite compressive face local buckling failure mode in flexure 


\subsubsection{Tensile Testing}

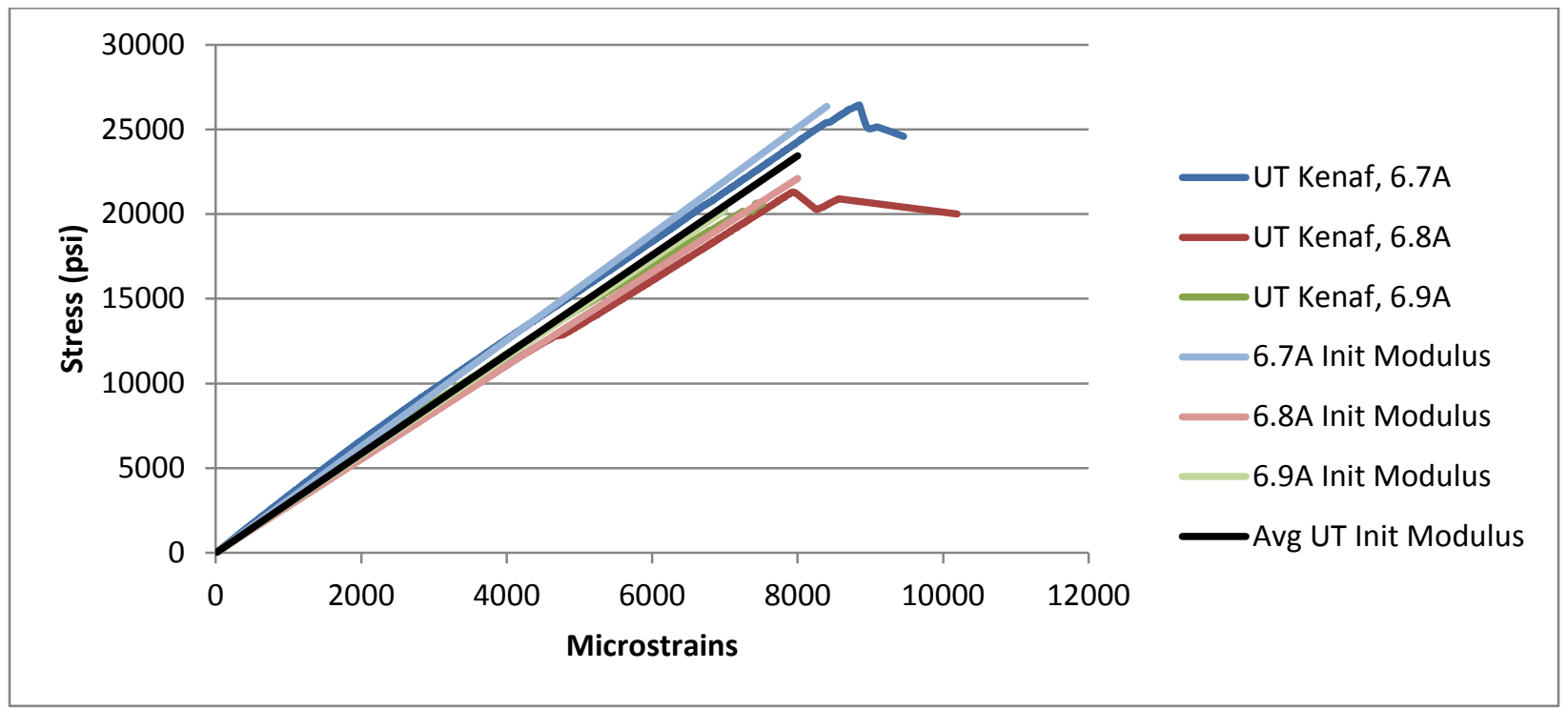

Figure 5-19 - Stress-strain plots for dry, tensile, untreated kenaf samples

As representative plots of the tensile stress-strain behavior of kenaf fiber composites, plots of the three dry samples are shown in Figure 5-19, along with the calculated initial slopes and the average initial slope of all dry, tensile, untreated kenaf samples. The tensile strength, stiffness, and strain testing results (after normalization to 40\% FVF) are plotted in Figure 5-20, Figure 5-21, and Figure 5-22, respectively. On average, the unidirectional kenaf reinforced composite had a tensile strength, stiffness, and ultimate strain of around $25 \mathrm{ksi}, 3.3 \mathrm{Msi}$, and $8,000 \mu \varepsilon$, respectively, at $40 \% \mathrm{FVF}$.

The glass reinforced composites, again, had some unexpected variation, particularly with regard to the dry samples having considerably lower ultimate strengths and strains than the 50 hour and saturated samples. As the stiffness is nearly identical in each of the three conditions, clearly the low strength and strain are directly related. This indicates that the dry samples were more brittle than the 50 hour and saturated samples, likely implying that some matrix plasticization took place.

The OSB samples were, again, not even on the same scale of strength and stiffness as the fiber reinforced composites and their results do not require further analysis for this study. 


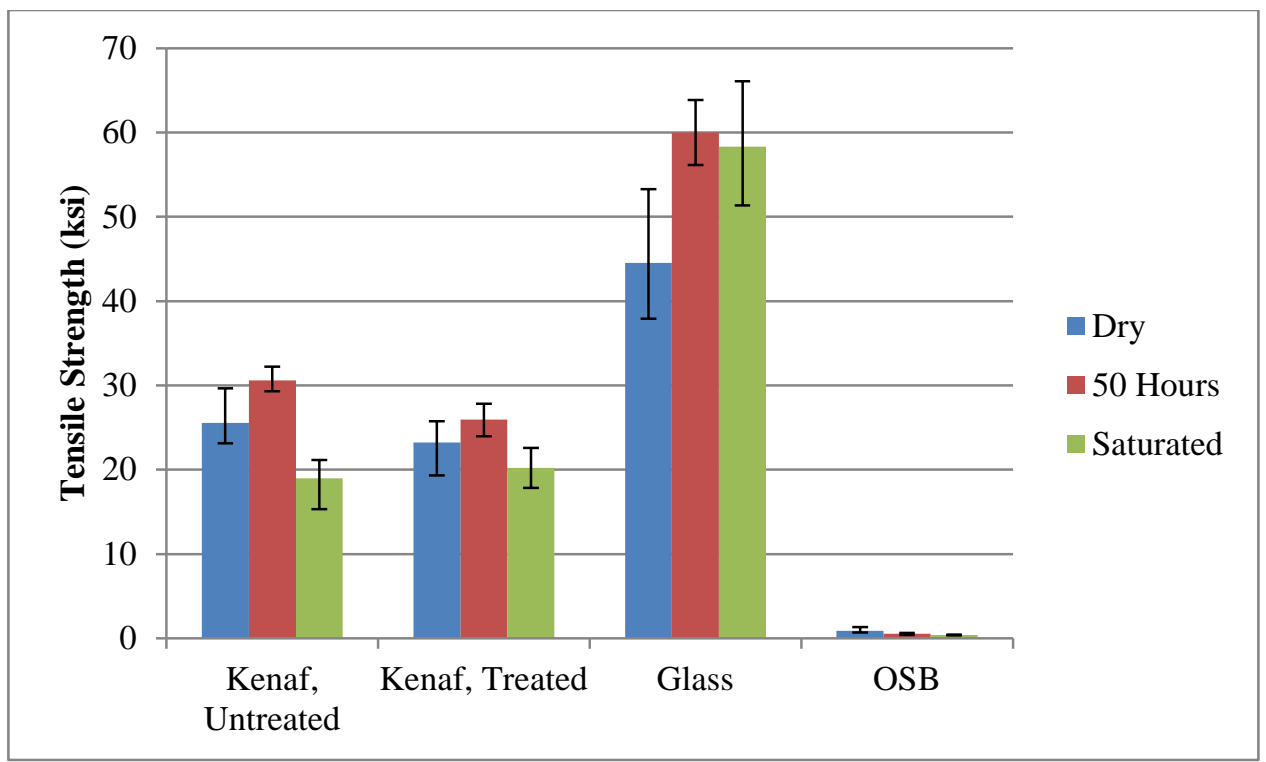

Figure 5-20 - ASTM D3039 tensile strength comparison (with max/min bars)

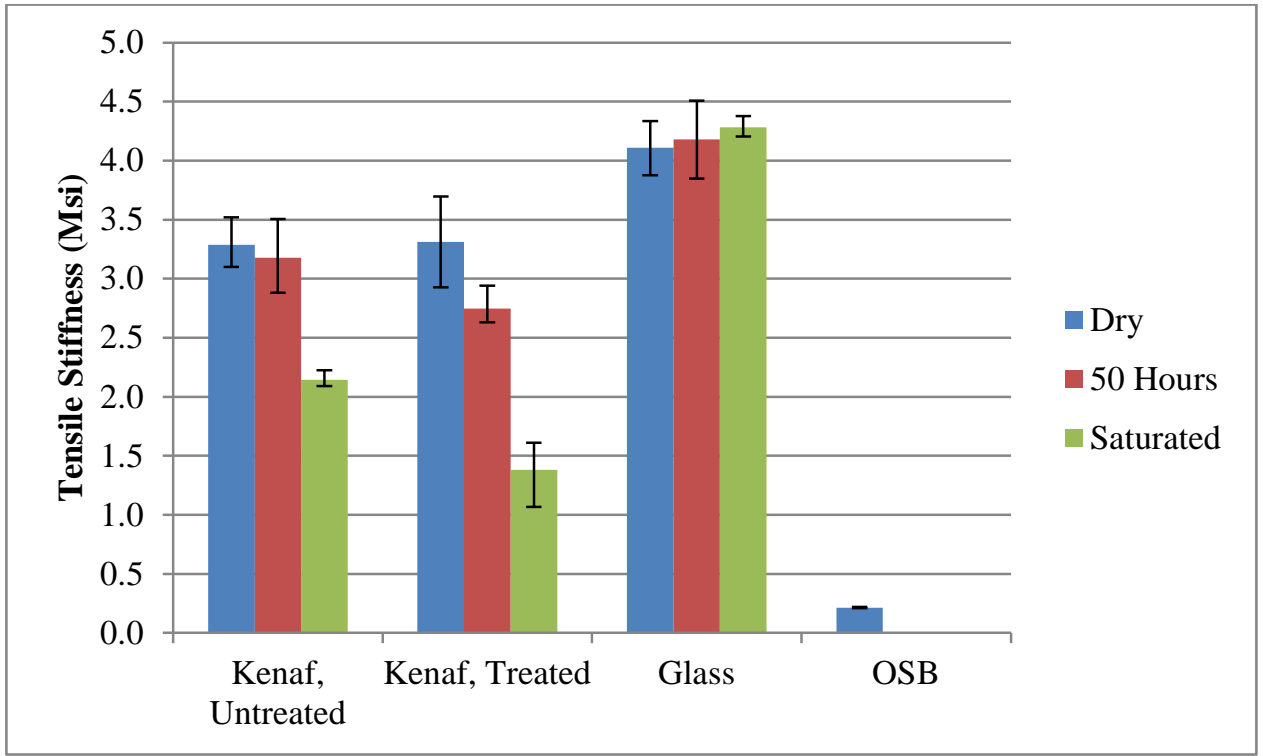

Figure 5-21 - ASTM D3039 tensile chord modulus comparison (with max/min bars) 


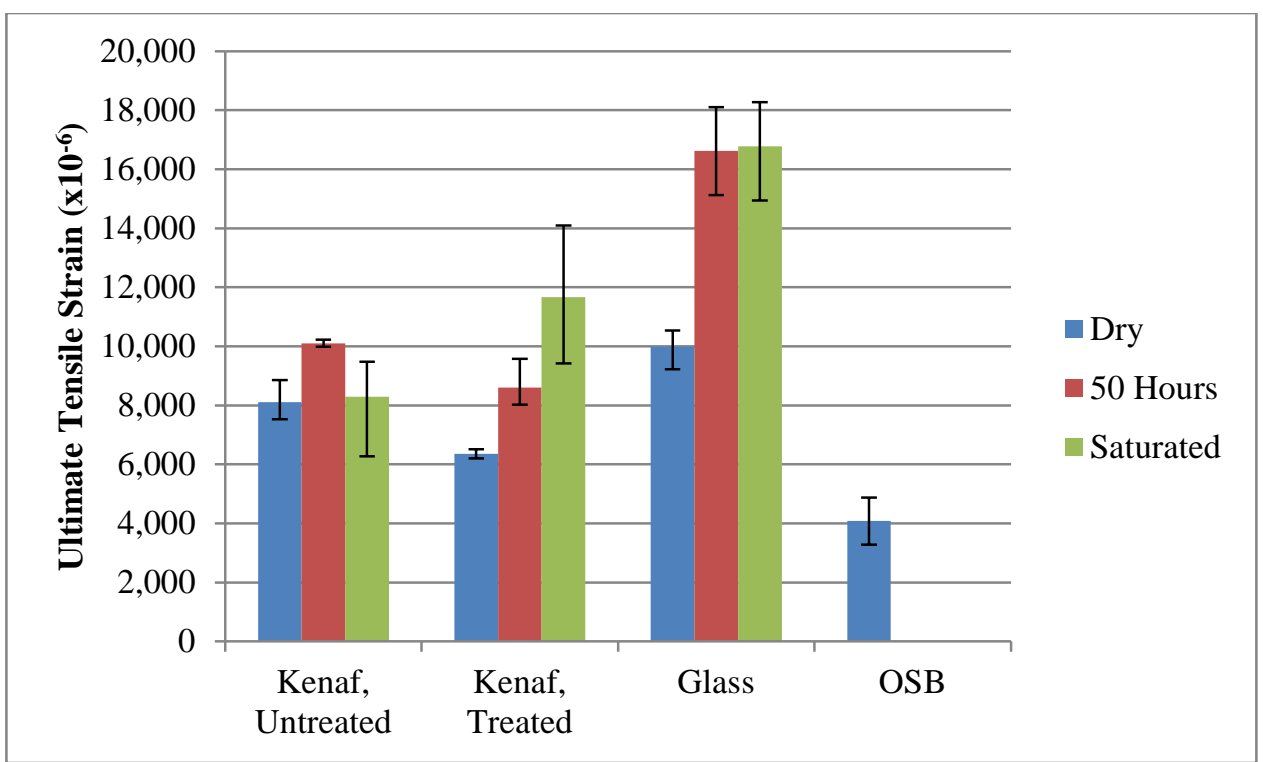

Figure 5-22 - ASTM D3039 ultimate tensile strain comparison (with max/min bars)

The treated and untreated kenaf reinforced materials again produced fairly similar results, suggesting that only relatively small differences exist between their strength, stiffness, and bonding properties. Like with the glass reinforced composite, both of the kenaf materials had, on average, higher strains after exposure to moisture, supporting the hypothesis of matrix plasticization. Both of the materials also had reductions in their stiffness with exposure to moisture, but the treated kenaf materials had greater losses at both 50 hours and saturation. Both also appeared to gain some strength after 50 hours of absorption and then experienced a reduction at saturation, with the untreated kenaf experiencing both a greater stiffness gain at 50 hours and a greater stiffness loss at saturation. Some of this poorer behavior in the presence of moisture observed for the treated kenaf could again be due to its potentially better bonding and reduced room for fiber expansion, although this effect is not as clearly seen as with the flexural samples previously. In support of the hypothesis that fiber swelling increases ductility by increasing the microfibrillar angle, the reduced stiffness is complemented by increased ultimate strain.

If saturated, it appears that the kenaf reinforced composites would typically only possess around $78 \%$ of their original tensile strength and around $55 \%$ of their original tensile stiffness, with around a $25 \%$ increase in their ultimate tensile strain.

The failure mode of the kenaf composites in tension was, unsurprisingly, tensile rupture. Occasionally the tensile failure would occur at different locations on the opposite faces, with a split down the fabric layer interface connecting the two failures (see Figure 5-23). There did not appear to be significant fiber pull-out or dry fibers, which are usually easy to see in failed tensile samples if present (see Figure 5-24). 


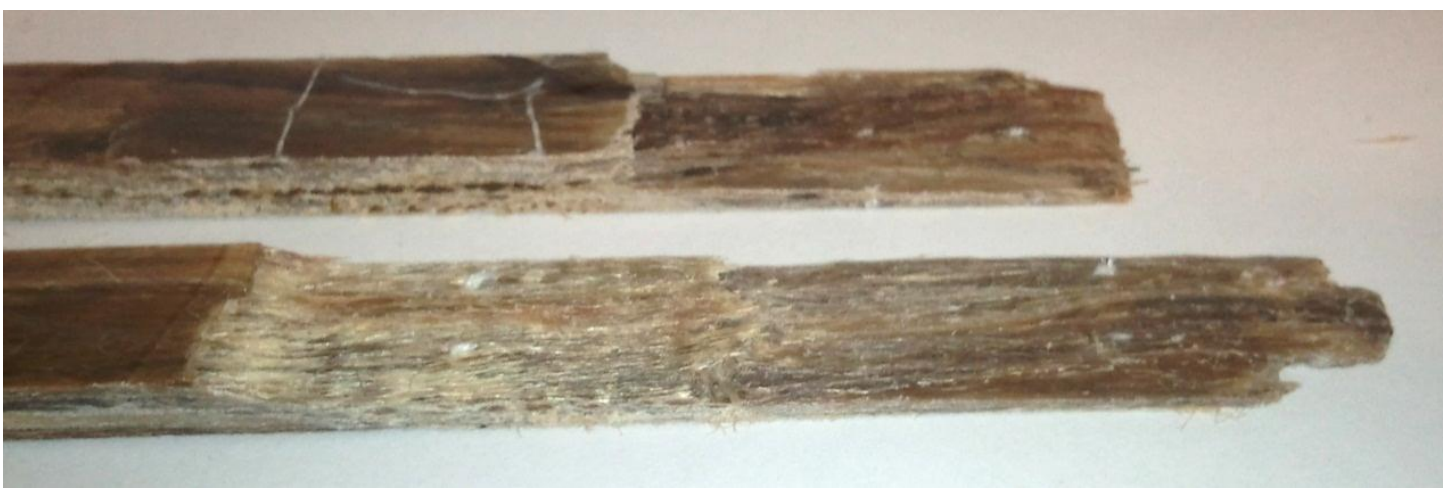

Figure 5-23 - Kenaf composite tensile failure with splitting along fabric interface

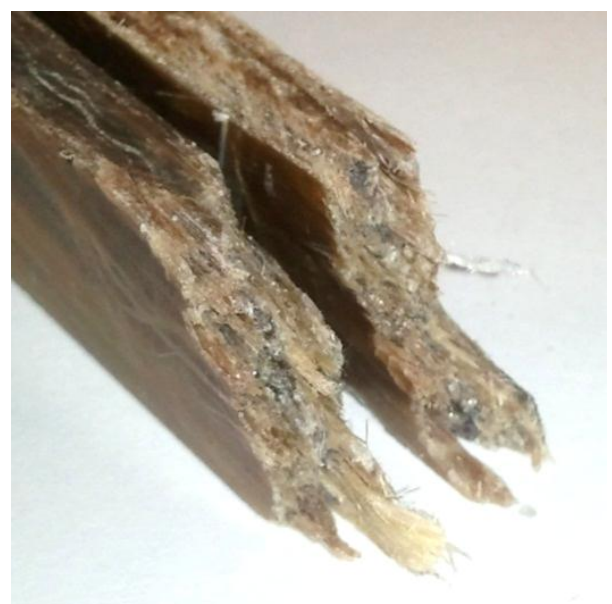

Figure 5-24 - Typical kenaf composite tensile failure due to rupture 


\subsubsection{Compressive Testing}

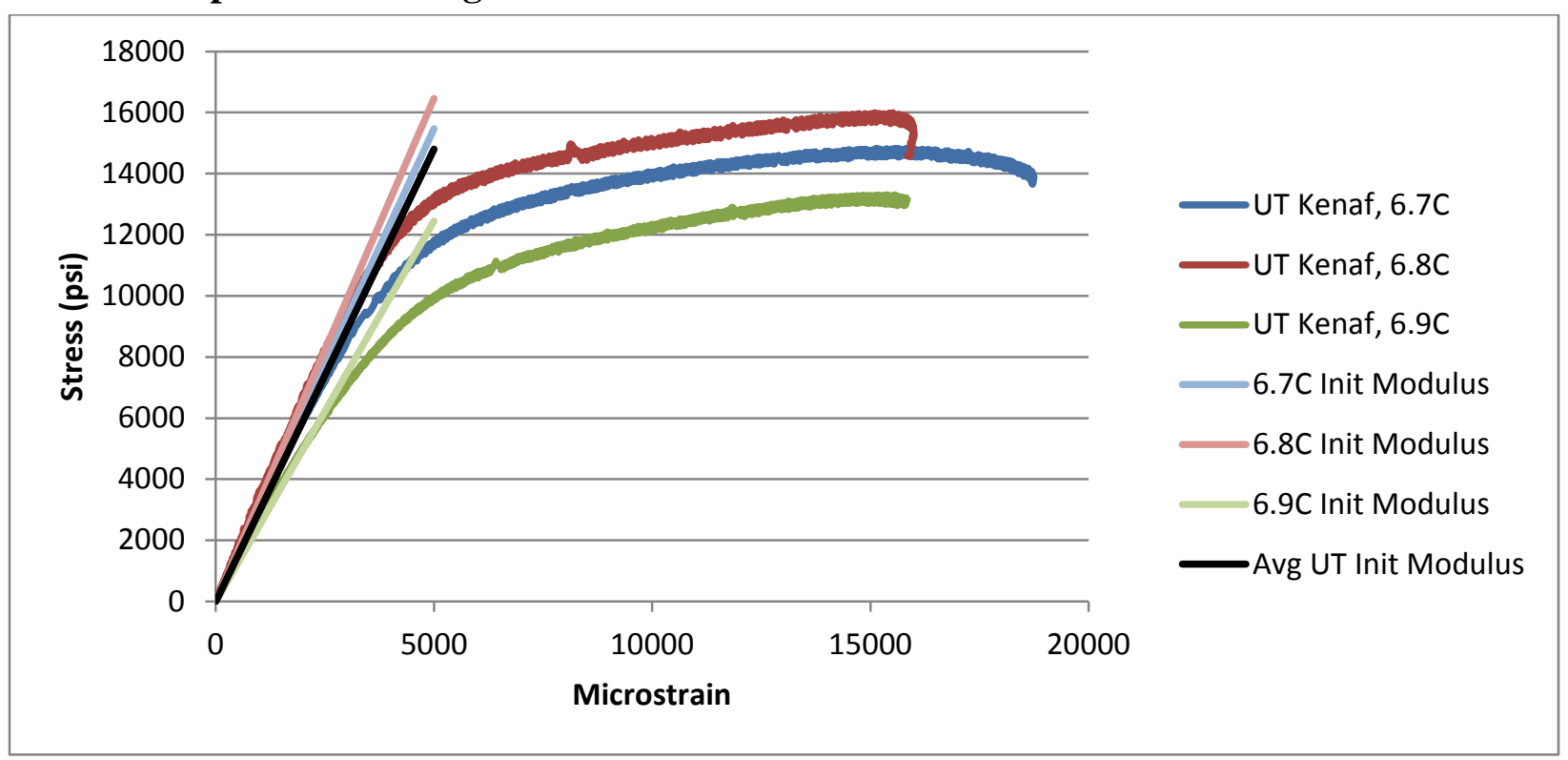

Figure 5-25 - Stress-strain plots for dry, ASTM D695-based compressive, untreated kenaf samples

As representative plots of the compressive stress-strain behavior of kenaf fiber composites, plots of the three dry ASTM D695-based samples are shown in Figure 5-25, along with the calculated initial slopes and the average initial slope of all dry, compressive, untreated kenaf samples. The compressive strength, stiffness, and strain testing results of the ASTM D695based testing (after normalization to 40\% FVF) are plotted in Figure 5-26, Figure 5-27, and Figure 5-28, respectively. On average, the unidirectional kenaf reinforced composite had a compressive strength, stiffness, and ultimate strain of around $17 \mathrm{ksi}, 3.35 \mathrm{Msi}$, and 13,000 $\mu \varepsilon$, respectively, at $40 \% \mathrm{FVF}$.

But for a few outliers in terms of compressive strength, the glass fiber reinforced samples all produced fairly comparable results regardless of their immersion time. Even with the strength results (in which the 50 hour immersed samples had a $25 \%$ higher average strength than either the dry or saturated samples), if only the maximum results are compared (the top lines of the black bars), the strengths would have been fairly close, showing that a few poor samples were responsible for creating the disparity. These poor samples were more likely due to manufacturing defects than directly related to the dry or saturated test conditions. While the compressive stiffness of the glass reinforced samples was quite close to their tensile stiffness, their compressive strength (even at maximum) was only around half of the tensile strength, which is a fairly common trend in published properties of unidirectional composites (Barbero 2011). 


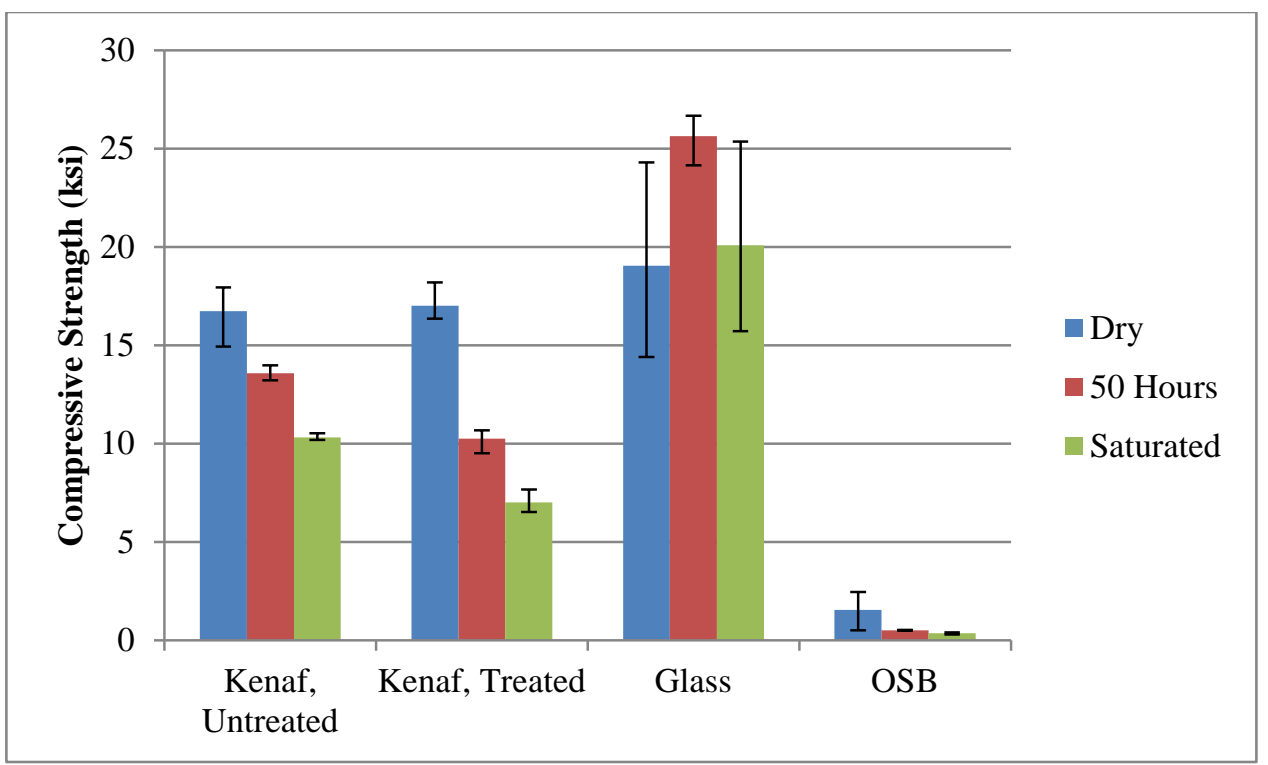

Figure 5-26 - ASTM D695 compressive strength comparison (with max/min bars)

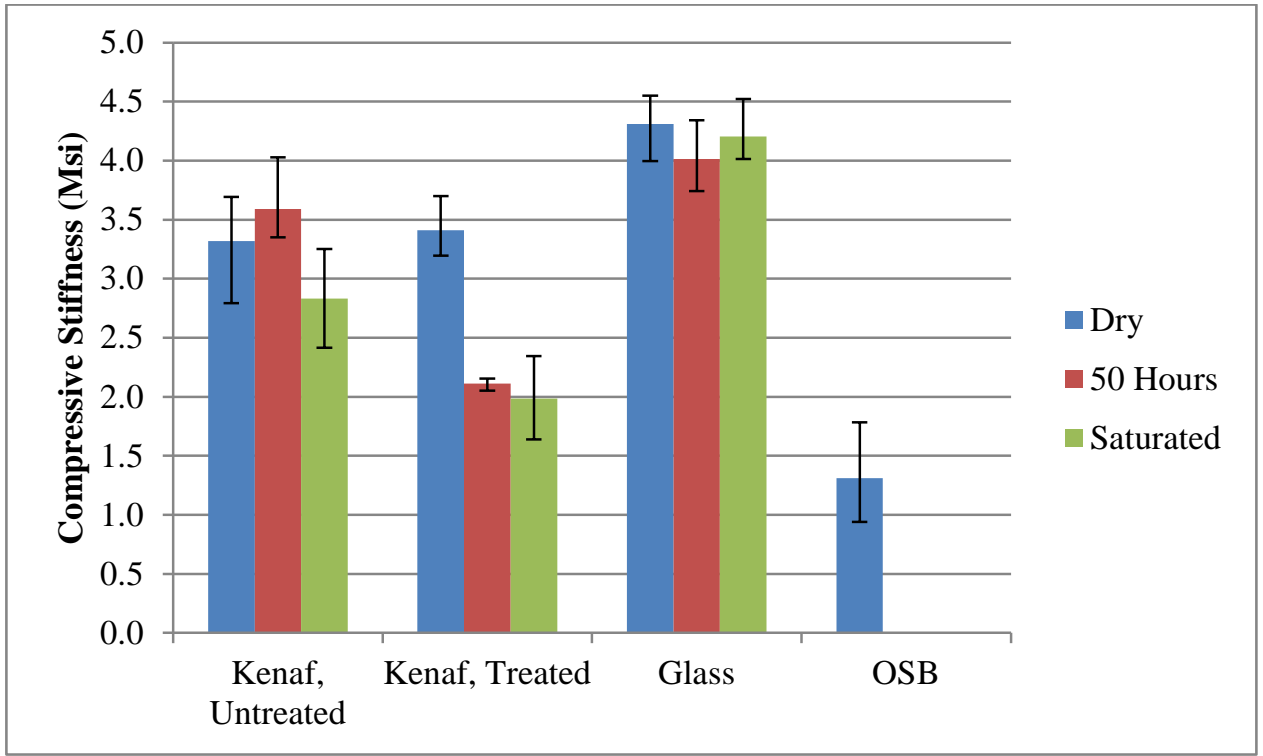

Figure 5-27 - ASTM D695 modulus of elasticity comparison (with max/min bars) 


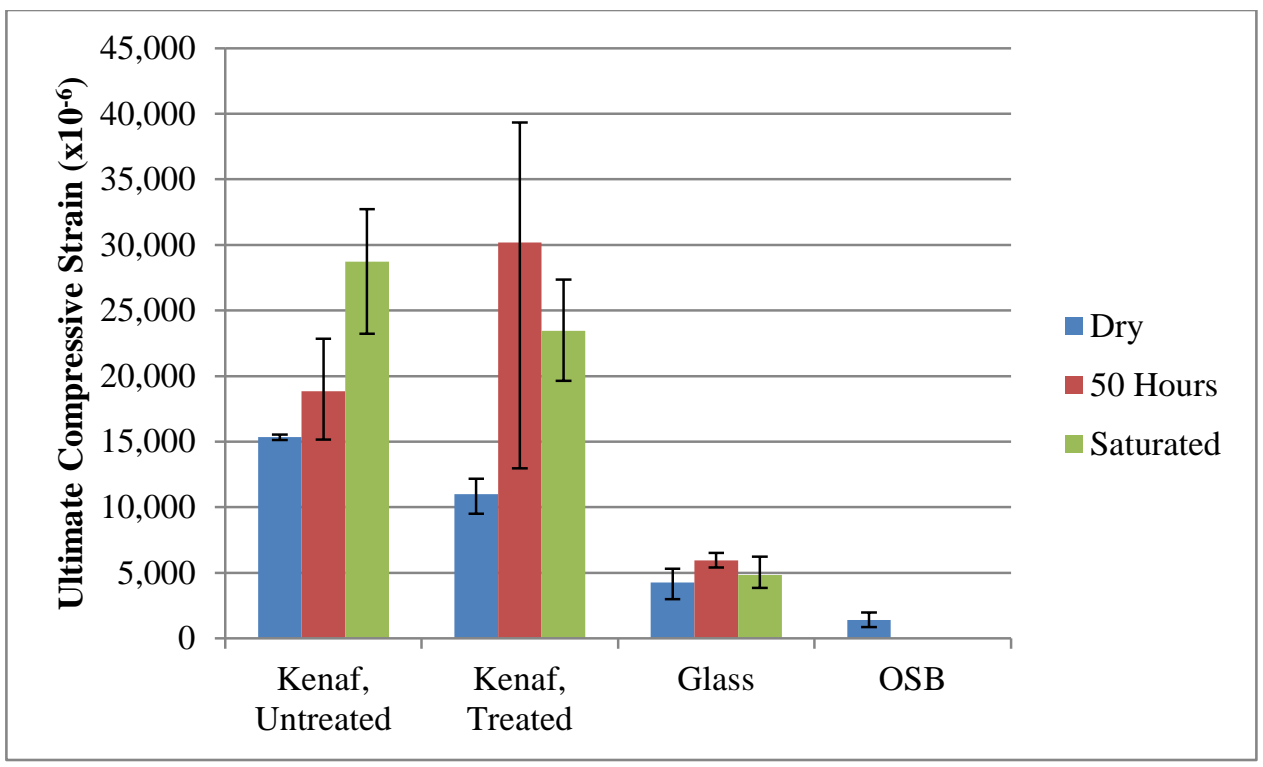

Figure 5-28 - ASTM D695 ultimate compressive strain comparison (with max/min bars)

Most of the kenaf reinforced composites failed in a shear mode with a diagonal line of failure at the unsupported top of the sample (see Figure 5-29), although a few had the shear failure near the center, and a few had more of a brooming failure at the top. The failure mode was not noticeably different between the dry and saturated samples. Shah and Lakkad (1981) also reported the prevalence of this shear failure when compressive testing jute reinforced composites and found that it less common in glass reinforced or hybrid glass/jute reinforced composites. As with (most of) the flexural and tensile samples, the treated kenaf samples also experienced greater strength and stiffness reductions than the untreated kenaf samples in the presence of moisture potentially due to a greater prevalence of matrix microcracking.

As was seen with the kenaf tension samples, the compression samples also experienced a significant increase in strain with exposure to moisture. However, unlike with the tension samples, even the dry strains were 3-4 times higher than the glass ultimate compressive strain. This phenomenon is likely due to the angled helical pattern of microfibrils making up the natural fiber structure allowing for significant compression deformation. Compared to the relatively brittle column action of glass fibers, it is to be expected that the kenaf fibers would have a more ductile compression behavior. As with the tensile test results, the reduced stiffness values seem to roughly correlate with increased strain values, suggesting an increase in microfibrillar angle as the fibers swell.

If saturated, it appears that the kenaf reinforced composites would typically only possess around $50 \%$ of their original compressive strength and around $72 \%$ of their original compressive stiffness, with around a 100\% increase in their ultimate compressive strain. 


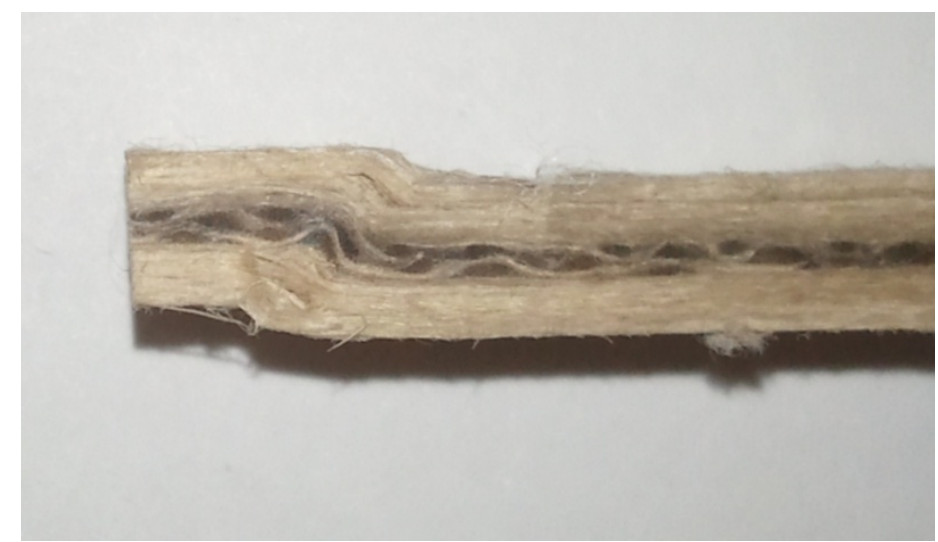

Figure 5-29 - ASTM D695 compressive test resulting in shear failure at loading end

As previously stated, the D695-based compressive test was used for its reputation of obtaining good modulus of elasticity values, while the D3410-based compressive test was used for its reputation of obtaining good strength values. The compressive strength, stiffness, and strain testing results of the ASTM D3410-based testing (after normalization to 40\% FVF) are plotted in Figure 5-30, Figure 5-31, and Figure 5-32, respectively.

As discussed by Blandford, the D3410 compressive tests resulted in much lower compressive stiffness results. As the tests were running, it was obvious that the thin, unsupported samples had a tendency to bend and deform at an angle between the compressive surfaces, losing the perpendicular line of loading and skewing the strain measurements. As a result, the stiffness and strain measurements can be reasonably ignored. On the other hand, the strength values do seem to match up reasonably well with those from the D695-based tests. A plot of the average compressive strength results from the two different tests is shown in Figure 5-33. Although this average plot does seem to provide some better normalization for the glass reinforced composite results, there is no other significant information gained by considering the ASTM D3410-based compressive test results along with the ASTM D695-based results. 


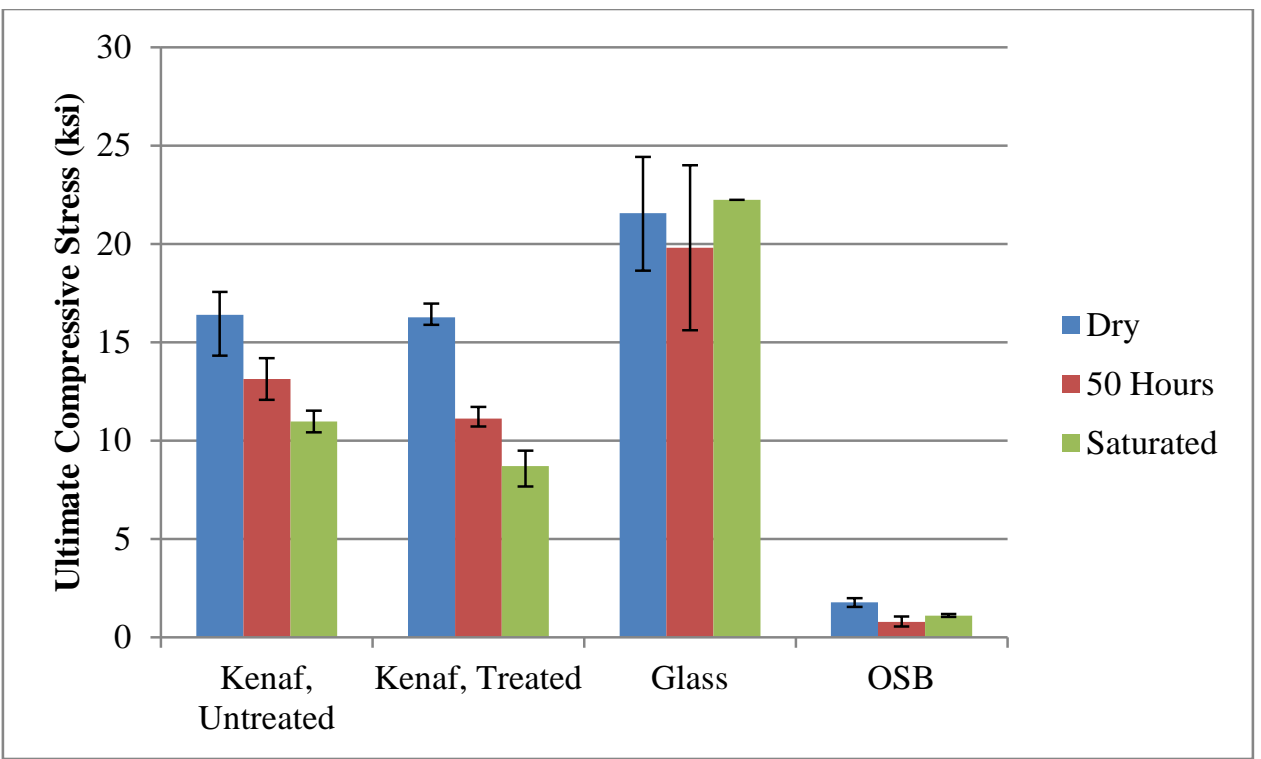

Figure 5-30 - ASTM D3410 ultimate compressive stress comparison (with max/min bars)

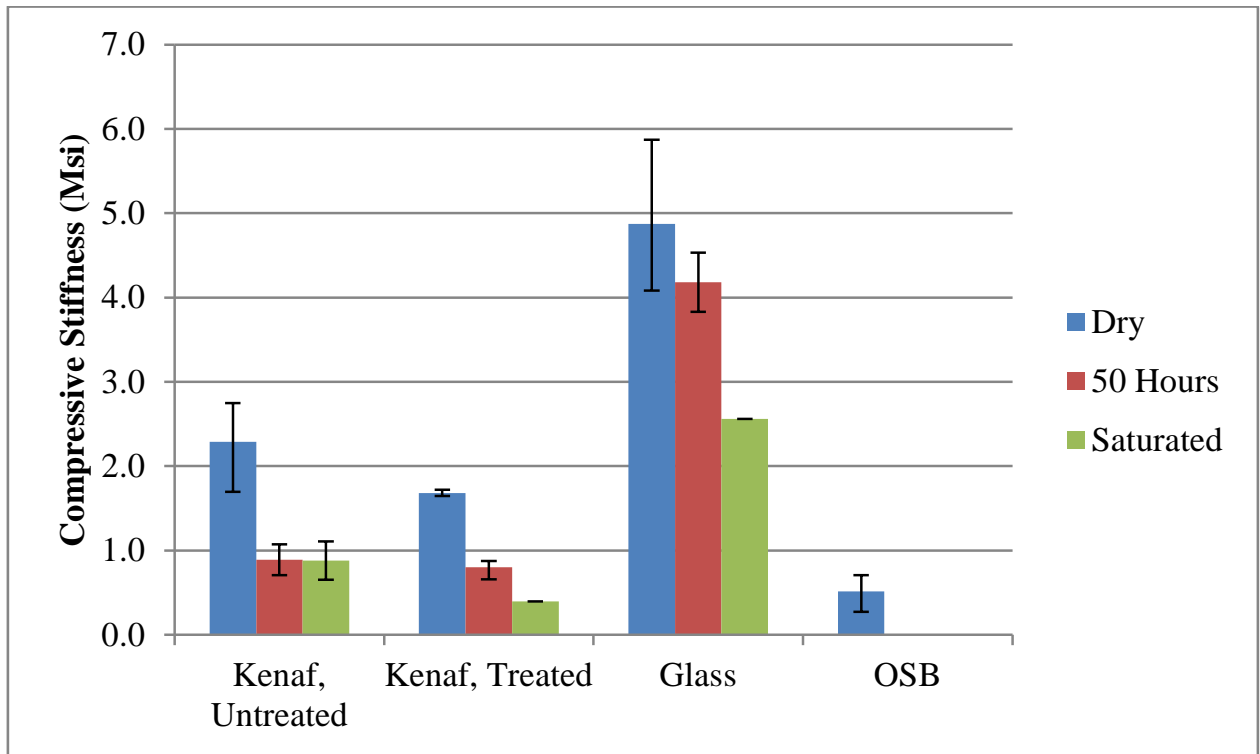

Figure 5-31 - ASTM D3410 compressive chord modulus comparison (with max/min bars) 


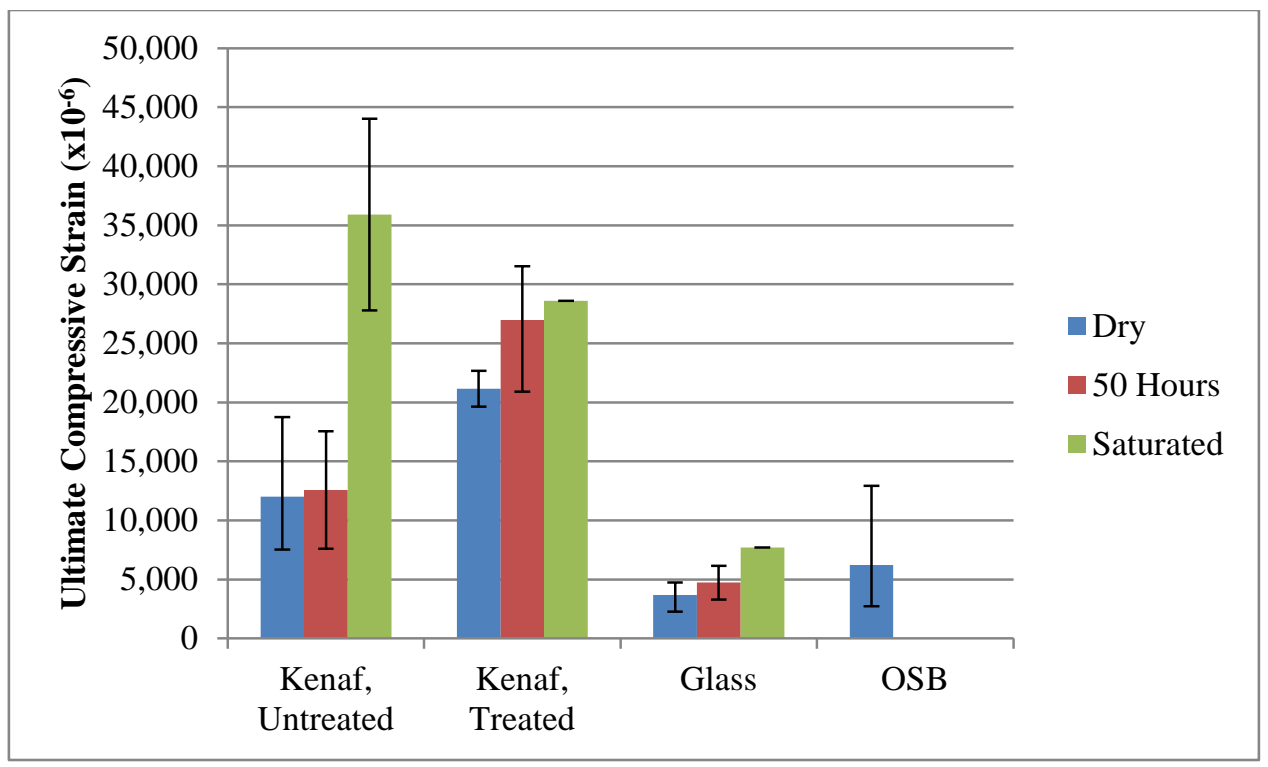

Figure 5-32 - ASTM D3410 ultimate compressive strain comparison (with max/min bars)

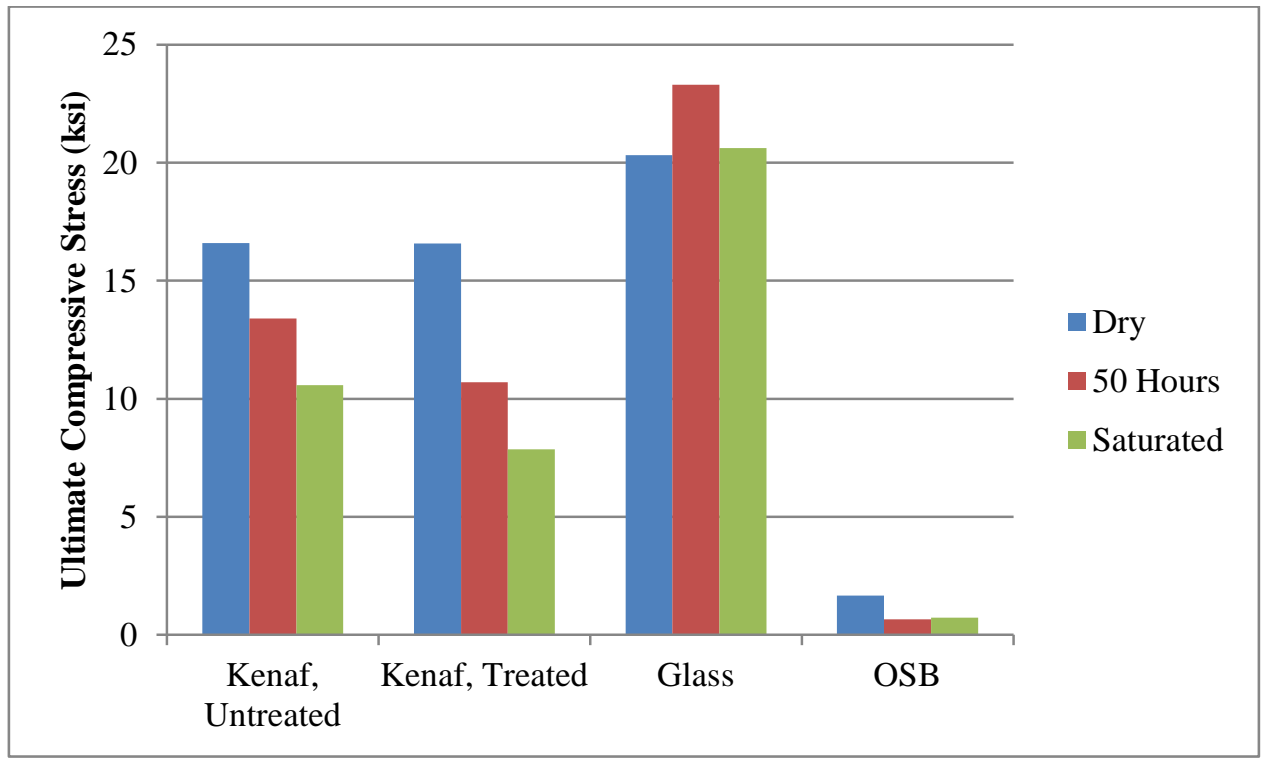

Figure 5-33 - Average compressive strength results

While many of the ASTM D3410-based samples had unconventional failure modes due to their tendency to fold over while testing, several had the shear compressive failure mode seen with the ASTM D695-based compressive testing (see Figure 5-34). 


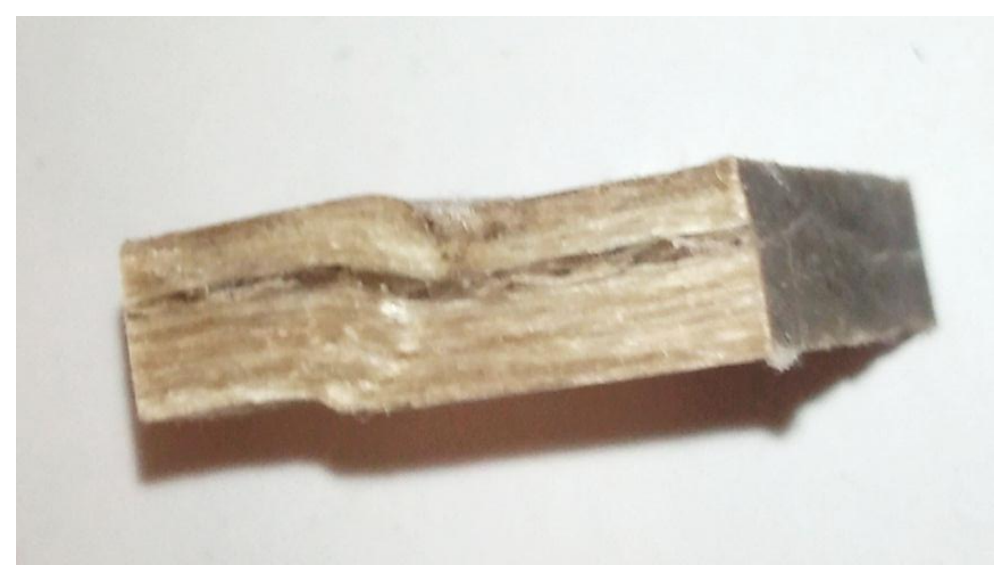

Figure 5-34 - ASTM D3410-based compressive test sample exhibiting shear failure mode

\subsubsection{Shear Testing}

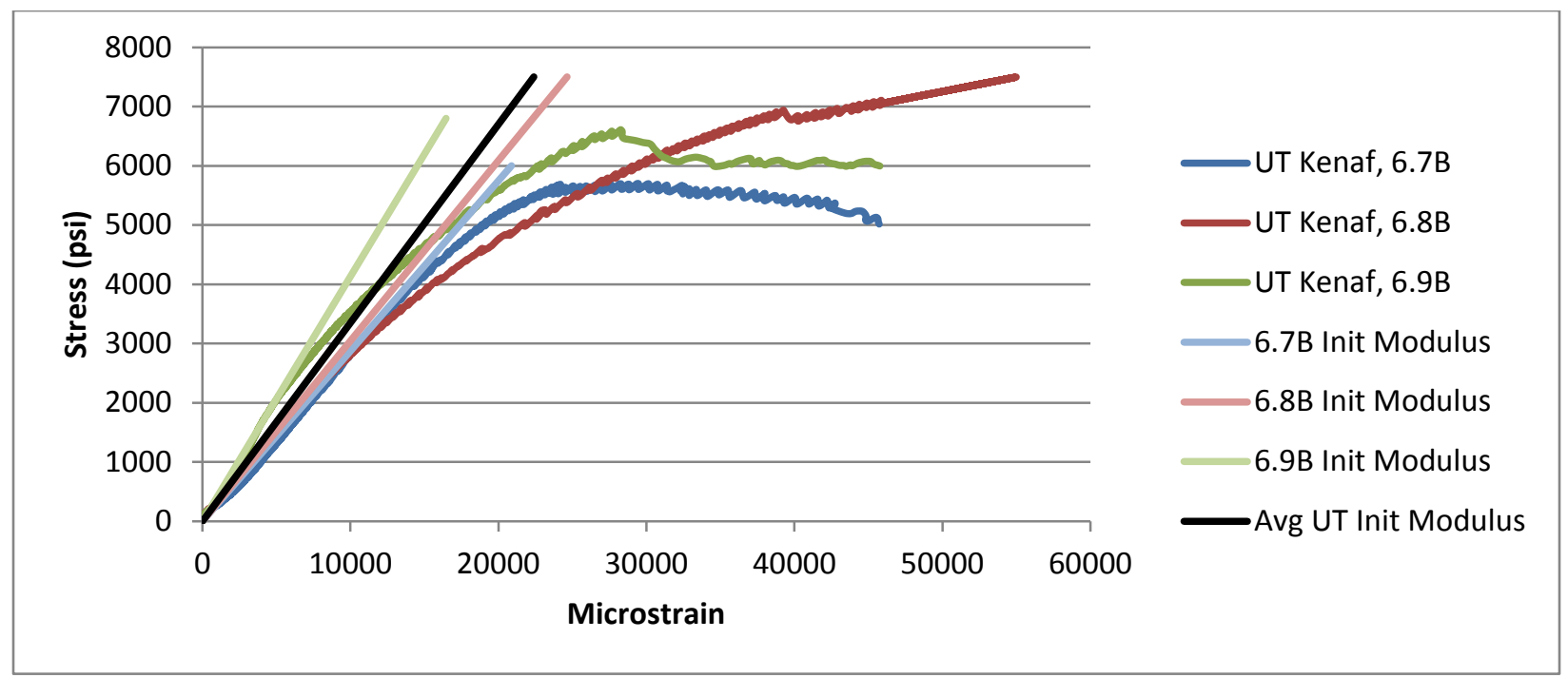

Figure 5-35 - Stress-strain plots for dry, shear, untreated kenaf samples

As representative plots of the shear stress-strain behavior of kenaf fiber composites, plots of the three dry, untreated kenaf samples are shown in Figure 5-35, along with the calculated initial slopes and the average initial slope of all dry, shear, untreated kenaf samples. The shear strength, stiffness, and strain testing results (after normalization to 40\% FVF) are plotted in Figure 5-36, Figure 5-37, and Figure 5-38, respectively. On average, the bidirectional kenaf reinforced composite had an in-plane shear strength, stiffness, and ultimate strain of around $6 \mathrm{ksi}, 0.35 \mathrm{Msi}$, and $30,000 \mu \varepsilon$, respectively, at $40 \% \mathrm{FVF}$.

The glass reinforced composite results again had a surprising degree of variation, this time in their ultimate shear strength and ultimate shear strain values. The ultimate shear strain values are of less of a concern as they are at least partly dependent on the lower shear strength and could also potentially be due to poor bonding between the gage and the recently wet composite surface. Despite the presence of notches, which create a larger cut surface area to 
potentially aid moisture penetration, the glass reinforced shear samples neither absorbed more moisture nor at a faster rate than any of the other samples. However, if the assumption that the resin system has been degraded due to the immersion conditioning (made when analyzing the flexural test results) is combined with the generalization that shear performance in composites is matrix dominated, these losses begin to appear logical. There is no other particularly cogent reasoning behind why there is a significant drop in the strength, however, other than the previously mentioned difficulties manufacturing which may have led to defects and areas of higher- or lower-than-expected fiber volume fraction.

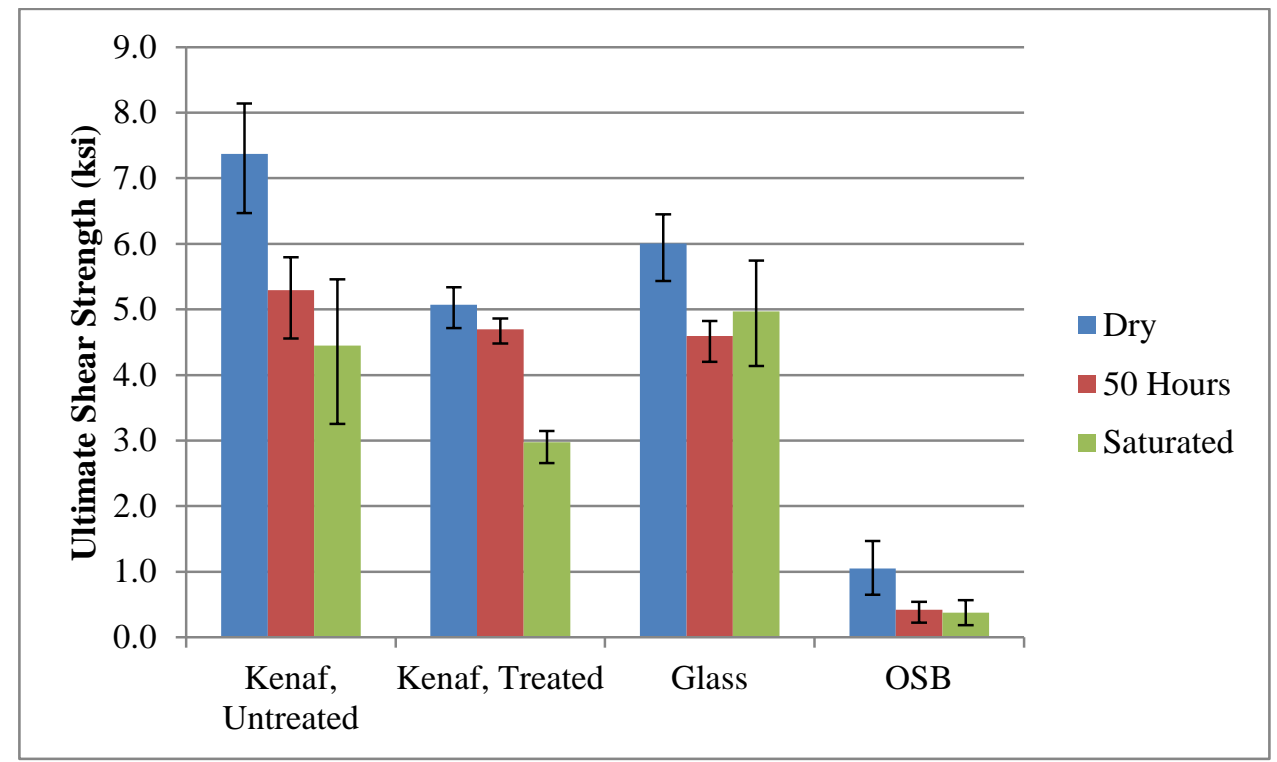

Figure 5-36 - ASTM D5379 ultimate in-plane shear strength comparison (with max/min bars)

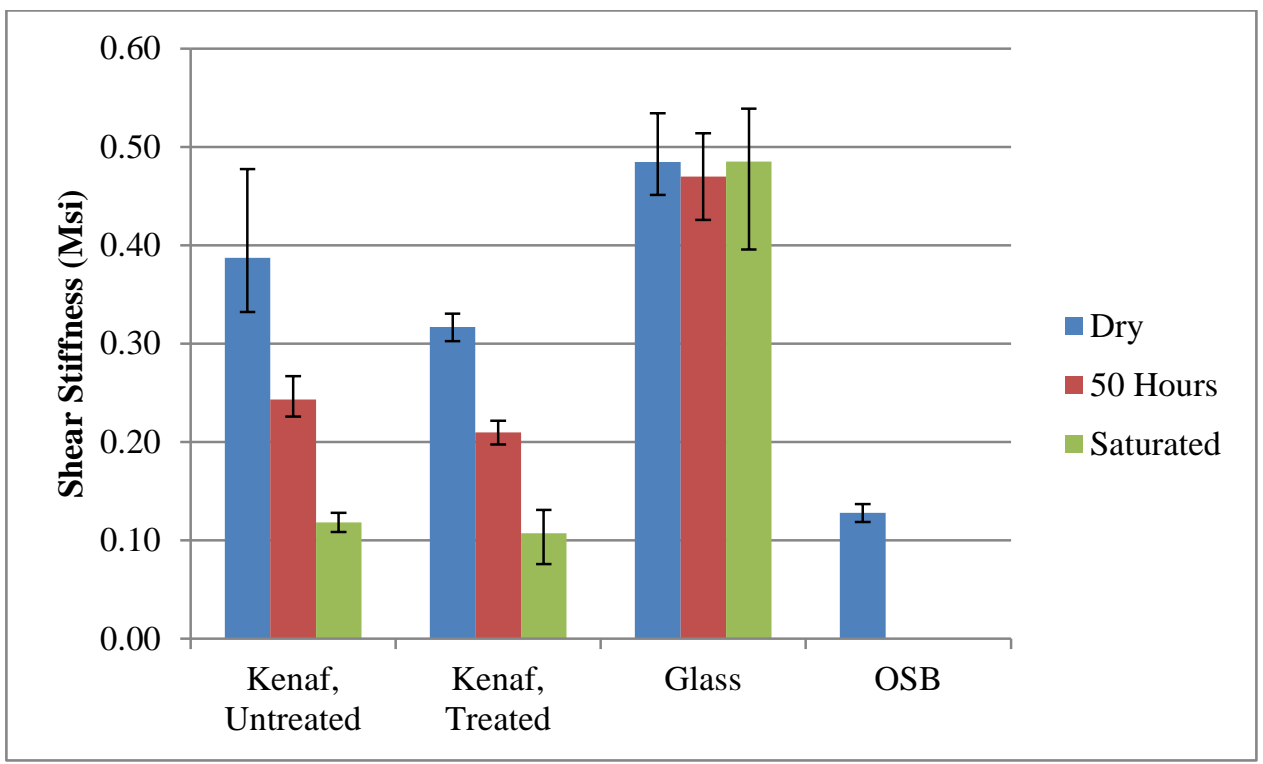

Figure 5-37 - ASTM D5379 in-plane shear chord modulus comparison (with max/min bars) 


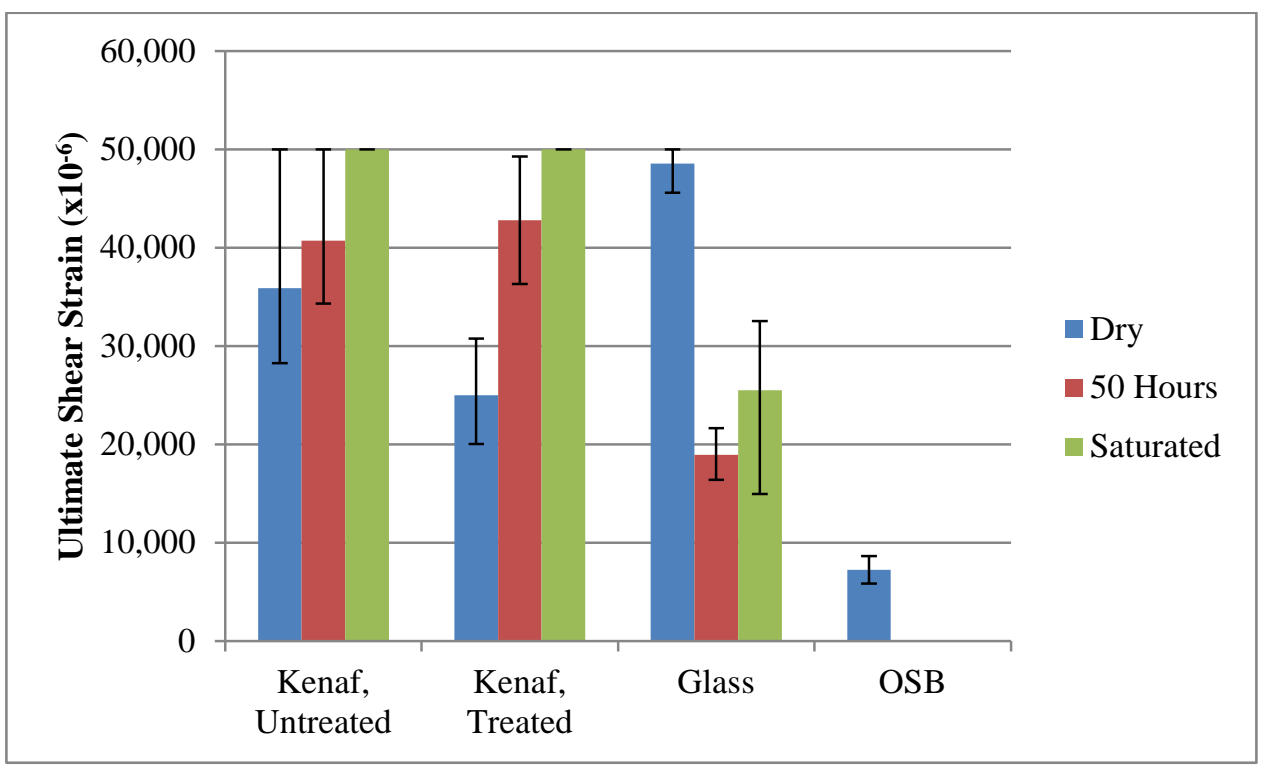

Figure 5-38 - ASTM D5379 ultimate in-plane shear strain comparison (with max/min bars)

The idea that shear performance is matrix dominated in composites would also explain why, despite considerable differences between the strengths of the kenaf and glass reinforced composites in every other test method, the shear strengths of both natural and synthetic reinforcements are relatively comparable. Many of the same themes observed in other tests are again present when analyzing the untreated and treated kenaf behavior: general performance between the two is fairly comparable, both have decreasing stiffnesses with moisture exposure corresponding to increasing ultimate strains, and the reductions in strength and stiffness are more noticeable in the treated kenaf than in the untreated kenaf. Somewhat surprising, however, is the significantly higher dry strength and stiffness for the untreated kenaf than for the treated kenaf. This phenomenon may again be a result of the slightly higher thickness ( $10 \%)$ of the untreated samples not entirely being corrected for during fiber volume fraction normalization. This hypothesis would again reflect the axiom that shear performance is matrix dominated.

If saturated, it appears that the kenaf reinforced composites would typically only possess around $60 \%$ of their original in-plane shear strength and around 32\% of their original in-plane shear stiffness, with around a 67\% increase in their ultimate in-plane shear strain (assuming saturated ultimate strain is near the test-dictated maximum of $50,000 \mu \varepsilon$ ).

Almost all of the shear samples exhibited the desired failure mode; that is, a failure between the two notches. For the kenaf fiber reinforced composites, this failure was not only noticeable from the load-strain readings, but also due to the appearance of a transverse crack on the fabric surface (adjacent to the 90-direction layer), as shown in Figure 5-39. 


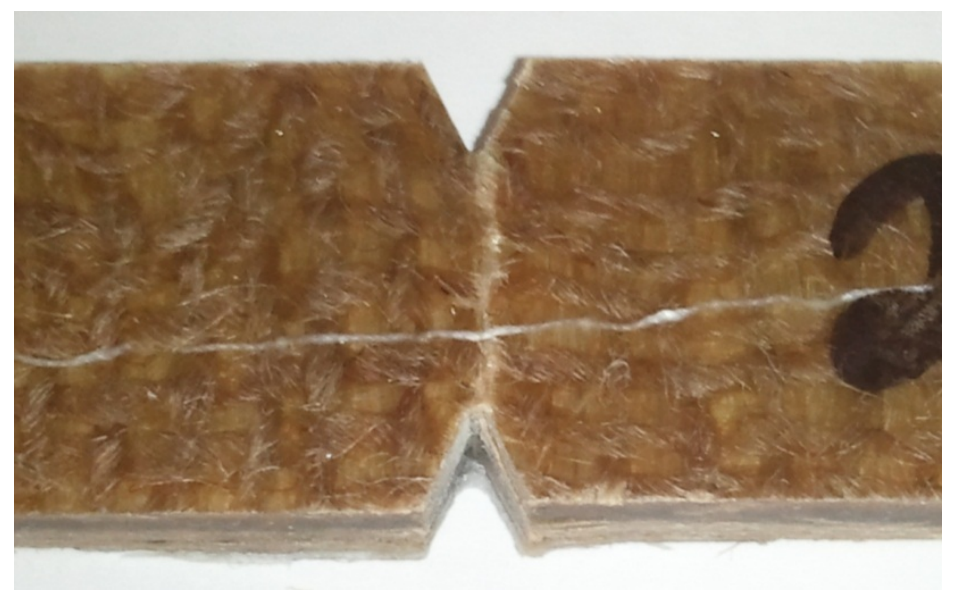

Figure 5-39 - Typical kenaf reinforced composite failure between notches, as seen on fabric surface

\subsubsection{Specific Properties}

A compilation from the previous sections of the average, dry-condition strength and stiffness properties obtained for the glass, untreated kenaf, and kenaf reinforced composites is shown in Table 5-3. From the data in this table, it appears that there is no clear advantage in mechanical properties between composites reinforced with the untreated or treated kenaf fibers. It also appears that, with the exception of shear strength, the composites reinforced with glass fibers exceed the performance of the kenaf reinforced composites, albeit sometimes by only a narrow margin.

Table 5-3 - Comparison of dry, normalized FVF mechanical properties

\begin{tabular}{|l|c|c|c|c|c|c|}
\hline \multicolumn{1}{|c|}{ Property } & \multicolumn{2}{c|}{ Glass } & \multicolumn{2}{c|}{ Untreated Kenaf } & \multicolumn{2}{c|}{ Treated Kenaf } \\
\hline Units & MPa & ksi & MPa & ksi & MPa & ksi \\
\hline Flexural Strength & 289 & 42.0 & 183 & 26.6 & 201 & 29.1 \\
\hline Tensile Strength & 307 & 44.5 & 176 & 25.6 & 160 & 23.3 \\
\hline Shear Strength & 41.4 & 6.01 & 50.8 & 7.37 & 35.0 & 5.07 \\
\hline Compressive Strength & 131 & 19.1 & 115 & 16.7 & 117 & 17.0 \\
\hline Units & GPa & Msi & GPa & Msi & GPa & Msi \\
\hline Flexural Stiffness & 11.44 & 1.66 & 7.42 & 1.08 & 7.59 & 1.10 \\
\hline Tensile Stiffness & 28.33 & 4.11 & 22.66 & 3.29 & 22.83 & 3.31 \\
\hline Shear Stiffness & 3.34 & 0.48 & 2.67 & 0.39 & 2.19 & 0.32 \\
\hline Compressive Stiffness & 29.73 & 4.31 & 22.89 & 3.32 & 23.52 & 3.41 \\
\hline
\end{tabular}

However, when this data is examined from the viewpoint of specific properties, as in the mechanical properties divided by the respective composite density, the results are quite different. This is due to the fact that the glass fibers have a much higher density than the kenaf fibers (around $2.59 \mathrm{~g} / \mathrm{cc}$ as compared to $1.4 \mathrm{~g} / \mathrm{cc}$ ). As a result, the glass fiber reinforced composites tend to have a density (at $40 \% \mathrm{FVF}$ ) of around $1.76 \mathrm{~g} / \mathrm{cc}$, while the kenaf reinforced composites tend to have a density of around $1.30 \mathrm{~g} / \mathrm{cc}$. The specific properties are calculated by dividing the 
properties in Table 5-3 by the different materials' respective densities, and are shown in Table 5-4.

From these weight-adjusted values, it appears that the kenaf reinforced composite materials are very competitive with the glass reinforced composite materials, with the glass only holding an advantage in tensile strength and, to a lesser extent, in flexural strength and stiffness. When the mechanical results are viewed from this viewpoint, it may be concluded that structural members manufactured with kenaf fibers would, pound for pound, possess mechanical properties in dry conditions that are comparable to those manufactured with glass fibers.

Table 5-4 - Comparison of specific properties (strength or stiffness divided by composite density)

\begin{tabular}{|c|c|c|c|c|c|c|}
\hline Property & \multicolumn{2}{|c|}{ Glass } & \multicolumn{2}{|c|}{ Untreated Kenaf } & \multicolumn{2}{|c|}{ Treated Kenaf } \\
\hline Units* & [1] & [2] & [1] & [2] & [1] & [2] \\
\hline Flexural Strength & 165 & 41 & 141 & 35 & 154 & 39 \\
\hline Tensile Strength & 175 & 44 & 135 & 34 & 123 & 31 \\
\hline Shear Strength & 24 & 5.9 & 39 & 9.8 & 27 & 6.7 \\
\hline Compressive Strength & 75 & 19 & 89 & 22 & 90 & 23 \\
\hline Units* & [3] & [4] & [3] & [4] & [3] & [4] \\
\hline Flexural Stiffness & 6.5 & 1.6 & 5.7 & 1.4 & 5.8 & 1.5 \\
\hline Tensile Stiffness & 16.1 & 4.0 & 17.4 & 4.4 & 17.5 & 4.4 \\
\hline Shear Stiffness & 1.9 & 0.5 & 2.0 & 0.5 & 1.7 & 0.4 \\
\hline Compressive Stiffness & 16.9 & 4.2 & 17.6 & 4.4 & 18.0 & 4.5 \\
\hline
\end{tabular}

\subsection{Conclusions}

A comparison was made between the mechanical properties of an untreated kenaf fiber reinforced composite, a chemically treated kenaf fiber reinforced composite, a glass fiber reinforced composite, and OSB. The material strengths and stiffnesses in the loading modes of flexure, tension, compression, and shear were all evaluated. In addition to evaluating the dry mechanical properties, the mechanical properties after 50 hours of immersion and at saturation were also evaluated, since a major concern with natural fiber reinforced composites is their tendency to absorb moisture. All of the fiber reinforced composite results were normalized to a fiber volume fraction of $40 \%$ for direct comparison purposes.

There is generally little difference between the untreated kenaf reinforced composites and the treated kenaf reinforced composites when only the dry results are examined; neither material appears to have a significant and consistent advantage when the flexure, tension, compression, and shear properties are all considered together. However, there does appear to be a difference between the two materials once they have been immersed in water for between 50 and 2700 hours. While the untreated kenaf samples ended up absorbing more moisture overall, the treated kenaf samples reached their saturation point more quickly and tended to have greater reductions in their mechanical properties when tested after immersion conditioning. This is likely due to the treated kenaf samples having an improved chemical compatibility with the resin and thus having reduced gaps at the fiber-matrix interface; the untreated kenaf, on the other hand, would have had more room for the fibers to swell before the matrix was damaged. 
Another trend observed in several of the tests was the tendency for the kenaf samples exposed to moisture to have reduced stiffnesses corresponding to increased strains to failure. This is also likely directly related to the fiber swelling issue, as moisture would not only cause some degree of matrix plasticization, but would also cause the microfibrillar angle of the natural fibers to increase, reducing overall stiffness but increasing ductility.

On average, the bidirectional kenaf reinforced composite had a flexural strength and stiffness of around $28 \mathrm{ksi}$ and $1.09 \mathrm{Msi}$, respectively, compared to $42 \mathrm{ksi}$ and $1.66 \mathrm{Msi}$ for the glass reinforced composite. If immersed in water to the point of saturation, the kenaf reinforced composites would typically only possess around $60 \%$ of that flexural strength and around $80 \%$ of that flexural stiffness.

On average, the unidirectional kenaf reinforced composite had a tensile strength, stiffness, and ultimate strain of around $25 \mathrm{ksi}, 3.3 \mathrm{Msi}$, and 8,000 $\mu \varepsilon$, respectively, compared to $44 \mathrm{ksi}, 4.11 \mathrm{Msi}$, and 10,000 $\mu \varepsilon$ for the glass reinforced composite. If immersed in water to the point of saturation, the kenaf reinforced composites would typically only possess around $78 \%$ of that tensile strength and around $55 \%$ of that tensile stiffness, with around a $25 \%$ increase in the ultimate tensile strain.

On average, the unidirectional kenaf reinforced composite had a compressive strength, stiffness, and ultimate strain of around $17 \mathrm{ksi}, 3.35 \mathrm{Msi}$, and 13,000 $\mu \varepsilon$, respectively, compared to $19 \mathrm{ksi}, 4.31 \mathrm{Msi}$, and 5,000 $\mu \varepsilon$ for the glass reinforced composite. If immersed in water to the point of saturation, the kenaf reinforced composites would typically only possess around $50 \%$ of that compressive strength and around $72 \%$ of that compressive stiffness, with around a $100 \%$ increase in the ultimate compressive strain.

On average, the bidirectional kenaf reinforced composite had an in-plane shear strength, stiffness, and ultimate strain of around $6 \mathrm{ksi}, 0.35 \mathrm{Msi}$, and 30,000 $\mu \varepsilon$, respectively, compared to $6 \mathrm{ksi}, 0.48 \mathrm{Msi}$, and $48,000 \mu \varepsilon$ for the glass reinforced composite. If immersed in water to the point of saturation, the kenaf reinforced composites would typically only possess around $60 \%$ of that shear strength and around 32\% of that shear stiffness, with around a 67\% increase in the ultimate shear strain.

When the above mechanical properties are viewed as specific properties, by dividing the strength or stiffness by the density of the material, the kenaf reinforced composites possess very similar values to those of the glass reinforced composites for nearly every property. The kenaf fiber reinforced composites cannot reasonably even be compared to the OSB, as the composites' mechanical properties and moisture performance are typically many times better than the OSB properties. In future work, testing against high-quality plywood or other engineered wood would provide a better comparison for the natural fiber reinforced composites.

In terms of their suitability for structural applications, these results show that a kenaf reinforced composite structural component would provide very competitive strength and stiffness properties when compared to a glass reinforced composite structural component of the same weight. Additionally, while reducing the moisture content is still a major concern, the reduction is not so severe as to limit the materials from being used in applications where no long term moisture exposure is expected to occur, similar to the way OSB and many other engineered wood products are used today. 


\section{WEATHERING AND ENVIRONMENTAL TESTING}

\subsection{Abstract}

Since natural fiber composites are more vulnerable to environmental effects than synthetic fiber composites, a series of weathering and environmental tests were conducted on kenaf fiber reinforced composites, and the results are compared to those of glass FRP and OSB. The environmental conditions studied include real-time outdoor weathering, elevated temperatures, immersion in different $\mathrm{pH}$ solutions, and UV exposure. In all of these tests, some amount of degradation was found in the kenaf fiber reinforced composites; however, in almost every case the degradation was not greater than $50 \%$ of the flexural strength or stiffness. Through these tests, it was found that exposure to elevated temperatures (up to $150^{\circ} \mathrm{F}$ ) had very little effect on the kenaf FRP composites, and that immersion in different $\mathrm{pH}$ solutions only resulted in slight differences in flexural performance (with amount of degradation in the order of alkali > water > acid). The UV exposure testing for 1000 hours was found to produce results similar to the year-long outdoor exposure testing, and may be a suitable accelerated aging test for future research work on kenaf FRPs.

\subsection{Introduction and Scope}

The use of natural fiber reinforced composites in infrastructure applications presents a number of aging and environmental challenges. Natural fibers do not possess the same environmental resistance characteristics of glass or carbon fibers, although proper surface treatments and a good fiber/matrix interface can potentially significantly improve their environmental durability. While natural fiber reinforced composites will most likely be restricted in their usage to interior structural applications (at least in early stages of development) they must still be able to survive some environmental exposure during construction as well as accidental or catastrophic short-term exposures without significant and unexpected reductions in their mechanical properties.

While one of the most effective ways to analyze a material's weathering and aging performance is to place it in an outdoor environment and allow it to age in real-time, this is obviously not the most efficient way to assess a material's durability, especially during new product development. A number of "accelerated aging" methods can be used to simulate one or more characteristics of real-time aging in an outdoor environment, such as testing in wet conditions, acidic or alkali conditions, elevated temperatures, or in a controlled UV exposure chamber. For example, while it is likely that, in end-use, a construction material will not ever be fully immersed in a strong alkali solution, a test in which the material is immersed in a strong alkali can simulate the accumulation of years of salt spray in just a matter of days. Several samples made of the kenaf materials introduced in the previous chapters, as well as glass fiber reinforced composites and OSB were tested in these environmental conditions, in addition to real-time outdoor weathering conditions. Most of the elevated temperature and varying $\mathrm{pH}$ environmental condition tests were conducted by P.K.R. Majjigapu as a part of his Master's research work at WVU, and a more extensive review of those procedures and results can be found in his problem report (Majjigapu 2013). 


\subsection{Materials and Manufacturing Processes}

\subsubsection{Materials}

The loose kenaf fibers discussed in the previous chapters were cut, cleaned, combed, and stitched to a light bidirectional kenaf fabric (at a weight ratio of around 5-to-1 fibers to fabric) in order to create essentially unidirectional kenaf fiber mats.

A roll of unidirectional E-glass fibers loosely stitched (stitching not intended as reinforcement) in the transverse direction was used for the manufacture of the glass reinforced composite samples.

The resin system used for all of the composites in this chapter was Ashland's Derakane 510A vinyl ester, as discussed in the previous chapters. A MEKP catalyst and a Cobalt Naphthenate promoter were added to the resin at concentrations of $0.75 \%$ weight and $0.25 \%$ weight, respectively. Vinyl ester has been shown to weather environmental effects, such as moisture, alkaline, and saline environments, both at ambient and elevated temperatures, with only minor changes in the glass transition temperature, whereas other resins, such as polyester, degraded badly in some conditions (Chin et al. 1998).

The OSB used was a standard $1 / 4$ inch utility panel purchased from a local hardware store.

As a secondary test to demonstrate the potential use of coatings on natural fiber reinforced composites, some of the treated kenaf samples were painted with a urethane protective coating before undergoing the weathering and accelerated aging tests. The urethane coating used was Minwax Helmsman Spar Urethane, and was applied according to the manufacturer's instructions on the product label.

\subsubsection{Fiber Treatment}

As discussed at length in the previous chapters, the untreated kenaf fibers were simply cut and combed before stitching. The treated kenaf fibers, on the other hand, were immersed in $5 \% \mathrm{NaOH}$ for 40 minutes, rinsed in 5\% acetic acid (white vinegar), rinsed in distilled water, and then oven dried at $160^{\circ} \mathrm{F}$ for 6-8 hours. After drying, the fibers were combed before stitching.

No surface treatments were applied to the glass fibers or OSB panels.

\subsubsection{Sample Preparation}

Before manufacturing the fiber reinforced composites, each of the fiber mats was dried in an oven at $160^{\circ} \mathrm{F}$ for 1 hour and sealed in a plastic bag. Due to each unidirectional kenaf fiber bundle being stitched to a piece of fabric, the [0/90] layup used for all of the tests in this chapter was actually [0/fabric/90/fabric], or $\left[(0)_{4} /\right.$ fabric/(90) 4 /fabric], accounting for relative quantities by weight. The plates manufactured with glass fibers followed the same basic structure as the kenaf mats, just without the fabric layers.

The 10 inch by 15 inch kenaf and glass reinforced composite plates were all manufactured by heated compression molding. Technick Products Techlube 25 was used as the mold release agent and all of the steel mold parts were covered with aluminum foil to ease the removal of the plate from the mold. The top plate was free floating within the inside edge of the side bars in order to provide the best possible compression, albeit at the expense of consistent plate thickness. The resin was applied to each of the fiber mats by hand and spread evenly with a 
paintbrush. Once installed in the compression molding device, the composite mat was compressed to around 40 psi and raised to $150^{\circ} \mathrm{F}$ for 2 hours. After 2 hours, the heating elements were switched off and pressure was maintained for 24 additional hours.

Due to the structure of the glass fibers (tightly packed bundles of unidirectional fibers loosely stitched from one to another) and the decision to use a mold which applied constant pressure rather than a constant thickness, more of the resin was squeezed out of the mold during the manufacture of the glass fiber reinforced composites. This led to glass fiber volume fractions more on the order of $50-55 \%$ (and, as a result, thinner plates) instead of the $35-40 \%$ achieved with kenaf fibers using the exact same fiber volumes and molding parameters. Because of this inconsistency, nearly all of the mechanical testing results in this chapter are divided by their respective fiber volume fraction and then multiplied by $40 \%$ (an average VF) in order to normalize the results for a better comparison of properties.

For nearly every test material (glass, OSB, untreated kenaf, treated kenaf) at each test condition, three samples were tested and the results were averaged. All of the coupons were cut from larger plates using a table saw. A carbide blade was used to cut the OSB and natural fiber reinforced samples and a diamond-tipped blade was used to cut the glass reinforced samples. Unless otherwise noted, all of the samples were cut, stored, and tested at room temperature (65$80^{\circ} \mathrm{F}$ ) and typical indoor humidity (50-60\% RH).

The coupons were cut according to the guidelines ASTM D 790, as they were all tested in flexure. In general, the dimensions used were 5 inches long by 0.5 inch wide by the thickness (between 0.11-0.30 inches). No strain gages are required for flexural modulus calculations.

\subsection{Experimental Methodology}

\subsubsection{Real-time Weathering Tests}

In order to assess the actual weathering ability of the natural fiber reinforced composites instead of just relying on accelerated aging methods and approximations, 210 samples were placed in an outdoor location for 12 months.

\subsubsection{Test Apparatus}

An aluminum fixture was built with a series of rails that would allow the 5-inch samples to be slid into place and securely fastened with an individual rail clamp for each row (shown in Figure 6-1). The fixture was built to hold the samples at an angle of $15^{\circ}$ from horizontal to avoid pooling from precipitation, and a spacer built into the rails allows for easy water drainage.

An Omega temperature and humidity datalogger was hung from one corner of the test fixture, and set to take a single temperature reading each hour. The device needs to be left open in order to collect humidity readings, but since it was not designed to be left open for prolonged outdoor use, the humidity measurements were not taken. 


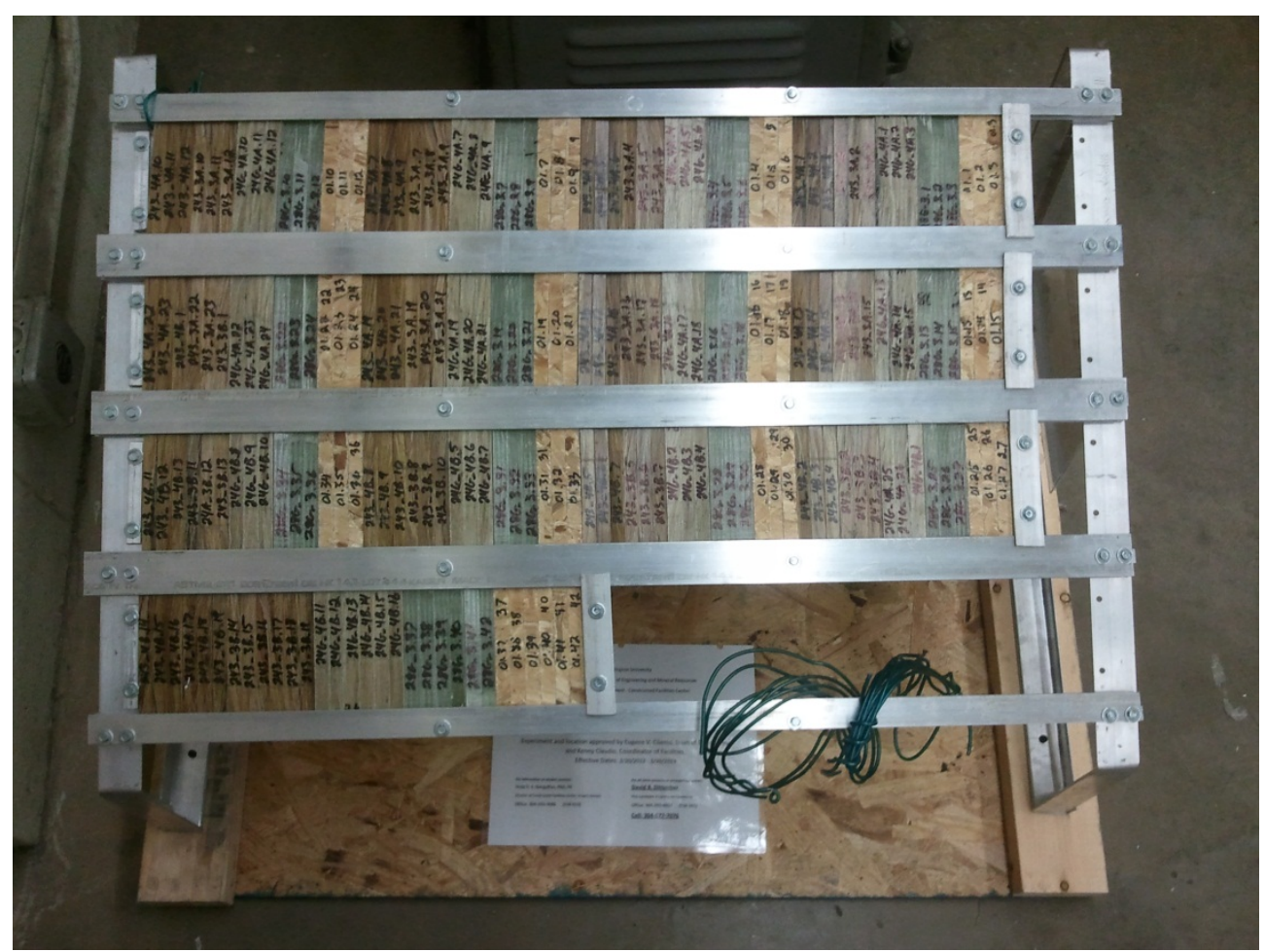

Figure 6-1 - Real-time weathering test fixture

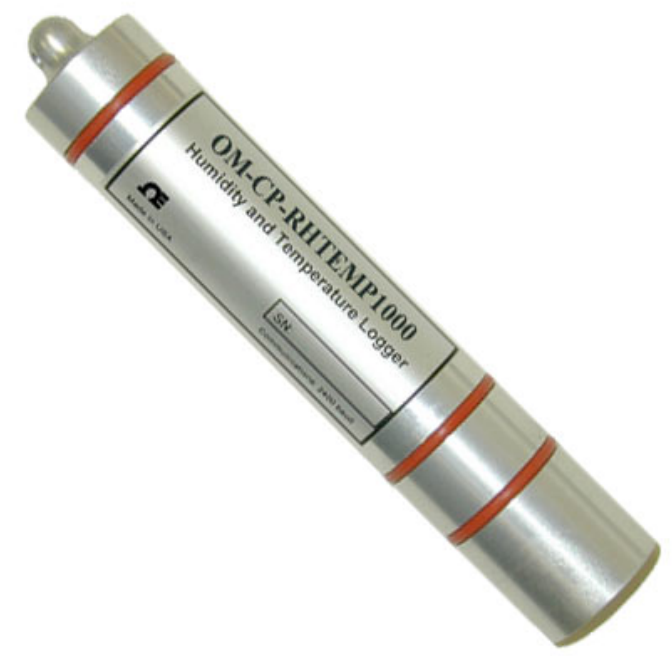

Figure 6-2 - Omega temperature datalogger (image from www.omega.com)

The fixture was loaded with the samples and secured in a rooftop location on the WVU Engineering Science Building (Evansdale Campus, Morgantown, WV), facing south. None of the surrounding structures impeded precipitation or sunlight (except for perhaps just before twilight) from reaching the samples. The temperature datalogger was used in place of local 
meteorologist highs and lows due to the potentially higher temperatures at the sample location from the surrounding roofing material.

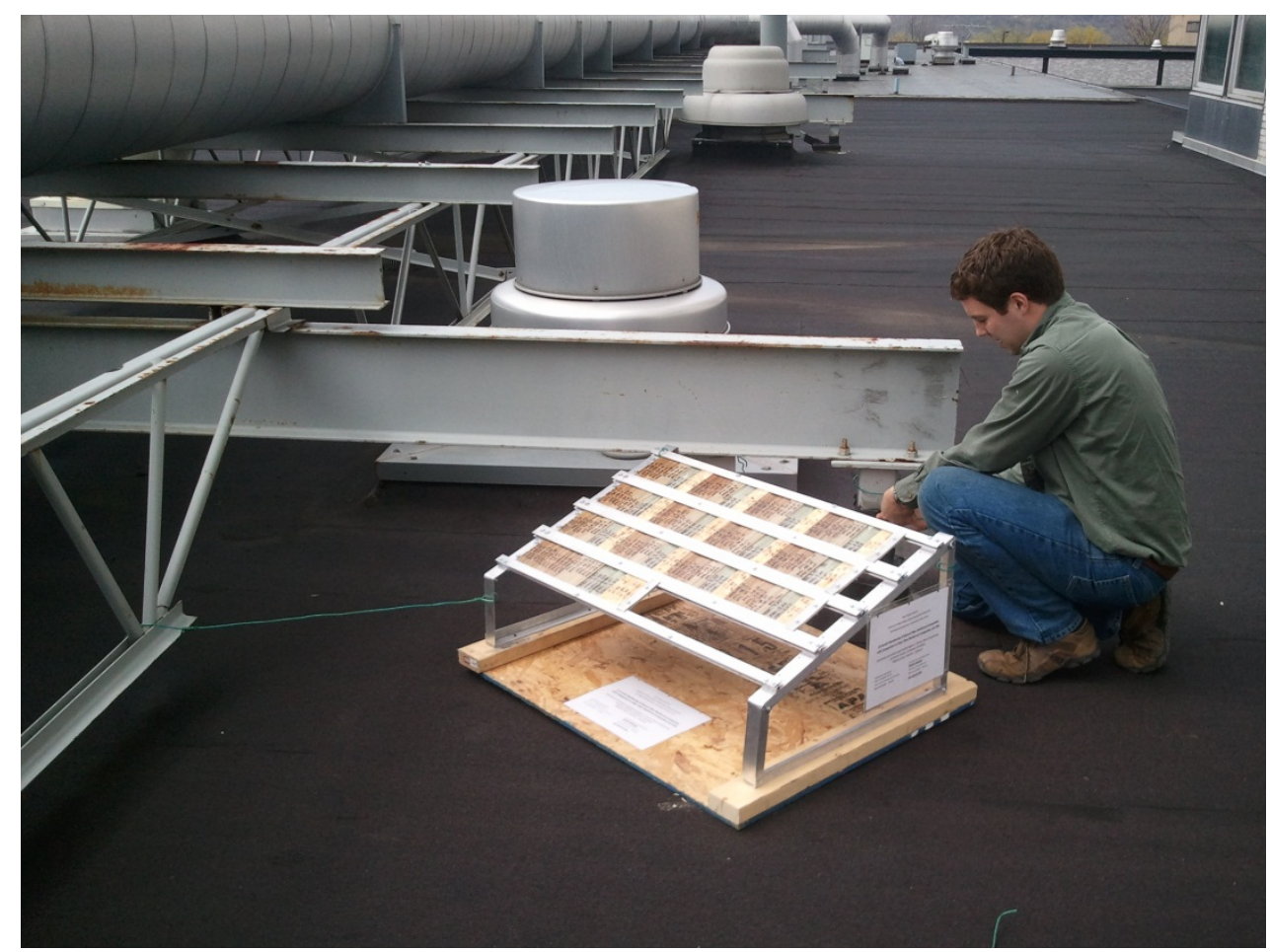

Figure 6-3 - Real-time weathering test fixture installed on roof-top location

\subsubsection{Test Procedure}

The fixture was installed on the rooftop on March 30, 2012 at approximately 11:00 AM. For twelve, one-month increments, a photograph was taken of the entire fixture and three samples of each material type (untreated kenaf composite, treated kenaf composite, treated and coated kenaf composite, glass fiber composite, and OSB) were removed from the fixture. The hourly temperature readings from the Omega datalogger were also recorded each time some samples were removed.

Each of the samples that were removed from the fixture was visually inspected, photographed, weighed, and measured to assess any swelling or weight gain/loss that may have occurred. Finally, each sample was tested in flexure on the same day as removal from the fixture, and the average flexural strength and stiffness of each material was recorded.

In addition to the temperature datalogger recordings, daily meteorologist temperatures (for comparison), humidity, and precipitation readings were recorded from the Morgantown Airport, approximately 3 miles from the test site. 


\subsubsection{Accelerated Aging Tests}

\subsubsection{Elevated Temperature Effects}

For the elevated temperature testing, samples were placed in a thermal chamber preheated to either $100^{\circ} \mathrm{F}\left(38^{\circ} \mathrm{C}\right)$ or $150^{\circ} \mathrm{F}\left(66^{\circ} \mathrm{C}\right)$, and left in the chamber for various times, ranging from 3 hours to 12 days. Three samples of each material were tested for each of the temperatures and each of the durations. After removal from the chamber, the samples were allowed to cool to room temperature, and were tested within a few days. While this procedure perhaps does not accurately portray the performance of the material at an elevated temperature, it should still be useful for assessing whether permanent damage was incurred. More details on the elevated temperature testing methodology can be found in the problem report by Majjigapu (2013).

\subsubsection{2 pH Environmental Effects}

For the $\mathrm{pH}$ testing, an acidic solution was prepared from muriatic acid ( $\mathrm{HCl})$ purchased from Lowes, and an alkali solution was prepared from sodium hydroxide $(\mathrm{NaOH})$ micropearls purchased from Fisher Scientific. The acid was mixed with water until a pH of 3.0 was reached, and the $\mathrm{NaOH}$ micropearls were mixed with water until a $\mathrm{pH}$ of 13.0 was reached. Samples were then immersed in the solutions for the duration of the tests, except for periodical removal to record weight changes. One set of samples from each solution was removed after 50 hours, while the second set was allowed to approximately reach saturation (1200 hours). Three samples of each material were tested in each of the solutions and for each of the durations. The removed samples were rinsed in water, patted dry, and tested in flexure within a day of removal. The flexural test results were also compared to the flexural tests of samples immersed in water for 50 hours and until saturation from previous testing (see Chapters 4 and 5). More details on the $\mathrm{pH}-$ immersion testing methodology can be found in the problem report by Majjigapu (2013).

\subsubsection{UV Exposure Effects}

The UV exposure testing was conducted by the quality control lab at Bedford Reinforced Plastics (BRP), Bedford, PA. The test methodology followed the Cycle A procedure of ASTM D4329 using a fluorescent UVA-340 lamp and UV testing apparatus. Each test cycle consisted of 8 hours of UV exposure at $140^{\circ} \mathrm{F}\left(60^{\circ} \mathrm{C}\right)$ followed by 4 hours of condensation via water spraying at $122^{\circ} \mathrm{F}\left(50^{\circ} \mathrm{C}\right)$. For each of the materials tested, one sample each was tested for 500 hours (41.7 cycles), 1000 hours (83.3 cycles), and 1500 hours (125 cycles).

\subsection{Results and Discussion}

\subsubsection{Real-time Weathering Tests}

\subsubsection{Weathering Conditions}

The weather conditions recorded for the tests were a combination of on-site measurements (temperature, recorded hourly) and meteorology records (humidity and precipitation, accessed on weatherunderground.com). On average, the temperatures recorded at the site had maximum values that were $4.4^{\circ} \mathrm{F}$ higher than the meteorology records, minimum values that were $2.6^{\circ} \mathrm{F}$ higher, and average values that were $1.9^{\circ} \mathrm{F}$ higher, likely due to local heating from the surrounding roofing material and full exposure to the sun. The locally recorded 
temperatures and meteorology published precipitation values are compared to Morgantown's historical averages in Table 6-1. Accounting for the slightly higher local values, as explained above, the testing year of 2012-2013 had fairly average temperatures, although it was a dry year, with only around $80 \%$ of the typical precipitation.

Table 6-1 - Weathering conditions during experiment compared to Morgantown historical averages

\begin{tabular}{|c|c|c|c|c|c|c|c|c|}
\hline Morgantown, WV & \multicolumn{4}{|c|}{ Actual Conditions } & \multicolumn{4}{|c|}{ Historical Average Conditions* } \\
\hline Month & $\begin{array}{c}\text { Temp } \\
\text { Avg }\left({ }^{\circ} F\right)\end{array}$ & $\begin{array}{c}\text { Temp } \\
\text { Hi }\left({ }^{\circ} \mathbf{F}\right)\end{array}$ & $\begin{array}{l}\text { Temp } \\
\text { Lo }\left({ }^{\circ} \mathbf{F}\right)\end{array}$ & $\begin{array}{l}\text { Precip. } \\
\text { (in) }\end{array}$ & $\begin{array}{c}\text { Temp } \\
\operatorname{Avg}\left({ }^{\circ} F\right)\end{array}$ & $\begin{array}{l}\text { Temp } \\
\text { Hi }\left({ }^{\circ} F\right)\end{array}$ & $\begin{array}{c}\text { Temp } \\
\text { Lo }\left({ }^{\circ} \mathbf{F}\right)\end{array}$ & $\begin{array}{l}\text { Precip. } \\
\text { (in) }\end{array}$ \\
\hline April '12 & 57 & 71 & 47 & 1.40 & 52 & 64 & 39 & 3.59 \\
\hline May '12 & 70 & 85 & 59 & 3.72 & 61 & 72 & 49 & 4.57 \\
\hline June '12 & 73 & 88 & 61 & 1.38 & 70 & 80 & 59 & 4.15 \\
\hline July ‘12 & 78 & 93 & 68 & 4.02 & 73 & 83 & 63 & 4.38 \\
\hline August '12 & 75 & 91 & 63 & 2.58 & 72 & 82 & 62 & 3.42 \\
\hline September '12 & 67 & 81 & 57 & 3.30 & 66 & 76 & 55 & 3.16 \\
\hline October '12 & 57 & 68 & 48 & 5.84 & 54 & 65 & 43 & 2.82 \\
\hline November '12 & 44 & 56 & 35 & 0.65 & 44 & 54 & 34 & 3.57 \\
\hline December '12 & 42 & 49 & 35 & 5.25 & 34 & 42 & 25 & 3.33 \\
\hline January '13 & 37 & 44 & 30 & 3.03 & 30 & 39 & 21 & 3.26 \\
\hline February '13 & 34 & 42 & 26 & 1.43 & 33 & 43 & 23 & 3.05 \\
\hline March '13 & 39 & 48 & 31 & 2.24 & 41 & 52 & 29 & 3.94 \\
\hline April-March & 56 & 69 & 47 & 34.95 & 53 & 63 & 42 & 43.24 \\
\hline
\end{tabular}

* From www.weather.com

Since flexural testing of the samples after removal from the weathering fixture was the main metric for determining material weathering resistance, the weathering conditions on the day of removal along with the four preceding days are of greater importance, since higher moisture content has been shown to lead to reduced flexural behavior. The same-day accumulated precipitation and average temperatures as well as the 5-day accumulated precipitation and average temperatures are shown in Table 6-2. Also included in this table are the total accumulated freeze-thaw cycles experienced by the samples before testing. A freeze-thaw cycle was considered to have occurred when the minimum recorded temperature of one day was $32^{\circ} \mathrm{F}$ or below and the maximum temperature of the following day was above $32^{\circ} \mathrm{F}$. The most significant outliers from this data are likely the samples tested at the end of October, following a particularly rainy five days, and the samples collected at the end of January, which had fairly high precipitation in the preceding days in combination with a fairly high number of freeze-thaw cycles. 
Table 6-2 - Precipitation and average temperature from same day as well as the four days preceding sample removal; accumulated freeze-thaw cycles for each set of samples

\begin{tabular}{|c|c|c|c|c|c|c|}
\hline $\begin{array}{l}\text { Months } \\
\text { exposed }\end{array}$ & $\begin{array}{c}\text { Previous } \\
\text { Month }\end{array}$ & $\begin{array}{l}\text { Same-Day } \\
\text { Precip. } \\
\text { (in) }\end{array}$ & $\begin{array}{l}\text { Same-Day } \\
\text { Avg. Temp. } \\
\left.\text { ( }{ }^{\circ} \mathrm{F}\right)\end{array}$ & $\begin{array}{l}\text { 5-Day } \\
\text { Precip. } \\
\text { (in) }\end{array}$ & $\begin{array}{l}\text { 5-Day } \\
\text { Avg. Temp. } \\
\left({ }^{\circ} \mathrm{F}\right)\end{array}$ & $\begin{array}{c}\text { Accumulated } \\
\text { Freeze-Thaw } \\
\text { Cycles }\end{array}$ \\
\hline 1 & April '12 & 0.00 & 71 & 0.13 & 59 & 0 \\
\hline 2 & May ‘12 & 0.00 & 72 & 0.62 & 76 & 0 \\
\hline 3 & June ‘12 & 0.36 & 84 & 0.39 & 75 & 0 \\
\hline 4 & July '12 & 0.12 & 74 & 0.55 & 75 & 0 \\
\hline 5 & August '12 & 0.27 & 75 & 0.31 & 75 & 0 \\
\hline 6 & September '12 & 0.01 & 61 & 1.14 & 61 & 0 \\
\hline 7 & October '12 & 0.76 & 37 & 4.62 & 40 & 0 \\
\hline 8 & November '12 & 0.05 & 58 & 0.17 & 47 & 12 \\
\hline 9 & December '12 & 0.00 & 29 & 0.41 & 31 & 26 \\
\hline 10 & January '13 & 0.11 & 31 & 1.73 & 45 & 40 \\
\hline 11 & February '13 & 0.03 & 35 & 0.91 & 38 & 56 \\
\hline 12 & March '13 & 0.00 & 40 & 0.42 & 36 & 72 \\
\hline
\end{tabular}

\subsubsection{Experimental Results}

After removal from the weathering fixture and before flexural testing, the samples were measured, weighed, and photographed. The \% weight changes recorded each month for the kenaf and glass reinforced samples are shown in Figure 6-4. The percent apparent density changes each month were calculated according to Equation (6-1), and for the kenaf and glass reinforced samples are shown in Figure 6-5. Photographs taken of the samples each month are included in Appendix B. Weight and density changes can occur due to many factors: moisture absorption or desorption, sample swelling, erosion, decomposition, and fungal growth.

$$
\Delta \rho=\frac{\text { mass }_{\text {new }}}{(L \times w \times t)_{\text {new }}} / \frac{\text { mass }_{\text {initial }}}{(L \times w \times t)_{\text {initial }}}-1
$$

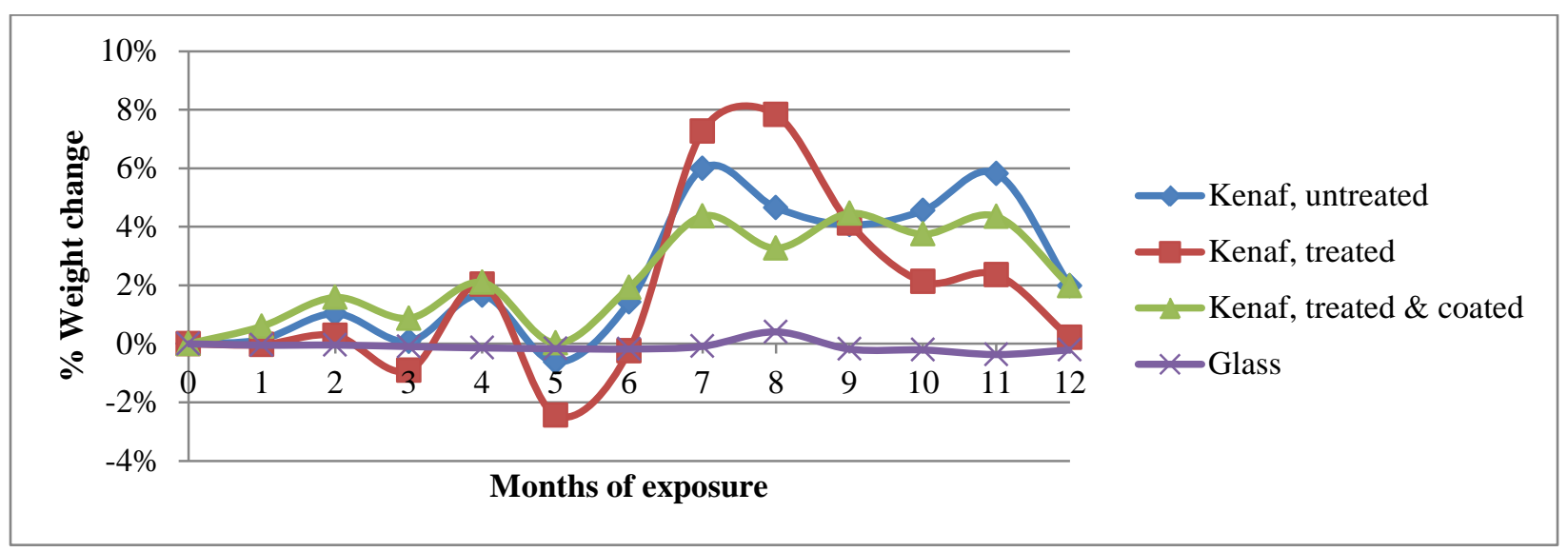

Figure 6-4 - Real-time weathering \% weight change, by month 


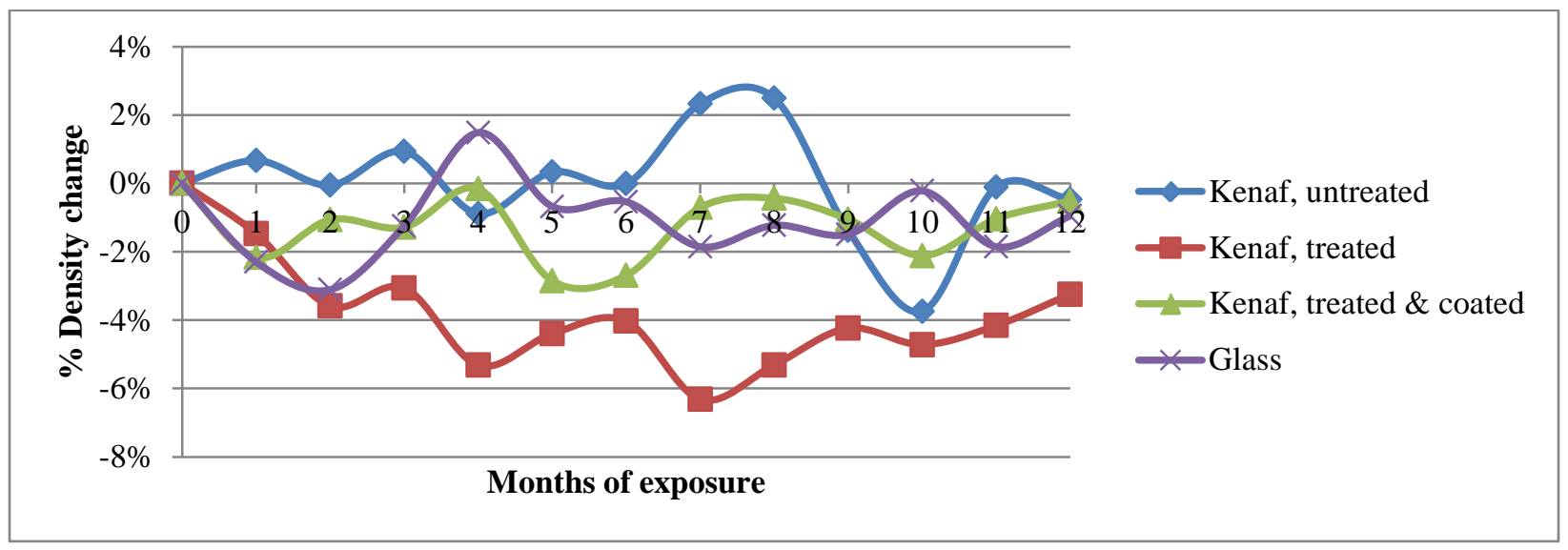

Figure 6-5 - Real-time weathering \% apparent density change, by month

The OSB samples' weight and density changes are not shown in these plots due to their variability and their existence on an entirely different scale. For example, before the high amount of rain in October, the density of the OSB samples had decreased by more than $27 \%$ while gaining around $4 \%$ weight, while after the rain their density jumped back to a decrease of only around $4 \%$ while their weight gain increased to over 50\%. Over 12 months, the OSB samples had experienced weight gains as high as 55\% and density losses of as much as $30 \%$.

Just as the OSB samples reflected a major change in weight and density as a result of a particularly wet October, so too did the natural fiber reinforced samples. All three kenafreinforced variations experienced a spike in weight gain around months 7 and 8, almost certainly due to the heavier precipitation and the cooler days slowing desorption. The small weight gain spikes in months 2 and 4 also correspond to higher rainfall in the preceding 5 days than in the surrounding months. As was seen throughout several of the other tests in this dissertation, the moisture absorption performance is generally fairly similar between the kenaf fiber reinforced samples over long durations, with the urethane coating providing some short-term moisture resistance and the untreated kenaf reaching a somewhat higher equilibrium.

Due to the inaccuracy of calculating volume based on the average of three measurements per dimension as well as the general small magnitude of the dimensions themselves, it is reasonable to conclude that an error of around $2 \%$ is common, which reduces the significance of most of the changes shown in Figure 6-5. Of particular interest, though, is the behavior of the treated, uncoated kenaf fiber samples. While the other samples tended to fluctuate in their density changes near their initial density, the treated kenaf fiber samples continued to lose density until they reached equilibrium around month 9 of about $-4 \%$. Since this curve does not correspond particularly well with Figure 6-4, it would appear that the treated kenaf fiber samples were experiencing significant volume gains largely independent of their moisture absorption. These gains could be due to some sort of fungal growth or fiber decomposition leading to swelling (for which there was also some visual evidence, to be discussed later), and are somewhat expected given that the chemical surface treatment removes the waxy, protective coating from the fibers. 
After the measurements and weights were recorded, all of the samples were tested in flexure. The flexural strength results, by month, are shown in Figure 6-6 and the flexural stiffness results are shown in Figure 6-7.

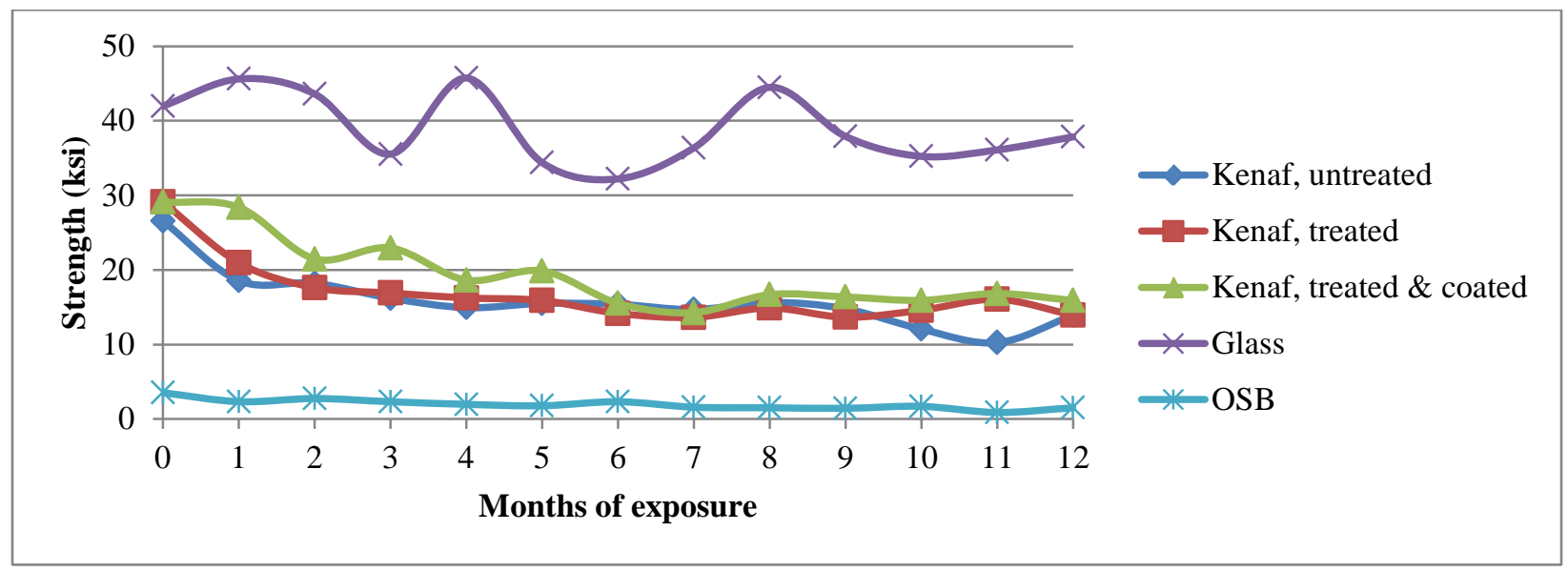

Figure 6-6 - Real-time weathering flexural strength degradation, by month

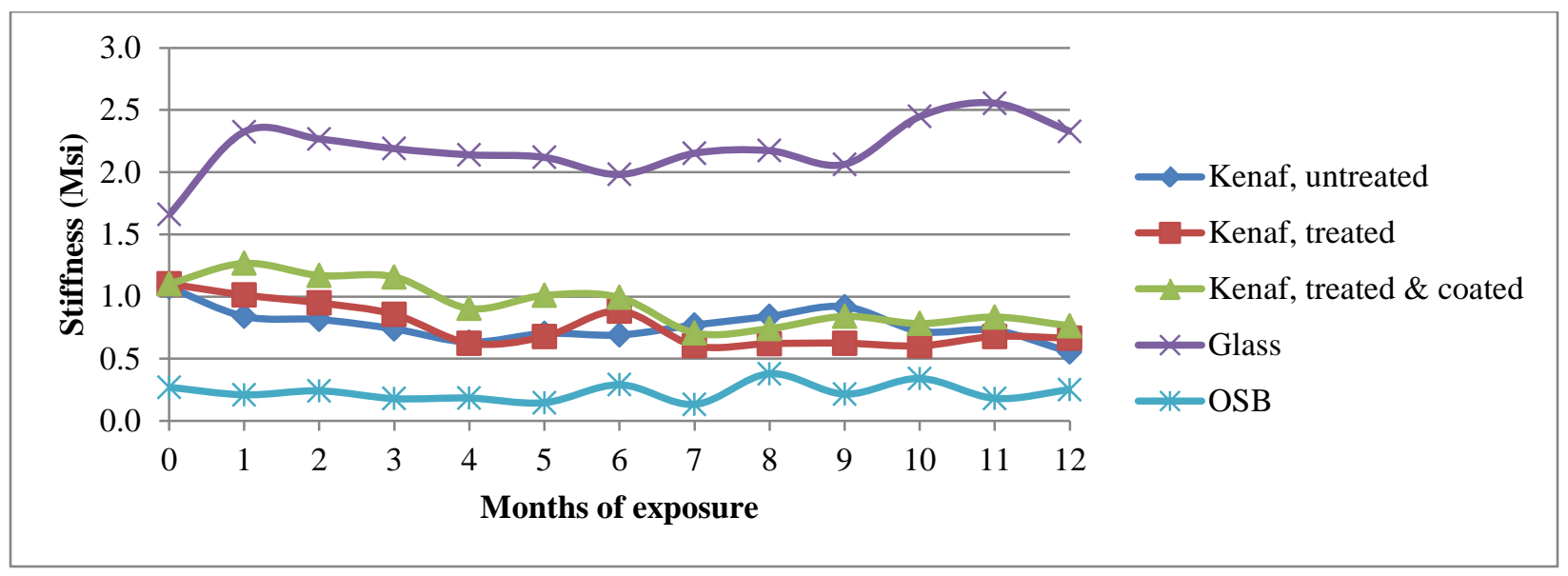

Figure 6-7 - Real-time weathering flexural stiffness degradation, by month

As was often seen during mechanical testing, the glass fiber reinforced samples tended to have some inherent variation, independent of the testing conditions, due to manufacturing errors (see previous chapter). While GFRP composites can experience weathering changes due to moisture, age curing, the presence of voids, and the type of resins system, the variations in Figure 6-6 and Figure 6-7 are within the range of data scatter found in previous testing. The one consistent conclusion that can be made from the glass FRP data is that they are significantly less affected by outdoor exposure than the kenaf reinforced composites.

The OSB showed the greatest visual indication of weathering, with the samples swelling significantly, fragmenting, and fading or darkening, depending on the precipitation leading up to the monthly removal of samples for testing (see Appendix B). While the material was clearly 
damaged, from Figure 6-7 it would appear that the stiffness increased with outdoor exposure. This conclusion is likely faulty, however, as the "initial straight line portions" of the loaddeflection plots for the OSB were essentially non-existent due to the signal noise at very low loading, making for a very low accuracy for the stiffness calculations. The high degree of thickness swelling (up to 60-70\%) also would tend to inflate the OSB strength and stiffness values, as the moment of inertia increased by over 4 times in some cases. Since strength and stiffness calculations were based on original dimensions, this increase in moment of inertia would benefit the material properties, despite the internal damage it caused.

Both the treated and untreated kenaf fiber reinforced materials exhibited fairly similar weathering behavior, with the only noticeable differences being the slightly lower strength of the untreated kenaf in the last few months and the slightly higher asymptotic stiffness of the untreated kenaf in the last 6 months of testing. This improved long-term stiffness behavior is reasonable in light of the hypothesis that the treated kenaf fibers are more susceptible to fungal growth and decomposition, as evidenced by their decreased density. The reduced long term strength of the untreated kenaf fibers is likely due to their tendency to have higher moisture absorption equilibrium, although the rate is slower, and this higher moisture content would manifest itself as increased strength reduction due to the number of freeze-thaw cycles in the last few months of testing. The urethane coating provided a significant improvement to the treated kenaf strength and stiffness, although the improvement was most obvious over the first six months and essentially negligible for strength (though stiffness was still improved) over the last six months.

All of the kenaf samples seemed to have approached an asymptotic strength and stiffness after about 6-7 months, as the tests after those months showed little additional decrease in properties. This reduced strength is at around $45 \%$ of the original strength for the untreated kenaf and $50 \%$ of the original strength for the treated and treated/coated kenaf samples, while the reduced stiffness was at around $60 \%$ of the original stiffness for the treated kenaf and $70 \%$ of the original stiffness for the untreated and treated/coated kenaf samples.

There are several factors that would lead to this long-term strength and stiffness degradation. One is matrix cracking damage due to moisture absorption and swelling, which by itself was shown to cause up to a $40 \%$ loss in strength and a $20 \%$ loss in stiffness in the previous chapter. Another is the absorption/desorption cycling, and the additional damage caused by this moisture fatiguing. Mercier et al. (2008) reported that glass/epoxy samples were not damaged during the first absorption/desorption cycle in humid air, but that this was not the case for samples exposed to immersion cycling, which seemed to have some interfacial debonding. If glass/epoxy samples experienced some interfacial debonding as a result of moisture fatiguing, the effect would be much more magnified in the high-absorbing kenaf fiber reinforced composites. With the relatively high moisture content in kenaf fiber composites, freeze-thaw cycling would introduce progressive damage. Finally, UV light can damage both the matrix and fibers, and fungal and bacterial growth can occur on the organic fibers, leading to decomposition.

Several of these damage modes were manifested visually on the surface of the kenaf composite samples. As shown in Figure 6-8, residual stresses in the 0 and 90 layers, exacerbated by moisture and temperature changes, led to splitting in the middle fabric layer at the ends of several samples. Figure 6-9 shows cracks along the longitudinal fiber layer, indicating interfacial failure due to UV degradation, moisture cycling, or the first several freeze-thaw cycles. Finally, 
an unidentified black material in the transverse layers that led to additional localized swelling was first noticed after 10 months, although only in the uncoated kenaf samples (Figure 6-10). These spots are perhaps a similar fungal hyphae to the ones found to grow along cut edges by Singh et al. (2000).

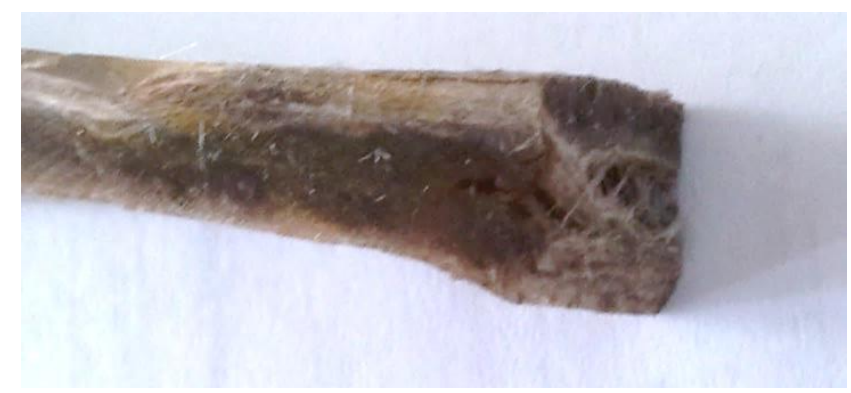

Figure 6-8 - Weathering-induced end splitting or "brooming" (9 months)

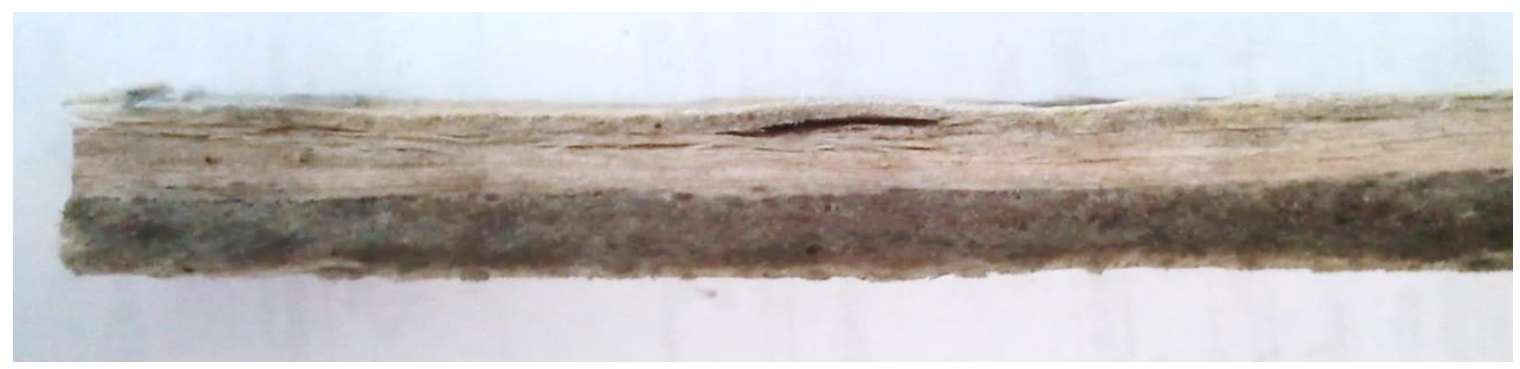

Figure 6-9 - Weathering-induced cracks along longitudinal fiber layers (9 months)

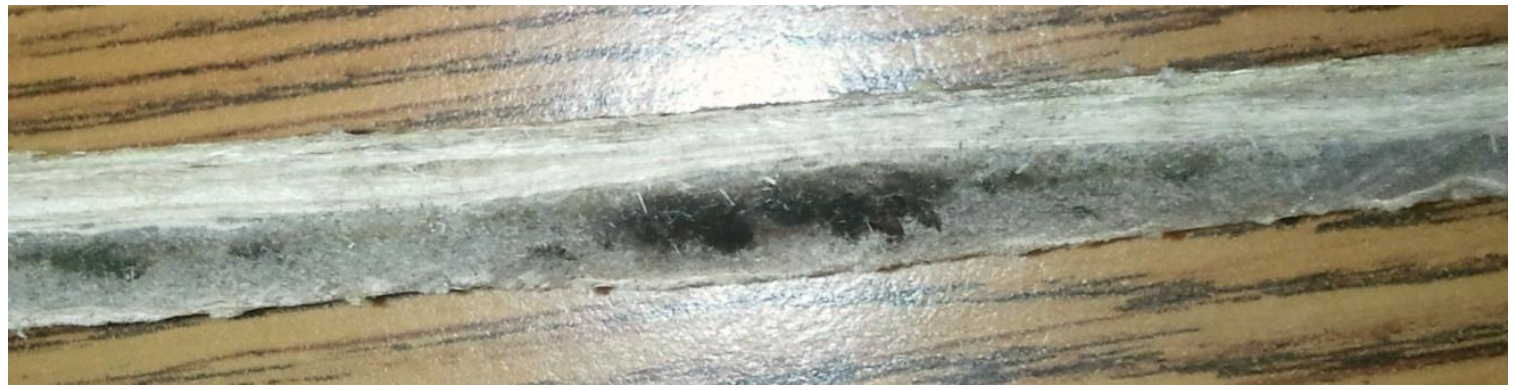

Figure 6-10 - Weathering-induced black areas with higher swelling in transverse fiber layers (11 months)

\subsubsection{Accelerated Aging Tests}

\subsubsection{Elevated Temperature Effects}

The flexural strength results of the elevated temperature tests are plotted below for the untreated kenaf (Figure 6-11), treated kenaf (Figure 6-12), and glass fiber (Figure 6-13) reinforced composites. The flexural stiffness results of the elevated temperature tests are also plotted for the untreated kenaf (Figure 6-14), treated kenaf (Figure 6-15), and glass fiber (Figure 
6-16) reinforced composites. In all of these plots, the RT baseline results are the average, normalized results from the previous chapter. Due to the relatively small sample size (3 tests averaged for each data column), the error bars indicate the maximum and minimum values obtained during testing instead of the standard deviation.

Because the samples were allowed to cool to room temperature before testing, these results do not necessarily assess the performance of the composites at elevated temperatures, but rather assess whether damage is incurred by exposing the materials to these temperatures for a certain length of time. Since the composites were manufactured at $150^{\circ} \mathrm{F}$, it was unlikely that any such damage would occur. Ochi (2008) actually found that there was little change in tensile strength in kenaf/PLA composites at temperatures less than $150^{\circ} \mathrm{C}$. The differences in the results between the composites exposed to $100^{\circ} \mathrm{F}$ and $150^{\circ} \mathrm{F}$ are largely negligible and random, indicating no significant change in mechanical behavior across that temperature range. With that in mind, and based on the general uniformity of the results, using higher temperatures for the tests or testing the samples immediately after or while still at elevated temperature would likely provide more meaningful elevated temperature data.

From the results that were obtained, however, there are a few useful conclusions. One is that, while the untreated kenaf shows no significant loss in strength due to the higher temperatures, the treated kenaf appears to have lost, on average, as much as $20 \%$ of its strength (Figure 6-12). However, since this loss is most severe for the shorter temperature durations, this loss is more likely due to manufacturing or testing errors, rather than the material's response to short-duration elevated temperatures. The glass FRP strength results once again illustrate the high variation in the glass composites manufactured as a part of this research, as discussed in the previous chapter.

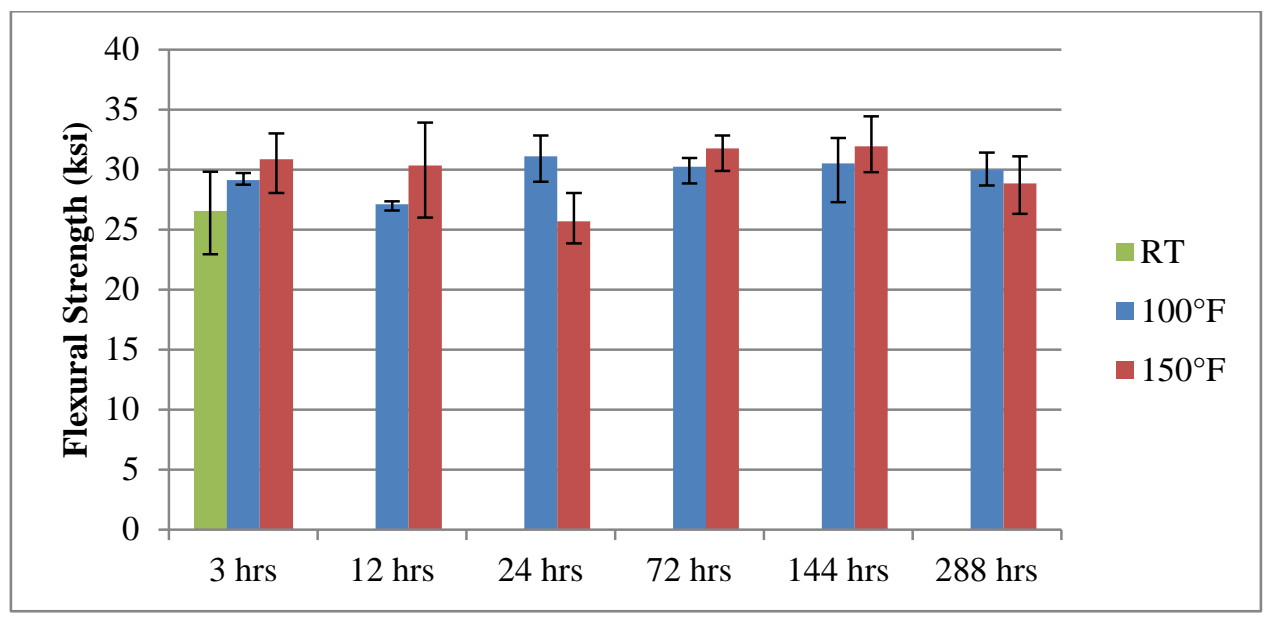

Figure 6-11 - Untreated kenaf strength vs. temperature (with max/min bars) 


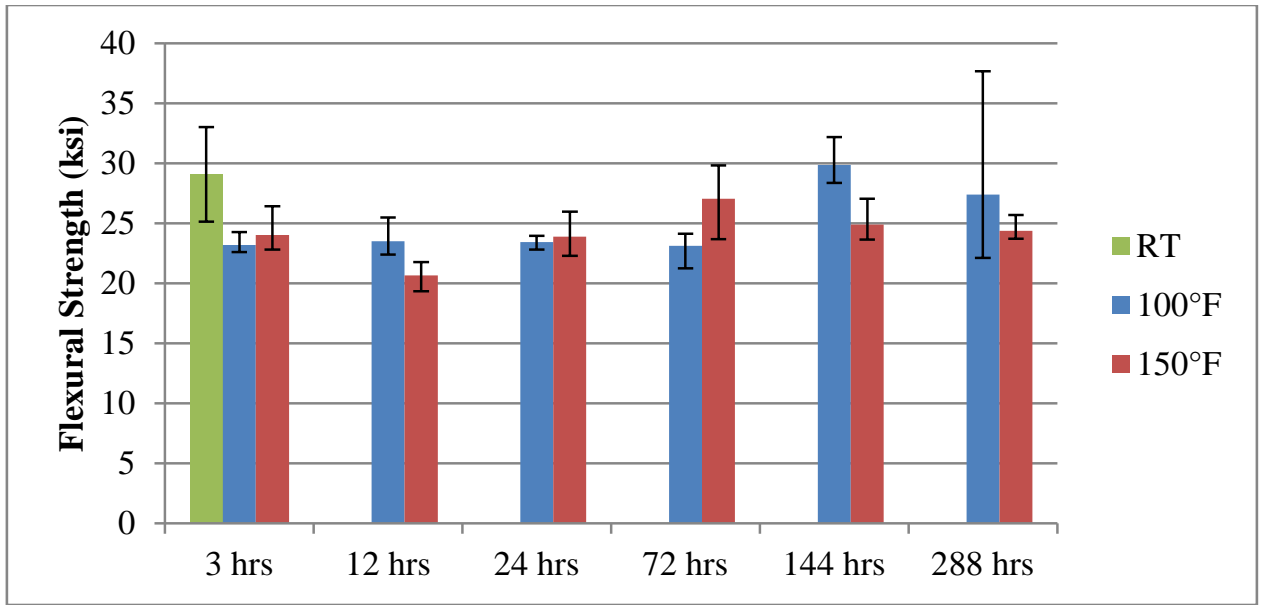

Figure 6-12 - Treated kenaf strength vs. temperature (with max/min bars)

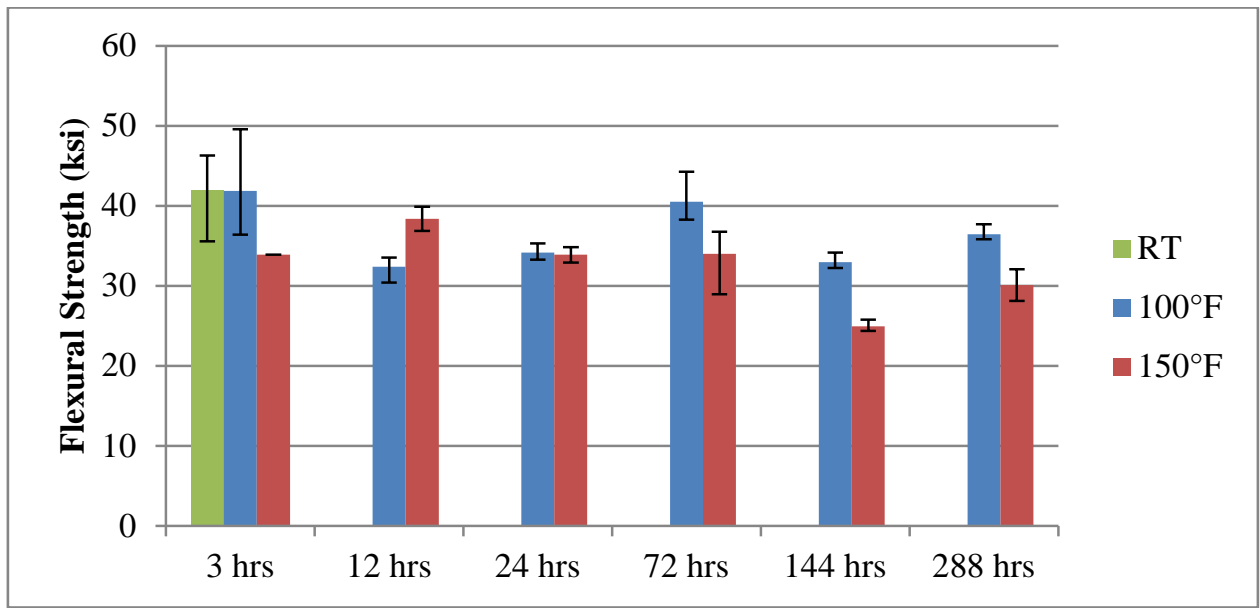

Figure 6-13 - Glass FRP strength vs. temperature (with max/min bars)

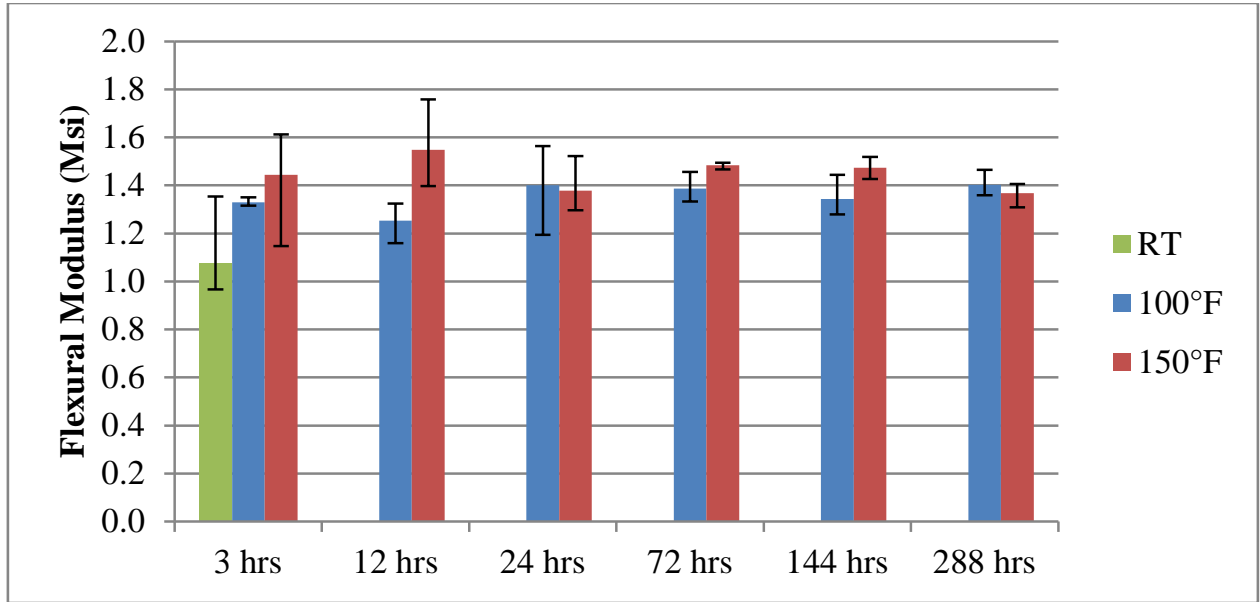

Figure 6-14 - Untreated kenaf stiffness vs. temperature (with max/min bars) 


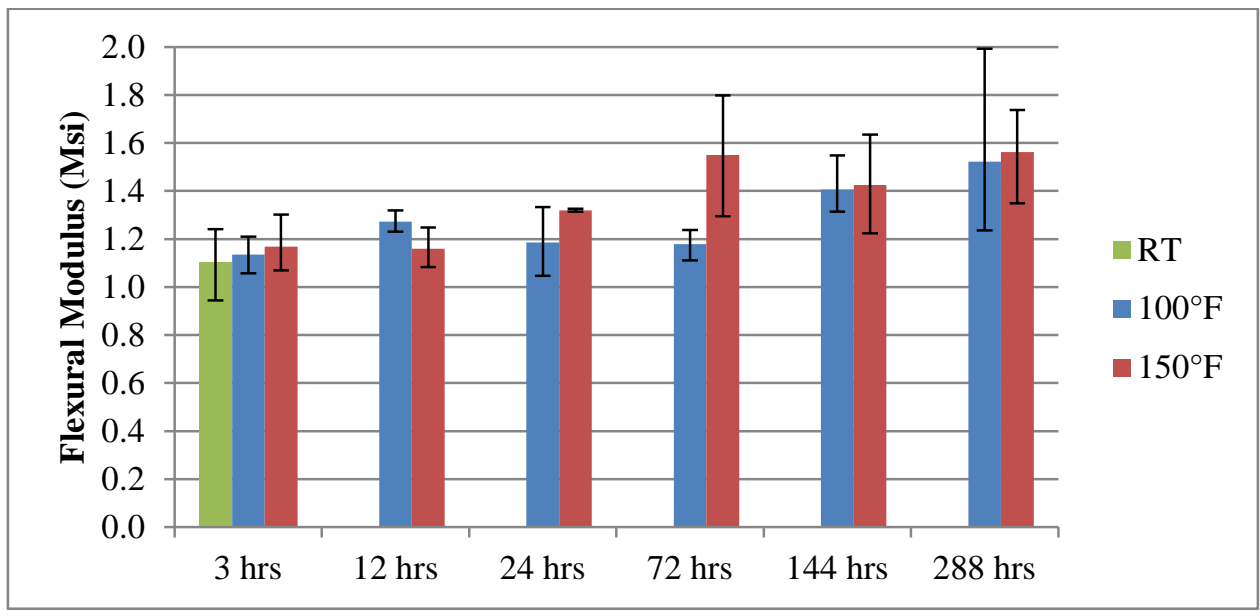

Figure 6-15 - Treated kenaf stiffness vs. temperature (with max/min bars)

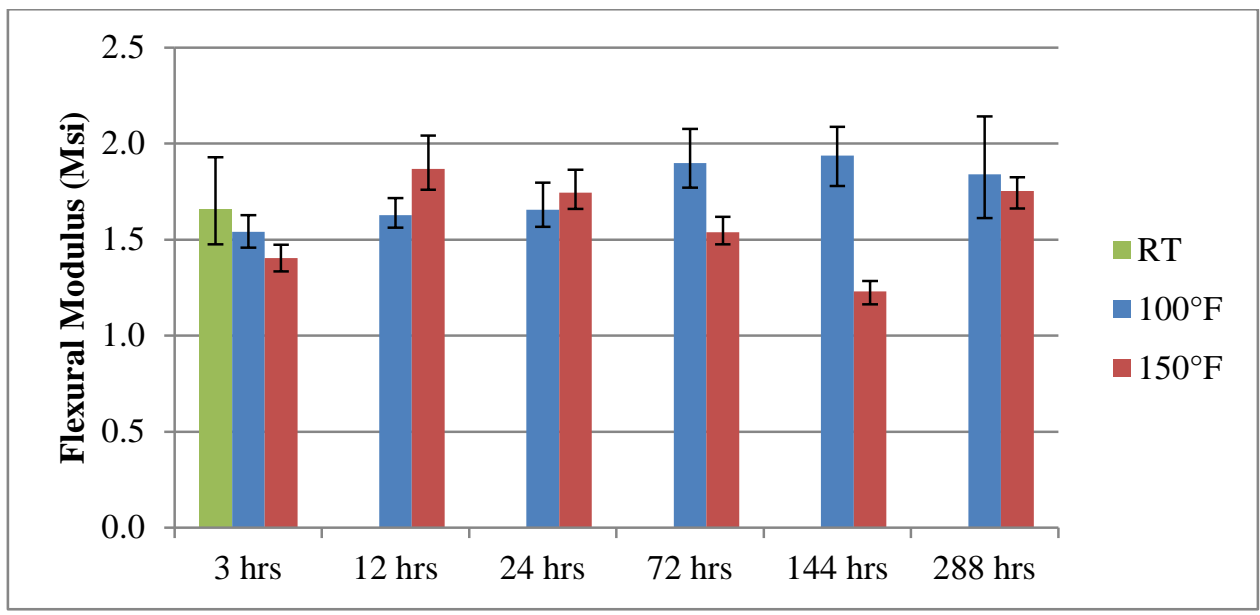

Figure 6-16 - Glass FRP stiffness vs. temperature (with max/min bars)

The other most useful conclusion is derived from the three stiffness plots: it appears that, with the exception of a few of the glass FRP samples, the time exposed to elevated temperatures caused the samples to stiffen slightly. This could possibly be due to the resin not reaching full cure using the manufacturing method described in the above methodology, and therefore the extra time at an elevated temperature may have helped complete the chemical reaction of curing. Based on these results, modifying the curing procedure or including a post-cure time could potentially increase the stiffness of the NFRP composites in this study by as much as $30-40 \%$. Based on this testing, it would appear that there is no concern with using the kenaf NFRP composites in applications where the temperature would rarely exceed $150^{\circ} \mathrm{F}$, as with many infrastructural applications. A more in-depth discussion of the elevated temperature testing can be found in the problem report by Majjigapu (2013). 


\subsubsection{2 pH Environmental Effects}

The glass fiber strength and stiffness results, with respect to their immersion medium and duration, are shown in Figure 6-17 and Figure 6-18, respectively. The treated and untreated, coated and uncoated kenaf fiber strength and stiffness results are shown in Figure 6-19 and Figure 6-20, respectively. In all of these plots, the dry baseline results are the average, normalized results from the previous chapter. As with the elevated temperature testing, the $\mathrm{pH}$ environmental test results are the averages of only three samples for each data column, and thus the error bars represent the maximum and minimum values instead of the standard deviations. Recall that the alkali fluid was a solution of water and sodium hydroxide at a $\mathrm{pH}$ of 13 and the acidic fluid was a solution of water and hydrochloric acid at a $\mathrm{pH}$ of 3 . Also, note that the water (pH 7) samples were immersed for 2700 hours, until saturation, while the acidic and alkali samples were only immersed for 1200 hours, until mostly saturated.

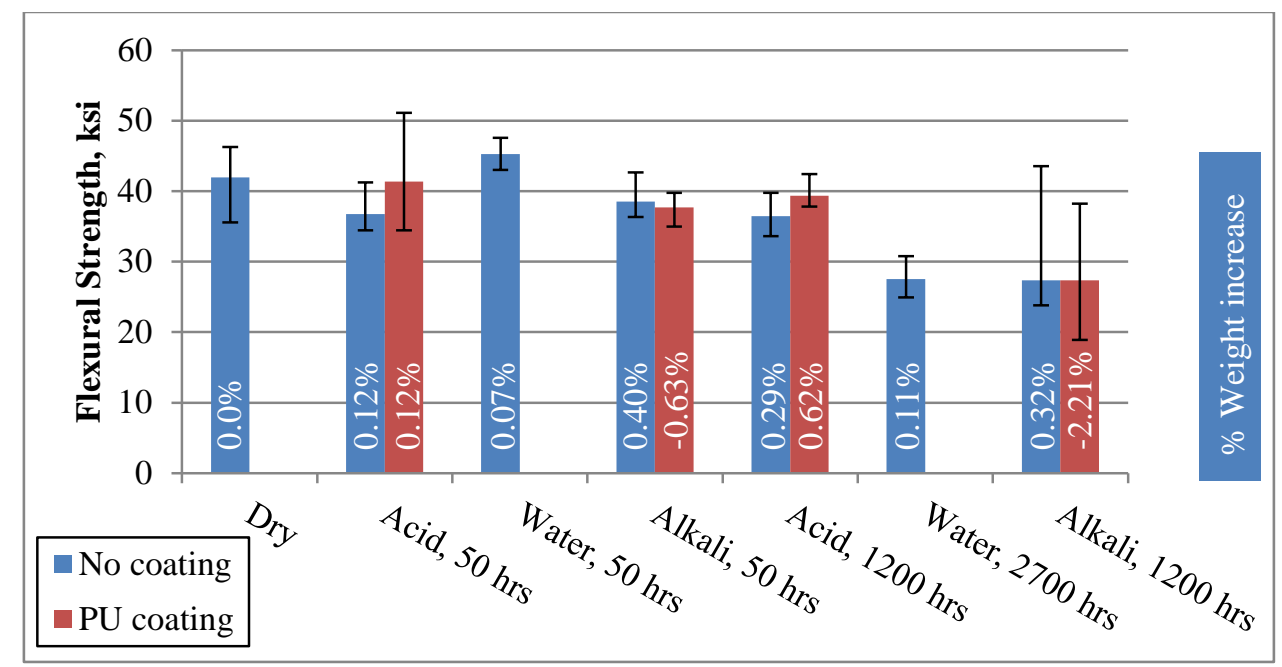

Figure 6-17 - Glass FRP strength vs. immersion treatment (with max/min bars; \% weight increase)

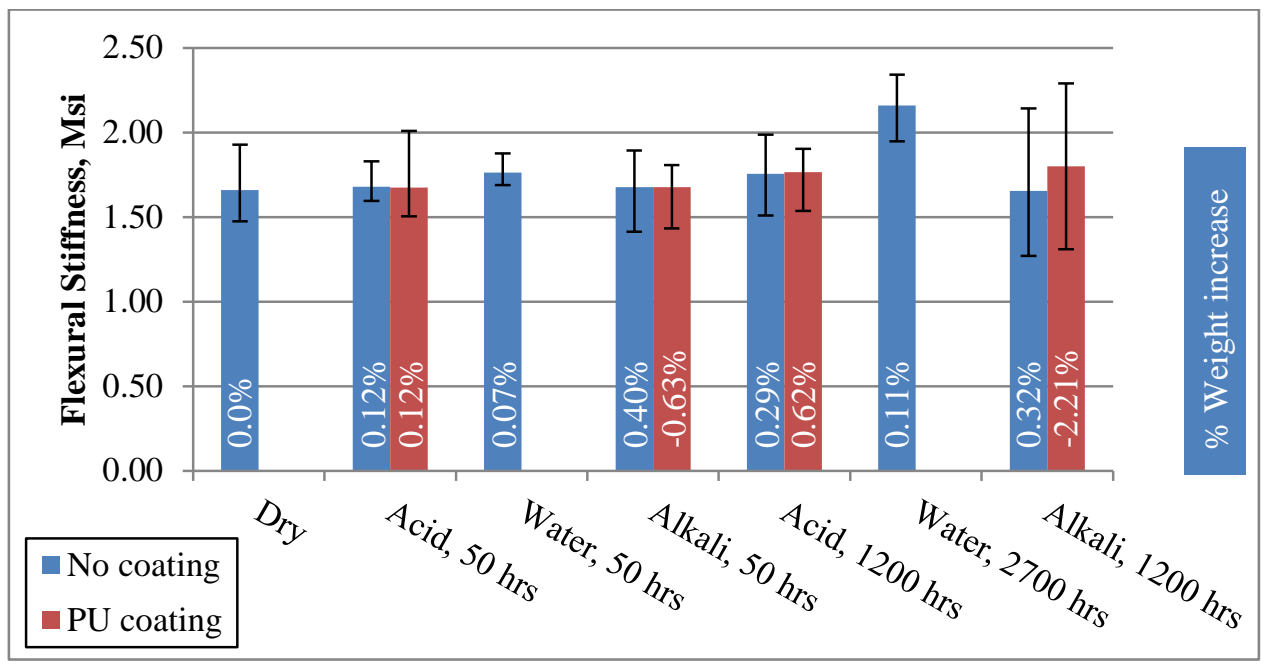

Figure 6-18 - Glass FRP stiffness vs. immersion treatment (with max/min bars; \% weight increase) 


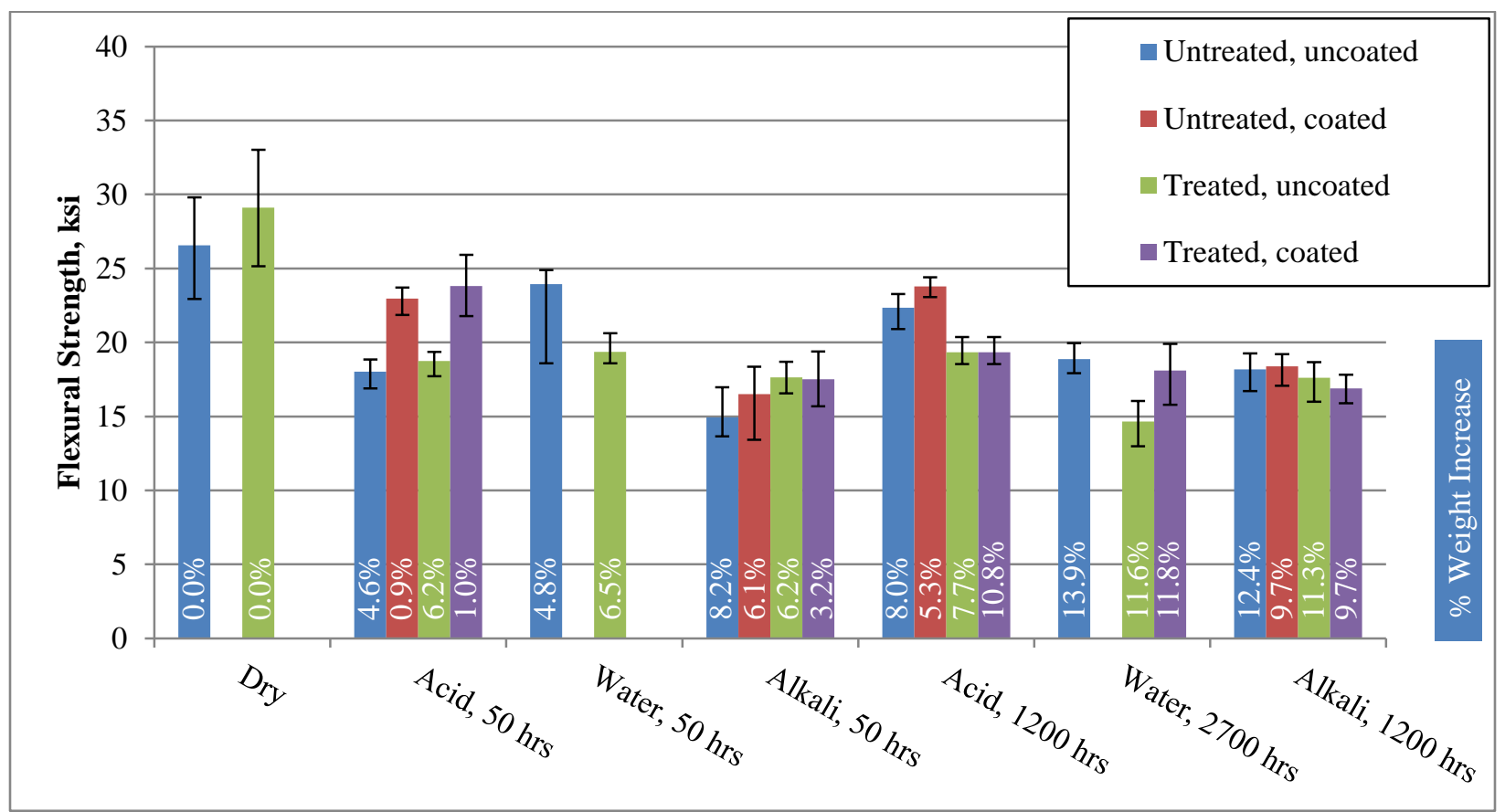

Figure 6-19 - Kenaf FRP strength vs. immersion treatment (with max/min bars; \% weight increase)

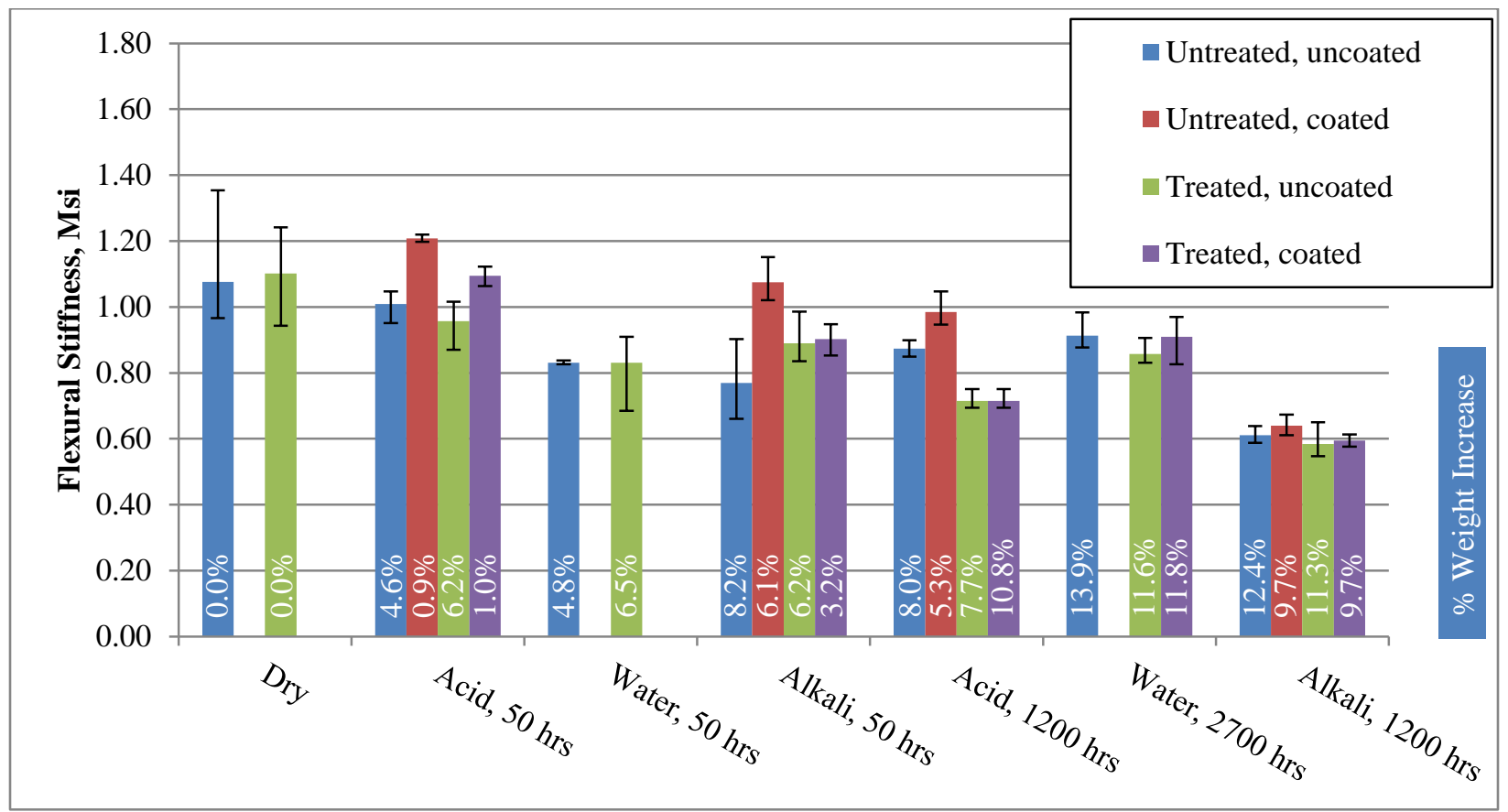

Figure 6-20 - Kenaf FRP stiffness vs. immersion treatment (with max/min bars; \% weight increase) 
As expected, the glass samples were generally unaffected by the immersion testing, regardless of whether or not they were urethane-coated. The urethane coating actually seems to have dissolved in the alkali environment, as shown by the weight reduction in the coated samples. Given the variation found in the glass reinforced composite samples elsewhere in this research, the fairly constant stiffness is somewhat surprising, with the exception of the "saturated" samples immersed in water. (For the glass FRP samples, "saturation" actually refers to when the kenaf fiber samples tested alongside the glass samples reached saturation, as the glass FRP absorbs essentially no moisture.) Since there is no reason for these particular samples to have higher stiffnesses as a result of their moisture exposure, this outlier is probably due to manufacturing error. With regards to the glass FRP strength results, the maximum values are actually much more consistent than the average values, suggesting that, once again, the variation is likely due to manufacturing errors. In general, however, there does appear to be some reduction in strength due to water and alkali immersion; alkalis, in particular, are known to degrade glass FRP composites (Barbero 2011).

For all of the kenaf FRP composites, there is a definite decrease in both strength and stiffness with longer duration immersions, regardless of the $\mathrm{pH}$ of the testing medium. In most cases the degradation in the acidic and alkali solutions is not significantly worse than the degradation in water, suggesting that the moisture absorption is far more responsible for causing damage than the chemistry of the solution. However, the alkali solution seemed to consistently have a larger affect than the acidic solution and is therefore the more important consideration when selecting potential applications for kenaf FRP composites. Since the alkali solution is similar to the chemical treatment applied to the fibers, the long exposure likely allows more of the hemicellulose and lignin to be dissolved from the exposed fibers, reducing fiber strength and stiffness and damaging the fiber-matrix interface, in addition to the general moisture absorption effects. The urethane coating did not seem to have much of an effect on long-duration immersion (as was seen with the water absorption testing in the previous chapter), but appeared to help with short-term environmental resistance, particularly in the acidic solution.

A more in-depth discussion of the $\mathrm{pH}$ immersion testing can be found in the problem report by Majjigapu (2013).

\subsubsection{UV Exposure Effects}

One of the most common effects of UV exposure is surface discoloration. While the UV exposure testing conducted by BRP also included some moisture and thermal cycling, there was significant surface discoloration as seen in Figure 6-21 (in each image, the sample on the left is the baseline surface coloration). This discoloration is fairly similar to the discoloration seen in the real-time weathering samples after a few months of exposure (see Appendix B). While the urethane coating adjusts the material coloration a little bit itself, it does appear to help resist UVinduced discoloration.

The post-UV exposure flexural strength and stiffness results for each of the materials and test durations are shown in Figure 6-22 and Figure 6-23, respectively. The left-most columns in each grouping represent the baseline, un-exposed average testing results from the previous chapter. 


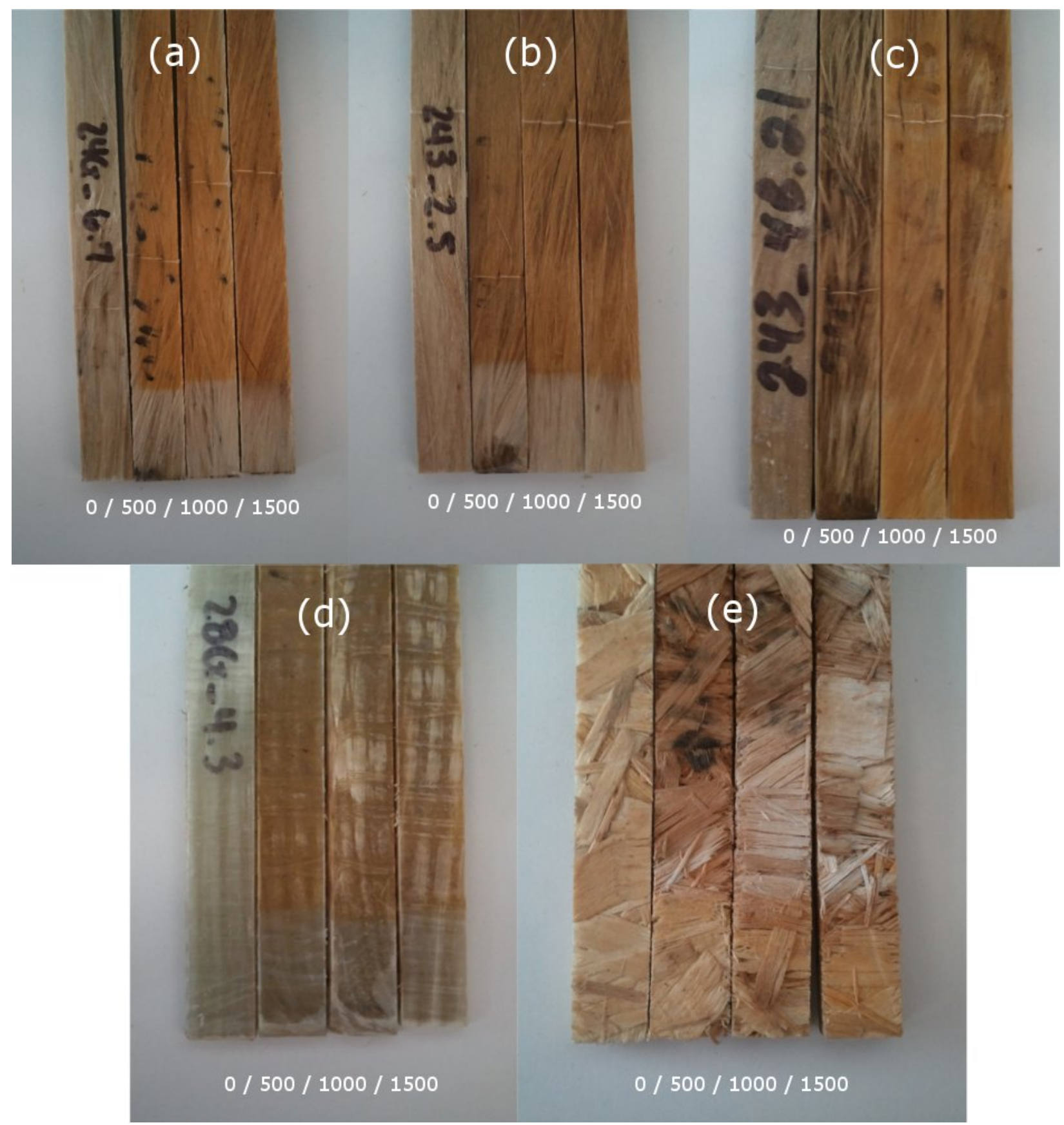

Figure 6-21 - UV-induced color change for (a) untreated kenaf FRP, (b) treated kenaf FRP, (c) treated/coated kenaf FRP, (d) glass FRP, and (e) OSB 


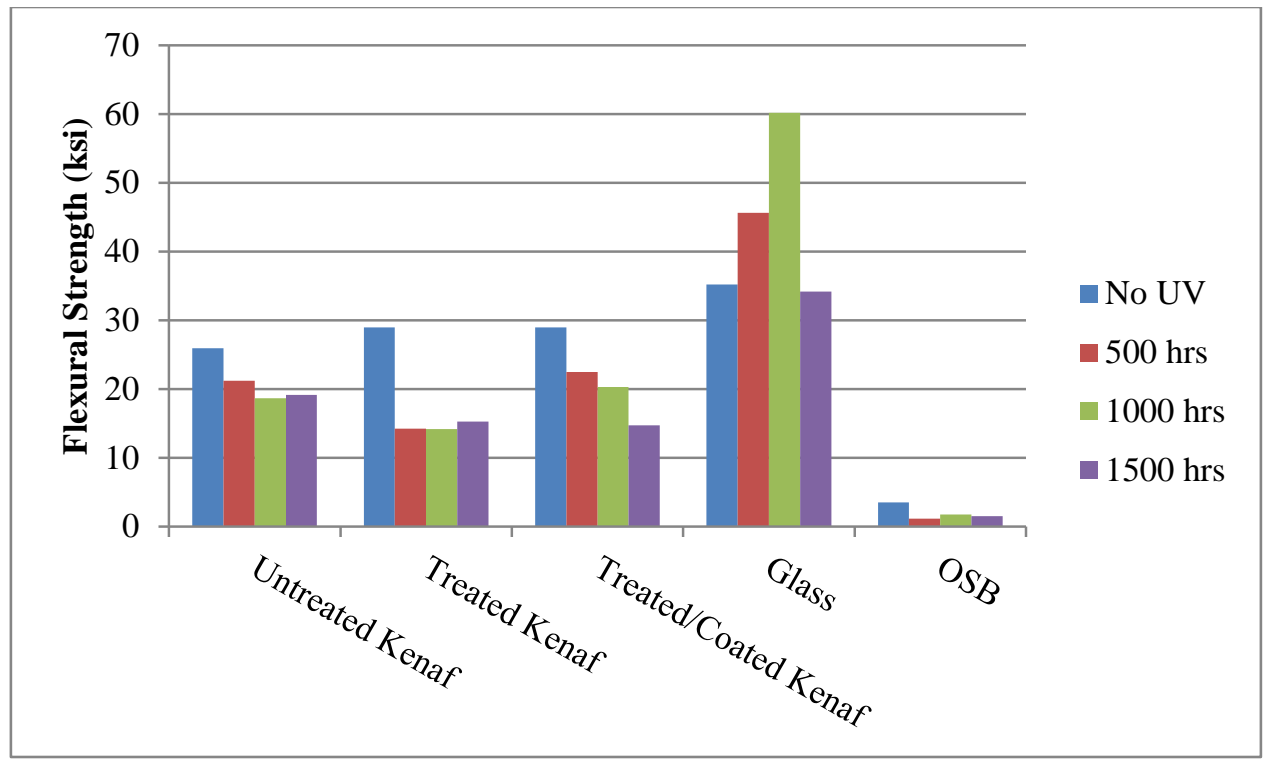

Figure 6-22 - Post-UV conditioning flexural strength results

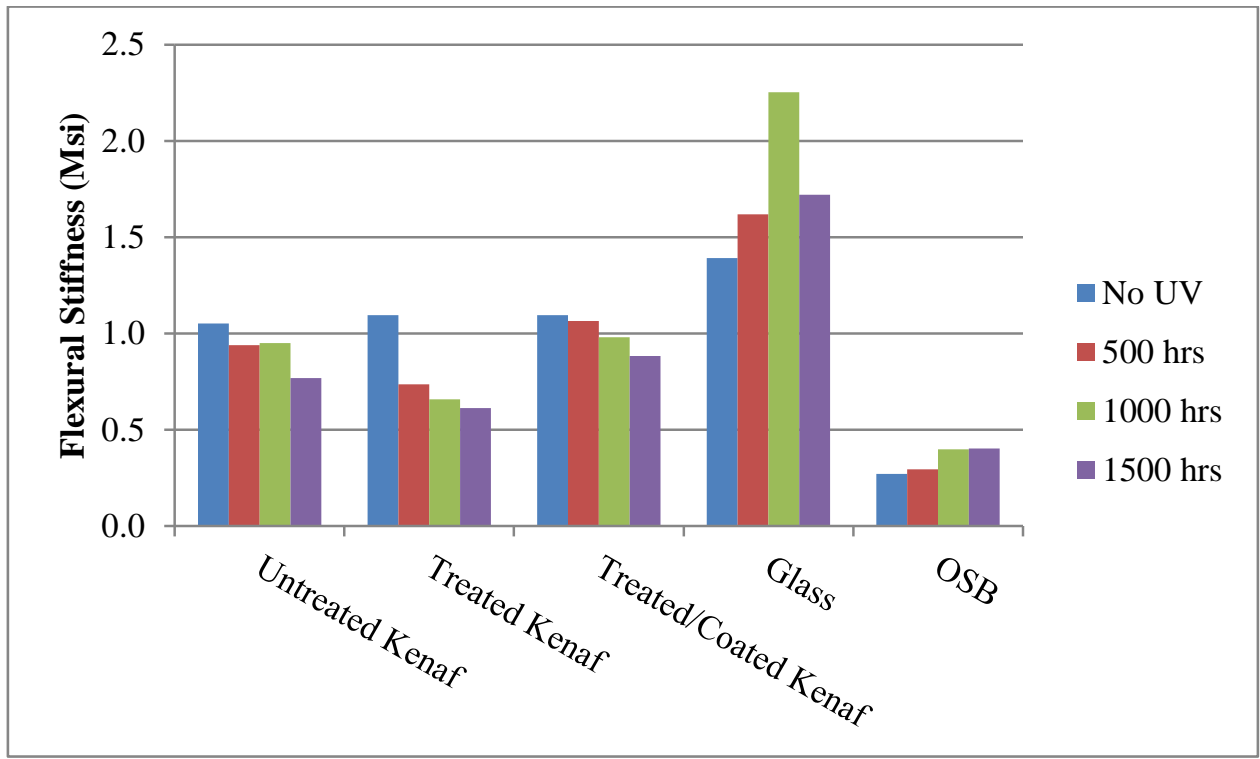

Figure 6-23 - Post-UV conditioning flexural stiffness results

Yet again, the high degree of variation in the glass FRP samples is difficult to explain. In this case, it would appear that the samples tested for 1000 hours must have had a significantly higher FVF than the other glass FRP samples, as both the strength and stiffness were far higher than the average values. While there could have been some post-cure effect due to the elevated temperature and time passed between manufacturing and testing, it is extremely unlikely that it would result in such a significant increase in mechanical properties and would appear in only one set of data. Based on the results, however, it seems reasonable to conclude that the glass FRP composites are minimally affected by the UV exposure testing, but for a slight discoloration. 
The increase in stiffness in the OSB samples is again likely due to their cross-sectional change and resulting increase in moment of inertia, rather than an actual increase in material stiffness. The low strength properties indicate that there was actually significant damage to the OSB, with the elevated temperature, water misting, and UV light all contributing to the material degradation.

The kenaf reinforced samples produced results much more similar to what was expected: all of the samples experienced reductions to their strength and stiffness as a result of UV exposure testing. This test does not differentiate between UV damage and damage incurred due to moisture absorption during the misting cycle, however. As was found with moisture absorption testing, the treated kenaf fibers seem to be more susceptible to environmental and absorption damage and thus had more significant reductions to both their strengths and stiffnesses than the untreated kenaf samples (around 50\% reduction as compared to 30\%). Generally, the longer duration tests produced larger degradations to the flexural properties, but in most cases the difference between 500 and 1500 hours of exposure was not significant, with the exception of the treated and coated kenaf samples. As was seen during other environmental tests, the urethane coating did an excellent job at resisting environmental damage over the shorter duration test, but failed to provide much long-term resistance.

\subsection{Design Considerations}

Many civil engineering design codes make use of environmental effect factors to account for reductions in material properties that may occur given a particular application's potential for exposure to moisture, temperature, or other effects. As the kenaf fiber reinforced composites are somewhat analogous to wood in their fundamental structure (tightly bound, oriented cellulosic fibers) a comparison was made between the environmental effects assessed in this chapter and the published environmental effect factors in the 2012 National Design Specification (NDS) for Wood Construction. The kenaf composite environmental effect factors were simply calculated by dividing the worst case scenario for each environmental condition by the baseline value and rounding down, rather than by statistical analysis, and are therefore on the conservative side.

Table 6-3 - Moisture effect factors, $C_{M}$, from NDS 2012, Table 4A for dimensional lumber and from experimental testing of saturated kenaf composites

\begin{tabular}{|l|c|c|c|}
\hline $\begin{array}{c}\text { Moisture effect } \\
\text { factors }\end{array}$ & $\begin{array}{c}\text { Dimensional } \\
\text { lumber }\end{array}$ & $\begin{array}{c}\text { Untreated kenaf } \\
\text { composite }\end{array}$ & $\begin{array}{c}\text { Treated kenaf } \\
\text { composite }\end{array}$ \\
\hline $\mathbf{F}_{\mathbf{b}}$ & 0.85 & 0.70 & 0.50 \\
\hline $\mathbf{F}_{\mathbf{t}}$ & 1.00 & 0.75 & 0.85 \\
\hline $\mathbf{F}_{\mathbf{v}}$ & 0.97 & 0.60 & 0.60 \\
\hline $\mathbf{F}_{\mathbf{c}}$ & 0.67 & - & - \\
\hline $\mathbf{F}_{\mathbf{c}}$ & 0.80 & 0.60 & 0.45 \\
\hline $\mathbf{E}_{\text {and }} \mathbf{E}_{\mathbf{m i n}}$ & 0.90 & - & - \\
\hline $\mathbf{E}_{\mathbf{b}}$ & - & 0.85 & 0.75 \\
\hline $\mathbf{E}_{\mathbf{t}}$ & - & 0.65 & 0.40 \\
\hline $\mathbf{G}^{\mathbf{E}_{\mathbf{c}}}$ & - & 0.30 & 0.30 \\
\hline
\end{tabular}


Table 6-3 lists the moisture effect factors (or "wet service factors") for dimensional lumber (NDS 2012), untreated kenaf composites, and treated kenaf composites, based on the moisture absorption data from the previous chapter. As a whole, the kenaf factors are much lower than the dimensional lumber factors, although according to NDS these factors apply to lumber in which the moisture content will exceed 19\% for extended time periods, not necessarily fully immersed samples, as the kenaf composites were tested. In every area but tensile strength, the untreated kenaf composite has equivalent or better property retention in a water-immersed environment than the treated kenaf composite.

The temperature factors for dimensional lumber (NDS 2012), untreated kenaf composites, and treated kenaf composites are shown in Table 6-4, with respect to the maximum temperature to which the member will experience sustained exposure (up to $150^{\circ} \mathrm{F}$ ). Since the kenaf composites were tested at $100^{\circ} \mathrm{F}$ and $150^{\circ} \mathrm{F}$, the $100^{\circ} \mathrm{F}$ results were applied to the $\mathrm{T} \leq$ $100^{\circ} \mathrm{F}$ category, while the $150^{\circ} \mathrm{F}$ results were applied to both of the $\mathrm{T} \geq 100^{\circ} \mathrm{F}$ categories. In general, the kenaf composites seem to possess fairly similar strength performance at these elevated temperatures and have no need for stiffness reduction. The untreated kenaf composites have almost no strength loss due to elevate temperatures, while the treated kenaf were found to experience a strength degradation of as much as $30 \%$.

Table 6-4 - Temperature factors, $C_{t}$, from NDS 2012, Table 2.33 for dimensional lumber and from experimental testing of kenaf composites for up to 12 days of exposure

\begin{tabular}{|c|c|c|c|}
\hline $\mathrm{T} \leq 100^{\circ} \mathrm{F}$ & $\begin{array}{c}\text { Dimensional } \\
\text { lumber }\end{array}$ & $\begin{array}{l}\text { Untreated kenaf } \\
\text { composite }\end{array}$ & $\begin{array}{c}\text { Treated kenaf } \\
\text { composite }\end{array}$ \\
\hline$F_{t}, E, E_{\min }$ & 1.00 & - & - \\
\hline$F_{v}, F_{c}, F_{c \perp}$ & 1.00 & - & - \\
\hline $\mathbf{F}_{\mathbf{b}}$ & 1.00 & 1.00 & 0.80 \\
\hline $\mathbf{E}_{\mathbf{b}}$ & - & 1.00 & 1.00 \\
\hline
\end{tabular}

$100^{\circ} \mathrm{F}<\mathrm{T} \leq 125^{\circ} \mathrm{F}$

\begin{tabular}{|l|c|c|c|}
\hline $\mathbf{F}_{\mathbf{t}}, \mathbf{E}, \mathbf{E}_{\min }$ & 0.90 & - & - \\
\hline $\mathbf{F}_{\mathbf{v}}, \mathbf{F}_{\mathbf{c}}, \mathbf{F}_{\mathbf{c} \perp}$ & 0.80 & - & - \\
\hline $\mathbf{F}_{\mathbf{b}}$ & 0.80 & 0.95 & 0.70 \\
\hline $\mathbf{E}_{\mathbf{b}}$ & - & 1.00 & 1.00 \\
\hline
\end{tabular}

$125^{\circ} \mathrm{F}<\mathrm{T} \leq 150^{\circ} \mathrm{F}$

\begin{tabular}{|l|c|c|c|}
\hline $\mathbf{F}_{\mathbf{t}}, \mathbf{E}, \mathbf{E}_{\min }$ & 0.90 & - & - \\
\hline $\mathbf{F}_{\mathbf{v}}, \mathbf{F}_{\mathbf{c}}, \mathbf{F}_{\mathbf{c} \perp}$ & 0.70 & - & - \\
\hline $\mathbf{F}_{\mathbf{b}}$ & 0.70 & 0.95 & 0.70 \\
\hline $\mathbf{E}_{\mathbf{b}}$ & - & 1.00 & 1.00 \\
\hline
\end{tabular}

The final two design tables (UV exposure: Table 6-5; 1-year outdoor exposure: Table 6-6) do not have equivalent factors in the NDS, but nevertheless a simple way of summarizing the results of these environmental tests. As it so happens, the degradation due to 1000 hours of UV exposure is actually quite similar to the degradation due to a year of outdoor exposure, with the exception of the bending strength of the untreated kenaf composite, which retained a higher 
strength than expected during the UV testing. For future tests, it appears that the 1000 hour UV testing may be a suitable accelerated aging method for quick evaluation of different fiber treatments or coatings. These factors again confirm the general finding that the treated kenaf composites have slightly poorer environmental resistance than the untreated kenaf composites. Over longer periods of time (greater than 1 year), this trend may continue, or the treated kenaf composite may end up with better weathering durability; future testing will explore this issue.

Table 6-5 - Design factors, $\mathrm{C}_{\mathrm{UV}}$, based on ASTM D4329, Cycle A UV exposure for over 1000 hours

\begin{tabular}{|l|c|c|}
\hline Design Factors & $\begin{array}{c}\text { Untreated kenaf } \\
\text { composite }\end{array}$ & $\begin{array}{c}\text { Treated kenaf } \\
\text { composite }\end{array}$ \\
\hline $\mathbf{F}_{\mathbf{b}}$ & 0.70 & 0.50 \\
\hline $\mathbf{E}_{\mathbf{b}}$ & 0.70 & 0.55 \\
\hline
\end{tabular}

Table 6-6 - Design Factors, $\mathrm{C}_{\mathrm{OE}}$, based on outdoor exposure in WV for 1 year

\begin{tabular}{|l|c|c|}
\hline Design Factors & $\begin{array}{c}\text { Untreated kenaf } \\
\text { composite }\end{array}$ & $\begin{array}{c}\text { Treated kenaf } \\
\text { composite }\end{array}$ \\
\hline $\mathbf{F}_{\mathbf{b}}$ & 0.50 & 0.50 \\
\hline $\mathbf{E}_{\mathbf{b}}$ & 0.70 & 0.60 \\
\hline
\end{tabular}

\subsection{Conclusions}

The flexural performance of the kenaf fiber reinforced composites due to a number of environmental conditions was evaluated, including: outdoor weathering, elevated temperatures, immersion in different $\mathrm{pH}$ solutions, and UV exposure.

The outdoor weathering test was conducted in Morgantown, WV, for one year duration. Over the course of that year, the temperatures were slightly higher than average and the accumulated precipitation was slightly lower. The weight and density of the samples were found to largely dependent on the weather conditions of the five days preceding removal and testing. One of the most significant density findings was that the treated kenaf samples experienced a 4$6 \%$ density loss, possibly indicating fungal growth and/or decomposition, while the untreated and coated kenaf samples tended to have very little density change.

The flexural results of the outdoor weathering test showed that, while the glass FRP samples had excellent weathering resistance, all of the kenaf fiber reinforced samples tended to lose strength and stiffness with increased exposure duration. A number of factors could have contributed to these losses, including: moisture-induced swelling, absorption/desorption cycling, freeze-thaw cycling, fungal or bacteria growth, and decomposition. Visual indications of damage in the kenaf FRP samples included end-splitting, longitudinal cracking, and black patches on cut surfaces. The urethane coating was found to provide some environmental resistance, although its effect decreased over time. Over the course of a year, the strength reduction factor was 0.50 for both the untreated kenaf composites and treated kenaf composites, and the stiffness reduction factors were 0.70 and 0.60 , respectively. 
The elevated temperature tests did not show much flexural performance reduction in any of the samples, most likely due to the low temperatures at which the tests were conducted and the delay between elevated temperature exposure and flexural testing. One of the most interesting findings, however, was the increase in stiffness found in almost every test condition, which suggests that post-curing may be able to improve the kenaf FRP bending stiffness by as much as $30-40 \%$. While no stiffness degradation was observed, the strength degradation factors for composites exposed to temperatures between $100-150^{\circ} \mathrm{F}$ for extended durations are 0.95 and 0.70 for untreated kenaf composites and treated kenaf composites, respectively.

Immersion in any medium ( $\mathrm{pH} \mathrm{3,7,} \mathrm{or} \mathrm{13)} \mathrm{for} \mathrm{increasing} \mathrm{durations} \mathrm{resulted} \mathrm{in} \mathrm{decreasing}$ flexural strengths and stiffnesses for the kenaf FRP composites, whether with treated or untreated fibers, and whether with or without urethane coatings. The alkali exposure tended to cause slightly greater degradation, while the acidic exposure caused slightly lesser. The urethane coating did help somewhat with shorter-duration tests, but clearly only slowed rather than ceased absorption. The urethane coating also has a tendency to dissolve in the alkali environment. The moisture degradation factors for long-term immersion in water range between 0.60-0.75 and 0.45-0.85 for untreated and treated kenaf composite strengths, respectively, and between 0.300.85 and $0.30-0.75$ for untreated and treated kenaf composite stiffnesses.

UV exposure testing discolored the composite surface, just as was seen with the outdoor weathering samples, and caused significant strength and stiffness degradation in the kenaf FRP composites, with the untreated kenaf faring slightly better than the treated kenaf. The urethane coating provided excellent short-term environmental resistance, but again proved ineffective over long durations. Over the course of 1000 hours of UV testing, the strength reductions factors were 0.70 and 0.50 for untreated kenaf composites and treated kenaf composites, respectively, and the stiffness reduction factors were 0.70 and 0.55 . These factors were fairly similar to the year-long outdoor weathering reduction factors, suggesting that this test could be reasonably substituted as an approximate accelerated aging test in future research. 


\section{STRUCTURAL COMPONENT TESTING}

\subsection{Abstract}

In order for the stitched fiber bundle manufacturing method of the previous chapters to be scaled up for full-size structural components, a method of overlapping fiber ends without a significant loss of strength and stiffness is needed. It was found that meshing together fibers with 1.5-2.5 in of overlap provided similar strength and stiffness properties as continuous fibers. Large mats of fiber bundles joined using this method were manufactured into NFRP SIPs with polyisocyanurate foam cores by Fiber-Tech Industries. The vacuum-based manufacturing method was found to provide only around $82 \%$ of the strength of the composites manufactured by compression molding (likely due to the presence of voids and dry fibers), although the stiffness was unchanged. The foam cores proved to be poorly matched to the strength and stiffness of the NFRP composite facesheets, as significant corner delaminations occurred during shipping and the core was responsible for premature shear or delamination failures in both flexure and compressive testing. Despite the poor performance due to the core material, the NFRP SIPs offer the potential to be 2-4 times stronger and more than 2.5 times stiffer than the OSB SIPs that are already common in the construction industry, if significant improvements can be made to the core and the core-facesheet interface.

\subsection{Introduction and Scope}

Once a material's mechanical and durability properties are known, there are still several important steps necessary before products can begin to be manufactured with that material. Several of these steps include selecting appropriate applications given the material constraints, overcoming any complications associated with scaling up a design to a component-scale product, and making adaptations and accommodations to suit the manufacturing entity that will be making the product.

As an extension of the previous chapters' research, the kenaf fiber reinforced mats were manufactured at a large size at WVU-CFC and sent to a composites manufacturer to be turned into structural insulated panels. Due to the time-intensive nature of the current method of handcrafting the mats, only two $4 \times 1$ foot panels will be manufactured and tested: one in flexure and one in longitudinal compression, the two most likely loading conditions for a structural panel used in construction.

\subsection{Materials and Manufacturing Processes}

\subsubsection{Materials}

The loose kenaf fibers discussed in the previous chapters were cut, cleaned, combed, and stitched to a light bidirectional kenaf fabric (at a weight ratio of around 5-to-1 fibers to fabric) in order to create essentially unidirectional kenaf fiber mats.

The resin system used for the joint testing samples in this chapter was Ashland's Derakane 510A-40 vinyl ester, as discussed in the previous chapters. A MEKP catalyst and a 
Cobalt Naphthenate promoter were added to the resin at concentrations of $0.75 \%$ weight and $0.25 \%$ weight, respectively.

The resin system used by Fiber-Tech Industries, Inc to manufacture the structural insulated panels was a similar vinyl ester, Ashland's Derakane 510 N. Compared to the Derakane 510A-40 vinyl ester resin, the $510 \mathrm{~N}$ offers slightly reduced fire retardance, lower density, slightly increased stiffness, and slightly reduced clear casting strength, although higher laminate strength properties, according to the material technical data sheet. It also relies on MEKP and Cobalt Naphthenate additives for curing.

The polyisocyanurate foam system used by Fiber-Tech Industries was Elliott Company’s ELFOAM P200, with a density of $2.0 \mathrm{lb} / \mathrm{ft}^{3}$ and an insulating R-value/inch of $5.4 \mathrm{Hr}-\mathrm{ft}^{2}{ }^{\circ} \mathrm{F} / \mathrm{BTU}$. This foam was selected by the manufacturer as a commonly used insulating foam system. In order to obtain the desired thickness of 3.5 inches, a 2 inch thick block and a 1.5 inch thick block were glued together using the Derakane $510 \mathrm{~N}$ resin. The foam strength and stiffness properties are shown in Table 7-1.

Table 7-1 - Manufacturer-given foam core strength and stiffness properties (along with average axial values)

\begin{tabular}{|l|c|c|}
\hline Property & Parallel Value & Perpendicular Value \\
\hline Compressive Strength & $27 \mathrm{psi}$ & $18 \mathrm{psi}$ \\
\hline Compressive Modulus & $700 \mathrm{psi}$ & $334 \mathrm{psi}$ \\
\hline Shear Strength & $22 \mathrm{psi}$ & $16 \mathrm{psi}$ \\
\hline Shear Modulus & $220 \mathrm{psi}$ & $177 \mathrm{psi}$ \\
\hline Tensile Strength & $41 \mathrm{psi}$ & $26 \mathrm{psi}$ \\
\hline Tensile Modulus & $1225 \mathrm{psi}$ & $463 \mathrm{psi}$ \\
\hline Approximate axial strength & $34 \mathrm{psi}$ & $22 \mathrm{psi}$ \\
\hline Approximate axial modulus & $963 \mathrm{psi}$ & $399 \mathrm{psi}$ \\
\hline
\end{tabular}

\subsubsection{Fiber Treatment}

The fibers used for the joint testing samples were treated with $5 \% \mathrm{NaOH}$ for 16 hours, in order to take advantage of the opportunity to get some further assessment of that treatment condition, although the \% gain or loss for each configuration should be approximately the same for other treatment methods.

Due to the inconclusive mechanical property gains shown for chemical treatments and a lack of available equipment for safe, large-scale chemical treatments, the fibers stitched into mats to be sent to Fiber-Tech Industries were untreated.

\subsubsection{Sample Preparation}

\subsubsection{Joint Design}

Since natural fibers are an organic material, they can only be obtained in restricted lengths. Kenaf is one of the longest natural fiber sources, with fibers up to a maximum of 10 feet. However, they can only be efficiently prepared for composite manufacturing at a maximum length of around 12-15 inches. Since most applications will require aligned fibers in lengths 
greater than this, it is necessary to create "joints" where one bundle of aligned fibers overlaps with the next bundle.

To determine the best possible joint configuration, test panels were created with unidirectional [0/fabric/fabric/0] layups, where the 0 -fibers had different joints in the center of the span as shown in Figure 7-1. From the test panels, samples were cut to 8 inches by 0.5 inches, with an average thickness of around 0.19 inches. Samples were tested in tension following the basic procedure of ASTM D3039, with strain measured via an extensometer.

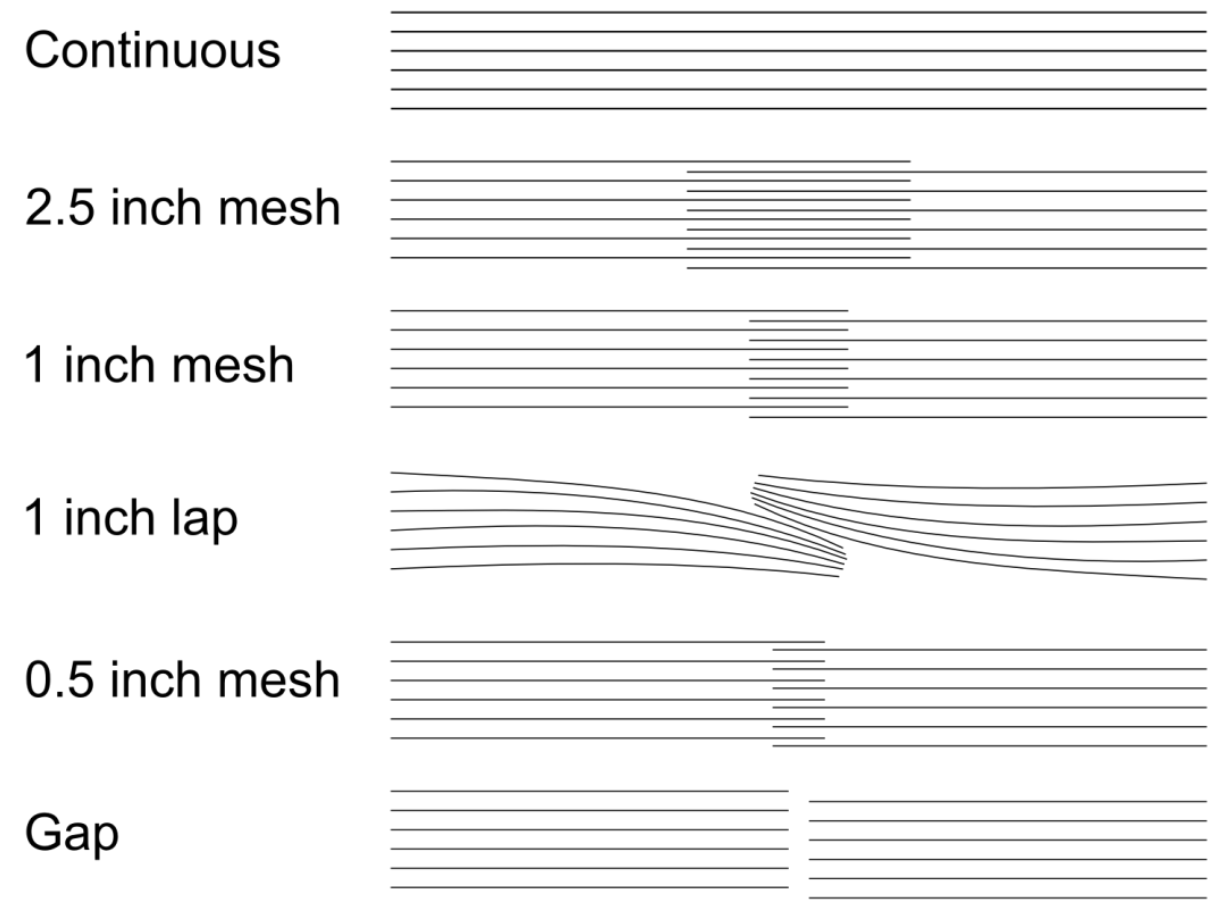

Figure 7-1 - Different fiber 'joint' types tested

The tensile strength results are shown in Figure 7-2, modulus results in Figure 7-3, and strain results in Figure 7-4. There appears to be no loss in strength for a mesh overlap of at least 2.5 inches, although this large of an overlap is somewhat inefficient. A mesh overlap of at least an inch results in a strength reduction to about $75 \%$ of the continuous fibers. There also appears to be no stiffness loss whatsoever for a mesh overlap of at least one inch.

The lap-joint overlap had reduced strength and stiffness compared to the meshed fibers (although, as expected with a continuous plane of resin, higher strain to failure), indicating that the mesh-type joint is superior. The 0.5 inch mesh also had reduced strength and stiffness compared to the 1 inch mesh. And, as a baseline, the 'gap' joint was essentially just testing the strength and stiffness of the resin, albeit with some added stress concentrations due to the fiber endings. 


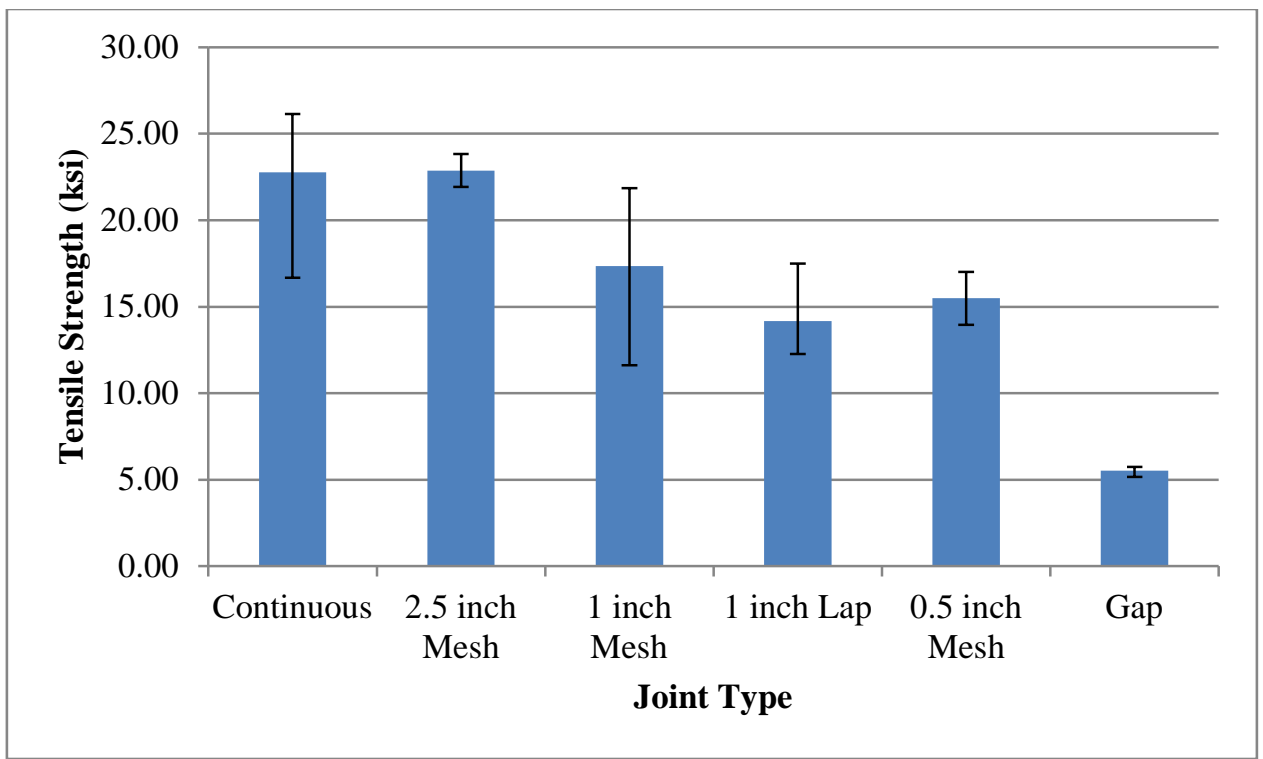

Figure 7-2 - Tensile strength comparison between different fiber 'joint' designs

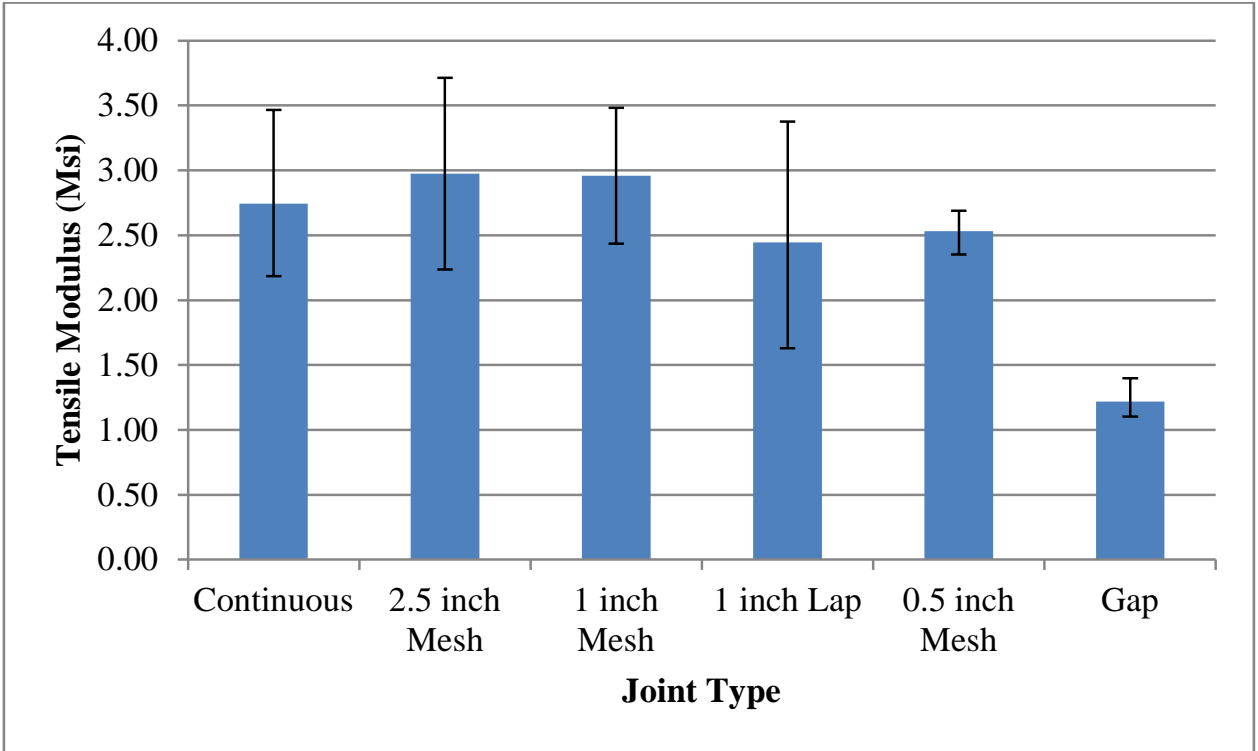

Figure 7-3 - Tensile modulus comparison between different fiber 'joint' designs 


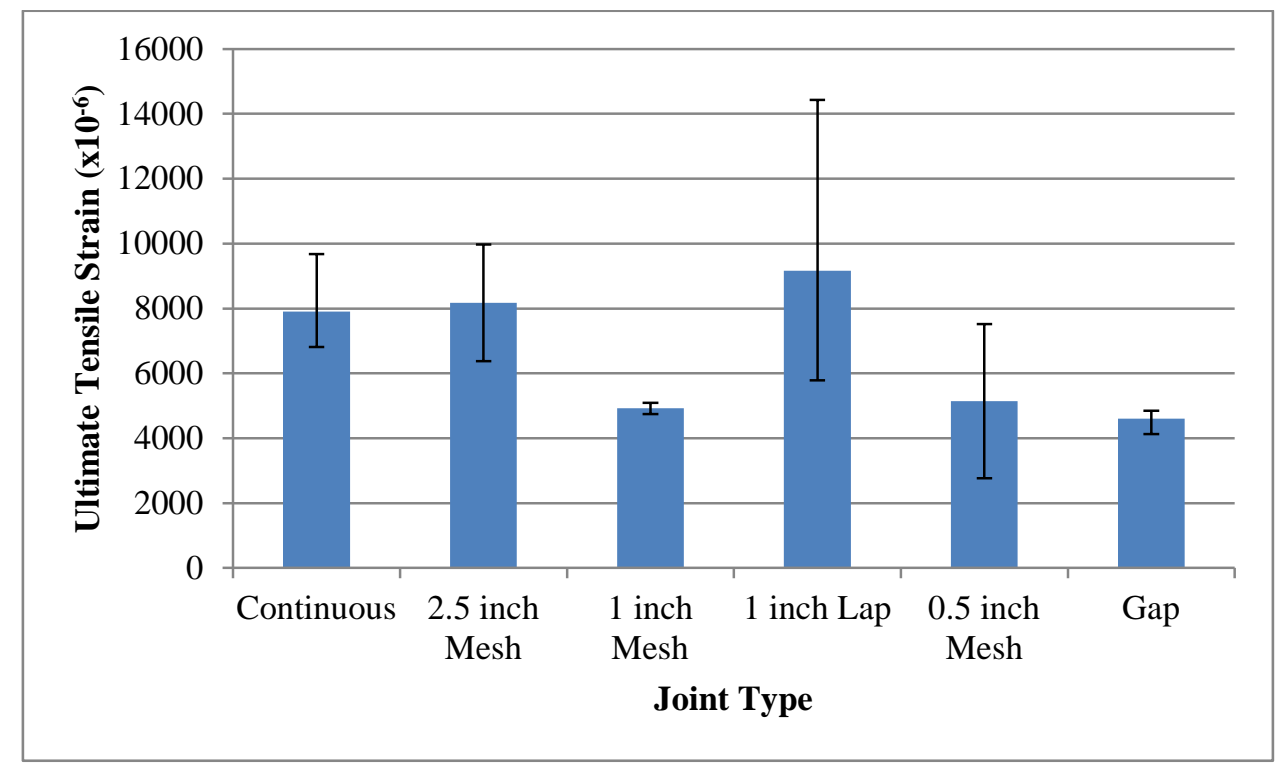

Figure 7-4 - Ultimate Tensile strain comparison between different fiber 'joint' designs

While the 2.5 inch mesh and the 1 inch lap joints did not seem to have a significant effect on the strain to failure, the other joints had reduced strains to about $60 \%$ of the continuous fiber ultimate strain.

From the above results, it was determined that an optimum kenaf fiber composite component should have joints of at least 1.5 inches in order to avoid significant losses in strength and stiffness without wasting material. From these preliminary tests, it does not seem like any design reductions to laminate strength or stiffness due to overlaps are necessary, although with more extensive testing a small reduction may prove to be required for at least the strength.

\subsubsection{Fiber-Tech Industries}

In order to test the ability of kenaf fiber reinforced composites to be used in larger structural components, manufacturing assistance was sought from Fiber-Tech Industries, Inc in Spokane, WA. Fiber-Tech Industries is a major manufacturer of solid fiberglass reinforced plastic panels, as well as panels with foam or wood cores.

The constraints of the available equipment at WVU mandated that, when stitching together fiber bundles into unidirectional mats, the mat width could not exceed around 12-15 inches (see Figure 7-5). Therefore, a method was devised in which several unidirectional mats could be laid next to each other in each of the 0 - and 90-directions with a few inches of fabric under-lap helping to keep the mats in place during compression.

In order to get two $4 \times 1$ foot sandwich panels made, four $60 \times 13$ inch mats (with fibers in the 60 inch direction) and ten $26 \times 12$ inch mats (with fibers in the 26 inch direction) were stitched (see Figure 7-6). The joints were designed to have at least 1.5 in overlap, and the fiber ends were meshed together to avoid creating resin rich planes. This allowed Fiber-Tech to manufacture two sets of [0/90] 60 x 26 inch mat layers; one for each side of the foam core. The 
average weight of a stitched $26 \times 12$ inch mat was 0.582 lbs and the average weight of a stitched $60 \times 13$ inch mat was 1.461 lbs.

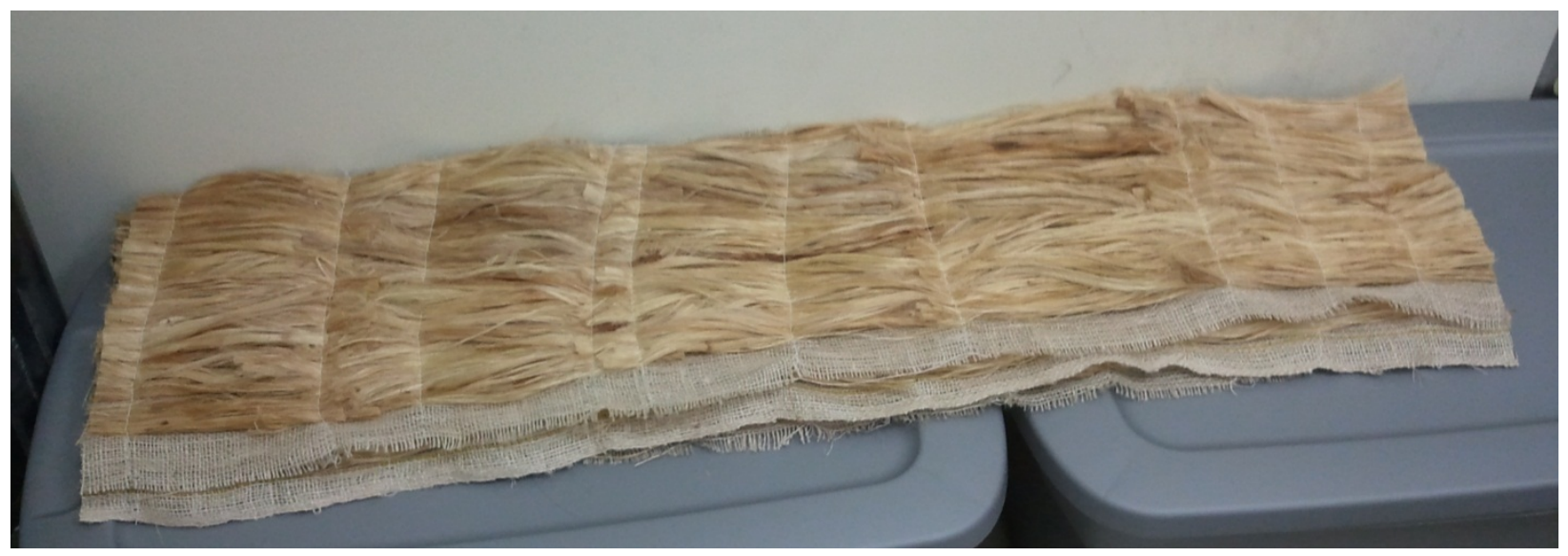

Figure 7-5 - 12 in $x 48$ in stitched mats with 12 in long fibers

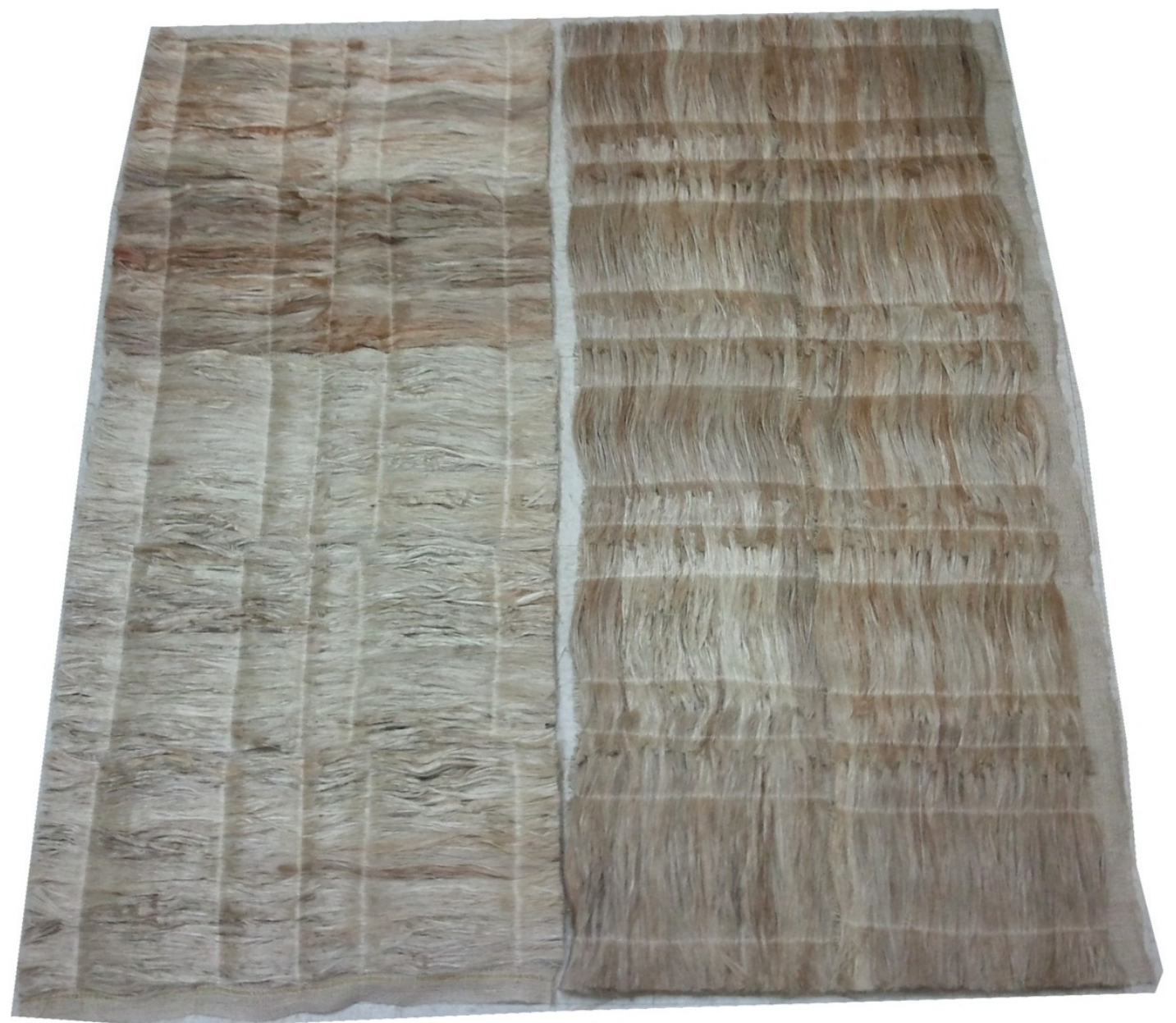

Figure 7-6-60" x 26” 90-degree and 0-degree layers made up of 5 and 2 separate mats, respectively 


\section{Mat Placement}

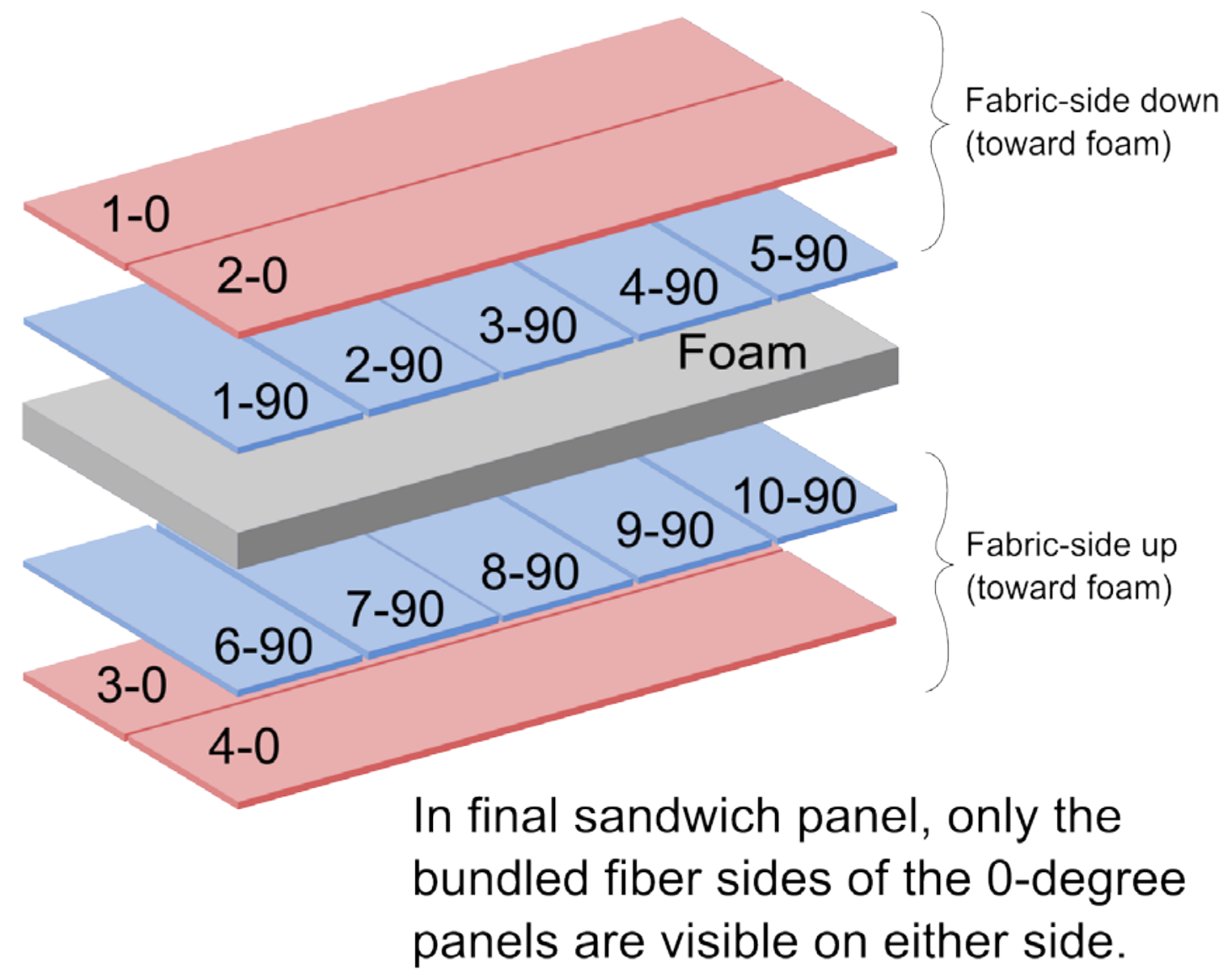

\section{Mat Overlapping}

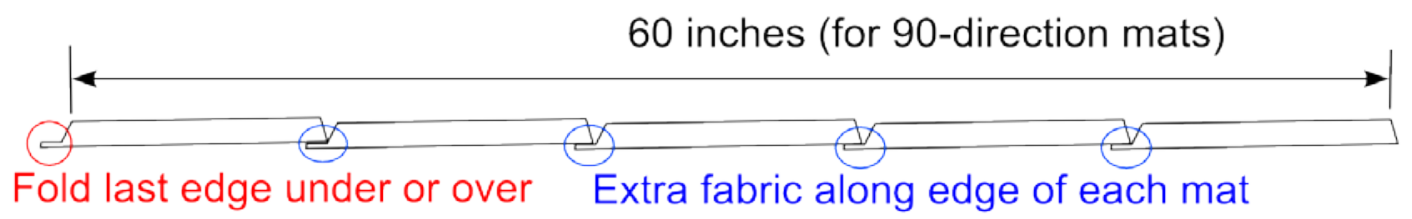

Arrows on fabric on 0-degree layers should all point the same direction. Arrows on fabric on 90-degree layers should all point the same direction. On finished products, please mark the edges the arrows pointed toward.

Figure 7-7 - Mat placement diagrams for manufacturing of two adjacent 60” $x$ 13” sandwich panels 
The mat placement diagram of Figure 7-7 shows the layup and relative positions of each mat to the foam core used by Fiber-Tech to manufacture the structural insulated panels. FiberTech used a form of vacuum molding to do the manufacturing. First, a polyester film was laid down on a table heated to $100^{\circ} \mathrm{F}$. Above this table, a cardboard box was set up with two rows of 1/8-inch holes cut into it at 1/2-inch spacing and for the total width of the panels. The box was filled with 10 pounds of Derakane 510N and passed over the polyester film once, allowing the resin to trickle down onto the table surface. The first pair of 0 -direction kenaf fiber mats was laid down on the table, followed by two more passes of the resin box, the placement of the 90direction mats, and a few more passes of the resin box until it was emptied. The pre-cut foam panels were placed on top of this layer, the resin box was refilled, and the process was repeated in reverse to place the top fiber mats. A clear polyester film was laid on top of the whole assembly, followed by a cut-to-size plywood board with spacer blocks attached on the sides to control the thickness of the panels once pressure is applied. A vacuum was pulled on the entire assembly to a pressure of about $12-14 \mathrm{psi}$, the table temperature was increased to $120^{\circ} \mathrm{F}$, and an oven placed over top of the panel was heated to $150^{\circ} \mathrm{F}$. The vacuum pressure was released after 30 minutes, and the panel was allowed to cool for an additional two hours before being split into two and cut to size by table saw.

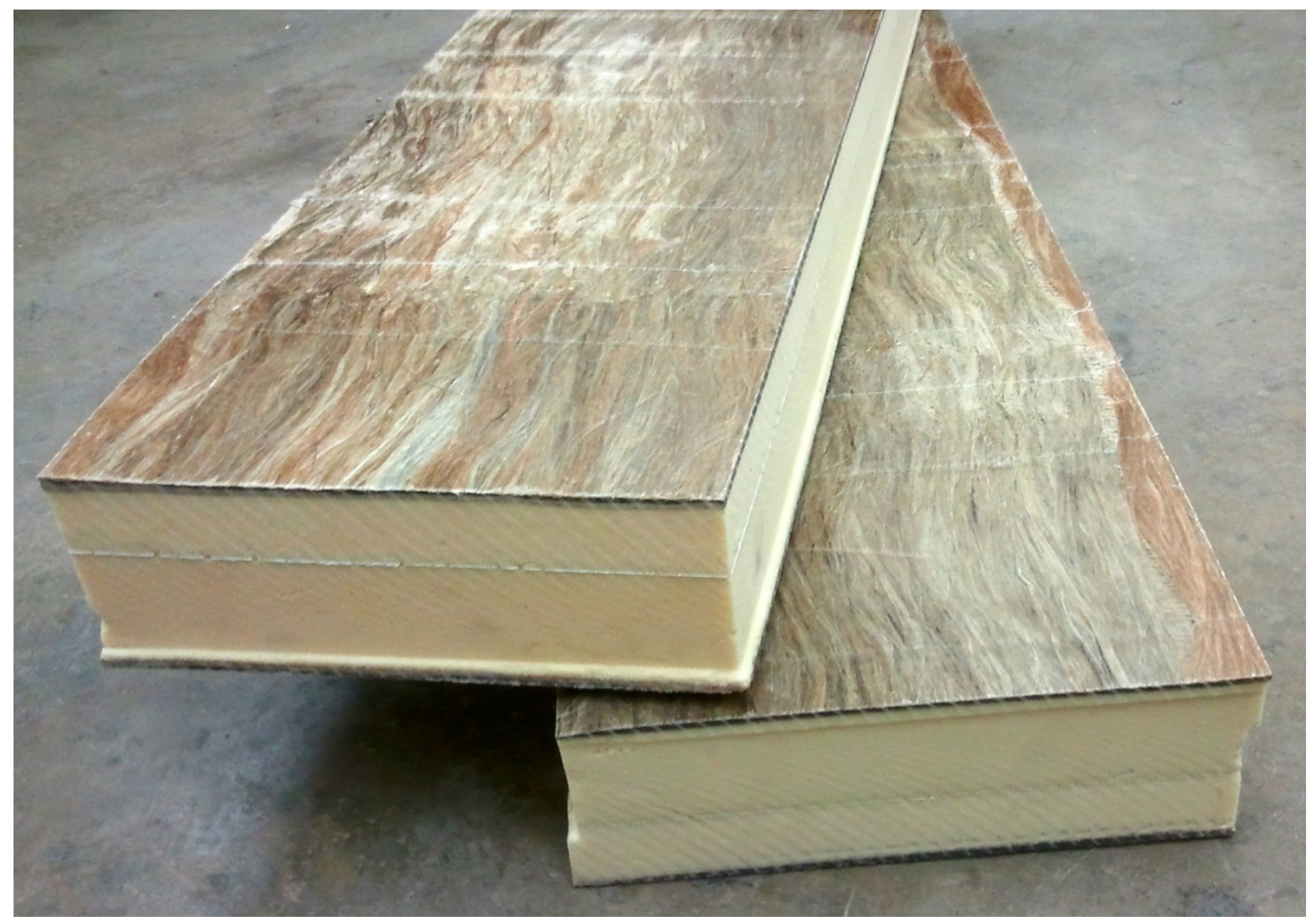

Figure 7-8 - Completed structural insulated panels from Fiber-Tech 
The completed panels were then shipped from Spokane to WVU, and are shown in Figure 7-8. Overall, the quality of the panels was fair, with two main areas of concern: the surfaces were, in some places, pretty rough, indicating some dry fibers or at least ones with poor cover, and there were several fairly extensive corner delaminations between the panels and the foam core (the worst of which is shown in Figure 7-9). From speaking to the manufacturer, it appears that a couple of the corners had splits of about 2 inches long present after cutting the panels; therefore, the rest of the splitting must have taken place during transit. This delamination was somewhat expected, as some of the smaller compression panels made at WVU also had some warping effects due to the unbalanced layup and poorly controlled cooling. More than likely, the small splits induced during the cutting of the panels propagated due to residual stresses from the unbalanced layup and thermal effects as the panels traveled across the country during a particularly cold January. In the future, using a thinner, balanced layup, slower cooling procedure, and avoiding shipping during the hottest and coldest times of the year (if possible) would help prevent these delaminations from occurring. Regardless, a better bond between the facesheets and core material is needed.

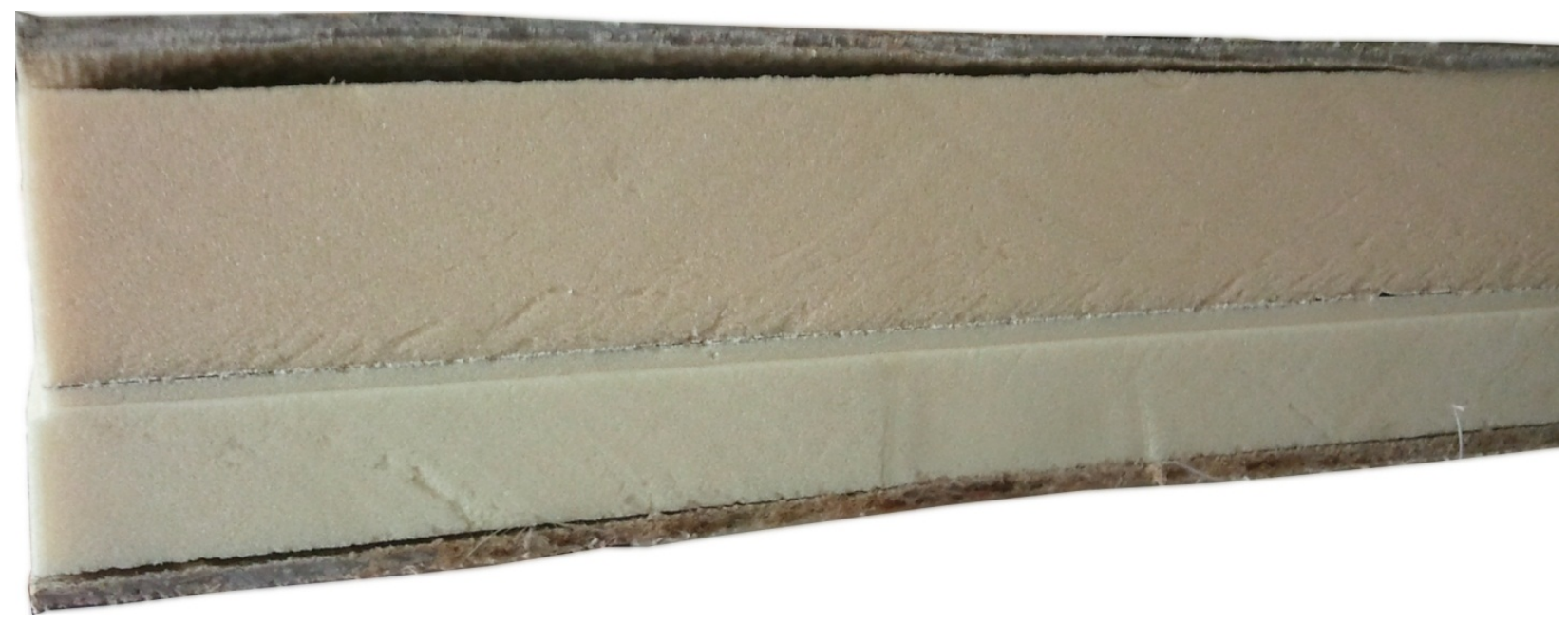

Figure 7-9 - As-received delamination at composite/foam interface (worst corner)

Using the weights of the mats along with the total panel weight and dimensions and the foam, resin, and fiber densities, the fiber volume fraction was calculated to be approximately $37 \%$, although the average is likely slightly higher ( $40 \%)$ throughout the majority of the panels, as there are a few obviously resin rich regions along the edges. Additionally, the areas of overlap may possess greater than 50\% FVF, while the areas with only continuous fibers were found (again, by weight and volume calculations) to possess a FVF of only around 27\%.

\subsection{Experimental Methodology}

\subsubsection{Flexural coupons}

Along with the completed panels, Fiber-Tech Industries provided some extra material from the facesheets (with the foam removed) cut from the ends of the panels during the trimming 
process. From this extra material, nine flexural samples were cut with the average dimensions of $6.1 \times 0.51 \times 0.27$ in (see Figure 7-10). These samples were tested in three point bending over a span of 4.30 in ( $~ 0.27 \times 16$, as specified by ASTM D790) at a crosshead rate of $0.25 \mathrm{in} / \mathrm{min}$.

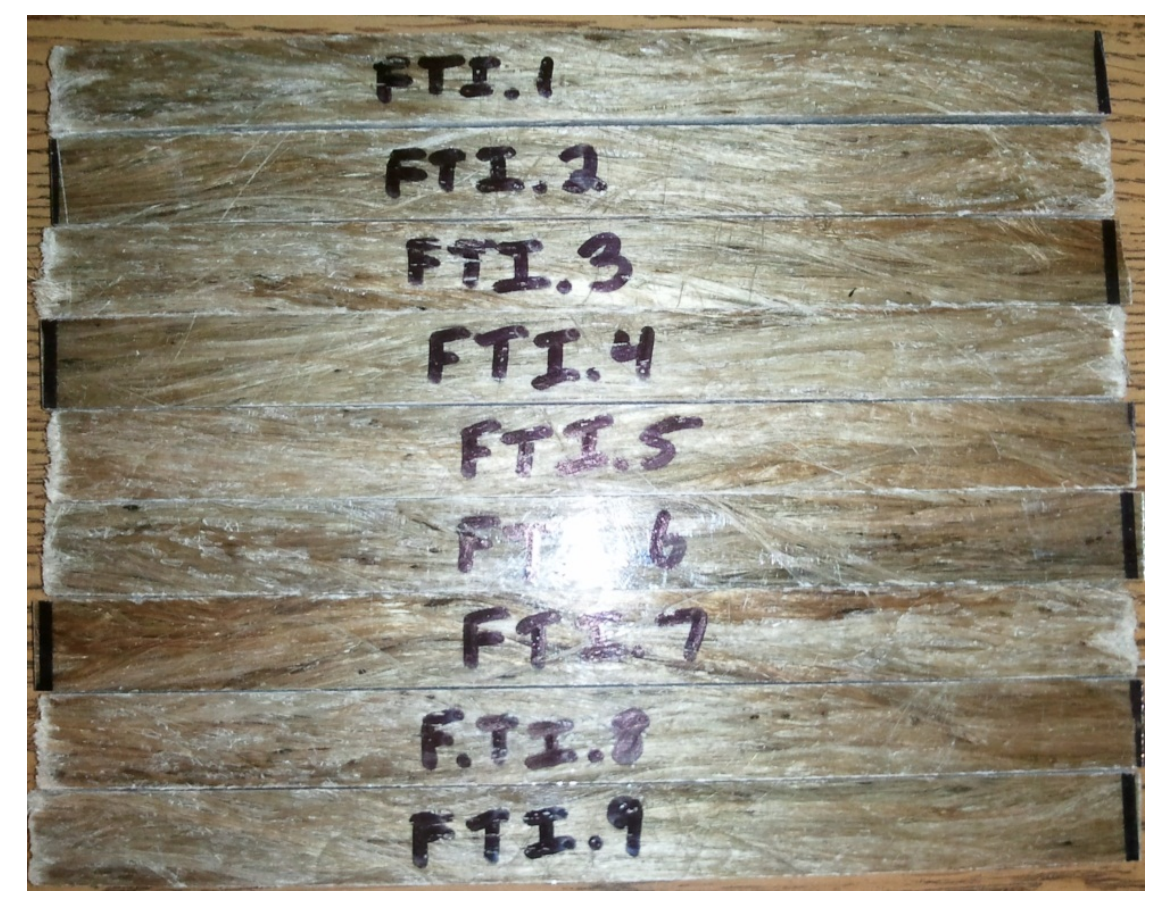

Figure 7-10 - Flexural coupons from Fiber-Tech Panel cut-off material

\subsubsection{Flexure test setup}

The OSB SIPs ended up being slightly thicker overall than the NFRP SIPs (4.5 in to 4 in), with facesheets of nearly twice the thickness (0.5 in to $\sim 0.25$ in), as shown in Figure 7-11. Despite these dimensional discrepancies, the panels were tested in the exact same configurations in both flexure and compression. In an initial test to assess the stiffness of the panels at a second span, the panels were placed across a 27 in span and loaded up to 500 lbs. The primary test (to failure) was conducted across a 45 in span (see Figure 7-12). Load was applied to the samples via a $1 / 2$ in thick, 6 in wide steel plate that spanned beyond the width of the sample. Elastomeric pads of $1 / 4$ in thickness were placed between the loading plate and the sample to reduce stress concentrations and to protect the top-surface, midspan strain gages.

The NFRP SIP intended for flexural testing was gaged with nine strain gages: a pair of longitudinal gages at or very near the midspan and mid-width of each facesheet; a pair of longitudinal gages at the midspan, $\sim 1$ in from the edge of the bottom facesheet; a transverse gage at or very near the midspan and mid-width of each facesheet; and a biaxial shear gage near the midspan and mid-width of the bottom facesheet. Additionally, a load cell was placed between the actuator and the loading plate to record the load and a LVDT was attached at or near midspan and mid-width of the bottom facesheet via a string and pulley system to record the bending deflection. While the OSB SIP test did not include any gages, the load and deflection were recorded in the same manner. 


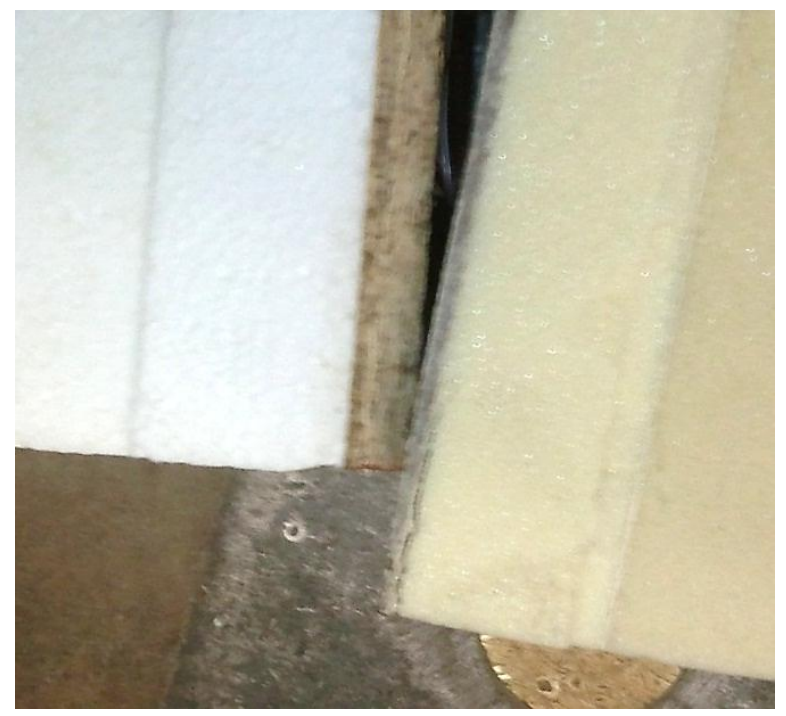

Figure 7-11 - Skin panel thickness comparison between OSB and NFRP SIPs

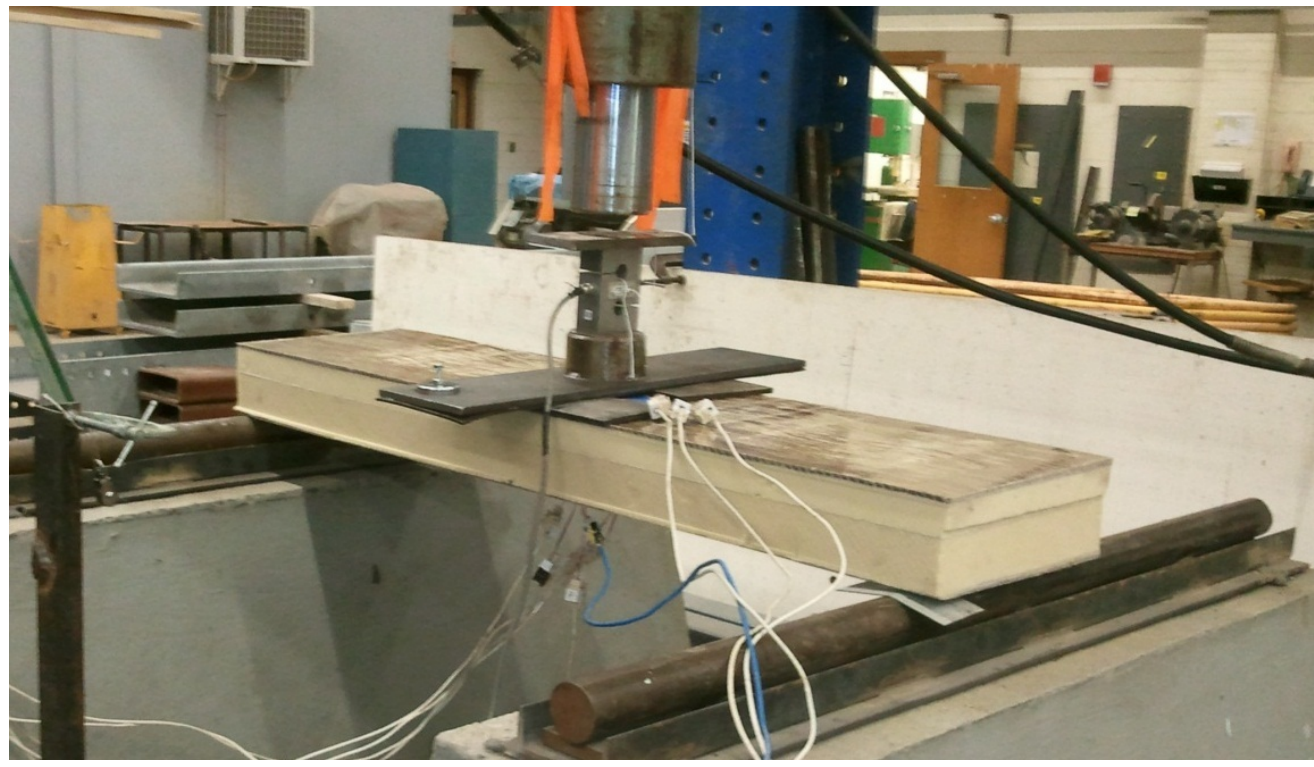

Figure 7-12 - Flexural test setup of NFRP SIP

\subsubsection{Compressive test setup}

As with the flexural testing, a pair of compression tests was run on each of an NFRP SIP panel and an OSB SIP panel. The first test consisted of an applied ramp load to around $1500 \mathrm{lbs}$, with wooden bracers restricting the panels from first mode buckling at the midspan of each facesheet (Figure 7-13). For the second test, the bracers were removed and the columns were allowed to buckle and deform in their natural modes as they were loaded to failure. The overall length of each column was 48 inches, while the NFRP and OSB panels had widths of 12.5 and 12.0 in, respectively. The panel thicknesses were approximately the same as the flexure samples: 4.0 in for the NFRP SIP and 4.5 in for the OSB SIP. The load was applied through a $1 / 2$ in thick 
steel plate that extended beyond all of the edges of the loading surface of the samples. Elastomeric pads of $1 / 4$ in thickness were placed between the loading plate and the top surface of the SIP, and between the bottom surface of the SIP and the concrete floor to reduce stress concentrations and to more evenly distribute the load.

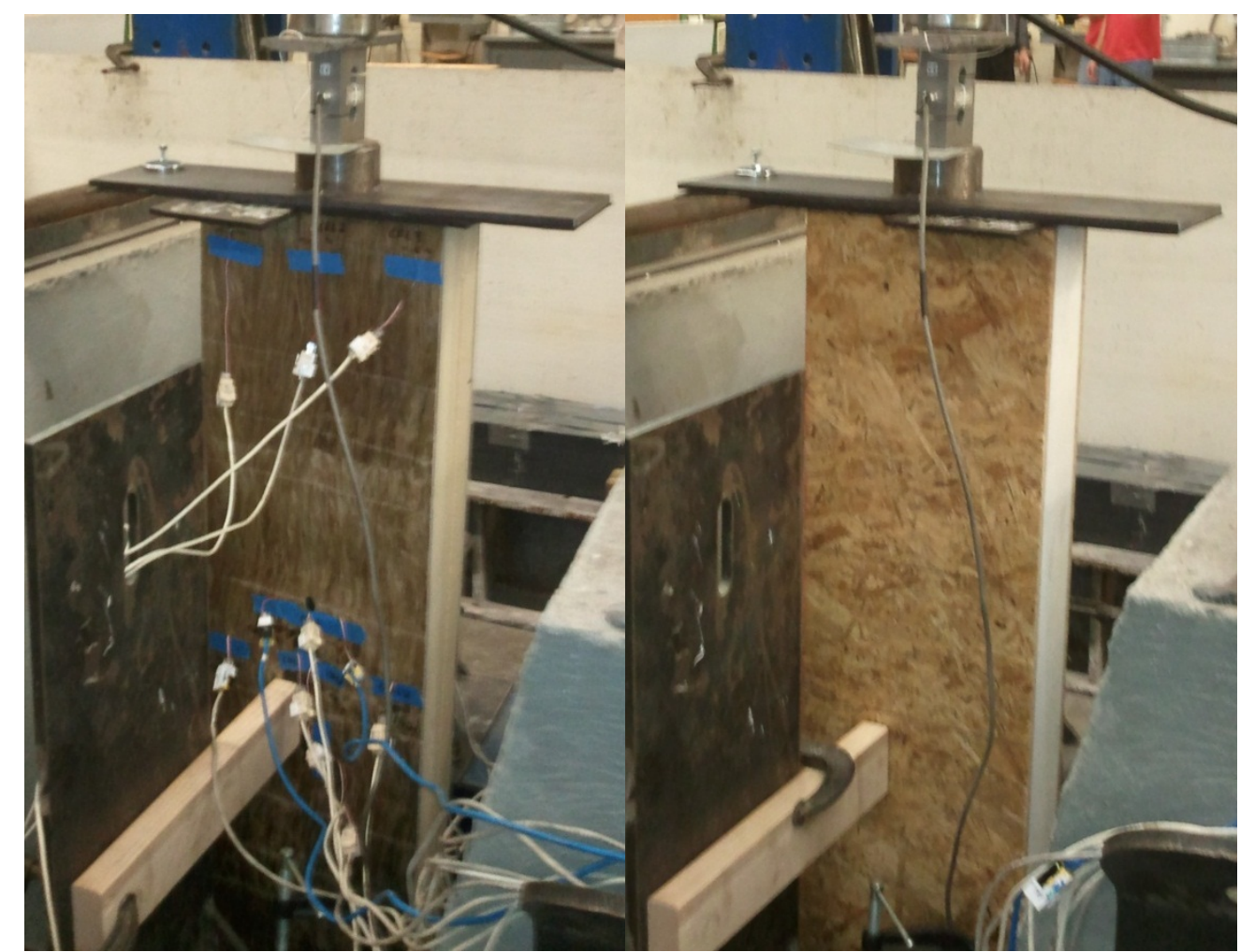

Figure 7-13 - Compressive test setup for both NFRP and OSB SIPs (before removal of midspan support)

The NFRP SIP intended for compressive testing was gaged with fourteen strain gages: a trio of longitudinal gages at or very near the midspan and mid-width of one facesheet (facesheet 'A,' the panel shown facing the camera in Figure 7-13); a pair of longitudinal gages at the midspan, $\sim 1$ in from each side edge of the facesheet $\mathrm{A}$; a trio of longitudinal gages at the center and $\sim 1$ in from each side edge, in a line about $\sim 1.5$ in below the top edge of facesheet A; a pair of longitudinal gates at or very near the midspan and mid-width of the reverse facesheet, facesheet 'B;' a transverse gage at or very near the midspan and mid-width of each facesheet (A and $\mathrm{B}$ ); and a pair of biaxial shear gages near the midspan and mid-width of the facesheet $\mathrm{A}$. Additionally, a load cell was placed between the actuator and the loading plate to record the load. While the OSB SIP test did not include any gages, the load was recorded in the same manner in order to determine the ultimate load capacity (while the absence of deflection measurements for the OSB panel renders the data collected from the first, braced test useless, this test was still conducted so that both panels would have experienced similar pre-loading before the second, unbraced test to failure). 


\subsection{Results and Discussion}

\subsubsection{Flexural coupon results}

One immediately noticeable problem with the coupon samples cut from the excess panel material was the pervasive presence of voids and dry fibers throughout the thickness (see Figure 7-14 for the worst observed cases). As a manufacturing process, the method of using vacuum bagging to exert compression forces on composite panels is unable to provide the same pressure as manufacturing in a hydraulic press. While the samples manufactured at WVU were loaded with around 40psi, the vacuum bagging was only able to achieve around a third of that. As a result, the resin was not forced to permeate throughout the reinforcement to the same extent as in the compression molded samples, resulting in more voids and dry fibers.

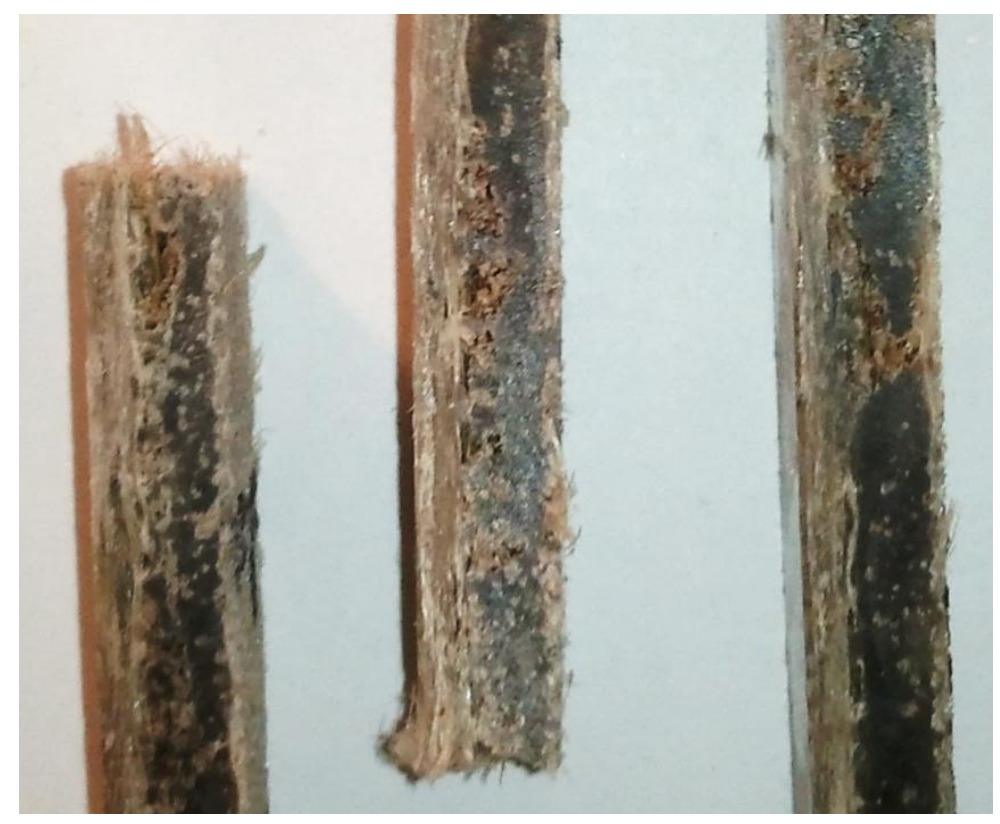

Figure 7-14 - Dry fibers and voids in Fiber-Tech Industries panels (worst cases)

Based on the fiber/fabric weight measurements taken before sending the mats to FiberTech Industries for manufacturing, the published resin density, and the measured composite density, the fiber volume fraction for these samples was approximated as around $27 \%$. Therefore, to compare the strength and stiffness results to those obtained from compression molding they need to be normalized to a $40 \%$ FVF. The average flexural strength and stiffness measurements for the nine samples, their normalized values, and their comparison to earlier compression molded samples are shown in Table 7-2. Based on these results, there is no decrease in stiffness due to the vacuum bagging manufacturing method, while the strength was decreased by $18 \%$. Therefore, in predicting the strength and stiffness behavior of these panels, the natural fiber $40 \%$ FVF composite stiffnesses measured in previous chapters should be multiplied by $\mathbf{0 . 6 7 5}$ (FVF adjustment only), while the composite strengths should be multiplied by $\mathbf{0 . 4 1 5}$ (0.675 FVF adjustment x 0.82 manufacturing method adjustment x 0.75 worst-case, 1.5 in joint overlap strength reduction). This reduction should end up being comfortably on the conservative side, 
since the volume fraction at the joints (where the 0.75 strength factor is applied) should actually be upwards of $50 \%$, due to the overlapping fiber bundles, while in the areas of low FVF (27\%), the overlap strength reduction would not apply. If the panels were manufactured at a FVF of $40 \%$ in the continuous sections, the stiffness and strength reduction factors for these panels would be 1.0 and 0.615 , respectively.

Table 7-2 - Flexural coupon testing results and comparison to compression molded samples (at 40\% FVF)

\begin{tabular}{|l|r|r|r|}
\hline & Average Value & Unit & Standard Deviation \\
\hline Flexural Strength ( 27\% FVF) & 14.9 & $\mathrm{ksi}$ & 1.11 \\
\hline Normalized Strength (40\% FVF) & 21.8 & $\mathrm{ksi}$ & 1.99 \\
\hline Compression Molded Strength & 26.6 & $\mathrm{ksi}$ & 2.16 \\
\hline \% Compression Molded Strength & $82 \%$ & 0.05 \\
\hline Flexural Stiffness ( 27\% FVF) & 0.75 & Msi & 0.07 \\
\hline Normalized Stiffness (40\% FVF) & 1.09 & Msi & 0.10 \\
\hline Compression Molded Stiffness & 1.08 & Msi & \\
\hline \% Compression Molded Stiffness & $101 \%$ &
\end{tabular}

\subsubsection{Flexure test results}

The load vs deflection curve recorded for the NFRP SIP in flexure over a 27 in span is shown in Figure 7-15. The SIP was loaded to just over 500 lbs in order to obtain a good idea of the stiffness behavior at a span other than the span to failure (45 in). The strain measurements taken at each strain location for this test are shown in Figure 7-16.

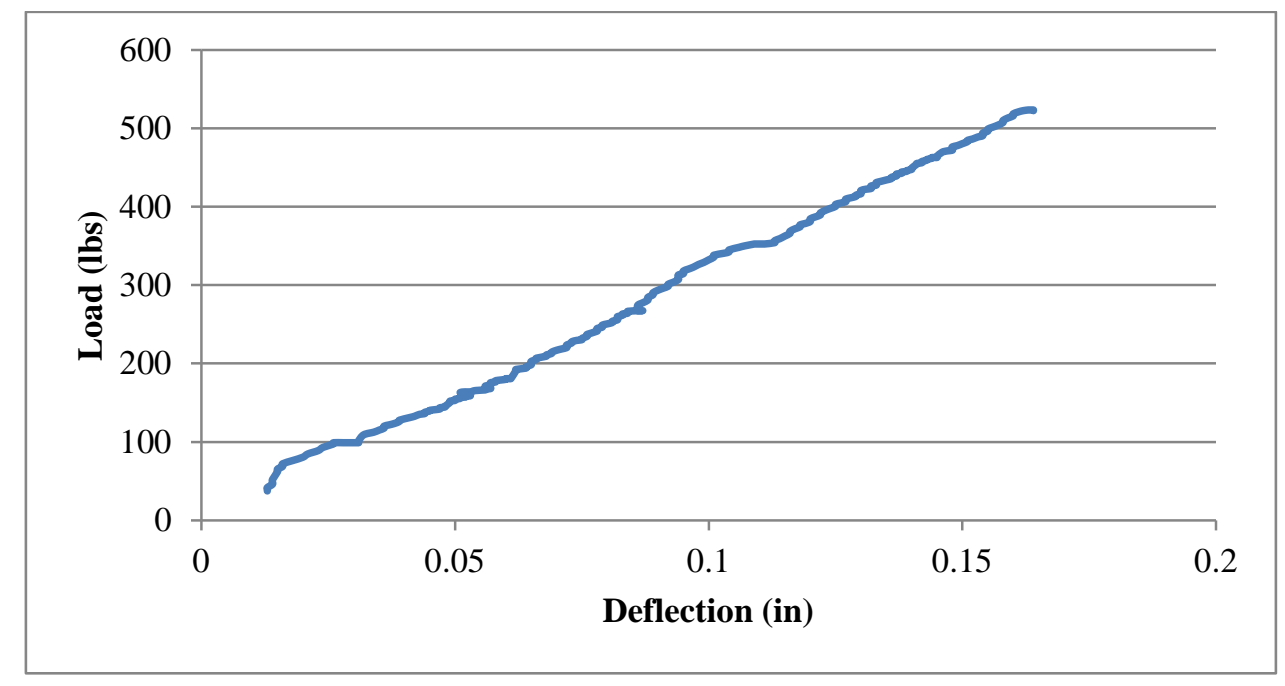

Figure 7-15 - Load vs. deflection for flexure of NFRP SIP over 27 in span (not to failure) 


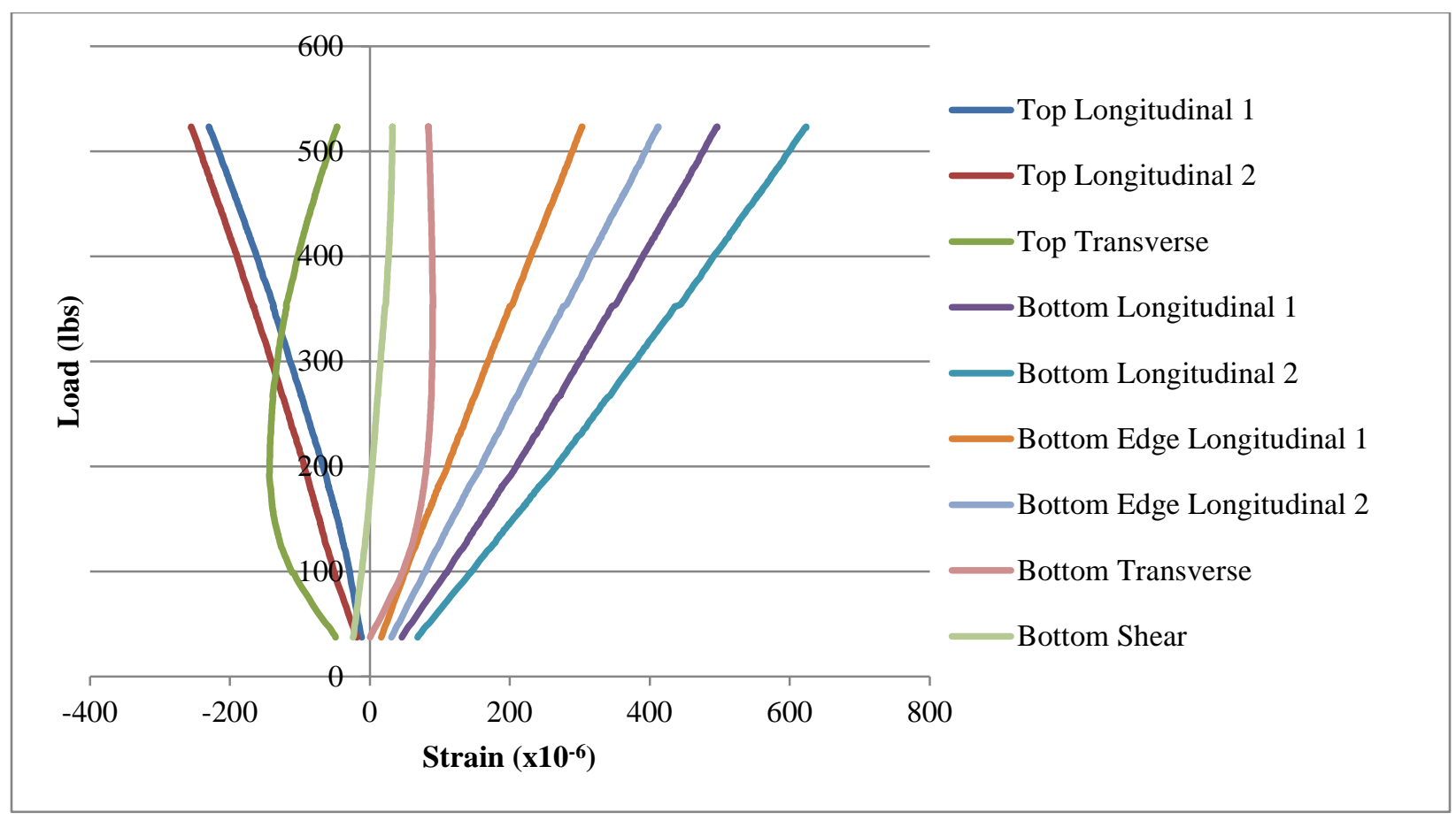

Figure 7-16 - Strain measurements from flexure of NFRP SIP over 27 in span (not to failure)

From Figure 7-16, it appears that the longitudinal strain has a fairly high variation depending on the locations of the gages, and can be off by over $20 \%$ for parallel gages located less than an inch apart. This disparity in strain measurements is thought to be due to a combination of the variation introduced by the organic nature of the natural fibers (with slightly different strengths and stiffnesses for each fiber), the variation introduced by handmanufacturing the mats (with inherent FVF variations across the width and through the thickness), and the variation introduced by poor resin coverage on the surface (some locations on the same surface had dry or nearly-dry fibers showing, while others had smooth resin coverage). Despite the NFRP having nearly identical stiffnesses in tension and compression, the tensile gages recorded much higher strain than the compression gages. This was probably partly due to local stiffening in the compression gages just below the load patch and partly due to the presence of a significant corner delamination between the core and the lower facesheet, which would shift the neutral axis to above the mid-thickness of the panel. The fairly high transverse strain on the top panel was more due to the gage's location directly beneath the loading plate rather than the Poisson's ratio effect. As expected, the in-plane shear strain was negligible due to the balanced [0/90] layup of each panel.

The load vs. deflection curve recorded for the NFRP SIP in flexure over a 45 in span is shown in Figure 7-17. At this span, the panel was loaded to failure at $692 \mathrm{lbs}$. The strain measurements taken at each strain location for this test are shown in Figure 7-18. The load vs. deflection curve for the OSB SIP in flexure over a 45 in span is shown in Figure 7-19, and the load at failure was 1090 lbs. 


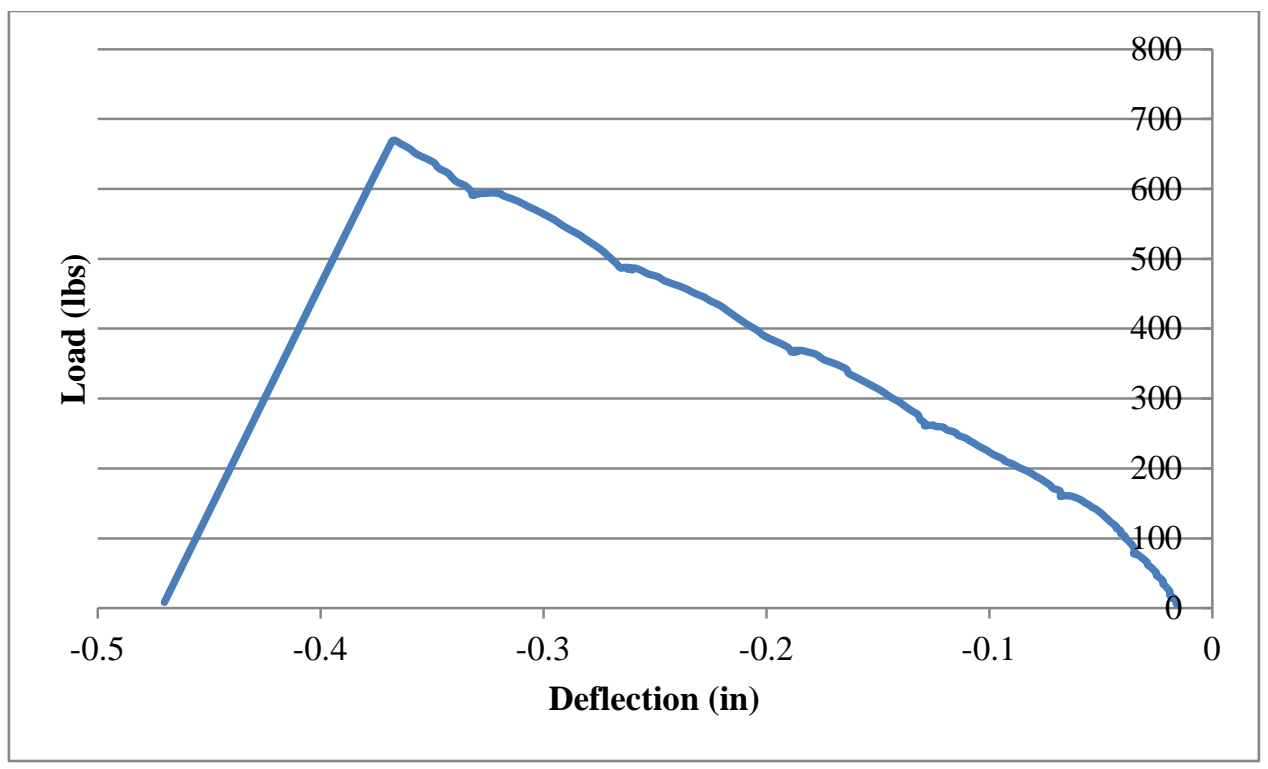

Figure 7-17 - Load vs. deflection for flexure of NFRP SIP over 45 in span (to failure)

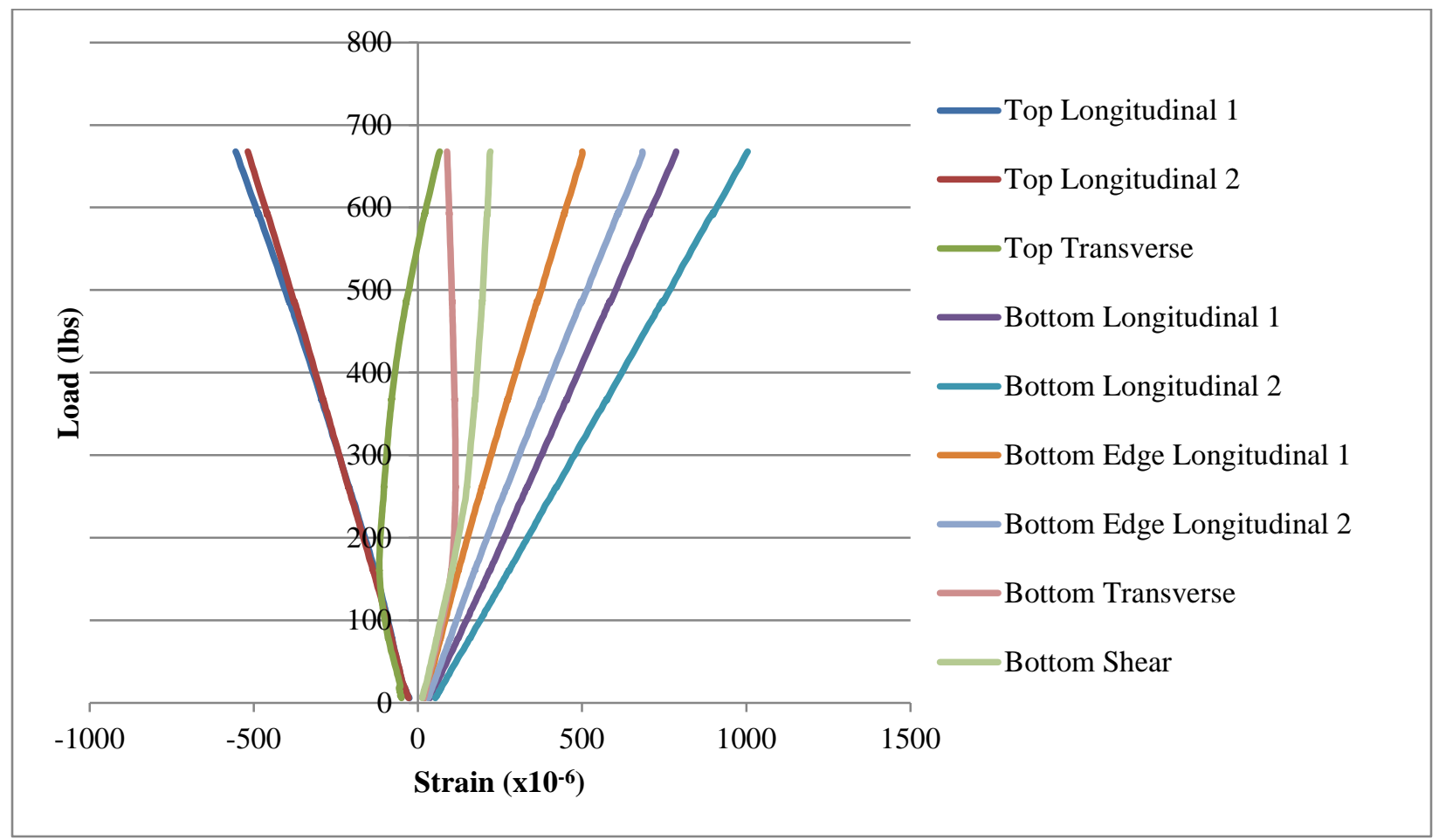

Figure 7-18 - Strain measurements from flexure of NFRP SIP over 45 in span (to failure) 


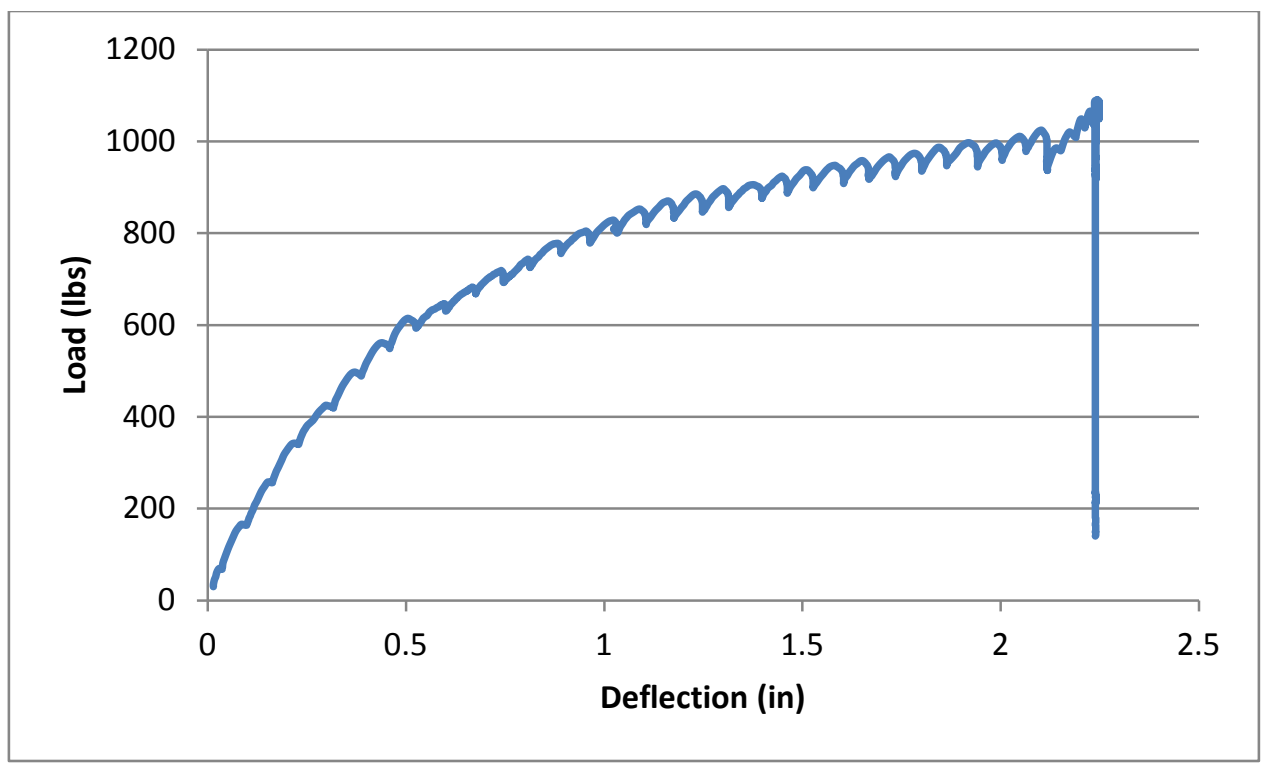

Figure 7-19 - Load vs. deflection for flexure of OSB SIP over 45 in span (to failure)

From the curve in Figure 7-17, it appears that there is some non-linearity fairly early in the loading of the NFRP SIP, which is likely due to the poor load transfer of the overly-flexible core. The strain behavior of this test was very similar to the strain behavior over the 27 in span, with the top facesheet and the bottom facesheet reaching average longitudinal strains of $-537 \mu \epsilon$ and $744 \mu \epsilon$, respectively, at failure. Based on coupon testing, the NFRP composite would not be expected to fail until it reached a strain of around $8000 \mu \epsilon$ in tension or $15000 \mu \epsilon$ in compression. Unlike the NFRP SIP, the OSB SIP exhibited a fairly linear modulus of elasticity over the first $50 \%$ of its loading (Figure 7-19), followed by severe stiffness reduction, as it ended up carrying $58 \%$ more load than the NFRP SIP but had nearly 5 times the deflection at failure.

According to Daniel (2009), "Possible composite sandwich beam failure modes include tensile or compressive failure of the facesheets, debonding at the core/facesheet interface, indentation failure under localized loading, core failure, wrinkling of the compression facesheet, and global buckling." Once a single failure mode has initiated the failure process, several other failure modes may occur before the final failure. Daniel (2009) found that the core material properties were the most influential factors for determining failure initiation and mode. In short beams, such as those tested in this study ( $d / t$ of around 10$)$, the core is mainly subjected to shear forces, leading to failure when the shear stress reaches the shear strength of the material (Daniel 2009). Unless large pre-existing cracks are present at the interface, the core debonding failure mode will usually be preceded by another mode of failure (Triantafillou and Gibson 1989). In the case of the NFRP panels, one large pre-existing delamination was present at the corner of the bottom (tension) face and was responsible (along with the poor shear strength of the core) for the failure mode of the panel.

The failure modes of each of the panels were very similar, as seen in Figure 7-20 (NFRP SIP) and Figure 7-21 (OSB SIP), and were a combination of shearing through the core along with delamination at the facesheet/core interface. In the NFRP SIP, a splitting noise could be 
heard as the panel was loaded and the pre-existing corner delamination spread across the width of the panel. The OSB SIP, on the other hand, failed suddenly at a very high deflection.

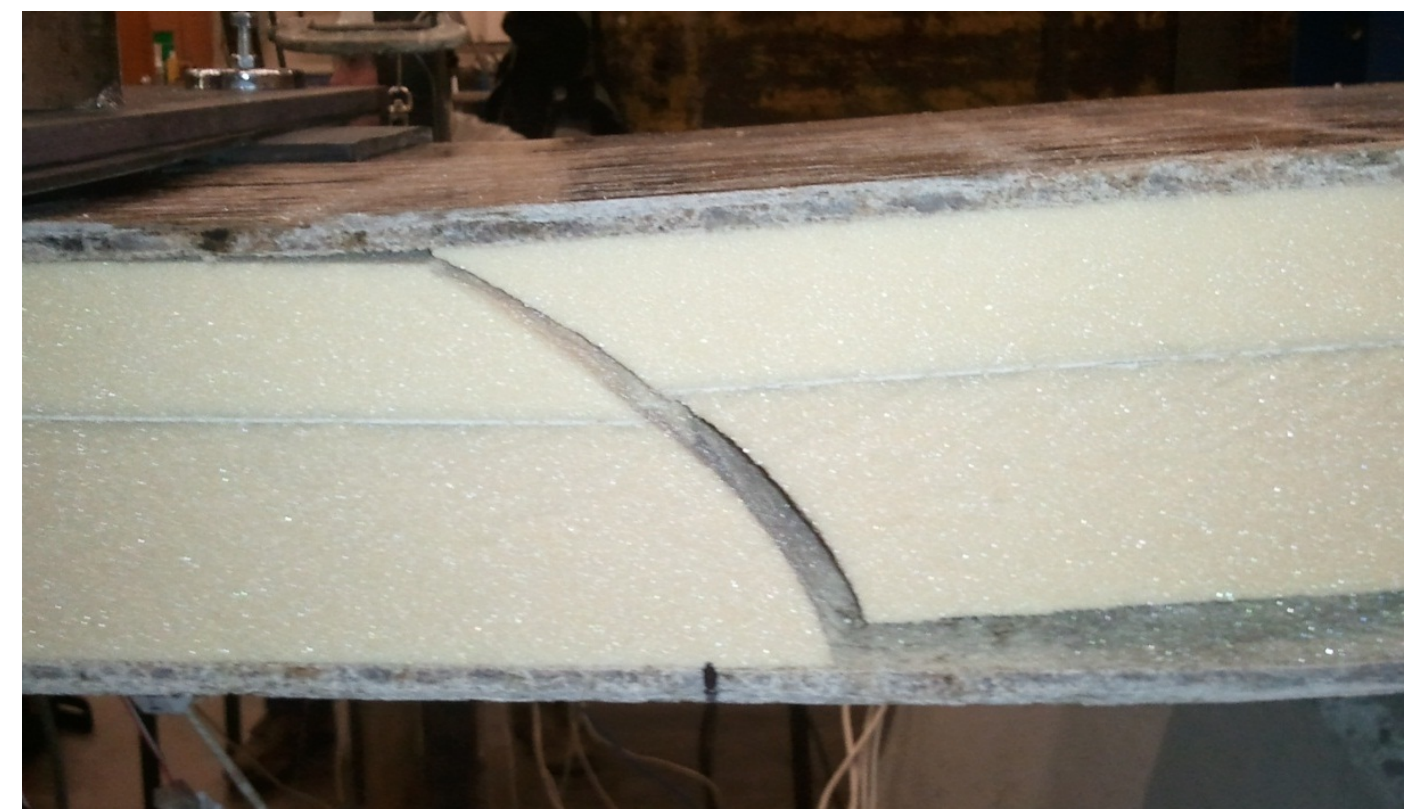

Figure 7-20 - Shear and delamination flexural failures in foam core of NFRP SIP

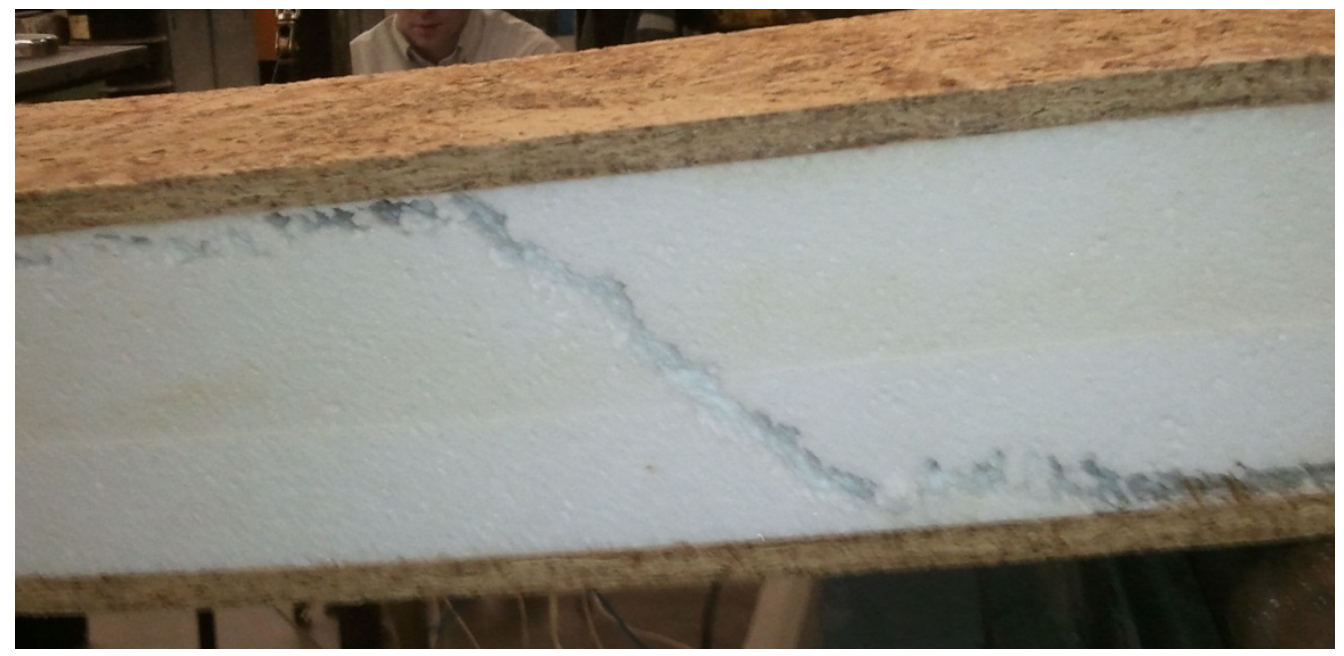

Figure 7-21 - Shear and delamination flexural failures in foam core of OSB SIP

Correlating the NFRP SIP experimental results with theoretical values of stiffness and strength was difficult due to the low strength and stiffness of the core material, the pre-existing delaminations, and the resulting core-centric failure mode.

The bending deflection can be calculated as shown in Equation (7-1), where $P$ is the applied 3-point bending load, L is the span length, EI is the flexural stiffness (modulus of 
elasticity multiplied by moment of inertia), and AG is the shear stiffness (shear area multiplied by the modulus of rigidity).

$$
\delta=\frac{P L^{3}}{48(E I)}+\frac{P L}{4(A G)}
$$

The flexural stiffness, EI, can be approximated for the section according to Equation (7-2), where $b$ is the panel width, $t$ is the thickness, $E$ is the axial modulus of elasticity, and the $f$ and $c$ subscripts indicate the facesheet and core, respectively. This equation was derived from the moment of inertia formula and parallel axis theorem, and is valid only for a 3-layer, symmetric sandwich composite, as was tested in this experiment.

$$
(E I)=\frac{E_{f} t_{f}^{3} b}{6}+\frac{E_{f} t_{f} b\left(t_{f}+t_{c}\right)^{2}}{2}+\frac{E_{c} t_{c}^{3} b}{12}
$$

To calculate the bending modulus of elasticity, $E_{B}$, independent of the moment of inertia, the transformed area moment of inertia, in which the width of the foam core section is multiplied by $E_{c} / E_{f}$, must be used. For the NFRP SIP, the moment of inertia is $24.14 \mathrm{in}^{4}$ and the bending modulus is 1.95 Msi. For the OSB SIP (using OSB properties from experimental results in a previous chapter and typical, medium-quality polystyrene properties, as the exact density/quality of the polystyrene core was not known), the moment of inertia is $48.27 \mathrm{in}^{4}$ and the bending modulus is $0.770 \mathrm{Msi}$. The higher moment of inertia is due to the thicker facesheets on the OSB SIP. This theoretical bending modulus is reasonable, given that the basic properties of SIPs listed in (NTA 2011) dictate that $E_{b}$ for strong axis bending should have a value of 0.6588 Msi.

Usually, the core shear modulus in sandwich panels is taken as the shear modulus of the composite structure. In the case of the OSB panel, this method works fairly well, and using the required core shear modulus of 405 psi from (NTA 2011), the theoretical deflections from Equation (7-1) at a $200 \mathrm{lb}$ load (within the linear range of its deflection) are 0.0102 in due to bending (first term) and 0.1157 in due to shear (second term). This total theoretical deflection of 0.1259 in is only $8.5 \%$ higher than the experimental deflection of 0.116 in. However, if the core shear modulus of the polyisocyanurate foam (220 psi) is used in the theoretical deflection calculation of the NFRP SIP, the result is 2-3 times higher than the experimental deflections at spans of 27 and 45 in. Due to the extremely high disparity between the core and facesheet shear moduli in the NFRP SIP, it seems reasonable to conclude that the sandwich panel shear modulus will be higher than the modulus of the poor-quality core alone. By iterating $G$ to get the minimum average error for each of the test spans, it was found that a value of around 575 psi allows for theoretical deflection prediction of around 22\% error for each of the spans (22\% low for 27 in span; 22\% high for 45 in span). This method obviously only provides a rough approximation, but the conclusions are reasonable: shear deflection is higher than bending deflection, due to the low shear modulus of the core material, and the shear modulus of the NFRP SIP is higher than the shear modulus of the core due to the high shear modulus of the NFRP material. If the assumptions that Equation (7-2) results in an accurate flexural stiffness and that dividing that stiffness by the transformed area method gives an accurate bending modulus are true, then, despite the poor core material, the bending modulus of elasticity of the NFRP SIP is 2.54 times higher than the bending modulus of the OSB SIP.

In predicting the flexural strength of the SIPs, the two simplest failure modes to explore are core shear failure (the controlling failure mode for both SIPs) and facesheet failure due to 
bending stress, $\sigma=M c / I$. According to Daniel (2009), the shear stress can be assumed constant throughout the core, and simplified as shown in Equation (7-3).

$$
\tau=\frac{P}{2 b t_{c}}
$$

For the NFRP SIP, with $\tau=16$ psi, the predicted core-shearing failure load would therefore be at a load, $P$, of 1344 lbs. The experimental failure load was $692 \mathrm{lbs}$, around $51 \%$ of the predicted. This prediction is not nearly as poor as it would seem at first glance due to the fact that there was a significant corner delamination, resulting in: a) $b<12$ in (from a visual inspection, it appeared the delamination extended for at least 4-5 inches across the width at its deepest point), and b) an initial crack along which the shearing/delamination failure would begin to propagate at a load much lower than theoretical failure, further reducing $b$. Again, the actual mechanical properties of the polystyrene core of the OSB SIP were not known, and the shear strength can range from 10-40 psi, depending on the density and quality. Using the actual failure load of 1100 lbs to back-calculate the shear strength results in a value of 13-14 psi, which would be reasonable for a mid-to-low end polystyrene core.

If the SIPs were designed such that the failure mode would not be due to core shearing/delaminating, then their failure strength could be reasonably predicted using $\sigma=M c / I$, or Equation (7-4), modified for three-point bending, where $c$ is the distance from the neutral axis to the outside surface of a facesheet and $I$ is the transformed-area moment of inertia.

$$
\sigma=\frac{P L c}{4 I}
$$

For the NFRP SIP, the lowest axial strength would be compression on the top surface. Applying the strength reduction factor of section 7.5.1 to the untreated NFRP compression strength from a previous chapter, the theoretical compression strength of the facesheet would be $0.415 \times 16.743=6.948 \mathrm{ksi}$. Using this stress in Equation (7-4), the predicted failure load for the NFRP SIP would be 7,450 lbs. For the OSB SIP, the lowest axial strength would be tension on the bottom surface. From previous testing, the dry, OSB tensile strength is around $0.923 \mathrm{ksi}$. Based on this facesheet strength, the predicted failure load for the OSB SIP would be 1,760 lbs. Therefore, if both panels were designed such that failure was not initiated in the core, the theoretical flexural strength of the NFRP SIP would be 4.23 times higher than the theoretical flexural strength of the OSB SIP.

In addition to potentially being over 2.5 times stiffer and 4 times stronger in bending, the NFRP skins are also only around $90 \%$ of the weight of the OSB skins, due to their reduced thickness. Clearly, the potential for NFRP composites to be used as facesheets on SIPs in flexural applications is great, but the challenge lies in designing a core system that is cheap and lightweight, yet does not allow such high shear deformation and does not fail prematurely.

\subsubsection{Compressive test results}

Since the deflection was not captured directly during the compressive tests, an approximate deflection value can be obtained by multiplying the average of all of the longitudinal strain gages by the column length (48 in). A plot of the applied load vs. this straindetermined deflection for the braced NFRP SIP compressive test is shown in Figure 7-22. The 
sample was only loaded up to around $1500 \mathrm{lbs}$ in this test, not to failure. The strain measurements taken at each strain location for this test are shown in Figure 7-23.

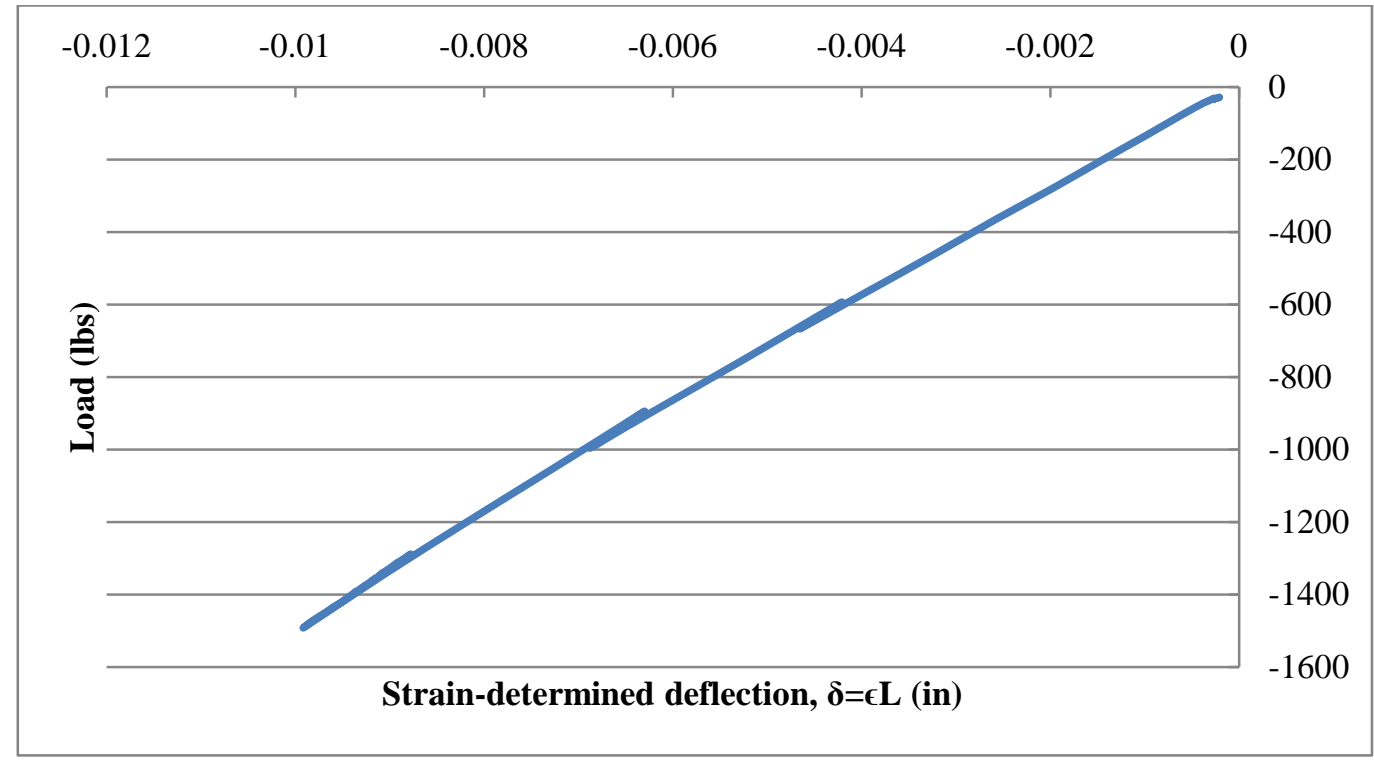

Figure 7-22 - Load vs. strain-determined deflection of NFRP SIP in braced compressive test (not to failure)

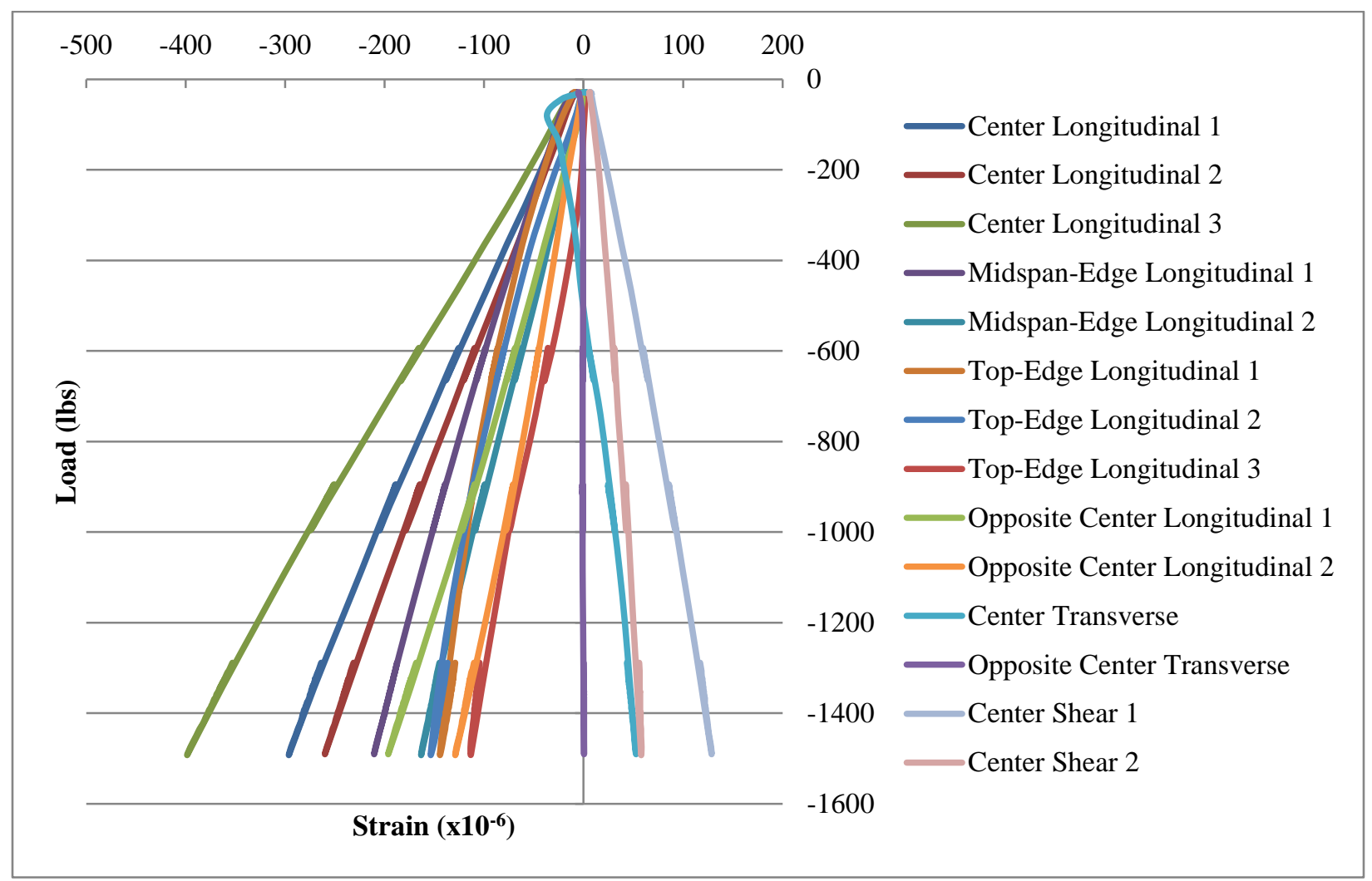

Figure 7-23 - Strain measurements from braced compressive test of NFRP SIP (not to failure) 
From Figure 7-22, it appears that the load vs. strain behavior is linear, at least over the range of this test. As was observed with the flexural strain results, a fairly high variation is seen in the longitudinal strain results of Figure 7-23, with side A (the side with most of the gages) experiencing, on average, significantly higher strains than side B (gages listed as “opposite”). The strain is also generally higher in the gages at the center of the panel than near the edges or the top. Also similar to the flexural test results, the transverse and shear gages picked up relatively little strain, albeit more than in flexural testing.

A comparative plot of the longitudinal gage averages for each of the two facesheets is shown in Figure 7-24. The stress is calculated by neglecting the compressive load carried by the core ( $<0.5 \%$ of the total load), and assuming that it is equally distributed between the two facesheets. Based on this figure, the compressive modulus is $1.07 \mathrm{Msi}$ for side A and 1.67 Msi for side $\mathrm{B}$, as compared to a theoretical value of $0.675 \times 3.3=2.23 \mathrm{Msi}$. The difference between the two different observed moduli for the two facesheets is probably more due to unequal load distribution than to significant differences in their compressive stiffnesses. The overall reduced stiffness of the facesheets compared to the expected stiffness is likely due to some bending behavior of the facesheets due to the flexible core and presence of several delaminations, despite bracing.

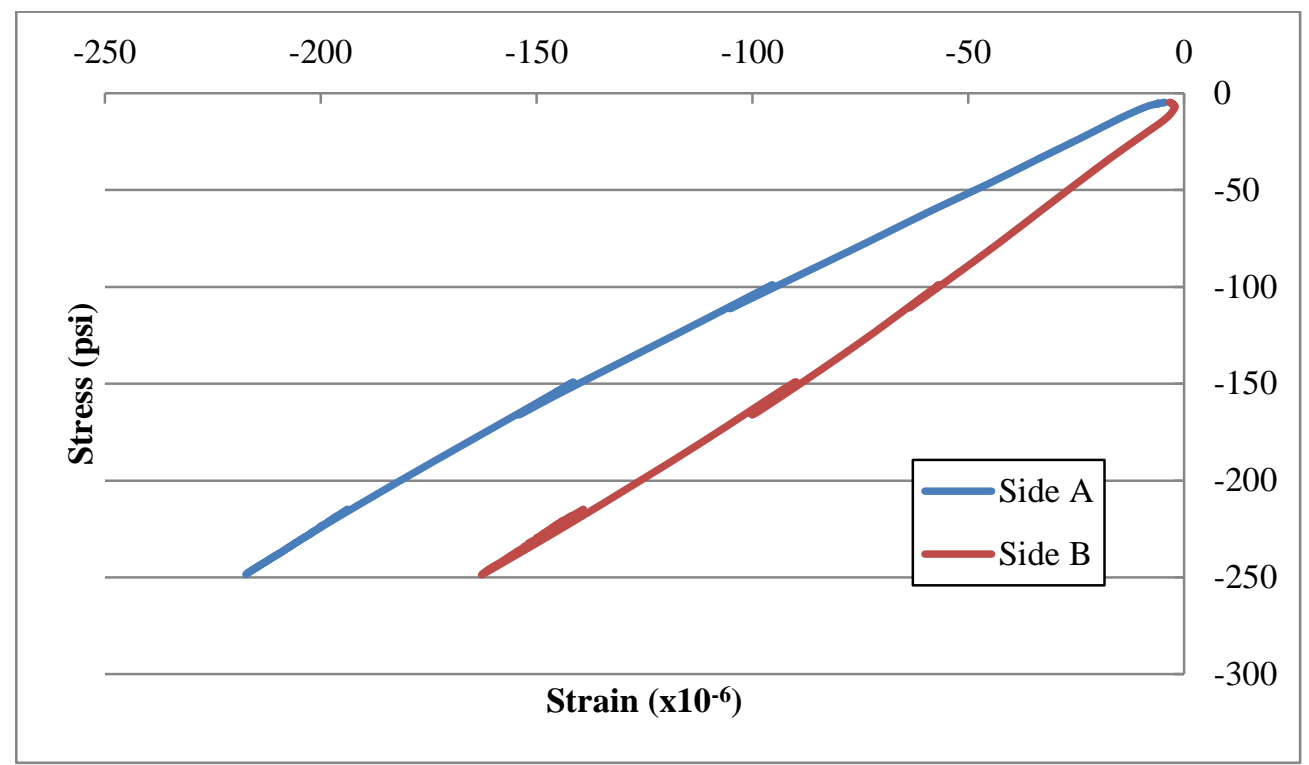

Figure 7-24 - Stress vs. strain for each facesheet during braced compressive test of NFRP SIP (not to failure)

A plot of the applied load vs. the strain-determined deflection for the un-braced NFRP SIP compressive test is shown in Figure 7-25. Again, this plot is extremely linear, despite the sample being loaded to failure at 5100 lbs. The strain measurements taken at each strain location for this test are shown in Figure 7-26. 


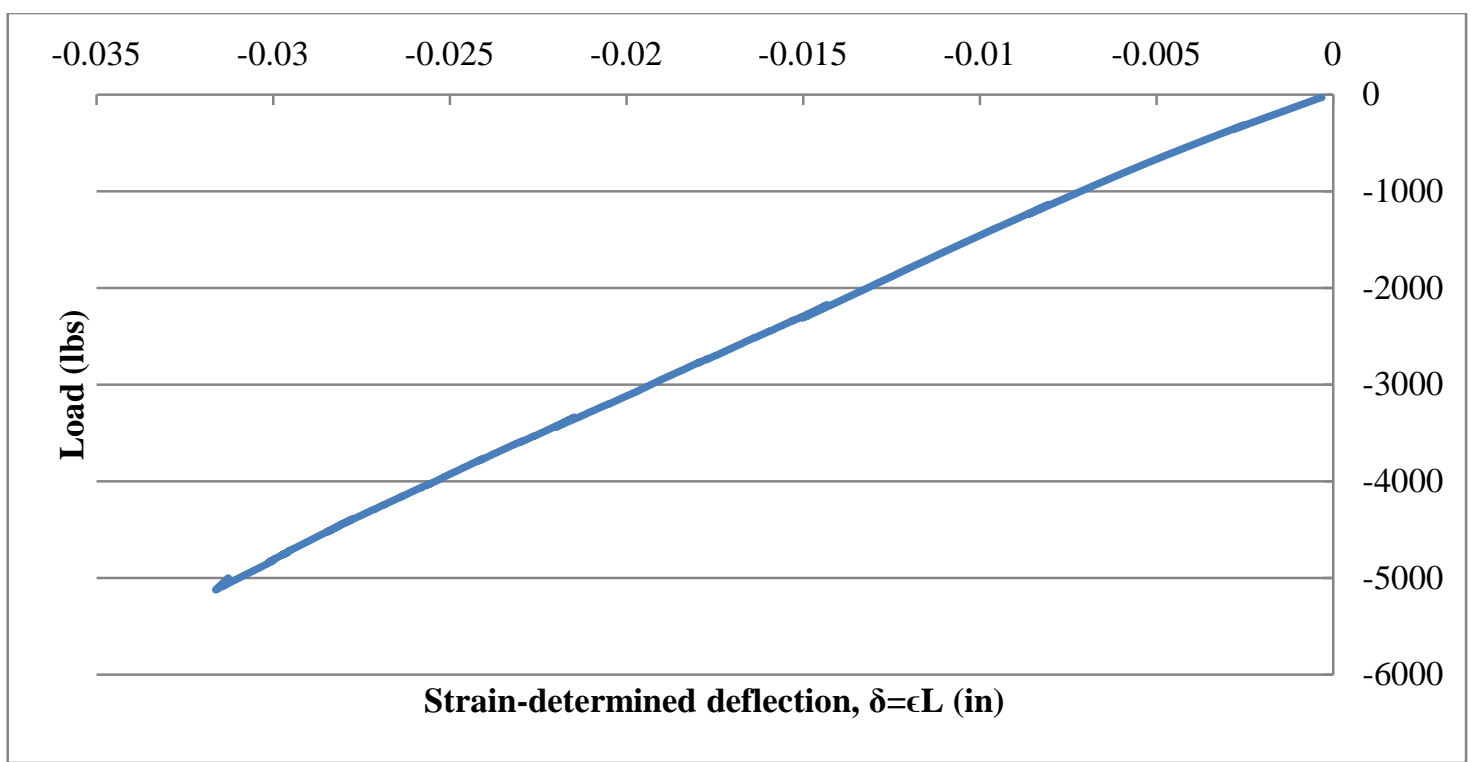

Figure 7-25 - Load vs. strain-determined deflection of NFRP SIP in un-braced compressive test (to failure)

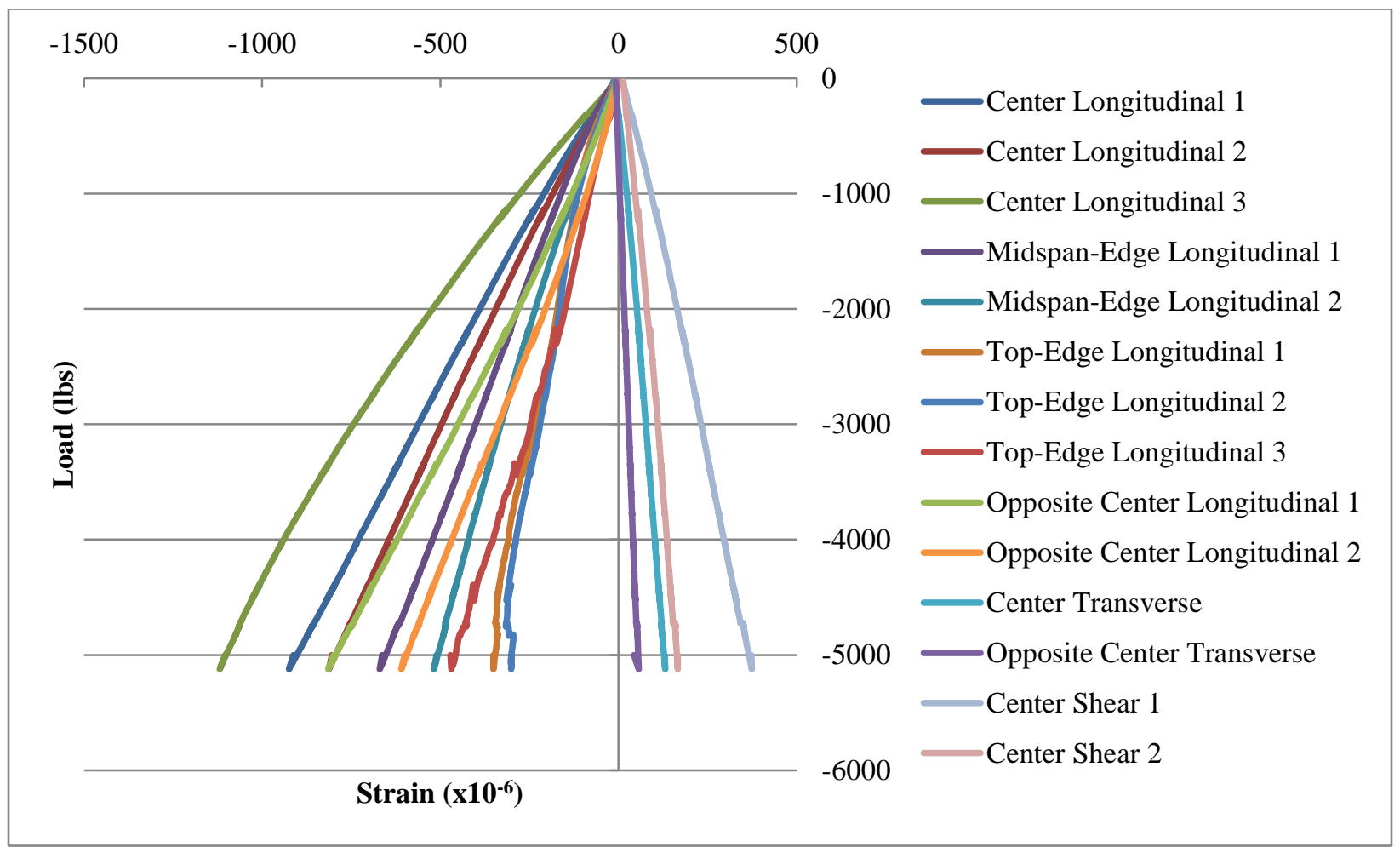

Figure 7-26 - Strain measurements from un-braced compressive test of NFRP SIP (to failure)

A comparative plot of the longitudinal gage averages for each of the two facesheets is shown in Figure 7-27. The strain behavior of this test started out fairly similarly to the strain behavior of the braced compressive test, but as the test progressed, it appears some bowing of the sandwich structure toward side A was taking place, as side A gained stiffness and side B lost 
stiffness. At failure, the average longitudinal strains were $-646 \mu \epsilon$ and $-712 \mu \epsilon$, respectively, for facesheets $\mathrm{A}$ and $\mathrm{B}$, and the failure stress is around 850 psi. Based on coupon testing, the NFRP composite would not be expected to fail until it reached a compressive strain of around $15000 \mu \epsilon$. The compressive moduli of the facesheets in the un-braced test were found to be $1.12 \mathrm{Msi}$ for facesheet A and 1.47 Msi for facesheet B, indicating that the bracing did not have a significant effect on the stiffness behavior, at least early in loading.

The un-braced OSB panel failed when it reached a load of 9640 lbs. Since no deflection or strain measurements were taken, the only material property that can be determined from this test is the compressive strength, which is around 800 psi if the foam is again assumed to carry only a negligible axial load.

The compressive failure modes of the two panels were quite different, with side B of the NFRP SIP delaminating suddenly and completely (Figure 7-28) while the OSB SIP had a crushing/buckling failure at the bottom end, with some delamination in the failure region (Figure 7-29). Based on OSB experimental strength properties, the OSB SIP would be predicted to fail at a compressive stress of between 1200-1600 psi. If the test were set up such that the load were not equally distributed, such that between $75-100 \%$ of the load were transmitted through one panel, this predicted stress is very reasonable. The failure mode of the OSB SIP supports the theory of unevenly distributed load, as the failure was not symmetric and clearly initiated in one of the panels.

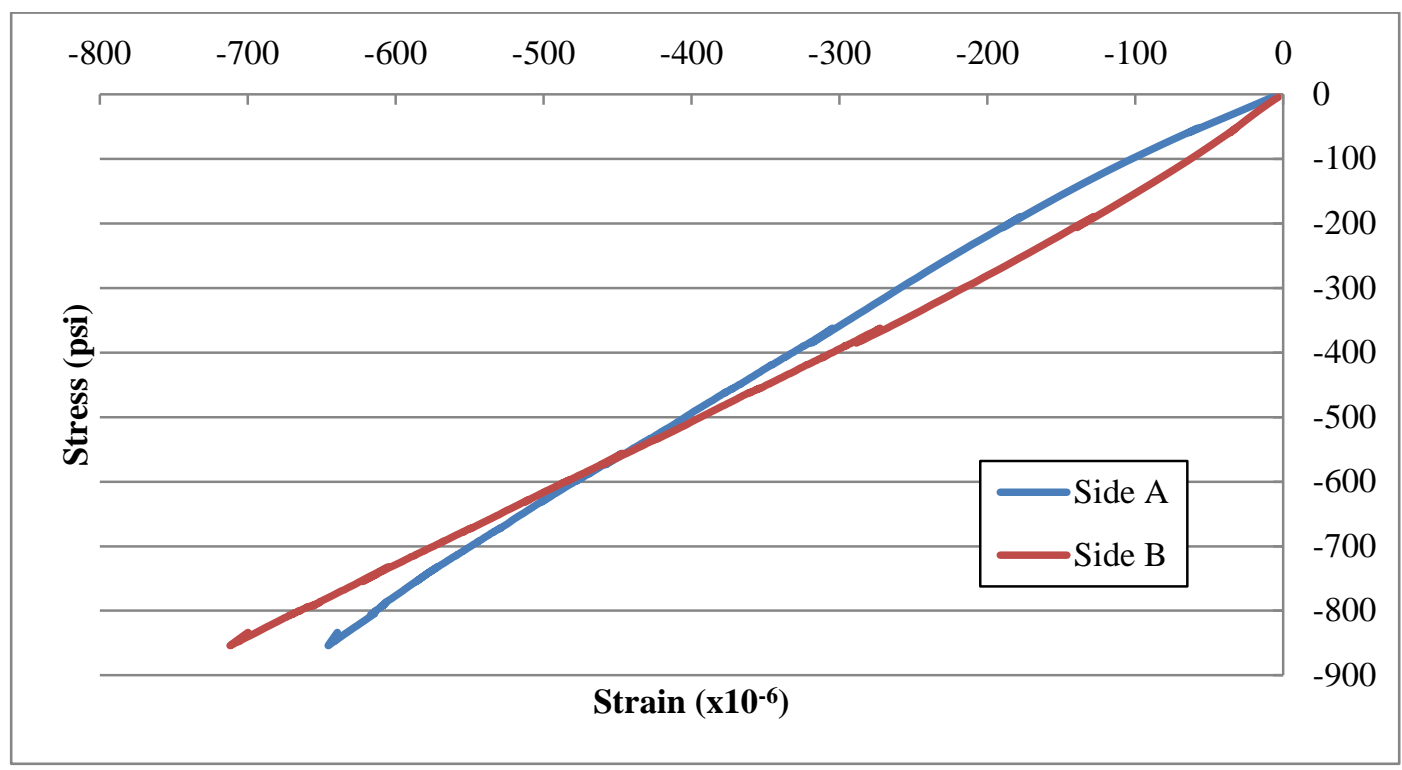

Figure 7-27 - Stress vs. strain for each facesheet during un-braced compressive test of NFRP SIP (to failure) 


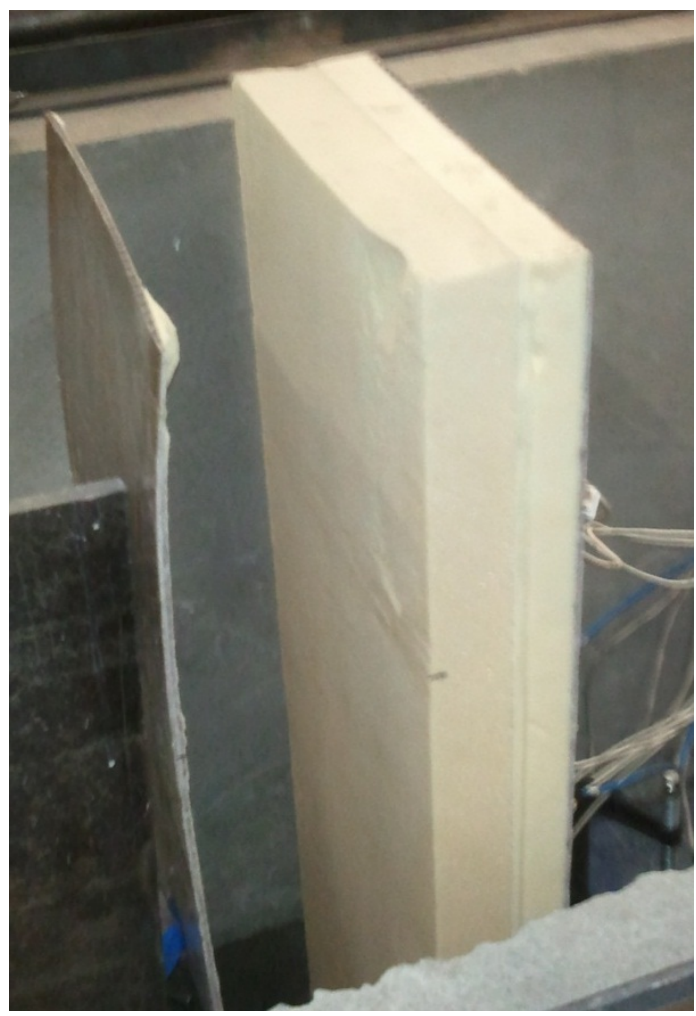

Figure 7-28 - Complete panel delamination compressive failure of NFRP SIP

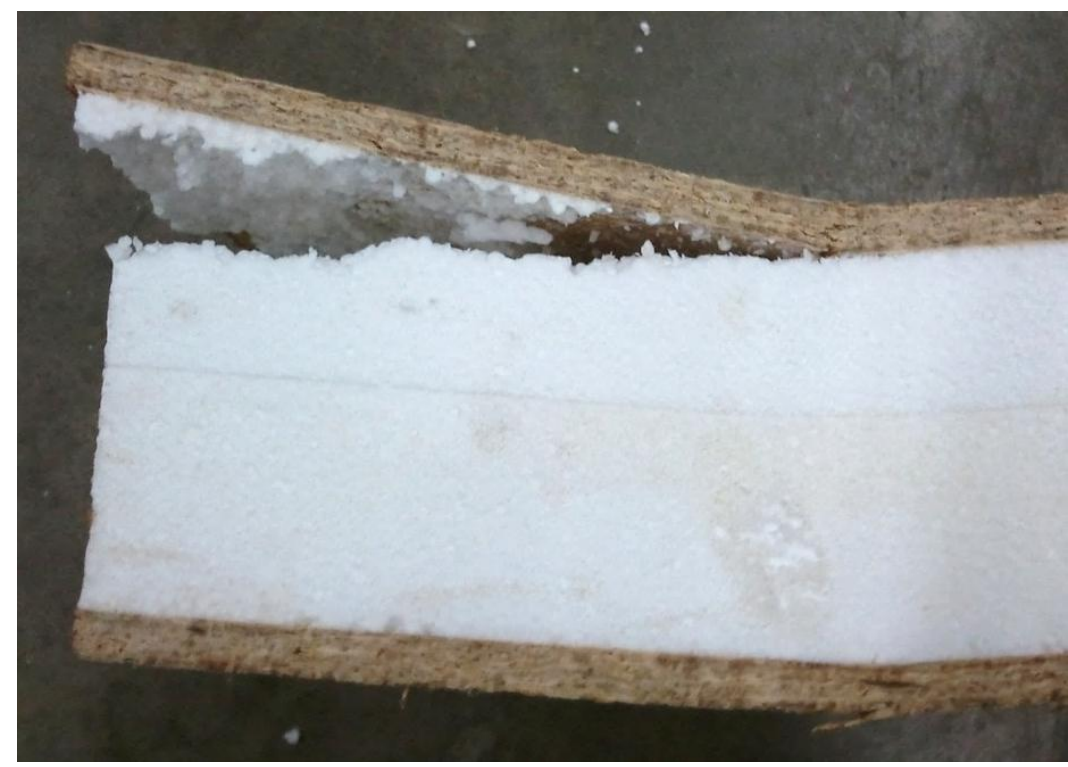

Figure 7-29 - End-buckling compressive failure of OSB SIP

The NFRP SIP panels were not so unevenly loaded, however, as made evident by their fairly similar strain data. However, if this worst case loading scenario is considered, then the stress in the over-loaded panel would be around 1700 psi. The ultimate strength of the NFRP 
panels is predicted to be $0.415 \times 16.743=6.948 \mathrm{ksi}$. Therefore, neglecting buckling behavior, the NFRP SIP would be able to take 4-8 times more loading before material rupture, depending on the load distribution between the two panels, for an ultimate load of over 41,000 lbs. Compared to the theoretical ultimate load before material rupture for the OSB SIP of around 19,000 lbs, the NFRP SIP has the potential to carry 2.17 times the load of the OSB SIP, neglecting buckling and core behaviors.

For the theoretical buckling failure mode of the NFRP SIP, the boundary conditions are assumedly somewhere between pinned-pinned (effective length factor, $K=1.0)$ and fixed $(K=$ 0.5 ). If the effective length factor, $K$, is assumed to be 0.75 , then Euler's formula, shown in Equation (7-5), results in a predicted buckling load of 374,000 lbs for a 48 in long, unsupported NFRP SIP. Clearly, the SIP would theoretically fail due to material rupture long before it failed in buckling.

$$
P_{c r}=\frac{\pi^{2} E I}{(K L)^{2}}
$$

If a single, 0.25 in thick panel is considered as itself a 48 in long column, the critical buckling load is calculated to be $P_{c r}=0.0001 \mathrm{lbs}$. This essentially means that the panels alone have no bowing resistance, and when placed upright have a tendency to bow under their own self weight. Therefore, the only force resisting individual panel buckling is the adhesion of the panels to the foam core; when this interface begins to fail, the panel quickly buckles away from the SIP assembly, as was observed during testing.

Although the adhesion capacity between the NFRP composite and the polyisocyanurate core is not known and cannot be calculated without additional measurements and testing, there is a common quality between the delaminations seen in the flexure and compressive testing. In both cases, the strain in the panel which experienced the most severe delamination reached a value of around $\pm 720 \mu \epsilon$ (around $\pm 680 \mu \epsilon$ at the facesheet-core interface) just before failure. This strain appears to be the point at which the adhesion cannot handle the shear stress between the stress differential of the panel and core materials. One of the first steps in further research with NFRP SIPs should be working to improve this interface, such that it will not shear before the panels reach near their individual failure strains. One potential method of improving the delamination resistance capacity is the introduction of "shear keys" horizontally along the facesheet-core interface (Mitra and Raja 2012).

\subsection{Conclusions}

While the specific experimental NFRP SIP results were somewhat poorer than expected due to the poor mechanical properties of the selected core material and the pre-existing corner delaminations, as prototypes, the panels do highlight the potential gains that may be had with further research. The NFRP panels' negative qualities of fairly high strain variation, the presence of voids and dry fibers, and residual stresses leading to corner delaminations are of less importance than the positive qualities of their good mechanical properties and light weight.

By using a 1.5-2.5 inch meshed overlap when manufacturing the stitched fiber bundles, the resulting composite properties are not significantly reduced, providing nearly the same strength and stiffness as composites reinforced with continuous fibers. This overlap method allows the kenaf fiber bundle reinforcement method to be scaled up to any size of component. 
Large mats made of these fiber bundles were manufactured into NFRP SIP panels with polyisocyanurate foam cores by Fiber-Tech Industries. The vacuum-based manufacturing method used by Fiber-Tech was found to reduce the flexural strength by $18 \%$ compared to the compression-molded samples, but it made no difference on the flexural stiffness.

In flexural testing, the NFRP SIP panels only withstood around 70\% of the three point bending load that the OSB SIP panels resisted, but if they were redesigned with a better core compatibility, they have the potential to be over four times stronger in flexure. Even with the poor core material, they already offer a bending modulus of elasticity that is 2.5 times higher than that of the OSB SIP. The failure mode of core shearing/debonding was essentially independent of the good mechanical properties of the NFRP facesheets.

In compressive testing, the NFRP SIP panels only withstood around $50 \%$ of the axial load that the OSB SIP panels resisted, but their failure was again due to the core material and the facesheet-core interface, and not to the NFRP panels themselves. If the interface adhesion was improved, the NFRP SIP panels have the potential to carry more than twice the axial load of the OSB SIPs.

While the experimental results of these NFRP SIP prototypes were poorer than expected due to the weak and flexible core material, their potential as structural facesheets is excellent if the core and adhesion issues can be addressed. 


\section{MODELING NFRP COMPOSITES}

\subsection{Abstract}

Due to the variation in their constituent properties and their poor bonding with synthetic resins, composites reinforced with natural fibers may not necessarily be as easily modeled as those with glass fibers. A variety of stiffness models and failure criteria using constituent material properties and based on classical laminate theory were applied and evaluated with respect to experimental results. Additionally, the mechanical performance of the kenaf reinforced composites when saturated with water was also examined. One of the most important findings was that the flexural stiffness of the natural fiber reinforced composite should theoretically be increased by up to $40 \%$ if a better bond between the fiber and matrix can be obtained. While the unbalanced lay-up of the flexural samples presented some additional challenges, the natural fiber reinforced composites could be just as easily and accurately modeled as glass fiber reinforced composites, except for moisture uptake scenarios.

\subsection{Introduction and Scope}

\subsubsection{Mathematical Modeling of Composites}

The underlying purpose for the application of mathematical models and the comparison with experimental results is to determine what models, if any, can be most accurately used to predict the mechanical behavior (stress, strain, and stiffness) of the material to a particular loading condition. There are numerous ways to model material behavior, ranging from very simple and well-established models for isotropic materials, to very complex and relatively untested models, used mostly for complex loading situations of anisotropic materials.

One of the most common ways to model composite materials is the application of some variation of classical laminate plate theory, often referred to as CLT. Using this method, along with its corollaries, the stress, strain, and stiffness can be calculated at any point within a multilayered plate under general load cases, as long as the thickness of the plate is assumed to be small compared to the other directions (span/thickness $>10$ ) and the stress through the thickness is zero $\left(\sigma_{3}=0\right)$. Additional assumptions include: the material is transversely isotropic; there is perfect bonding between matrix and reinforcement, and between laminae; plane sections remain plane; and other related assumptions common to beam and plate theories.

Modeling work on natural fiber reinforced composites available in literature is very limited, particularly for the kind of composite mat manufactured as a part of this study. There are many potential difficulties inherent with modeling natural fiber composites, particularly: fibers are organic, and thus have different properties in different directions as well as high variability in strength and stiffness in addition to moisture content effects; bonding between the hydrophilic fibers and hydrophobic matrix may be quite poor without proper sizing in some situations; and the mechanical behavior of the resulting material can be non-linear, greatly complicating the modeling procedure. For example, Ochi (2008) applied a simple rule of mixtures model and was only able to predict kenaf / PLA composite strength to within 50-75\% error. In another study, Schrass-Christian and Billington (2008) developed an Euler simple-step model to predict the non-linear tensile and flexural behavior of their hemp/cellulose acetate (HCA) biocomposite. 
While their model fit the stress-strain curve well, it was also extremely reliant on using experimental results to determine the modulus of elasticity at different strains. The purpose of this chapter, therefore, is to indicate what kind of models might work best with the kenaf fiber reinforced panels manufactured in the previous chapters while requiring the least empirical data, and to use the results of these models to try to draw further conclusions about the fiber behavior, matrix behavior, and bonding between the two.

\subsubsection{Properties and Assumptions}

All of the calculations in this chapter were conducted assuming Ashland's Derakane 510A-40 was the matrix material. The material properties shown in Table 8-1 were either taken from the material technical datasheet or assumed based on published values of a similar material.

Table 8-1 - Derakane 510A-40 mechanical properties used for modeling

\begin{tabular}{|c|c|c|}
\hline Property & Value & Unit \\
\hline Tensile modulus $^{\mathrm{a}}, \mathrm{E}_{\mathrm{T}}$ & 490 & ksi \\
\hline Compressive modulus ${ }^{\mathrm{b}}, \mathrm{E}_{\mathrm{C}}$ & 490 & ksi \\
\hline Flexural modulus ${ }^{\mathrm{a}}, \mathrm{E}_{\mathrm{B}}$ & 520 & ksi \\
\hline Shear modulus ${ }^{c}, G$ & 178 & ksi \\
\hline Poisson's ratio $^{d}, v$ & 0.380 & \\
\hline Tensile strength ${ }^{a}, F_{T}$ & 12.3 & ksi \\
\hline Compressive strength $^{\mathrm{d}}, \mathrm{F}_{\mathrm{C}}$ & 17.0 & ksi \\
\hline Flexural strength ${ }^{\mathrm{a}}, \mathrm{F}_{\mathrm{B}}$ & 21.7 & ksi \\
\hline Shear strength $^{\mathrm{d}}, \mathrm{F}_{\tau}$ & 12.0 & ksi \\
\hline Tensile yield strain ${ }^{\mathrm{a}}, \varepsilon_{\text {yield }}$ & 0.04-0.05 & \\
\hline Density $^{\mathrm{a}}, \rho$ & 0.711 & oz/in ${ }^{3}$ \\
\hline \multicolumn{3}{|c|}{$\begin{array}{l}\text { Sources: }{ }^{\mathrm{a}} \text { Ashland Technical Datasheet; }{ }^{\mathrm{b}} \text { Assumed } \mathrm{E}_{\mathrm{C}}=\mathrm{E}_{\mathrm{T}} ;{ }^{\mathrm{c}} \text { Assumed } \mathrm{G}= \\
\mathrm{E}_{\mathrm{T}} /(2(1+v)) ;{ }^{\mathrm{d}} \text { Assumed from Derakane } 411 \text { properties in Barbero, } 2011\end{array}$} \\
\hline
\end{tabular}

The fiber and fabric constituent mechanical properties were, for the most part, taken from the experimental results of single fiber tensile tests, and are shown in Table 8-2. These properties are based on fibers stored at room temperature and indoor RH. Recall from previous experimental discussions that the kenaf fiber stress/strain behavior is not linear; however, the initial modulus values are accurate for at least the first $50-60 \%$ of the stress/strain to failure for nearly all samples tested. Also recall that the tensile and compression laminates had a different layup from the flexure and shear laminates ([0 $0_{4} / \mathrm{fabric} /$ fabric $\left./ 0_{4}\right]$ versus $\left[0_{4} /\right.$ fabric $/ 90_{4} /$ fabric $]$ ), as shown in Figure 5-1. The adjusted modulus and strength values were modified by multiplying the minimum-diameter based area by $150 \%$, as discussed in section 4.5.2. 
Table 8-2 - Fiber and fabric constituent mechanical properties used for modeling

\begin{tabular}{|c|c|c|c|}
\hline Fiber & Property & Value & Unit \\
\hline \multirow{7}{*}{ Untreated kenaf } & Longitudinal modulus ${ }^{\mathrm{a}}, \mathrm{E}_{1}$ & 13.0 & Msi \\
\hline & Longitudinal strength ${ }^{\mathrm{a}}, \mathrm{F}_{1}$ & 100 & ksi \\
\hline & Adjusted modulus ${ }^{\mathrm{b}}, \mathrm{E}_{1}$ & 8.67 & Msi \\
\hline & Adjusted strength $^{\mathrm{b}}, \mathrm{F}_{1}$ & 66.7 & ksi \\
\hline & Failure strain $^{\mathrm{a}}, \varepsilon_{\text {ult }}$ & 0.011 & \\
\hline & Poisson's ratio $^{c}, v$ & 0.3 & \\
\hline & Density $^{\mathrm{d}}, \rho$ & 0.809 & oz/in ${ }^{3}$ \\
\hline \multirow{7}{*}{ Treated kenaf } & Longitudinal modulus ${ }^{\mathrm{a}}, \mathrm{E}_{1}$ & 15.0 & Msi \\
\hline & Longitudinal strength $^{\mathrm{a}}, \mathrm{F}_{1}$ & 110 & ksi \\
\hline & Adjusted modulus ${ }^{\mathrm{b}}, \mathrm{E}_{1}$ & 10.0 & Msi \\
\hline & Adjusted strength ${ }^{\mathrm{b}}, \mathrm{F}_{1}$ & 73.3 & ksi \\
\hline & Failure strain ${ }^{\mathrm{a}}, \varepsilon_{\text {ult }}$ & 0.010 & \\
\hline & Poisson's ratio $^{c}, v$ & 0.3 & \\
\hline & Density $^{\mathrm{d}}, \rho$ & 0.809 & oz/in ${ }^{3}$ \\
\hline \multirow{5}{*}{ Glass } & Longitudinal modulus ${ }^{\mathrm{a}}, \mathrm{E}_{1}$ & 10.0 & Msi \\
\hline & Longitudinal strength ${ }^{\mathrm{a}}, \mathrm{F}_{1}$ & 180 & ksi \\
\hline & Failure strain $^{\mathrm{a}}, \varepsilon_{\text {ult }}$ & 0.020 & \\
\hline & Poisson's ratio ${ }^{\mathrm{e}}, v$ & 0.22 & \\
\hline & Density $^{\mathrm{e}}, \rho$ & $1.445-1.497$ & $\mathrm{oz} / \mathrm{in}^{3}$ \\
\hline \multirow{5}{*}{ Kenaf fabric } & Longitudinal modulus ${ }^{\mathrm{f}}, \mathrm{E}_{1}$ & 0.93 & Msi \\
\hline & Longitudinal strength ${ }^{\mathrm{f}}, \mathrm{F}_{1}$ & 20 & ksi \\
\hline & Failure strain ${ }^{\mathrm{f}}, \varepsilon_{\text {ult }}$ & $0.030-0.060$ & \\
\hline & Poisson's ratio $^{c}, v$ & 0.3 & \\
\hline & Density $^{\mathrm{d}}, \rho$ & 0.809 & $\mathrm{oz} / \mathrm{in}^{3}$ \\
\hline
\end{tabular}

The coupon dimensions of Table 8-3 and the laminate properties from Table 8-4 were used to provide the baseline experimentally determined material properties for comparison between predicted and experimental results. Note that the width for the shear samples in Table 8-3 is the notched width. 
Table 8-3 - Average coupon dimensions from mechanical testing used for modeling

\begin{tabular}{|l|l|c|c|c|l|}
\hline Reinforcing material & Coupon type & Length (in) & Width (in) & Thickness (in) & \multicolumn{1}{c|}{ Standard } \\
\hline \multirow{4}{*}{ Untreated kenaf } & Flexure & 5.00 & 0.50 & 0.24 & ASTM D 790 \\
\cline { 2 - 6 } & Tension & 7.97 & 0.49 & 0.24 & ASTM D 3039 \\
\cline { 2 - 6 } & Compression & 3.31 & 0.50 & 0.21 & ASTM D 695 \\
\cline { 2 - 6 } & Shear & 3.00 & 0.48 & 0.24 & ASTM D 5379 \\
\hline \multirow{5}{*}{ Treated kenaf } & Flexure & 4.97 & 0.50 & 0.20 & ASTM D 790 \\
\cline { 2 - 6 } & Tension & 8.00 & 0.50 & 0.20 & ASTM D 3039 \\
\cline { 2 - 6 } & Compression & 3.31 & 0.51 & 0.19 & ASTM D 695 \\
\cline { 2 - 6 } & Shear & 3.00 & 0.47 & 0.20 & ASTM D 5379 \\
\hline \multirow{5}{*}{ Glass } & Flexure & 5.00 & 0.52 & 0.12 & ASTM D 790 \\
\cline { 2 - 6 } & Tension & 7.94 & 0.50 & 0.12 & ASTM D 3039 \\
\cline { 2 - 6 } & Compression & 3.31 & 0.50 & 0.12 & ASTM D 695 \\
\cline { 2 - 6 } & Shear & 3.00 & 0.47 & 0.16 & ASTM D 5379 \\
\hline
\end{tabular}

Table 8-4 - Laminate properties from mechanical testing used for modeling (normalized at $40 \%$ FVF)

\begin{tabular}{|c|c|c|c|c|c|c|}
\hline \multirow{2}{*}{$\begin{array}{c}\text { Reinforcing Material: } \\
\text { Property }\end{array}$} & \multicolumn{2}{|c|}{ Untreated kenaf } & \multicolumn{2}{|c|}{ Treated kenaf } & \multicolumn{2}{|c|}{ Glass (no fabric layers) } \\
\hline & Value & Unit & Value & Unit & Value & Unit \\
\hline Tensile modulus ${ }^{\mathrm{a}}, \mathrm{E}_{\mathrm{T}}$ & 3.29 & Msi & 3.31 & Msi & 4.11 & Msi \\
\hline Compressive modulus $^{\mathrm{a}}, \mathrm{E}_{\mathrm{C}}$ & 3.32 & Msi & 3.41 & Msi & 4.31 & Msi \\
\hline Flexural modulus ${ }^{\mathrm{b}}, \mathrm{E}_{\mathrm{B}}$ & 1.08 & Msi & 1.10 & Msi & 1.66 & Msi \\
\hline In-plane shear modulus ${ }^{\mathrm{b}}, \mathrm{G}$ & 0.387 & Msi & 0.317 & Msi & 0.485 & Msi \\
\hline Tensile strength $^{\mathbf{a}}, \mathbf{F}_{\mathbf{T}}$ & 25.6 & ksi & 23.3 & ksi & 44.5 & ksi \\
\hline Compressive strength ${ }^{\mathrm{a}}, \mathrm{F}_{\mathrm{C}}$ & 16.7 & ksi & 17.0 & ksi & 19.1 & ksi \\
\hline Flexural strength $^{\mathrm{b}}, \mathrm{F}_{\mathrm{B}}$ & 26.6 & ksi & 29.1 & ksi & 42.0 & ksi \\
\hline In-plane shear strength ${ }^{b}, F_{s}$ & 7.4 & ksi & 5.1 & ksi & 6.0 & ksi \\
\hline Tensile failure strain $^{\mathrm{a}}, \boldsymbol{\varepsilon}_{\mathrm{T} \text {,ult }}$ & 0.0081 & & 0.0064 & & 0.0100 & \\
\hline Compressive failure strain ${ }^{\mathrm{a}}, \varepsilon_{\mathrm{C} \text {,ult }}$ & 0.0154 & & 0.0110 & & 0.0043 & \\
\hline In-plane shear failure strain ${ }^{b}, \gamma_{\text {ult }}$ & 0.0359 & & 0.0250 & & 0.0485 & \\
\hline Density, $\rho$ & 0.753 & oz/in ${ }^{3}$ & 0.753 & $\mathrm{oz} / \mathrm{in}^{3}$ & 1.015 & oz/in ${ }^{3}$ \\
\hline
\end{tabular}




\subsection{Stiffness Prediction}

\subsubsection{Lamina Engineering Constants}

\subsubsection{Rule of mixtures}

One of the simplest ways to determine quantitative properties of materials made up of two or more phases is the use of volume or mass fractions. This method is commonly used to determine several different lamina properties in fiber reinforced composite materials and is called the rule of mixtures (ROM). The rule of mixtures method for the longitudinal modulus is shown in equation (8-1), where $E_{1}$ is the lamina longitudinal modulus, $E_{f}$ is the fiber longitudinal modulus, $E_{m}$ is the matrix modulus, and $V_{f}$ is the volume fraction of fibers.

$$
E_{1}=E_{f} V_{f}+E_{m}\left(1-V_{f}\right)
$$

The ROM longitudinal modulus results for 40\% fiber volume fraction (FVF) laminae of the untreated kenaf, treated kenaf, and glass reinforced composites are shown in Table 8-5 (as "Pred. $E_{1}$ "). The ROM results for a $20 \%$ FVF lamina (20\% FVF each direction $=40 \%$ FVF total) of kenaf fabric reinforced are also shown. When these predicted stiffnesses are compared to experimental results (average modulus of tensile and compressive tests), the glass reinforced composites prove to be within $20 \%$ of the predicted stiffness. On the other hand, the kenaf reinforced laminae do not exhibit anywhere near as high of stiffness as the ROM predicts. This reduced stiffness is due to the presence of the fabric layers in the center of the tension and compression "unidirectional" kenaf fiber samples as well as the under-approximation of the areas of the single fiber tensile tests. Using the increased single fiber areas and the adjusted modulus values from Table 8-2, the adjusted predicted $\mathrm{E}_{1}$ values in Table 8-5 are much closer to the experimental values. The remainder of the difference between the predicted and experimental values is likely due to the presence of the fabric layers in the "unidirectional" kenaf fiber samples, and will be accounted for in the laminate mechanics.

Table 8-5 - Longitudinal modulus rule of mixtures results for $40 \%$ FVF (in Msi)

\begin{tabular}{|l|c|c|c|c|c|}
\hline Reinforcing Material & Exp. E $_{\mathbf{1}}$ & Pred. E $_{\mathbf{1}}$ & E/P Ratio & Adj. Pred. E & Adj. E/P Ratio \\
\hline Untreated kenaf & 3.310 & 5.494 & 0.60 & 3.762 & 0.88 \\
\hline Treated kenaf & 3.360 & 6.294 & 0.53 & 4.294 & 0.78 \\
\hline Glass & 4.210 & 4.294 & 0.98 & n/a & n/a \\
\hline Kenaf fabric & n/a & 0.578 & n/a & n/a & n/a \\
\hline
\end{tabular}

The stiffness properties in the transverse direction for each lamina can be obtained by applying the inverse rule of mixtures (IROM), as shown in equation (8-2). By the IROM, each of the fiber reinforced laminae have $\mathrm{E}_{2} \approx 0.8 \mathrm{Msi}$, while the kenaf fabric lamina has $\mathrm{E}_{2}=0.541$ Msi. For approximation purposes, since no fabric-only layups were tested for confirmation and since its overall contribution to laminate behavior is small, the kenaf fabric will be assumed to possess equal longitudinal and transverse stiffnesses of the average of its ROM and IROM values. While the ROM is generally regarded as an accurate prediction of $E_{1}$ behavior, the IROM is notorious for underpredicting $\mathrm{E}_{2}$ values. Alternative models have been shown to 
provide better approximations of $\mathrm{E}_{2}$ and other lamina properties; therefore, the IROM $\mathrm{E}_{2}$ values for the fiber reinforced laminae will not be used.

$$
\frac{1}{E_{2}}=\frac{\left(1-V_{f}\right)}{E_{m}}+\frac{V_{f}}{E_{f}}
$$

\subsubsection{Additional models and properties}

There are a variety of other models commonly used to determine other lamina engineering constants. One of the most accurate, yet most complex, is the period microstructure model (PMM). Barbero (2011) discusses the PMM in depth, and provides an online tool for easy calculation of lamina properties, among many other composite operations, at cadec-online.com. The PMM results for all of the fiber reinforced lamina moduli are shown in Table 8-6.

Several of the fabric properties needed to be calculated by other methods presented by Barbero (2011) due to limited available data on the fabric itself. The cylindrical assemblage model (CAM) was used to obtain an approximation of $\mathrm{G}_{12}$ and the stress partitioning parameter technique (SPP) was used to obtain an approximation of $\mathrm{G}_{23}$ for the fabric reinforced lamina.

Table 8-6 - Lamina engineering constants for $40 \%$ FVF used for modeling

\begin{tabular}{|l|c|c|c|c|}
\hline Property & Untreated kenaf & Treated kenaf & Glass & Kenaf fabric \\
\hline $\mathbf{E}_{\mathbf{1}}$ (Msi) & $3.762^{\mathrm{a}}$ & $4.294^{\mathrm{a}}$ & $4.294^{\mathrm{a}}$ & $0.559^{\mathrm{b}}$ \\
\hline $\mathbf{E}_{\mathbf{2}}$ (Msi) & $0.925^{\mathrm{c}}$ & $0.940^{\mathrm{c}}$ & $0.940^{\mathrm{c}}$ & $0.559^{\mathrm{b}}$ \\
\hline $\mathbf{v}_{\mathbf{1 2}}$ & $0.344^{\mathrm{c}}$ & $0.344^{\mathrm{c}}$ & $0.344^{\mathrm{c}}$ & $0.348^{\mathrm{a}}$ \\
\hline $\mathbf{v}_{\mathbf{2 3}}$ & $0.573^{\mathrm{c}}$ & $0.577^{\mathrm{c}}$ & $0.577^{\mathrm{c}}$ & $0.500^{\mathrm{d}}$ \\
\hline $\mathbf{G}_{\mathbf{1 2}}$ (ksi) & $378.1^{\mathrm{c}}$ & $382.8^{\mathrm{c}}$ & $382.8^{\mathrm{c}}$ & $413.6^{\mathrm{e}}$ \\
\hline $\mathbf{G}_{\mathbf{2 3}}$ (ksi) & $293.8^{\mathrm{c}}$ & $298.1^{\mathrm{c}}$ & $298.1^{\mathrm{c}}$ & $375.4^{\mathrm{f}}$ \\
\hline \multicolumn{2}{|l|}{ Sources: ${ }^{\mathrm{a}} \mathrm{ROM}$; ${ }^{\mathrm{b}}$ Average of ROM and IROM; ${ }^{\mathrm{C}} \mathrm{PMM} ;{ }^{\mathrm{d}}$ Assumed; ${ }^{\mathrm{e}} \mathrm{CAM}$; ${ }^{\mathrm{I}} \mathrm{SPP}$} \\
\hline
\end{tabular}

\subsubsection{Ply Mechanics / Macromechanics}

\subsubsection{Reduced stiffness matrices}

Following the procedure in Barbero (2011), an intermediate step before comparing the laminate engineering constants (resulting from the application of CLT) to experimental results is the determination of the ply constitutive equations for each of the different lamina types: untreated kenaf fiber reinforced, treated kenaf fiber reinforced, kenaf fabric reinforced, and glass fiber reinforced. The compliance equations of a transversely isotropic material can be written in matrix form as shown in equations (8-3) and (8-4).

$$
\begin{gathered}
\left\{\begin{array}{l}
\epsilon_{1} \\
\epsilon_{2} \\
\gamma_{6}
\end{array}\right\}=[S]\left\{\begin{array}{l}
\sigma_{1} \\
\sigma_{2} \\
\sigma_{6}
\end{array}\right\}= \\
\left\{\begin{array}{ccc}
1 / E_{1} & -v_{21} / E_{2} & 0 \\
-v_{12} / E_{1} & 1 / E_{2} & 0 \\
0 & 0 & 1 / G_{12}
\end{array}\right]\left\{\begin{array}{l}
\sigma_{1} \\
\sigma_{2} \\
\sigma_{6}
\end{array}\right\} \\
\left\{\begin{array}{l}
\gamma_{4} \\
\gamma_{5}
\end{array}\right\}=\left[\begin{array}{cc}
1 / G_{23} & 0 \\
0 & 1 / G_{13}
\end{array}\right]\left\{\begin{array}{l}
\sigma_{4} \\
\sigma_{5}
\end{array}\right\}
\end{gathered}
$$


For thin plates, $\sigma_{4}$ and $\sigma_{5}$ are neglected; this assumption will be made at this point for the current laminae.

The [3x3] compliance matrix, also typically called the [S] matrix, of equation (8-3) may be inverted to produce the reduced stiffness matrix, [Q] of equation (8-5), where $\Delta$ is calculated as shown in equation (8-6).

$$
\begin{gathered}
\left\{\begin{array}{l}
\sigma_{1} \\
\sigma_{2} \\
\sigma_{6}
\end{array}\right\}=[Q]\left\{\begin{array}{l}
\epsilon_{1} \\
\epsilon_{2} \\
\gamma_{6}
\end{array}\right\}=\left[\begin{array}{ccc}
E_{1} / \Delta & v_{12} E_{2} / \Delta & 0 \\
v_{12} E_{2} / \Delta & E_{2} / \Delta & 0 \\
0 & 0 & G_{12}
\end{array}\right]\left\{\begin{array}{l}
\epsilon_{1} \\
\epsilon_{2} \\
\gamma_{6}
\end{array}\right\} \\
\Delta=1-v_{12} v_{21}=1-v_{12}^{2} E_{2} / E_{1}
\end{gathered}
$$

For any off-axis layers, [Q] must be transformed by the use of transformation matrices in order to obtain $[\bar{Q}]$, which is the stiffness matrix of the $[\mathrm{Q}]$ lamina in the correctly oriented direction. For 0-degree layers, for example, $[\mathrm{Q}]=[\overline{\mathrm{Q}}]$. The stiffness matrix coefficients for the laminae to be modeled are shown in Table 8-7.

Table 8-7 - Reduced lamina stiffness matrix coefficients (Msi)

\begin{tabular}{|l|c|c|c|c|c|c|}
\hline \multicolumn{1}{|c|}{ Lamina } & $\overline{\mathbf{Q}}_{1 \mathbf{1 1}}$ & $\overline{\mathbf{Q}}_{\mathbf{1 2}}$ & $\overline{\mathbf{Q}}_{\mathbf{2 2}}$ & $\overline{\mathbf{Q}}_{\mathbf{1 6}}$ & $\overline{\mathbf{Q}}_{\mathbf{2 6}}$ & $\overline{\mathbf{Q}}_{\mathbf{6 6}}$ \\
\hline UT Kenaf, 0 & 3.875 & 0.328 & 0.953 & 0 & 0 & 0.378 \\
\hline UT Kenaf, 90 & 0.953 & 0.328 & 3.875 & 0 & 0 & 0.378 \\
\hline T Kenaf, 0 & 4.408 & 0.332 & 0.965 & 0 & 0 & 0.383 \\
\hline T Kenaf, 90 & 0.965 & 0.332 & 4.408 & 0 & 0 & 0.383 \\
\hline Glass, 0 & 4.408 & 0.332 & 0.965 & 0 & 0 & 0.383 \\
\hline Glass, 90 & 0.965 & 0.332 & 4.408 & 0 & 0 & 0.383 \\
\hline Kenaf Fabric & 0.636 & 0.221 & 0.636 & 0 & 0 & 0.414 \\
\hline
\end{tabular}

\subsubsection{ABD matrices}

Once the correctly oriented reduced stiffness matrices are obtained, the plate stiffness equations can be calculated by combining the stiffness matrices according to the summations shown in equation (8-7), where $k$ is the lamina number from bottom to top and $\bar{z}$ is the coordinate of the middle surface of each lamina, measured from the midplane of the laminate.

$$
\begin{gathered}
A_{i j}=\sum_{k=1}^{N}\left(\bar{Q}_{i j}\right)_{k}\left(z_{k}-z_{k-1}\right)=\sum_{k=1}^{N}\left(\bar{Q}_{i j}\right)_{k} t_{k} ; i, j=1,2,6 \\
B_{i j}=\frac{1}{2} \sum_{k=1}^{N}\left(\bar{Q}_{i j}\right)_{k}\left(z_{k}^{2}-z_{k-1}^{2}\right)=\sum_{k=1}^{N}\left(\bar{Q}_{i j}\right)_{k} t_{k} \bar{z}_{k} ; i, j=1,2,6 \\
D_{i j}=\frac{1}{3} \sum_{k=1}^{N}\left(\bar{Q}_{i j}\right)_{k}\left(z_{k}^{3}-z_{k-1}^{3}\right)=\sum_{k=1}^{N}\left(\bar{Q}_{i j}\right)_{k}\left(t_{k} \bar{z}_{k}^{2}+\frac{t_{k}^{3}}{12}\right) ; i, j=1,2,6
\end{gathered}
$$


The $[A],[B]$, and [D] submatrices combine to form the plate stiffness matrix, commonly referred to as the ABD matrix, in equation (8-8). The inverse ABD matrix, called the $\alpha \beta \delta$ matrix or plate compliance matrix, is shown in relation to the plate forces and deformations in equation (8-9).

$$
\begin{gathered}
\left\{\begin{array}{c}
N_{x} \\
N_{y} \\
N_{x y} \\
M_{x} \\
M_{y} \\
M_{x y}
\end{array}\right\}=\left[\begin{array}{llllll}
A_{11} & A_{12} & A_{16} & B_{11} & B_{12} & B_{16} \\
A_{12} & A_{22} & A_{26} & B_{12} & B_{22} & B_{26} \\
A_{16} & A_{26} & A_{66} & B_{16} & B_{26} & B_{66} \\
B_{11} & B_{12} & B_{16} & D_{11} & D_{12} & D_{16} \\
B_{12} & B_{22} & B_{26} & D_{12} & D_{22} & D_{26} \\
B_{16} & B_{26} & B_{66} & D_{16} & D_{26} & D_{66}
\end{array}\right]\left\{\begin{array}{c}
\epsilon_{x}^{0} \\
\epsilon_{y}^{0} \\
\gamma_{x y}^{0} \\
\kappa_{x} \\
\kappa_{y} \\
\kappa_{x y}
\end{array}\right\} \\
\left\{\begin{array}{c}
\epsilon_{x}^{0} \\
\epsilon_{y}^{0} \\
\gamma_{x y}^{0} \\
\kappa_{x} \\
\kappa_{y} \\
\kappa_{x y}
\end{array}\right\}=\left[\begin{array}{llllll}
\alpha_{11} & \alpha_{12} & \alpha_{16} & \beta_{11} & \beta_{12} & \beta_{16} \\
\alpha_{12} & \alpha_{22} & \alpha_{26} & \beta_{12} & \beta_{22} & \beta_{26} \\
\alpha_{16} & \alpha_{26} & \alpha_{66} & \beta_{16} & \beta_{26} & \beta_{66} \\
\beta_{11} & \beta_{12} & \beta_{16} & \delta_{11} & \delta_{12} & \delta_{16} \\
\beta_{12} & \beta_{22} & \beta_{26} & \delta_{12} & \delta_{22} & \delta_{26} \\
\beta_{16} & \beta_{26} & \beta_{66} & \delta_{16} & \delta_{26} & \delta_{66}
\end{array}\right]\left\{\begin{array}{c}
N_{x} \\
N_{y} \\
N_{x y} \\
M_{x} \\
M_{y} \\
M_{x y}
\end{array}\right\}
\end{gathered}
$$

The ABD and $\alpha \beta \delta$ matrices for each of the laminate types are shown in Table 8-8 and Table 8-9, respectively.

Table 8-8 - ABD matrices for flexural coupon layups and dimensions, $A$ : (ksi-in), $B$ : $\left(\mathrm{ksi}^{-i{ }^{2}}\right), D$ : (ksi-in $\left.{ }^{3}\right)$

\begin{tabular}{|l|r|r|r|r|r|r|}
\hline \multirow{4}{*}{ Untreated Kenaf } & 494.0 & 73.5 & 0 & -20.93 & -0.25 & 0 \\
\cline { 2 - 7 } & 73.5 & 494.0 & 0 & -0.25 & 12.74 & 0 \\
\cline { 2 - 7 } & 0 & 0 & 72.6 & 0 & 0 & -0.87 \\
\cline { 2 - 7 } & -20.93 & -0.25 & 0 & 2.677 & 0.347 & 0 \\
\cline { 2 - 7 } & -0.25 & 12.74 & 0 & 0.347 & 1.869 & 0 \\
\cline { 2 - 7 } Treated Kenaf & 0 & 0 & -0.87 & 0 & 0 & 0.328 \\
\hline \multirow{5}{*}{ Glass } & 455.3 & 62.0 & 0 & -17.05 & -0.18 & 0 \\
\cline { 2 - 7 } & 62.0 & 455.3 & 0 & -0.18 & 10.49 & 0 \\
\cline { 2 - 7 } & 0 & 0 & 61.3 & 0 & 0 & -0.61 \\
\cline { 2 - 7 } & -17.05 & -0.18 & 0 & 1.728 & 0.203 & 0 \\
\cline { 2 - 7 } & -0.18 & 10.49 & 0 & 0.203 & 1.177 & 0 \\
\cline { 2 - 7 } & 0 & 0 & -0.61 & 0 & 0 & 0.192 \\
\hline \multirow{5}{*}{} & 322.4 & 39.8 & 0 & -6.20 & 0.00 & 0 \\
\cline { 2 - 7 } & 39.8 & 322.4 & 0 & 0.00 & 6.20 & 0 \\
\cline { 2 - 7 } & 0 & 0 & 45.9 & 0 & 0 & 0.00 \\
\cline { 2 - 7 } & -6.20 & 0.00 & 0 & 0.387 & 0.048 & 0 \\
\cline { 2 - 7 } & 0.00 & 6.20 & 0 & 0.048 & 0.387 & 0 \\
\cline { 2 - 7 } & 0 & 0 & 0.00 & 0 & 0 & 0.055 \\
\hline
\end{tabular}


Table 8-9 - $\alpha \boldsymbol{B} \delta$ matrices for flexural coupon layups and dimensions, $\alpha$ : (ksi-in $)^{-1}, 6:\left(\mathrm{ksi}^{-i n^{2}}\right)^{-1}, \delta:\left(\mathrm{ksi}^{-i n^{3}}\right)^{-1}$

\begin{tabular}{|c|c|c|c|c|c|c|}
\hline \multirow{6}{*}{ Untreated Kenaf } & 0.0031 & -0.0004 & 0 & 0.0246 & -0.0013 & 0 \\
\hline & -0.0004 & 0.0025 & 0 & -0.0008 & -0.0171 & 0 \\
\hline & 0 & 0 & 0.0142 & 0 & 0 & 0.0378 \\
\hline & 0.0246 & -0.0008 & 0 & 0.579 & -0.099 & 0 \\
\hline & -0.0013 & -0.0171 & 0 & -0.099 & 0.670 & 0 \\
\hline & 0 & 0 & 0.0378 & 0 & 0 & 3.152 \\
\hline \multirow{6}{*}{ Treated Kenaf } & 0.0036 & -0.0004 & 0 & 0.0356 & -0.0017 & 0 \\
\hline & -0.0004 & 0.0028 & 0 & -0.001 & -0.0252 & 0 \\
\hline & 0 & 0 & 0.0169 & 0 & 0 & 0.0537 \\
\hline & 0.0356 & -0.001 & 0 & 0.947 & -0.149 & 0 \\
\hline & -0.0017 & -0.0252 & 0 & -0.149 & 1.100 & 0 \\
\hline & 0 & 0 & 0.0537 & 0 & 0 & 5.380 \\
\hline \multirow{6}{*}{ Glass } & 0.0046 & -0.0006 & 0 & 0.0734 & 0 & 0 \\
\hline & -0.0006 & 0.0046 & 0 & 0 & -0.0734 & 0 \\
\hline & 0 & 0 & 0.0218 & 0 & 0 & 0 \\
\hline & 0.0734 & 0 & 0 & 3.820 & -0.472 & 0 \\
\hline & 0 & -0.0734 & 0 & -0.472 & 3.820 & 0 \\
\hline & 0 & 0 & 0 & 0 & 0 & 18.141 \\
\hline
\end{tabular}

\subsubsection{Deformation response}

Once the $\alpha \beta \delta$ matrix is defined, the laminate's strain and curvature responses to a given loading can be easily obtained. For balanced symmetric laminates, laminate moduli can be derived for in-plane loads (equation (8-10)) and laminate bending moduli can be derived for bending loads (equation (8-11)).

$$
\begin{gathered}
E_{x}=\frac{1}{t \alpha_{11}}=\frac{A_{11} A_{22}-A_{12}^{2}}{t A_{22}} \\
E_{y}=\frac{1}{t \alpha_{22}}=\frac{A_{11} A_{22}-A_{12}^{2}}{t A_{11}} \\
G_{x y}=\frac{1}{t \alpha_{66}}=\frac{A_{66}}{t} \\
v_{x y}=-\frac{\alpha_{12}}{\alpha_{11}}=\frac{A_{12}}{A_{22}} \\
E_{x}^{b}=\frac{12}{t^{3} \delta_{11}}=\frac{12\left(D_{11} D_{22}-D_{12}^{2}\right)}{t^{3} D_{22}} \\
E_{y}^{b}=\frac{12}{t^{3} \delta_{22}}=\frac{12\left(D_{11} D_{22}-D_{12}^{2}\right)}{t^{3} D_{11}} \\
G_{x y}^{b}=\frac{12}{t^{3} \delta_{66}}=\frac{12 D_{66}}{t^{3}}
\end{gathered}
$$




$$
v_{x y}^{b}=-\frac{\delta_{12}}{\delta_{11}}=\frac{D_{12}}{D_{22}}
$$

The laminate moduli are shown in Table 8-10 and compared to experimental values using the experimental coupon dimensions from Table 8-3 and lamina properties from Table 8-4. While the shear value is slightly off ( 20\%) in the case of the untreated kenaf and glass composites, the flexure stiffness prediction is a much bigger concern, as it over-predicts the stiffness by over $100 \%$ for the kenaf composites. The obvious problem in applying these lamina moduli simplifications is that, in the case of the shear and flexure layups, the laminate is not balanced symmetric. As a result, the $\mathrm{B}$-matrix is not [0]. $\mathrm{A}_{16}, \mathrm{~A}_{26}, \mathrm{D}_{16}$, and $\mathrm{D}_{26}$ are, however, since the laminates are still cross-ply. This issue of flexural stiffness prediction of severely asymmetric laminates is explored in detail in the next few sections.

On the other hand, the equivalent laminate moduli for the tension and compression samples are very close to the experimental values for both the kenaf-reinforced and glassreinforced samples. The error of $\leq 7 \%$ is reasonably within the expected range of combined error stemming from material assumptions, layup inconsistency, error in volume fraction measurements, error in dimensional measurements, and testing equipment error.

Table 8-10 - Application of equivalent laminate moduli equations (in Msi)

\begin{tabular}{|c|c|c|c|c|c|c|c|}
\hline Material & Coupon properties & $\mathbf{E}_{\mathbf{x}}$ & $\mathbf{E}_{\mathbf{y}}$ & $\mathbf{G}_{\mathrm{xy}}$ & $v_{x y}$ & Exp. & Error \\
\hline \multirow{4}{*}{ Untreated Kenaf } & Tension & 3.121 & 0.860 & 0.303 & 0.345 & 3.287 & $-5 \%$ \\
\hline & Compression & 3.121 & 0.860 & 0.303 & 0.345 & 3.319 & $-6 \%$ \\
\hline & Shear & 2.013 & 2.013 & 0.303 & 0.149 & 0.387 & $-22 \%$ \\
\hline & Flexure ( ${ }^{\mathrm{b}}$-moduli) & 2.268 & 1.583 & 0.284 & 0.186 & 1.076 & $111 \%$ \\
\hline \multirow{4}{*}{ Treated Kenaf } & Tension & 3.547 & 0.873 & 0.306 & 0.345 & 3.311 & $7 \%$ \\
\hline & Compression & 3.547 & 0.873 & 0.306 & 0.345 & 3.411 & $4 \%$ \\
\hline & Shear & 2.234 & 2.234 & 0.306 & 0.136 & 0.317 & $-3 \%$ \\
\hline & Flexure ( ${ }^{b}$-moduli) & 2.539 & 1.729 & 0.288 & 0.173 & 1.102 & $130 \%$ \\
\hline \multirow{4}{*}{ Glass } & Tension & 4.294 & 0.940 & 0.383 & 0.344 & 4.108 & $5 \%$ \\
\hline & Compression & 4.294 & 0.940 & 0.383 & 0.344 & 4.312 & $0 \%$ \\
\hline & Shear & 2.646 & 2.646 & 0.383 & 0.124 & 0.485 & $-21 \%$ \\
\hline & Flexure ( ${ }^{\mathrm{b}}$-moduli) & 2.646 & 2.646 & 0.284 & 0.186 & 1.659 & $59 \%$ \\
\hline
\end{tabular}

Note: Tension/Compression layup is $\left[0_{4} / \mathrm{fab} / \mathrm{fab} / 0_{4}\right]$, and Shear/Flexure layup is $\left[0_{4} / \mathrm{fab} / 90_{4} / \mathrm{fab}\right]$

\subsubsection{Neutral axis adjustment}

From examining the ABD and $\alpha \beta \delta$ matrices in Table 8-8 and Table 8-9, it appears that the biggest problem in using the lamina moduli equations would be the $B_{11} / \beta_{11}$ term, which would relate longitudinal bending moment and longitudinal strain. Since the laminate bending moduli simplification assumes [B] is [0], its predicted flexural modulus, $\mathrm{E}_{\mathrm{x}}$, would end up being inaccurately high, as it ignores this extension along the longitudinal axis. To provide some correction for this introduced inaccuracy, the $\mathrm{B}_{11} / \beta_{11}$ term can be reduced or eliminated by 
calculating the ABD matrices using the distances from the laminae centers to the bending neutral axis, instead of to the laminate midplane.

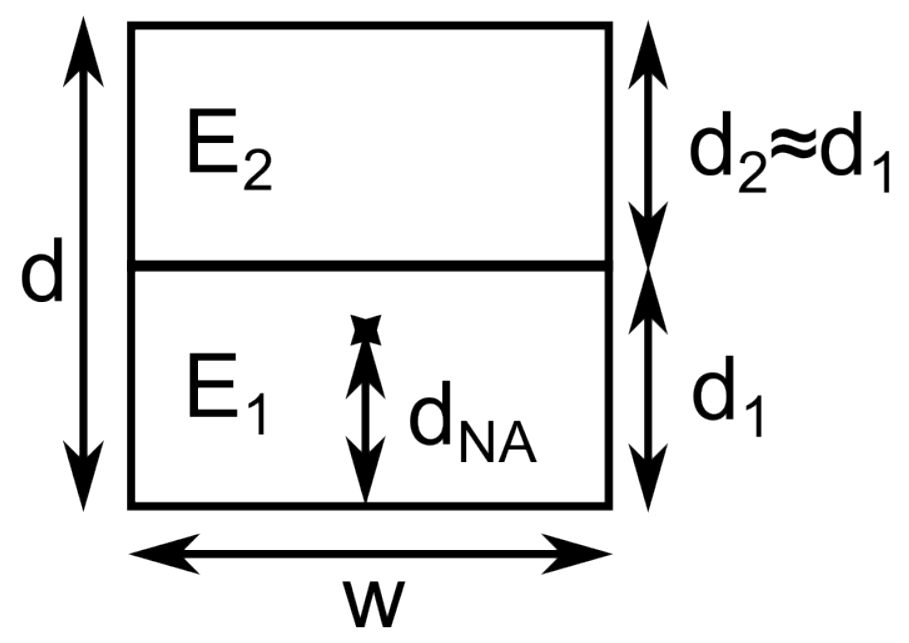

Figure 8-1 - Two-ply neutral axis calculation

Before the ABD matrices can be adjusted, the neutral axis of bending needs to be determined for each of the laminate types. The calculation is simpler for the glass fiber flexural coupons, where only two layers need to be accounted for: the longitudinal layer on the bottom and the transverse layer on the top, as shown in Figure 8-1. For $\mathrm{d}_{1} \approx \mathrm{d}_{2}$ and $\mathrm{E}_{1}>\mathrm{E}_{2}$, the neutral axis will be located at $\mathrm{d}_{\mathrm{NA}}<\mathrm{d}_{1}$. Using the transformed section method with $n_{1}=\mathrm{E}_{1} / \mathrm{E}_{2}$ :

$$
d_{N A}=\frac{d \times w \times\left((3 / 4 d \times 1 / 2)+\left(1 / 4 d \times 1 / 2 \times n_{1}\right)\right)}{d \times w \times(1 / 2)+\left(1 / 2 \times n_{1}\right)}=\frac{d\left(n_{1}+3\right)}{4\left(n_{1}+1\right)}
$$




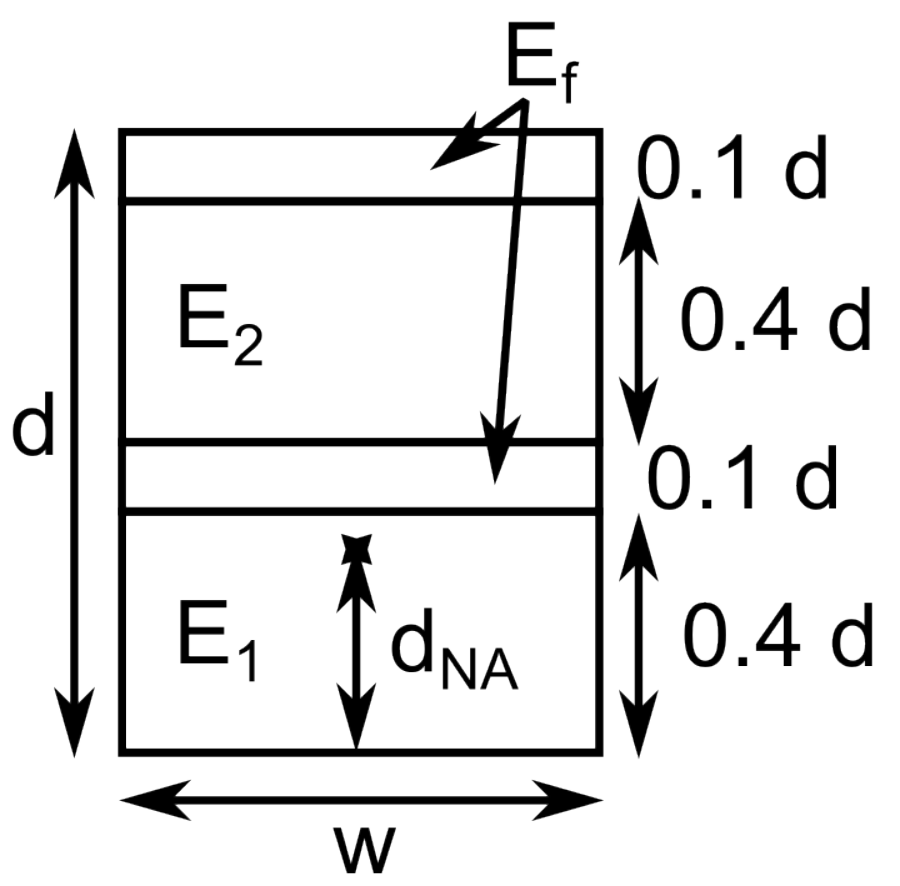

Figure 8-2 - Four-ply neutral axis calculation

The general lamina thicknesses for the kenaf samples with four plies (including the two fabric layers) are as shown in Figure 8-2. For $n_{1}=\mathrm{E}_{1} / \mathrm{E}_{\mathrm{f}}$ and $n_{2}=\mathrm{E}_{2} / \mathrm{E}_{\mathrm{f}}$, the transformed section method will result in a neutral axis location of:

$$
\begin{gathered}
d_{N A}=\frac{d \times w \times\left((0.95 d \times 0.1)+\left(0.7 d \times 0.4 \times n_{2}\right)+(0.45 d \times 0.1)+\left(0.2 d \times 0.4 \times n_{1}\right)\right)}{d \times w \times\left((0.1)+\left(0.4 \times n_{2}\right)+(0.1)+\left(0.4 \times n_{1}\right)\right)} \\
=\left(\frac{0.14+0.08 n_{1}+0.28 n_{2}}{0.2+0.4 n_{1}+0.4 n_{2}}\right) d
\end{gathered}
$$

The adjusted neutral axis depths, due to layups and lamina moduli, are shown in Table 8-11, with respect to laminate thickness.

Table 8-11 - Adjusted neutral axis depths based on unbalanced laminates

\begin{tabular}{|l|l|l|c|}
\hline Samples & $\mathbf{n}_{\mathbf{1}}$ & $\mathbf{n}_{\mathbf{2}}$ & Neutral axis depth \\
\hline Untreated Kenaf & 6.73 & 1.65 & $0.321 \times \mathrm{d}$ \\
\hline Treated Kenaf & 7.68 & 1.68 & $0.311 \times \mathrm{d}$ \\
\hline Glass & 4.57 & $\mathrm{n} / \mathrm{a}$ & $0.340 \times \mathrm{d}$ \\
\hline
\end{tabular}

\subsubsection{Adjusted equivalent laminate moduli}

Once the bending neutral axis location is determined, $\bar{z}$ in equations (8-7) can be adjusted to be the distance from the neutral axis, instead of from the midplane, resulting in the ABD matrix of Table 8-12 and the $\alpha \beta \delta$ matrix of Table 8-13. 
Table 8-12 - ABD matrices from Table 8-8 adjusted for neutral axis

\begin{tabular}{|l|r|r|r|r|r|r|}
\hline \multirow{4}{*}{ Untreated Kenaf } & 494.0 & 73.5 & 0 & 0.29 & 2.91 & 0 \\
\cline { 2 - 7 } & 73.5 & 494.0 & 0 & 2.91 & 33.96 & 0 \\
\cline { 2 - 7 } & 0 & 0 & 72.6 & 0 & 0 & 2.25 \\
\cline { 2 - 7 } & 0.29 & 2.91 & 0 & 1.790 & 0.462 & 0 \\
\cline { 2 - 7 } & 2.91 & 33.96 & 0 & 0.462 & 3.875 & 0 \\
\cline { 2 - 7 } & 0 & 0 & 2.25 & 0 & 0 & 0.387 \\
\hline \multirow{5}{*}{ Glass } & 455.3 & 62.0 & 0 & 0.16 & 2.17 & 0 \\
\cline { 2 - 7 } & 62.0 & 455.3 & 0 & 2.17 & 27.70 & 0 \\
\cline { 2 - 7 } & 0 & 0 & 61.3 & 0 & 0 & 1.70 \\
\cline { 2 - 7 } & 0.16 & 2.17 & 0 & 1.089 & 0.278 & 0 \\
\cline { 2 - 7 } & 2.17 & 27.70 & 0 & 0.278 & 2.620 & 0 \\
\cline { 2 - 7 } & 0 & 0 & 1.70 & 0 & 0 & 0.233 \\
\hline & 322.4 & 39.8 & 0 & -0.01 & 0.76 & 0 \\
\cline { 2 - 7 } & 39.8 & 322.4 & 0 & 0.76 & 12.39 & 0 \\
\cline { 2 - 7 } & 0 & 0 & 45.9 & 0 & 0 & 0.88 \\
\cline { 2 - 6 } & -0.01 & 0.76 & 0 & 0.268 & 0.063 & 0 \\
\cline { 2 - 6 } & 0.76 & 12.39 & 0 & 0.063 & 0.744 & 0 \\
\cline { 2 - 6 } & 0 & 0 & 0.88 & 0 & 0 & 0.072 \\
\hline
\end{tabular}

Table 8-13 - $\alpha \beta \delta$ matrices from Table 8-9 adjusted for neutral axis

\begin{tabular}{|c|c|c|c|c|c|c|}
\hline \multirow{6}{*}{ Untreated Kenaf } & 0.0021 & -0.0005 & 0 & -0.0003 & 0.0029 & 0 \\
\hline & -0.0005 & 0.0052 & 0 & 0.0034 & -0.0459 & 0 \\
\hline & 0 & 0 & 0.0168 & 0 & 0 & -0.0976 \\
\hline & -0.0003 & 0.0034 & 0 & 0.579 & -0.099 & 0 \\
\hline & 0.0029 & -0.0459 & 0 & -0.099 & 0.670 & 0 \\
\hline & 0 & 0 & -0.0976 & 0 & 0 & 3.152 \\
\hline \multirow{6}{*}{ Treated Kenaf } & 0.0023 & -0.0005 & 0 & -0.0002 & 0.0039 & 0 \\
\hline & -0.0005 & 0.0063 & 0 & 0.0046 & -0.0667 & 0 \\
\hline & 0 & 0 & 0.0205 & 0 & 0 & -0.1496 \\
\hline & -0.0002 & 0.0046 & 0 & 0.947 & -0.149 & 0 \\
\hline & 0.0039 & -0.0667 & 0 & -0.149 & 1.100 & 0 \\
\hline & 0 & 0 & -0.1496 & 0 & 0 & 5.380 \\
\hline \multirow{6}{*}{ Glass } & 0.0032 & -0.0007 & 0 & 0.0001 & 0.0091 & 0 \\
\hline & -0.0007 & 0.0088 & 0 & 0.0091 & -0.1468 & 0 \\
\hline & 0 & 0 & 0.0285 & 0 & 0 & -0.3483 \\
\hline & 0.0001 & 0.0091 & 0 & 3.820 & -0.472 & 0 \\
\hline & 0.0091 & -0.1468 & 0 & -0.472 & 3.820 & 0 \\
\hline & 0 & 0 & -0.3483 & 0 & 0 & 18.141 \\
\hline
\end{tabular}

This correction provides some improvement to the model's ability to fit the experimental results, as the $B_{11} / \beta_{11}$ terms have been significantly reduced. This occurs at the expense of increases to $B_{12} / \beta_{12}$ and $B_{22} / \beta_{22}$, although those terms do not affect the laminate bending behavior in the presence of an applied longitudinal moment as much as $B_{11} / \beta_{11}$. The adjusted laminate bending moduli are shown in Table 8-14. The adjusted values are a great improvement over the original values, but they still overestimate the bending stiffness, particularly for the 
kenaf fiber composites. This could be due to complications applying the equivalent laminate moduli to the severely unbalanced laminates, despite the neutral axis adjustment, or it could be simply due to poor bonding in the kenaf fiber composites, given that the glass fiber composite results are relatively close to experimental.

Table 8-14 - Neutral axis adjusted equivalent bending laminate moduli (in Msi)

\begin{tabular}{|l|c|c|c|c|}
\hline \multicolumn{1}{|c|}{ Samples } & Original $\mathbf{E}_{\mathbf{b x}}$ & ${\text { Adjusted } \mathbf{E}_{\mathbf{b x}}}$ & $\mathbf{E}_{\mathbf{b}}$, experimental & Adj. $\mathbf{E}_{\mathbf{b x}}$ error \\
\hline Untreated Kenaf & 2.268 & 1.506 & 1.076 & $+40 \%$ \\
\hline Treated Kenaf & 2.539 & 1.589 & 1.102 & $+44 \%$ \\
\hline Glass & 2.646 & 1.823 & 1.659 & $+10 \%$ \\
\hline
\end{tabular}

\subsubsection{Basic Mechanics of Materials}

Using the adjusted neutral axis from the previous section, a simple way to approximate the bending stiffness of the laminate is by summing the products of the extensional moduli and the moments of inertia of each lamina, and dividing by the laminate moment of inertia, as shown in equation (8-14).

$$
E_{b}=\frac{\sum_{i=1}^{n} E_{i} I_{i}}{I}
$$

The bending modulus results from applying this method are shown in Table 8-15. This method seems to under-predict the flexural moduli, and it is the only tested method of stiffness calculation that does so. For the glass fiber composite, the predicted result is significantly lower than the experimental result, while for the kenaf fiber composites, the predicted results are very close to the actual results. This indicates that either something is occurring with the glass composite samples to provide them with extra stiffness or else this model as a whole tends to under-predict bending moduli, and the kenaf samples are not as stiff as their constituent materials would suggest.

Table 8-15 - Summed stiffness method laminate bending moduli (in Msi)

\begin{tabular}{|l|c|c|c|}
\hline \multicolumn{1}{|c|}{ Samples } & $\mathbf{E}_{\mathbf{b}}$, predicted & $\mathbf{E}_{\mathbf{b}}$, experimental & Error \\
\hline Untreated Kenaf & 1.068 & 1.076 & $-0.7 \%$ \\
\hline Treated Kenaf & 1.092 & 1.102 & $-0.9 \%$ \\
\hline Glass & 1.385 & 1.659 & $-16 \%$ \\
\hline
\end{tabular}

\subsubsection{Finite Element}

\subsubsection{Model settings}

As yet another method of modeling the stiffness values, finite element models were created in ANSYS of each of the laminate types. The models were built around SHELL 181, four-node elements with full-integration and sectional layups. The materials were set up as linear orthotropic materials with the extensional moduli, Poisson's ratios, and shear moduli of Table 
8-6. Element size was controlled at 0.03 inches, and boundary conditions and loads were applied at the nodal level, including symmetry conditions.

A nominal load of $10 \mathrm{lbs}$ was applied to each model, and the deflection was taken as the average of the nodal displacements across the line of maximum deflection. From the load $(P)$ and deflection $(\delta)$ data, the longitudinal modulus (tension, compression) was calculated from equation (8-15), where $L$ is the full sample length and $A$ is the cross-sectional area. The bending modulus was calculated by equation (8-16), where $m$ is $P / \delta, b$ is the sample width, and $d$ is the sample thickness. The XY-shear strain $(\gamma)$ at the applied load was collected from the shear models and the shear modulus was calculated by equation (8-17).

$$
\begin{gathered}
E_{L}=\frac{P L}{A \delta} \\
E_{B}=\frac{L^{3} m}{4 b d^{3}} \\
G=\frac{P}{A \gamma}
\end{gathered}
$$

\subsubsection{Results}

As examples of the FE displacement results, plots of the compression (Figure 8-3), tension (Figure 8-4), flexure (Figure 8-5), and shear (Figure 8-6) displacements for the treated kenaf composite samples are shown.

Table 8-16 lists the moduli obtained from the FE results, as well as their error with regard to the experimental results. As with the laminate theory model, the tension and compression results are fairly close to the experimental values, while shear and flexure are less accurate. In this case, however, the glass shear and flexure are still relatively close to the experimental values, while the kenaf fiber results are still off by up to $41 \%$. These results lend additional weight to the hypothesis that the natural fiber composites' stiffness would be easier to model in tension and compression, where the quality of the fiber-matrix interface has less impact, while the poor bonding would result in lower than predicted flexural and shear modulus results. 


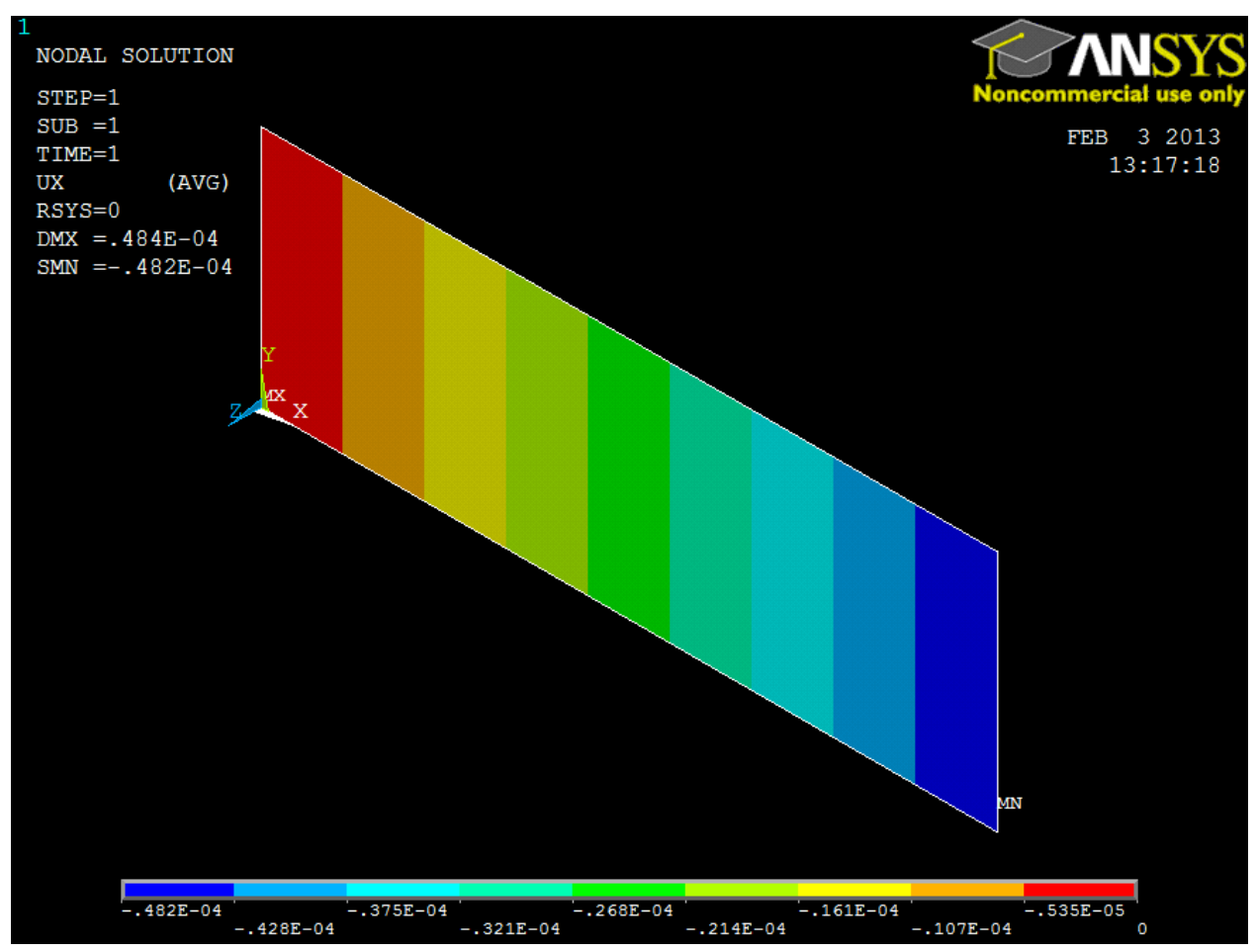

Figure 8-3 - Characteristic FE compression test results, treated kenaf composite, $X$-displacement

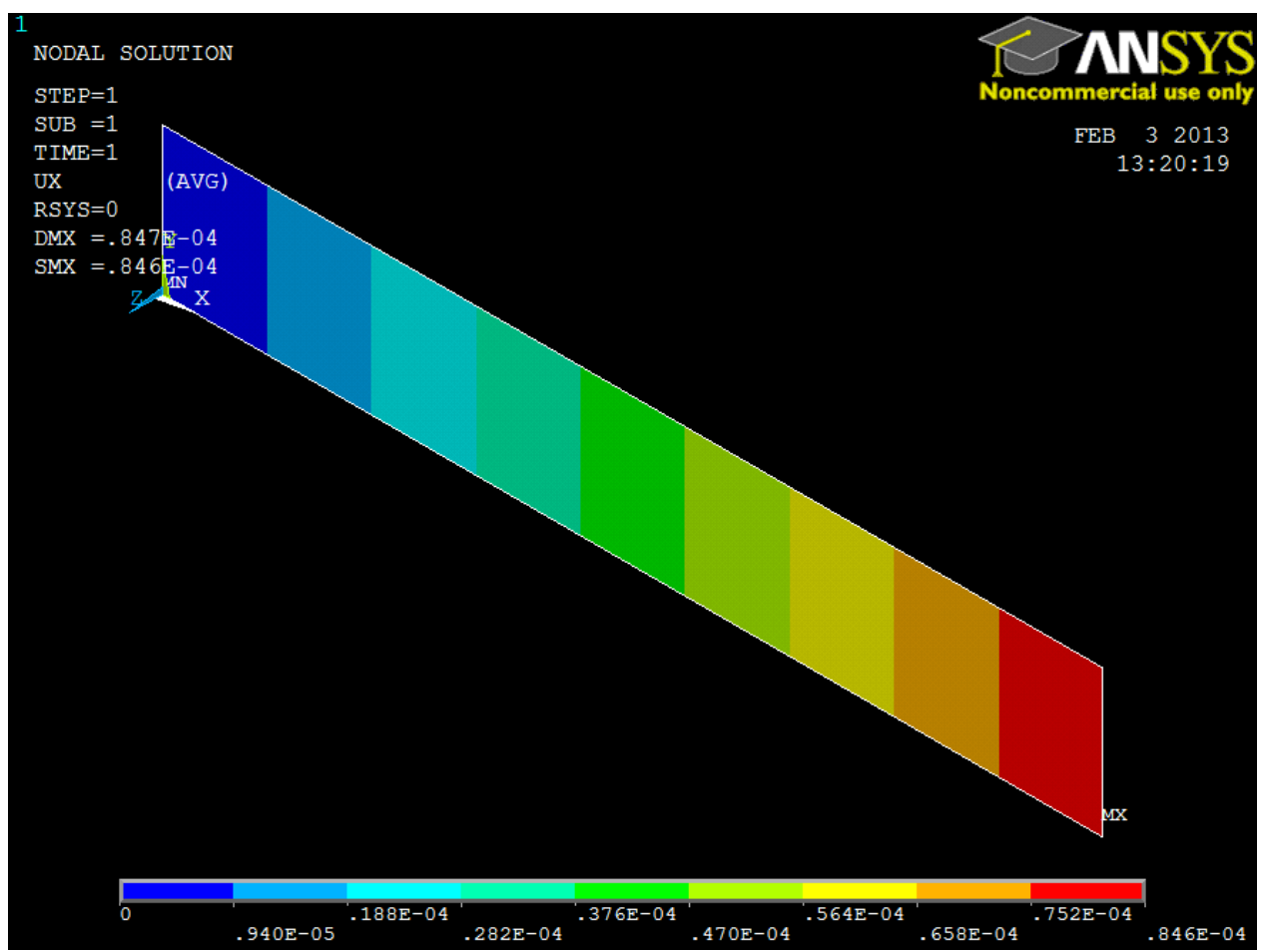

Figure 8-4 - Characteristic FE tension test results, treated kenaf composite, X-displacement 


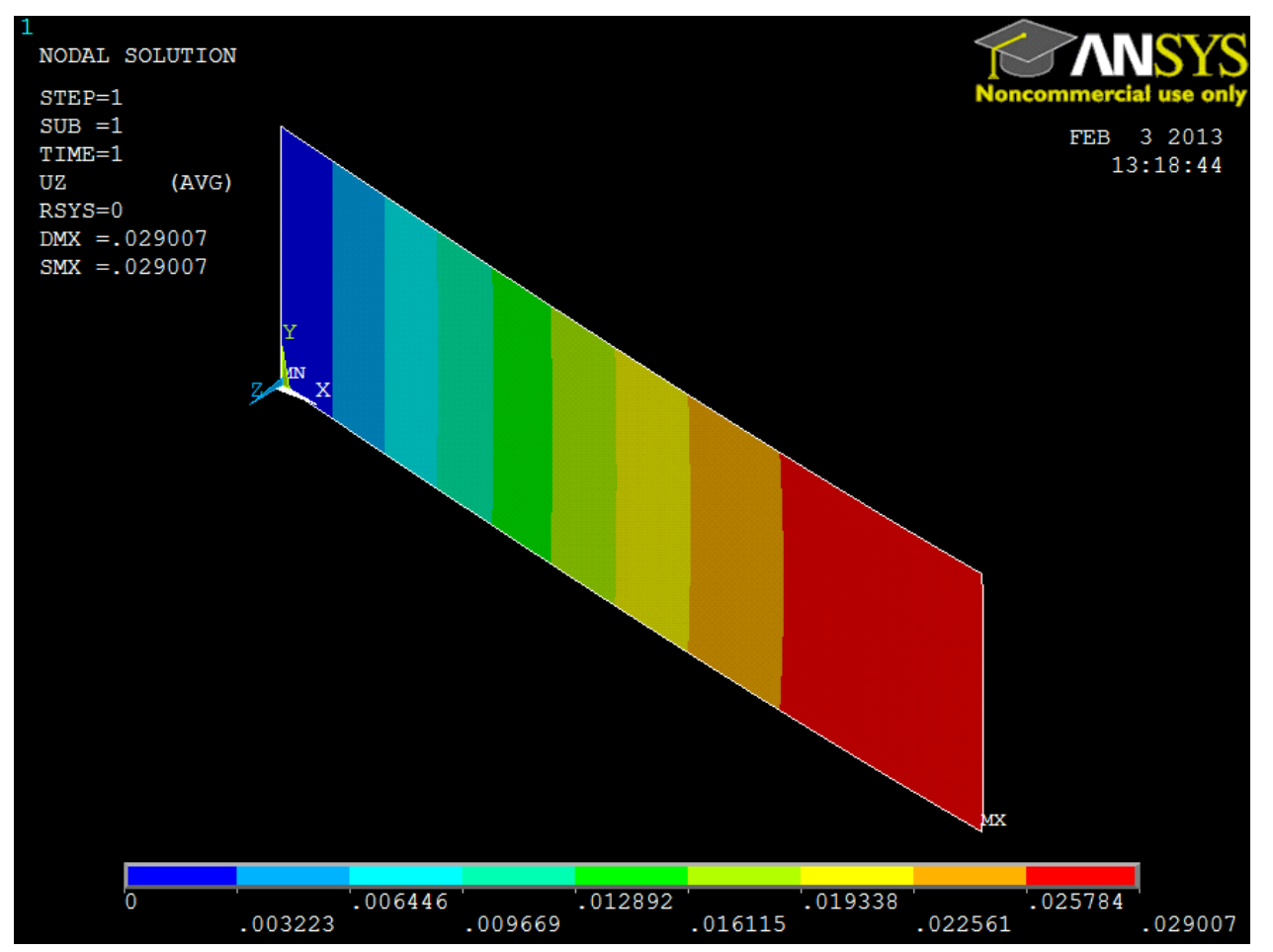

Figure 8-5 - Characteristic FE flexure test results, treated kenaf composite, Z-displacement

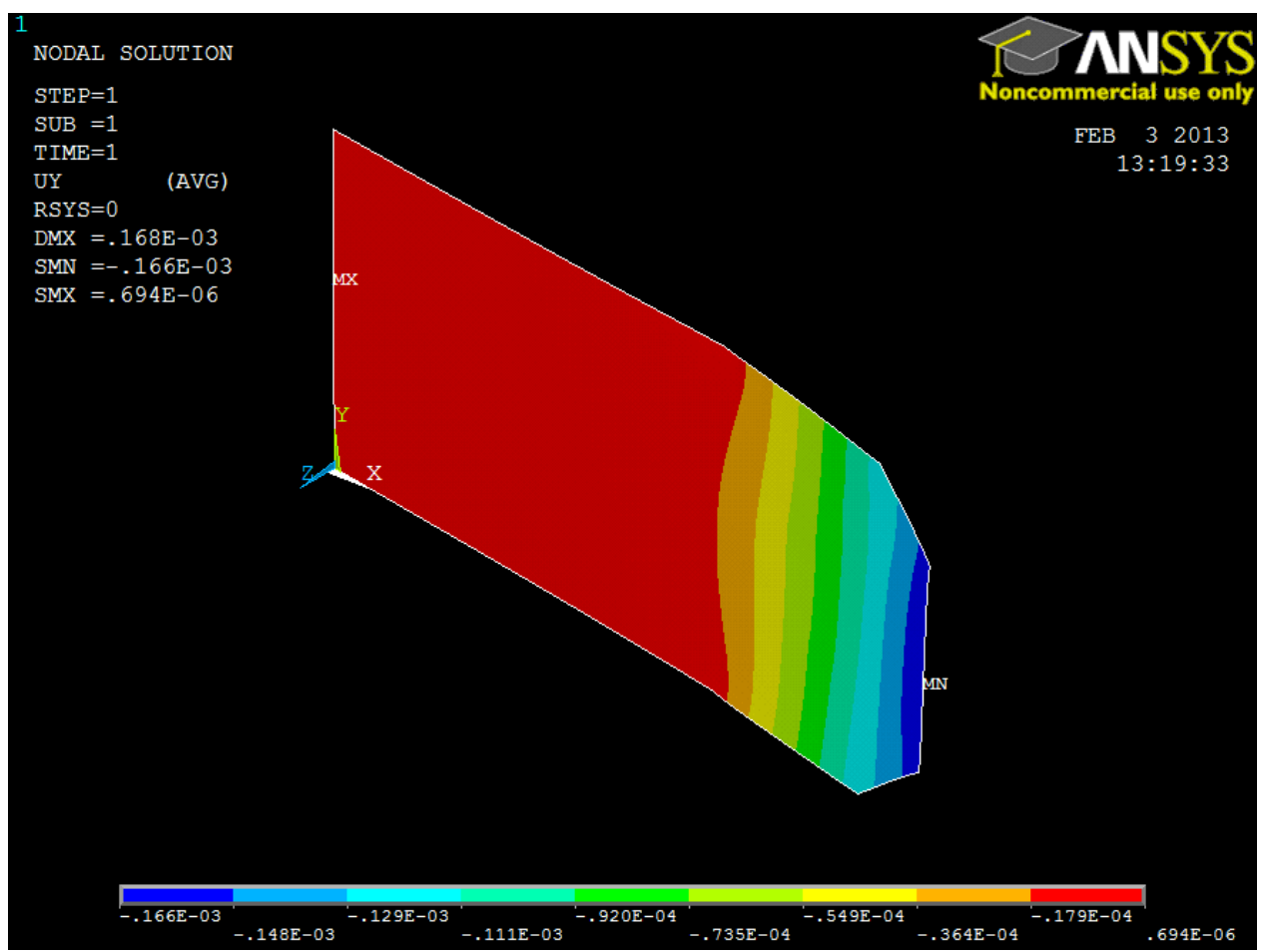

Figure 8-6 - Characteristic FE shear test results, treated kenaf composite, Y-displacement 
Table 8-16 - Finite element laminate moduli results (in Msi)

\begin{tabular}{|c|l|c|c|c|}
\hline Reinforcing material & Samples & FE moduli & Experimental moduli & FE error \\
\hline \multirow{4}{*}{ Untreated Kenaf } & Compression & 3.121 & 3.319 & $-6.0 \%$ \\
\cline { 2 - 5 } & Tension & 3.121 & 3.287 & $-5.0 \%$ \\
\cline { 2 - 5 } & Shear & 0.448 & 0.387 & $16 \%$ \\
\cline { 2 - 5 } & Flexure & 1.474 & 1.076 & $37 \%$ \\
\hline \multirow{5}{*}{ Treated Kenaf } & Compression & 3.547 & 3.411 & $4.0 \%$ \\
\cline { 2 - 5 } & Tension & 3.547 & 3.311 & $7.1 \%$ \\
\cline { 2 - 5 } & Shear & 0.434 & 0.317 & $37 \%$ \\
\cline { 2 - 5 } & Flexure & 1.553 & 1.102 & $41 \%$ \\
\hline \multirow{5}{*}{ Glass } & Compression & 4.294 & 4.310 & $-0.4 \%$ \\
\cline { 2 - 5 } & Tension & 4.294 & 4.108 & $4.5 \%$ \\
\cline { 2 - 5 } & Shear & 0.433 & 0.485 & $-11 \%$ \\
\cline { 2 - 5 } & Flexure & 1.7874 & 1.659 & $7.7 \%$ \\
\hline
\end{tabular}

\subsubsection{Other Methods}

\subsubsection{Average extensional modulus}

In the appendices of a NASA technical note by Chamis (1974) describing how to analyze three-point-bend tests for materials with different behavior in tension and compression, it is presented that the flexural modulus, $E_{F}$, can be approximated as the average extensional modulus through the beam thickness, as shown in (8-18). The resulting approximations for each of the laminate types are shown in Table $8-17$, with $\mathrm{N}=4$ for the kenaf samples and $\mathrm{N}=2$ for the glass samples.

$$
E_{F} \approx \frac{1}{h} \sum_{i=1}^{N} h_{i} E_{i}
$$

Table 8-17 - Average extensional modulus method laminate bending moduli (in Msi)

\begin{tabular}{|l|c|c|c|}
\hline \multicolumn{1}{|c|}{ Samples } & $\mathbf{E}_{\mathrm{F}}$, predicted & $\mathbf{E}_{\mathrm{F}}$, experimental & Error \\
\hline Untreated Kenaf & 1.987 & 1.076 & $+85 \%$ \\
\hline Treated Kenaf & 2.205 & 1.102 & $+100 \%$ \\
\hline Glass & 2.617 & 1.659 & $+58 \%$ \\
\hline
\end{tabular}

While this model offers a small improvement over the unmodified neutral axis laminate theory moduli, it still grossly overestimates the flexural stiffness values. The summed stiffness method of section 8.3.3 provides a much better approximation with roughly the same level of calculation. 


\subsubsection{Variation of Castigliano's method}

A second procedure from the same publication (Chamis 1974) uses strain energy and Castigliano's method to determine the deformation of a beam with varying moduli in tension and compression. Once a relationship is established between the midspan deflection and the applied load, the predicted slope of the P- $\delta$ curve can be derived and used to predict the initial flexural modulus. The following derivations modify this theory to work for beams composed of laminae with different moduli, rather than just for a single material with different tensile/compressive behavior.

The uniaxial stress-strain relations for each lamina are

$$
\begin{aligned}
& \sigma_{1}=\varepsilon_{1} E_{1}, \sigma_{2}=\varepsilon_{2} E_{2}, \text { etc... } \\
& \tau_{1}=\gamma_{1} G_{1}, \tau_{2}=\gamma_{2} G_{2}, \text { etc... }
\end{aligned} \text { for lamina } 1,2, \ldots
$$

while the strain displacement relationship is

$$
\varepsilon=-z \frac{d^{2} w}{d x^{2}}
$$

where positive $\mathrm{z}$ is the distance above the neutral axis, as shown in Figure 8-7.

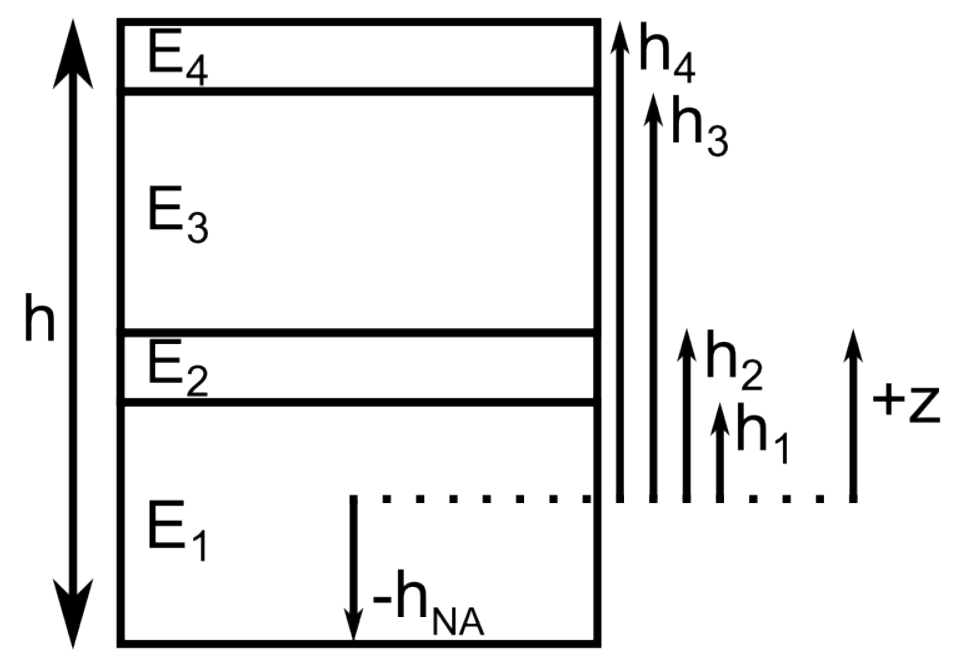

Figure 8-7 - Laminate coordinate system and thicknesses

The bending moment for the section, assuming that the neutral axis will be located within the first lamina will be

$$
M=b \int_{-h_{N A}}^{0} z \sigma_{1} d z+b \int_{0}^{h_{1}} z \sigma_{1} d z+b \int_{h_{1}}^{h_{2}} z \sigma_{2} d z+\cdots
$$

where the integration from $\left(-h_{N A}, 0\right)$ is the tension part of lamina 1 , and the integration from $\left(0, \mathrm{~h}_{1}\right)$ is the compression part of lamina 1 . While this distinction between the tension and compression sections does not make a mathematical difference on this derivation, it was made to show that the bending moment is the sum of the tensile stresses and the compressive stresses, 
multiplied by their respective areas. All of the other lamina act only in compression and thus can be represented by a single integral across their thickness. Given that the stress of each lamina is represented as

$$
\sigma_{i}=-z E_{i} \frac{d^{2} w}{d x^{2}}
$$

and the relationship between the bending moment and the flexural rigidity, $D$, is

$$
M=-D \frac{d^{2} w}{d x^{2}}
$$

the flexural rigidity can be written independent of the $\mathrm{d}^{2} \mathrm{w} / \mathrm{dx}{ }^{2}$ term as

$$
D=b \int_{-h_{N A}}^{0} z^{2} E_{1} d z+b \int_{0}^{h_{1}} z^{2} E_{1} d z+b \int_{h_{1}}^{h_{2}} z^{2} E_{2} d z+\cdots
$$

(tension side) (compression side)

where, again, the integration of the first lamina is split into two for clarity. The flexural rigidities of the three sample types are shown in Table 8-18.

Table 8-18 - Flexural rigidities of given materials and laminate thicknesses

\begin{tabular}{|l|c|}
\hline \multicolumn{1}{|c|}{ Samples } & Flexural rigidity, D (kip-in ${ }^{2}$ ) \\
\hline Untreated Kenaf & 0.8524 \\
\hline Treated Kenaf & 0.5199 \\
\hline Glass & 0.1356 \\
\hline
\end{tabular}

If each side of the bending moment equation (8-23) is multiplied by $\mathrm{z}$, the result can be simplified as

$$
M z=-D z \frac{d^{2} w}{d x^{2}} \text { or } M z=D \varepsilon
$$

accounting for the relationship of equation (8-20). Substituting in the stress-strain relations of equations (8-19) results in equations (8-26), where the stress in each lamina is related to the lamina stiffness and the location within the lamina thickness.

$$
\begin{array}{lll}
\sigma_{1}=M z \frac{E_{1}}{D} & 0 \leq x \leq l, & -h_{N A} \leq z \leq h_{1} \\
\sigma_{2}=M z \frac{E_{2}}{D} & 0 \leq x \leq l, & h_{1} \leq z \leq h_{2} \\
\sigma_{3}=\text { etc } \ldots & &
\end{array}
$$

For the region $0 \leq x \leq(l / 2), M=-P x / 2$, and after substituting in this relationship in equations (8-27), the stress is directly related to the applied 3-point bending load, the location within the beam, and the lamina stiffness. 


$$
\begin{aligned}
& \sigma_{1}= \begin{cases}\text { in tension, } & \frac{-P x z}{2 D} E_{1} \\
\text { in compression, } \frac{-P x z}{2 D} E_{1} & -h_{N A} \leq z \leq 0\end{cases} \\
& \sigma_{2}=\frac{-P x z}{2 D} E_{2} h_{1} \leq z \leq h_{2} \\
& \sigma_{3}=\text { etc } \ldots
\end{aligned}
$$

In a similar method, the shear stress can be related to the applied 3-point bending load, the location within the beam, and the laminate stiffness as shown in equations (8-28).

$$
\begin{aligned}
\tau_{1} & =\frac{P E_{1}}{4 D}\left(t_{1}^{2}-z^{2}\right) & & -h_{N A} \leq z \leq h_{1} \\
\tau_{2} & =\frac{P E_{2}}{4 D}\left(t_{2}^{2}-z^{2}\right) & & h_{1} \leq z \leq h_{2} \\
\tau_{3} & =\text { etc } \ldots & &
\end{aligned}
$$

The total energy stored in the sample is

$$
\begin{gathered}
U=2 b \int_{0}^{l / 2} \frac{1}{2}\left(\int_{-h_{N A}}^{0}\left(\sigma_{1} \varepsilon_{1}+\tau_{1} \gamma_{1}\right) d z+\int_{0}^{h_{1}}\left(\sigma_{1} \varepsilon_{1}+\tau_{1} \gamma_{1}\right) d z+\int_{h_{1}}^{h_{2}}\left(\sigma_{2} \varepsilon_{2}+\tau_{2} \gamma_{2}\right) d z\right. \\
\left.+\int_{h_{2}}^{h_{3}}\left(\sigma_{3} \varepsilon_{3}+\tau_{3} \gamma_{3}\right) d z+\int_{h_{3}}^{h_{4}}\left(\sigma_{4} \varepsilon_{4}+\tau_{4} \gamma_{4}\right) d z\right) d x
\end{gathered}
$$

which, after substituting in the stress-strain relations of equations (8-19) results in equation (8-30).

$$
\begin{aligned}
U=2 b \int_{0}^{l / 2} \frac{1}{2} & \left(\int_{-h_{N A}}^{0}\left(\sigma_{1}^{2} / E_{1}+\tau_{1}^{2} / G_{1}\right) d z+\int_{0}^{h_{1}}\left(\sigma_{1}^{2} / E_{1}+\tau_{1}^{2} / G_{1}\right) d z\right. \\
& +\int_{h_{1}}^{h_{2}}\left(\sigma_{2}^{2} / E_{2}+\tau_{2}^{2} / G_{2}\right) d z+\int_{h_{2}}^{h_{3}}\left(\sigma_{3}^{2} / E_{3}+\tau_{3}^{2} / G_{3}\right) d z \\
& \left.+\int_{h_{3}}^{h_{4}}\left(\sigma_{4}^{2} / E_{4}+\tau_{4}^{2} / G_{4}\right) d z\right) d x
\end{aligned}
$$

By further substituting in equations (8-27) and (8-28) for the stress terms and differentiating $U$ with respect to $P$ according to Castigliano's first theorem (procedure conducted in MATLAB), the maximum deflection of the samples at the midpoint of the span, $w$, is calculated in terms of the applied load $P$. The midpoint deflections for the three materials are shown in Table 8-19.

Table 8-19 - Midpoint deflection of given materials and dimensions

\begin{tabular}{|l|c|}
\hline \multicolumn{1}{|c|}{ Samples } & Midpoint deflection, w (in) \\
\hline Untreated Kenaf & $1.6045 \times \mathrm{P}$ \\
\hline Treated Kenaf & $1.4868 \times \mathrm{P}$ \\
\hline Glass & $1.8709 \times \mathrm{P}$ \\
\hline
\end{tabular}


Analogous to running an experimental 3-point bending test, the slope of the initial linear portion, $m$, of the $\mathrm{P}-\delta$ curve can be obtained by dividing $P$ by the midpoint deflection in Table 8-19, canceling out the load variable. The slope $m$ can then be used with the flexural modulus equation (8-16) to obtain the stiffness prediction value for each sample type by the modified Castigliano's method, as shown in Table 8-20. The results from this method seem to correlate fairly well with the flexural moduli obtained by both the adjusted neutral axis equivalent laminate moduli method and the finite element method.

Table 8-20 - Castigliano's method laminate bending moduli (in Msi)

\begin{tabular}{|l|c|c|c|}
\hline \multicolumn{1}{|c|}{ Samples } & $\mathbf{E}_{\mathbf{B}}$, predicted & $\mathbf{E}_{\mathbf{B}}$, experimental & Error \\
\hline Untreated Kenaf & 1.442 & 1.076 & $+34 \%$ \\
\hline Treated Kenaf & 1.511 & 1.102 & $+37 \%$ \\
\hline Glass & 1.694 & 1.659 & $+2.1 \%$ \\
\hline
\end{tabular}

\subsubsection{Conclusions}

A comparison of all the different stiffness modeling error results for each for each of the sample types is shown in Table 8-21. One obvious trend is that the compression and tension results are modeled almost exactly the same by the equivalent laminate moduli and FE methods, and their values are very close to the experimental values. This is because the balanced layup of those samples makes for a simpler analysis.

The shear results of the equivalent laminate moduli method and the FE method produced significantly different results for the natural fiber composite samples, with the ELM method under-predicting the shear stiffness by as much as $22 \%$ and the FE method over-predicting by as much as $37 \%$. For the glass fiber samples, both methods under-predicted the shear modulus. This range of error on the shear samples is probably due to the greater contribution of the material values that required some assumptions (shear moduli and Poisson's ratios). Because of the unbalanced layup of the shear samples, the FE results are probably more accurate predictions than the ELM results, indicating that the untreated kenaf samples may not be achieving their full shear stiffness potential due to poor bonding.

The flexure results were the most intriguing, as one of the models worked quite well with only the natural fiber samples while others worked well only with the glass samples. The unmodified ELM results can be dismissed, as that method is only intended to be applied to balanced, symmetric layups. From the remainder of the results, the Adjusted ELM, FE, and Castigliano's methods all produced relatively similar results, with the Castigliano's method providing a slightly closer prediction to the experimental results. These methods were also the most rigorous analyses. The average extensional modulus method failed to predict any of the samples very closely, and should be dismissed as a method for predicting either natural fiber or glass unbalanced layup stiffness. The summed stiffness method was the only method to underpredict any values, and was very accurate in predicting the experimental natural fiber stiffness values. While the glass sample stiffness was under-predicted by $16 \%$, this method could still be used as a fast, relatively conservative method of predicting unbalanced layup stiffness, and with further testing on different layups and materials, could be confirmed to provide fairly accurate results for the poor bonding present in natural fiber composites. 
Table 8-21 - Comparison of stiffness model errors

\begin{tabular}{|c|c|c|c|c|c|c|c|}
\hline 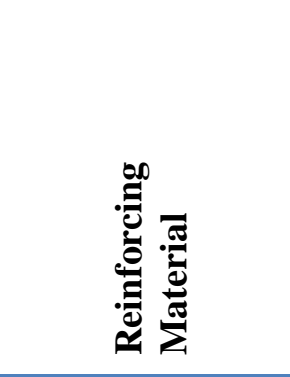 & $\underset{\mathscr{E}}{\stackrel{\mathscr{E}}{\overparen{E}}}$ & 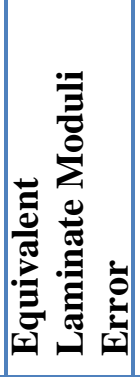 & 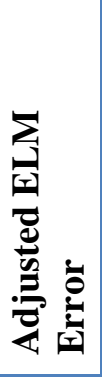 & 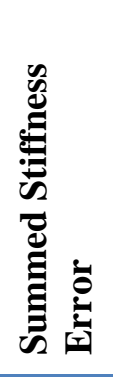 & 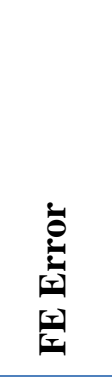 & 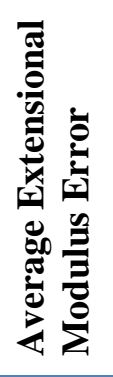 & 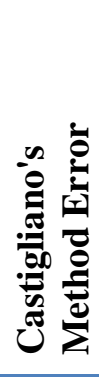 \\
\hline \multirow{4}{*}{ Untreated Kenaf } & Compression & $-6 \%$ & & & $-6.0 \%$ & & \\
\hline & Tension & $-5 \%$ & & & $-5.0 \%$ & & \\
\hline & Shear & $-22 \%$ & & & $16 \%$ & & \\
\hline & Flexure & $111 \%$ & $40 \%$ & $-0.7 \%$ & $37 \%$ & $85 \%$ & $34 \%$ \\
\hline \multirow{4}{*}{ Treated Kenaf } & Compression & $4 \%$ & & & $4.0 \%$ & & \\
\hline & Tension & $7 \%$ & & & $7.1 \%$ & & \\
\hline & Shear & $-3 \%$ & & & $37 \%$ & & \\
\hline & Flexure & $130 \%$ & $44 \%$ & $-0.9 \%$ & $41 \%$ & $100 \%$ & $37 \%$ \\
\hline \multirow{4}{*}{ Glass } & Compression & $0 \%$ & & & $-0.4 \%$ & & \\
\hline & Tension & $5 \%$ & & & $4.5 \%$ & & \\
\hline & Shear & $-21 \%$ & & & $-11 \%$ & & \\
\hline & Flexure & $59 \%$ & $10 \%$ & $-16 \%$ & $7.7 \%$ & $58 \%$ & $2.1 \%$ \\
\hline
\end{tabular}

In light of all of the stiffness results, it would appear that either FE or ELM methods can be used to predict longitudinal stiffness behavior of balanced natural fiber laminates, nearly as well as they predict the behavior of glass laminates. In flexure, Castigliano's, ELM, or FE methods all likely produce values of the same accuracy, even with unbalanced layups. Assuming these models are the most accurate, it appears that there is still fairly significant stiffness improvement available in the natural fiber composites if the bonding is improved. This hypothesis is supported by the fact that the tension and compression experimental results, where the bonding is not as important since the fibers span the entire length of the samples, are easily predicted by common modeling methods while the flexural and shear results, where the fibermatrix interface has a greater effect on the stiffness behavior, are over-predicted by as much as $40 \%$ by common modeling methods. If the physico-chemical interface is improved between the natural fibers and synthetic resin, the shear and stiffness properties could therefore potentially increase by as much as $40 \%$, while only small gains would likely result in the tensile and compressive stiffnesses.

\subsection{Strength Prediction}

\subsubsection{Lamina Strength and Strain Properties}

Before laminate failure criteria can be applied, the lamina ultimate strength properties have to be determined. 
In order to predict the longitudinal lamina tensile strength, the following assumptions are made (Barbero 2011):

1. Fiber strength is uniform,

2. Both the fibers and the matrix behave linearly until failure,

3. The fibers are more brittle than the matrix, and

4. The fibers are stiffer than the matrix.

Longitudinal tensile failure occurs, therefore, when the fibers reach their apparent strength, $F_{f t}$, while the matrix is still intact. This can be represented by a variation of the rule of mixtures, as shown in equation (8-31), where $\sigma_{m}^{*}$ is the stress in the matrix at failure, and can be calculated by equation (8-32).

$$
\begin{gathered}
F_{1 t}=F_{f t} V_{f}+\sigma_{m}^{*}\left(1-V_{f}\right) \\
\sigma_{m}^{*}=F_{f t} \frac{E_{m}}{E_{f}}
\end{gathered}
$$

The $F_{1 t}$ properties from the ROM method are shown in Table 8-22 and compared to the experimental tensile test strengths (with the acknowledgement that approximately $1 / 5$ of the kenaf fiber reinforcement by weight is fabric).

Table 8-22 - Longitudinal lamina tensile strengths

\begin{tabular}{|l|c|c|}
\hline Reinforcing Material & $\mathbf{F}_{\mathbf{1 t}} \mathbf{( k s i )}$ & Experimental (ksi) \\
\hline Untreated kenaf & 28.942 & 25.6 \\
\hline Treated kenaf & 31.475 & 23.3 \\
\hline Glass & 77.292 & 44.5 \\
\hline Kenaf fabric & 12.430 & n/a \\
\hline
\end{tabular}

The kenaf fiber predicted lamina strengths are reasonable with respect to the experimental tensile results. The glass predicted lamina strength, on the other hand, was high compared to the experimental value. This could be due to damage incurred during manufacturing ( $25-50 \%$ reduction possible (Barbero 2011$)$ ) or manufacturing errors resulting in misalignment of fibers, miscalculation of FVF, or asymmetric layup. The kenaf fabric strength is based on a number of logical, but unconfirmed assumptions, and thus will be rounded to 12.0 for the rest of the strength modeling.

The remainder of the lamina strength properties can be obtained by a combination of constituent material strengths and stiffnesses, lamina stiffnesses, fracture mechanics, and empirical formulas (Barbero 2011). These properties can be most easily obtained by the use of the CADEC online software, with the following assumptions shown in Table 8-23 made for all laminae. 
Table 8-23 - Assumptions for determining lamina properties with CADEC software

\begin{tabular}{|c|c|}
\hline Property & Value \\
\hline Transition thickness, $t$ & $0.6^{\mathrm{a}}$ \\
\hline Fracture plane angle, $\alpha_{0}$ & $54^{\circ} \mathrm{b}$ \\
\hline Weibull shape parameter, m (kenaf) & $1.96^{c}$ \\
\hline Weibull shape parameter, m (glass) & $4.00^{d}$ \\
\hline SD of misalignment angle, $\alpha_{\sigma}$ & $0.40^{\mathrm{e}}$ \\
\hline
\end{tabular}

While the standard deviation of the misalignment angle, $\alpha_{\sigma}$, seems low compared to what would be expected given the fiber orientations visible on the plate surface, this value cannot be experimentally obtained for kenaf by the method recommended in the text since it is based on the assumption that fibers are round and regular, which does not hold true for natural fibers. The kenaf fabric strength properties were assumed as reasonable values based on kenaf fiber lamina strengths. The CADEC-based lamina strength properties are shown in Table 8-24.

Table 8-24 - Lamina strength properties for each lamina reinforcing material

\begin{tabular}{|c|c|c|c|c|}
\hline Strength (ksi) & Untreated kenaf & Treated kenaf & Glass & Kenaf fabric \\
\hline $\mathbf{F}_{\mathbf{1 t}}$ & 28.941 & 31.475 & 77.288 & 12 \\
\hline $\mathbf{F}_{\mathbf{1 c}}$ & 18.723 & 18.768 & 18.768 & 12 \\
\hline $\mathbf{F}_{\mathbf{2 t}}$ & 9.602 & 9.602 & 9.581 & 12 \\
\hline $\mathbf{F}_{\mathbf{2}}$ & 13.272 & 13.242 & 13.242 & 12 \\
\hline $\mathbf{F}_{\mathbf{4}}$ & 4.821 & 4.81 & 4.81 & 4.8 \\
\hline $\mathbf{F}_{\mathbf{5}}$ & 4.821 & 4.81 & 4.81 & 4.8 \\
\hline $\mathbf{F}_{\mathbf{6}}$ & 9.359 & 9.339 & 9.339 & 6 \\
\hline
\end{tabular}

Since lamina failure strains were not obtained experimentally (due to the constraints of the kenaf fiber mat manufacturing method, it is not possible to obtain experimental lamina properties for fibers alone), they are approximated as linear elastic according to equation (8-33).

$$
\begin{aligned}
& \epsilon_{1 t}=F_{1 t} / E_{1} \quad \epsilon_{1 c}=F_{1 c} / E_{1} \\
& \epsilon_{2 t}=F_{2 t} / E_{2} \quad \epsilon_{2 c}=F_{2 c} / E_{2} \\
& \gamma_{4 u}=F_{4} / G_{23} \quad \gamma_{5 u}=F_{5} / G_{13} \quad \gamma_{6 u}=F_{6} / G_{12}
\end{aligned}
$$

The resulting approximate failure strains, which are likely conservative given that actual stress-strain behavior is not linear up to failure, are shown in Table 8-25. 
Table 8-25 - Lamina failure strain properties for each lamina reinforcing material

\begin{tabular}{|c|c|c|c|c|}
\hline Failure strain & Untreated kenaf & Treated kenaf & Glass & Kenaf fabric \\
\hline $\boldsymbol{\epsilon}_{\mathbf{1 t}}$ & 0.0077 & 0.0073 & 0.0180 & 0.0215 \\
\hline $\boldsymbol{\epsilon}_{\mathbf{1 c}}$ & 0.0050 & 0.0044 & 0.0044 & 0.0215 \\
\hline $\boldsymbol{\epsilon}_{\mathbf{2 t}}$ & 0.0104 & 0.0102 & 0.0102 & 0.0215 \\
\hline $\boldsymbol{\epsilon}_{\mathbf{2 c}}$ & 0.0143 & 0.0141 & 0.0141 & 0.0215 \\
\hline$\gamma_{\mathbf{4 u}}$ & 0.0164 & 0.0161 & 0.0161 & 0.0128 \\
\hline$\gamma_{\mathbf{5 u}}$ & 0.0128 & 0.0126 & 0.0126 & 0.0116 \\
\hline$\gamma_{\mathbf{6 u}}$ & 0.0248 & 0.0244 & 0.0244 & 0.0145 \\
\hline
\end{tabular}

\subsubsection{Laminate Strain and Stress}

In laminate theory, midsurface strains and curvatures are obtained from equation (8-34), where $N_{x}, N_{y}$, and $N_{x y}$ are the axial and shear forces per unit length; $M_{x}, M_{y}$, and $M_{x y}$ are the moments per unit length; and $V_{x}, V_{y}$ are the shear forces per unit length.

$$
\left\{\begin{array}{c}
\epsilon_{x}^{0} \\
\epsilon_{y}^{0} \\
\gamma_{x y}^{0} \\
\kappa_{x} \\
\kappa_{y} \\
\kappa_{x y}
\end{array}\right\}=\left[\begin{array}{llllll}
\alpha_{11} & \alpha_{12} & \alpha_{16} & \beta_{11} & \beta_{12} & \beta_{16} \\
\alpha_{12} & \alpha_{22} & \alpha_{26} & \beta_{12} & \beta_{22} & \beta_{26} \\
\alpha_{16} & \alpha_{26} & \alpha_{66} & \beta_{16} & \beta_{26} & \beta_{66} \\
\beta_{11} & \beta_{12} & \beta_{16} & \delta_{11} & \delta_{12} & \delta_{16} \\
\beta_{12} & \beta_{22} & \beta_{26} & \delta_{12} & \delta_{22} & \delta_{26} \\
\beta_{16} & \beta_{26} & \beta_{66} & \delta_{16} & \delta_{26} & \delta_{66}
\end{array}\right]\left\{\begin{array}{c}
N_{x} \\
N_{y} \\
N_{x y} \\
M_{x} \\
M_{y} \\
M_{x y}
\end{array}\right\}
$$

The experimental failure strengths can be translated into laminate loads per unit length, for given laminate layups and average coupon dimensions, as shown in Table 8-26.

Table 8-26 - Laminate load conditions leading to failure

\begin{tabular}{|l|c|c|c|c|}
\hline Sample type & Laminate load & Untreated kenaf & Treated kenaf & Glass \\
\hline Tension & $N_{x}$ (kip/in) & 6.144 & 4.660 & 5.340 \\
\hline Compression & $N_{X}$ (kip/in) & -3.507 & -3.230 & -2.292 \\
\hline \multirow{2}{*}{ Flexure } & $M_{x}$ (kips) & -0.255 & -0.194 & -0.101 \\
\cline { 2 - 5 } & $V_{x}$ (kip/in) & 0.064 & 0.059 & 0.047 \\
\hline \multirow{2}{*}{ Shear } & $N_{x y}$ (kip/in) & 1.776 & 1.020 & 0.960 \\
\hline
\end{tabular}

Once the laminate strain vector $\left\{\epsilon_{x}^{0}, \epsilon_{y}^{0}, \gamma_{x y}^{0}, \kappa_{x}, \kappa_{y}, \kappa_{x y}\right\}$ has been calculated for each sample type, the strain vector $\left\{\epsilon_{x}, \epsilon_{y}, \gamma_{x y}\right\}$ at any point in the thickness can be calculated as:

$$
\left\{\begin{array}{c}
\epsilon_{x} \\
\epsilon_{y} \\
\gamma_{x y}
\end{array}\right\}=\left\{\begin{array}{c}
\epsilon_{x}^{0} \\
\epsilon_{y}^{0} \\
\gamma_{x y}^{0}
\end{array}\right\}+z\left\{\begin{array}{c}
\kappa_{x} \\
\kappa_{y} \\
\kappa_{x y}
\end{array}\right\}
$$


The stress at the same point can be calculated by pre-multiplying the strain vectors by the transformed reduced stiffness matrices $[\overline{\mathrm{Q}}]$ and $\left[\overline{\mathrm{Q}}^{*}\right]$ corresponding to the specific lamina $(k)$ as shown in equation (8-36).

$$
\begin{gathered}
\left\{\begin{array}{c}
\sigma_{x} \\
\sigma_{y} \\
\sigma_{x y}
\end{array}\right\}^{k}=\left[\begin{array}{lll}
\bar{Q}_{11} & \bar{Q}_{12} & \bar{Q}_{16} \\
\bar{Q}_{12} & \bar{Q}_{22} & \bar{Q}_{26} \\
\bar{Q}_{16} & \bar{Q}_{26} & \bar{Q}_{66}
\end{array}\right]^{k}\left\{\begin{array}{c}
\epsilon_{x} \\
\epsilon_{y} \\
\gamma_{x y}
\end{array}\right\} \\
\left\{\begin{array}{ll}
\sigma_{y z} \\
\sigma_{x z}
\end{array}\right\}^{k}=\left[\begin{array}{ll}
\bar{Q}_{44}^{*} & \bar{Q}_{45}^{*} \\
\bar{Q}_{45}^{*} & \bar{Q}_{55}^{*}
\end{array}\right]^{k}\left\{\begin{array}{l}
\gamma_{y z} \\
\gamma_{x z}
\end{array}\right\}
\end{gathered}
$$

In order to apply the following failure criteria, a MATLAB script was written to calculate the stresses $\left\{\sigma_{x}, \sigma_{y}, \sigma_{x y}, \sigma_{y z}, \sigma_{x z}\right\}$ and the strains $\left\{\epsilon_{x}, \epsilon_{y}, \gamma_{x y}, \gamma_{y z}, \gamma_{x z}\right\}$ at the top and bottom of each lamina (resulting in stresses at 8 points for 4-layer laminates and 4 points for 2-layer laminates) for each material (untreated kenaf, treated kenaf, glass) and for each sample type (tensile, compressive, flexure, shear). Due to the quantity of these results they are not reproduced here, but they are used in applying the following failure criteria.

\subsubsection{Maximum Stress/Strain Criteria}

The maximum stress and maximum strain criteria make use of R-ratios (equation (8-37)), where a value of less than 1.0 indicates that the laminate has failed.

$$
R=\frac{\sigma_{\text {ultimate }}}{\sigma_{\text {applied }}} \text { or } R=\frac{\epsilon_{\text {ultimate }}}{\epsilon_{\text {applied }}}
$$

In applying the maximum strength criterion, the stresses in each lamina are compared to the ultimate strengths of Table 8-24 according to equation (8-38), where the minimum strength R-ratio controls the failure.

$$
\begin{array}{lll}
R_{1}=F_{1 t} / \sigma_{1} ; \text { if } \sigma_{1}>0 \text { or } & R_{1}=-F_{1 c} / \sigma_{1} ; \text { if } \sigma_{1}<0 & \\
R_{2}=F_{2 t} / \sigma_{2} ; \text { if } \sigma_{2}>0 \text { or } & R_{2}=-F_{2 c} / \sigma_{2} ; \text { if } \sigma_{2}<0 & \\
R_{4}=F_{4} / a b s\left(\sigma_{4}\right) & R_{5}=F_{5} / a b s\left(\sigma_{5}\right) & R_{6}=F_{6} / a b s\left(\sigma_{6}\right)
\end{array}
$$

In applying the maximum strain criterion, the maximum strain in each lamina is compared to the ultimate strains of Table 8-25 according to equation (8-39), where the minimum strain R-ratio controls the failure.

$$
\begin{array}{lll}
R_{1}=\epsilon_{1 t} / \epsilon_{1} ; \text { if } \epsilon_{1}>0 \text { or } & R_{1}=-\epsilon_{1 c} / \epsilon_{1} ; \text { if } \epsilon_{1}<0 & \\
R_{2}=\epsilon_{2 t} / \epsilon_{2} ; \text { if } \epsilon_{2}>0 \text { or } & R_{2}=-\epsilon_{2 c} / \epsilon_{2} ; \text { if } \epsilon_{2}<0 & \\
R_{4}=\gamma_{4 u} / a b s\left(\epsilon_{4}\right) & R_{5}=\gamma_{5 u} / a b s\left(\epsilon_{5}\right) & R_{6}=\gamma_{6 u} / a b s\left(\epsilon_{6}\right)
\end{array}
$$

For each of the test types, several of the strength/strain ratios produce very high or infinite values. Therefore, only a few ratios need to be considered for each lamina. The applicable max stress and max strain ratios for each material and test type are shown in Table 8-27. The lowest ratio is the one that controls failure, and for each material and test type these 
are highlighted in green. The max stress and strain failure criteria frequently resulted in similar or identical ratios since the ultimate strengths were used to calculate the ultimate strains.

The tensile and compressive ultimate strengths/strains for the kenaf reinforced composites ended up being very close ( $\mathrm{R}$ near to 1.0 ) to the ultimate strengths/strains predicted by the max stress and max strain failure criteria. For the glass reinforced composites, the predicted compressive value was also quite close to the experimental value, however the tensile value was high (likely for the reasons mentioned in section 8.4.1). For shear, the values were less accurate (within 22\% for kenaf reinforced composites, but off by $56 \%$ for glass), similar to the trend seen with stiffness prediction. Also as with stiffness prediction, the flexural strength/strain prediction results ended up generally being the least accurate, with all three of the materials' experimental results exceeding the predicted results by $50-200 \%$.

Perhaps an oversimplified explanation for this could be that strength prediction with composites is rarely accurate, and the maximum stress and strain criteria are some of the simplest failure criteria available (although their long history and fundamental concepts imply that they are still valuable tools). On the other hand, the calculated strains experienced by the fibers in flexure ( $3 \% \max )$ were quite a lot higher than both the theoretical ultimate strains and the measured strains from single fiber tensile tests and unidirectional axial tests (around 1\%). This seemingly higher strain would most likely stem from one of two causes: either the fibers are slipping somewhat within the matrix and thus relaxing their stress/strain or somewhere in the analysis process the adjusted neutral axis is unaccounted for. The SEM and modulus prediction results provide support for the first possibility, as it is clear that the bonding is less than optimal between the natural fibers and the resin. However, this fails to explain why the glass fiber flexural samples, which should have good bonding with the vinyl ester resin, also have an experimental strength of around twice their predicted strength.

The main issue, therefore, most likely lies with a failure to account for the severely unbalanced layup and the associated adjustments to the neutral axis and moment of inertia. For example, a footnote in ASTM D790 mentions that the flexural strength calculated using their formula is only the apparent flexural strength based on homogeneous beam theory. As a result, the experimental results based on this equation do not indicate the actual stress at the maximum fiber surfaces, only the apparent strength of an analogous homogeneous beam, while the failure criteria results account for laminate theory and therefore indicate the actual stress. Unfortunately, the best way to confirm this would be to include gages at the top and bottom of each flexural sample, and therefore is a recommended exercise for future flexural tests of severely unbalanced laminates. This disconnect between the two definitions of 'flexural stress' is likely responsible for the majority of the error between the experimental and predicted failure strengths/strains. 
Table 8-27 - Applicable stress and strain ratios for each material, test type, and failure mode at $40 \%$ FVF

\begin{tabular}{|c|c|c|c|c|c|c|c|}
\hline \multirow{2}{*}{ Test type/Failure mode } & \multirow{2}{*}{ Strength Ratio } & \multicolumn{2}{|c|}{ Untreated kenaf } & \multicolumn{2}{|c|}{ Treated kenaf } & \multicolumn{2}{|c|}{ Glass } \\
\hline & & Min Stress & Min Strain & Min Stress & Min Strain & Min Stress & Min Strain \\
\hline Tension $[0 / f a b / f a b / 0]$ & Controlling Value: & 0.938 & 0.938 & 1.12 & 1.12 & 1.74 & 1.74 \\
\hline \multirow{2}{*}{$\begin{array}{l}\text { Tensile failure in load } \\
\text { direction }\end{array}$} & $\mathrm{R}_{1}$ in 0-fiber layers & 0.938 & 0.938 & 1.12 & 1.12 & 1.74 & 1.74 \\
\hline & $\mathrm{R}_{1}$ in fabric layers & 2.61 & 2.62 & 3.26 & 3.27 & $\mathrm{n} / \mathrm{a}$ & $\mathrm{n} / \mathrm{a}$ \\
\hline \multirow{2}{*}{$\begin{array}{l}\text { Compression failure } \\
\text { perpendicular to load }\end{array}$} & $\mathrm{R}_{2}$ in 0-fiber layers & 2969 & 5.08 & 3692 & 6.22 & High & 3.95 \\
\hline & $\mathrm{R}_{2}$ in fabric layers & 671 & 7.60 & 836 & 9.48 & $\mathrm{n} / \mathrm{a}$ & $\mathrm{n} / \mathrm{a}$ \\
\hline Compression [0/fab/fab/0] & Controlling Value: & 0.930 & 0.930 & 0.912 & 0.912 & 0.983 & 0.983 \\
\hline \multirow{2}{*}{$\begin{array}{l}\text { Compression failure in load } \\
\text { direction }\end{array}$} & $\mathrm{R}_{1}$ in 0-fiber layers & 0.930 & 0.930 & 0.912 & 0.912 & 0.983 & 0.983 \\
\hline & $\mathrm{R}_{1}$ in fabric layers & 4.01 & 4.01 & 4.47 & 4.48 & $\mathrm{n} / \mathrm{a}$ & $\mathrm{n} / \mathrm{a}$ \\
\hline \multirow{2}{*}{$\begin{array}{l}\text { Tensile failure perpendicular } \\
\text { to load }\end{array}$} & $\mathrm{R}_{2}$ in 0-fiber layers & 3293 & 5.63 & 3669 & 6.19 & High & 6.66 \\
\hline & $\mathrm{R}_{2}$ in fabric layers & 1029 & 11.6 & 1146 & 13.0 & $\mathrm{n} / \mathrm{a}$ & $\mathrm{n} / \mathrm{a}$ \\
\hline Flexure [0/fab/90/fab] & Controlling Value: & 0.666 & 0.672 & 0.633 & 0.639 & 0.465 & 0.462 \\
\hline \multirow{3}{*}{$\begin{array}{l}\text { Tensile/Compressive failure } \\
\text { in direction of applied } \\
\text { moment }\end{array}$} & $\mathrm{R}_{1}$ in 0-fiber layers & 0.666 & 0.672 & 0.633 & 0.639 & 0.575 & 0.591 \\
\hline & $\mathrm{R}_{2}$ in 90-fiber layers & 0.712 & 0.701 & 0.662 & 0.652 & 0.465 & 0.462 \\
\hline & $\mathrm{R}_{1}$ in fabric layers & 0.825 & 0.894 & 0.780 & 0.849 & $\mathrm{n} / \mathrm{a}$ & $\mathrm{n} / \mathrm{a}$ \\
\hline \multirow{3}{*}{$\begin{array}{l}\text { Compressive/Tensile failure } \\
\text { perpendicular to applied } \\
\text { moment }\end{array}$} & $\mathrm{R}_{2}$ in 0-fiber layers & 8.91 & 5.11 & 7.87 & 5.25 & 3.90 & 4.94 \\
\hline & $\mathrm{R}_{1}$ in 90-fiber layers & 8.31 & 2.93 & 8.07 & 2.92 & 7.64 & 6.31 \\
\hline & $\mathrm{R}_{2}$ in fabric layers & 3.68 & 6.64 & 3.31 & 6.95 & $\mathrm{n} / \mathrm{a}$ & $\mathrm{n} / \mathrm{a}$ \\
\hline \multirow{3}{*}{ Interlaminar shear failure } & $\mathrm{R}_{5}$ in 0 -fiber layers & 10.9 & 10.9 & 9.80 & 9.80 & 9.18 & 9.18 \\
\hline & $\mathrm{R}_{5}$ in 90-fiber layers & 14.0 & 10.9 & 12.6 & 9.80 & 11.8 & 9.18 \\
\hline & $\mathrm{R}_{5}$ in fabric layers & 9890 & 9890 & 9063 & 9063 & $\mathrm{n} / \mathrm{a}$ & $\mathrm{n} / \mathrm{a}$ \\
\hline Shear $[0 / f a b / 90 / f a b]$ & Controlling Value: & 0.781 & 0.781 & 1.13 & 1.13 & 1.56 & 1.56 \\
\hline \multirow{3}{*}{ In-plane shear failure } & $\mathrm{R}_{6}$ in 0-fiber layers & 1.05 & 1.05 & 1.52 & 1.52 & 1.56 & 1.56 \\
\hline & $\mathrm{R}_{6}$ in 90-fiber layers & 0.781 & 0.781 & 1.13 & 1.13 & 1.56 & 1.56 \\
\hline & $\mathrm{R}_{6}$ in fabric layers & 435 & 435 & 640 & 640 & $\mathrm{n} / \mathrm{a}$ & $\mathrm{n} / \mathrm{a}$ \\
\hline
\end{tabular}




\subsubsection{Interacting Failure Criterion}

The previous failure criteria operate on the assumption that each failure mode acts independently of the others. The interacting failure criterion proposed by Barbero (2011) is separated into two failure modes: the fiber dominated failure mode (equation (8-40), where $\langle x\rangle$ is the positive part of $\mathrm{x}$ ) and the matrix dominated failure mode (equation (8-41), with the additional variable definitions of equation (8-42)).

$$
\begin{gathered}
R_{1}=\left\langle F_{1 t} / \sigma_{1}\right\rangle+\left\langle-F_{1 c} / \sigma_{1}\right\rangle \\
\left(f_{22} \sigma_{2}^{2}+f_{44} \sigma_{4}^{2}+f_{55} \sigma_{5}^{2}+f_{66} \sigma_{6}^{2}\right) R_{2}^{2}+\left(f_{2} \sigma_{2}\right) R_{2}-1=0 \\
f_{2}=\frac{1}{F_{2 t}}-\frac{1}{F_{2 c}} \quad f_{44}=\frac{1}{\left(F_{4}\right)^{2}} \\
f_{22}=\frac{1}{F_{2 t} F_{2 c}} \quad f_{55}=\frac{1}{\left(F_{5}\right)^{2}} \\
f_{66}=\frac{1}{\left(F_{6}\right)^{2}}
\end{gathered}
$$

The tension and compression tests only contained stresses from the fiber dominated mode, while the shear tests only contained one of the stresses from the matrix dominated mode. Therefore, for these tests, the interacting failure criterion reduces to the max stress criterion.

For the flexure tests, the minimum stress ratios for the fiber dominated failure mode are also the same as those by the max stress criterion. However, there are interacting stresses in the $\mathrm{R}_{2}$ strength ratio in both the 0 - and 90-direction laminae, where equation (8-41) can be simplified to equation (8-43). Table 8-28 shows the adjusted R-ratios from the interacting failure criterion.

$$
\left(f_{22} \sigma_{2}^{2}+f_{55} \sigma_{5}^{2}\right) R_{2}^{2}+\left(f_{2} \sigma_{2}\right) R_{2}-1=0
$$

Table 8-28 - Applicable stress ratios from interacting failure criterion

\begin{tabular}{|l|l|c|c|c|}
\hline Flexure & \multicolumn{1}{|c|}{ Strength Ratio } & Untreated kenaf & Treated kenaf & Glass \\
\hline \multirow{2}{*}{ 0-Layer } & R1: fiber-dominated & 0.666 & 0.633 & 0.575 \\
\cline { 2 - 5 } & R2: matrix-dominated & 6.01 & 5.42 & 3.55 \\
\hline \multirow{2}{*}{ 90-Layer } & R1: fiber-dominated & 8.31 & 8.07 & 7.64 \\
\cline { 2 - 5 } & R2: matrix-dominated & 0.515 & 0.479 & 0.336 \\
\hline
\end{tabular}

The major adjustment caused by applying the interacting failure criterion is the shift of the controlling strength ratio from tension in the bottom of the 0-layer to compression in the top of the 90-layer. This correction is accurate, as the failure mode observed in many of the flexural tests was either compression in the top surface (due to fiber microbuckling) or a combination of top-surface compression and bottom-surface tensile rupture. 


\subsubsection{Conclusions}

While strength prediction is historically much less accurate than stiffness prediction, even simple models like the max stress and max strain criteria can predict the behavior of simple layups with reasonable accuracy. From the application of these models, it would appear that there are no extra complications involved in predicting the strength of natural fiber reinforced composites as compared to glass fiber reinforced composites, with the exception of potentially some fiber slippage due to poor bonding which could lead to higher apparent strain. Additionally, when testing severely unbalanced laminates according to ASTM D790, it would greatly aid advanced analysis to include compressive and tensile gages in order to determine actual stresses and strains, instead of just apparent properties.

\subsection{Moisture Effects}

While many composite analysis texts discuss the issue of moisture absorption effects (and their computational analog, temperature effects), the approach invariably starts from the assumption that the matrix material is responsible for most, if not all, of the absorption. This assumption is generally a very sound one for most synthetic fiber composites; however, it is clearly not the case with composites reinforced with natural fibers. As an additional complication beyond this faulty base assumption, the natural fiber reinforced composites have significant stiffness degradation in the presence of moisture due to two vaguely related factors: increased compliance in the fibers inherent in a wet organic material and reduced stiffness from a matrix damaged by exhaustive microcracking due to fiber swelling.

\subsubsection{Stiffness Behavior}

Due to the somewhat unconventional mechanical behavior of natural fiber reinforced composites immersed in water, a simple empirical relationship is the only available explanation for the stiffness degradation without conducting a far more extensive test program on different materials and layups, and at different moisture concentrations. The following relationship is only applicable for the materials and layups described earlier in this report, at around $40 \%$ overall FVF, and at saturation (theoretically the worst-case scenario for moisture absorption).

For the untreated kenaf fibers, saturation was approximately reached after around 2700 hours at, on average, around 14\% water absorption by weight. For the treated kenaf fibers, saturation was approximately reached after around 500 hours at, on average, around $11 \%$ water absorption by weight. However, since the samples were being tested simultaneously, the kenaf fiber samples remained immersed in water for an additional 2200 hours, during which time they gained negligible weight. At least part of the reason for this disparity in time is the lower FVF of the untreated kenaf fiber composites (before $40 \%$ normalization), which would have left the fibers with better resin cover, slowing the moisture absorption rate considerably.

Since the stiffness reduction of the natural fiber composites is largely due to two mostly separate parameters (fiber plasticization, matrix microcracking from fiber swelling), it is therefore theorized that this can be modeled by the fibers and matrix individually experiencing stiffness reductions. These reductions are labeled MRF (matrix reduction factor) and FRF (fiber reduction factor). In order to determine the MRF and FRF for each of the test types, the following procedure was applied to both the untreated kenaf reinforced composite and the treated kenaf reinforced composite models: 
1. Adjust the FRF (to a value less than 1.0). This value is multiplied by the fiber stiffness (including fabric stiffness) from Table 8-2 previously used for all modeling. Recalculate all lamina stiffnesses based on this new value. Recalculate ABD matrix and all equivalent laminate moduli.

2. Iterate Step 1 until $\mathrm{E}_{\mathrm{x}}$ value is close to empirical saturated tensile modulus. Logically, this fiber stiffness should be fairly close to the experimental fiber stiffness, since in unidirectional tension the fiber stiffness largely controls the lamina stiffness.

3. Adjust the MRF (to a value less than 1.0). This value is multiplied by the matrix stiffness properties from Table 8-1 previously used for all modeling. Recalculate all lamina stiffnesses based on this new value. Recalculate ABD matrix and all equivalent laminate moduli.

4. Iterate Step 2 until $G_{x y}$ value is close to empirical saturated in-plane shear modulus. Logically, this matrix stiffness should be fairly close to the experimental matrix stiffness, since in in-plane shear the matrix stiffness largely controls the lamina stiffness. Since matrix stiffness reduction is due to microcracking damage incurred from swelling, the MRF obtained here should be kept constant for all test types.

5. Recheck the tensile FRF for accuracy, and iterate steps 1-4 if necessary.

6. Adjust the FRF again (to a value less than 1.0, but greater than the tensile FRF) in order to find the compressive FRF. Since fiber swelling applies a positive strain in the axial direction, the compressive FRF will be greater than the tensile FRF, since at least some of the compressive strain will simply counteract the swelling. Recalculate lamina stiffnesses, ABD matrix, and equivalent lamina moduli for each iteration.

7. Iterate Step 6 until $\mathrm{E}_{\mathrm{x}}$ value is close to empirical compressive modulus.

8. Adjust FRF to halfway between compressive and tensile FRF since in both shear and flexure some fibers will be in tension and some in compression. Use this midpoint FRF value to recalculate $E_{b x}$ and $G_{x y}$ for theoretical flexure and in-plane shear stiffnesses.

The results of the above procedure are shown in Table 8-29 for untreated kenaf reinforced composite and in Table 8-30 for treated kenaf reinforced composite.

The above methodology seems to explain fairly well how the reduction in stiffness of both the fibers and the matrix result in the experimentally obtained values. The flexure predicted values are probably by far the least accurate (especially since the dry predictions obtained by the equivalent laminate moduli were $40 \%$ high as compared to $16 \%$ low). It is also interesting to note that, while the matrix reduction is approximately the same for both material types, the treated fibers experienced a much greater stiffness reduction. This could potentially be due to the treated fibers being kept at saturation for a long time, resulting in some biodegradation, while the untreated fibers only just reached saturation before testing. 
Table 8-29 - Reduced stiffness of untreated kenaf reinforced composite at saturation

\begin{tabular}{|l|c|c|c|c|c|}
\hline Test Type & MRF & FRF & Pred. E or G (Msi) & Exp. E or G (Msi) & \% Error \\
\hline Tension & 0.4 & 0.7 & 2.114 & 2.14 & $1.2 \%$ \\
\hline Compression & 0.4 & 0.9 & 2.692 & 2.83 & $4.9 \%$ \\
\hline Flexure & 0.4 & 0.8 & 0.932 & 0.91 & $2.4 \%$ \\
\hline Shear & 0.4 & 0.8 & 0.127 & 0.12 & $5.8 \%$ \\
\hline
\end{tabular}

Table 8-30 - Reduced stiffness of treated kenaf reinforced composite at saturation

\begin{tabular}{|l|c|c|c|c|c|}
\hline Test Type & MRF & FRF & Pred. E or G (Msi) & Exp. E or G (Msi) & \% Error \\
\hline Tension & 0.4 & 0.4 & 1.419 & 1.38 & $2.8 \%$ \\
\hline Compression & 0.4 & 0.6 & 2.081 & 1.98 & $5.1 \%$ \\
\hline Flexure & 0.4 & 0.5 & 0.724 & 0.86 & $15.8 \%$ \\
\hline Shear & 0.4 & 0.5 & 0.124 & 0.11 & $12.7 \%$ \\
\hline
\end{tabular}

\subsubsection{Strength Behavior}

Unlike with moisture-induced stiffness reduction, most composite texts acknowledge that strength is affected via moisture-induced strain (swelling). The change in dimension, $\Delta L$, of a body due to mechanical strain, thermal strain, and moisture strain, can be approximated as shown in equation (8-44), where $\epsilon$ is the mechanical strain, $\beta$ is the coefficient of moisture expansion, $\Delta m$ is the change in moisture concentration, $\alpha$ is the coefficient of thermal expansion, $\Delta T$ is the change in temperature, and $L_{0}$ is the initial length (Barbero 2011).

$$
\Delta L=(\epsilon+\beta \Delta m+\alpha \Delta T) L_{0}
$$

From the swelling measurements taken after samples had reached saturation, the average $\beta$-coefficients (in the longitudinal and transverse lamina orientations) were determined according to equations (8-45) and the results are shown in Table 8-31. The thickness swell was used to determine $\beta_{2}$ instead of the width swell due to the width swelling of every laminate being limited by the presence of at least two bi-directional fabric layers.

$$
\begin{gathered}
\beta_{1}=\frac{\% \text { length swell }}{\% \text { moisture }} \\
\beta_{2}=\frac{\% \text { thickness swell }}{\% \text { moisture }}
\end{gathered}
$$


Table 8-31 - Coefficients of moisture expansion for kenaf laminae

\begin{tabular}{|l|c|c|}
\hline Variable & Untreated kenaf & Treated kenaf \\
\hline $\boldsymbol{\Delta} \boldsymbol{m}$ & $13.72 \%$ & $11.30 \%$ \\
\hline $\boldsymbol{\beta}_{\mathbf{1}}$ & 0.0077 & 0.0287 \\
\hline $\boldsymbol{\beta}_{\mathbf{2}}$ & 0.7060 & 1.3585 \\
\hline $\boldsymbol{\beta}_{\mathbf{1}} \boldsymbol{\Delta} \boldsymbol{m}$ & 0.0011 & 0.0032 \\
\hline $\boldsymbol{\beta}_{\mathbf{2}} \boldsymbol{\Delta} \boldsymbol{m}$ & 0.0969 & 0.1535 \\
\hline
\end{tabular}

Using the coefficients of moisture expansion and the saturated \% moisture absorption, the theoretical stress given any mechanical strain can be determined as shown in equation (8-46) (neglecting thermal terms, and transforming $\beta$-coefficients into the laminate coordinate system) (Barbero 2011).

$$
\left\{\begin{array}{c}
\sigma_{x} \\
\sigma_{y} \\
\sigma_{x y}
\end{array}\right\}=\left[\begin{array}{lll}
\bar{Q}_{11} & \bar{Q}_{12} & \bar{Q}_{16} \\
\bar{Q}_{12} & \bar{Q}_{22} & \bar{Q}_{26} \\
\bar{Q}_{16} & \bar{Q}_{26} & \bar{Q}_{66}
\end{array}\right]\left(\left\{\begin{array}{c}
\epsilon_{x} \\
\epsilon_{y} \\
\gamma_{x y}
\end{array}\right\}-\left\{\begin{array}{c}
\alpha_{x} \\
\alpha_{y} \\
\alpha_{x y}
\end{array}\right\} \Delta T-\left\{\begin{array}{l}
\beta_{x} \\
\beta_{y} \\
\beta_{x y}
\end{array}\right\} \Delta m\right)
$$

While the laminate stresses, and the corresponding maximum stress/strain failure criteria, based on this equation are computable, by the time all of the assumptions (lamina failure strengths, reduced lamina failure strengths at saturation, reduced stiffness due to saturation from section 8.5.1, etc.) are compounded along with the inherent variation of the data and small sample size (3 samples each type and material), the results would be of little value. The most important conclusion to take away from this section is that, while predicting the strength and stiffness of composites saturated to this level is complex or impossible, obtaining the strength and stiffness values from testing is fairly easy and, in-practice, natural fiber composites should never be used in situations where they are immersed in water for long periods of time anyway.

\subsection{Conclusions}

In this chapter experimentally-determined and manufacturer-given constituent material properties were used by various models to determine the stiffness and strength of a $40 \%$ FVF laminate, and the results were compared to experimentally-obtained values. The basis for the majority of the modeling was the laminate theory published by Barbero (2011).

From the stiffness modeling, it was found that the Equivalent Laminate Modulus and Finite Element methods obtained identical and excellent tensile and compressive results and reasonable stiffness and flexural results, and that Castigliano's methods could also be used to obtain similar flexural results. An adjustment to the neutral axis location in order to account for the extremely unbalanced laminate was shown to significantly improve the flexural stiffness prediction of the Equivalent Laminate Modulus method, which is in fact intended only for symmetric, balanced laminates. A simple Summed Stiffness Method was shown to predict the flexural stiffness much more accurately than the other "good" methods, although the fact that the other three, mathematically more rigorous methods agree indicates that more than likely the problem is with the material and not the models, and thus the Summed Stiffness Method may only be accurate by accident. If this assumption is valid, then the bonding between the fibers and the matrix is poor, as was implied by SEM and mechanical testing in previous chapters, and, 
with better bonding, the flexural stiffness of the material could be increased by as much as $40 \%$. This assumption is not invalidated by the accuracy of the tensile and compressive results, as both of those tests rely more on the fiber properties alone and less on the interaction between the fibers and matrix.

While strength modeling of composites as a whole can be a very difficult exercise, it was found that the maximum stress and strain criteria could predict the failure stress and strain quite accurately for the tensile and compressive tests. For the flexural tests, the prediction was quite low, although this is likely more due to a disconnection between the definitions of flexural strength and apparent flexural strength, and less to the failure of the constituent materials to be modeled accurately. The strength model was also able to indicate the closeness between tensile surface and compressive surface failures of the flexural samples that was observed so often with mechanical testing.

Finally, the saturated stiffness of the material was modeled by empirically applying stiffness reduction factors to the fibers and matrix individually, and the average coefficients of moisture expansion were calculated. Given the high degree of moisture absorption and the resulting high moisture-induced strains, no good method has yet been determined for nonempirical prediction of saturated strength and stiffness performance.

But for their moisture absorption, it does not appear that natural fiber reinforced composites present any greater challenge in the prediction of their mechanical properties than do glass fiber reinforced composites, and in some cases their laminate behavior was predicted even more accurately. 


\section{SUSTAINABILITY OF NFRP COMPOSITES}

\subsection{Abstract}

The sustainability of natural fiber reinforced polymer composites was examined from two perspectives: environmental and socioeconomic. The environmental sustainability was assessed by a comparison of five wall designs, including a kenaf FRP structural insulated panel design and several other common construction methods. The embodied energy and contributed $\mathrm{CO}_{2}$ of the materials needed for each of these wall designs were calculated using two different methods, with one based on the cost of the materials and the other on the weight of the materials. Both methods produced similar results, in which a stick-framing wall design or an OSB wall design were the most eco-friendly and the FRP SIPs were the least eco-friendly. While the kenaf fibers themselves possess excellent sustainability characteristics, the vinyl ester resin used in the composites is comparatively environmentally hazardous. Consistent throughout all the designs, however, was a correlation between the respective costs of the materials and the respective environmental impacts.

The socioeconomic study looked at the sustainability of natural fiber reinforced composite materials as housing materials in developing countries. A literature study on the country of Bangladesh showed that the jute and kenaf market would benefit from the introduction of a value-added product like NFRP. The high rate of homeless and inadequately housed in Bangladesh could also improve greatly if a new, affordable and durable material were introduced. The two major hurdles needing to be overcome before NFRPs can be adopted as housing materials in Bangladesh are the cost and availability of a resin system and the moisture resistance/durability of the fibers.

\subsection{Introduction}

According to Graedel and Allenby (2003), industrial ecology of manufacturing involves "the design of industrial processes, products, and services from the dual perspectives of product competitiveness and environmental concerns." Designing products therefore entails considering both technical and sociological issues. On the sociological side, industrial ecology also involves considering human culture, individual choices, and societal institutions, as well as how these things define the industry-environment interactions (Graedel and Allenby 2003). One part of this larger context is examining the impact that products and industries supporting human society have, not just on the ecosystem, but also on the available financial, labor, energy, and raw material resources available, both now and in the future. This multi-dimensional focus on efficiency is at the heart of the concept of sustainable engineering.

One way of assessing the environmental impact of a given product or practice, or of making comparisons between similar products or practices, is by conducting a life cycle assessment (LCA; also called "life cycle analysis"). LCAs have become so common in the last few decades that their practice is now governed by international standards (ISO 14040, for example). While conducting a full LCA is a practice beyond the scope of this dissertation, the basic principles can be translated on a smaller scale and combined with online tools to make reasonable comparisons between comparable civil engineering products at the pre-construction stage. 
In addition to environmental concerns, industrial ecology dictates that the impacts of products and practices on human society must also be considered. Natural fiber reinforced composites can potentially provide eco-friendly and affordable building materials worldwide (as discussed in Chapter 2), particularly in some of the poorest regions of the world where industrial natural fiber crops are also most prevalent. In addition to natural FRPs entering the market as building material alternatives, an increased demand for these fibers, both in textile and composite forms, could have a significant economic impact on these developing countries and regions. In an effort to better evaluate the potential global impact of natural fiber reinforced composites, a literature review of the current fiber production, housing practices, and housing needs in Bangladesh was evaluated in the context of kenaf FRP composites, since the fibers used most often throughout this study originated there.

\subsection{Environmental Impacts}

In order to conduct a meaningful evaluation of environmental impacts, a comparison between different construction systems needs to be based on a unit that accounts for the variations in geometries, architecture, strengths, and stiffnesses the systems possess. Therefore a comparison based on unit mass or volume is incapable of providing an accurate description of relative environmental impact. In the following assessment, the perimeter wall for a simple building is designed for 5 different construction systems: OSB structural insulated panels (SIPs), kenaf FRP SIPs, glass FRP SIPs, stick-framing, and insulating concrete forms (ICFs).

In order to provide uniformity between the systems, each is designed according to the 2012 International Residential Code (IRC) for one- and two-family dwellings. In each of the designs, it is assumed that the foundation, floor, roof supports, roof, exterior cladding, windows, doors, hardware, fasteners, and utilities will all be roughly the same for each construction system, and can thus be neglected. Therefore, the design will only consider wall systems, including structural elements, insulation, and interior and exterior surface panels. To define loading, the building is assumed to be constructed in the state of West Virginia, where the maximum wind speed is $90 \mathrm{mph}$ (from Figure R301.2(4)A in the IRC) and the maximum snow load is 30 pcf (from Figure R301.2(5)). The building is a single story, $50 \mathrm{ft}$ long and $30 \mathrm{ft}$ wide, with $10 \mathrm{ft}$ walls. All of the analyses only consider material inputs, ignoring construction labor, maintenance labor, and demolition labor, as well as in-use and end-of-life costs, energy, and materials.

\subsubsection{Designing equivalent SIP systems}

Before the materials needed for the construction of the building can be defined for each of the SIP systems, glass and kenaf SIPs need to be designed such that they most efficiently meet the mechanical requirements for OSB SIPs in the IRC. In conducting this analysis, it is assumed that the OSB SIP will have an expanded polystyrene (EPS) core, while the KFRP and GFRP SIPs will have polyurethane / polyisocyanurate cores (PUR/PIR), similar to the SIPs tested in Chapter 7. Unlike the tested panels, however, the assumption will be made that the core system has been designed such that material rupture of the facesheet $\left(\sigma_{u l t}=P / A\right.$ for axial loading and $\sigma_{u l t}=M c / I$ for flexural loading) controls each of the SIP systems. This assumption is significant, as a number of failure modes are likely to occur in sandwich panel systems before material rupture of a facesheet, particularly with extremely thin facesheets and flexible cores. 
However, attempting to account for all of these different failure modes at this stage of design is unnecessarily complicated, given the number of other assumptions made.

Section R613 of the IRC is about structural insulated panel wall construction. Based on the minimum requirements for OSB SIPs in this section, the OSB SIP for the building design will be assumed to have the properties shown in Table 9-1. The core and facesheet axial stiffnesses are not specified in the IRC, but are necessary for the following analyses.

Table 9-1 - OSB SIP Properties and sources in 2012 IRC

\begin{tabular}{|l|l|l|}
\hline OSB SIP Property & Value & Source \\
\hline Facesheet thickness & $7 / 16 \mathrm{in}$ & Table R613.3.2 \\
\hline Facesheet density & $34 \mathrm{pcf}$ & Table R613.3.2 \\
\hline Facesheet bending strength & $2.72 \mathrm{ksi}$ & Table R613.3.2 \\
\hline Facesheet tensile (axial) strength & $1.42 \mathrm{ksi}$ & Table R613.3.2 \\
\hline Facesheet bending stiffness & $664 \mathrm{ksi}$ & Table R613.3.2 \\
\hline Facesheet axial stiffness & $750 \mathrm{ksi}$ & Testing average \\
\hline SIP thickness & $4.5 \mathrm{in}$ & Table R613.5(1) \\
\hline Resulting core thickness & $3.625 \mathrm{in}$ & \\
\hline Core stiffness & $0.300 \mathrm{ksi}$ & Typical value for medium quality EPS \\
\hline
\end{tabular}

Table 9-2 - Insulating properties for construction materials included in the following designs

\begin{tabular}{|l|c|}
\hline Material & R-Value \\
\hline Expanded polystyrene foam & $4.00 /$ in \\
\hline PUR/PIR foam & $6.25 /$ in \\
\hline Fiberglass batts & $3.71 /$ in \\
\hline Wood sheathing (OSB) & $1.25 /$ in \\
\hline GFRP & $4.00 /$ in \\
\hline NFRP (assumed) & $4.00 /$ in \\
\hline Drywall & $0.90 /$ in \\
\hline 3.5 in wood stud & 4.38 \\
\hline Poured concrete & $0.08 /$ in \\
\hline
\end{tabular}

The insulating properties for all of the materials used in the following comparisons are given in Table 9-2, where the units of the R-values are hr- $\mathrm{ft}^{2}{ }^{\circ} \mathrm{F} / \mathrm{btu}$ (Martin 2011). A higher Rvalue therefore corresponds to better insulation. With the EPS core, the OSB SIP is able to insulate with an R-value of 15.6, as shown in Equation (9-1), where FS stands for facesheet, $R$ is the R-value, and $t$ is the thickness.

$$
\left(R_{F S} \times t_{F S}\right) \times 2+\left(R_{\text {core }} \times t_{\text {core }}\right)=(1.25 \times 7 / 16) \times 2+(4.00 \times 3.625)=\mathbf{1 5 . 6}
$$

If, as was found with the structural component testing, the OSB is assumed to carry essentially all of the axial load due to the low modulus of the foam core, then the axial capacity of the OSB SIP for a 1-ft wide section can be calculated by setting the facesheet axial strength equal to $P / A$, and solving for $\mathrm{P}$ as shown in Equation (9-2). 


$$
P=\sigma_{u l t} \times A=1.42 \times(7 / 16 \times 12) \times 2=14.91 \text { kips } / \boldsymbol{f t}
$$

The bending moment capacity of the OSB SIP can be calculated for a 1-ft wide section based on the facesheet axial strength, as shown in Equation (9-3). The bending moment of inertia per 1-ft wide section is calculated according to Equation (7-2), dividing each term by the axial modulus of elasticity of the facesheets, and is $43.5 \mathrm{in}^{4}$ for the OSB SIP. The axial strength is used instead of the flexural strength because the SIP is assumed to act as a perfectly adhered sandwich panel in bending, where load transfer through the core is optimal, and deflections are assumed to be relatively small. With these assumptions, the concave panel of the SIP in bending will be completely in compression, while the convex panel will be completely in tension; therefore, the axial strength, and not the bending strength, applies.

$$
M=\sigma_{u l t} \times I / c=1.42 \times(43.5 / 2.25)=27.5 \text { kip-in } / f t
$$

The OSB SIP bending stiffness (of the entire SIP structure, not the facesheets alone), can

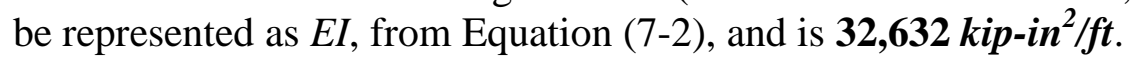

Based on the 2012 IRC, an OSB SIP possessing the insulating R-value, axial capacity, bending moment capacity, and bending stiffness shown above is sufficient as a 4.5 in thick, 10-ft high wall panel for a one-story residential dwelling in West Virginia (with proper connections and fasteners, external weathering protection, etc.). Using these values as the minimum allowable values, designs can be optimized for SIPs which have GFRP or KFRP as the facesheets and PUR/PIR as the core material.

The first step of the design process was to determine the minimum facesheet thickness that would meet the axial capacity of Equation (9-2). Next, the core thickness was calculated by solving the bending stiffness equation for $t_{c}$, with $E I=32,632 \mathrm{kip}^{-i n^{2}} / \mathrm{ft}$. The resulting panel dimensions were checked against the bending moment capacity of Equation (9-3) and the insulating capacity of Equation (9-1), and adjusted if needed. Since this first iteration resulted in a panel with extremely thin facesheets and a core thickness of around 6 inches, the facesheet thickness was iterated until a design was found which met all of the above criteria in addition to having a total thickness of 4.5 in or less. Since the FRP facesheets are more expensive, heavy, and environmentally damaging than the core material (shown later in this chapter), the most efficient design is one where the facesheet thickness is minimized within these constraints. The axial strength and stiffness properties used for the FRP facesheets were the compressive properties for $40 \%$ FVF obtained in Chapter 5, including a reduction factor of 0.75 on the axial strength of the KFRP panel due to the discontinuous fiber reinforcement, as described in Chapter 7. The SIP dimensions and capacities resulting from this design process are shown in Table 9-3. 
Table 9-3 - GFRP and KFRP SIP design properties, meeting or exceeding 2012 IRC OSB SIP specifications

\begin{tabular}{|c|c|c|c|}
\hline Property & OSB & GFRP & KFRP \\
\hline Facesheet thickness, single (in) & 0.4375 & 0.07 & 0.09 \\
\hline Core thickness (in) & 3.625 & 4.25 & 4.2 \\
\hline SIP thickness (in) & 4.5 & 4.39 & 4.38 \\
\hline Facesheet weight, both $\left(\mathbf{l b} / \mathbf{f t}^{2}\right)$ & 2.92 & 1.28 & 1.22 \\
\hline Core weight $\left(\mathbf{l b} / \mathbf{f t}^{2}\right)$ & 0.42 & 0.66 & 0.65 \\
\hline Total weight $\left(\mathbf{l b} / \mathbf{f t}^{2}\right)$ & 3.34 & 1.94 & 1.87 \\
\hline R-value (hr-ft ${ }^{2}{ }^{\circ}$ F/Btu) & 15.6 & 27.1 & 27.0 \\
\hline Moment of inertia (in $/ \mathbf{f t})$ & 43.5 & 7.9 & 10.0 \\
\hline Axial capacity (kips/ft) & 14.9 & 26.9 & 26.8 \\
\hline Bending moment capacity (kip-in/ft) & 27.5 & 57.3 & 56.4 \\
\hline Bending stiffness (kip-in $/$ ftt) & 32,632 & 32,997 & 32,872 \\
\hline
\end{tabular}

\subsubsection{Material Usage for each Wall Design}

\subsubsection{OSB, GFRP, and KFRP SIPs}

Calculating the material usage for the SIPs is fairly simple once the GFRP and KFRP designs in the previous section were completed. For the $30 \mathrm{x} 50 \mathrm{ft}$ building, the total perimeter is $160 \mathrm{ft}$. For a $10 \mathrm{ft}$ tall wall, the total SIP area is approximately $1600 \mathrm{ft}^{2}$. The respective total facesheet and core weights can be calculated by multiplying this area by the weights in Table 9-3. The fiber and resin weights in each of the FRP composites can be calculated by the weight fractions corresponding to 40\% FVF: $55 \%$ fiber and 45\% resin for the GFRP, and 44\% fiber and $56 \%$ resin for the KFRP.

The joints between panels are typically connected via splines ( 3 in wide) of the facing materials inserted just inside each of the panel edges and connected by screws or other fasteners. For $4 \mathrm{ft}$ wide panels, the $160 \mathrm{ft}$ perimeter would include 38 such joints, for a total of 76 of 3 in $\mathrm{x}$ $10 \mathrm{ft}$ facesheet panels.

In order to provide connections at the floor and roof interfaces, a number of fitted lumber pieces are inserted within the edges of the facesheets. A single 1.5 in thick bottom plate of lumber is needed at the floor connection, and a double, 3.0 in thick top plate is needed at the roof connection. Because of the varying core thicknesses and the requirement that the plates match the core thicknesses, the total volume of lumber needed for each of the SIP systems varies. In addition to the bottom and top plates, 2 of 1.5 in thick lumber end plates are needed at each corner of the building to seal the cores.

The weights of each material needed for each of the SIP wall designs are shown in Table 9-4, rounded to the nearest pound. 
Table 9-4 - Weight (in lbs) of raw material for each SIP wall design for 30 x $50 \mathrm{ft}$ building

\begin{tabular}{|l|r|r|r|}
\hline \multicolumn{1}{|c|}{ Material } & OSB SIP & GFRP SIP & KFRP SIP \\
\hline OSB & 4949 & - & - \\
\hline Glass Fibers & - & 1193 & - \\
\hline Kenaf Fibers & - & - & 955 \\
\hline Vinyl Ester & - & 976 & 1215 \\
\hline EPS & 672 & - & - \\
\hline PUR/PIR Foam & - & 1056 & 1040 \\
\hline Lumber & 778 & 912 & 902 \\
\hline TOTAL (lbs) & $\mathbf{6 4 0 0}$ & $\mathbf{4 1 3 8}$ & $\mathbf{4 1 1 1}$ \\
\hline
\end{tabular}

\subsubsection{Stick-framed}

For a stick-framed design to serve all of the same purposes as the SIP designs, it must include the framing, insulation, and interior and exterior wall sheathings.

Based on Table R602.3.1, a reasonable stud pattern for this design and load conditions is $2 \mathrm{x} 4 \mathrm{~s}$ at 16 in spacing. For the $160 \mathrm{ft}$ perimeter, this would add up to around 126 of 115.5 in studs (10 ft height - (1.5 in x 3 stud thicknesses)). In addition to the vertical studs, a single thickness bottom plate and a double thickness top plate are also needed around the entire perimeter.

One of the more common insulating methods for stick-framing is the use of fiberglass insulating batts, which are placed in the spaces between the studs prior to the attachment of the interior wall sheathing. According to the stud spacing, this would result in around 118 batts of 14.5 in width and 115.5 in height, each. For optimum insulating properties, the batts should be the same thickness as the stud width of 3.5 in.

For exterior sheathing, since it is assumed that cladding and weather protection will need to be added to each design, $1 / 2$ in thick OSB panels are sufficient for the entirety of the $160 \mathrm{ft}$ perimeter. The interior perimeter is approximated at $160 \mathrm{ft}$, although in reality it will be slightly less, for the $1 / 2$ in drywall panels. The total stick-framing design weights for each material are shown in Table 9-5.

Table 9-5 - Weight (in lbs) of raw material for stick-framing design for 30 x $50 \mathrm{ft}$ building

\begin{tabular}{|l|r|r|r|}
\hline \multicolumn{1}{|c|}{ Material } & Volume (ft $\mathbf{~}^{\mathbf{}}$ ) & Density (pcf) & Weight (lbs) \\
\hline Lumber & 61.71 & 36.8 & 2271 \\
\hline Fiberglass Batts & 400.27 & 0.76 & 304 \\
\hline OSB & 66.67 & 34.0 & 2267 \\
\hline Drywall & 66.67 & 38.4 & 2560 \\
\hline TOTAL & & & $\mathbf{7 4 0 2}$ \\
\hline
\end{tabular}

The assembly R-value of the above design can be calculated according to Equation (9-4) (Martin 2011), in which the R-value at the studs is 5.46 (OSB + stud + drywall), the R-value at 
the cavity is 14.06 (OSB + fiberglass batt + drywall), the $\%_{\text {studs }}$ is $0.096(1.5 / 16)$, and the $\%_{\text {cavity }}$ is 0.904 .

$$
R_{\text {assembly }}=\frac{1}{\%_{\text {studs }} / R_{\text {studs }}+{ }^{\%_{\text {cavity }} / R_{\text {cavity }}}}=12.25
$$

\subsubsection{Insulating Concrete Forms}

Exterior concrete wall construction is covered in detail in section R611 of the 2012 IRC. In order to have the best comparison to the previous designs, the smallest allowable wall type and thickness, 4 in flat, was selected from Table R611.3. Along with this reinforced concrete wall, 2 in thick, stay-in-place EPS foam forms will be used on both the interior and exterior surfaces, for a total wall thickness of over 8 inches. While this thickness is significantly higher than for the previous designs, it is essentially the minimum thickness allowed according to the IRC (including stay-in-place forms).

In addition to the concrete material and EPS foam, vertical and horizontal steel reinforcement is needed within the poured concrete. According to Table R611.6(1), for $90 \mathrm{mph}$ wind loading, $10 \mathrm{ft}$ height, and 4 in thick wall, \#4 steel bars are needed at 42 in spacing as vertical reinforcement, for a total of around 50 bars for the entire building (500 linear feet). According to section R611.6.2, four \#4 steel bars are needed for horizontal reinforcement around the entire structure, one each located at 12 in above the bottom, 12 in below the top, and at 1/3 and $2 / 3$ the total height (640 linear feet, total).

With stay-in-place forms, the surface on the interior of the building must be protected according to section R611.4.2; the exterior surface will be assumed to be protected in the same manner as all of the other designs. For the interior surface, $1 / 2$ in thick drywall will be installed. The total insulating concreted form (ICF) design weights for each material are shown in Table 9-6.

Table 9-6 - Weight (in lbs) of raw material for insulating concrete forms design for 30 x 50 ft building

\begin{tabular}{|l|r|r|r|}
\hline Material & Volume (ft $\mathbf{~}^{\mathbf{3}}$ ) & Density (pcf) & Weight (lbs) \\
\hline Concrete & 531.78 & 144 & 76576 \\
\hline Steel & 1.55 & 490 & 762 \\
\hline EPS & 533.33 & 0.9 & 480 \\
\hline Drywall & 66.67 & 38.4 & 2560 \\
\hline TOTAL & & & $\mathbf{8 0 3 7 8}$ \\
\hline
\end{tabular}

The insulating capacity R-value of the ICF wall design, including 4 in of concrete, 4 in of EPS, and 0.5 in of drywall, is $\mathbf{1 6 . 7 7 .}$

The results of each of the preceding wall designs will be used in the following cost and environmental impact study. 


\subsubsection{Environmental Impact for each Wall Design}

\subsubsection{Background on Life Cycle Analyses}

The concept of LCA originated around 1969 when Coca-Cola began rethinking their packaging systems, and pioneered a new way of looking at a products' impacts throughout its entire lifespan, not just during its manufacture (Baumann and Tillman 2004). Over the next two decades LCA studies began to proliferate, although many of the early studies also focused primarily on packaging and waste. At the beginning of its usage, LCA was primarily focused on increasing the efficiency, in terms of both cost and energy, of products already widely used. The oil crisis, which emerged in 1973, contributed to interest in LCA as the depletion of natural resources and rising costs for energy usage became serious national concerns in the US (Baumann and Tillman 2004). As time passed, however, the focus on environmental impacts also became of great importance.

Particularly in its early years, LCA was vulnerable to claims that the science behind the assessment methodology was seriously lacking and that, therefore, results could be easily manipulated in support of or in opposition toward a particular product. As a result of this criticism, the entire LCA methodology has become much more standardized, particularly by the International Organization for Standardization in 1993 (ISO 14040).

The basic LCA process (according to ISO 14040) begins with the goal and scope definition, which determines the direction and extent to which the LCA will be conducted. The second step is the life cycle inventory analysis (LCI), in which data is collected and calculations are made to determine the product system's inputs and outputs, including natural resources, energy, water, and emissions, throughout the entire lifetime of the product. The third step is the life cycle impact assessment (LCIA) which assigns the LCI findings to potential environmental impacts, resulting in a numerical evaluation of the product's influence on a number of environmental concerns. The final step is the interpretation of results, in which the raw data is evaluated and conclusions are drawn as to the most significant findings and the resulting recommendations for improvement.

Despite LCA studies' growing popularity and standardization, there are still problems associated with the scale of information needing to be considered when conducting a full LCA. One major LCA looked at the manufacturing for a generic American automobile, considering all of the components of processing of raw materials, manufacturing of parts, and assembly (Hendrickson et al. 2006). Some problems were introduced when the study was forced to only examine a select few steel mills and plastics plants and to assume that they were "representative" of all plants, although evidence for this assumption was not provided. Additionally, much of the transportation involving materials, fuels, and minor components was overlooked. Despite these shortcomings, the study took two years and cost almost \$8 million (Hendrickson et al. 2006). Clearly, "full" LCAs are best left to major industries where the results of the studies will have major financial implications.

In terms of building construction, studies have shown that the embodied energy involved in the construction phase may be between $10-50 \%$ of the total energy used in the operational lifetime of the building, indicating the importance of not only designing for operational energy efficiency, but also material and construction efficiency (Crawford 2011). With this in mind, the following brief sustainability study will focus only on the raw materials involved in building 
construction, as this data is much easier to obtain and verify than data related to the operational lifetime and disposal of buildings. To narrow down the focus of the study even further, only the relative costs, embodied energies, and equivalent $\mathrm{CO}_{2}$ contributions will be examined for each of the construction designs. Energy use is easy to communicate and contains information related to the total environmental impact, and energy data is also relatively easy to collect (Baumann and Tillman 2004).

The purpose of this abbreviated study is therefore to determine the relative environmental impact of five different wall design systems by looking at the embodied energy and equivalent $\mathrm{CO}_{2}$ contributed throughout the production of their most easily identifiable constituent materials. The functional unit for this study was selected as the IRC-specified minimum exterior wall design for a single story, 30x50 ft building constructed in West Virginia, USA. This unit was selected since units such as mass or volume would fail to accurately portray the relative construction efficiency for varying materials.

Most LCAs include process flowcharts illustrating all of the different system inputs and outputs at the inventory analysis stage. An example of a KFRP SIP partial process flowchart is shown in Figure 9-1. While this flowchart really only focuses on the production of the kenaf fibers themselves, it illustrates how quickly the sprawl of LCAs can grow. For example, none of the processes required for production of the FRP resin or the insulating foam core are shown, although each of these would individually possess a process flowchart at least as complex as Figure 9-1. The fertilizer, pesticides, various equipment, chemicals, and adhesives are all also the results of multiple-input manufacturing processes, involving numerous raw materials and energy sources. Additionally, the flowchart only focuses on the manufacturing of the product, and does not go into detail on any of the materials or energy consumed, nor byproducts resulting from any phase beyond the kenaf SIP leaving the manufacturing plant. Because of the overwhelming amount of data implicit with full LCA studies, some simplified methods are available to calculate approximate environmental impacts from a more manageable amount of data. 


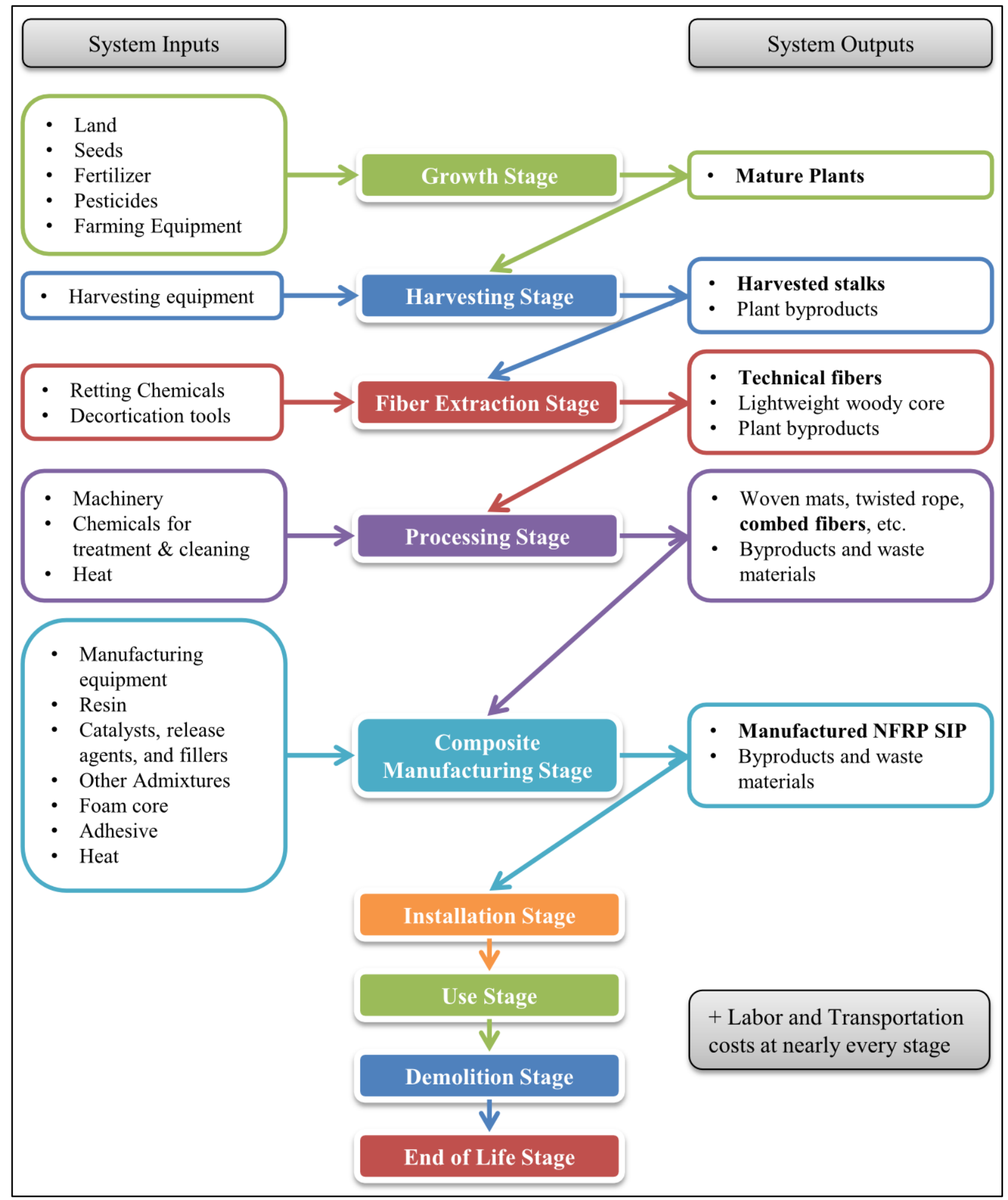

Figure 9-1 - Partial process flow diagram for NFRP SIP 


\subsubsection{Background on EIO-LCA}

Economic Input-Output Life Cycle Assessment (EIO-LCA) is a program developed by researchers at Carnegie Mellon University which, based on a dollar amount and a selected industry sector, returns the approximate economic and environmental impacts that would typically occur as a result of an investment of that level in that sector (Carnegie Mellon University Green Design Institute 2013).

When compared to full LCA, EIO-LCA has the advantages of being faster and cheaper in addition to being based on the entire US economy, which provides a more comprehensive evaluation. On the other hand, the major disadvantage is that it uses aggregate data from the product sector rather than the exact process data (Hendrickson et al. 2006). The Carnegie Mellon model also appears to be applicable for a single country (either the US or a select few others) instead of globally, and uses relatively outdated data (2002 or before) (Carnegie Mellon University Green Design Institute 2013). The data used in the EIO-LCA model is sourced largely from federal departments, bureaus, and agencies and related federal census data. The industryby-industry input-output matrix was developed by the US Department of Commerce (Hendrickson et al. 2006).

Because of the way the EIO-LCA model is built, comparing two similar products or processes will produce results with less uncertainty than simply characterizing the impact of a single product, since most errors and uncertainty will likely be shared by both sets of products or processes (Hendrickson et al. 2006). Hendrickson et al. (2006) admit that significant differences can exist between the simplified EIO-LCA and a full LCA depending on the product or process being studied and the analysis constraints, but in several test cases discussed in their book, the results were fairly close.

The sectors selected in the EIO-LCA model for each of the construction materials needed by any of the five wall designs are shown in Table 9-7. The calculations of total expenditures for

each of these sectors and for each of the wall designs, along with the results of the EIO-LCA study, are discussed in the following section. 
Table 9-7 - EIO-LCA Sectors and detailed sectors for each raw material

\begin{tabular}{|l|l|}
\hline \multirow{2}{*}{ Material } & EIO-LCA Sector / Detailed Sector \\
\hline \multirow{2}{*}{ OSB } & Wood, paper, and printing \\
\cline { 2 - 2 } Drywall & Sawmills and wood preservation \\
\hline \multirow{2}{*}{ Glass Fibers } & Wood, paper, and printing \\
\hline \multirow{2}{*}{ Kenaf Fibers } & Plastic, rubber, and nonmetallic mineral products \\
\cline { 2 - 2 } & Lime and gypsum product manufacturing \\
\cline { 2 - 2 } Vinyl Ester Resin, & Rrtificial and synthetic fibers and filaments manufacturing \\
\cline { 2 - 2 } & Textiles, apparel, and leather \\
\cline { 2 - 2 } & Riber, yarn, and thread mills \\
\hline \multirow{2}{*}{ EPS } & Plastics material and resin manufacturing \\
\hline \multirow{2}{*}{ PUR/PIR Foam } & Plastic, rubber, and nonmetallic mineral products \\
\cline { 2 - 2 } & Polystyrene foam product manufacturing \\
\cline { 2 - 2 } & Urethane and other foam product (except polystyrene) manufacturing \\
\hline \multirow{2}{*}{ Fiberglass Batts } & Plastic, rubber, and nonmetallic mineral products \\
\cline { 2 - 2 } & Mineral wool manufacturing \\
\hline \multirow{2}{*}{ Concrete } & Plastic, rubber, and nonmetallic mineral products \\
\cline { 2 - 2 } & Ready-mix concrete manufacturing \\
\hline \multirow{2}{*}{ Steel } & Ferrous and nonferrous metal production \\
\cline { 2 - 2 } & Iron and steel mills \\
\hline
\end{tabular}

\subsubsection{Cost, embodied energy, and $\mathrm{CO}_{2}$ contribution of each wall design}

In order to begin applying the EIO-LCA model, the approximate dollar amount of product from each sector needed to be determined for the functional unit. These unit-weight cost estimates for each of the materials used in the five construction designs are shown in Table 9-8, with the source details listed in Table 9-9. To provide a second comparison between environmental impacts in addition to the EIO-LCA, published embodied energy and $\mathrm{CO}_{2}$ contribution per unit weight for each of the materials were also collected and are compiled in Table 9-8.

As often as possible, the values represented in Table 9-8 are the averages published from at least two sources. The downside of this method is that it fails to take into account quality or differences between different manufacturers/suppliers and product lines. As with the EIO-LCA, the cost, embodied energy, and $\mathrm{CO}_{2}$ contribution values are therefore more averages for a particular material type than specific to the exact materials that would be used for the wall construction.

Several of the cost values were estimated based on prices listed on a global bulk trading website (Alibaba.com 2013), and therefore are typically a rough average of the listed costs for obtaining these materials from Asia. In many cases these costs may end up being on the low end of actual US prices, since transportation to the US is not included. According to Bast Fibers, LLC, the bulk price for the exact kenaf fibers used in manufacturing the KFRP composites in the 
previous chapters would potentially be around $\$ 0.55$ / lb, including transportation from Bangladesh and importation to the US. Since this value was only slightly higher than other published costs for raw kenaf fibers, this value was included in the average price shown in Table 9-8. On the other hand, the bulk price for Derakane 510A quoted by Ashland, Inc. was \$3.19 / lb, which was significantly higher than other generic vinyl ester prices. This cost is probably much higher than an industry repeat customer would actually pay, and was therefore left out of the average pricing information for generic vinyl ester.

No source was found for the environmental impact data for vinyl ester resin, so the numbers for epoxy resins were used instead. Since epoxy resins encompass a wide variety of different resin systems (technically including vinyl ester), these numbers may be high for vinyl esters.

Finally, in some cases it was unclear whether the cost and environmental impact values for the chemical products (resins and foams) cited by the sources were regarding the liquid, precuring material or the post-curing, lower density material, so these values may be quite high as well.

Table 9-8 - Embodied Energy, $\mathrm{CO}_{2}$ contribution, and cost by weight for each material

\begin{tabular}{|c|c|c|c|c|c|c|}
\hline \multirow{2}{*}{ Material } & \multicolumn{2}{|c|}{ Embodied Energy } & \multirow{2}{*}{$\begin{array}{c}\mathrm{CO} 2 \\
\mathrm{~kg} / \mathrm{kg}(\mathrm{lb} / \mathrm{lb})\end{array}$} & \multicolumn{2}{|c|}{ Cost } & \multirow{2}{*}{ Sources } \\
\hline & MJ/kg & btu/lb & & $\$ / \mathbf{k g}$ & $\$ / \mathbf{l b}$ & \\
\hline \multicolumn{7}{|c|}{ Fibers } \\
\hline Glass Fibers & 53.0 & 22813 & 2.78 & $\$ 1.75$ & $\$ 0.80$ & [1-6] \\
\hline Kenaf Fibers & 22.9 & 9839 & 0.88 & $\$ 0.94$ & $\$ 0.43$ & {$[2,6,7]$} \\
\hline Flax Fibers & 9.0 & 3888 & 0.39 & - & - & {$[1,3,5]$} \\
\hline Hemp Fibers & 6.0 & 2594 & 0.55 & - & - & [3-5] \\
\hline \multicolumn{7}{|c|}{ Resins } \\
\hline Polyester Resin & 58.0 & 24963 & 2.39 & $\$ 1.50$ & $\$ 0.68$ & {$[1,6,9,10]$} \\
\hline Envirez & 50.0 & 21500 & - & - & - & [1] \\
\hline Epoxy Resin & 127.1 & 54681 & 5.91 & - & - & {$[3,5,10]$} \\
\hline Vinyl Ester Resin & - & - & - & $\$ 4.09$ & \$ 1.86 & [6] \\
\hline \multicolumn{7}{|c|}{ Insulations } \\
\hline EPS Foam & 99.4 & 42749 & 3.23 & $\$ 1.50$ & $\$ 0.68$ & {$[5,6,12,14]$} \\
\hline PUR/PIR Foam & 101.3 & 43559 & 3.48 & $\$ 1.50$ & $\$ 0.68$ & {$[6,12,14]$} \\
\hline Fiberglass Batts & 28.0 & 12046 & 1.35 & $\$ 0.70$ & $\$ 0.32$ & {$[6,12]$} \\
\hline \multicolumn{7}{|c|}{ Other Materials } \\
\hline OSB & 22.5 & 9658 & 0.83 & $\$ 0.47$ & $\$ 0.21$ & {$[6,12,13]$} \\
\hline Lumber (2x4) & 9.6 & 4141 & 0.55 & $\$ 0.40$ & $\$ 0.18$ & {$[5,6,12]$} \\
\hline Drywall & 6.8 & 2904 & 0.38 & $\$ 0.44$ & $\$ 0.20$ & {$[6,12]$} \\
\hline Concrete & 1.1 & 486 & 0.13 & $\$ 0.04$ & $\$ 0.02$ & {$[5,12,15]$} \\
\hline Steel Rebar & 23.3 & 10024 & 1.59 & $\$ 0.66$ & $\$ 0.30$ & {$[5,6,12]$} \\
\hline \multicolumn{7}{|c|}{ Processes } \\
\hline Hand Lay-up & 19.3 & 8300 & - & - & - & [1] \\
\hline RTM, VARTM, Cold Press & 11.5 & 4950 & - & - & - & [1] \\
\hline Pultrusion & 1.8 & 775 & - & - & - & [1] \\
\hline Truck Transportation (/mile) & 0.00166 & 0.71212 & $9.66 \mathrm{E}-05$ & - & - & {$[1,5]$} \\
\hline Sea Transportation (/mile) & 0.00016 & 0.06739 & $2.41 \mathrm{E}-05$ & - & - & {$[1,5]$} \\
\hline
\end{tabular}


Table 9-9 - Sources corresponding to data in Table 9-8

\begin{tabular}{|l|l|}
\hline$\#$ & \multicolumn{1}{|c|}{ Source } \\
\hline [1] & (Marriott et al. 2009) \\
\hline [2] & (Mohanty et al. 2002) \\
\hline [3] & (Joshi et al. 2004) \\
\hline [4] & (Pervaiz and Sain 2003) \\
\hline [5] & (Ashby 2013) \\
\hline [6] & Estimated from (Alibaba.com 2013) \\
\hline [7] & Correspondence with Bast Fibers, LLC \\
\hline [8] & (Wang et al. 2012) \\
\hline [9] & (Centre for Building Performance Research 2007) \\
\hline [10] & (Patel 2003) \\
\hline [11] & Correspondence with Ashland, Inc. \\
\hline [12] & (Hammond and Jones 2013) \\
\hline [13] & (European Commission 2005) \\
\hline [14] & (GreenSpec 2013) \\
\hline [15] & (Erlin et al. 2005) \\
\hline
\end{tabular}

Once the cost data was compiled, the EIO-LCA could be computed, using the industry sectors from Table 9-7, the average cost per unit weight data from Table 9-8, and the estimated weight of each material for each design from the previous sections. Since the EIO-LCA is scaled to work with industry-sized quantities, and since the model is linear with respect to cost, the input values were multiplied by $\$ 1,000,000$ and the outputs were scaled accordingly.

The EIO-LCA can be used to collect far more information than just the embodied energy and $\mathrm{CO}_{2}$ contribution for a particular analysis; for example, several of the different output tables for each of the five designs are shown in Appendix C. However, since embodied energy and $\mathrm{CO}_{2}$ contribution were the two easiest environmental impact values to compare with other sources, these values are the only ones shown in the comparison in Table 9-10. Also listed in this table are the insulating factors, weights, and material costs of each of the designs, as well as the comparative environmental impacts of each design calculated using the data from Table 9-8.

Overall, the results from these two environmental assessments match reasonably well and lead to essentially the same conclusions. However, the data for several of the particular materials seems to be skewed; the OSB, drywall, and the two insulating foam systems seem to be the least accurate. Since the foam values were all quite high based on the values from Table 9-8, it seems probable that at least a few of the values used in obtaining the averages were for the foams in pre-foamed format, in which case a pound of un-foamed polystyrene or polyurethane would possess a much higher environmental impact than a pound of expanded foam. The drywall values from the EIO-LCA were based off of the gypsum production sector, but since drywall is obviously not $100 \%$ gypsum, the embodied energy and $\mathrm{CO}_{2}$ contribution are unreasonably high. Finally, one of the sources for the embodied energy and $\mathrm{CO}_{2}$ contribution of OSB was nearly twice as high as the other, and if the high result were thrown out as erroneous, the values obtained in Table 9-10 would match much more closely.

One of the common trends found from this analysis is that the cost seems to correspond surprisingly well with the embodied energy and the contributed $\mathrm{CO}_{2}$, particularly once the 
results from the two studies are averaged (see Table 9-11). Based on these averages, each of these construction methods tends to require around 30,000 btu of energy and produces around 4 lbs of $\mathrm{CO}_{2}$ for every dollar spent. The only significant outlier in Table 9-11 is the high rate of $\mathrm{CO}_{2}$ produced by the designing with ICFs, although this is not particularly surprising given that cement production is notorious for producing massive amounts of carbon dioxide. This correlation between cost and environmental impact is reasonable (particularly from the viewpoint of embodied energy) in light of the fact that some form of energy is required at each step of production for any material, and energy costs money. This correlation makes it far less disconcerting that the embodied energy and contributed $\mathrm{CO}_{2}$ of the FRP SIP designs are so high; if costs can be driven down by improved efficiency in manufacturing and incorporation of lowercost materials, then the environmental impact will likely also decrease proportionally. This cost/environmental impact relationship is also encouraging in the construction industry, since sustainable practices are unlikely to become popular unless they are also cost-efficient. A survey of 20 small and medium building firms in Australia found that the biggest concerns with adoption of sustainable practices were all focused either on the cost and availability of adopting the practices or on negative feelings associated with government-mandated changes (Thorpe et al. 2008). 
Table 9-10 - Embodied Energy, $\mathrm{CO}_{2}$ contribution, and cost comparisons for the five wall designs

\begin{tabular}{|c|c|c|c|c|c|c|c|}
\hline \multirow[b]{2}{*}{$\begin{array}{c}\text { Wall System / } \\
\text { Material }\end{array}$} & \multirow[b]{2}{*}{$\begin{array}{c}\text { R- } \\
\text { value } \\
\text { (/in) }\end{array}$} & \multirow[b]{2}{*}{$\begin{array}{l}\text { Weight } \\
\text { (lbs) }\end{array}$} & \multirow[b]{2}{*}{ Cost (\$) } & \multicolumn{2}{|c|}{ From EIO-LCA } & \multicolumn{2}{|c|}{ (From data in Table 9-8) } \\
\hline & & & & $\begin{array}{c}\text { Embodied Energy } \\
\left(10^{6} \mathrm{btu}\right)\end{array}$ & $\begin{array}{c}\text { Contributed } \mathrm{CO}_{2} \\
\text { (lbs) }\end{array}$ & $\begin{array}{c}\text { Embodied Energy } \\
\left(10^{6} \text { btu }\right)\end{array}$ & $\begin{array}{c}\text { Contributed } \mathrm{CO}_{2} \\
\text { (lbs) }\end{array}$ \\
\hline \multicolumn{8}{|c|}{ OSB Structural Insulated Panel Design } \\
\hline OSB & 1.25 & 4949 & \$ $1,057.37$ & 27.2 & 3124 & 47.8 & 4108 \\
\hline EPS & 4.00 & 672 & $\$ \quad 458.18$ & 9.1 & 1261 & 28.7 & 2167 \\
\hline Lumber & 1.25 & 778 & $\$ \quad 140.07$ & 1.8 & 227 & 3.2 & 428 \\
\hline OSB SIP Total & 15.60 & 6400 & $\$ 1,655.62$ & 38.1 & 4620 & 79.8 & 6703 \\
\hline \multicolumn{8}{|c|}{ Glass FRP Structural Insulated Panel Design } \\
\hline Glass Fibers & - & 1193 & $\$ 949.74$ & 25.2 & 3696 & 27.2 & 3314 \\
\hline Vinyl Ester & 4.00 & 976 & $\$ 1,815.96$ & 72.2 & 10032 & 53.4 & 5770 \\
\hline PUR/PIR Foam & 6.25 & 1056 & $\$ 720.00$ & 12.2 & 1797 & 46.0 & 3675 \\
\hline Lumber & 1.25 & 912 & $\$ \quad 164.22$ & 2.1 & 266 & 3.8 & 502 \\
\hline GFRP SIP Total & 27.10 & 4138 & $\$ 3,649.92$ & 111.8 & 15774 & 130.4 & 13261 \\
\hline \multicolumn{8}{|c|}{ Kenaf FRP Structural Insulated Panel Design } \\
\hline Kenaf Fibers & - & 955 & $\$ \quad 405.72$ & 9.1 & 1492 & 9.4 & 839 \\
\hline Vinyl Ester & 4.00 & 1215 & $\$ 2,259.86$ & 90.0 & 12474 & 66.4 & 7181 \\
\hline PUR/PIR Foam & 6.25 & 1040 & $\$ 709.09$ & 12.0 & 1771 & 45.3 & 3619 \\
\hline Lumber & 1.25 & 902 & $\$ \quad 162.29$ & 2.0 & 262 & 3.7 & 496 \\
\hline KFRP SIP Total & 27.00 & 4111 & $\$ 3,536.95$ & 112.8 & 15994 & 124.9 & 12135 \\
\hline \multicolumn{8}{|c|}{ Stick-Framing Design } \\
\hline Lumber & 1.25 & 2271 & 408.80 & 5.1 & 660 & 9.4 & 1249 \\
\hline Fiberglass Batts & 3.71 & 304 & $\$ \quad 97.35$ & 2.1 & 297 & 3.7 & 411 \\
\hline OSB & 1.25 & 2267 & $\$ \quad 484.24$ & 12.4 & 1434 & 21.9 & 1881 \\
\hline Drywall & 0.90 & 2560 & $\$ \quad 512.00$ & 21.7 & 5984 & 7.4 & 973 \\
\hline Stick-Framing Total & 12.25 & 7402 & $\$ 1,502.39$ & 41.4 & 8382 & 42.4 & 4514 \\
\hline \multicolumn{8}{|c|}{ Insulating Concrete Form Design } \\
\hline Concrete & 0.08 & 76576 & \$ $1,531.52$ & 34.1 & 9218 & 37.2 & 10108 \\
\hline Steel & - & 762 & $\$ \quad 228.50$ & 9.4 & 1839 & 7.6 & 1207 \\
\hline EPS & 4.00 & 480 & $\$ \quad 327.27$ & 6.5 & 902 & 20.5 & 1548 \\
\hline Drywall & 0.90 & 2560 & $\$ \quad 512.00$ & 21.7 & 5984 & 7.4 & 973 \\
\hline ICF Total & 16.77 & 80378 & $\$ 2,599.30$ & 71.8 & 17952 & 72.8 & 13836 \\
\hline
\end{tabular}


Table 9-11 - Environmental impact divided by total cost of construction materials

\begin{tabular}{|c|c|c|c|}
\hline Design & Weight / Cost & Embodied Energy / Cost & $\mathrm{CO}_{2} /$ Cost \\
\hline & lbs $/ \$$ & btu/\$ & lbs $\mathrm{CO}_{2} / \$$ \\
\hline OSB SIP & 3.9 & 35606 & 3.42 \\
\hline GFRP SIP & 1.1 & 33179 & 3.98 \\
\hline KFRP SIP & 1.2 & 33602 & 3.98 \\
\hline Stick Framing & 4.9 & 27889 & 4.29 \\
\hline ICF & 30.9 & 27815 & 6.11 \\
\hline & Average & 31618 & 4.36 \\
\hline & Standard Deviation & 3558 & 1.03 \\
\hline
\end{tabular}

That being said, the environmental impacts of the FRP SIPs were somewhat higher than expected, particularly since the kenaf fiber SIP replaces a synthetic material with a natural one. It is not difficult to discern why these SIPs are so harmful to the environment, however: the vinyl ester resin is responsible for nearly $65 \%$ of the embodied energy and $\mathrm{CO}_{2}$ in the GFRP SIP and nearly $80 \%$ of the embodied energy and $\mathrm{CO}_{2}$ in the KFRP SIP. The higher quantity of resin needed for the KFRP is due to the thicker panel requirements due to the lower axial stiffness of KFRP as compared to GFRP at the same fiber volume fraction. This high environmental impact of the resin system is directly in line with the findings of Fowler et al. (2006), who concluded that the greater environmental detriment in synthetic composites is from the petroleum-based matrix materials and that the development of high quality bio-matrix materials is of higher environmental concern than the development of natural reinforcements. It is not a guarantee, however, that a bioresin would possess better environmental properties, however, since the manufacturing process could be just as detrimental as for synthetic resins. Resin system aside, however, the kenaf fibers possessed only around $40 \%$ of the embodied energy and $\mathrm{CO}_{2}$ contribution of the glass fibers, even at a higher volume (albeit at a lower overall weight). These findings illustrate not only the importance of replacing synthetic resin systems with natural ones as resin technology progresses, but also the environmental gains that can be made by replacing glass fibers with natural fibers.

OSB SIPs seem to offer a roughly equivalent environmental impact as the stick-framed designs, without significantly increasing the cost (of raw materials). In addition to their higher insulation factor, better home-sealing, and quicker assembly, this makes their use in construction of single-family dwellings very attractive. ICFs have by far the highest $\mathrm{CO}_{2}$ contribution (mostly due to cement production) and are extremely heavy, but can offer good insulating properties and very high strengths.

\subsubsection{Additional Considerations}

While looking at the raw materials that go into a particular construction design is an interesting and informative exercise, it fails to consider the whole cradle-to-grave approach of a full LCA. In addition to the production of the construction materials, the construction stage itself will involve additional energy consumption, materials, and equipment. Stick-framing, in particular, is notorious for resulting in a high quantity of wasted material and taking a long time to complete. During the "use stage," the higher insulating properties of the FRP SIPs (assuming 
they were coupled with a well-insulated roof and well-insulated windows and doors) would provide significant cost and energy savings in their favor as compared to the less well-insulated wall designs. Demolition and disposal costs and environmental impacts are difficult to project, although the GFRPs may potentially sit in a landfill for decades without decomposing. While the KFRP fibers would biodegrade over time, the synthetic resin would still potentially present decomposition and toxicity problems.

In order for the real long-term environmental impact of natural fiber reinforced composite structural materials to be established, more published data needs to be generated and a more thorough LCA needs to be conducted. The natural FRPs currently do not appear to be any more environmentally friendly than glass FRPs and would likely have significantly higher environmental impacts than conventional construction materials. However, if they were incorporated in biopolymer matrices (assuming biopolymers could be produced to be more ecofriendly than synthetic polymers) and produced on a larger, more efficient scale, their environmental impacts could potentially be substantially reduced. When coupled with some of their other properties, such as the worldwide presence of natural fibers in poor regions of the world (as discussed in the next section), these environmental impact findings are not particularly discouraging for natural FRPs.

\subsection{Socioeconomic impacts}

In the following case study, the potential socioeconomic impacts and sustainability of natural fiber reinforced composite materials in housing applications are studied in the country of Bangladesh. Bangladesh was selected for this study due to its rampant poverty, overpopulation, and housing issues as well as its serving as the country of origin for the fibers studied throughout this research.

In the case study, the three socioeconomic and sustainability questions asked were:

- What is the level and quality of current fiber production in the country?

- What current national housing issues is the country facing?

- How might natural fiber reinforced composites help to house the needy in this country?

\subsubsection{Bangladesh Case Study}

\subsubsection{Current natural fiber production}

Jute and kenaf are the two most commonly grown fibers in Bangladesh, with jute holding the largest share of the market. Kenaf production is only about $1 / 200$ th the scale of the production of jute (Food and Agriculture Organization of the United Nations 2010). Kenaf fibers have traditionally been cultivated for cordage and more recently for the pulp and paper industries (Taylor 1995). Since kenaf is such a small fraction of the total natural fiber production in Bangladesh, most of the available statistics are regarding either jute or the natural fiber industry as a whole.

The yield rate of jute is typically around $750 \mathrm{~kg}$ of fiber (+950 kg of stalk byproduct) per acre (Bangladesh Bureau of Statistics 2010c). According to statistics from the Bangladeshi 
government, each acre requires around 56 laborers to produce the fiber crop (Bangladesh Bureau of Statistics 2010c). The 'farm-gate' price, that is, the price of the product if purchased directly from a farm, is around $23.6 \mathrm{Tk}$ (taka) per $\mathrm{kg}$, or around $\$ 0.30 / \mathrm{kg}$ (Bangladesh Bureau of Statistics 2010c). The production gains are, on average, around $31 \%$ of the farm-gate price, which calculates to around \$70 profit per acre, per crop (Bangladesh Bureau of Statistics 2010c). Production costs include: land leasing, land preparation labor, seeds, weeding labor, insecticides, fertilizer, harvesting labor, retting labor, peeling labor, and drying labor. Leasing and weeding costs alone make up about half of the expense (Bangladesh Bureau of Statistics 2010c).

Bangladesh is the second largest supplier of raw jute and kenaf fibers in the world, behind only India, but is by far the greatest exporter, since India essentially consumes all of the raw fibers in industries within its own country (Food and Agriculture Organization of the United Nations 2010). In 2009-2010, Bangladesh supplied around 1.09 million metric tons of jute and kenaf, as compared to 1.78 million metric tons from India and a total of 2.88 million tons worldwide (Food and Agriculture Organization of the United Nations 2010). Of those 1.09 million metric tons, 0.315 million were exported in the raw form (Food and Agriculture Organization of the United Nations 2010). There were an estimated 1,028,832 acres of jute produced in Bangladesh in 2009-2010 (Bangladesh Bureau of Statistics 2010a). Agriculture as a whole has a 19\% share of the Bangladesh GDP, and jute is the most important cash (non-food) crop grown in Bangladesh (Bangladesh Bureau of Statistics 2010c). Only 1.25\% of the national gross value added was from the jute textile industry, while chemicals, petroleum, and plastic (also of interest for the production of natural fiber reinforced composites) accounted for 9.18\% (Bangladesh Bureau of Statistics 2010b).

The jute industry had experienced a decrease in production with the growth of the synthetic fiber industry over the last few decades, but has been growing again more recently, with the export of jute tripling between 2000 and 2008 (Bangladesh Bureau of Statistics 2010c). Simultaneously, the jute export prices more than doubled between 2006 and 2010 (Food and Agriculture Organization of the United Nations 2010).

From the above statistics, it is clear that the production of natural fibers is an abundant industry in Bangladesh, and the knowledge and experience to increase production is readily available. However, since Bangladesh exports much of their natural fiber crop in its raw format, they end up selling the product with very little value added. If those fibers could be manufactured into more fully-developed products incorporating other locally available raw materials, the natural fiber industry seems like it would quickly become a much more significant part of the national economy, providing needed income and jobs to the critically overpopulated country.

\subsubsection{Current national housing situation}

Bangladesh is one of the most populous countries in the world, with more than 1,000 people per square kilometer (Habitat for Humanity 2011). By comparison, West Virginia has around 30 people per square kilometer, while New Jersey has around 450 (Wikipedia.org 2013). Bangladesh is also one of the poorest countries in the world with a per-capita GDP of only \$260 (Hoek-Smit 1998).

In Bangladesh, land is the main asset for income generation, and most rural jobs involve agriculture. However, much of the rural population does not own enough land to generate a 
usable income, and thus the rural homeless incidence rate is very high (Tipple and Speak 2009). The poverty level has been on the decline recently, going from $40 \%$ in 2005 to $31.5 \%$ in 2010 , but due to the large number of rural migrants moving to cities (28\% urbanization in 2010), an increasing portion of those in poverty are urban (Habitat for Humanity 2011). For example, in Dhaka, the capital and largest city, a third of the population lives in slums or informal settlements (Habitat for Humanity 2011).

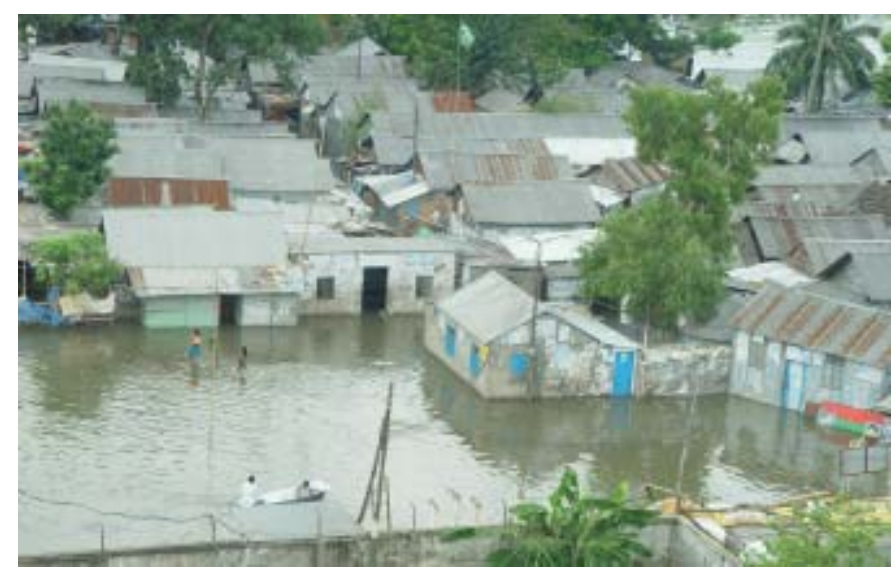

Figure 9-2 - Flooded housing in Bangladesh (Ahmed 2005)

Bangladesh is located on a low-lying delta, and thus is subject to frequent floods and cyclones (as shown in Figure 9-2). During heavy flooding, more than $60 \%$ of the land is inundated (Ahmed 2005). Many homeless people have become so by losing their original homestead to eviction or disasters, such as floods or landslides, and as a result have nowhere to set up a new home. The Bangladesh Bureau of Statistics therefore defines the homeless as, "[the] floating population ... the mobile and vagrant category of rootless people who have no permanent dwelling whatever" (Tipple and Speak 2009). The homeless population estimation for Bangladesh was 2.6 million of 133.4 million (1.96\%) in 2002 (Tipple and Speak 2009). Of these, around $10 \%$ lived on the streets, while the other $90 \%$ lived in inadequate housing (Tipple and Speak 2009). In 1991, the per capita dwelling floor space was $54.9 \mathrm{ft}^{2}\left(5.1 \mathrm{~m}^{2}\right)$ with an occupancy level of $5.48 \mathrm{pp} /$ dwelling unit (Hoek-Smit 1998), as compared to a per capita dwelling floor space of almost $740 \mathrm{ft}^{2}\left(68.7 \mathrm{~m}^{2}\right)$ in the US (Green and Malpezzi 2003). At that time it was estimated that close to half of all of the housing units in the country were made of temporary materials and needed replacement within a 1-5 year period (Hoek-Smit 1998).

High land prices make finding traditional housing difficult for low-income families. The Bangladeshi government acts more as a facilitator of housing than as a provider, leaving construction to the private sector and non-governmental organizations (Habitat for Humanity 2011). The private developers, however, tend to only produce housing that is affordable to the upper and middle-income groups (Habitat for Humanity 2011). The construction costs for a small, 300 sq. ft. house would be in the order of 150,000 Tk, or about $\$ 1,840$ (Hoek-Smit 1998). Habitat for Humanity (2011) works to provide houses that start as a single room with a veranda and a latrine, which can later be upgraded (via a loan) with a second room. They also work to renovate or repair existing homes. 
Ahmed (2005) provides an excellent review of the current housing practices common in Bangladesh, with suggestions on how to improve housing designs. His handbook was written because of the tendency to apply the same housing design models irrespective of the context, which has led to many problems with housing in Bangladesh due to the frequent floods.

According to Ahmed (2005), the three most common types of housing can be classified as "kutcha," "semi-pucca," and "pucca." Kutcha houses have earthen plinth foundations with bamboo or timber posts supporting woven fiber wall mats and thatch roofs (see Figure 9-3). Semi-pucca houses are an improvement on this, with a foundation resting on either an earthen plinth with a brick perimeter wall or a concrete slab. The walls of a semi-pucca house are woven bamboo mats, corrugated iron, or brick, with corrugated iron roofs (see Figure 9-4). Pucca houses are entirely brick and concrete structures, with reinforced concrete roofs (see Figure 9-5). Good quality timber, although in high demand, is expensive and does not grow in the floodplains.

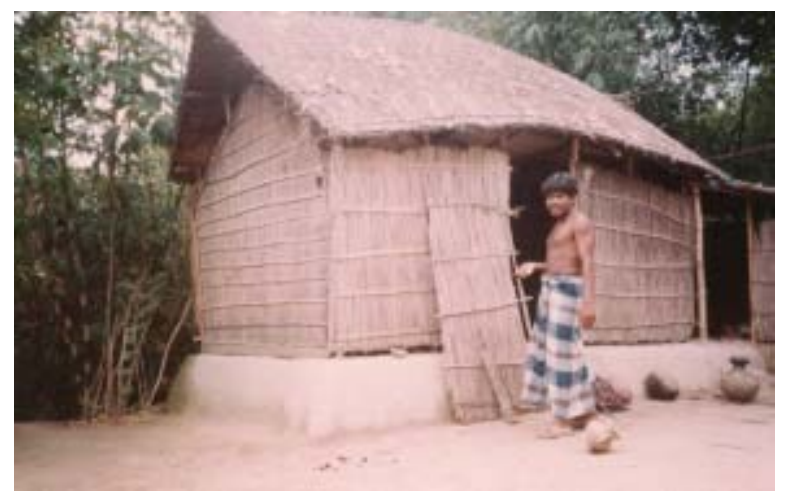

Figure 9-3 - Kutcha-style house in Bangladesh (Ahmed 2005)

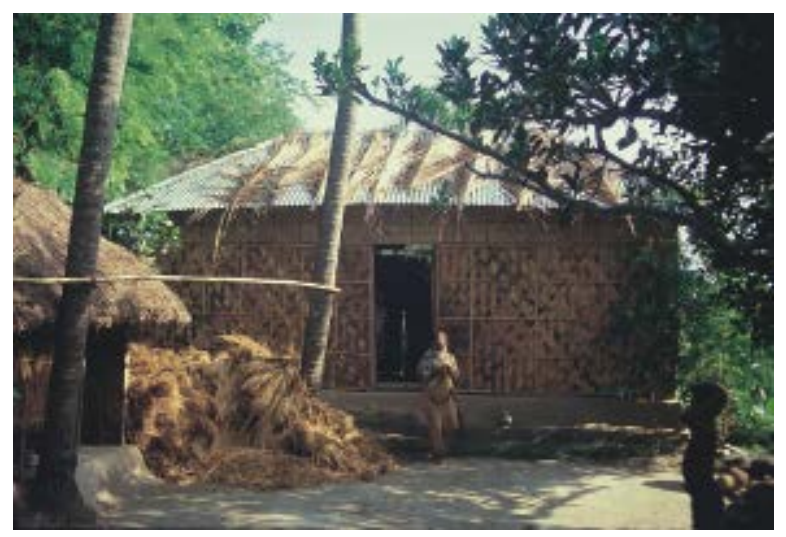

Figure 9-4 - Semi-pucca-style house in Bangladesh (Ahmed 2005) 


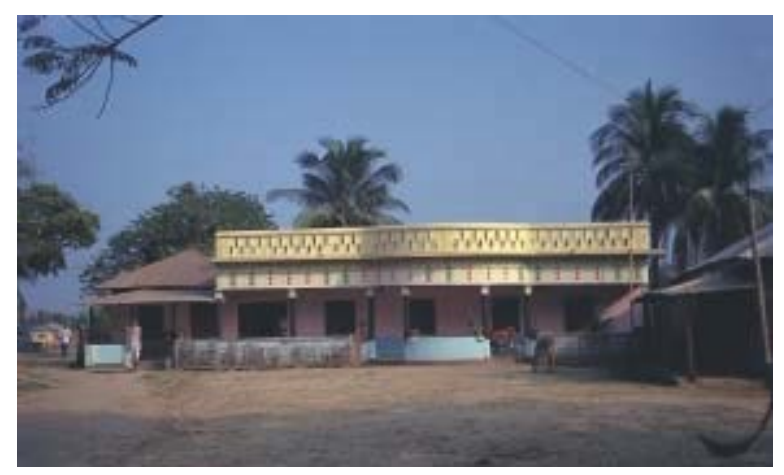

Figure 9-5 - Pucca-style house in Bangladesh (Ahmed 2005)

The woven fiber wall mats only have a lifespan of around 2-5 years, with decay accelerated if exposed to flooding (Ahmed 2005). Strong currents can also completely detach the mats from the posts. The corrugated iron sheets respond in a similar fashion, with corrosion accelerated by flooding. Brick and earthen constructions can also weaken, particularly along mortar joints, or collapse completely. To extend the life of construction materials in flood areas, homeowners often apply chemical treatments to wall mats and thatch roofs and paint the bottoms of posts with bitumen to reduce moisture absorption and prevent decay (Ahmed 2005).

Bangladesh is a country in dire need of new and context-appropriate housing solutions, and the only realistic way for this to be accomplished is for these solutions to be addressed using equipment and raw materials that are available and affordable within their borders.

\subsubsection{Potential impacts on housing the needy}

Before discussing the applicability of natural fiber reinforced composites to address the housing needs in Bangladesh, it needs to be clearly understood just what is at stake for the homeless population, both in Bangladesh and in other developing countries worldwide.

Despres (1991) lists ten characteristics of home, with the implication that without a home, the homeless lack these:

- $\quad$ Permanence and continuity

- $\quad$ Center for family relationships

- $\quad$ Security and control

- $\quad$ Mirror of personal views

- $\quad$ Influence and place for change

- $\quad$ Retreat from the surrounding world

- $\quad$ Personal status indicator

- $\quad$ Center for activity

- $\quad$ Concrete structure

- $\quad$ Place to own

Beyond social and psychological implications, the physical needs connected with housing are also great. According to Tipple and Speak (2009), "to live in the conditions endured on the street or in the most inadequate housing is to confront pathogens and physical dangers daily."

There are two approaches in explaining the causes of homelessness: one views it as a result of the individual's inadequacies or behaviors, while the other views it as a result of 
'structural' or systemic problems, where either the housing market has failed to provide adequate, affordable housing or wider, global economic factors are the cause (Tipple and Speak 2009). In developed countries, the response to homelessness is more often the former, and as a result the focus is on enabling the homeless to have access to existing housing and services so that they can begin to, or regain, function as a normal part of society. The main problem with homelessness in developing countries is that there is just not enough housing to go around and what is available is too expensive for a large portion of the population (Tipple and Speak 2009).

There are many different levels of homelessness, ranging from roofless, to houseless, to insecure accommodation, to inferior or substandard housing (Tipple and Speak 2009). Adequate shelter was defined by the United Nations Committee on Economic, Social and Cultural Rights as, "adequate privacy, adequate space, adequate security, adequate lighting and ventilation, adequate basic infrastructure and adequate location with regard to work and basic facilities - all at a reasonable cost" (Tipple and Speak 2009). Accomplishing these objectives, therefore, should be the overarching goal of any housing work in developing countries, although the definition of adequacy in each case may vary depending on the social and cultural characteristics of each location and people group. This goal may be too lofty in many cases, as Struyk (1987) argues that the best tool available to governments for improving their housing situation is the lowering of minimum acceptable building standards. While this may sound harsh at first, there may be reasonable ways to reduce building expenses within the framework of the local cultures without sacrificing the social, psychological, and physical gains of housing.

This mention of varying social and cultural characteristics brings up a critical issue when addressing housing in developing countries. Tipple and Speak (2009) state it well when they say, "The linking of home to kinship, the role of the extended family, the weaker position of women in society, the different attitudes to ownership and the broad range of political contexts in developing countries all serve to make our current understanding, based on industrialized perceptions, values, culture and society, inappropriate for the developing countries context." Encountering resistance when introducing new housing technology to the homeless in developing countries may seem unfathomable, but in many places there are significantly different social and cultural values that need to be understood before attempting to improve the quality of living.

In addition to making sure the technology itself is appropriate, the way in which it is introduced needs also be considered. When introducing new technology or innovations in housing to a people group, the stages of adoption (knowledge, persuasion, decision, and confirmation) must be followed to ensure that the change is a positive one to the community (LaMore 2004). For example, prefabricated technology would replace the need for volunteer labor, which can be a significant factor in building community between neighbors (LaMore 2004).

With this perspective of homelessness in developing countries in mind, there are several factors related to natural fiber reinforced composites potentially being used to address housing needs in Bangladesh, as well as other developing countries. The first is the availability of materials: in 2009/2010, developing countries (with India included in this category, according to this study) were responsible for producing $99.75 \%$ of the world's total amount of jute, kenaf and “allied fibres" (Food and Agriculture Organization of the United Nations 2010). From the previous sections, it would appear that Bangladesh has a thriving natural fiber industry that 
would benefit greatly from the introduction of value-added products. The biggest hurdle impeding this advance is the resin system used in the composites. Without a bio-based resin system that could be manufactured sustainably from locally available materials, the entire industry would be dependent on importing expensive chemicals that would render the entire production economically unfeasible. Therefore, while the availability of materials is the first positive factor, it is also the first major challenge.

The second major factor is the availability of labor. In order for a natural fiber reinforced composite product to be feasible in a developing country, it would need to be low-tech and involve a high degree of manual labor. According to Tipple and Speak (2009), "Housing construction through labour-based technologies using local materials generates more jobs per unit of expenditure than most other forms of activity." New homes also generate multiple other kinds of jobs beyond just construction work. There are a large number of able workers in Bangladesh; many of the developing countries throughout the world are also the most populous. The manufacture of the kenaf FRP panels conducted in this research involved very low technology (oven, sewing machine, heated press), and with the exception of the resin system could be very easily adapted to the equipment and labor force in Bangladesh or other developing countries.

The final major factor is the need for housing. While natural FRP composites could be used in a variety of applications, the most critical need in Bangladesh would be to use them as a housing material. In this way, locally grown materials could be manufactured by the local labor force into a product that could be used to address the local housing needs. While the research in this dissertation showed that kenaf FRPs possessed sufficient mechanical properties to be used as structural materials in infrastructure applications, it also highlighted that a major issue with them is their tendency to absorb moisture, swell, and warp. In fact, this idea of using natural fibers to address housing needs is by no means a new one. In the 1970s, Winfield (1974; 1979) worked to design jute/polyester composite homes in India and Bangladesh with some success, but he also had trouble with moisture absorption. While his materials had nowhere near the strength and stiffness properties achieved with the kenaf reinforced composite in this research, implementation of the current kenaf FRP system at this stage would likely only achieve the same result. The kenaf FRP composites are far too susceptible to moisture to be used as external building materials, and with the frequent flooding and storms, they are likely also too susceptible to be used as internal building materials in Bangladesh.

Therefore, while kenaf FRPs offer a huge potential to significantly impact the economy and quality of life of the people of Bangladesh, there are two major issues that need to be addressed first: replacing the vinyl ester resin with a bio-resin (or at least a more affordable resin that can be easily obtained locally) and improving the moisture resistance and durability of the fibers. If reasonable solutions can be found to these two challenging, but not insurmountable, issues, then developing countries would have a practical and sustainable way to address their housing needs, while providing a boost to their local economies.

\subsection{Conclusions}

The environmental impacts of five different wall designs were compared by looking at the embodied energy and carbon dioxide contribution of the raw materials. This partial LCA was conducted using a functional unit of the primary materials needed to build an external wall for a 
$30 \times 50 \mathrm{ft}$ single story building in West Virginia, USA, designed according to the 2012 International Residential Code. The five different variations of this functional unit were OSB SIPs, GFRP SIPs, KFRP SIPs, stick-framing, and insulating concrete forms. Two simplified methods of obtaining the embodied energy were explored: a cost-based EIO-LCA and a weightbased analysis of published typical values. As the methods conducted in this chapter are only very simplified forms of LCAs, these results should be considered approximations and not definitive conclusions.

The GFRP and KFRP SIPs were designed to meet or exceed the mechanical and insulating properties of the OSB SIP, without exceeding the overall thickness. In both cases, the facesheet material only needed to be around $20 \%$ of the thickness of the OSB. The limiting material property was the bending stiffness of the panel, as both the GFRP and KFRP SIPs far exceeded the OSB SIP in axial capacity (1.8x), bending capacity (2.0x), and insulating capacity (1.7x). Additionally, both FRP SIPs ended up lighter than the OSB SIPs $(<60 \%)$, although even at this reduced weight would cost nearly twice as much in raw materials. The stick framing design ended up being heavier but cheaper than the SIP designs, albeit with reduced insulating properties. The ICF cost about midway between the stick-framing/OSB SIP and the FRP SIPs, but at 10-20 times heavier total weight.

Overall, the two different methods of examining environmental impacts produced fairly similar results for each of the wall designs, although the impacts of the individual constituent materials were off by as 3-4 times, particularly for the foam cores. This may have been due to a discrepancy in the data referring to the EPS or PUR/PIR in either a foamed or an un-foamed state.

Despite these small differences, both models concur that either the stick-framing or OSB SIP designs have the least environmental impact, while the FRP SIP designs have the worst, by as much as 3-3.5 times. The ICF design had a relatively low embodied energy, but a high $\mathrm{CO}_{2}$ contribution due to the cement content. The poor embodied energy and high $\mathrm{CO}_{2}$ contribution of the FRP SIPs was due almost entirely to the vinyl ester (epoxy) resin system. Comparing fibers alone, however, the kenaf fibers possessed only $40 \%$ of the embodied energy and $\mathrm{CO}_{2}$ contribution of the glass fibers, despite the functional unit containing a higher volume of kenaf fibers.

By dividing the embodied energy of each design by the cost of the materials, a very consistent correlation was found for all five of the designs, with about 30,000 btus of energy per each dollar of material. In terms of $\mathrm{CO}_{2}$, the ICF produced about $6 \mathrm{lbs}$ of $\mathrm{CO}_{2}$ for every dollar of material, while the other four designs only produced around $4 \mathrm{lbs} / \$$. Based on these results, it seems reasonable to conclude that the poor environmental impacts of the KFRP SIPs will improve along with reductions in the cost of the constituent materials, which are parallel objectives for future research.

The literature-based case study of the current natural fiber production and housing needs in Bangladesh examined the concept of sustainability from a context of socioeconomics. Bangladesh appears to be one of the world leaders in bast fiber production, but does not possess enough industries to turn all of their fiber crops into value-added products. The lack of quality housing is a serious issue throughout the country due to overpopulation and frequent flooding, and the current housing materials need frequent replacement. With these facts in mind, it would appear that Bangladesh would be a prime location for the implementation of natural FRP housing 
materials if, and only if: 1.) a bio-based resin could be made from locally-available raw materials, and 2.) the moisture resistance and durability of the natural fibers can be improved. 


\section{CONCLUSIONS}

\subsection{Introduction}

This dissertation examined kenaf natural fiber reinforced composites and their potential to be adapted as structural materials from several different perspectives, including mechanical properties, weathering and environmental durability, scaling from coupon size to component size, theoretical modeling, and sustainability impacts. The main conclusions from each of the primary chapters are briefly restated within this chapter in order to provide a comprehensive summary of the research completed. These conclusions represent the original contribution of this work to the field of structural engineering.

The main objective of this work was to show that natural fiber reinforced composites can no longer be so easily dismissed as potential primary structural components. Due to their desirable cost, weight, and environmental characteristics, as well as their widespread availability, these materials could viably compete in the near future with glass fiber reinforced composites and other construction materials in infrastructure markets. This potential would most quickly become a reality if the resin system was replaced with a cheaper and more eco-friendly polymer and if the moisture absorption capacity of the fibers was significantly reduced.

\subsection{Main Chapter Conclusions}

\subsubsection{Literature Review Conclusions}

Of the wide variety of natural fibers available, those from the bast fiber family, such as flax, hemp, jute, kenaf, and ramie, seem to possess the most promising properties for structural applications. The single fiber tension tests conducted in this research showed that the kenaf fibers possessed fairly typical strength properties ( $\sim 0 \mathrm{ksi}$, after area adjustment, as compared to a range in literature of 35-135 ksi) but relatively high stiffness properties ( $\sim 9$ Msi, as compared to a range of 2.1-7.7 Msi) for the species. The composite mechanical properties were higher than all of the NFRP values published in literature with the exception of the results of Ochi (2008) and Van de Weyenberg et al. (2006). However, both of these were conducted on very thin laminates (10-50\% the thickness of the NFRPs in this research work), which can produce properties that do not scale up accordingly with thicker samples.

The main challenges facing natural FRPs are associated with moisture absorption, fire resistance, mechanical properties and durability, variability, and manufacturing / processing. Fire resistance can most likely be addressed with proven resin additives or coatings, variability can be accounted for by quality control and safety factors, and manufacturing methods can be fairly easily adjusted to the constraints of working with natural fibers. The remaining concerns with natural FRPs seem to hinge on the interface between the fibers and the matrix: good bonding at the interface will provide better mechanical performance and better encapsulation of the fibers against moisture and environmental effects. A large proportion of natural fiber research is currently targeting this problem, with many fiber surface treatments or compatibilizers showing improvements. However, it would appear that some of the methods are situation-dependent, and may not provide equal improvements for all types of fibers and resin systems. 


\subsubsection{Preliminary Testing Conclusions}

The most important conclusion to be drawn from the preliminary test results is that the fiber architecture significantly affects the strength and stiffness of natural fiber reinforced composites. The process of spinning and weaving fibers into fabrics increases the maximum strain, but sacrifices much of the inherent linear stiffness of the fibers. The unprocessed fibers obtained from Bangladesh possessed much higher stiffness properties than any of the natural fabric yarns.

The second significant result of the first round of testing was the great improvement the vinyl ester resin offered over the phenolic adhesive system as a binding agent for the natural fibers.

Implications for future research: By using unprocessed, "loose” fibers instead of woven fabrics, the structural properties of natural fibers are more efficiently utilized in composites. Future work should focus on adopting this fiber configuration to different products and processes, particularly for mass-manufacturing methods. While this fiber configuration may present problems with processes like pultrusion, which relies on continuous lengths of fibers, it may be possible to stitch the loose fibers between layers of a relatively stiff fabric which might enable manufacturing while retaining the individual fiber strength and stiffness properties in the final product.

\subsubsection{Fiber Surface Treatment Characterization Conclusions}

Of the resin systems explored, both of the vinyl esters seemed to provide much better quality composites than the polyurethane. This was likely due more to the moisture in the fibers reacting with the polyurethane than with a performance failure of the resin system, as the polyurethane plate had obvious foaming problems.

Getting an accurate picture of the how successful the different fiber surface treatments were at improving the fiber/matrix interface was difficult given the variety of alkalization methodologies tested and the inherent variability of working with organic materials. While the 16 hour treatment was not explored until later in the research process and thus was not included in some of the other chapters' testing matrices, there is some evidence that this method best accomplishes the objective of removing the water absorbent materials and improving the fiber/matrix interface. To more easily compare the untreated, 5\% $\mathrm{NaOH}$ for 40 minute, and 5\% $\mathrm{NaOH}$ for 16 hour results, all of the different values collected for these samples throughout the chapters are shown in Table 10-1.

From Table 10-1, several new, general conclusions can be drawn. First, it would appear that alkalization improves the individual fiber strength and stiffness, although this is probably at least partially due to the removal of non-cellulose, non-structural material from the fiber. The treated fiber would therefore have a smaller cross-section with essentially the same load / deformation response. This hypothesis is also supported by the descending average minimum fiber diameter: $\sim 50 \mu \mathrm{m}$ for untreated fibers, $\sim 41 \mu \mathrm{m}$ for the $5 \% \mathrm{NaOH}, 40$ minute treated fibers, and $\sim 35 \mu \mathrm{m}$ for the $5 \% \mathrm{NaOH}, 16$ hour treated fibers.

This higher cellulose content would also explain the improvement in flexural properties seen in the treated fibers, with the longer $\mathrm{NaOH}$ treatment showing a greater improvement than 
the shorter $\mathrm{NaOH}$ treatment. However, these improvements in fiber properties essentially absent from the tensile, compressive, and shear tests. At this point, the only logical explanation for these inconsistent results is data scatter. Further testing in each of these test configurations should explore whether this trend continues.

Table 10-1 - Comparison between fiber treatment methods (improvement over untreated in green, retrogression in red)

\begin{tabular}{|l|r|r|r|r|r|}
\hline & \multirow{2}{*}{ Untreated } & \multicolumn{2}{|c|}{$\mathbf{5 \% , 4 0}$ minutes } & \multicolumn{2}{|c|}{$\mathbf{5 \% , 1 6 ~ h o u r s ~}$} \\
\cline { 3 - 6 } & & Value & \% Untreated & Value & \% Untreated \\
\hline Single Fiber Strength, ksi & 61.03 & 82.17 & $135 \%$ & 104.56 & $171 \%$ \\
Single Fiber Stiffness, Msi & 6.77 & 10.02 & $148 \%$ & 10.68 & $158 \%$ \\
\hline Flexural Strength, ksi & 25.97 & 28.96 & $112 \%$ & 29.62 & $114 \%$ \\
Flexural Stiffness, Msi & 1.05 & 1.10 & $104 \%$ & 1.58 & $150 \%$ \\
Saturated \% Moisture & $13.2 \%$ & $12.4 \%$ & $94 \%$ & $9.6 \%$ & $73 \%$ \\
\hline 50 hr Reduced Strength, \% & $105 \%$ & $71 \%$ & $68 \%$ & & \\
50 hr Reduced Stiffness, \% & $90 \%$ & $81 \%$ & $90 \%$ & & \\
Saturated Reduced Strength, \% & $72 \%$ & $48 \%$ & $67 \%$ & $82 \%$ & $114 \%$ \\
Saturated Reduced Stiffness, \% & $86 \%$ & $75 \%$ & $87 \%$ & $52 \%$ & $60 \%$ \\
\hline Tensile Strength, ksi & 25.57 & 23.26 & $91 \%$ & 22.76 & $89 \%$ \\
Tensile Stiffness, Msi & 3.29 & 3.31 & $101 \%$ & 2.74 & $83 \%$ \\
Shear Strength, ksi & 7.37 & 5.07 & $69 \%$ & & \\
Shear Stiffness, Msi & 0.39 & 0.32 & $82 \%$ & & \\
Compressive Strength, ksi & 16.74 & 17.01 & $102 \%$ & & \\
Compressive Stiffness, ksi & 3.32 & 3.41 & $103 \%$ & & \\
\hline
\end{tabular}

The moisture absorption results seem to indicate a slight improvement in moisture resistance with treatment, although even this improved saturation point is still nowhere near the low saturation point desired for exposed structures. The poorer mechanical properties after absorption, despite the lower saturation point, are actually a good sign that the alkalization is accomplishing its purpose. Better bonding between the fibers and matrix would lead to a higher rate of microcracking damage due to swelling; therefore, the better post-immersion mechanical properties of the untreated fibers most likely correlate with poorer bonding, as expected with untreated fibers.

Implications for future research: Resin systems to be used with natural fibers need to have no negative reactions when put in contact with moisture during the curing process. Treatment with alkalis for longer durations seems to improve some mechanical and moisture resistance properties, but at the expense of environmental resistance properties. More tests exploring alkali treatment of kenaf fibers for $16+$ hours would help better define the effects of alkali surface treatments.

\subsubsection{Mechanical Characterization Conclusions}

The mechanical results from the dry environment showed little difference between the untreated and treated (5\% $\mathrm{NaOH}$ for 40 minutes) kenaf FRP composites. As discussed in the previous section, the treated fiber composites had poorer post-immersion mechanical properties, most likely due to the better fiber-matrix interface experiencing increased matrix cracking from 
swelling. In general and for every case except for the shear stiffness (reduced to $\sim 33 \%$ ), the strength of the saturated kenaf FRPs was reduced to $\sim 60 \%$ and the stiffness to $\sim 75 \%$ of the dry properties. While this reduction is significant, it is not so bad as to restrict NFRPs from being used in interior applications, similar to the way many engineered wood products are used in construction. Moisture was also found to increase ductility of the natural fiber composites, but at the expense of reduced stiffness.

At 40\% FVF, the glass FRPs exceeded kenaf FRPs' mechanical properties. However, once these properties are divided by the respective composite densities to get the specific mechanical properties, the kenaf FRPs were found to be extremely competitive ( $\pm 15 \%$ or better), with the exception of tensile stiffness ( $25 \%$ lower). This lower tensile strength is to be expected given the great difference in tensile strength between glass and natural fibers, but the fact that the other properties are very comparable shows that kenaf FRPs can be designed to essentially match glass FRP performance, pound for pound. While self-weight is not necessarily a significant concern in many structural applications, FRPs are often used in applications where light weight is a concern and the comparable specific properties of natural FRPs is critical for these specialized uses.

Implications for future research: While the 40 minute $\mathrm{NaOH}$ treatment did not seem to have a particularly positive impact on the mechanical properties or moisture resistance, another round of testing exploring the composites made from fibers treated for 16+ hours would help to better define the range of possible impacts from alkali treatments. Even as things currently stand, however, the kenaf fiber composites are ready to compete mechanically with glass fiber composites in dry environments, and potential applications need to be explored.

\subsubsection{Weathering and Environmental Testing Conclusions}

The outdoor, real-time weathering tests produced a number of interesting findings. From comparing weather patterns and measurements, it appears that the greatest impact on weight and dimensional changes in the kenaf reinforced composites is precipitation. The treated kenaf had an unexpected loss in density which could be attributed to fungal growth and decomposition. Since the fiber surface lignin and waxes help provide a natural resistance, most likely their removal through the treatment process was responsible for this change.

While a urethane coating helped provide some environmental resistance, as shown by an improved retention of material properties, its effectiveness decreased over time. Visual indications of damage became common as the exposure time increased, and included splitting and cracking along fiber layers as well as some black patches on surfaces with exposed fiber ends. The difference in mechanical property retention between the treated and untreated kenaf composites was relatively small, with essentially the same strength reduction (50\%) while the untreated kenaf retained a slightly higher stiffness (70\% vs. 60\%).

Elevated temperature exposure up to $150^{\circ} \mathrm{F}$ for as long as 288 consecutive hours did not appear to cause any permanent damage, and in fact may have helped to post-cure the resin as higher flexural stiffnesses were obtained.

As a general trend, alkali immersion tended to cause a slightly greater degradation in the kenaf FRP strength and stiffness than water immersion, while acid immersion tended to cause a slightly lesser degradation. Since alkalis are used to chemically dissolve some of the natural fiber material as surface treatments, it is not unexpected that the natural FRPs would sustain higher 
damage in alkali environments. The urethane coating provided a short term barrier (best for $\sim 50$ hours of immersion or 5-6 months of outdoor exposure), but had little effect on the longerduration tests. For compression, flexure, and tension, the typical reduction factor for kenaf composite strength at saturation is around 0.5-0.8, and for stiffness is around 0.4-0.8.

UV exposure testing according to ASTM D4329, Cycle A for 1000+ hours was found to closely mimic a year's worth of outdoor, real-time weathering flexural property degradation, and is a suitable method for continued exploration of durability improvements. A reasonable approximation for the uncoated kenaf FRP outdoor aging factor is around 0.5 for strength and 0.7 for stiffness after one year. While the strength and stiffness may have still been decreasing somewhat after 12 months, their rates of decrease had slowed appreciably, implying that continued exposure might not result in significant further losses.

Implications for future research: Despite the urethane coating and alkali chemical treatment, kenaf fiber composites still degrade in outdoor environments much more quickly than glass fiber composites. There is a lot of improvement possible through the selection of better coatings, treatments, and resin systems, and these options to improve the weathering behavior need to be further explored.

\subsubsection{Structural Component Testing Conclusions}

An overlap of around 1.5-2.5 inches, with the fiber ends meshed together, allows for components to be manufactured at any length without significant loss of mechanical properties, despite the inherent length limitations of the organic fibers. A low-pressure manufacturing method, such as the one used by Fiber-Tech Industries, was found to reduce flexural strength by up to $18 \%$ as compared to high-pressure molding, but had little impact on the stiffness.

While the core material used in the KFRP SIPs was not of sufficient quality to prevent core failure modes, the facesheet panels themselves offer a high potential in SIP applications. The KFRP SIP component tested had facesheets only around $1 / 2$ as thick as the OSB facesheets but, if they were to fail in facesheet rupture, would possess four times the strength and 2.5 times the stiffness in flexure. Under axial loading, the core shearing and debonding again controlled failure, despite the fact that the KFRP facesheets ought to be able to resist twice the load of the OSB facesheets.

If a more compatible core material were bonded to the kenaf composite facesheets, the KFRP SIPs would be versatile construction materials, thanks to their high strength and stiffness properties, light weight, and potentially high insulating properties.

Implications for future research: A core material with better mechanical properties and better adhesion to the KFRP face sheets would be able to provide a significant improvement to the SIP strength and stiffness. Researching KFRP adherence to other potential core materials, as well as exploring manufacturing processes where higher compression may be able to achieve better fiber volume fractions and a lower void content, would make the KFRP SIP systems much more viable products.

\subsubsection{Modeling NFRP Composites Conclusions}

The stiffness properties of the kenaf reinforced composites in tension, compression, flexure, and shear can be predicted with the same reasonable accuracy as glass reinforced composites using the modified equivalent laminate modulus method and the finite element 
method, with the exception that the predicted flexural stiffness was around $40 \%$ higher than the experimental. Castigliano's theory was found to confirm this high predicted flexural stiffness, suggesting that, with better bonding between the fibers and resin, the flexural stiffness of the material could theoretically be increased by as much as $40 \%$. Since the tensile and compressive tests are more dependent on the fiber properties themselves than the fiber/matrix interaction, these stiffnesses were still able to be predicted accurately despite the limited bonding.

As with the stiffness prediction, strength prediction by the maximum stress and strain criteria was fairly accurate for tensile and compressive loading. Strength prediction for the bending loading was quite low, although this was likely more due to a mixed definition of flexural strength (versus "apparent flexural strength") than to the inability of the kenaf composites to be accurately modeled. Despite the low strength prediction, the model did indicate similar strength ratios between the tensile and compressive faces of the flexural samples, which explain the frequent variation in failure mode (tensile, compressive, or combined).

One area in which kenaf reinforced composites exhibit additional modeling challenges beyond synthetic fiber reinforced composites is in predicting properties after moisture absorption. The analysis of synthetic composites typically assumes that absorption is due to the matrix and that the magnitudes of absorption and swelling are small, neither of which assumption apply to kenaf composites. But for their moisture absorption, it does not appear that natural fiber reinforced composites present any greater challenge in the prediction of their mechanical properties than do glass fiber reinforced composites, and in some cases their laminate behavior was predicted even more accurately.

Implications for future research: The modeling results again highlighted the potential increase in stiffness possible by better bonding, and the use of chemical treatments and sizings to address this issue should continue to be explored. An improvement in flexural stiffness of $40 \%$ would make kenaf fiber reinforced composites a desirable alternative to glass fiber reinforced composites, especially since stiffness is usually the main concern when building with FRPs.

\subsubsection{Sustainability of NFRP Composites Conclusions}

Based on the guidelines of the 2012 IRC, and assuming the facesheet rupture failure mode will control, GFRP and KFRP SIPs were designed to meet or exceed the required mechanical properties of OSB SIPs. In this design, the bending stiffness ended up being the controlling factor, while the GFRP and KFRP SIPs were able to exceed the axial, bending, and insulating capacities of OSB SIPs by over 170\%, at a lower overall weight.

However, from an analysis of raw material costs, embodied energy, and contributed $\mathrm{CO}_{2}$ through a simplified LCA, it was found that the GFRP and KFRP SIPs were by far more expensive and environmentally hazardous. The source of the high cost and embodied energy was clearly the vinyl ester resin system. While the kenaf fibers had lower cost and better environmental properties than the glass fibers, these advantages were lost in the KFRP SIP due to the need for a thicker composite leading to a larger overall quantity of resin. By comparison, a stick framing design was fairly comparable to the OSB SIP design in terms of cost and environmental impact, while the insulating concrete form design was about midway between these cheaper designs and the expensive FRP SIP designs, albeit with the highest $\mathrm{CO}_{2}$ contribution due to the cement content. 
One unexpected finding from the cost and environmental impact analyses was a strong correlation between the cost of the raw materials and their embodied energy. For all five designs, the embodied energy per dollar ended up being around 30,000 btus/\$. Based on this correlation and the underlying principle that expense is tied to efficiency of production, it seems reasonable to predict that, if production methods are improved and if the vinyl ester resin is replaced with a more economical resin system, the overall environmental impact and cost of natural fiber composite SIPs will decrease similarly.

A case study of the viability of kenaf fiber reinforced composite housing materials in Bangladesh found that the country possesses a massive natural fiber market and a critical need for new housing. However, the adoption of kenaf fiber reinforced composites to meet this need is entirely dependent on developing a bio-based resin that can be made from locally-available raw materials and improving the moisture resistance and durability of the kenaf fibers.

Implications for future research: For kenaf FRP SIPs to become significant and useful building materials on a global scale, improving the moisture resistance through chemical treatments and coatings (and as a result, improving the bonding and mechanical properties) as well as finding a low-cost, compatible, more eco-friendly resin system are the two most critical issues to be addressed in future research.

\subsection{Specific Contributions}

With the overarching goal of developing natural fiber reinforced composites into viable and competitive housing materials in mind, the following specific findings from this research are original contributions to this field:

- Natural fiber reinforced composites can possess specific mechanical properties competitive with glass fiber reinforced composites simply by using a reinforcement configuration of aligned fibers as opposed to fabrics in a good quality resin. Any improvements to mechanical properties that result from improving the bonding between the fibers and resin system will further increase their appeal.

- Sodium hydroxide chemical treatments can improve mechanical properties and reduce moisture absorption both for individual kenaf fibers and composites reinforced with aligned fibers. The extent of this modification is related to the duration of treatment, with a treatment of $5 \% \mathrm{NaOH}$ for 16 hours showing the greatest promise so far. There is some indication that these improvements may come at the expense of reduced mechanical property retention of NFRPs in wet environments, however.

- NFRPs degrade much more quickly than glass FRPs in outdoor environmental conditions. However, in both immersed and outdoor environments, the strength and stiffness properties appear to reduce exponentially more slowly, which would potentially be beneficial for long-term exposures. The use of coatings can improve weathering and moisture resistances. A urethane coating may not be the ideal choice, however, due to urethane's own moisture-related problems. 
- By overlapping and meshing fiber ends together, composites can be manufactured with minimal reductions in strength and stiffness despite discontinuous reinforcement. Implementation of this method is necessary if the limited-length fibers are to be used as reinforcement in larger structural components. If chemical treatments can further increase fiber/resin bonding, the required length for overlapping will be decreased.

- Natural FRPs possess strength and stiffness properties exceeding those necessary to be used as facesheets in SIP components. The greater challenges lie in manufacturing them at a low enough cost to be competitive with other SIP options and in matching them with a core material with sufficient strength and adherence to avoid premature core failures.

- Natural FRPs made from aligned loose fibers match up well with the theoretical stiffness prediction methods of classical lamination theory and finite element method in unidirectional tension and compression, but are far less accurate for the more bond-dependent flexural and shear properties. Natural FRP strength prediction using maximum stress and strain criteria is again relatively accurate in unidirectional tension and compression and significantly less so in flexure and shear. However, strength prediction for synthetic FRPs also has a tendency to be inaccurate.

- While natural fibers provide an improvement over glass fibers in terms of both cost and environmental impacts, the costs and environmental impacts of the composite materials are much more highly influenced by the resin systems than the reinforcements. For natural fibers to truly be sustainable and competitive building materials, they must eventually be paired with low-cost, eco-friendly resin systems. In the meantime, their substitution for glass fibers can still provide some cost and environmental gains.

\subsection{Recommendations}

While identifying low-cost, eco-friendly resin systems would provide the greatest improvement in terms of the cost and environmental impacts of natural composites as structural materials, such polymer developments have been and will continue to be researched for decades. In order to provide the most immediate (1-3 years) impact in the field of natural fiber reinforced composites, further research should focus on the following areas:

- Chemical treatments of fibers: Since the 16-hour treated fibers showed some greater promise in the late stages of this research, a new matrix of different sodium hydroxide treatments centered on that duration should be explored. While the long-term weathering and moisture absorption properties are extremely important to assess, other useful impacts of these chemical treatments on both the individual fibers and fiber/matrix bonding can be assessed much more quickly by single fiber tensile tests, flexural tests, short-duration immersion tests, and ASTM D4329 UV exposure tests. Additionally, chemical treatments other than sodium hydroxide should be explored, particularly treatments involving less hazardous chemical waste. 
- Resin systems: While cost-effectively incorporating bio-resins may not be a realistic immediate objective, there are resin systems other than vinyl esters which could offer similar mechanical and environmental resistance properties at much improved cost and environmental impact. Polyester, in particular, could offer marginally reduced properties at less than $1 / 3$ the cost, $1 / 2$ the embodied energy, and $1 / 2$ the contributed $\mathrm{CO}_{2}$, if it could be made to bond well with natural fibers.

- Coatings: Only one coating (spar urethane) was evaluated throughout this research and it proved to only have marginal success at resisting weathering and moisture absorption. Exploration of other coatings, including just covering the cut edges of the samples with the same polymer as the resin system, should result in better moisture resistance of the composite. If a better coating is combined with a more effective chemical treatment process, the long-term durability and moisture resistance of the kenaf fiber reinforced composites could be significantly improved.

- Core materials: Even with their current limitations, the kenaf FRPs could potentially provide excellent facesheets in lightweight sandwich panel configurations intended for indoor applications. If a better-matched core material could be adhered between the FRP panels, this immediate structural application could generate the interest and funding necessary for further research and development.

- Long-term performance evaluation: While studies in this area may require more than 3 years to conduct, the long term performance of the materials (weathering, aging, fatigue, etc.) would be a critical concern needing thorough experimentation before the materials could be safely used in primary structural applications. 


\section{APPENDIX A - MECHANICAL TESTING RESULTS}

Table A-1 - Dry, non-normalized mechanical testing results

\begin{tabular}{|c|c|c|c|c|}
\hline DRY & Kenaf, untreated & Kenaf, treated & Glass & OSB \\
\hline $\begin{array}{l}\text { Flexural Strength, ksi } \\
\text { (ASTM D790) }\end{array}$ & 22.953 & 28.486 & 49.782 & 3.533 \\
\hline $\begin{array}{l}\text { Flexural Stiffness, Msi } \\
\text { (ASTM D790) }\end{array}$ & 0.929 & 1.078 & 1.968 & 0.270 \\
\hline $\begin{array}{l}\text { Tensile Strength, ksi } \\
\text { (ASTM D3039) }\end{array}$ & 22.790 & 22.520 & 63.476 & 0.923 \\
\hline $\begin{array}{l}\text { Tensile Stiffness, Msi } \\
\text { (ASTM D3039) }\end{array}$ & 2.930 & 3.206 & 5.858 & 0.213 \\
\hline $\begin{array}{l}\text { Tensile Strain, } \mu \varepsilon \\
\text { (ASTM D3039) }\end{array}$ & 8101 & 6360 & 9987 & 4078 \\
\hline $\begin{array}{l}\text { Shear Strength, ksi } \\
\text { (ASTM D5379) }\end{array}$ & 6.382 & 4.782 & 7.033 & 1.047 \\
\hline $\begin{array}{l}\text { Shear Stiffness, Msi } \\
\text { (ASTM D5379) }\end{array}$ & 0.335 & 0.299 & 0.567 & 0.128 \\
\hline $\begin{array}{l}\text { Shear Strain, } \mu \gamma \text { (ASTM } \\
\text { D5379) }\end{array}$ & 35902 & 25003 & 48531 & 7254 \\
\hline $\begin{array}{l}\text { Compression Strength, } \\
\text { ksi (ASTM D695) }\end{array}$ & 14.923 & 16.473 & 27.169 & 1.542 \\
\hline $\begin{array}{l}\text { Compression Stiffness, } \\
\text { Msi (ASTM D695) }\end{array}$ & 2.958 & 3.302 & 6.148 & 1.310 \\
\hline $\begin{array}{l}\text { Compression Strain, } \mu \varepsilon \\
\text { (ASTM D695) }\end{array}$ & 15357 & 10985 & 4251 & 1402 \\
\hline $\begin{array}{l}\text { Compression Strength, } \\
\text { ksi (ASTM D3410) }\end{array}$ & 14.610 & 15.759 & 30.752 & 1.792 \\
\hline $\begin{array}{l}\text { Compression Stiffness, } \\
\text { Msi (ASTM D3410) }\end{array}$ & 2.040 & 1.629 & 6.946 & 0.514 \\
\hline $\begin{array}{l}\text { Compression Strain, } \mu \varepsilon \\
\text { (ASTM D3410) }\end{array}$ & 12013 & 21159 & 3679 & 6208 \\
\hline
\end{tabular}


Table A-2 - 50 hours immersion, non-normalized mechanical testing results

\begin{tabular}{|c|c|c|c|c|}
\hline 50 HOURS & Kenaf, untreated & Kenaf, treated & Glass & OSB \\
\hline $\begin{array}{l}\text { Flexural Strength, ksi } \\
\text { (ASTM D790) }\end{array}$ & 20.683 & 18.936 & 53.654 & 1.728 \\
\hline $\begin{array}{l}\text { Flexural Stiffness, Msi } \\
\text { (ASTM D790) }\end{array}$ & 0.717 & 0.813 & 2.091 & 0.054 \\
\hline $\begin{array}{l}\text { Tensile Strength, ksi } \\
\text { (ASTM D3039) }\end{array}$ & 27.257 & 25.124 & 85.563 & 0.543 \\
\hline $\begin{array}{l}\text { Tensile Stiffness, Msi } \\
\text { (ASTM D3039) }\end{array}$ & 2.831 & 2.658 & 5.958 & $\mathrm{n} / \mathrm{a}$ \\
\hline $\begin{array}{l}\text { Tensile Strain, } \mu \varepsilon \\
\text { (ASTM D3039) }\end{array}$ & 10093 & 8603 & 16615 & $\mathrm{n} / \mathrm{a}$ \\
\hline $\begin{array}{l}\text { Shear Strength, ksi } \\
\text { (ASTM D5379) }\end{array}$ & 4.583 & 4.429 & 5.377 & 0.419 \\
\hline $\begin{array}{l}\text { Shear Stiffness, Msi } \\
\text { (ASTM D5379) }\end{array}$ & 0.211 & 0.198 & 0.550 & $\mathrm{n} / \mathrm{a}$ \\
\hline $\begin{array}{l}\text { Shear Strain, } \mu \gamma \text { (ASTM } \\
\text { D5379) }\end{array}$ & 40717 & 42791 & 18957 & $\mathrm{n} / \mathrm{a}$ \\
\hline $\begin{array}{l}\text { Compression Strength, } \\
\text { ksi (ASTM D695) }\end{array}$ & 12.097 & 9.937 & 36.542 & 0.512 \\
\hline $\begin{array}{l}\text { Compression Stiffness, } \\
\text { Msi (ASTM D695) }\end{array}$ & 3.202 & 2.045 & 5.724 & $\mathrm{n} / \mathrm{a}$ \\
\hline $\begin{array}{l}\text { Compression Strain, } \mu \varepsilon \\
\text { (ASTM D695) }\end{array}$ & 18857 & 30192 & 5945 & $\mathrm{n} / \mathrm{a}$ \\
\hline $\begin{array}{l}\text { Compression Strength, } \\
\text { ksi (ASTM D3410) }\end{array}$ & 11.706 & 10.779 & 28.247 & 0.796 \\
\hline $\begin{array}{l}\text { Compression Stiffness, } \\
\text { Msi (ASTM D3410) }\end{array}$ & 0.793 & 0.776 & 5.963 & $\mathrm{n} / \mathrm{a}$ \\
\hline $\begin{array}{l}\text { Compression Strain, } \mu \varepsilon \\
\text { (ASTM D3410) }\end{array}$ & 12569 & 26982 & 4726 & $\mathrm{n} / \mathrm{a}$ \\
\hline
\end{tabular}


Table A-3 - Saturated, non-normalized mechanical testing results

\begin{tabular}{|c|c|c|c|c|}
\hline SATURATED & Kenaf, untreated & Kenaf, treated & Glass & OSB \\
\hline $\begin{array}{l}\text { Flexural Strength, ksi } \\
\text { (ASTM D790) }\end{array}$ & 16.296 & 14.344 & 32.632 & 1.852 \\
\hline $\begin{array}{l}\text { Flexural Stiffness, Msi } \\
\text { (ASTM D790) }\end{array}$ & 0.789 & 0.839 & 2.560 & 0.189 \\
\hline $\begin{array}{l}\text { Tensile Strength, ksi } \\
\text { (ASTM D3039) }\end{array}$ & 16.920 & 19.575 & 83.162 & 0.413 \\
\hline $\begin{array}{l}\text { Tensile Stiffness, Msi } \\
\text { (ASTM D3039) }\end{array}$ & 1.911 & 1.338 & 6.108 & $\mathrm{n} / \mathrm{a}$ \\
\hline $\begin{array}{l}\text { Tensile Strain, } \mu \varepsilon \\
\text { (ASTM D3039) }\end{array}$ & 8284 & 11669 & 16774 & $\mathrm{n} / \mathrm{a}$ \\
\hline $\begin{array}{l}\text { Shear Strength, ksi } \\
\text { (ASTM D5379) }\end{array}$ & 3.849 & 2.804 & 5.818 & 0.373 \\
\hline $\begin{array}{l}\text { Shear Stiffness, Msi } \\
\text { (ASTM D5379) }\end{array}$ & 0.102 & 0.101 & 0.568 & $\mathrm{n} / \mathrm{a}$ \\
\hline $\begin{array}{l}\text { Shear Strain, } \mu \gamma \text { (ASTM } \\
\text { D5379) }\end{array}$ & 50000 & 50000 & 25493 & $\mathrm{n} / \mathrm{a}$ \\
\hline $\begin{array}{l}\text { Compression Strength, } \\
\text { ksi (ASTM D695) }\end{array}$ & 9.190 & 6.783 & 28.625 & 0.359 \\
\hline $\begin{array}{l}\text { Compression Stiffness, } \\
\text { Msi (ASTM D695) }\end{array}$ & 2.524 & 1.920 & 5.995 & $\mathrm{n} / \mathrm{a}$ \\
\hline $\begin{array}{l}\text { Compression Strain, } \mu \varepsilon \\
\text { (ASTM D695) }\end{array}$ & 28724 & 23440 & 4824 & $\mathrm{n} / \mathrm{a}$ \\
\hline $\begin{array}{l}\text { Compression Strength, } \\
\text { ksi (ASTM D3410) }\end{array}$ & 9.782 & 8.440 & 31.704 & 1.111 \\
\hline $\begin{array}{l}\text { Compression Stiffness, } \\
\text { Msi (ASTM D3410) }\end{array}$ & 0.786 & 0.385 & 3.649 & $\mathrm{n} / \mathrm{a}$ \\
\hline $\begin{array}{l}\text { Compression Strain, } \mu \varepsilon \\
\text { (ASTM D3410) }\end{array}$ & 35914 & 28608 & 7689 & $\mathrm{n} / \mathrm{a}$ \\
\hline
\end{tabular}


Table A-4 - Dry, VF-normalized mechanical testing results

\begin{tabular}{|c|c|c|c|c|}
\hline$\underline{\text { DRY, VF }}$ & Kenaf, untreated & Kenaf, treated & Glass & OSB \\
\hline $\begin{array}{l}\text { Flexural Strength, ksi } \\
\text { (ASTM D790) }\end{array}$ & 26.570 & 29.108 & 41.976 & 3.533 \\
\hline $\begin{array}{l}\text { Flexural Stiffness, Msi } \\
\text { (ASTM D790) }\end{array}$ & 1.076 & 1.102 & 1.659 & 0.270 \\
\hline $\begin{array}{l}\text { Tensile Strength, ksi } \\
\text { (ASTM D3039) }\end{array}$ & 25.569 & 23.259 & 44.517 & 0.923 \\
\hline $\begin{array}{l}\text { Tensile Stiffness, Msi } \\
\text { (ASTM D3039) }\end{array}$ & 3.287 & 3.311 & 4.108 & 0.213 \\
\hline $\begin{array}{l}\text { Tensile Strain, } \mu \varepsilon \\
\text { (ASTM D3039) }\end{array}$ & 8101 & 6360 & 9987 & 4078 \\
\hline $\begin{array}{l}\text { Shear Strength, ksi } \\
\text { (ASTM D5379) }\end{array}$ & 7.375 & 5.070 & 6.008 & 1.047 \\
\hline $\begin{array}{l}\text { Shear Stiffness, Msi } \\
\text { (ASTM D5379) }\end{array}$ & 0.387 & 0.317 & 0.485 & 0.128 \\
\hline $\begin{array}{l}\text { Shear Strain, } \mu \gamma \text { (ASTM } \\
\text { D5379) }\end{array}$ & 35902 & 25003 & 48531 & 7254 \\
\hline $\begin{array}{l}\text { Compression Strength, } \\
\text { ksi (ASTM D695) }\end{array}$ & 16.743 & 17.013 & 19.054 & 1.542 \\
\hline $\begin{array}{l}\text { Compression Stiffness, } \\
\text { Msi (ASTM D695) }\end{array}$ & 3.319 & 3.411 & 4.312 & 1.310 \\
\hline $\begin{array}{l}\text { Compression Strain, } \mu \varepsilon \\
\text { (ASTM D695) }\end{array}$ & 15357 & 10985 & 4251 & 1402 \\
\hline $\begin{array}{l}\text { Compression Strength, } \\
\text { ksi (ASTM D3410) }\end{array}$ & 16.392 & 16.276 & 21.567 & 1.792 \\
\hline $\begin{array}{l}\text { Compression Stiffness, } \\
\text { Msi (ASTM D3410) }\end{array}$ & 2.288 & 1.683 & 4.871 & 0.514 \\
\hline $\begin{array}{l}\text { Compression Strain, } \mu \varepsilon \\
\text { (ASTM D3410) }\end{array}$ & 12013 & 21159 & 3679 & 6208 \\
\hline
\end{tabular}

\section{Volume Fraction Correction}

\begin{tabular}{lrllll}
\hline 1. Divided by VF: & $34.6 \%$ & $39.1 \%$ & $47.4 \%$ & n/a & (Flexure) \\
& $34.6 \%$ & $37.7 \%$ & $46.8 \%$ & n/a & (Shear) \\
& $35.7 \%$ & & $38.7 \%$ & $57.0 \%$ & n/a $\quad$ (T \& C) \\
2. Normalized at: & $40 \%$ & FVF & & &
\end{tabular}


Table A-5 - 50 hours immersion, VF-normalized mechanical testing results

\begin{tabular}{|c|c|c|c|c|}
\hline 50 HOURS, VF & Kenaf, untreated & Kenaf, treated & Glass & OSB \\
\hline $\begin{array}{l}\text { Flexural Strength, ksi } \\
\text { (ASTM D790) }\end{array}$ & 23.942 & 19.349 & 45.241 & 1.728 \\
\hline $\begin{array}{l}\text { Flexural Stiffness, Msi } \\
\text { (ASTM D790) }\end{array}$ & 0.830 & 0.831 & 1.763 & 0.054 \\
\hline $\begin{array}{l}\text { Tensile Strength, ksi } \\
\text { (ASTM D3039) }\end{array}$ & 30.581 & 25.947 & 60.006 & 0.543 \\
\hline $\begin{array}{l}\text { Tensile Stiffness, Msi } \\
\text { (ASTM D3039) }\end{array}$ & 3.176 & 2.745 & 4.178 & $\mathrm{n} / \mathrm{a}$ \\
\hline $\begin{array}{l}\text { Tensile Strain, } \mu \varepsilon \\
\text { (ASTM D3039) }\end{array}$ & 10093 & 8603 & 16615 & $\mathrm{n} / \mathrm{a}$ \\
\hline $\begin{array}{l}\text { Shear Strength, ksi } \\
\text { (ASTM D5379) }\end{array}$ & 5.296 & 4.696 & 4.593 & 0.419 \\
\hline $\begin{array}{l}\text { Shear Stiffness, Msi } \\
\text { (ASTM D5379) }\end{array}$ & 0.243 & 0.210 & 0.470 & $\mathrm{n} / \mathrm{a}$ \\
\hline $\begin{array}{l}\text { Shear Strain, } \mu \gamma \text { (ASTM } \\
\text { D5379) }\end{array}$ & 40717 & 42791 & 18957 & $\mathrm{n} / \mathrm{a}$ \\
\hline $\begin{array}{l}\text { Compression Strength, } \\
\text { ksi (ASTM D695) }\end{array}$ & 13.572 & 10.263 & 25.627 & 0.512 \\
\hline $\begin{array}{l}\text { Compression Stiffness, } \\
\text { Msi (ASTM D695) }\end{array}$ & 3.592 & 2.112 & 4.014 & $\mathrm{n} / \mathrm{a}$ \\
\hline $\begin{array}{l}\text { Compression Strain, } \mu \varepsilon \\
\text { (ASTM D695) }\end{array}$ & 18857 & 30192 & 5945 & $\mathrm{n} / \mathrm{a}$ \\
\hline $\begin{array}{l}\text { Compression Strength, } \\
\text { ksi (ASTM D3410) }\end{array}$ & 13.134 & 11.133 & 19.810 & 0.796 \\
\hline $\begin{array}{l}\text { Compression Stiffness, } \\
\text { Msi (ASTM D3410) }\end{array}$ & 0.890 & 0.801 & 4.182 & $\mathrm{n} / \mathrm{a}$ \\
\hline $\begin{array}{l}\text { Compression Strain, } \mu \varepsilon \\
\text { (ASTM D3410) }\end{array}$ & 12569 & 26982 & 4726 & $\mathrm{n} / \mathrm{a}$ \\
\hline
\end{tabular}

\section{Volume Fraction Correction}

\begin{tabular}{lrllll}
\hline 1. Divided by VF: & $34.6 \%$ & $39.1 \%$ & $47.4 \%$ & $\mathrm{n} / \mathrm{a}$ & (Flexure) \\
& $34.6 \%$ & $37.7 \%$ & $46.8 \%$ & $\mathrm{n} / \mathrm{a}$ & (Shear) \\
& $35.7 \%$ & $38.7 \%$ & $57.0 \%$ & $\mathrm{n} / \mathrm{a}$ & (T \& C) \\
2. Normalized at: & $40 \%$ & FVF & & &
\end{tabular}


Table A-6 - Saturated, VF-normalized mechanical testing results

\begin{tabular}{|l|r|r|r|r|}
\hline \multicolumn{1}{|c|}{ VATUR } & Kenaf, untreated & Kenaf, treated & \multicolumn{1}{c|}{ Glass } & \multicolumn{1}{c|}{ OSB } \\
\hline \hline $\begin{array}{l}\text { Flexural Strength, ksi } \\
\text { (ASTM D790) }\end{array}$ & 18.864 & 14.658 & 27.516 & 1.852 \\
\hline $\begin{array}{l}\text { Flexural Stiffness, Msi } \\
\text { (ASTM D790) }\end{array}$ & 0.913 & 0.857 & 2.158 & 0.189 \\
\hline \hline $\begin{array}{l}\text { Tensile Strength, ksi } \\
\text { (ASTM D3039) }\end{array}$ & 18.984 & 20.217 & 58.323 & 0.413 \\
\hline $\begin{array}{l}\text { Tensile Stiffness, Msi } \\
\text { (ASTM D3039) }\end{array}$ & 2.145 & 1.382 & 4.284 & $\mathrm{n} / \mathrm{a}$ \\
\hline $\begin{array}{l}\text { Tensile Strain, } \mu \varepsilon \\
\text { (ASTM D3039) }\end{array}$ & 8284 & 11669 & 16774 & $\mathrm{n} / \mathrm{a}$ \\
\hline \hline $\begin{array}{l}\text { Shear Strength, ksi } \\
\text { (ASTM D5379) }\end{array}$ & 4.448 & 2.973 & 4.970 & 0.373 \\
\hline $\begin{array}{l}\text { Shear Stiffness, Msi } \\
\text { (ASTM D5379) }\end{array}$ & 0.118 & 0.107 & 0.485 & $\mathrm{n} / \mathrm{a}$ \\
\hline $\begin{array}{l}\text { Shear Strain, } \mu \gamma(\text { ASTM } \\
\text { D5379) }\end{array}$ & 50000 & 50000 & 25493 & $\mathrm{n} / \mathrm{a}$ \\
\hline \hline $\begin{array}{l}\text { Compression Strength, } \\
\text { ksi (ASTM D695) }\end{array}$ & 10.310 & 7.005 & 20.075 & 0.359 \\
\hline $\begin{array}{l}\text { Compression Stiffness, } \\
\text { Msi (ASTM D695) }\end{array}$ & 2.832 & 1.983 & 4.204 & $\mathrm{n} / \mathrm{a}$ \\
\hline $\begin{array}{l}\text { Compression Strain, } \mu \varepsilon \\
\text { (ASTM D695) }\end{array}$ & 28724 & 23440 & 4824 & $\mathrm{n} / \mathrm{a}$ \\
\hline \hline $\begin{array}{l}\text { Compression Strength, } \\
\text { ksi (ASTM D3410) }\end{array}$ & 10.974 & 8.717 & 22.234 & 1.111 \\
\hline $\begin{array}{l}\text { Compression Stiffness, } \\
\text { Msi (ASTM D3410) }\end{array}$ & 0.882 & 0.398 & 2.559 & $\mathrm{n} / \mathrm{a}$ \\
\hline $\begin{array}{l}\text { Compression Strain, } \mu \varepsilon \\
\text { (ASTM D3410) }\end{array}$ & 35914 & 28608 & 7689 & $\mathrm{n} / \mathrm{a}$ \\
\hline \hline
\end{tabular}

\section{Volume Fraction Correction}

\begin{tabular}{lrllll}
\hline 1. Divided by VF: & $34.6 \%$ & $39.1 \%$ & $47.4 \%$ & $\mathrm{n} / \mathrm{a}$ & (Flexure) \\
& $34.6 \%$ & $37.7 \%$ & $46.8 \%$ & $\mathrm{n} / \mathrm{a}$ & (Shear) \\
& $35.7 \%$ & $38.7 \%$ & $57.0 \%$ & $\mathrm{n} / \mathrm{a}$ & (T \& C) \\
2. Normalized at: & $40 \%$ & FVF & & &
\end{tabular}




\section{APPENDIX B - MONTHLY WEATHERING SAMPLE IMAGES}

The photographs in this section were taken of the weathering samples after they were removed each month and before they were tested in flexure.

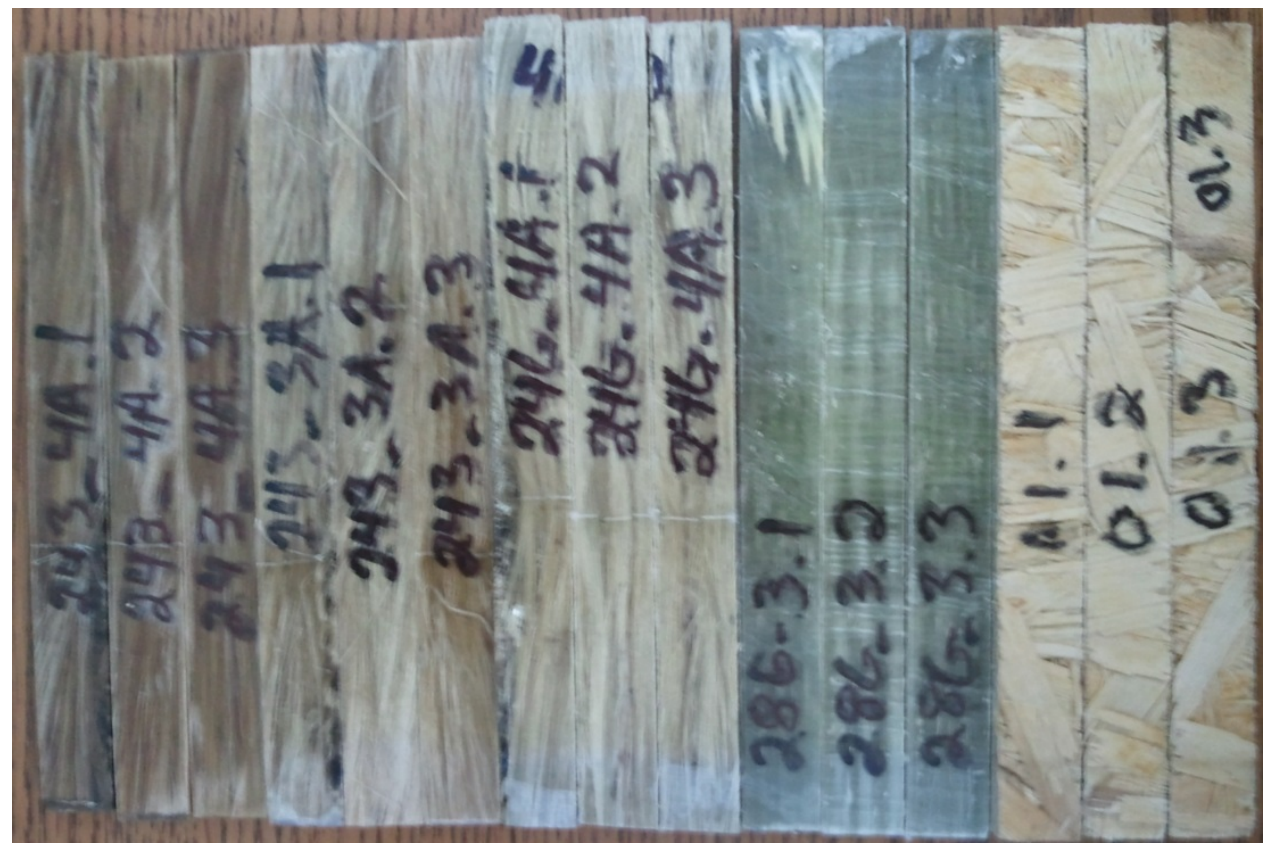

Figure B-1 - Samples collected at the end of April 2012; 1 month exposure

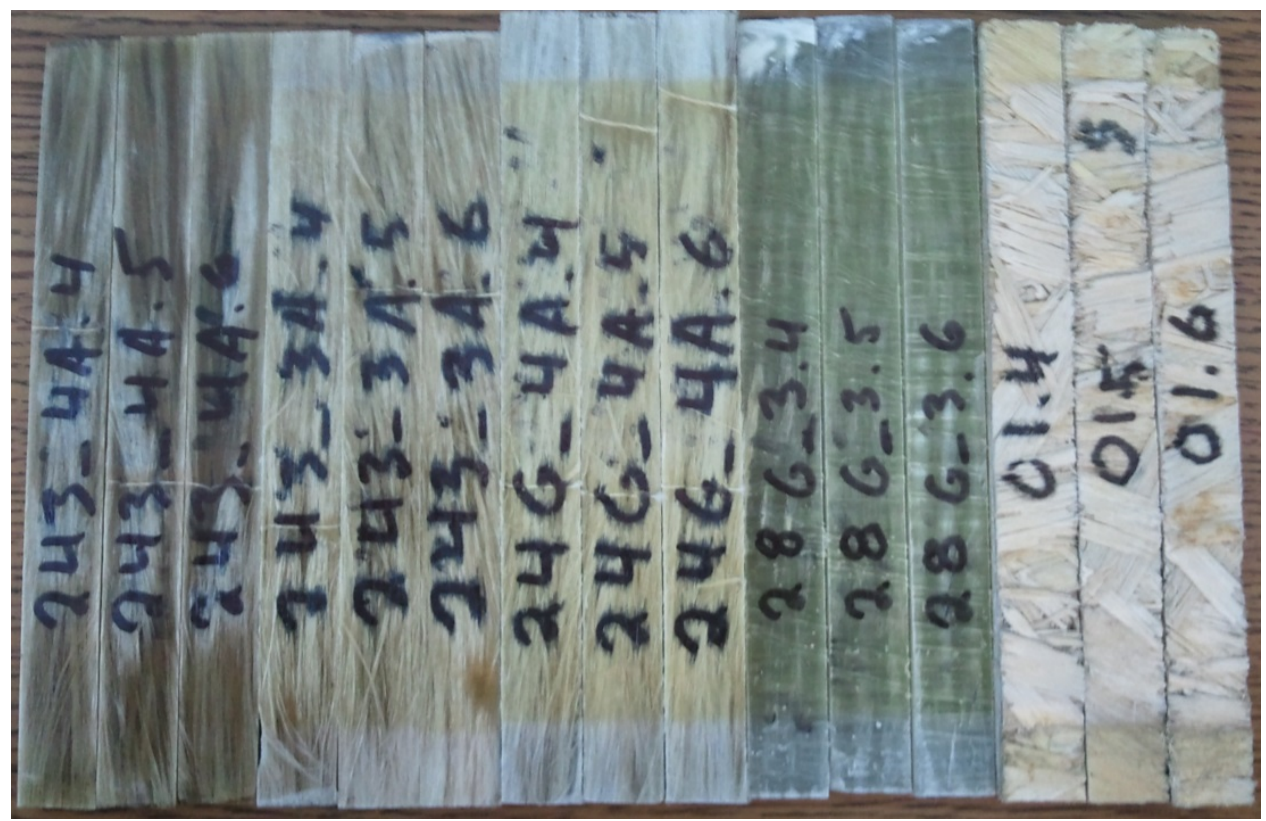

Figure B-2 - Samples collected at the end of May 2012; 2 months exposure 


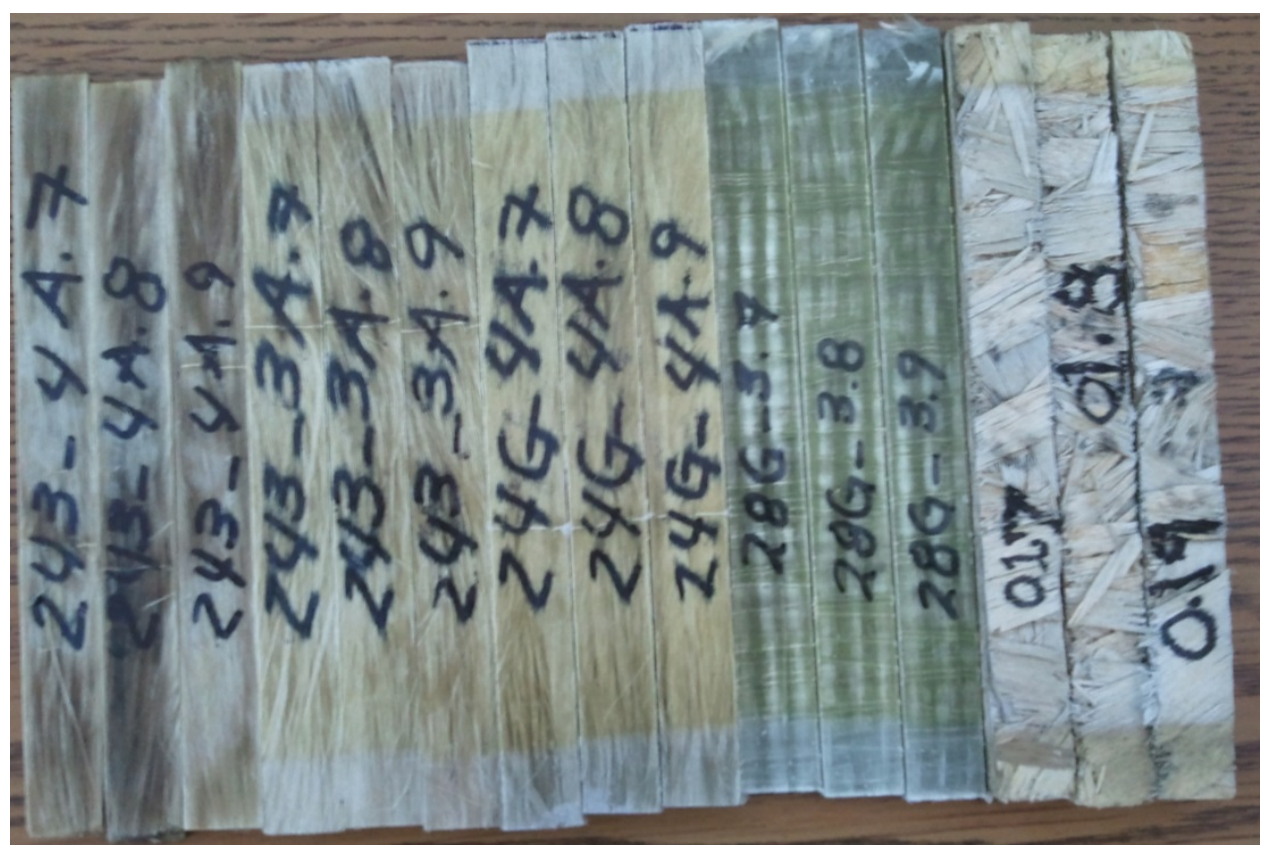

Figure B-3 - Samples collected at the end of June 2012; 3 months exposure

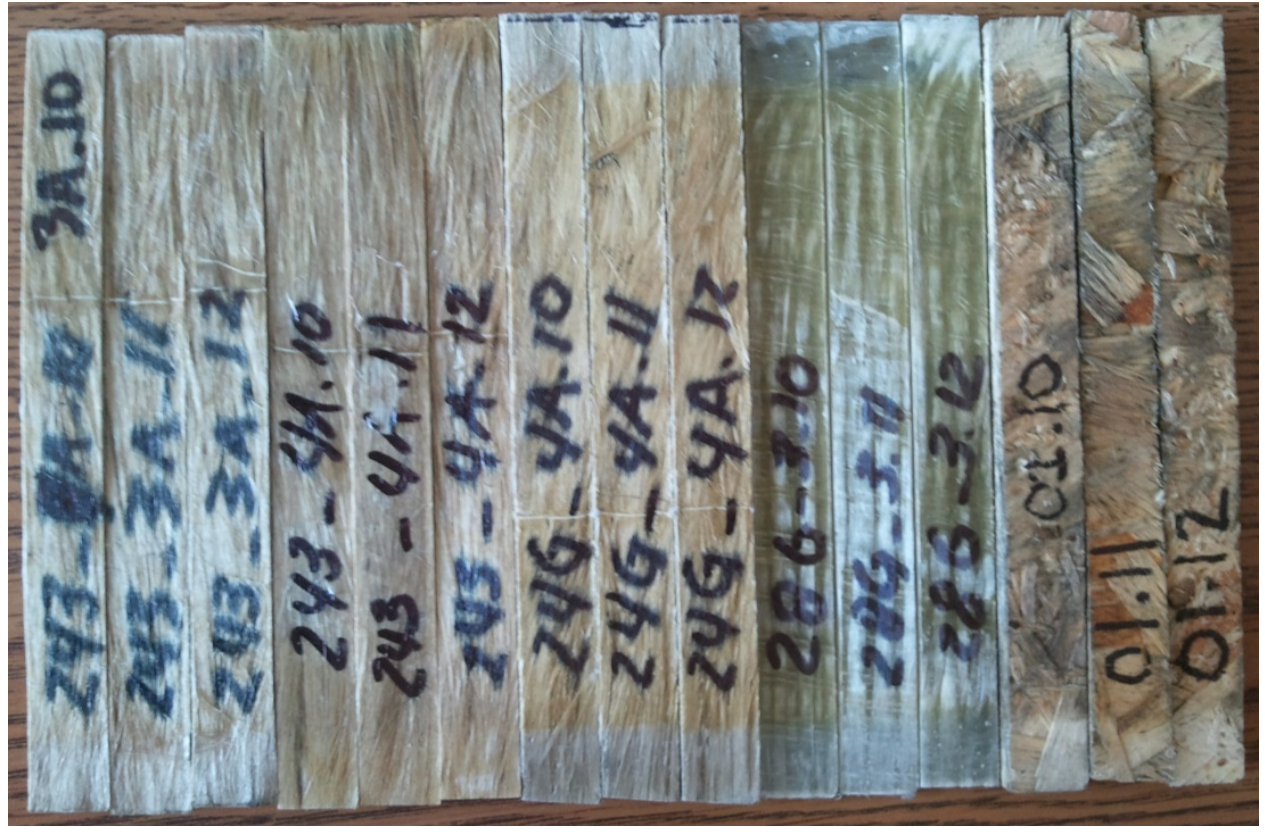

Figure B-4 - Samples collected at the end of July 2012; 4 months exposure 


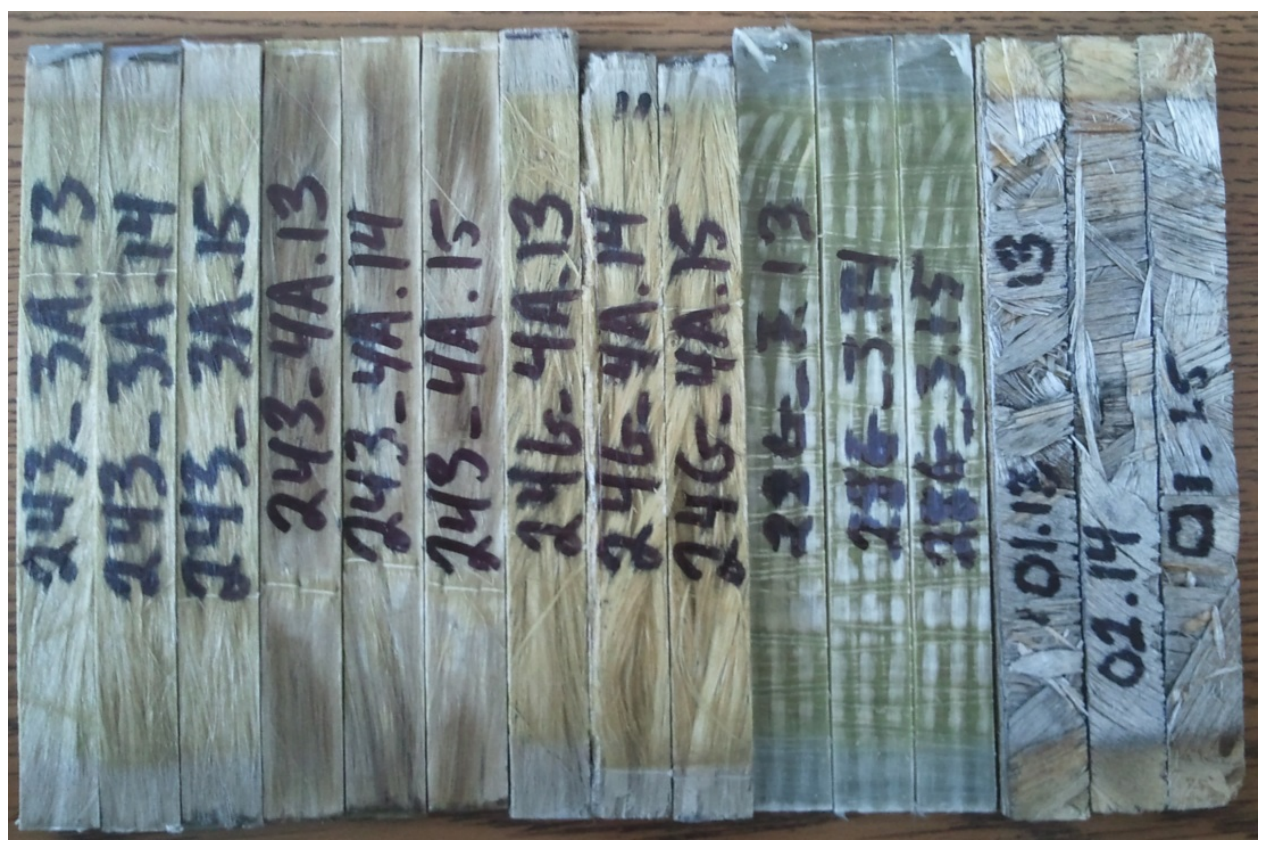

Figure B-5 - Samples collected at the end of August 2012; 5 months exposure

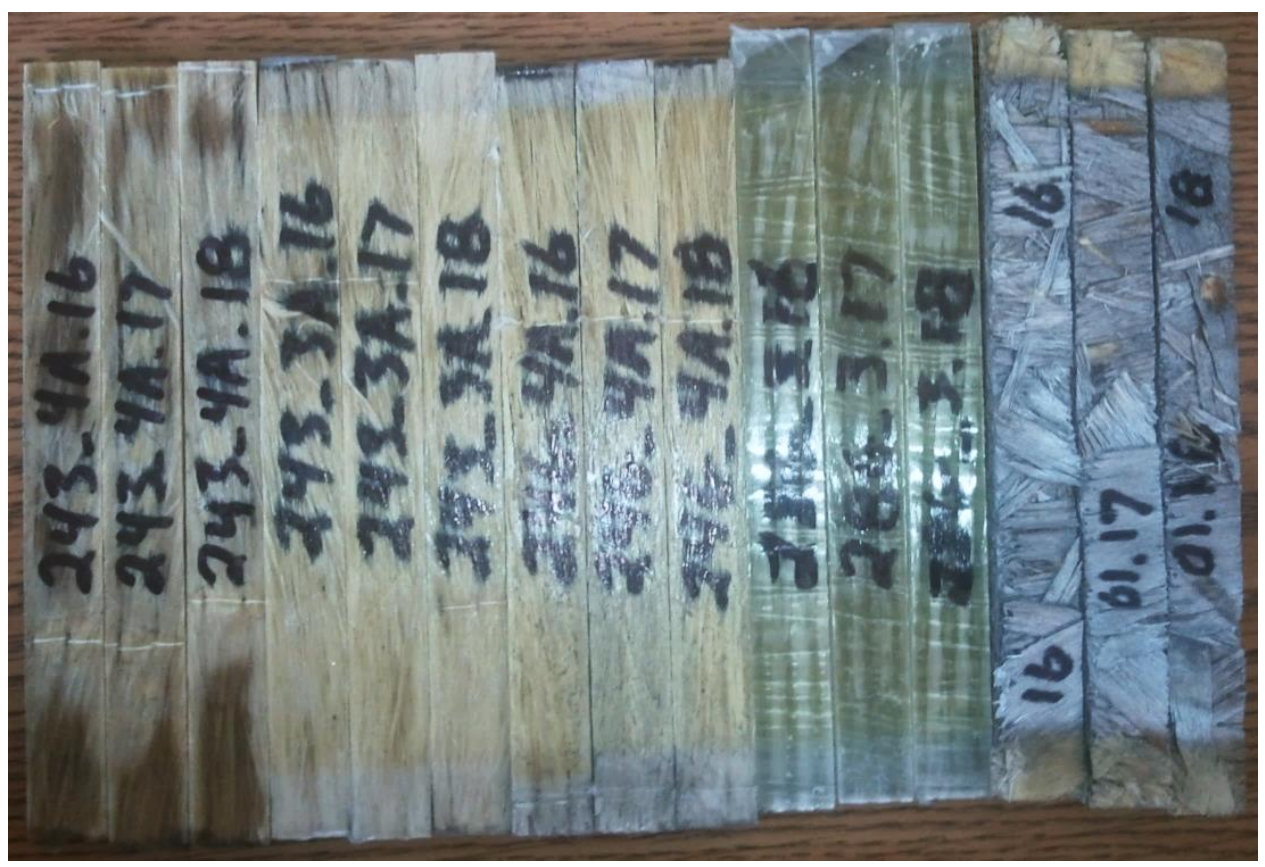

Figure B-6 - Samples collected at the end of September 2012; 6 months exposure 


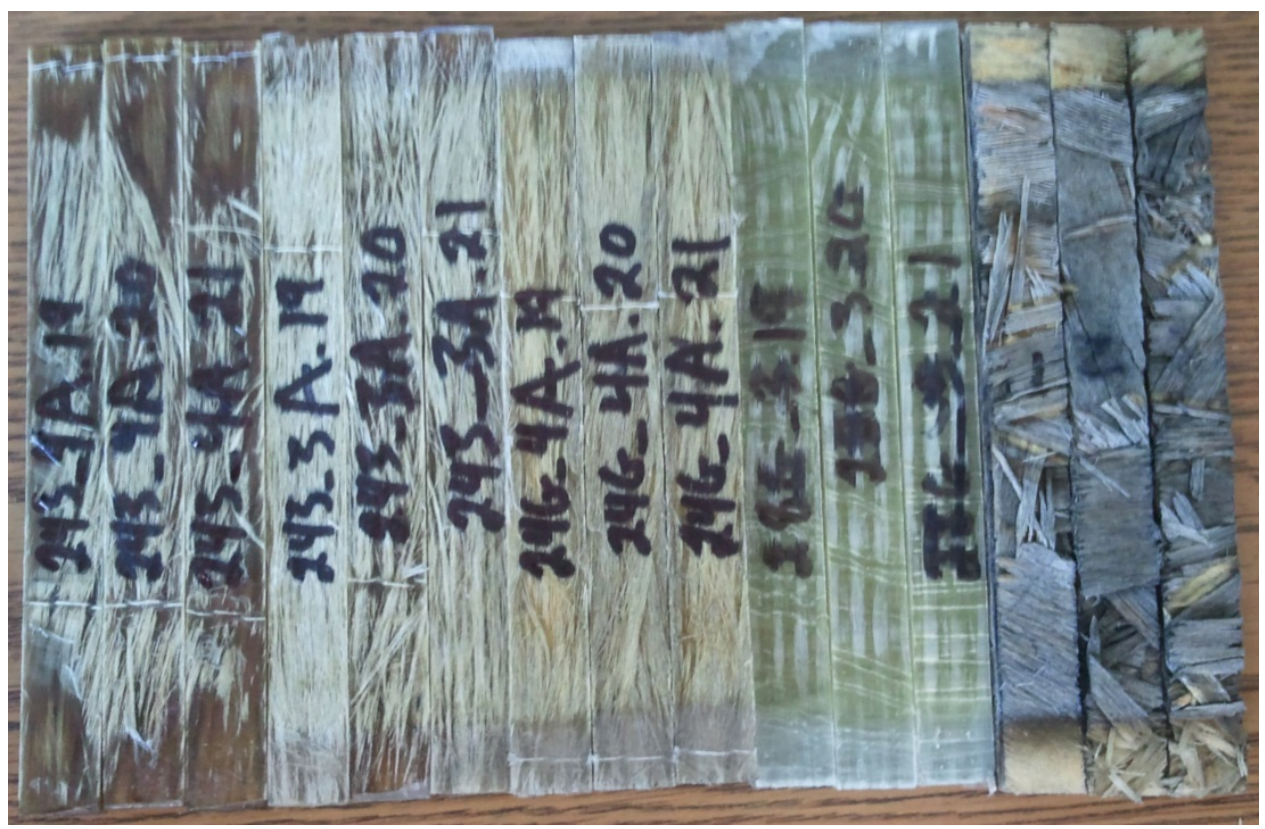

Figure B-7 - Samples collected at the end of October 2012; 7 months exposure

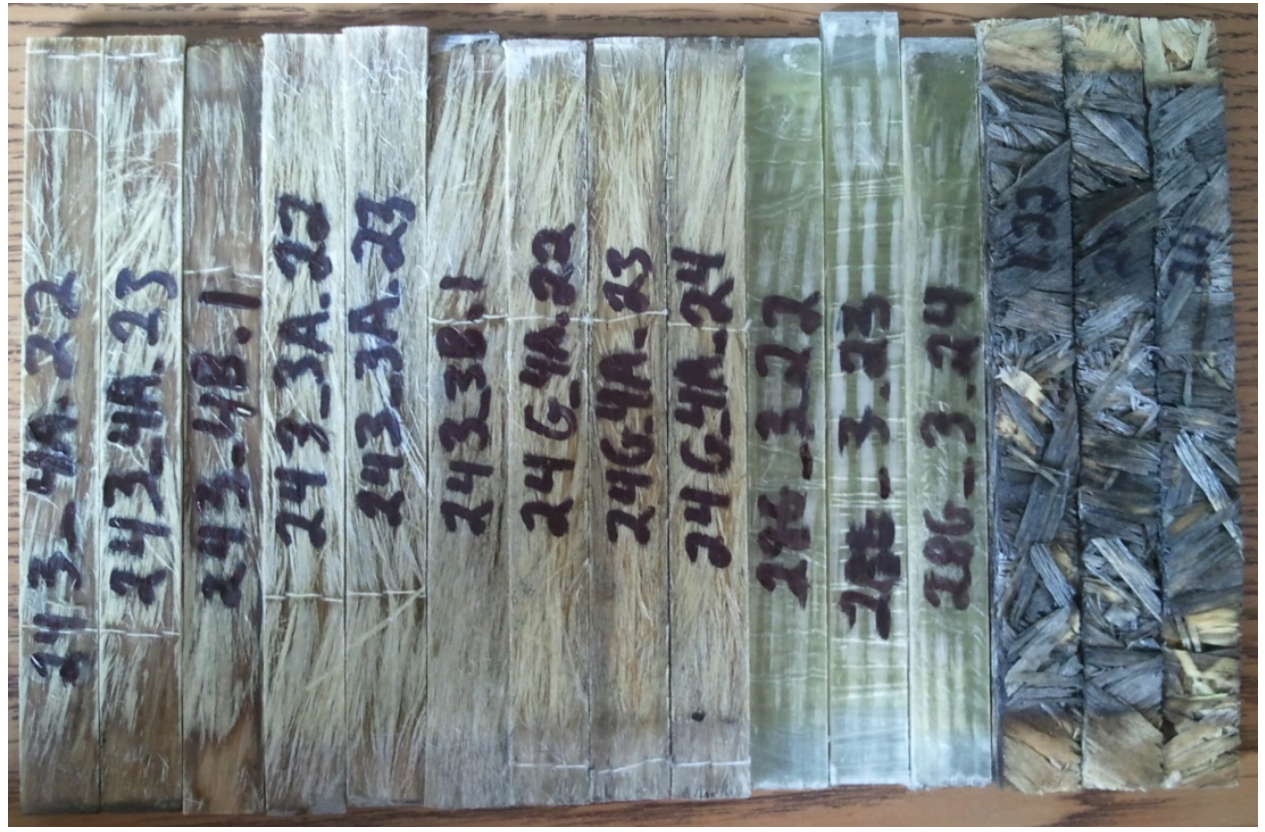

Figure B-8 - Samples collected at the end of November 2012; 8 months exposure 


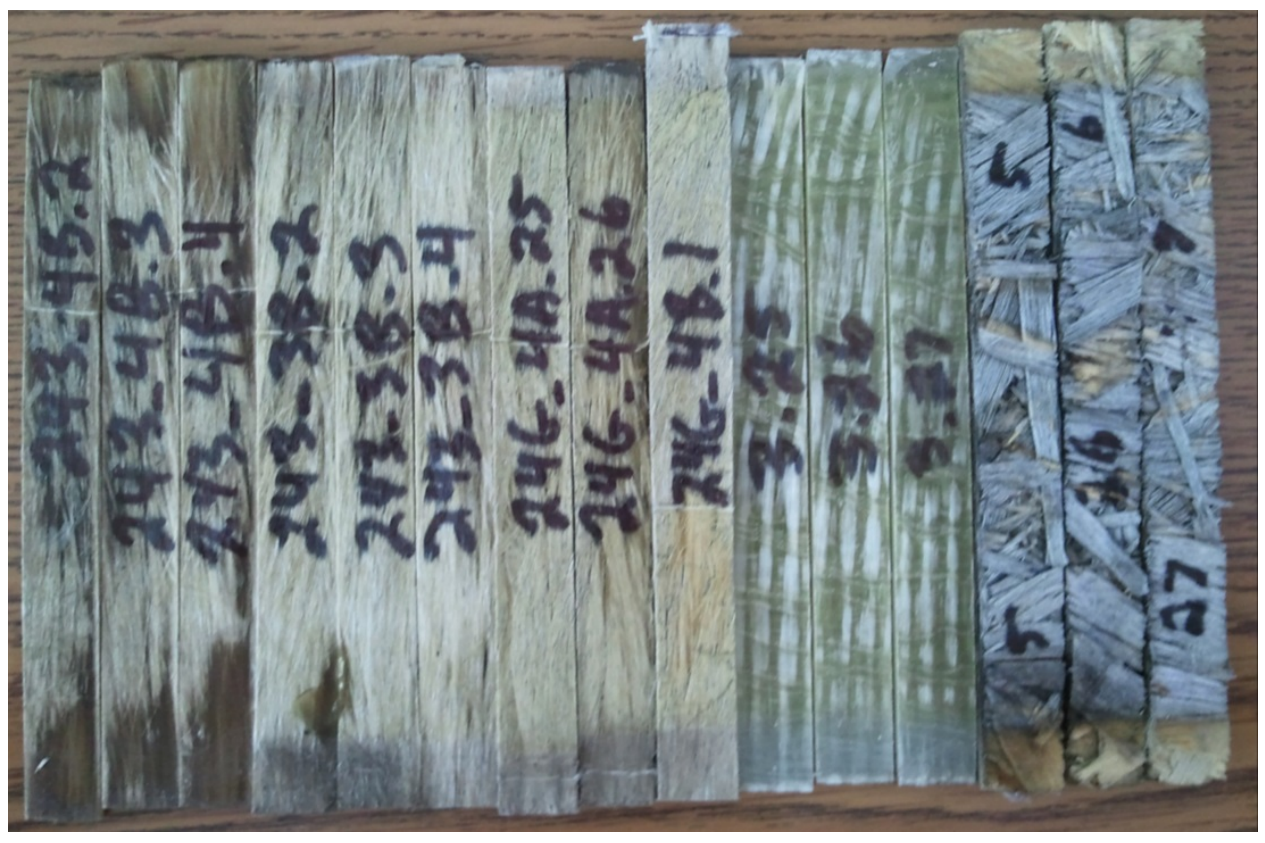

Figure B-9 - Samples collected at the end of December 2012; 9 months exposure

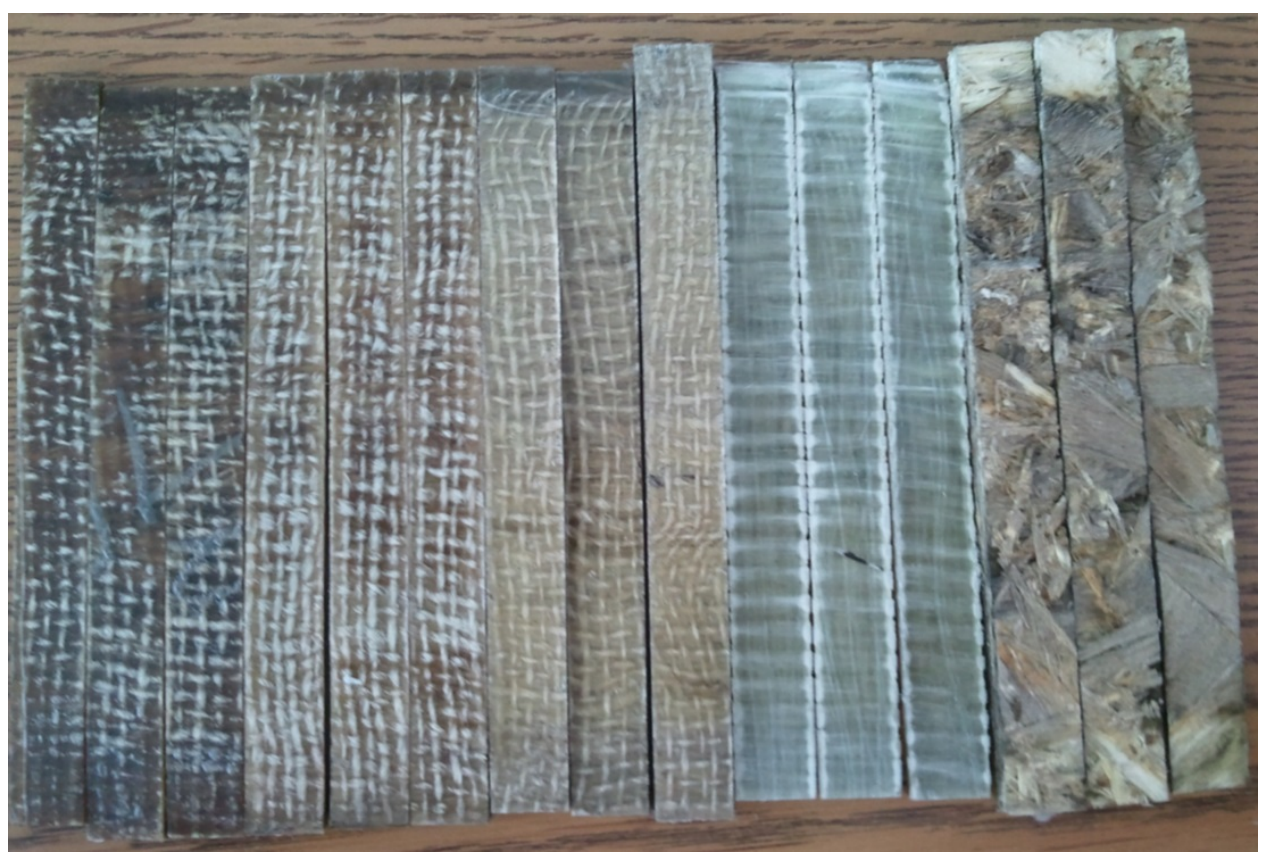

Figure B-10 - Reverse side of samples collected at the end of December 2012; 9 months exposure 


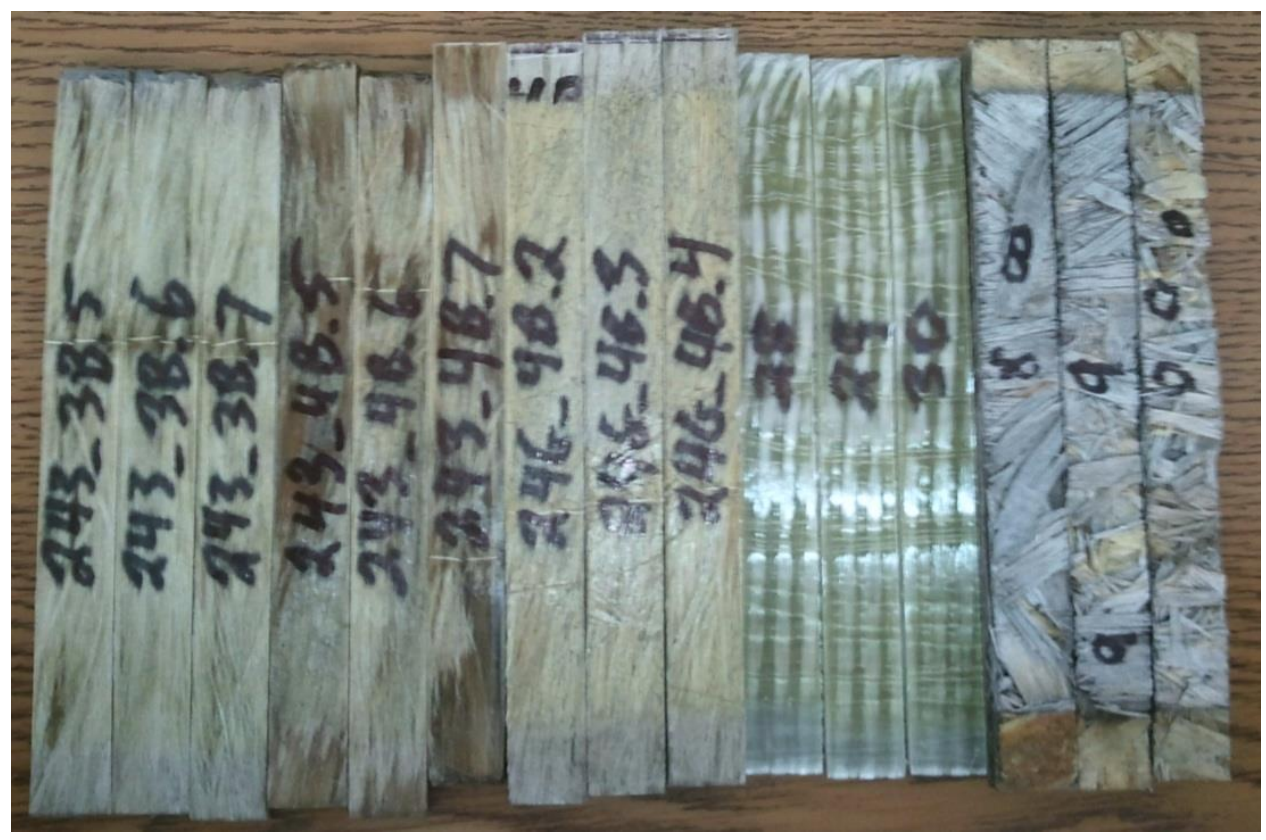

Figure B-11 - Samples collected at the end of January 2013; 10 months exposure

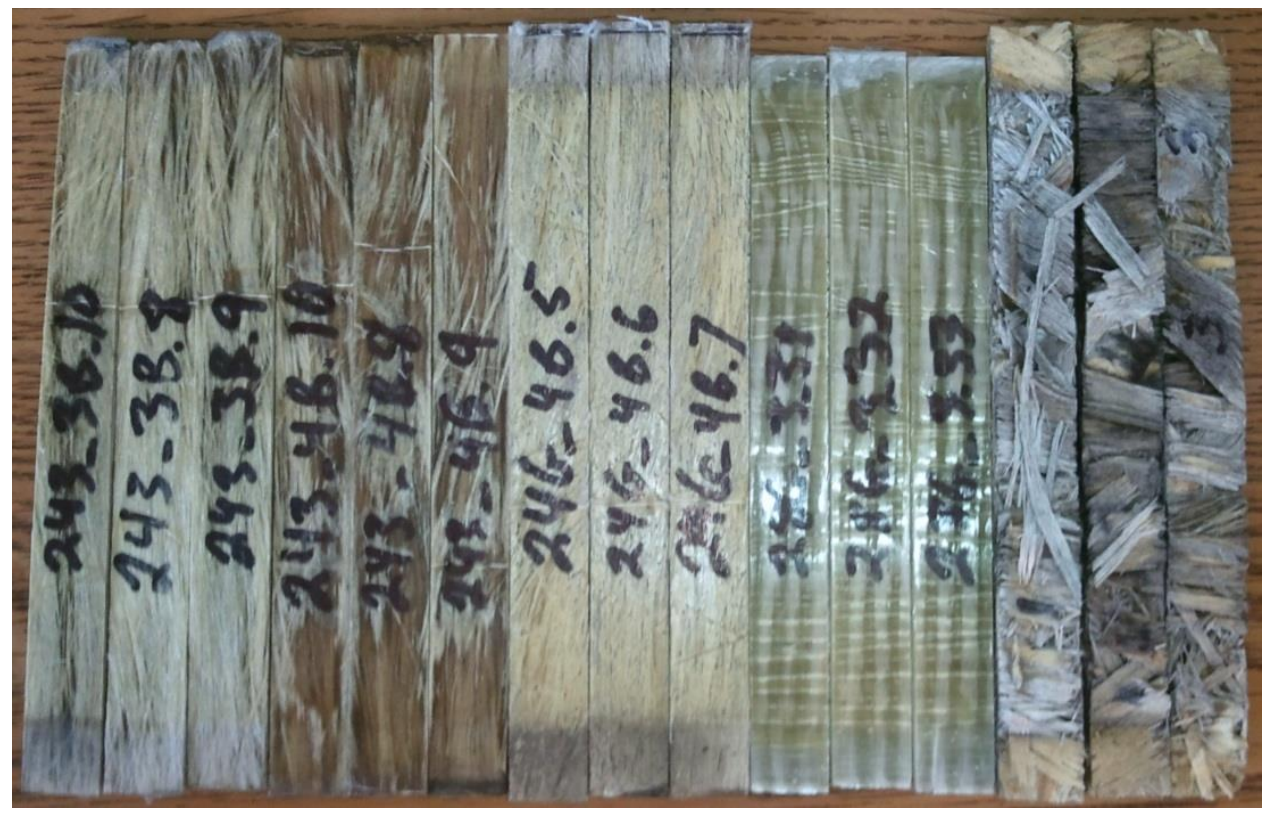

Figure B-12 - Samples collected at the end of February 2012; 11 months exposure 


\section{APPENDIX C - EIO-LCA DETAILED RESULTS}

The Economic Input-Output Life Cycle Assessment (EIO-LCA) tool (Carnegie Mellon University Green Design Institute 2013) provides significantly more information than just the total embodied energy and $\mathrm{CO}_{2}$ contribution. For example, the top ten sectors in terms of economic activity, embodied energy by source, and greenhouse gas emissions are shown in this appendix for each of the wall designs analyzed.

Table C-1 - Key to column headings in EIO-LCA detailed results

\begin{tabular}{|l|l|}
\hline \multicolumn{1}{|c|}{ Column Heading } & \multicolumn{1}{c|}{ Meaning } \\
\hline Total Economic & The complete economic supply chain of purchases needed to produce the level of output. \\
\hline Total Value Added & $\begin{array}{l}\text { The total value added by sector, representing the difference between output and supply } \\
\text { chain purchases. }\end{array}$ \\
\hline Employee Comp VA & The portion of the value added in the form of employee compensation (labor costs). \\
\hline Net Tax VA & The portion of the value added associated with taxes paid, minus any subsidies. \\
\hline Profits VA & The portion of the value added in the form of profits. \\
\hline Direct Economic (\$) & $\begin{array}{l}\text { The purchases made by the industries being analyzed, as opposed to the total supply chain } \\
\text { of purchases. }\end{array}$ \\
\hline Direct Economic (\%) & Percent of total purchases made directly by the industries being analyzed. \\
\hline Total Energy & Total energy use from all fuels and electricity from all sectors. \\
\hline Coal & Coal used by each sector. \\
\hline NatGas & Natural gas used by each sector. \\
\hline Petrol & Petroleum-based fuels used by each sector. \\
\hline Bio/Waste & Biomass/Waster fuel used by each sector. \\
\hline NonFossElec & Non-fossil electricity used by each sector. \\
\hline Total (lbs CO $\left.\mathbf{O}_{2} \mathbf{e}\right)$ & $\begin{array}{l}\text { Global Warming Potential }\left(\mathrm{GWP}^{2}\right) \text { is a weighting of greenhouse gas emissions into the air } \\
\text { from the production of each sector. }\end{array}$ \\
\hline $\mathbf{C O} \mathbf{O}_{2}$ Fossil & $\begin{array}{l}\text { Emissions of Carbon Dioxide }\left(\mathrm{CO}_{2}\right) \text { into the air from each sector from fossil fuel } \\
\text { combustion sources. }\end{array}$ \\
\hline $\mathbf{C O} \mathbf{O}_{2}$ Process & $\begin{array}{l}\text { Emissions of Carbon Dioxide }\left(\mathrm{CO}_{2}\right) \text { into the air from each sector from sources other than } \\
\text { fossil fuel combustion sources. }\end{array}$ \\
\hline $\mathbf{C H}{ }_{4}$ & Emissions of Methane $\left(\mathrm{CH}{ }_{4}\right)$ into the air from each sector. \\
\hline $\mathbf{N}_{2} \mathbf{O}$ & Emissions of Nitrous Oxide $\left(\mathrm{N}_{2} \mathrm{O}\right)$ into the air from each sector. \\
\hline $\mathbf{H F C / P F C s ~}$ & $\begin{array}{l}\text { Emissions of all high-GWP gases such as hydrofluorocarbons, perfluorocarbons, and } \\
\text { sulfur hexafluoride into the air from each sector }\end{array}$ \\
\hline
\end{tabular}


Table C-2 - OSB SIP Design: Economic activity for raw materials

\begin{tabular}{|c|c|c|c|c|c|c|c|}
\hline Top Ten Sectors & $\begin{array}{c}\text { Total } \\
\text { Economic }\end{array}$ & $\begin{array}{c}\text { Total Value } \\
\text { Added }\end{array}$ & $\begin{array}{l}\text { Employee } \\
\text { Comp VA }\end{array}$ & $\begin{array}{l}\text { Net Tax } \\
\text { VA }\end{array}$ & $\begin{array}{c}\text { Profits } \\
\text { VA }\end{array}$ & $\begin{array}{c}\text { Direct } \\
\text { Economic }\end{array}$ & $\begin{array}{c}\text { Direct } \\
\text { Economic }\end{array}$ \\
\hline Total for all sectors & $\$ 4,170.00$ & $\$ 1,650.00$ & $\$ 902.00$ & $\$ 97.40$ & $\$ 646.00$ & $\$ 2,740.00$ & $65.7 \%$ \\
\hline Reconstituted wood product manufacturing & $\$ 1,060.00$ & $\$ 367.00$ & $\$ 203.00$ & $\$ 5.01$ & $\$ 159.00$ & $\$ 1,050.00$ & $99.5 \%$ \\
\hline Polystyrene Foam Product Manufacturing & $\$ 412.00$ & $\$ 162.00$ & $\$ 81.10$ & $\$ 11.90$ & $\$ 68.70$ & $\$ 411.00$ & $99.9 \%$ \\
\hline Logging & $\$ 303.00$ & $\$ 104.00$ & $\$ 47.80$ & $\$ 7.06$ & $\$ 48.90$ & $\$ 173.00$ & $57.1 \%$ \\
\hline Sawmills and wood preservation & $\$ 249.00$ & $\$ 56.10$ & $\$ 44.00$ & $\$ 1.06$ & $\$ 11.10$ & $\$ 226.00$ & $90.6 \%$ \\
\hline Plastics material and resin manufacturing & $\$ 150.00$ & $\$ 26.00$ & $\$ 16.30$ & $\$ 1.16$ & $\$ 8.56$ & $\$ 121.00$ & $80.1 \%$ \\
\hline Wholesale trade & $\$ 131.00$ & $\$ 91.50$ & $\$ 49.60$ & $\$ 21.40$ & $\$ 20.40$ & $\$ 57.70$ & $43.9 \%$ \\
\hline Management of companies and enterprises & $\$ 115.00$ & $\$ 70.90$ & $\$ 60.00$ & $\$ 1.74$ & $\$ 9.13$ & $\$ 45.70$ & $39.9 \%$ \\
\hline Power generation and supply & $\$ 83.70$ & $\$ 57.10$ & $\$ 17.40$ & $\$ 10.10$ & $\$ 29.60$ & $\$ 56.00$ & $66.9 \%$ \\
\hline Oil and gas extraction & $\$ 80.40$ & $\$ 40.80$ & $\$ 5.36$ & $\$ 7.18$ & $\$ 28.20$ & $\$ 1.84$ & $2.3 \%$ \\
\hline Petroleum refineries & $\$ 77.40$ & $\$ 6.18$ & $\$ 2.21$ & $\$ 0.25$ & $\$ 3.72$ & $\$ 20.20$ & $26.1 \%$ \\
\hline
\end{tabular}

Table C-3 - OSB SIP Design: Embodied energy by source for raw materials

\begin{tabular}{|c|c|c|c|c|c|c|}
\hline Top Ten Sectors & Total Energy & Coal & NatGas & Petrol & Bio/Waste & NonFossElec \\
\hline & $10^{6}$ btu & $10^{6}$ btu & $10^{6}$ btu & $10^{6}$ btu & $10^{6}$ btu & $10^{6} \mathrm{btu}$ \\
\hline Total for all sectors & 38.10 & 7.32 & 11.75 & 5.76 & 7.89 & 5.34 \\
\hline Reconstituted wood product manufacturing & 13.17 & 0.10 & 3.91 & 0.94 & 5.15 & 3.03 \\
\hline Power generation and supply & 8.63 & 6.28 & 1.84 & 0.31 & 0.00 & 0.20 \\
\hline Plastics material and resin manufacturing & 2.16 & 0.09 & 1.13 & 0.47 & 0.23 & 0.25 \\
\hline Other basic organic chemical manufacturing & 1.81 & 0.23 & 0.69 & 0.25 & 0.55 & 0.10 \\
\hline Polystyrene Foam Product Manufacturing & 1.46 & 0.15 & 0.86 & 0.02 & 0.01 & 0.42 \\
\hline Sawmills and wood preservation & 1.27 & 0.00 & 0.12 & 0.07 & 0.88 & 0.20 \\
\hline Petroleum refineries & 1.26 & 0.00 & 0.34 & 0.82 & 0.06 & 0.04 \\
\hline Petrochemical manufacturing & 1.21 & 0.02 & 0.48 & 0.46 & 0.20 & 0.05 \\
\hline Oil and gas extraction & 0.72 & 0.00 & 0.58 & 0.06 & 0.00 & 0.07 \\
\hline Truck transportation & 0.71 & 0.00 & 0.00 & 0.70 & 0.00 & 0.01 \\
\hline
\end{tabular}


Table C-4 - OSB SIP Design: Greenhouse gases in equivalent $\mathrm{lbs}$ of $\mathrm{CO}_{2}$ for raw materials

\begin{tabular}{|c|c|c|c|c|c|c|}
\hline Top Ten Sectors & Total & $\mathrm{CO}_{2}$ Fossil & $\mathrm{CO}_{2}$ Process & $\mathrm{CH}_{4}$ & $\mathbf{N}_{2} \mathbf{O}$ & HFC/PFCs \\
\hline & lbs $\mathrm{CO}_{2} \mathrm{e}$ & lbs $\mathrm{CO}_{2} \mathrm{e}$ & lbs $\mathrm{CO}_{2} \mathrm{e}$ & lbs $\mathrm{CO}_{2} \mathrm{e}$ & lbs $\mathrm{CO}_{2} \mathrm{e}$ & lbs $\mathrm{CO}_{2} \mathrm{e}$ \\
\hline Total for all sectors & 4620 & 3872 & 199 & 323 & 193 & 45 \\
\hline Power generation and supply & 1643 & 1619 & 0 & 4 & 10 & 10 \\
\hline Reconstituted wood product manufacturing & 647 & 647 & 0 & 0 & 0 & 0 \\
\hline Oil and gas extraction & 277 & 78 & 51 & 149 & 0 & 0 \\
\hline Plastics material and resin manufacturing & 218 & 218 & 0 & 0 & 0 & 0 \\
\hline Other basic organic chemical manufacturing & 184 & 165 & 0 & 0 & 19 & 0 \\
\hline Petroleum refineries & 174 & 174 & 0 & 1 & 0 & 0 \\
\hline Petrochemical manufacturing & 153 & 128 & 18 & 7 & 0 & 0 \\
\hline Polystyrene Foam Product Manufacturing & 134 & 134 & 0 & 0 & 0 & 0 \\
\hline Truck transportation & 122 & 122 & 0 & 0 & 0 & 0 \\
\hline Lime and gypsum product manufacturing & 104 & 39 & 65 & 0 & 0 & 0 \\
\hline
\end{tabular}

Table C-5 - GFRP SIP Design: Economic activity for raw materials

\begin{tabular}{|c|c|c|c|c|c|c|c|}
\hline Top Ten Sectors & $\begin{array}{l}\text { Total } \\
\text { Economic }\end{array}$ & $\begin{array}{l}\text { Total Value } \\
\text { Added }\end{array}$ & $\begin{array}{l}\text { Employee } \\
\text { Comp VA }\end{array}$ & $\begin{array}{l}\text { Net Tax } \\
\text { VA }\end{array}$ & $\begin{array}{l}\text { Profits } \\
\text { VA }\end{array}$ & $\begin{array}{l}\text { Direct } \\
\text { Economic }\end{array}$ & $\begin{array}{l}\text { Direct } \\
\text { Economic }\end{array}$ \\
\hline Total for all sectors & $\$ 10,400.00$ & $\$ 3,600.00$ & $\$ 2,070.00$ & $\$ 203.00$ & $\$ 1,330.00$ & $\$ 6,500.00$ & $63 \%$ \\
\hline Plastics material and resin manufacturing & $\$ 1,680.00$ & $\$ 290.00$ & $\$ 182.00$ & $\$ 12.90$ & $\$ 95.50$ & $\$ 1,570.00$ & $93 \%$ \\
\hline Other basic organic chemical manufacturing & $\$ 910.00$ & $\$ 143.00$ & $\$ 115.00$ & $\$ 8.28$ & $\$ 19.60$ & $\$ 651.00$ & $72 \%$ \\
\hline $\begin{array}{l}\text { Urethane and Other Foam Product } \\
\text { (except Polystyrene) Manufacturing }\end{array}$ & $\$ 652.00$ & $\$ 216.00$ & $\$ 129.00$ & $\$ 2.42$ & $\$ 84.60$ & $\$ 651.00$ & $100 \%$ \\
\hline $\begin{array}{l}\text { Artificial and synthetic fibers and filaments } \\
\text { manufacturing }\end{array}$ & $\$ 631.00$ & $\$ 130.00$ & $\$ 109.00$ & $\$ 2.58$ & $\$ 17.80$ & $\$ 612.00$ & $97 \%$ \\
\hline Petrochemical manufacturing & $\$ 594.00$ & $\$ 91.90$ & $\$ 19.50$ & $\$ 3.55$ & $\$ 68.80$ & $\$ 498.00$ & $84 \%$ \\
\hline Management of companies and enterprises & $\$ 480.00$ & $\$ 297.00$ & $\$ 251.00$ & $\$ 7.29$ & $\$ 38.20$ & $\$ 235.00$ & $49 \%$ \\
\hline Petroleum refineries & $\$ 440.00$ & $\$ 35.10$ & $\$ 12.60$ & $\$ 1.39$ & $\$ 21.20$ & $\$ 201.00$ & $46 \%$ \\
\hline Oil and gas extraction & $\$ 396.00$ & $\$ 201.00$ & $\$ 26.40$ & $\$ 35.40$ & $\$ 139.00$ & $\$ 16.60$ & $4 \%$ \\
\hline Fiber, yarn, and thread mills & $\$ 382.00$ & $\$ 82.50$ & $\$ 75.30$ & $\$ 1.18$ & $\$ 6.08$ & $\$ 368.00$ & $97 \%$ \\
\hline Wholesale trade & $\$ 359.00$ & $\$ 250.00$ & $\$ 135.00$ & $\$ 58.50$ & $\$ 55.80$ & $\$ 164.00$ & $46 \%$ \\
\hline
\end{tabular}


Table C-6 - GFRP SIP Design: Embodied energy by source for raw materials

\begin{tabular}{|c|c|c|c|c|c|c|}
\hline Top Ten Sectors & Total Energy & Coal & NatGas & Petrol & Bio/Waste & NonFossElec \\
\hline & $10^{6} \mathrm{btu}$ & $10^{6} \mathrm{btu}$ & $10^{6} \mathrm{btu}$ & $10^{6} \mathrm{btu}$ & $10^{6} \mathrm{btu}$ & $10^{6} \mathrm{btu}$ \\
\hline Total for all sectors & $\mathbf{1 1 1 . 8 4}$ & 19.05 & 42.84 & 24.45 & 14.31 & 11.28 \\
\hline Plastics material and resin manufacturing & 24.07 & 1.01 & 12.51 & 5.23 & 2.56 & 2.74 \\
\hline Other basic organic chemical manufacturing & 21.33 & 2.66 & 8.15 & 2.95 & 6.43 & 1.15 \\
\hline Power generation and supply & 17.06 & 12.42 & 3.63 & 0.60 & 0.00 & 0.40 \\
\hline Petrochemical manufacturing & 13.93 & 0.19 & 5.47 & 5.34 & 2.32 & 0.62 \\
\hline Petroleum refineries & 7.15 & 0.00 & 1.91 & 4.63 & 0.35 & 0.25 \\
\hline Artificial and synthetic fibers and filaments manufacturing & 4.10 & 0.85 & 1.82 & 0.23 & 0.16 & 1.04 \\
\hline Oil and gas extraction & 3.53 & 0.00 & 2.88 & 0.30 & 0.00 & 0.35 \\
\hline Fiber, yarn, and thread mills & 1.36 & 0.07 & 0.24 & 0.03 & 0.13 & 0.90 \\
\hline Truck transportation & 1.20 & 0.00 & 0.00 & 1.19 & 0.00 & 0.01 \\
\hline Pipeline transportation & 1.18 & 0.00 & 0.90 & 0.00 & 0.00 & 0.29 \\
\hline
\end{tabular}

Table C-7 - GFRP SIP Design: Greenhouse gases in equivalent lbs of $\mathrm{CO}_{2}$ for raw materials

\begin{tabular}{|c|c|c|c|c|c|c|}
\hline Top Ten Sectors & Total & $\mathrm{CO}_{2}$ Fossil & $\mathrm{CO}_{2}$ Process & $\mathrm{CH}_{4}$ & $\mathbf{N}_{2} \mathbf{O}$ & HFC/PFCs \\
\hline & lbs $\mathrm{CO}_{2} \mathrm{e}$ & lbs $\mathrm{CO}_{2} \mathrm{e}$ & lbs $\mathrm{CO}_{2} \mathrm{e}$ & lbs $\mathrm{CO}_{2} \mathrm{e}$ & lbs $\mathrm{CO}_{2} \mathrm{e}$ & lbs $\mathrm{CO}_{2} \mathrm{e}$ \\
\hline Total for all sectors & 15774 & 12738 & 711 & 1267 & 763 & 301 \\
\hline Power generation and supply & 3234 & 3190 & 0 & 9 & 20 & 21 \\
\hline Plastics material and resin manufacturing & 2420 & 2420 & 0 & 0 & 0 & 0 \\
\hline Other basic organic chemical manufacturing & 2160 & 1938 & 0 & 0 & 222 & 0 \\
\hline Petrochemical manufacturing & 1756 & 1470 & 203 & 84 & 0 & 0 \\
\hline Oil and gas extraction & 1366 & 385 & 251 & 730 & 0 & 0 \\
\hline Petroleum refineries & 990 & 988 & 0 & 3 & 0 & 0 \\
\hline Artificial and synthetic fibers and filaments manufacturing & 425 & 425 & 0 & 0 & 0 & 0 \\
\hline Fertilizer Manufacturing & 299 & 74 & 100 & 0 & 125 & 0 \\
\hline Industrial gas manufacturing & 293 & 34 & 0 & 0 & 0 & 260 \\
\hline Cotton farming & 253 & 63 & 0 & 0 & 190 & 0 \\
\hline
\end{tabular}


Table C-8 - KFRP SIP Design: Economic activity for raw materials

\begin{tabular}{|c|c|c|c|c|c|c|c|}
\hline Top Ten Sectors & $\begin{array}{c}\text { Total } \\
\text { Economic }\end{array}$ & $\begin{array}{c}\text { Total Value } \\
\text { Added }\end{array}$ & $\begin{array}{l}\text { Employee } \\
\text { Comp VA }\end{array}$ & $\begin{array}{c}\text { Net Tax } \\
\text { VA }\end{array}$ & $\begin{array}{c}\text { Profits } \\
\text { VA }\end{array}$ & $\begin{array}{c}\text { Direct } \\
\text { Economic }\end{array}$ & $\begin{array}{c}\text { Direct } \\
\text { Economic }\end{array}$ \\
\hline Total for all sectors & $\$ 10,200.00$ & $\$ 3,490.00$ & $\$ 1,970.00$ & $\$ 199.00$ & $\$ 1,320.00$ & $\$ 6,310.00$ & $62 \%$ \\
\hline Plastics material and resin manufacturing & $\$ 1,920.00$ & $\$ 333.00$ & $\$ 208.00$ & $\$ 14.80$ & $\$ 109.00$ & $\$ 1,810.00$ & $94 \%$ \\
\hline Other basic organic chemical manufacturing & $\$ 875.00$ & $\$ 138.00$ & $\$ 111.00$ & $\$ 7.96$ & $\$ 18.90$ & $\$ 623.00$ & $71 \%$ \\
\hline Petrochemical manufacturing & $\$ 682.00$ & $\$ 105.00$ & $\$ 22.40$ & $\$ 4.07$ & $\$ 79.00$ & $\$ 589.00$ & $86 \%$ \\
\hline $\begin{array}{l}\text { Urethane and Other Foam Product (except } \\
\text { Polystyrene) Manufacturing }\end{array}$ & $\$ 638.00$ & $\$ 211.00$ & $\$ 126.00$ & $\$ 2.36$ & $\$ 82.80$ & $\$ 637.00$ & $100 \%$ \\
\hline Petroleum refineries & $\$ 451.00$ & $\$ 36.00$ & $\$ 12.90$ & $\$ 1.43$ & $\$ 21.70$ & $\$ 215.00$ & $48 \%$ \\
\hline Fiber, yarn, and thread mills & $\$ 450.00$ & $\$ 97.20$ & $\$ 88.70$ & $\$ 1.38$ & $\$ 7.16$ & $\$ 434.00$ & $96 \%$ \\
\hline Management of companies and enterprises & $\$ 441.00$ & $\$ 273.00$ & $\$ 231.00$ & $\$ 6.71$ & $\$ 35.20$ & $\$ 202.00$ & $46 \%$ \\
\hline Oil and gas extraction & $\$ 403.00$ & $\$ 204.00$ & $\$ 26.90$ & $\$ 36.00$ & $\$ 141.00$ & $\$ 16.20$ & $4 \%$ \\
\hline Wholesale trade & $\$ 355.00$ & $\$ 247.00$ & $\$ 134.00$ & $\$ 57.90$ & $\$ 55.20$ & $\$ 164.00$ & $46 \%$ \\
\hline Sawmills and wood preservation & $\$ 170.00$ & $\$ 38.40$ & $\$ 30.10$ & $\$ 0.73$ & $\$ 7.59$ & $\$ 159.00$ & $94 \%$ \\
\hline
\end{tabular}

Table C-9 - KFRP SIP Design: Embodied energy by source for raw materials

\begin{tabular}{|c|c|c|c|c|c|c|}
\hline Top Ten Sectors & Total Energy & Coal & NatGas & Petrol & Bio/Waste & NonFossElec \\
\hline & $10^{6} \mathrm{btu}$ & $10^{6} \mathrm{btu}$ & $10^{6} \mathrm{btu}$ & $10^{6} \mathrm{btu}$ & $10^{6} \mathrm{btu}$ & $10^{6} \mathrm{btu}$ \\
\hline Total for all sectors & 112.79 & 18.10 & 43.79 & 25.78 & 14.50 & 10.99 \\
\hline Plastics material and resin manufacturing & 27.58 & 1.16 & 14.41 & 5.99 & 2.94 & 3.14 \\
\hline Other basic organic chemical manufacturing & 20.47 & 2.56 & 7.83 & 2.83 & 6.18 & 1.11 \\
\hline Power generation and supply & 16.68 & 12.13 & 3.55 & 0.59 & 0.00 & 0.39 \\
\hline Petrochemical manufacturing & 16.02 & 0.22 & 6.27 & 6.13 & 2.66 & 0.71 \\
\hline Petroleum refineries & 7.33 & 0.00 & 1.95 & 4.75 & 0.36 & 0.26 \\
\hline Oil and gas extraction & 3.58 & 0.00 & 2.93 & 0.31 & 0.00 & 0.35 \\
\hline Fiber, yarn, and thread mills & 1.61 & 0.08 & 0.29 & 0.03 & 0.15 & 1.06 \\
\hline Pipeline transportation & 1.21 & 0.00 & 0.92 & 0.00 & 0.00 & 0.29 \\
\hline Truck transportation & 1.18 & 0.00 & 0.00 & 1.18 & 0.00 & 0.01 \\
\hline Alkalies and chlorine manufacturing & 1.16 & 0.14 & 0.65 & 0.01 & 0.11 & 0.25 \\
\hline
\end{tabular}


Table C-10 - KFRP SIP Design: Greenhouse gases in equivalent lbs of $\mathrm{CO}_{2}$ for raw materials

\begin{tabular}{|c|c|c|c|c|c|c|}
\hline Top Ten Sectors & Total & $\mathrm{CO}_{2}$ Fossil & $\mathrm{CO}_{2}$ Process & $\mathrm{CH}_{4}$ & $\mathbf{N}_{2} \mathbf{O}$ & HFC/PFCs \\
\hline & lbs $\mathrm{CO}_{2} \mathrm{e}$ & lbs $\mathrm{CO}_{2} \mathrm{e}$ & lbs $\mathrm{CO}_{2} \mathrm{e}$ & lbs $\mathrm{CO}_{2} \mathrm{e}$ & lbs $\mathrm{CO}_{2} \mathrm{e}$ & lbs $\mathrm{CO}_{2} \mathrm{e}$ \\
\hline Total for all sectors & 15994 & 12848 & 746 & 1294 & 801 & 304 \\
\hline Power generation and supply & 3168 & 3124 & 0 & 9 & 19 & 20 \\
\hline Plastics material and resin manufacturing & 2772 & 2772 & 0 & 0 & 0 & 0 \\
\hline Other basic organic chemical manufacturing & 2077 & 1861 & 0 & 0 & 214 & 0 \\
\hline Petrochemical manufacturing & 2015 & 1687 & 233 & 96 & 0 & 0 \\
\hline Oil and gas extraction & 1390 & 392 & 255 & 744 & 0 & 0 \\
\hline Petroleum refineries & 1014 & 1012 & 0 & 3 & 0 & 0 \\
\hline Fertilizer Manufacturing & 319 & 79 & 107 & 0 & 133 & 0 \\
\hline Cotton farming & 304 & 76 & 0 & 0 & 229 & 0 \\
\hline Industrial gas manufacturing & 299 & 35 & 0 & 0 & 0 & 264 \\
\hline Pipeline transportation & 235 & 108 & 0 & 127 & 0 & 0 \\
\hline
\end{tabular}

Table C-11 - Stick-Framing Design: Economic activity for raw materials

\begin{tabular}{|c|c|c|c|c|c|c|c|}
\hline Top Ten Sectors & $\begin{array}{c}\text { Total } \\
\text { Economic }\end{array}$ & $\begin{array}{c}\text { Total Value } \\
\text { Added }\end{array}$ & $\begin{array}{l}\text { Employee } \\
\text { Comp VA }\end{array}$ & $\begin{array}{c}\text { Net Tax } \\
\text { VA }\end{array}$ & $\begin{array}{c}\text { Profits } \\
\text { VA }\end{array}$ & $\begin{array}{c}\text { Direct } \\
\text { Economic }\end{array}$ & $\begin{array}{c}\text { Direct } \\
\text { Economic }\end{array}$ \\
\hline & $\$$ & $\$$ & $\$$ & $\$$ & $\$$ & $\$$ & $\%$ \\
\hline Total for all sectors & $\$ 3,650.00$ & $\$ 1,490.00$ & $\$ 802.00$ & $\$ 79.70$ & $\$ 610.00$ & $\$ 2,490.00$ & $68 \%$ \\
\hline Reconstituted wood product manufacturing & $\$ 491.00$ & $\$ 170.00$ & $\$ 94.30$ & $\$ 2.33$ & $\$ 73.50$ & $\$ 489.00$ & $99 \%$ \\
\hline Lime and gypsum product manufacturing & $\$ 488.00$ & $\$ 199.00$ & $\$ 88.20$ & $\$ 2.94$ & $\$ 108.00$ & $\$ 487.00$ & $100 \%$ \\
\hline Sawmills and wood preservation & $\$ 463.00$ & $\$ 104.00$ & $\$ 81.70$ & $\$ 1.97$ & $\$ 20.60$ & $\$ 441.00$ & $95 \%$ \\
\hline Logging & $\$ 316.00$ & $\$ 108.00$ & $\$ 50.00$ & $\$ 7.38$ & $\$ 51.10$ & $\$ 190.00$ & $60 \%$ \\
\hline Wholesale trade & $\$ 108.00$ & $\$ 75.00$ & $\$ 40.70$ & $\$ 17.60$ & $\$ 16.80$ & $\$ 48.10$ & $45 \%$ \\
\hline Mineral wool manufacturing & $\$ 97.80$ & $\$ 45.00$ & $\$ 22.30$ & $\$ 0.72$ & $\$ 22.00$ & $\$ 97.60$ & $100 \%$ \\
\hline Forest nurseries, forest products, and timber tracts & $\$ 92.20$ & $\$ 35.80$ & $\$ 4.05$ & $\$ 7.02$ & $\$ 24.70$ & $\$ 27.00$ & $29 \%$ \\
\hline Truck transportation & $\$ 91.20$ & $\$ 41.40$ & $\$ 27.50$ & $\$ 1.65$ & $\$ 12.30$ & $\$ 63.50$ & $70 \%$ \\
\hline Management of companies and enterprises & $\$ 90.20$ & $\$ 55.90$ & $\$ 47.30$ & $\$ 1.37$ & $\$ 7.20$ & $\$ 44.30$ & $49 \%$ \\
\hline Power generation and supply & $\$ 73.40$ & $\$ 50.00$ & $\$ 15.30$ & $\$ 8.82$ & $\$ 25.90$ & $\$ 50.60$ & $69 \%$ \\
\hline
\end{tabular}


Table C-12 - Stick-Framing Design: Embodied energy by source for raw materials

\begin{tabular}{|c|c|c|c|c|c|c|}
\hline Top Ten Sectors & Total Energy & Coal & NatGas & Petrol & Bio/Waste & NonFossElec \\
\hline & $10^{6}$ btu & $10^{6} \mathrm{btu}$ & $10^{6} \mathrm{btu}$ & $10^{6}$ btu & $10^{6} \mathrm{btu}$ & $10^{6} \mathrm{btu}$ \\
\hline Total for all sectors & 41.42 & 8.71 & 15.73 & 6.41 & 6.06 & 4.52 \\
\hline Lime and gypsum product manufacturing & 12.99 & 2.22 & 8.00 & 1.61 & 0.05 & 1.08 \\
\hline Power generation and supply & 7.56 & 5.51 & 1.61 & 0.27 & 0.00 & 0.18 \\
\hline Reconstituted wood product manufacturing & 6.09 & 0.05 & 1.82 & 0.44 & 2.39 & 1.40 \\
\hline Sawmills and wood preservation & 2.36 & 0.00 & 0.23 & 0.12 & 1.63 & 0.38 \\
\hline Paperboard Mills & 2.17 & 0.20 & 0.45 & 0.09 & 1.28 & 0.15 \\
\hline Truck transportation & 1.13 & 0.00 & 0.00 & 1.12 & 0.00 & 0.01 \\
\hline Mineral wool manufacturing & 1.00 & 0.06 & 0.71 & 0.00 & 0.00 & 0.23 \\
\hline Petroleum refineries & 0.72 & 0.00 & 0.19 & 0.47 & 0.04 & 0.03 \\
\hline Other nonmetallic mineral mining & 0.65 & 0.00 & 0.33 & 0.19 & 0.00 & 0.14 \\
\hline Oil and gas extraction & 0.56 & 0.00 & 0.45 & 0.05 & 0.00 & 0.05 \\
\hline
\end{tabular}

Table C-13 - Stick-Framing Design: Greenhouse gases in equivalent lbs of $\mathrm{CO}_{2}$ for raw materials

\begin{tabular}{|c|c|c|c|c|c|c|}
\hline Top Ten Sectors & Total & $\mathrm{CO}_{2}$ Fossil & $\mathrm{CO}_{2}$ Process & $\mathrm{CH}_{4}$ & $\mathbf{N}_{2} \mathbf{O}$ & HFC/PFCs \\
\hline & lbs $\mathrm{CO}_{2} \mathrm{e}$ & lbs $\mathrm{CO}_{2} \mathrm{e}$ & lbs $\mathrm{CO}_{2} \mathrm{e}$ & lbs $\mathrm{CO}_{2} \mathrm{e}$ & lbs $\mathrm{CO}_{2} \mathrm{e}$ & lbs $\mathrm{CO}_{2} \mathrm{e}$ \\
\hline Total for all sectors & 8382 & 4774 & 3058 & 332 & 189 & 29 \\
\hline Lime and gypsum product manufacturing & 4576 & 1701 & 2882 & 0 & 0 & 0 \\
\hline Power generation and supply & 1441 & 1419 & 0 & 4 & 9 & 9 \\
\hline Reconstituted wood product manufacturing & 299 & 299 & 0 & 0 & 0 & 0 \\
\hline Oil and gas extraction & 216 & 61 & 40 & 116 & 0 & 0 \\
\hline Truck transportation & 194 & 194 & 0 & 0 & 0 & 0 \\
\hline Cement manufacturing & 145 & 60 & 84 & 0 & 0 & 0 \\
\hline Paperboard Mills & 110 & 110 & 0 & 0 & 0 & 0 \\
\hline All other crop farming & 105 & 22 & 0 & 0 & 83 & 0 \\
\hline Petroleum refineries & 100 & 100 & 0 & 0 & 0 & 0 \\
\hline Mineral wool manufacturing & 96 & 96 & 0 & 0 & 0 & 0 \\
\hline
\end{tabular}


Table C-14 - ICF Design: Economic activity for raw materials

\begin{tabular}{|c|c|c|c|c|c|c|c|}
\hline Top Ten Sectors & $\begin{array}{c}\text { Total } \\
\text { Economic }\end{array}$ & $\begin{array}{c}\text { Total Value } \\
\text { Added }\end{array}$ & $\begin{array}{l}\text { Employee } \\
\text { Comp VA }\end{array}$ & $\begin{array}{l}\text { Net Tax } \\
\text { VA }\end{array}$ & $\begin{array}{c}\text { Profits } \\
\text { VA }\end{array}$ & $\begin{array}{c}\text { Direct } \\
\text { Economic }\end{array}$ & $\begin{array}{c}\text { Direct } \\
\text { Economic }\end{array}$ \\
\hline Total for all sectors & $\$ 5,930.00$ & $\$ 2,560.00$ & $\$ 1,440.00$ & $\$ 125.00$ & $\$ 995.00$ & $\$ 4,270.00$ & $72 \%$ \\
\hline Ready-mix concrete manufacturing & $\$ 1,520.00$ & $\$ 509.00$ & $\$ 333.00$ & $\$ 9.16$ & $\$ 167.00$ & $\$ 1,520.00$ & $100 \%$ \\
\hline Lime and gypsum product manufacturing & $\$ 489.00$ & $\$ 200.00$ & $\$ 88.40$ & $\$ 2.95$ & $\$ 109.00$ & $\$ 486.00$ & $99 \%$ \\
\hline Polystyrene Foam Product Manufacturing & $\$ 294.00$ & $\$ 116.00$ & $\$ 58.00$ & $\$ 8.49$ & $\$ 49.10$ & $\$ 294.00$ & $100 \%$ \\
\hline Cement manufacturing & $\$ 286.00$ & $\$ 136.00$ & $\$ 47.40$ & $\$ 3.53$ & $\$ 85.20$ & $\$ 272.00$ & $95 \%$ \\
\hline Truck transportation & $\$ 230.00$ & $\$ 104.00$ & $\$ 69.20$ & $\$ 4.15$ & $\$ 30.90$ & $\$ 185.00$ & $80 \%$ \\
\hline Iron and steel mills & $\$ 222.00$ & $\$ 60.20$ & $\$ 44.10$ & $\$ 1.41$ & $\$ 14.70$ & $\$ 203.00$ & $91 \%$ \\
\hline Management of companies and enterprises & $\$ 199.00$ & $\$ 123.00$ & $\$ 104.00$ & $\$ 3.03$ & $\$ 15.90$ & $\$ 99.80$ & $50 \%$ \\
\hline Wholesale trade & $\$ 168.00$ & $\$ 117.00$ & $\$ 63.50$ & $\$ 27.40$ & $\$ 26.20$ & $\$ 91.80$ & $55 \%$ \\
\hline Sand, gravel, clay, and refractory mining & $\$ 126.00$ & $\$ 66.00$ & $\$ 39.10$ & $\$ 2.81$ & $\$ 24.00$ & $\$ 120.00$ & $95 \%$ \\
\hline Power generation and supply & $\$ 107.00$ & $\$ 73.20$ & $\$ 22.30$ & $\$ 12.90$ & $\$ 37.90$ & $\$ 43.40$ & $41 \%$ \\
\hline
\end{tabular}

Table C-15 - ICF Design: Embodied energy by source for raw materials

\begin{tabular}{|c|c|c|c|c|c|c|}
\hline Top Ten Sectors & Total Energy & Coal & NatGas & Petrol & Bio/Waste & NonFossElec \\
\hline & $10^{6} \mathrm{btu}$ & $10^{6} \mathrm{btu}$ & $10^{6} \mathrm{btu}$ & $10^{6} \mathrm{btu}$ & $10^{6} \mathrm{btu}$ & $10^{6} \mathrm{btu}$ \\
\hline Total for all sectors & 71.75 & 25.69 & 21.99 & 12.89 & 4.37 & 6.72 \\
\hline Cement manufacturing & 15.83 & 9.57 & 0.82 & 2.54 & 1.45 & 1.47 \\
\hline Lime and gypsum product manufacturing & 12.99 & 2.23 & 8.02 & 1.61 & 0.05 & 1.09 \\
\hline Power generation and supply & 11.09 & 8.06 & 2.36 & 0.39 & 0.00 & 0.26 \\
\hline Iron and steel mills & 6.90 & 4.09 & 1.88 & 0.07 & 0.03 & 0.83 \\
\hline Ready-mix concrete manufacturing & 3.71 & 0.60 & 2.15 & 0.48 & 0.17 & 0.31 \\
\hline Truck transportation & 2.84 & 0.00 & 0.00 & 2.82 & 0.00 & 0.03 \\
\hline Paperboard Mills & 2.39 & 0.22 & 0.49 & 0.10 & 1.41 & 0.16 \\
\hline Other basic organic chemical manufacturing & 1.68 & 0.21 & 0.64 & 0.23 & 0.51 & 0.09 \\
\hline Sand, gravel, clay, and refractory mining & 1.43 & 0.11 & 0.47 & 0.59 & 0.00 & 0.25 \\
\hline Petroleum refineries & 1.10 & 0.00 & 0.29 & 0.71 & 0.05 & 0.04 \\
\hline
\end{tabular}


Table C-16 - ICF Design: Greenhouse gases in equivalent lbs of $\mathrm{CO}_{2}$ for raw materials

\begin{tabular}{|c|c|c|c|c|c|c|}
\hline Top Ten Sectors & Total & $\mathrm{CO}_{2}$ Fossil & $\mathrm{CO}_{2}$ Process & $\mathrm{CH}_{4}$ & $\mathbf{N}_{2} \mathbf{O}$ & HFC/PFCs \\
\hline & lbs $\mathrm{CO}_{2} \mathrm{e}$ & lbs $\mathrm{CO}_{2} \mathrm{e}$ & lbs $\mathrm{CO}_{2} \mathrm{e}$ & lbs $\mathrm{CO}_{2} \mathrm{e}$ & lbs $\mathrm{CO}_{2} \mathrm{e}$ & lbs $\mathrm{CO}_{2} \mathrm{e}$ \\
\hline Total for all sectors & 17952 & 9724 & 7524 & 532 & 83 & 73 \\
\hline Cement manufacturing & 6336 & 2640 & 3696 & 0 & 0 & 0 \\
\hline Lime and gypsum product manufacturing & 4576 & 1705 & 2882 & 0 & 0 & 0 \\
\hline Power generation and supply & 2105 & 2075 & 0 & 6 & 13 & 13 \\
\hline Iron and steel mills & 1379 & 521 & 851 & 8 & 0 & 0 \\
\hline Truck transportation & 488 & 488 & 0 & 0 & 0 & 0 \\
\hline Ready-mix concrete manufacturing & 466 & 466 & 0 & 0 & 0 & 0 \\
\hline Oil and gas extraction & 326 & 92 & 60 & 174 & 0 & 0 \\
\hline Coal mining & 198 & 22 & 0 & 176 & 0 & 0 \\
\hline Sand, gravel, clay, and refractory mining & 176 & 176 & 0 & 0 & 0 & 0 \\
\hline Other basic organic chemical manufacturing & 170 & 152 & 0 & 0 & 17 & 0 \\
\hline
\end{tabular}




\section{REFERENCES}

Ahmed, K. I. (2005). "Handbook on Design and Construction of Housing for Flood-Prone Rural Areas of Bangladesh." Asian Disaster Preparedness Center, Bangkok.

Ahmed, K. S., and Vijayarangan, S. (2007). "Experimental Characterization of Woven JuteFabric-Reinforced Isothalic Polyester Composites." Journal of Applied Polymer Science, 104(4), 2650-2662.

Åkesson, D., Skrifvars, M., Seppälä, J. V., and Walkenström, P. (2006). "Preparation of Natural Fibre Composites from Biobased Thermoset Resins." 27th Risø International Symposium on Materials Science: Polymer Composite Materials for Wind Power Turbines.

Alam, M. K., Khan, M. A., and Lehmann, E. H. (2006). "Comparative Study of Water Absorption Behavior in Biopol ${ }^{\circledR}$ and Jute-reinforced Biopol ${ }^{\circledR}$ Composite using Neutron Radiography Technique." Journal of Reinforced Plastics and Composites, 25(11), 1179-1187.

Alibaba.com. (2013). "Alibaba.com: Global Trade Starts Here." http://www.alibaba.com/ (March 14, 2013).

Ashby, M. F. (2013). Materials and the Environment: Eco-Informed Material Choice. Butterworth-Heinemann, Oxford, UK.

Ayers, S. R., Van Erp, G. M., Heldt, T. J., and Cattell, C. (2004). "Implementation of Innovative Fibre Composite Structures in Australian Civil Engineering." Polymer Composites III 2004: Transportation Infrastructure, Defense, and Novel Applications of Composites, R. C. Creese, and H. GangaRao, eds., DEStech, Lancaster, PA, 145-159.

Bakare, I. O., Okieimen, F. E., Pavithran, C., Abdul Khalil, H. P. S., and Brahmakumar, M. (2010). "Mechanical and Thermal Properties of Sisal Fiber-Reinforced Rubber Seed Oil-based Polyurethane Composites." Materials and Design, 31(9), 4274-4280.

Baksi, S., Srikanth, G., Babu, M. S., and Biswas, S. (2010). "Composites as Building Material Indian Scenario." Technology Information, Forecasting and Assessment Council; Department of Science and Technology, Govt. of India.

Bangladesh Bureau of Statistics. (2010a). "Estimates of Jute, 2009-2010." http://www.bbs.gov.bd/ (March 17, 2013).

Bangladesh Bureau of Statistics. (2010b). "Report on Survey of Manufacturing Industries 200506." http://www.bbs.gov.bd/ (March 17, 2013).

Bangladesh Bureau of Statistics. (2010c). "Report on the Cost of Production of Jute Crop, 20082009." http://www.bbs.gov.bd/ (March 17, 2013).

Barbero, E. J. (2011). Introduction to Composite Materials Design. Taylor \& Francis, Boca Raton.

Baumann, H., and Tillman, A. (2004). The Hitch Hiker's Guide to LCA. Studentlitteratur AB, Lund, Sweden.

Beckwith, S. W. (2003). "Natural Fiber Reinforcement Materials: Lower Cost Technology for Composites Applications." Composites Fabrication, Nov/Dec 12-16. 
Belgacem, M. N., and Gandini, A. (2005). "The Surface Modification of Cellulose Fibres for Use as Reinforcing Elements in Composite Materials." Composite Interfaces, 12(1-2), 41-75.

Bismarck, A., Mishra, S., and Lampke, T. (2005). "Plant Fibers as Reinforcement for Green Composites." Natural Fibers, Biopolymers, and Biocomposites, A. K. Mohanty, M. Misra, and L. T. Drzal, eds., Taylor \& Francis, Boca Raton, 37-108.

Blandford, M. M. (2010). "Critical Buckling Strength Prediction of Pultruded GFRP Composite Columns". M.S. Thesis. West Virginia University.

Bourbigot, S., Le Bras, M., Duquesne, S., and Rochery, M. (2004). "Recent Advances for Intumescent Polymers." Macromolecular Materials and Engineering, 289(6), 499-511.

Brouwer, W. D. (2000). "Natural Fibre Composites: Where Can Flax Compete With Glass?" SAMPE Journal, 36(6), 18-23.

Burgueño, R., Quagliata, M. J., Mohanty, A. K., Mehta, G., Drzal, L. T., and Misra, M. (2004). "Load-Bearing Natural Fiber Composite Cellular Beams and Panels." Composites Part A: Applied Science and Manufacturing, 35(6), 645-656.

Burgueño, R., Quagliata, M. J., Mehta, G. M., Mohanty, A. K., Misra, M., and Drzal, L. T. (2005). "Sustainable Cellular Biocomposites from Natural Fibers and Unsaturated Polyester Resin for Housing Panel Applications." Journal of Polymers and the Environment, 13(2), 139149.

Carnegie Mellon University Green Design Institute. (2013). "Economic Input-Output Life Cycle Assessment (EIO-LCA) US 2002 (428) model." http://www.eiolca.net (March 14, 2013).

Centre for Building Performance Research. (2007). "Table of Embodied Energy Coefficients." http://www.victoria.ac.nz/cbpr/resources/index.aspx (March 14, 2013).

Chabba, S., and Netravali, A. N. (2004). "'Green' Composites Using Modified Soy Protein Concentrate Resin and Flax Fabrics and Yarns." JSME International Journal, Series A: Solid Mechanics and Material Engineering, 47(4), 556-560.

Chamis, C. C. (1974). "Analysis of the Three-Point-Bend Test for Materials with Unequal Tension and Compression Properties." Rep. No. NASA TN D-7572, National Aeronautics and Space Administration, Lewis Research Center, Cleveland, $\mathrm{OH}$.

Chandrashekhara, K. (2004). "Energy Efficient and Sustainable Building Enclosures Using Biobased Materials." NSF-PATH Housing Research Agenda, Volume 2, M. Syal, ed.,187-191.

Chang, W. P., Kim, K. J., and Gupta, R. K. (2009a). "Moisture Absorption Behavior of Wood/Plastic Composites Made with Ultrasound-Assisted Alkali-Treated Wood Particulates." Composite Interfaces, 16(7-9), 937-951.

Chang, W. P., Kim, K. J., and Gupta, R. K. (2009b). "Ultrasound-Assisted Surface-Modification of Wood Particulates for Improved Wood/Plastic Composites." Composite Interfaces, 16(7-9), 687-709.

Chapple, S., and Anandjiwala, R. (2010). "Flammability of Natural Fiber-Reinforced Composites and Strategies for Fire Retardancy: A Review." Journal of Thermoplastic Composite Materials, 23(6), 871-893. 
Chin, J. W., Haight, M. R., Hughes, W. L., and Nguyen, T. (1998). "Environmental Effects on Composite Matrix Resins Used in Construction." Durability of Fibre Reinforced Polymer (FRP) Composites for Construction (CDCC'98), 229-241.

Christian, S. J., and Billington, S. (2009). "Sustainable Biocomposites for Construction." COMPOSITES \& POLYCON 2009.

Cramer, S. M. (2004). "Structural Design and Materials - Research Needed to Reinvent Housing in the United States." NSF-PATH Housing Research Agenda, Volume 1, M. Syal, ed.,36-47.

Crawford, R. H. (2011). Life Cycle Assessment in the Built Environment. Spon Press, New York, NY.

Czigany, T. (2004). "An Acoustic Emission Study of Flax Fiber-Reinforced Polypropylene Composites " Journal of Composite Materials, 38(9), 769-778.

Daniel, I. M. (2009). "Influence of Core Properties on the Failure of Composite Sandwich Beams." Journal of Mechanics of Materials and Structures, 4(7-8), 1271-1286.

Dash, B. N., Rana, A. K., Mishra, H. K., Nayak, S. K., and Tripathy, S. S. (2000). "Novel LowCost Jute-Polyester Composites. III. Weathering and Thermal Behavior." Journal of Applied Polymer Science, 78(9), 1671-1679.

Davids, W. G., Dagher, H. J., Cassidy, E. D., Martin, K., and Gardner, D. (2004). "FRPReinforced Oriented Strand Board Panels in Wood-Framed Shear Wall Construction." NSFPATH Housing Research Agenda, Volume 2, M. Syal, ed.,162-171.

De, D., Adhikari, B., and De, D. (2007). "Grass Fiber Reinforced Phenol Formaldehyde Resin Composite: Preparation, Characterization and Evaluation of Properties of Composite." Polymers for Advanced Technologies, 18(1), 72-81.

Defosse, M. (2004). "Natural Fibers’ Growing Needs." PlasticsToday.Com.

Deliglio, T. (2010). "Stronger, Lighter, Cheaper... Naturally." Modern Plastics Worldwide, September 30-31.

Despres, C. (1991). "The Meaning of Home: Literature Review and Directions for Future Research and Theoretical Development." Journal of Architectural and Planning Research, 8(2), 96-115.

Dittenber, D. B., and GangaRao, H. V. S. (2011). "Critical Review of Recent Publications on Use of Natural Composites in Infrastructure." Journal of Composites Part A: Applied Science and Manufacturing, 43(8), 1419-1429.

Dodd, R. B., and Akin, D. E. (2005). "Recent Developments in Retting and Measurement of Fiber Quality in Natural Fibers: Pro and Cons." Natural Fibers, Biopolymers, and Biocomposites, A. K. Mohanty, M. Misra, and L. T. Drzal, eds., Taylor \& Francis, Boca Raton, 141-158.

Doherty, W., Halley, P., Edye, L., Rogers, D., Cardona, F., Park, Y., and Woo, T. (2007). "Studies on Polymers and Composites from Lignin and Fiber Derived from Sugar Cane." Polymers for Advanced Technologies, 18(8), 673-678. 
Drzal, L. T., Mohanty, A. K., Burgueño, R., and Misra, M. (2004). "Biobased Structural Composite Materials for Housing and Infrastructure Applications: Opportunities and Challenges." NSF-PATH Housing Research Agenda, Volume 2, M. Syal, ed.,129-140.

Dweib, M. A., O'Donnell, A., Wool, R. P., Hu, B., and Shenton III, H. W. (2005). "Houses Using Soy Oil and Natural Fiber Biocomposites." Natural Fibers, Biopolymers, and Biocomposites, A. K. Mohanty, M. Misra, and L. T. Drzal, eds., Taylor \& Francis, Boca Raton, 751-774.

Erlin, B., Hime, W., and Pistilli, M. (2005). "The Cost of Doing Business with Concrete." http://www.concreteconstruction.net/cement/the-cost-of-doing-business-with-concrete.aspx (March 14, 2013).

European Commission. (2005). "European Reference Life-Cycle Database: Oriented Strand Board; OSB III; Production Mix, at Plant." http://elcd.jrc.ec.europa.eu/ELCD3/ (March 14, 2013).

Evans, J. (2011). "Alternative Feedstocks... How Viable?" Plastics Engineering, February 13-17.

Evernden, M., and Mottram, T. J. (2009). "A Case for Houses in the UK to be Constructed of Fibre Reinforced Polymer Components." 11th International Conference on Non-conventional Materials and Technologies.

Food and Agriculture Organization of the United Nations. (2010). "Jute, Kenaf, Sisal, Abaca, Coir and Allied Fibres: Statistics." Rep. No. JU/HF/ST/2010/1, Trade and Marketing Division, FAO, Rome.

Fowler, P. A., Hughes, J. M., and Elias, R. M. (2006). "Biocomposites: Technology, Environmental Credentials and Market Forces." Journal of the Science of Food and Agriculture, 86(12), 1781-1789.

GangaRao, H. V. S., Batra, S., and Tolikonda, V. (2009). "Bending Properties of Flax-Vinyl Ester Composite." West Virginia University, Constructed Facilities Center, Morgantown, WV.

George, G., Joseph, K., Boudenne, A., and Thomas, S. (2010). "Recent Advances in Green Composites." Key Engineering Materials, 425 107-166.

Graedel, T. E., and Allenby, B. R. (2003). Industrial Ecology. Prentice Hall, Upper Saddle River, NJ.

Graham, F. (2011). "Rice, Straw and Coconut the New Alternatives to Wood." BBC News, http://bbc.co.uk (April 30, 2013).

Green, R. K., and Malpezzi, S. (2003). Primer on U.S. Housing Markets and Housing Policy. The Urban Institute, Washington, DC.

GreenSpec. (2013). "Oil-derived Insulation." http://www.greenspec.co.uk/insulation-oilderived.php (March 14, 2013).

Grozdanov, A., Avella, M., Buzarovska, A., Gentile, G., and Errico, M. E. (2010). "Reuse of Natural Fiber Reinforced Eco-Composites in Polymer Mortars." Polymer Engineering and Science, 50(4), 762-766.

Habitat for Humanity. (2011). "Bangladesh." Pamphlet, 1-3. 
Hagstrand, P. O., and Oksman, K. (2001). "Mechanical Properties and Morphology of Flax Fiber Reinforced Melamine-Formaldehyde Composites." Polymer Composites, 22(4), 568-578.

Hammond, G., and Jones, C. (2013). "GreenSpec: Embodied Energy." http://www.greenspec.co.uk/embodied-energy.php (March 14, 2013).

Haq, M., Burgueño, R., Mohanty, A. K., and Misra, M. (2009). "Processing Techniques for BioBased Unsaturated-Polyester/Clay Nanocomposites: Tensile Properties, Efficiency, and Limits." Composites Part A: Applied Science and Manufacturing, 40(4), 394-403.

Hendrickson, C. T., Lave, L. B., and Matthews, H. S. (2006). Environmental Life Cycle Assessment of Goods and Services: An Input-Output Approach. Resources for the Future, Washington, DC.

Henton, D. E., Gruber, P., Lunt, J., and Randall, J. (2005). "Polylactic Acid Technology." Natural Fibers, Biopolymers, and Biocomposites, A. K. Mohanty, M. Misra, and L. T. Drzal, eds., Taylor \& Francis, Boca Raton, 527-578.

Hoang, T. Q. T., Lagattu, F., and Brillaud, J. (2010). "Natural Fiber-Reinforced Recycled Polypropylene: Microstructural and Mechanical Properties." Journal of Reinforced Plastics and Composites, 29(2), 209-217.

Hoek-Smit, M. C. (1998). "Housing Finance in Bangladesh: Improving Access to Housing Finance by Middle and Lower Income Groups." The Government of Bangladesh, Bangladesh.

Hu, R., and Lim, J. K. (2007). "Fabrication and Mechanical Properties of Completely Biodegradable Hemp Fiber Reinforced Polylactic Acid Composites." Journal of Composite Materials, 41(13), 1655-1669.

Hu, W., Ton-That, M. T., Perrin-Sarazin, F., and Denault, J. (2010). "An Improved Method for Single Fiber Tensile Test of Natural Fibers." Polymer Engineering \& Science, 50(4), 819-825.

Huang, X., and Netravali, A. (2009). "Biodegradable Green Composites Made Using Bamboo Micro/Nano-Fibrils and Chemically Modified Soy Protein Resin." Composites Science and Technology, 69(7-8), 1009-1015.

Humphreys, M. F. (2009). "The Use of Polymer Composites in Construction." International Conference on FRP Composites for Infrastructure Applications.

Ibrahim, M. M., Dufresne, A., El-Zawawy, W. K., and Agblevor, F. A. (2010a). "Banana Fibers and Microfibrils as Lignocellulosic Reinforcements in Polymer Composites." Carbohydrate Polymers, 81(4), 811-819.

Ibrahim, N. A., Hadithon, K. A., and Abdan, K. (2010b). "Effect of Fiber Treatment on Mechanical Properties of Kenaf Fiber-Ecoflex Composites." Journal of Reinforced Plastics and Composites, 29(14), 2192-2198.

Jin, L., and Nava, H. (2002). "Opportunities and Challenges in Natural Fiber Reinforced Thermoset Composites." COMPOSITES 2002.

John, M. J., and Anandjiwala, R. D. (2008). "Recent Developments in Chemical Modification and Characterization of Natural Fiber-Reinforced Composites." Polymer Composites, 29(2), 187207. 
Johnston, D., and Gibson, S. (2008). Green from the Ground Up: Sustainable, Healthy, and Energy-Efficient Home Construction. Taunton, Newtown, CT.

Joseph, S., Oommen, Z., and Thomas, S. (2006). "Environmental Durability of Banana-FiberReinforced Phenol Formaldehyde Composites." Journal of Applied Polymer Science, 100(3), 2521-2531.

Joshi, S. V., Drzal, L. T., Mohanty, A. K., and Arora, S. (2004). "Are Natural Fiber Composites Environmentally Superior to Glass Fiber Reinforced Composites?" Composites Part A: Applied Science and Manufacturing, 35(3), 371-376.

Kalia, S., Kaith, B. S., and Kaur, I. (2009). "Pretreatments of Natural Fibers and their Application as Reinforcing Material in Polymer Composites - A Review." Polymer Engineering and Science, 49 1253-1272.

Kim, W., Argento, A., Lee, E., Flanigan, C., Houston, D., Harris, A., and Mielewski, D. F. (2012). "High Strain-rate Behavior of Natural Fiber-reinforced Polymer Composites." Journal of Composite Materials, 46(9), 1051-1065.

Koebel, C. T. (2004). "Housing Technology, Community, and the Economy." NSF-PATH Housing Research Agenda, Volume 1, M. Syal, ed., 66-76.

Kreže, T., Iskrač, S., Smole, M. S., Stana-Kleinschek, K., Strnad, S., and Fakin, D. (2005). "Flax Fibers Sorption Properties Influenced by Different Pretreatment Processes." Journal of Natural Fibers, 2(3), 25-37.

Kumar, R., and Zhang, L. (2009). "Soy Protein Films With the Hydrophobic Surface Created through Non-Covalent Interactions." Industrial Crops and Products, 29(2-3), 485-494.

Kumar, R., and Zhang, L. (2008). "Water-Induced Hydrophobicity of Soy Protein Materials Containing 2,2-Diphenyl-2-Hydroxyethanoic Acid." Biomacromolecules, 9(9), 2430-2437.

Lackey, E., Vaghan, J. G., Inamdar, K., and Hancock, B. (2004). "Statistical Characterization of Pultruded Composites with Natural Fiber Reinforcements." COMPOSITES 2004.

LaMore, R. L. (2004). "What are the Potential Effects of Technology in Affordable Housing Construction on the Development of Social Capital By Community-based Development Organizations." NSF-PATH Housing Research Agenda, Volume 2, M. Syal, ed.,266-273.

Lavadiya, D. N., and Fronk, T. H. (2011). "Mechanical Properties of Bio-Fibers for Composite Materials." SAMPE 2011.

Lawson, R. M., and Richards, J. (2010). "Modular Design for High-Rise Buildings." Institution of Civil Engineers: Structures and Buildings SB3, 151-164.

Li, F., and Larock, R. C. (2005). "Synthesis, Properties, and Potential Applications of Novel Thermosetting Biopolymers from Soybean and Other Natural Oils." Natural Fibers, Biopolymers, and Biocomposites, A. K. Mohanty, ed., Taylor \& Francis, Boca Raton, 727-750.

Liang, R., and Hota, G. (2009). "Fiber Reinforced Composites for Civil Infrastructures." International Conference on Fiber Reinforced Polymer (FRP) Composites for Infrastructure Applications. 
Little, J. C., and Hodgson, A. T. (2004). "Structural Insulated Panels: Sustainable Design Incorporating Impact on Indoor Air Quality." NSF-PATH Housing Research Agenda, Volume 2, M. Syal, ed.,178-186.

Majjigapu, P. K. R. (2013). "Flexural Evaluation of Aged Kenaf Fiber Composites.” M.S. Problem Report. West Virginia University.

Malkapuram, R., Kumar, V., and Singh Negi, Y. (2009). "Recent Development in Natural Fiber Reinforced Polypropylene Composites." Journal of Reinforced Plastics and Composites, 28(10), 1169-1189.

Malveda, M., Blagoev, M., and Kumamoto, T. (2009). "Lactic Acid, Its Salts and Esters: Abstract." SRI Consulting.

MarketsandMarkets. (2011). "Global Lactic Acid \& Poly Lactic Acid (PLA) Market by Applications and Potential Opportunities (2009-2016): Report Description." MarketsandMarkets.

Marriott, J. M., Bilec, M. M., Harries, K. A., and Aktas, C. B. (2009). "Pultruded Green Composites." University of Pittsburgh, Department of Civil and Environmental Engineering, Pittsburgh, PA.

Martin, R. L. (2011). "R-Value Table: Insulation Values for Selected Materials." http://www.coloradoenergy.org/procorner/stuff/r-values.htm (March 13, 2013).

McIntosh, J., and Harrington, M. A. (2007). "Bio-SIPS: A Deeper Shade of Green?" Sustainable Building Conference: Transforming Our Built Environment.

Mehta, G., Mohanty, A. K., Drzal, L. T., Kamdem, D. P., and Misra, M. (2006). "Effect of Accelerated Weathering on Biocomposites Processed by SMC and Compression Molding." Journal of Polymers and the Environment, 14(4), 359-368.

Mercier, J., Bunsell, A., Castaing, P., and Renard, J. (2008). "Characterisation and Modelling of Aging of Composites." Composites Part A: Applied Science and Manufacturing, 39(2), 428-438.

Mitra, N., and Raja, B. R. (2012). "Improving Delamination Resistance Capacity of Sandwich Composite Columns with Initial Face/Core Debond." Composites Part B: Engineering, 43, 16041612.

Mo, X., Wang, D., and Sun, X. S. (2005). "Straw-Based Biomass and Biocomposites." Natural Fibers, Biopolymers, and Biocomposites, A. K. Mohanty, M. Misra, and L. T. Drzal, eds., Taylor \& Francis, Boca Raton, 473-496.

Mohanty, A. K., Liu, W., Tummala, P., Drzal, L. T., Misra, M., and Narayan, R. (2005a). "Soy Protein-Based Plastics, Blends, and Composites." Natural Fibers, Biopolymers, and Biocomposites, A. K. Mohanty, M. Misra, and L. T. Drzal, eds., Taylor \& Francis, Boca Raton, 699-726.

Mohanty, A. K., Misra, M., Drzal, L. T., Selke, S. E., Harte, B. R., and Hinrichsen, G. (2005b). "Natural Fibers, Biopolymers, and Biocomposites: An Introduction." Natural Fibers, Biopolymers, and Biocomposites, A. K. Mohanty, M. Misra, and L. T. Drzal, eds., Taylor \& Francis, Boca Raton, 1-36. 
Mohanty, A. K., Misra, M., and Drzal, L. T. (2002). "Sustainable Bio-Composites from Renewable Resources: Opportunities and Challenges in the Green Materials World." Journal of Polymers and the Environment, 10(1), 19-26.

Mohanty, A. K., Misra, M., and Drzal, L. T. (2001). "Surface Modifications of Natural Fibers and Performance of the Resulting Biocomposites: An Overview." Composite Interfaces, 8(5), 313-343.

Mohanty, A. K., Misra, M., and Hinrichsen, G. (2000). "Biofibres, Biodegradable Polymers and Biocomposites: An Overview." Macromolecular Materials and Engineering, 276-277 1-24.

Mueller, D. H., and Krobjilowski, A. (2003). "New Discovery in the Properties of Composites Reinforced with Natural Fibers." Journal of Industrial Textiles, 33(2), 111-130.

Mullens, M. A., and Hastak, M. (2004). "Defining a National Housing Research Agenda: Construction Management and Production." NSF-PATH Housing Research Agenda, Volume 1, M. Syal, ed., 19-35.

Munder, F., Furll, C., and Hempel, H. (2005). "Processing of Bast Fiber Plants for Industrial Application." Natural Fibers, Biopolymers, and Biocomposites, A. K. Mohanty, M. Misra, and L. T. Drzal, eds., Taylor \& Francis, Boca Raton, 109-140.

Mutnuri, B., Aktas, C. J., Marriott, J., Bilec, M., and GangaRao, H. (2010). "Natural Fiber Reinforced Pultruded Composites." COMPOSITES 2010.

Netravali, A. N. (2003). "'Green Composites from Cellulose Fabrics \& Soy Protein Resin." Rep. No. NTC Project: F01-CR01, National Textile Center.

Netravali, A. N., Huang, X., and Mizuta, K. (2007). "Advanced 'Green' Composites." Advanced Composite Materials: The Official Journal of the Japan Society of Composite Materials, 16(4), 269-282.

Newill, J. F., McKnight, S. H., Hoppel, C. P. R., and Cooper, G. R. (1999). "Effects of Coatings on Moisture Absorption of Composite Materials." Rep. No. ARL-TR-2099, Army Research Laboratory, Army Proving Ground, MD.

Nishino, T., Hirao, K., Kotera, M., Nakamae, K., and Inagaki, H. (2003). "Kenaf Reinforced Biodegradable Composite." Composites Science and Technology, 63(9), 1281-1286.

NTA, I. (2011). "Structural Insulated Panels (SIP)." Rep. No. SIPA120908-10, NTA, INC, Nappanee, Indiana.

Ochi, S. (2008). "Mechanical Properties of Kenaf Fibers and Kenaf/PLA Composites." Mechanics of Materials, 40(4-5), 446-452.

O'Dell, J. L. (1997). "Natural Fibers in Resin Transfer Molded Composites." The Fourth International Conference on Woodfiber-Plastic Composites, 280-285.

O'Donnell, A., Dweib, M. A., and Wool, R. P. (2004). "Natural Fiber Composites with Plant OilBased Resin." Composites Science and Technology, 64(9), 1135-1145.

Oksman, K. (2001). "High Quality Flax Fibre Composites Manufactured by the Resin Transfer Moulding Process." Journal of Reinforced Plastics and Composites, 20(7), 621-627. 
Oksman, K., Skrifvars, M., and Selin, J. -. (2003). "Natural Fibres as Reinforcement in Polylactic Acid (PLA) Composites." Composites Science and Technology, 63(9), 1317-1324.

Omar, T., Van Erp, G., and Key, P. (2007). "Modular Deployable Composite Shelters - Truss System." Advanced Composites in Construction.

Pasma, J. (2010). "Building Tighter Envelopes Yields Efficiencies: Energy Savings and Applications for Structural Insulated Panels." Structural Engineering and Design, (December), 30-33.

Patel, M. (2003). "Cumulative Energy Demand (CED) and Cumulative CO2 Emissions for Products of the Organic Chemical Industry." Energy, 28 721-740.

Patel, M., and Narayan, R. (2005). "How Sustainable are Biopolymers and Biobased Products? The Hope, the Doubt, and the Reality." Natural Fibers, Biopolymers, and Biocomposites, A. K. Mohanty, M. Misra, and L. T. Drzal, eds., Taylor \& Francis, Boca Raton, 833-854.

Pearce, D. (2003). "The Social and Economic Value of Construction: The Construction Industry's Contribution to Sustainable Development." nCRISP, the Construction Industry Research and Innovation Strategy Panel, London.

Peng, X., Fan, M., Hartley, J., and Al-Zubaidy, M. (2012). "Properties of Natural Fibre Composites made by Pultrusion Process." Journal of Composite Materials, 46(2), 237-246.

Pervaiz, M., and Sain, M. M. (2003). "Carbon Storage Potential in Natural Fiber Composites." Resources, Conservation and Recycling, 39(4), 325-340.

Plackett, D., and Sodergard, A. (2005). "Polylactide-Based Biocomposites." Natural Fibers, Biopolymers, and Biocomposites, A. K. Mohanty, M. Misra, and L. T. Drzal, eds., Taylor \& Francis, Boca Raton, 579-596.

Pott, G. T., Hueting, D., and van Duersen, J. (2000a). "A Commercially Attractive Method to Reduce Moisture Sensitivity of Lignocellulose Fibres, Without the Use of Chemicals." BIOSOURCE HEMP 2000.

Pott, G. T., Hueting, D., and van Duersen, J. (2000b). "Reduction of Moisture Sensitivity in Wood and Natural Fibres for Polymer Composites." 3rd International Wood and Natural Fibre Composites Symposium.

Qiu, R., Ren, X., Fifield, L. S., Simmons, K. L., and Li, K. (2011). "Hemp-Fiber-Reinforced Unsaturated Polyester Composites: Optimization of Processing and Improvement of Interfacial Adhesion." Journal of Applied Polymer Science, 121(2), 862-868.

Rahman, M. M., and Khan, M. A. (2007). "Surface Treatment of Coir (Cocos nucifera) Fibers and Its Influence on the Fibers' Physico-Mechanical Properties." Composites Science and Technology, 67(11-12), 2369-2376.

Ray, D., and Rout, J. (2005). "Thermoset Biocomposites." Natural Fibers, Biopolymers, and Biocomposites, A. K. Mohanty, M. Misra, and L. T. Drzal, eds., Taylor \& Francis, Boca Raton, 291-346.

Ray, A. K., Mondal, S., Das, S. K., and Ramachandrarao, P. (2005). "Bamboo - A Functionally Graded Composite-Correlation Between Microstructure and Mechanical Strength." Journal of Materials Science, 40(19), 5249-5253. 
Riedel, U., and Nickel, J. (1999). "Natural Fibre-Reinforced Biopolymers as Construction Materials - New Discoveries." Die Angewandte Makromolekulare Chemie, 272(1), 34-40.

Rong, M. Z., Zhang, M. Q., Liu, Y., Yang, G. C., and Zeng, H. M. (2001). "The Effect of Fiber Treatment on the Mechanical Properties of Unidirectional Sisal-Reinforced Epoxy Composites." Composites Science and Technology, 61(10), 1437-1447.

Rouison, D., Couturier, M., Sain, M., MacMillan, B., and Balcom, B. J. (2005). "Water Absorption of Hemp Fiber/Unsaturated Polyester Composites." Polymer Composites, 26(4), 509525.

Rowell, R. M., Sanadi, A. R., Caulfield, D. F., and Jacobson, R. E. (1997). "Utilization of Natural Fibers in Plastic Composites: Problems and Opportunities." Lignocellulosic-Plastics Composites, 23-51.

Rowell, R. M. (1997). "Potentials for Jute Based Composites." Jute India '97 Expo.

Saha, S. (2011). "A Study on Effect of Fiber Parameters on Mechanical Behaviour of Natural Fiber Based Polymer Composites". National Institute of Technology, Rourkela.

Saheb, D. N., and Jog, J. P. (1999). "Natural Fiber Polymer Composites: A Review." Advances in Polymer Technology, 18(4), 351-363.

Satyanarayana, K. G., Arizaga, G. G. C., and Wypych, F. (2009). "Biodegradable Composites Based on Lignocellulosic Fibers-An Overview." Progress in Polymer Science (Oxford), 34(9), 982-1021.

Satyanarayana, K. G., Pai, B. C., Sukumaran, K., and Pillai, S. G. K. (1990a). "Fabrication and Properties of Lignocellulosic Fiber-Incorporated Polyester Composites." Handbook of Ceramics and Composites, Volume 1, N. P. Cheremisinoff, ed., Marcel Dekker, Inc., New York, 339-386.

Satyanarayana, K. G., Sukumaran, K., Mukherjee, P. S., Pavithran, C., and Pillai, S. G. K. (1990b). "Natural Fibre-Polymer Composites." Cement and Concrete Composites, 12(2), 117136.

Schrass-Christian, S. J., and Billington, S. (2008). "Modeling Biocomposites Using Laminate Plate Theory." 6th International Conference on Computation of Shell and Spatial Structures, IASS-IACM.

Shah, A. N., and Lakkad, S. C. (1981). "Mechanical Properties of Jute-Reinforced Plastics." Fibre Science and Technology, 15(1), 41-46.

Shenton III, H. W., and Wool, R. P. (2004). "Bio-Based Composite Materials for Whole House Design: Potential Applications and Research Needs." NSF-PATH Housing Research Agenda, Volume 2, M. Syal, ed., 327-333.

Sherman, L. M. (1999). "Natural Fibers: The New Fashion in Automotive Plastics." Plastics Technology Online.

Singh, B., and Gupta, M. (2005a). "Natural Fiber Composites for Building Applications." Natural Fibers, Biopolymers, and Biocomposites, A. K. Mohanty, M. Misra, and L. T. Drzal, eds., Taylor \& Francis, Boca Raton, 261-290. 
Singh, B., and Gupta, M. (2005b). "Performance of Pultruded Jute Fibre Reinforced Phenolic Composites as Building Materials for Door Frame." Journal of Polymers and the Environment, 13(2), 127-137.

Singh, B., Gupta, M., and Verma, A. (2000). "The Durability of Jute Fibre-Reinforced Phenolic Composites." Composites Science and Technology, 60(4), 581-589.

SIPA. (2009). Green Buildings with SIPs. Structural Insulated Panel Association (SIPA).

Sorathia, U., Lyon, R., Gann, R. G., and Gritzo, L. (1997). "Materials and Fire Threat." Fire Technology, 33(3), 260-275.

Stamboulis, A., Baillie, C. A., Garkhail, S. K., Van Melick, H. G. H., and Peijs, T. (2000). "Environmental Durability of Flax Fibres and Their Composites Based on Polypropylene Matrix." Applied Composite Materials, 7(5-6), 273-294.

Struyk, R. J. (1987). "Assessing Housing Needs and Policy Alternatives in Developing Countries." The Urban Institute, Washington, DC.

Sundaraman, S., Shabeer, A., Chandrashekhara, K., and Schuman, T. (2008). "Impact Behavior of Fiber Reinforced Pultruded Soy-Epoxy Composites." Journal of Biobased Materials and Bioenergy, 2(1), 1-7.

Symington, M. C., Banks, W. M., West, O. D., and Pethrick, R. A. (2009). "Tensile Testing of Cellulose Based Natural Fibers for Structural Composite Applications." Journal of Composite Materials, 43(9), 1083-1108.

Tan, T., Santos, S. F., Savastano Jr., H., and Soboyejo, W. O. (2012). "Fracture and ResistanceCurve Behavior in Hybrid Natural Fiber and Polypropylene Fiber Reinforced Composites." Journal of Materials Science, 47 2864-2874.

Taylor, C. S. (1995). "New Crop FactSHEET: Kenaf."

http://www.hort.purdue.edu/newcrop/cropfactsheets/kenaf.html (June 29, 2012).

Thorpe, D., Ryan, N., and Charles, M. B. (2008). "Environmental Sustainability - A Driver for Innovation in Construction SMEs?" Third International Conference of the Cooperative Research Center for Construction Innovation.

Tipple, G., and Speak, S. (2009). The Hidden Millions: Homelessness in Developing Countries. Routledge, New York, NY.

Triantafillou, T. C., and Gibson, L. J. (1989). "Debonding in Foam-Core Sandwich Panels." Materials and Structures, 22 64-69.

Valadez-Gonzalez, A., Cervantes-Uc, J. M., Olayo, R., and Herrera-Franco, P. J. (1999). "Effect of Fiber Surface Treatment on the Fiber-Matrix Bond Strength of Natural Fiber Reinforced Composites." Composites Part B: Engineering, 30(3), 309-320.

Van de Weyenberg, I., Chi Truong, T., Vangrimde, B., and Verpoest, I. (2006). "Improving the Properties of UD Flax Fibre Reinforced Composites by Applying an Alkaline Fibre Treatment." Composites Part A: Applied Science and Manufacturing, 37(9), 1368-1376.

Van Dessel, S., and Messac, A. (2004). "Advanced Housing Technology: A Path Rooted in Economic and Environmental Concerns." NSF-PATH Housing Research Agenda, Volume 2, M. Syal, ed.,319-326. 
Van Erp, G., Cattell, C., and Ayers, S. (2006). "A Fair Dinkum Approach to Fibre Composites in Civil Engineering." Construction and Building Materials, 20(1-2), 2-10.

van Rijswijk, K., Brouwer, W. D., and Beukers, A. (2001). "Application of Natural Fibre Composites in the Development of Rural Societies." Structures and Materials Laboratory, Delft University of Technology, Delft, Netherlands.

Von Bernuth, R. D. (2004). "The Built Environment and Public Health." NSF-PATH Housing Research Agenda, Volume 2, M. Syal, ed.,274-277.

Wambua, P., Ivens, J., and Verpoest, I. (2003). "Natural Fibres: Can They Replace Glass in Fibre Reinforced Plastics?" Composites Science and Technology, 63(9), 1259-1264.

Wang, J., Shi, S. Q., and Liang, K. (2012). "Comparative Life-cycle Assessment of Sheet Molding Compound Reinforced by Natural Fiber vs. Glass Fiber (Paper PS-50)." Proceedings of the 55th International Conference of Society of Wood Science and Technology, Beijing, China, 1-10.

Wikipedia.org. (2013). "List of U.S. states by population density."

http://en.wikipedia.org/wiki/List_of_U.S._states_by_population_density (March 17, 2013).

Williams, T., Hosur, M., Theodore, M., Netravali, A., Rangari, V., and Jeelani, S. (2011). "Time Effects on Morphology and Bonding Ability in Mercerized Natural Fibers for Composite Reinforcement." International Journal of Polymer Science.

Williams, G. I., and Wool, R. P. (2000). "Composites from Natural Fibers and Soy Oil Resins." Applied Composite Materials, 7(5-6), 421-432.

Winfield, A. G., and Winfield, B. L. (1974). "Reinforced Plastics in Low Cost Housing." Fillers and Reinforcements for Plastics, 134 207-218.

Winfield, A. G. (1979). "Jute Reinforced Polyester Projects for UNIDO/Government of India." Plastics and Rubber International, 4(23).

Wood, K. (2009). "Researchers Nano-Enhance Natural Fiber: Mississippi State University uses Nanoparticle Impregnation to Improve the Performance of Kenaf Fiber in Composites." Composites World.

Xie, Y., Hill, C. A. S., Xiao, Z., Militz, H., and Mai, C. (2010). "Silane Coupling Agents Used for Natural Fiber/Polymer Composites: A Review." Composites Part A: Applied Science and Manufacturing, 41(7), 806-819.

Zafeiropoulos, N. E., Dijon, G. G., and Baillie, C. A. (2007). "A Study of the Effect of Surface Treatments on the Tensile Strength of Flax Fibres: Part I. Application of Gaussian Statistics." Composites Part A: Applied Science and Manufacturing, 38(2), 621-628. 